\title{
MATRIX 1 RESULTS OF THE FYO7 ENHANCED DOE HIGH-LEVEL WASTE MELTER THROUGHPUT STUDIES AT SRNL
}

\author{
F.C. Raszewski \\ T.B. Edwards \\ D.K. Peeler
}

September 2008

Environmental \& Chemical Process Technology Savannah River National Laboratory Aiken, SC 29808

Prepared for the U.S. Department of Energy Under Contract Number DE-AC09-08SR22470

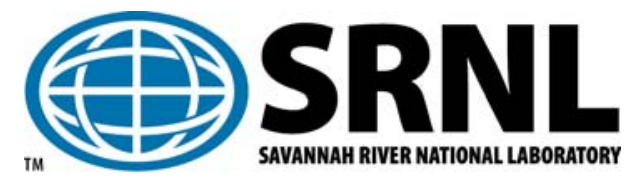




\section{DISCLAIMER}

This work was prepared under an agreement with and funded by the U.S. Government. Neither the U.S. Government or its employees, nor any of its contractors, subcontractors or their employees, makes any express or implied: 1. warranty or assumes any legal liability for the accuracy, completeness, or for the use or results of such use of any information, product, or process disclosed; or 2. representation that such use or results of such use would not infringe privately owned rights; or 3. endorsement or recommendation of any specifically identified commercial product, process, or service. Any views and opinions of authors expressed in this work do not necessarily state or reflect those of the United States Government, or its contractors, or subcontractors.

This document was prepared in conjunction with work accomplished under Contract No. DE-AC09-08SR22470 with the U.S. Department of Energy. 


\section{MATRIX 1 RESULTS OF THE FYO7 ENHANCED DOE HIGH-LEVEL WASTE MELTER THROUGHPUT STUDIES AT SRNL}

F.C. Raszewski

T.B. Edwards

D.K. Peeler

September 2008

Environmental \& Chemical Process Technology Savannah River National Laboratory Aiken, SC 29808 


\section{REVIEWS AND APPROVALS}

\section{AUTHORS:}

F.C. Raszewski, Process Engineering Technology

Date

T.B. Edwards, Statistical Consulting Section

Date

D.K. Peeler, Process Engineering Technology

Date

\section{TECHNICAL REVIEWER:}

K.M. Fox, Process Engineering Technology

Date

\section{APPROVERS:}

J.C. Griffin, Manager, E\&CPT Research Programs

Date

C.C. Herman, Manager, Process Engineering Technology

Date 


\section{EXECUTIVE SUMMARY}

High-level waste (HLW) throughput (i.e., the amount of waste processed per unit time) is a function of two critical parameters: waste loading (WL) and melt rate. For the Waste Treatment and Immobilization Plant (WTP) at the Hanford Site and the Defense Waste Processing Facility (DWPF) at the Savannah River Site (SRS), increasing HLW throughput would significantly reduce the overall mission life cycle costs for the Department of Energy (DOE).

It has been proposed that a team of glass formulation and processing experts at the Pacific Northwest National Laboratory (PNNL), Savannah River National Laboratory (SRNL), and Vitreous State Laboratory (VSL) at Catholic University of America develop a systematic approach to increase HLW throughput (by increasing WL with minimal or positive impacts on melt rate). ${ }^{1}$ Programmatically, this task is aimed at proof-of-principle testing and the development of tools to improve waste loading and melt rate, which will lead to higher waste throughput. The following four specific tasks have been proposed to meet this programmatic objective: 1) Integration and Oversight, 2) Crystal Accumulation Modeling (led by PNNL)/Higher Waste Loading Glasses (led by SRNL), 3) Melt Rate Evaluation and Modeling, and 4) Melter Scale Demonstrations. The details of these tasks can be found in the associated task plan WSRC-STI-2007-00483. The current study is focused on Task 2 (crystal accumulation modeling and higher waste loading glasses) and involves glass formulation and physical property testing by both PNNL and SRNL (as defined in the PNNL and SRNL test plans).

The intent of this report is to document the chemical composition and Product Consistency Test (PCT) results and statistical analysis of PNNL's Test Matrix 1 glasses. Note that this document is only a compilation of the data collected by SRNL for PNNL's glasses in support of this task and no conclusions will be drawn. 


\section{TABLE OF CONTENTS}

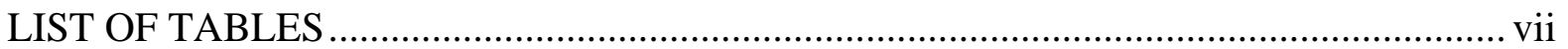

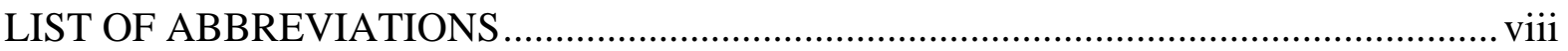

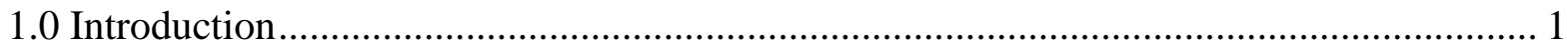

2.0 Experimental Procedures ...................................................................................... 1

2.1.1 Chemical Composition ............................................................................................... 1

2.1.2 Product Consistency Test (PCT) ……………………………………………...... 2

3.0 Quality Assurance (QA) ........................................................................................... 2

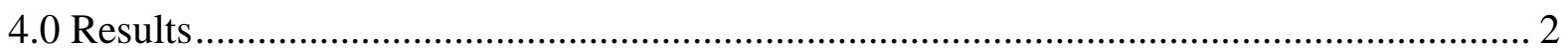

4.1 A Statistical Review of the Chemical Composition Measurements.................................. 2

4.1.1 Measurements in Analytical Sequence …………………………………………....... 3

4.1.2 Batch 1 and LRM Standard Results ..................................................................... 3

4.1.3 Composition Measurements by Glass Number.......................................................... 4

4.1.4 Measured versus Targeted Compositions ………………………………………..... 4

4.2 A Statistical Review of the PCT Results for the Test Matrix 1 Study Glasses ................ 5

4.2.1 Measurements in Analytical Sequence ………………………………………….... 5

4.2.2 Results for the Samples of the Multi-Element Solution Standard ............................. 5

4.2.3 Measurements by Glass Number................................................................................ 5

4.2.4 Normalized PCT Results................................................................................. 6

4.2.5 Effects of Heat Treatment on PCTs ..................................................................... 16

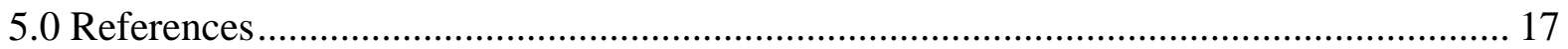




\section{LIST OF TABLES}

Table 1. Results from Samples of the Multi-Element Solution Standard............................................ 6

Table 2. Normalized PCTs by Glass ID/Compositional View ......................................................... 8 


\begin{tabular}{|c|c|}
\hline ANOVA & Analysis of Variance \\
\hline ARM & Approved Reference Material \\
\hline bc & Bias-Corrected \\
\hline CCC & Centerline Canister Cooled \\
\hline DOE & Department Of Energy \\
\hline DWPF & Defense Waste Processing Facility \\
\hline EA & Environmental Assessment \\
\hline EM & Environmental Management \\
\hline HLW & High-Level Waste \\
\hline ICP-AES & Inductively Coupled Plasma - Atomic Emission Spectroscopy \\
\hline ID & Identification \\
\hline LM & Lithium Metaborate \\
\hline LRM & Low-Activity Reference Material \\
\hline $\mathrm{NL}[\mathrm{B}]$ & Normalized Leachate for Boron \\
\hline $\mathrm{NL}[\mathrm{Li}]$ & Normalized Leachate for Lithium \\
\hline $\mathrm{NL}[\mathrm{Na}]$ & Normalized Leachate for Sodium \\
\hline $\mathrm{NL}[\mathrm{Si}]$ & Normalized Leachate for Silicon \\
\hline $\mathrm{PF}$ & Peroxide Fusion \\
\hline $\mathrm{PH}$ & Potassium Hydroxide \\
\hline PCCS & Product Control Composition System \\
\hline PCT & Product Consistency Test \\
\hline PNNL & Pacific Northwest National Laboratory \\
\hline ppm & Parts Per Million \\
\hline PSAL & Process Science Analytical Laboratory \\
\hline QA & Quality Assurance \\
\hline QAP & Quality Assurance Plan \\
\hline SB & Sludge Batch \\
\hline SME & Slurry Mix Evaporator \\
\hline SRNL & Savannah River National Laboratory \\
\hline SRS & Savannah River Site \\
\hline VSL & Vitreous State Laboratory \\
\hline WCP & Waste Compliance Plan \\
\hline WL & Waste Loading \\
\hline wt & Weight \\
\hline
\end{tabular}




\subsection{Introduction}

High-level waste (HLW) throughput (i.e., the amount of waste processed per unit time) is a function of two critical parameters: waste loading (WL) and melt rate. For the Waste Treatment and Immobilization Plant (WTP) at the Hanford Site and the Defense Waste Processing Facility (DWPF) at the Savannah River Site (SRS), increasing HLW throughput would significantly reduce the overall mission life cycle costs for the Department of Energy (DOE).

It has been proposed that a team of glass formulation and processing experts at Pacific Northwest National Laboratory (PNNL), SRNL, and the Vitreous State Laboratory (VSL) at Catholic University of America develop a systematic approach to increase HLW throughput (by increasing WL with minimal or positive impacts on melt rate). ${ }^{1}$ Programmatically, this task is aimed at proof-of-principle testing and the development of tools to improve waste loading and melt rate, which will lead to higher waste throughput. The following four specific tasks have been proposed to meet this programmatic objective: 1) Integration and Oversight, 2) Crystal Accumulation Modeling (led by PNNL)/Higher Waste Loading Glasses (led by SRNL), 3) Melt Rate Evaluation and Modeling, and 4) Melter Scale Demonstrations. The details of these tasks can be found in the associated task plan WSRC-STI-200700483. ${ }^{2}$ The current study is focused on Task 2 (crystal accumulation modeling and higher waste loading glasses) and involves glass formulation and physical property testing by both PNNL and SRNL (as defined in the PNNL and SRNL test plans). ${ }^{1-3}$

The intent of this report is to document the chemical composition and Product Consistency Test (PCT) results and statistical analysis ${ }^{4}$ of PNNL's Test Matrix 1 glasses. $^{\text {a }}$ Note that this document is only a compilation of the data collected by SRNL for PNNL's glasses in support of this task and no conclusions will be drawn.

\subsection{Experimental Procedures}

\subsubsection{Chemical Composition}

To confirm that the as-fabricated (quenched) glasses met the target compositions, a representative sample from each glass was submitted to the SRNL Process Science Analytical Laboratory (PSAL) for chemical analysis under the auspices of an analytical plan (SRNL-SCS-2007-00055). ${ }^{5}$ Three dissolution methods were utilized in measuring these chemical compositions: samples prepared by lithium metaborate (LM) dissolution were used to measure elemental concentrations of aluminum (Al), barium (Ba), bismuth (Bi), calcium (Ca), cadmium (Cd), cerium (Ce), chromium (Cr), iron (Fe), potassium $(\mathrm{K})$, lathanum $(\mathrm{La})$, magnesium $(\mathrm{Mg})$, manganese $(\mathrm{Mn})$, sodium $(\mathrm{Na})$, neodymium $(\mathrm{Nd})$, nickel (Ni), phosphorus (P), lead (Pb), sulfur $(\mathrm{S})$, titanium (Ti), zinc (Zn), and zirconium (Zr), while samples from glasses prepared by peroxide fusion (PF) dissolution were used to measure elemental concentrations of boron (B), lithium (Li), and silicon (Si) and a sample from a select glass was prepared by potassium hydroxide $(\mathrm{PH})$ to conduct a fluorine $(\mathrm{F})$ measurement. For each study glass, measurements were obtained from samples prepared in duplicate by each of these dissolution methods. All of the prepared samples were analyzed (twice for each element of interest) by Inductively Coupled Plasma - Atomic Emission Spectroscopy (ICP-AES). The instrument was recalibrated between the duplicate analyses.

\footnotetext{
${ }^{a}$ As stated in the SRNL and PNNL test plans for this study, 38 quenched and centerline canister cooled (ccc) Test Matrix 1 glasses were fabricated and treated at PNNL and sent to SRNL for chemical composition measurements and PCTs. Note that chemical composition measurements were only conducted on quenched glasses. Test Matrix 1 glasses should not be confused with the 28 Test Matrix 2 glasses that were fabricated at SRNL. The results of these glasses will be documented in a separate report (SRNS-STI-2008-00055).
} 
Samples of two glass standards were included in the analytical plans to provide an opportunity for checking the performance of the instrumentation over the course of the analyses and for potential bias correction. Specifically, several samples of Waste Compliance Plan (WCP) Batch 1 were included in the LM and PF portions of both analytical plans. ${ }^{6}$ The Low-Activity Reference Material (LRM) was included in the $\mathrm{KH}$ portion of the analytical plan. ${ }^{7}$ This standard contains fluorine, which was of interest for the KH-prepared samples of one of the study glasses.

Elemental concentrations were converted to oxide concentrations by multiplying the values for each element by the gravimetric factor for the corresponding oxide. During this process, an elemental concentration that was determined to be below the detection limit of the analytical procedures used by the PSAL was reduced to half of that detection limit as the oxide concentration was determined.

\subsubsection{Product Consistency Test (PCT)}

A 7-day PCT was performed in triplicate on each quenched and cCc glass to assess chemical durability using Method A of the PCT procedure (ASTM C1285-97). ${ }^{8}$ Also included in the experimental test matrix was the Environmental Assessment (EA) glass, the Approved Reference Material (ARM) glass, and blanks from the sample cleaning batch. ${ }^{9}$ Quenched and ccc samples were ground, washed, and prepared according to the standard procedure. ${ }^{10}$ The resulting solutions were sampled (filtered and acidified) and analyzed by PSAL under the auspices of an analytical plan (SRNL-SCS-2007-00059). ${ }^{11}$ Samples of a multi-element, standard solution were also included in the analytical plan (as a check on the accuracy of the ICP-AES). PCTs were also conducted in triplicate for samples of the Environmental Assessment (EA) glass and for samples of the Approved Reference Material (ARM) glass. Blanks (samples consisting only of ASTM Type I water) were also submitted for the PCT.

Normalized release rates were calculated based on target, measured, and bias-corrected (bc) compositions using the average of the logs of the leachate concentrations.

\subsection{Quality Assurance (QA)}

SRNL's work scope was performed in accordance with 1Q, QAP with supplemental quality assurance requirements as defined by L1, 8.21, Supplemental QA Requirements for DOE/RW-0333P also being applied (although not specified by the DOE-EM customer. Specific activities conducted in accordance with the SRNL DOE/RW-0333P compliant QA program include chemical composition measurements and PCTs. In addition, the $\mathrm{JMP}^{\mathrm{TM}}$ software used to support the analysis of the Test Matrix 1 glasses is controlled under WSRC-RP-99-00422 (Software QA Plan and Verification \& Validation for Commercial Statistical Packages Utilized by the Statistical Consulting Section of SRNL). ${ }^{4,12}$

\subsection{Results}

\subsection{A Statistical Review of the Chemical Composition Measurements}

Target compositions of each of 36 Test Matrix 1 glasses are provided in Table A1 (a two-part table) of Appendix A.

Table A2 in Appendix A provides the elemental concentration measurements derived from the samples prepared using LM, Table A3 in Appendix A provides the measurements derived from the samples prepared using PF, and Table A4 in Appendix A provides the measurements derived from 
the limited number of samples prepared using $\mathrm{KH}$. The values for the standards are also provided in these tables.

In the following sections, the analytical sequences of the measurements are explored, the measurements of the standards are investigated and used for bias correction, the measurements for each glass are reviewed, the average chemical compositions (measured and bias-corrected) for each glass are determined, and comparisons are made between the measurements and the targeted compositions for the glasses.

\subsubsection{Measurements in Analytical Sequence}

Figure A1 in Appendix A provides plots in analytical sequence for each analyte of the measurements generated by the PSAL grouped by preparation method. Different symbols and colors are used to represent each of the study and standard glasses. These plots include all of the measurement data from Tables A2, A3, and A4. While these plots provide only a limited opportunity for revealing patterns in the data, a review of these plots indicates no significant patterns or trends in the analytical process over the course of these measurements, and there appear to be no dramatic outliers in these chemical composition measurements.

\subsubsection{Batch 1 and LRM Standard Results}

In this section, the PSAL measurements of the chemical compositions of the Batch 1 and LRM samples are reviewed. These measurements are investigated across the ICP-AES analytical sets and blocks, and the results are used to bias correct the measurements for the study glasses.

Figure A2 in Appendix A provides statistical analyses of the results for each analyte grouped by preparation method by analytical set/block/sub-block. The results include analysis of variance (ANOVA) investigations looking for statistically significant differences between the means of these groups for each of the analytes for each of the standards. The results from the statistical tests for the Batch 1 standard may be summarized as follows: $\mathrm{Al}_{2} \mathrm{O}_{3}, \mathrm{BaO}, \mathrm{Ce}_{2} \mathrm{O}_{3}$ (a detection limit effect), $\mathrm{Cr}_{2} \mathrm{O}_{3}$, $\mathrm{Nd}_{2} \mathrm{O}_{3}, \mathrm{SiO}_{2}$, and $\mathrm{ZrO}_{2}$ have measurements that indicate a significant ICP-AES calibration effect on the block averages at the 5\% significance level. There was no indication of a statistically significant effect for the F results for the LRM standard; however, the measurements for this analyte did fall below the targeted value of $1 \mathrm{wt} \%$ for the LRM samples. The reference value for the oxide concentration of the standard is given in the header for each set of measurements in the figure.

Some of these results provide incentive for adjusting the measurements by the effects of the ICP-AES calibration and analytical process; therefore, the oxide measurements of the study glasses were bias corrected for the effect of the ICP-AES calibration on each of the analytical sets, blocks and subblocks. The basis for this bias correction is presented as part of Figure $\mathrm{A} 2$ - the average measurements for Batch 1 for each ICP-AES set/block/sub-block for $\mathrm{Al}_{2} \mathrm{O}_{3}, \mathrm{~B}_{2} \mathrm{O}_{3}, \mathrm{BaO}, \mathrm{CaO}, \mathrm{Cr}_{2} \mathrm{O}_{3}$, $\mathrm{Fe}_{2} \mathrm{O}_{3}, \mathrm{Li}_{2} \mathrm{O}, \mathrm{MgO}, \mathrm{MnO}, \mathrm{Na}_{2} \mathrm{O}, \mathrm{Nd}_{2} \mathrm{O}_{3}, \mathrm{NiO}, \mathrm{SiO}_{2}$, and $\mathrm{TiO}_{2}$ and the average measurement for LRM for each ICP-AES set/block/sub-block for F. The results for the standards were used to conduct the bias correction as long as the reference value for the analyte concentration in the standard glass was greater than or equal to $0.1 \mathrm{wt} \%$. Thus, applying this approach and based upon the information in the figures, bias correction was conducted for $\mathrm{Al}_{2} \mathrm{O}_{3}, \mathrm{~B}_{2} \mathrm{O}_{3}, \mathrm{BaO}, \mathrm{CaO}, \mathrm{Cr}_{2} \mathrm{O}_{3}, \mathrm{~F}, \mathrm{Fe}_{2} \mathrm{O}_{3}, \mathrm{~K}_{2} \mathrm{O}, \mathrm{Li}_{2} \mathrm{O}, \mathrm{MgO}$, $\mathrm{MnO}, \mathrm{Na}_{2} \mathrm{O}, \mathrm{Nd}_{2} \mathrm{O}_{3}, \mathrm{NiO}, \mathrm{SiO}_{2}$, and $\mathrm{TiO}_{2}$ measurements. No bias correction was conducted for $\mathrm{Bi}_{2} \mathrm{O}_{3}$, $\mathrm{CdO}, \mathrm{Ce}_{2} \mathrm{O}_{3}, \mathrm{La}_{2} \mathrm{O}_{3}, \mathrm{P}_{2} \mathrm{O}_{5}, \mathrm{PbO}, \mathrm{ZnO}$, or $\mathrm{ZrO}_{2}$. 
The bias correction was conducted as follows. For each analyte, let $\overline{\mathrm{a}}_{\mathrm{ij}}$ be the average measurement for the $\mathrm{i}^{\text {th }}$ analyte at analytical group $\mathrm{j}$ for the standard glass, and let $\mathrm{t}_{\mathrm{i}}$ be the reference value for the $\mathrm{i}^{\text {th }}$ analyte for the standard glass. (The averages and reference values are provided in Figure A2.). Let $\overline{\mathrm{c}}_{\mathrm{ijk}}$ be the average measurement for the $\mathrm{i}^{\text {th }}$ analyte at analytical group $\mathrm{j}$ for the $\mathrm{k}^{\text {th }}$ glass. The bias adjustment was conducted as follows

$$
\bar{c}_{i j k} \bullet\left(1-\frac{\bar{a}_{i j}-t_{i}}{\bar{a}_{i j}}\right)=\bar{c}_{i j k} \bullet \frac{t_{i}}{\bar{a}_{i j}}
$$

Bias-corrected measurements are indicated by a "bc" suffix, and such adjustments were performed for all of the oxides of this study except for $\mathrm{Bi}_{2} \mathrm{O}_{3}, \mathrm{CdO}, \mathrm{Ce}_{2} \mathrm{O}_{3}, \mathrm{La}_{2} \mathrm{O}_{3}, \mathrm{P}_{2} \mathrm{O}_{5}, \mathrm{PbO}, \mathrm{ZnO}$, and $\mathrm{ZrO}_{2}$. Both measured and measured " $\mathrm{bc}$ " values are included in the discussion that follows. In these discussions bias-corrected values for $\mathrm{Bi}_{2} \mathrm{O}_{3}, \mathrm{CdO}, \mathrm{Ce}_{2} \mathrm{O}_{3}, \mathrm{La}_{2} \mathrm{O}_{3}, \mathrm{P}_{2} \mathrm{O}_{5}, \mathrm{PbO}, \mathrm{ZnO}$, and $\mathrm{ZrO}_{2}$, which are the same as the original values, are included for completeness (e.g., to allow a sum of oxides to be computed for the bias-corrected results).

\subsubsection{Composition Measurements by Glass Number}

Figure A3 in Appendix A provides plots of the analyte concentration measurements by Lab ID within Glass ID (including Batch 1 and LRM) sorted by target values for the measured and bias-corrected (bc) values grouped by preparation method. Different symbols and colors are used to represent the different glasses. These plots demonstrate the individual measurements across the duplicates of each preparation method and the two ICP-AES calibrations. In general, the repeatability of the four individual measurements appears to be consistent.

\subsubsection{Measured versus Targeted Compositions}

The four measurements of each analyte were averaged to determine a representative chemical composition for each glass. These determinations were conducted both for the measured and for the bias-corrected data. A sum of oxides was also computed for each glass based upon both the measured and bias-corrected values except for the LRM glass. Figure A4 in Appendix A provides plots showing results for each glass for each oxide to help highlight the comparisons among the measured, bias-corrected, and targeted values. In general, the measured values are consistent with the target values; however, the measured values of $\mathrm{La}_{2} \mathrm{O}_{3}, \mathrm{P}_{2} \mathrm{O}_{5}, \mathrm{PbO}$ and $\mathrm{ZrO}_{2}$ are consistently low.

Table A5 in Appendix A provides a summary of the average compositions as well as the targeted compositions and some associated differences and relative differences. All of the sums of oxides (both measured and bias-corrected) for the study glasses fall within the interval of 95 to $105 \mathrm{wt} \%$ except for one of the Batch 1 glasses and for EM07-Si-37, which fell just below 95\%. Entries in Table A5 show the relative differences between the measured or bias-corrected values and the targeted values. These differences are shaded when they are greater than or equal to $5 \%$. Overall, there are only minor differences between the measured and targeted compositions for some of the oxides in some of glasses. 


\subsection{A Statistical Review of the PCT Results for the Test Matrix 1 Study Glasses}

Table B1 in Appendix B provides the elemental leachate concentration measurements determined by the PSAL for the solution samples generated by the PCTs by analytical set. One of the quality control checkpoints for the PCT procedure is solution-weight loss over the course of the 7-day test. No water loss issues were observed in any of the samples. Any measurement in Table B1 below the detection limit of the analytical procedure (indicated by a "<”) was replaced by $1 / 2$ of the detection limit in subsequent analyses. In addition to adjustments for detection limits, the values were adjusted for the dilution factors: the values for the study glasses, the blanks, and the ARM glass in Table B1 were multiplied by 1.6667 to determine the values in parts per million (ppm) and the values for EA were multiplied by 16.6667. Table B2 in Appendix B provides the resulting measurements.

In the following sections, the analytical sequence of the measurements over the four sets of PCTs is explored, the measurements of the standards are investigated and used to assess the overall accuracy of the ICP-AES measurement process, the measurements for each glass are reviewed, plots are provided that explore the effects of heat treatment on the PCTs for these glasses, and the PCTs are normalized using the compositions (targeted, measured, and bias-corrected) presented in Table A4.

\subsubsection{Measurements in Analytical Sequence}

Figures B1 and B2 in Appendix B provide plots of the leachate (ppm) concentrations in analytical sequence as generated by the PSAL for all of the data and for the data from only the study glasses, respectively. A different color and symbol are used to distinguish the Test Matrix 1 study glasses from the standards. No problems are seen in these plots.

\subsubsection{Results for the Samples of the Multi-Element Solution Standard}

Figure B3 in Appendix B provides analyses of the PSAL measurements of the samples of the multielement solution standard by analytical set by ICP-AES block. An analysis of variance (ANOVA) investigating for statistically significant differences among the block averages for these samples for each element of interest is included in these figures. These results indicate a statistically significant (at approximately a 5\% level) difference among the Li, $\mathrm{Na}$ and $\mathrm{Si}$ average measurements over these sets and blocks. No bias correction of the PCT results for the study glasses was conducted. This approach was taken since the triplicate PCTs for a single study glass were placed in different ICPAES blocks. Averaging the ppm's for each set of triplicates helps to minimize the impact of the ICPAES effects.

Table 1 summarizes the average measurements and the reference values for the 4 primary elements of interest. The results indicate consistent and accurate measurements from the PSAL processes used to conduct these analyses.

\subsubsection{Measurements by Glass Number}

Figure B4 in Appendix B provide plots of the leachate concentrations for each type of submitted sample by analytical set: the study glasses by heat treatment and the standards (EA, ARM, the multielement solution standard, and blanks). These plots allow for the assessment of the repeatability of the measurements, which suggests some scatter in the triplicate values for some analytes for some of the glasses. Also, note that some differences between the values for the two heat treatments for some glasses are evident. More will be said regarding comparisons between the heat treatments in the discussions that follow. 
Table 1. Results from Samples of the Multi-Element Solution Standard

\begin{tabular}{|c|c|c|c|c|c|}
\hline Analytical & Analytical & Avg B & Avg Li & Avg Na & Avg Si \\
\hline Set & Block & $\mathbf{( p p m )}$ & $\mathbf{( p p m )}$ & $\mathbf{( p p m )}$ & $\mathbf{( p p m )}$ \\
\hline 1 & 1 & 19.9 & 9.9 & 82.7 & 50.2 \\
\hline & 2 & 20.3 & 9.8 & 82.0 & 49.9 \\
\hline & 3 & 19.6 & 9.7 & 82.3 & 48.8 \\
\hline 2 & 1 & 21.0 & 9.7 & 80.0 & 48.5 \\
\hline & 2 & 20.0 & 9.7 & 79.6 & 47.9 \\
\hline & 3 & 20.1 & 9.6 & 77.7 & 48.3 \\
\hline 3 & 1 & 21.3 & 10.1 & 80.8 & 52.2 \\
\hline & 2 & 20.5 & 9.9 & 83.0 & 51.1 \\
\hline & 3 & 21.0 & 10.0 & 81.5 & 53.1 \\
\hline & 1 & 20.8 & 10.0 & 81.0 & 52.4 \\
\hline & 2 & 20.4 & 9.9 & 81.8 & 51.7 \\
\hline & 3 & 20.3 & 9.8 & 81.6 & 52.2 \\
\hline & $\begin{array}{c}\text { Grand } \\
\text { Average }\end{array}$ & 20.4 & 9.9 & 81.2 & 50.5 \\
\hline & $\begin{array}{c}\text { Reference } \\
\text { Value }\end{array}$ & 20 & 10 & 81 & 50 \\
\hline & $\begin{array}{c}\text { \% } \\
\text { difference }\end{array}$ & $2.15 \%$ & $-1.48 \%$ & $0.21 \%$ & $1.06 \%$ \\
\hline
\end{tabular}

\subsubsection{Normalized PCT Results}

PCT leachate concentrations are typically normalized using the cation composition (expressed as a weight percent) in the glass to obtain a grams-per-liter $(\mathrm{g} / \mathrm{L})$ leachate concentration. Two of the three replicate PCTs for a glass (whether the quenched or the cсc version) must be available for this computation to be completed. Otherwise, no PCT can be reported for the glass. The normalization of the PCTs is usually conducted using the measured compositions of the glasses. This is the preferred normalization process for the PCTs. For completeness, the targeted cation and the bias-corrected cation compositions were also used to conduct this normalization.

As is the usual convention, the common logarithm of the normalized PCT (normalized leachate, NL) for each element of interest was determined and used for comparison. To accomplish this computation, one must

1.Determine the common logarithm of the elemental parts per million (ppm) leachate concentration for each of the triplicates and each of the elements of interest (these values are provided in Table B2 of Appendix B),

2.Average the common logarithms over the triplicates for each element of interest, and then

Normalizing Using Measured Composition (preferred method)

3. Subtract a quantity equal to 1 plus the common logarithm of the average cation measured concentration (expressed as a weight percent of the glass) from the average computed in step 2 . 


\section{Or Normalizing Using Target Composition}

3. Subtract a quantity equal to 1 plus the common logarithm of the target cation concentration (expressed as a weight percent of the glass) from the average computed in step 2.

\section{Or Normalizing Using Measured Bias-Corrected Composition}

3. Subtract a quantity equal to 1 plus the common logarithm of the measured biascorrected cation concentration (expressed as a weight percent of the glass) from the average computed in step 2.

Figure B5 in Appendix B provides scatter plots for these results and offers an opportunity to investigate the consistency in the leaching across the elements for the glasses of this study. All combinations of the normalizations of the PCTs (i.e., those generated using the targeted, measured, and bias-corrected compositional views) and both heat treatments are represented in the series of scatter plots. Consistency in the leaching across the elements is typically demonstrated by a high degree of linear correlation among the values for pairs of these elements. The smallest correlation in this plot is between $\mathrm{Li}$ and $\mathrm{Si}$, with a value of $\sim 53 \%$, which is a lower correlation than typically seen for a set of PCTs.

Table 2 summarizes the normalized PCTs for the glasses of this study. The glasses are by glass identifier. Note that 4 values are shown for ARM and EA corresponding to the 4 analytical sets that were used to conduct all of these PCT measurements. 
Table 2. Normalized PCTs by Glass ID/Compositional View

\begin{tabular}{|c|c|c|c|c|c|c|c|c|c|c|}
\hline Glass ID & $\begin{array}{c}\text { Heat } \\
\text { Treatment }\end{array}$ & Comp View & $\begin{array}{c}\log N L \\
{[B(g / L)]}\end{array}$ & $\begin{array}{c}\log \mathrm{NL} \\
{[\mathrm{Li}(\mathrm{g} / \mathrm{L})]}\end{array}$ & $\begin{array}{c}\log \mathrm{NL} \\
{[\mathrm{Na}(\mathrm{g} / \mathrm{L})]}\end{array}$ & $\begin{array}{c}\log \mathrm{NL} \\
{[\mathrm{Si}(\mathrm{g} / \mathrm{L})]} \\
\end{array}$ & NL B(g/L) & NL Li (g/L) & $\mathrm{NL} \mathrm{Na}(\mathrm{g} / \mathrm{L})$ & NL Si (g/L) \\
\hline ARM & ref & reference & -0.3313 & -0.2550 & -0.2811 & -0.5742 & 0.466 & 0.556 & 0.524 & 0.267 \\
\hline ARM & ref & reference & -0.3429 & -0.2503 & -0.3315 & -0.5717 & 0.454 & 0.562 & 0.466 & 0.268 \\
\hline ARM & ref & reference & -0.1953 & -0.1574 & -0.2168 & -0.4856 & 0.638 & 0.696 & 0.607 & 0.327 \\
\hline ARM & ref & reference & -0.2230 & -0.1768 & -0.2201 & -0.4942 & 0.598 & 0.666 & 0.602 & 0.320 \\
\hline EA & ref & reference & 1.2552 & 0.9836 & 1.1413 & 0.5923 & 17.998 & 9.629 & 13.845 & 3.911 \\
\hline EA & ref & reference & 1.2408 & 0.9694 & 1.1223 & 0.5794 & 17.411 & 9.320 & 13.252 & 3.797 \\
\hline EA & ref & reference & 1.2790 & 0.9961 & 1.1460 & 0.6192 & 19.012 & 9.910 & 13.995 & 4.161 \\
\hline EA & ref & reference & 1.1777 & 0.9130 & 1.0644 & 0.5588 & 15.057 & 8.184 & 11.600 & 3.620 \\
\hline EM07-Al-06 & $\mathrm{CCC}$ & measured & -0.2335 & -0.2687 & -0.2942 & -0.5796 & 0.584 & 0.539 & 0.508 & 0.263 \\
\hline EM07-Al-06 & quenched & measured & -0.1695 & -0.2207 & -0.1966 & -0.4631 & 0.677 & 0.602 & 0.636 & 0.344 \\
\hline EM07-Al-06 & $\mathrm{CCC}$ & measured bc & -0.2324 & -0.2699 & -0.2951 & -0.6110 & 0.586 & 0.537 & 0.507 & 0.245 \\
\hline EM07-Al-06 & quenched & measured bc & -0.1684 & -0.2218 & -0.1975 & -0.4946 & 0.679 & 0.600 & 0.635 & 0.320 \\
\hline EM07-Al-06 & $\mathrm{CCC}$ & targeted & -0.2273 & -0.2850 & -0.2904 & -0.6007 & 0.593 & 0.519 & 0.512 & 0.251 \\
\hline EM07-Al-06 & quenched & targeted & -0.1633 & -0.2369 & -0.1928 & -0.4843 & 0.687 & 0.580 & 0.641 & 0.328 \\
\hline EM07-Al-15 & $\mathrm{CCC}$ & measured & -0.4037 & -0.3075 & -0.3968 & -0.4878 & 0.395 & 0.493 & 0.401 & 0.325 \\
\hline EM07-Al-15 & quenched & measured & -0.3592 & -0.2575 & -0.3502 & -0.5156 & 0.437 & 0.553 & 0.446 & 0.305 \\
\hline EM07-Al-15 & $\mathrm{CCC}$ & measured bc & -0.4026 & -0.3087 & -0.3977 & -0.5192 & 0.396 & 0.491 & 0.400 & 0.303 \\
\hline EM07-Al-15 & quenched & measured bc & -0.3581 & -0.2587 & -0.3511 & -0.5471 & 0.438 & 0.551 & 0.446 & 0.284 \\
\hline EM07-Al-15 & $\mathrm{CCC}$ & targeted & -0.3946 & -0.3189 & -0.4024 & -0.4913 & 0.403 & 0.480 & 0.396 & 0.323 \\
\hline EM07-Al-15 & quenched & targeted & -0.3501 & -0.2689 & -0.3558 & -0.5191 & 0.447 & 0.538 & 0.441 & 0.303 \\
\hline EM07-Al-20 & $\mathrm{CCC}$ & measured & -0.3362 & -0.1878 & -0.4210 & -0.4572 & 0.461 & 0.649 & 0.379 & 0.349 \\
\hline EM07-Al-20 & quenched & measured & -0.3106 & -0.1162 & -0.3491 & -0.3942 & 0.489 & 0.765 & 0.448 & 0.403 \\
\hline EM07-Al-20 & $\mathrm{CCC}$ & measured bc & -0.3300 & -0.1894 & -0.4190 & -0.4887 & 0.468 & 0.647 & 0.381 & 0.325 \\
\hline EM07-Al-20 & quenched & measured bc & -0.3044 & -0.1177 & -0.3471 & -0.4257 & 0.496 & 0.763 & 0.450 & 0.375 \\
\hline EM07-Al-20 & $\mathrm{CCC}$ & targeted & -0.3141 & -0.1908 & -0.4162 & -0.4580 & 0.485 & 0.644 & 0.384 & 0.348 \\
\hline EM07-Al-20 & quenched & targeted & -0.2885 & -0.1192 & -0.3443 & -0.3949 & 0.515 & 0.760 & 0.453 & 0.403 \\
\hline EM07-B-05 & $\mathrm{CCC}$ & measured & -0.2567 & -0.3210 & -0.2703 & -0.4689 & 0.554 & 0.478 & 0.537 & 0.340 \\
\hline EM07-B-05 & quenched & measured & -0.2849 & -0.3251 & -0.2460 & -0.4848 & 0.519 & 0.473 & 0.568 & 0.327 \\
\hline EM07-B-05 & $\mathrm{CCC}$ & measured bc & -0.2519 & -0.3172 & -0.2582 & -0.4803 & 0.560 & 0.482 & 0.552 & 0.331 \\
\hline
\end{tabular}


Table 2 cont. Normalized PCTs by Glass ID/Compositional View

\begin{tabular}{|c|c|c|c|c|c|c|c|c|c|c|}
\hline Glass ID & $\begin{array}{c}\text { Heat } \\
\text { Treatment } \\
\end{array}$ & Comp View & $\begin{array}{c}\log \mathrm{NL} \\
{[\mathrm{B}(\mathrm{g} / \mathrm{L})]}\end{array}$ & $\begin{array}{c}\log \mathrm{NL} \\
{[\mathrm{Li}(\mathrm{g} / \mathrm{L})]}\end{array}$ & $\begin{array}{c}\log \mathrm{NL} \\
{[\mathrm{Na}(\mathrm{g} / \mathrm{L})]}\end{array}$ & $\begin{array}{c}\log \mathrm{NL} \\
{[\mathrm{Si}(\mathrm{g} / \mathrm{L})]} \\
\end{array}$ & NL B(g/L) & NL Li (g/L) & NL Na $(\mathrm{g} / \mathrm{L})$ & NL Si $(g / L)$ \\
\hline EM07-B-05 & quenched & measured bc & -0.2801 & -0.3213 & -0.2339 & -0.4962 & 0.525 & 0.477 & 0.584 & 0.319 \\
\hline EM07-B-05 & $\mathrm{CCC}$ & targeted & -0.2349 & -0.3197 & -0.2547 & -0.4767 & 0.582 & 0.479 & 0.556 & 0.334 \\
\hline EM07-B-05 & quenched & targeted & -0.2632 & -0.3237 & -0.2304 & -0.4926 & 0.546 & 0.475 & 0.588 & 0.322 \\
\hline EM07-B-15 & $\mathrm{CCC}$ & measured & 0.2403 & 0.1483 & -0.0019 & -0.5608 & 1.739 & 1.407 & 0.996 & 0.275 \\
\hline EM07-B-15 & quenched & measured & 0.1320 & 0.0577 & -0.0647 & -0.5942 & 1.355 & 1.142 & 0.862 & 0.255 \\
\hline EM07-B-15 & $\mathrm{CCC}$ & measured bc & 0.2451 & 0.1520 & 0.0103 & -0.5722 & 1.758 & 1.419 & 1.024 & 0.268 \\
\hline EM07-B-15 & quenched & measured bc & 0.1367 & 0.0614 & -0.0526 & -0.6056 & 1.370 & 1.152 & 0.886 & 0.248 \\
\hline EM07-B-15 & $\mathrm{CCC}$ & targeted & 0.2421 & 0.1532 & 0.0100 & -0.5667 & 1.746 & 1.423 & 1.023 & 0.271 \\
\hline EM07-B-15 & quenched & targeted & 0.1338 & 0.0626 & -0.0529 & -0.6000 & 1.361 & 1.155 & 0.885 & 0.251 \\
\hline EM07-B-20 & $\mathrm{CCC}$ & measured & 0.9968 & 0.9231 & 0.7276 & -0.7238 & 9.926 & 8.378 & 5.341 & 0.189 \\
\hline EM07-B-20 & quenched & measured & 0.8219 & 0.7525 & 0.5635 & -0.7202 & 6.636 & 5.656 & 3.660 & 0.190 \\
\hline EM07-B-20 & $\mathrm{CCC}$ & measured bc & 1.0015 & 0.9269 & 0.7403 & -0.7352 & 10.035 & 8.451 & 5.500 & 0.184 \\
\hline EM07-B-20 & quenched & measured bc & 0.8266 & 0.7563 & 0.5761 & -0.7316 & 6.709 & 5.705 & 3.768 & 0.186 \\
\hline EM07-B-20 & $\mathrm{CCC}$ & targeted & 0.9979 & 0.9306 & 0.7358 & -0.7276 & 9.952 & 8.524 & 5.442 & 0.187 \\
\hline EM07-B-20 & quenched & targeted & 0.8230 & 0.7600 & 0.5716 & -0.7239 & 6.653 & 5.755 & 3.729 & 0.189 \\
\hline EM07-Bi-025 & $\mathrm{CCC}$ & measured & -0.2873 & -0.2934 & -0.3270 & -0.5180 & 0.516 & 0.509 & 0.471 & 0.303 \\
\hline EM07-Bi-025 & quenched & measured & -0.2886 & -0.2775 & -0.2825 & -0.5319 & 0.515 & 0.528 & 0.522 & 0.294 \\
\hline EM07-Bi-025 & $\mathrm{CCC}$ & measured bc & -0.2780 & -0.2859 & -0.3144 & -0.5206 & 0.527 & 0.518 & 0.485 & 0.302 \\
\hline EM07-Bi-025 & quenched & measured bc & -0.2793 & -0.2700 & -0.2698 & -0.5345 & 0.526 & 0.537 & 0.537 & 0.292 \\
\hline EM07-Bi-025 & $\mathrm{CCC}$ & targeted & -0.2712 & -0.2891 & -0.3250 & -0.5162 & 0.536 & 0.514 & 0.473 & 0.305 \\
\hline EM07-Bi-025 & quenched & targeted & -0.2725 & -0.2733 & -0.2805 & -0.5301 & 0.534 & 0.533 & 0.524 & 0.295 \\
\hline EM07-Bi-05 & $\mathrm{CCC}$ & measured & -0.3275 & -0.2815 & -0.3125 & -0.5219 & 0.470 & 0.523 & 0.487 & 0.301 \\
\hline EM07-Bi-05 & quenched & measured & -0.3553 & -0.2849 & -0.2941 & -0.5426 & 0.441 & 0.519 & 0.508 & 0.287 \\
\hline EM07-Bi-05 & CCC & measured bc & -0.3181 & -0.2740 & -0.3004 & -0.5246 & 0.481 & 0.532 & 0.501 & 0.299 \\
\hline EM07-Bi-05 & quenched & measured bc & -0.3459 & -0.2774 & -0.2820 & -0.5452 & 0.451 & 0.528 & 0.522 & 0.285 \\
\hline EM07-Bi-05 & $\mathrm{CCC}$ & targeted & -0.3049 & -0.2714 & -0.3022 & -0.5172 & 0.496 & 0.535 & 0.499 & 0.304 \\
\hline EM07-Bi-05 & quenched & targeted & -0.3327 & -0.2747 & -0.2838 & -0.5379 & 0.465 & 0.531 & 0.520 & 0.290 \\
\hline EM07-BL-1 & $\mathrm{CCC}$ & measured & -0.3527 & -0.3205 & -0.3504 & -0.4870 & 0.444 & 0.478 & 0.446 & 0.326 \\
\hline EM07-BL-1 & quenched & measured & -0.3300 & -0.3162 & -0.3192 & -0.5106 & 0.468 & 0.483 & 0.480 & 0.309 \\
\hline
\end{tabular}


Table 2 cont. Normalized PCTs by Glass ID/Compositional View

\begin{tabular}{|c|c|c|c|c|c|c|c|c|c|c|}
\hline Glass ID & $\begin{array}{c}\text { Heat } \\
\text { Treatment } \\
\end{array}$ & Comp View & $\begin{array}{c}\log \mathrm{NL} \\
{[\mathrm{B}(\mathrm{g} / \mathrm{L})]}\end{array}$ & $\begin{array}{c}\log \mathrm{NL} \\
{[\mathrm{Li}(\mathrm{g} / \mathrm{L})]}\end{array}$ & $\begin{array}{c}\log \mathrm{NL} \\
{[\mathrm{Na}(\mathrm{g} / \mathrm{L})]}\end{array}$ & $\begin{array}{c}\log \mathrm{NL} \\
{[\mathrm{Si}(\mathrm{g} / \mathrm{L})]}\end{array}$ & $\mathrm{NL} \mathrm{B}(\mathrm{g} / \mathrm{L})$ & NL Li (g/L) & NL Na (g/L) & NL Si (g/L) \\
\hline EM07-BL-1 & $\mathrm{CCC}$ & measured bc & -0.3516 & -0.3217 & -0.3513 & -0.5185 & 0.445 & 0.477 & 0.445 & 0.303 \\
\hline EM07-BL-1 & quenched & measured bc & -0.3289 & -0.3174 & -0.3201 & -0.5420 & 0.469 & 0.482 & 0.479 & 0.287 \\
\hline EM07-BL-1 & $\mathrm{ccC}$ & targeted & -0.3479 & -0.3291 & -0.3399 & -0.4963 & 0.449 & 0.469 & 0.457 & 0.319 \\
\hline EM07-BL-1 & quenched & targeted & -0.3252 & -0.3247 & -0.3087 & -0.5199 & 0.473 & 0.473 & 0.491 & 0.302 \\
\hline EM07-BL-2 & $\mathrm{ccC}$ & measured & -0.3366 & -0.3302 & -0.3374 & -0.5069 & 0.461 & 0.467 & 0.460 & 0.311 \\
\hline EM07-BL-2 & quenched & measured & -0.3306 & -0.3140 & -0.3053 & -0.5193 & 0.467 & 0.485 & 0.495 & 0.302 \\
\hline EM07-BL-2 & $\mathrm{ccC}$ & measured bc & -0.3304 & -0.3317 & -0.3353 & -0.5383 & 0.467 & 0.466 & 0.462 & 0.290 \\
\hline EM07-BL-2 & quenched & measured bc & -0.3244 & -0.3156 & -0.3033 & -0.5508 & 0.474 & 0.484 & 0.497 & 0.281 \\
\hline EM07-BL-2 & $\mathrm{ccc}$ & targeted & -0.3284 & -0.3396 & -0.3524 & -0.5200 & 0.469 & 0.457 & 0.444 & 0.302 \\
\hline EM07-BL-2 & quenched & targeted & -0.3224 & -0.3234 & -0.3203 & -0.5324 & 0.476 & 0.475 & 0.478 & 0.293 \\
\hline EM07-Ca-035 & $\mathrm{ccC}$ & measured & -0.3473 & -0.3104 & -0.3025 & -0.5918 & 0.450 & 0.489 & 0.498 & 0.256 \\
\hline EM07-Ca-035 & quenched & measured & -0.7477 & -0.5796 & -1.2019 & -1.5052 & 0.179 & 0.263 & 0.063 & 0.031 \\
\hline EM07-Ca-035 & $\mathrm{CCC}$ & measured bc & -0.3425 & -0.3066 & -0.2904 & -0.6032 & 0.454 & 0.494 & 0.512 & 0.249 \\
\hline EM07-Ca-035 & quenched & measured bc & -0.7430 & -0.5759 & -1.1897 & -1.5166 & 0.181 & 0.266 & 0.065 & 0.030 \\
\hline EM07-Ca-035 & $\mathrm{CCC}$ & targeted & -0.3302 & -0.3113 & -0.2960 & -0.5989 & 0.468 & 0.488 & 0.506 & 0.252 \\
\hline EM07-Ca-035 & quenched & targeted & -0.7306 & -0.5806 & -1.1954 & -1.5124 & 0.186 & 0.263 & 0.064 & 0.031 \\
\hline EM07-Ca-07 & $\mathrm{ccC}$ & measured & -0.3275 & -0.2409 & -0.2321 & -0.6603 & 0.470 & 0.574 & 0.586 & 0.219 \\
\hline EM07-Ca-07 & quenched & measured & -0.1761 & -0.1679 & -0.1268 & -0.5933 & 0.667 & 0.679 & 0.747 & 0.255 \\
\hline EM07-Ca-07 & CCC & measured bc & -0.3183 & -0.2333 & -0.2200 & -0.6629 & 0.481 & 0.584 & 0.603 & 0.217 \\
\hline EM07-Ca-07 & quenched & measured bc & -0.1668 & -0.1603 & -0.1147 & -0.5959 & 0.681 & 0.691 & 0.768 & 0.254 \\
\hline EM07-Ca-07 & $\mathrm{CCC}$ & targeted & -0.3064 & -0.2312 & -0.2186 & -0.6582 & 0.494 & 0.587 & 0.604 & 0.220 \\
\hline EM07-Ca-07 & quenched & targeted & -0.1549 & -0.1582 & -0.1133 & -0.5913 & 0.700 & 0.695 & 0.770 & 0.256 \\
\hline EM07-Cr-001 & $\mathrm{CCC}$ & measured & -0.2991 & -0.2656 & -0.3166 & -0.4497 & 0.502 & 0.543 & 0.482 & 0.355 \\
\hline EM07-Cr-001 & quenched & measured & -0.3175 & -0.2860 & -0.3068 & -0.4916 & 0.481 & 0.518 & 0.493 & 0.322 \\
\hline EM07-Cr-001 & $\mathrm{CCC}$ & measured bc & -0.2929 & -0.2671 & -0.3146 & -0.4811 & 0.509 & 0.541 & 0.485 & 0.330 \\
\hline EM07-Cr-001 & quenched & measured bc & -0.3112 & -0.2875 & -0.3048 & -0.5231 & 0.488 & 0.516 & 0.496 & 0.300 \\
\hline EM07-Cr-001 & $\mathrm{CCC}$ & targeted & -0.2868 & -0.2784 & -0.3184 & -0.4673 & 0.517 & 0.527 & 0.480 & 0.341 \\
\hline EM07-Cr-001 & quenched & targeted & -0.3052 & -0.2989 & -0.3087 & -0.5093 & 0.495 & 0.502 & 0.491 & 0.310 \\
\hline EM07-Cr-012 & $\mathrm{CCC}$ & measured & -0.3198 & -0.2804 & -0.3049 & -0.4573 & 0.479 & 0.524 & 0.496 & 0.349 \\
\hline EM07-Cr-012 & quenched & measured & -0.3349 & -0.2987 & -0.3130 & -0.5050 & 0.463 & 0.503 & 0.486 & 0.313 \\
\hline
\end{tabular}


Table 2 cont. Normalized PCTs by Glass ID/Compositional View

\begin{tabular}{|c|c|c|c|c|c|c|c|c|c|c|}
\hline Glass ID & $\begin{array}{c}\text { Heat } \\
\text { Treatment } \\
\end{array}$ & Comp View & $\begin{array}{c}\log \mathrm{NL} \\
{[\mathrm{B}(\mathrm{g} / \mathrm{L})]}\end{array}$ & $\begin{array}{c}\log \mathrm{NL} \\
{[\mathrm{Li}(\mathrm{g} / \mathrm{L})]} \\
\end{array}$ & $\begin{array}{c}\log \mathrm{NL} \\
{[\mathrm{Na}(\mathrm{g} / \mathrm{L})]}\end{array}$ & $\begin{array}{c}\log \mathrm{NL} \\
{[\mathrm{Si}(\mathrm{g} / \mathrm{L})]}\end{array}$ & $\mathrm{NL} \mathrm{B}(\mathrm{g} / \mathrm{L})$ & NL Li (g/L) & NL Na (g/L) & NL Si (g/L) \\
\hline EM07-Cr-012 & $\mathrm{CCC}$ & measured bc & -0.3136 & -0.2820 & -0.3029 & -0.4888 & 0.486 & 0.522 & 0.498 & 0.325 \\
\hline EM07-Cr-012 & quenched & measured bc & -0.3287 & -0.3002 & -0.3109 & -0.5365 & 0.469 & 0.501 & 0.489 & 0.291 \\
\hline EM07-Cr-012 & $\mathrm{CcC}$ & targeted & -0.3089 & -0.2921 & -0.3039 & -0.4718 & 0.491 & 0.510 & 0.497 & 0.337 \\
\hline EM07-Cr-012 & quenched & targeted & -0.3240 & -0.3103 & -0.3120 & -0.5195 & 0.474 & 0.489 & 0.488 & 0.302 \\
\hline EM07-Cr-02 & $\mathrm{CCC}$ & measured & -0.3504 & -0.3175 & -0.3537 & -0.4748 & 0.446 & 0.481 & 0.443 & 0.335 \\
\hline EM07-Cr-02 & quenched & measured & -0.3220 & -0.2892 & -0.3056 & -0.4843 & 0.476 & 0.514 & 0.495 & 0.328 \\
\hline EM07-Cr-02 & $\mathrm{CCC}$ & measured bc & -0.3492 & -0.3187 & -0.3546 & -0.5063 & 0.448 & 0.480 & 0.442 & 0.312 \\
\hline EM07-Cr-02 & quenched & measured bc & -0.3208 & -0.2904 & -0.3065 & -0.5158 & 0.478 & 0.512 & 0.494 & 0.305 \\
\hline EM07-Cr-02 & $\mathrm{ccC}$ & targeted & -0.3442 & -0.3327 & -0.3511 & -0.4908 & 0.453 & 0.465 & 0.446 & 0.323 \\
\hline EM07-Cr-02 & quenched & targeted & -0.3158 & -0.3044 & -0.3030 & -0.5004 & 0.483 & 0.496 & 0.498 & 0.316 \\
\hline EM07-F-02 & $\mathrm{ccC}$ & measured & -0.4223 & -0.3827 & -0.3825 & -0.5616 & 0.378 & 0.414 & 0.414 & 0.274 \\
\hline EM07-F-02 & quenched & measured & -0.3855 & -0.3350 & -0.3397 & -0.5719 & 0.412 & 0.462 & 0.457 & 0.268 \\
\hline EM07-F-02 & $\mathrm{ccC}$ & measured bc & -0.4132 & -0.3751 & -0.3704 & -0.5643 & 0.386 & 0.422 & 0.426 & 0.273 \\
\hline EM07-F-02 & quenched & measured bc & -0.3763 & -0.3275 & -0.3276 & -0.5746 & 0.420 & 0.470 & 0.470 & 0.266 \\
\hline EM07-F-02 & $\mathrm{cCC}$ & targeted & -0.3983 & -0.3701 & -0.3665 & -0.5561 & 0.400 & 0.426 & 0.430 & 0.278 \\
\hline EM07-F-02 & quenched & targeted & -0.3615 & -0.3224 & -0.3237 & -0.5664 & 0.435 & 0.476 & 0.475 & 0.271 \\
\hline EM07-Fe-05 & $\mathrm{CCC}$ & measured & -0.3502 & -0.3336 & -0.3372 & -0.5034 & 0.447 & 0.464 & 0.460 & 0.314 \\
\hline EM07-Fe-05 & quenched & measured & -0.3254 & -0.3469 & -0.3098 & -0.5296 & 0.473 & 0.450 & 0.490 & 0.295 \\
\hline EM07-Fe-05 & $\mathrm{CCC}$ & measured bc & -0.3490 & -0.3347 & -0.3381 & -0.5349 & 0.448 & 0.463 & 0.459 & 0.292 \\
\hline EM07-Fe-05 & quenched & measured bc & -0.3243 & -0.3481 & -0.3108 & -0.5611 & 0.474 & 0.449 & 0.489 & 0.275 \\
\hline EM07-Fe-05 & $\mathrm{CCC}$ & targeted & -0.3431 & -0.3478 & -0.3388 & -0.5201 & 0.454 & 0.449 & 0.458 & 0.302 \\
\hline EM07-Fe-05 & quenched & targeted & -0.3183 & -0.3611 & -0.3115 & -0.5463 & 0.481 & 0.435 & 0.488 & 0.284 \\
\hline EM07-Fe-15 & $\mathrm{CCC}$ & measured & -0.3015 & -0.2804 & -0.3088 & -0.4369 & 0.499 & 0.524 & 0.491 & 0.366 \\
\hline EM07-Fe-15 & quenched & measured & -0.2956 & -0.2465 & -0.2967 & -0.4952 & 0.506 & 0.567 & 0.505 & 0.320 \\
\hline EM07-Fe-15 & $\mathrm{cCC}$ & measured bc & -0.2953 & -0.2819 & -0.3097 & -0.4684 & 0.507 & 0.522 & 0.490 & 0.340 \\
\hline EM07-Fe-15 & quenched & measured bc & -0.2894 & -0.2480 & -0.2976 & -0.5267 & 0.514 & 0.565 & 0.504 & 0.297 \\
\hline EM07-Fe-15 & $\mathrm{CCC}$ & targeted & -0.2931 & -0.2937 & -0.3113 & -0.4526 & 0.509 & 0.509 & 0.488 & 0.353 \\
\hline EM07-Fe-15 & quenched & targeted & -0.2873 & -0.2598 & -0.2992 & -0.5109 & 0.516 & 0.550 & 0.502 & 0.308 \\
\hline EM07-Fe-20 & $\mathrm{cCC}$ & measured & -0.3256 & -0.3240 & -0.3229 & -0.4475 & 0.473 & 0.474 & 0.475 & 0.357 \\
\hline EM07-Fe-20 & quenched & measured & -0.2582 & -0.2019 & -0.2960 & -0.4821 & 0.552 & 0.628 & 0.506 & 0.330 \\
\hline
\end{tabular}


Table 2 cont. Normalized PCTs by Glass ID/Compositional View

\begin{tabular}{|c|c|c|c|c|c|c|c|c|c|c|}
\hline Glass ID & $\begin{array}{c}\text { Heat } \\
\text { Treatment } \\
\end{array}$ & Comp View & $\begin{array}{c}\log \mathrm{NL} \\
{[\mathrm{B}(\mathrm{g} / \mathrm{L})]}\end{array}$ & $\begin{array}{c}\log \mathrm{NL} \\
{[\mathrm{Li}(\mathrm{g} / \mathrm{L})]}\end{array}$ & $\begin{array}{c}\log \mathrm{NL} \\
{[\mathrm{Na}(\mathrm{g} / \mathrm{L})]}\end{array}$ & $\begin{array}{c}\log \mathrm{NL} \\
{[\mathrm{Si}(\mathrm{g} / \mathrm{L})]} \\
\end{array}$ & NL B(g/L) & NL Li (g/L) & $\mathrm{NL} \mathrm{Na}(\mathrm{g} / \mathrm{L})$ & NL Si $(g / L)$ \\
\hline EM07-Fe-20 & $\mathrm{CCC}$ & measured bc & -0.3244 & -0.3252 & -0.3238 & -0.4790 & 0.474 & 0.473 & 0.474 & 0.332 \\
\hline EM07-Fe-20 & quenched & measured bc & -0.2571 & -0.2031 & -0.2970 & -0.5136 & 0.553 & 0.627 & 0.505 & 0.307 \\
\hline EM07-Fe-20 & $\mathrm{CCC}$ & targeted & -0.3218 & -0.3377 & -0.3176 & -0.4549 & 0.477 & 0.460 & 0.481 & 0.351 \\
\hline EM07-Fe-20 & quenched & targeted & -0.2544 & -0.2156 & -0.2907 & -0.4895 & 0.557 & 0.609 & 0.512 & 0.324 \\
\hline EM07-K-03 & $\mathrm{CCC}$ & measured & -0.2399 & -0.2610 & -0.2584 & -0.5082 & 0.576 & 0.548 & 0.552 & 0.310 \\
\hline EM07-K-03 & quenched & measured & -0.3031 & -0.3245 & -0.2820 & -0.5539 & 0.498 & 0.474 & 0.522 & 0.279 \\
\hline EM07-K-03 & $\mathrm{CCC}$ & measured bc & -0.2352 & -0.2573 & -0.2463 & -0.5196 & 0.582 & 0.553 & 0.567 & 0.302 \\
\hline EM07-K-03 & quenched & measured bc & -0.2984 & -0.3208 & -0.2698 & -0.5653 & 0.503 & 0.478 & 0.537 & 0.272 \\
\hline EM07-K-03 & $\mathrm{CCC}$ & targeted & -0.2171 & -0.2573 & -0.2463 & -0.5131 & 0.607 & 0.553 & 0.567 & 0.307 \\
\hline EM07-K-03 & quenched & targeted & -0.2803 & -0.3208 & -0.2698 & -0.5588 & 0.524 & 0.478 & 0.537 & 0.276 \\
\hline EM07-K-06 & $\mathrm{CCC}$ & measured & 0.0864 & 0.0346 & 0.0213 & -0.4101 & 1.220 & 1.083 & 1.050 & 0.389 \\
\hline EM07-K-06 & quenched & measured & -0.0468 & -0.0913 & -0.0944 & -0.5059 & 0.898 & 0.810 & 0.805 & 0.312 \\
\hline EM07-K-06 & $\mathrm{CCC}$ & measured bc & 0.0957 & 0.0422 & 0.0334 & -0.4127 & 1.247 & 1.102 & 1.080 & 0.387 \\
\hline EM07-K-06 & quenched & measured bc & -0.0375 & -0.0838 & -0.0823 & -0.5085 & 0.917 & 0.825 & 0.827 & 0.310 \\
\hline EM07-K-06 & $\mathrm{CCC}$ & targeted & 0.1058 & 0.0451 & 0.0311 & -0.4070 & 1.276 & 1.109 & 1.074 & 0.392 \\
\hline EM07-K-06 & quenched & targeted & -0.0274 & -0.0808 & -0.0846 & -0.5028 & 0.939 & 0.830 & 0.823 & 0.314 \\
\hline EM07-Li-015 & $\mathrm{CCC}$ & measured & -0.4206 & -0.3141 & -0.3662 & -0.5442 & 0.380 & 0.485 & 0.430 & 0.286 \\
\hline EM07-Li-015 & quenched & measured & -0.4129 & -0.3277 & -0.3541 & -0.5883 & 0.386 & 0.470 & 0.442 & 0.258 \\
\hline EM07-Li-015 & $\mathrm{CCC}$ & measured bc & -0.4195 & -0.3153 & -0.3641 & -0.5757 & 0.381 & 0.484 & 0.432 & 0.266 \\
\hline EM07-Li-015 & quenched & measured bc & -0.4118 & -0.3289 & -0.3521 & -0.6198 & 0.387 & 0.469 & 0.444 & 0.240 \\
\hline EM07-Li-015 & $\mathrm{CCC}$ & targeted & -0.4098 & -0.3183 & -0.3679 & -0.5552 & 0.389 & 0.480 & 0.429 & 0.278 \\
\hline EM07-Li-015 & quenched & targeted & -0.4021 & -0.3319 & -0.3559 & -0.5993 & 0.396 & 0.466 & 0.441 & 0.252 \\
\hline EM07-Li-04 & $\mathrm{CCC}$ & measured & -0.3094 & -0.2721 & -0.2629 & -0.4647 & 0.491 & 0.534 & 0.546 & 0.343 \\
\hline EM07-Li-04 & quenched & measured & -0.2978 & -0.2741 & -0.2423 & -0.4928 & 0.504 & 0.532 & 0.572 & 0.322 \\
\hline EM07-Li-04 & $\mathrm{CCC}$ & measured bc & -0.3031 & -0.2736 & -0.2609 & -0.4961 & 0.498 & 0.533 & 0.548 & 0.319 \\
\hline EM07-Li-04 & quenched & measured bc & -0.2916 & -0.2757 & -0.2403 & -0.5242 & 0.511 & 0.530 & 0.575 & 0.299 \\
\hline EM07-Li-04 & $\mathrm{CCC}$ & targeted & -0.3000 & -0.2883 & -0.2683 & -0.4829 & 0.501 & 0.515 & 0.539 & 0.329 \\
\hline EM07-Li-04 & quenched & targeted & -0.2885 & -0.2903 & -0.2476 & -0.5110 & 0.515 & 0.512 & 0.565 & 0.308 \\
\hline EM07-Mn-01 & $\mathrm{CCC}$ & measured & -0.3601 & -0.3381 & -0.3433 & -0.4970 & 0.436 & 0.459 & 0.454 & 0.318 \\
\hline EM07-Mn-01 & quenched & measured & -0.3558 & -0.3401 & -0.3243 & -0.5130 & 0.441 & 0.457 & 0.474 & 0.307 \\
\hline
\end{tabular}


Table 2 cont. Normalized PCTs by Glass ID/Compositional View

\begin{tabular}{|c|c|c|c|c|c|c|c|c|c|c|}
\hline Glass ID & $\begin{array}{c}\text { Heat } \\
\text { Treatment } \\
\end{array}$ & Comp View & $\begin{array}{c}\log \mathrm{NL} \\
{[\mathrm{B}(\mathrm{g} / \mathrm{L})]}\end{array}$ & $\begin{array}{c}\log \mathrm{NL} \\
{[\mathrm{Li}(\mathrm{g} / \mathrm{L})]} \\
\end{array}$ & $\begin{array}{c}\log \mathrm{NL} \\
{[\mathrm{Na}(\mathrm{g} / \mathrm{L})]}\end{array}$ & $\begin{array}{c}\log \mathrm{NL} \\
{[\mathrm{Si}(\mathrm{g} / \mathrm{L})]}\end{array}$ & $\mathrm{NL} \mathrm{B}(\mathrm{g} / \mathrm{L})$ & NL Li (g/L) & NL Na (g/L) & NL Si (g/L) \\
\hline EM07-Mn-01 & $\mathrm{CCC}$ & measured bc & -0.3554 & -0.3344 & -0.3306 & -0.5084 & 0.441 & 0.463 & 0.467 & 0.310 \\
\hline EM07-Mn-01 & quenched & measured bc & -0.3510 & -0.3363 & -0.3116 & -0.5244 & 0.446 & 0.461 & 0.488 & 0.299 \\
\hline EM07-Mn-01 & $\mathrm{CcC}$ & targeted & -0.3568 & -0.3330 & -0.3383 & -0.5092 & 0.440 & 0.465 & 0.459 & 0.310 \\
\hline EM07-Mn-01 & quenched & targeted & -0.3525 & -0.3349 & -0.3193 & -0.5252 & 0.444 & 0.462 & 0.479 & 0.298 \\
\hline EM07-Mn-04 & $\mathrm{CCC}$ & measured & -0.3359 & -0.3177 & -0.3279 & -0.5039 & 0.461 & 0.481 & 0.470 & 0.313 \\
\hline EM07-Mn-04 & quenched & measured & -0.2971 & -0.2931 & -0.2954 & -0.5009 & 0.505 & 0.509 & 0.507 & 0.316 \\
\hline EM07-Mn-04 & $\mathrm{CCC}$ & measured bc & -0.3267 & -0.3102 & -0.3158 & -0.5065 & 0.471 & 0.490 & 0.483 & 0.312 \\
\hline EM07-Mn-04 & quenched & measured bc & -0.2879 & -0.2856 & -0.2833 & -0.5035 & 0.515 & 0.518 & 0.521 & 0.314 \\
\hline EM07-Mn-04 & $\mathrm{ccC}$ & targeted & -0.3222 & -0.3113 & -0.3114 & -0.5026 & 0.476 & 0.488 & 0.488 & 0.314 \\
\hline EM07-Mn-04 & quenched & targeted & -0.2834 & -0.2867 & -0.2788 & -0.4995 & 0.521 & 0.517 & 0.526 & 0.317 \\
\hline EM07-Na-05 & $\mathrm{CCC}$ & measured & -0.5376 & -0.2051 & -1.7232 & -0.5853 & 0.290 & 0.624 & 0.019 & 0.260 \\
\hline EM07-Na-05 & quenched & measured & -0.5199 & -0.2116 & -2.2201 & -0.5743 & 0.302 & 0.614 & 0.006 & 0.267 \\
\hline EM07-Na-05 & $\mathrm{ccC}$ & measured bc & -0.5329 & -0.2014 & -1.7110 & -0.5967 & 0.293 & 0.629 & 0.019 & 0.253 \\
\hline EM07-Na-05 & quenched & measured bc & -0.5152 & -0.2079 & -2.2080 & -0.5857 & 0.305 & 0.620 & 0.006 & 0.260 \\
\hline EM07-Na-05 & $\mathrm{CCC}$ & targeted & -0.5358 & -0.2060 & -1.6890 & -0.6051 & 0.291 & 0.622 & 0.020 & 0.248 \\
\hline EM07-Na-05 & quenched & targeted & -0.5182 & -0.2125 & -2.1860 & -0.5942 & 0.303 & 0.613 & 0.007 & 0.255 \\
\hline EM07-Na-10 & $\mathrm{CCC}$ & measured & -0.5000 & -0.2562 & -0.6397 & -0.5493 & 0.316 & 0.554 & 0.229 & 0.282 \\
\hline EM07-Na-10 & quenched & measured & -0.4477 & -0.1785 & -0.6481 & -0.5655 & 0.357 & 0.663 & 0.225 & 0.272 \\
\hline EM07-Na-10 & $\mathrm{CCC}$ & measured bc & -0.4909 & -0.2487 & -0.6270 & -0.5519 & 0.323 & 0.564 & 0.236 & 0.281 \\
\hline EM07-Na-10 & quenched & measured bc & -0.4386 & -0.1709 & -0.6354 & -0.5681 & 0.364 & 0.675 & 0.232 & 0.270 \\
\hline EM07-Na-10 & $\mathrm{CCC}$ & targeted & -0.4839 & -0.2507 & -0.6218 & -0.5492 & 0.328 & 0.561 & 0.239 & 0.282 \\
\hline EM07-Na-10 & quenched & targeted & -0.4316 & -0.1729 & -0.6302 & -0.5654 & 0.370 & 0.672 & 0.234 & 0.272 \\
\hline EM07-Na-20 & $\mathrm{CCC}$ & measured & -0.0018 & -0.2022 & 0.0013 & -0.4064 & 0.996 & 0.628 & 1.003 & 0.392 \\
\hline EM07-Na-20 & quenched & measured & -0.0603 & -0.3150 & -0.0103 & -0.4317 & 0.870 & 0.484 & 0.977 & 0.370 \\
\hline EM07-Na-20 & $\mathrm{CCC}$ & measured bc & 0.0029 & -0.1985 & 0.0140 & -0.4178 & 1.007 & 0.633 & 1.033 & 0.382 \\
\hline EM07-Na-20 & quenched & measured bc & -0.0556 & -0.3113 & 0.0024 & -0.4431 & 0.880 & 0.488 & 1.006 & 0.360 \\
\hline EM07-Na-20 & $\mathrm{CCC}$ & targeted & 0.0048 & -0.1966 & 0.0031 & -0.4033 & 1.011 & 0.636 & 1.007 & 0.395 \\
\hline EM07-Na-20 & quenched & targeted & -0.0537 & -0.3095 & -0.0084 & -0.4286 & 0.884 & 0.490 & 0.981 & 0.373 \\
\hline EM07-Ni-001 & $\mathrm{cCC}$ & measured & -0.3917 & -0.3202 & -0.3465 & -0.5242 & 0.406 & 0.478 & 0.450 & 0.299 \\
\hline EM07-Ni-001 & quenched & measured & -0.3832 & -0.3174 & -0.3324 & -0.5583 & 0.414 & 0.482 & 0.465 & 0.277 \\
\hline
\end{tabular}


Table 2 cont. Normalized PCTs by Glass ID/Compositional View

\begin{tabular}{|c|c|c|c|c|c|c|c|c|c|c|}
\hline Glass ID & $\begin{array}{c}\text { Heat } \\
\text { Treatment } \\
\end{array}$ & Comp View & $\begin{array}{c}\log \mathrm{NL} \\
{[\mathrm{B}(\mathrm{g} / \mathrm{L})]}\end{array}$ & $\begin{array}{c}\log \mathrm{NL} \\
{[\mathrm{Li}(\mathrm{g} / \mathrm{L})]}\end{array}$ & $\begin{array}{c}\log \mathrm{NL} \\
{[\mathrm{Na}(\mathrm{g} / \mathrm{L})]}\end{array}$ & $\begin{array}{c}\log \mathrm{NL} \\
{[\mathrm{Si}(\mathrm{g} / \mathrm{L})]}\end{array}$ & $\mathrm{NL} \mathrm{B}(\mathrm{g} / \mathrm{L})$ & NL Li (g/L) & $\mathrm{NL} N a(\mathrm{~g} / \mathrm{L})$ & NL Si (g/L) \\
\hline EM07-Ni-001 & $\mathrm{CCC}$ & measured bc & -0.3906 & -0.3214 & -0.3446 & -0.5557 & 0.407 & 0.477 & 0.452 & 0.278 \\
\hline EM07-Ni-001 & quenched & measured bc & -0.3822 & -0.3185 & -0.3304 & -0.5898 & 0.415 & 0.480 & 0.467 & 0.257 \\
\hline EM07-Ni-001 & $\mathrm{CCC}$ & targeted & -0.3861 & -0.3362 & -0.3467 & -0.5348 & 0.411 & 0.461 & 0.450 & 0.292 \\
\hline EM07-Ni-001 & quenched & targeted & -0.3776 & -0.3333 & -0.3325 & -0.5688 & 0.419 & 0.464 & 0.465 & 0.270 \\
\hline EM07-Ni-02 & CCC & measured & -0.3721 & -0.3065 & -0.3068 & -0.5124 & 0.425 & 0.494 & 0.493 & 0.307 \\
\hline EM07-Ni-02 & quenched & measured & -0.3648 & -0.3030 & -0.2862 & -0.5324 & 0.432 & 0.498 & 0.517 & 0.294 \\
\hline EM07-Ni-02 & $\mathrm{CCC}$ & measured bc & -0.3658 & -0.3080 & -0.3048 & -0.5439 & 0.431 & 0.492 & 0.496 & 0.286 \\
\hline EM07-Ni-02 & quenched & measured bc & -0.3586 & -0.3045 & -0.2841 & -0.5638 & 0.438 & 0.496 & 0.520 & 0.273 \\
\hline EM07-Ni-02 & $\mathrm{CCC}$ & targeted & -0.3591 & -0.3193 & -0.3154 & -0.5256 & 0.437 & 0.479 & 0.484 & 0.298 \\
\hline EM07-Ni-02 & quenched & targeted & -0.3519 & -0.3159 & -0.2947 & -0.5456 & 0.445 & 0.483 & 0.507 & 0.285 \\
\hline EM07-NM-0025 & CCC & measured & -0.3506 & -0.3193 & -0.3367 & -0.4769 & 0.446 & 0.479 & 0.461 & 0.333 \\
\hline EM07-NM-0025 & quenched & measured & -0.3184 & -0.2999 & -0.3154 & -0.5155 & 0.480 & 0.501 & 0.484 & 0.305 \\
\hline EM07-NM-0025 & CCC & measured bc & -0.3444 & -0.3208 & -0.3347 & -0.5084 & 0.452 & 0.478 & 0.463 & 0.310 \\
\hline EM07-NM-0025 & quenched & measured bc & -0.3123 & -0.3014 & -0.3133 & -0.5470 & 0.487 & 0.500 & 0.486 & 0.284 \\
\hline EM07-NM-0025 & $\mathrm{CCC}$ & targeted & -0.3390 & -0.3330 & -0.3408 & -0.4931 & 0.458 & 0.465 & 0.456 & 0.321 \\
\hline EM07-NM-0025 & quenched & targeted & -0.3069 & -0.3136 & -0.3194 & -0.5316 & 0.493 & 0.486 & 0.479 & 0.294 \\
\hline EM07-P-0 & $\mathrm{CCC}$ & measured & -0.3243 & -0.3105 & -0.3367 & -0.5029 & 0.474 & 0.489 & 0.461 & 0.314 \\
\hline EM07-P-0 & quenched & measured & -0.2557 & -0.3094 & -0.3205 & -0.5326 & 0.555 & 0.490 & 0.478 & 0.293 \\
\hline EM07-P-0 & $\mathrm{CCC}$ & measured bc & -0.3196 & -0.3067 & -0.3240 & -0.5143 & 0.479 & 0.493 & 0.474 & 0.306 \\
\hline EM07-P-0 & quenched & measured bc & -0.2509 & -0.3056 & -0.3078 & -0.5440 & 0.561 & 0.495 & 0.492 & 0.286 \\
\hline EM07-P-0 & $\mathrm{CCC}$ & targeted & -0.3285 & -0.3168 & -0.3326 & -0.5193 & 0.469 & 0.482 & 0.465 & 0.302 \\
\hline EM07-P-0 & quenched & targeted & -0.2599 & -0.3157 & -0.3164 & -0.5490 & 0.550 & 0.483 & 0.483 & 0.283 \\
\hline EM07-P-025 & $\mathrm{CCC}$ & measured & -0.3463 & 0.0006 & -0.2459 & -0.4952 & 0.451 & 1.001 & 0.568 & 0.320 \\
\hline EM07-P-025 & quenched & measured & -0.2659 & -0.2932 & -0.3102 & -0.5381 & 0.542 & 0.509 & 0.490 & 0.290 \\
\hline EM07-P-025 & $\mathrm{CCC}$ & measured bc & -0.3371 & 0.0082 & -0.2332 & -0.4978 & 0.460 & 1.019 & 0.585 & 0.318 \\
\hline EM07-P-025 & quenched & measured bc & -0.2567 & -0.2857 & -0.2975 & -0.5407 & 0.554 & 0.518 & 0.504 & 0.288 \\
\hline EM07-P-025 & $\mathrm{CCC}$ & targeted & -0.3271 & 0.0096 & -0.2385 & -0.4941 & 0.471 & 1.022 & 0.577 & 0.321 \\
\hline EM07-P-025 & quenched & targeted & -0.2467 & -0.2842 & -0.3028 & -0.5370 & 0.567 & 0.520 & 0.498 & 0.290 \\
\hline EM07-Si-30 & $\mathrm{CCC}$ & measured & 1.7386 & 1.5052 & 1.4258 & 0.0435 & 54.783 & 32.007 & 26.656 & 1.105 \\
\hline EM07-Si-30 & quenched & measured & 0.4355 & 0.2564 & 0.2719 & -0.3740 & 2.726 & 1.805 & 1.870 & 0.423 \\
\hline
\end{tabular}


Table 2 cont. Normalized PCTs by Glass ID/Compositional View

\begin{tabular}{|c|c|c|c|c|c|c|c|c|c|c|}
\hline Glass ID & $\begin{array}{c}\text { Heat } \\
\text { Treatment }\end{array}$ & Comp View & $\begin{array}{c}\log \mathrm{NL} \\
{[\mathrm{B}(\mathrm{g} / \mathrm{L})]}\end{array}$ & $\begin{array}{c}\log \mathrm{NL} \\
{[\mathrm{Li}(\mathrm{g} / \mathrm{L})]}\end{array}$ & $\begin{array}{c}\log \mathrm{NL} \\
{[\mathrm{Na}(\mathrm{g} / \mathrm{L})]}\end{array}$ & $\begin{array}{c}\log \mathrm{NL} \\
{[\mathrm{Si}(\mathrm{g} / \mathrm{L})]}\end{array}$ & NL B(g/L) & NL Li (g/L) & NL Na (g/L) & NL Si (g/L) \\
\hline EM07-Si-30 & $\mathrm{CCC}$ & measured bc & 1.7449 & 1.5037 & 1.4249 & 0.0121 & 55.574 & 31.895 & 26.601 & 1.028 \\
\hline EM07-Si-30 & quenched & measured bc & 0.4418 & 0.2549 & 0.2710 & -0.4054 & 2.765 & 1.798 & 1.866 & 0.393 \\
\hline EM07-Si-30 & $\mathrm{CCC}$ & targeted & 1.7519 & 1.4919 & 1.4251 & 0.0382 & 56.480 & 31.036 & 26.615 & 1.092 \\
\hline EM07-Si-30 & quenched & targeted & 0.4488 & 0.2430 & 0.2712 & -0.3794 & 2.811 & 1.750 & 1.867 & 0.417 \\
\hline EM07-Si-37 & $\mathrm{CCC}$ & measured & 0.0727 & -0.0492 & -0.0404 & -0.4456 & 1.182 & 0.893 & 0.911 & 0.358 \\
\hline EM07-Si-37 & quenched & measured & -0.0808 & -0.2123 & -0.1483 & -0.5076 & 0.830 & 0.613 & 0.711 & 0.311 \\
\hline EM07-Si-37 & $\mathrm{CCC}$ & measured bc & 0.0739 & -0.0503 & -0.0413 & -0.4770 & 1.185 & 0.891 & 0.909 & 0.333 \\
\hline EM07-Si-37 & quenched & measured bc & -0.0797 & -0.2134 & -0.1492 & -0.5391 & 0.832 & 0.612 & 0.709 & 0.289 \\
\hline EM07-Si-37 & $\mathrm{CCC}$ & targeted & 0.0733 & -0.0687 & -0.0455 & -0.4562 & 1.184 & 0.854 & 0.901 & 0.350 \\
\hline EM07-Si-37 & quenched & targeted & -0.0802 & -0.2318 & -0.1534 & -0.5183 & 0.831 & 0.586 & 0.702 & 0.303 \\
\hline EM07-Si-50 & $\mathrm{CCC}$ & measured & -0.3920 & -0.2844 & -0.4704 & -0.5246 & 0.406 & 0.519 & 0.339 & 0.299 \\
\hline EM07-Si-50 & quenched & measured & -0.4056 & -0.2669 & -0.4578 & -0.5574 & 0.393 & 0.541 & 0.349 & 0.277 \\
\hline EM07-Si-50 & $\mathrm{CCC}$ & measured bc & -0.3909 & -0.2856 & -0.4713 & -0.5561 & 0.407 & 0.518 & 0.338 & 0.278 \\
\hline EM07-Si-50 & quenched & measured bc & -0.4045 & -0.2680 & -0.4587 & -0.5888 & 0.394 & 0.539 & 0.348 & 0.258 \\
\hline EM07-Si-50 & $\mathrm{CCC}$ & targeted & -0.3872 & -0.2999 & -0.4649 & -0.5499 & 0.410 & 0.501 & 0.343 & 0.282 \\
\hline EM07-Si-50 & quenched & targeted & -0.4009 & -0.2823 & -0.4523 & -0.5826 & 0.397 & 0.522 & 0.353 & 0.261 \\
\hline EM07-Zr-001 & $\mathrm{CCC}$ & measured & -0.3467 & -0.3196 & -0.3045 & -0.4706 & 0.450 & 0.479 & 0.496 & 0.338 \\
\hline EM07-Zr-001 & quenched & measured & -0.3454 & -0.3311 & -0.2906 & -0.5006 & 0.451 & 0.467 & 0.512 & 0.316 \\
\hline EM07-Zr-001 & $\mathrm{CCC}$ & measured bc & -0.3373 & -0.3121 & -0.2918 & -0.4732 & 0.460 & 0.487 & 0.511 & 0.336 \\
\hline EM07-Zr-001 & quenched & measured bc & -0.3360 & -0.3236 & -0.2779 & -0.5032 & 0.461 & 0.475 & 0.527 & 0.314 \\
\hline EM07-Zr-001 & $\mathrm{CCC}$ & targeted & -0.3211 & -0.3119 & -0.3017 & -0.4665 & 0.477 & 0.488 & 0.499 & 0.342 \\
\hline EM07-Zr-001 & quenched & targeted & -0.3198 & -0.3234 & -0.2878 & -0.4965 & 0.479 & 0.475 & 0.515 & 0.319 \\
\hline EM07-Zr-05 & CCC & measured & -0.4363 & -0.3535 & -0.3840 & -0.5918 & 0.366 & 0.443 & 0.413 & 0.256 \\
\hline EM07-Zr-05 & quenched & measured & -0.4085 & -0.3331 & -0.3549 & -0.6040 & 0.390 & 0.464 & 0.442 & 0.249 \\
\hline EM07-Zr-05 & CCC & measured bc & -0.4271 & -0.3459 & -0.3713 & -0.5944 & 0.374 & 0.451 & 0.425 & 0.254 \\
\hline EM07-Zr-05 & quenched & measured bc & -0.3993 & -0.3255 & -0.3422 & -0.6066 & 0.399 & 0.473 & 0.455 & 0.247 \\
\hline EM07-Zr-05 & $\mathrm{CCC}$ & targeted & -0.4217 & -0.3429 & -0.3767 & -0.5915 & 0.379 & 0.454 & 0.420 & 0.256 \\
\hline EM07-Zr-05 & quenched & targeted & -0.3939 & -0.3225 & -0.3476 & -0.6037 & 0.404 & 0.476 & 0.449 & 0.249 \\
\hline
\end{tabular}




\subsubsection{Effects of Heat Treatment on PCTs}

Figure B6 in Appendix B provides a series of plots and statistical comparisons that show the effects of heat treatment on the common logarithm ppm-responses of the triplicate PCTs for each glass. The quenched version of a given glass yielded measurements indicating a significantly different mean $\log (\mathrm{ppm})$ response than the ccc version (at the $5 \%$ significance level) for a given element if the Prob $>|\mathbf{t}|$ value in the figure is 0.05 or smaller.

Many of these glasses exhibited a statistically significant difference between the ccc and quenched versions of one or more of the PCT elements. In a few cases (EM07-B-15, EM07-Si-30, and EM07$\mathrm{Si}$-37), the ccc versions of the glasses were less durable than their quenched counterparts for all four elements (B, Li, Na and Si). Figure B7 in Appendix B provides plots of the normalized PCT responses for both of the heat treatments. These plots provide a basis for judging the practical impact of differences in the PCT response due to the heat treatment of the glass. 


\subsection{References}

1. K. Gerdes, "Enhanced DOE High-Level Waste Melter Throughput Studies [Work Authorization / Task Change Request (TCR)]," Project Manager at the U.S. Department of Energy Headquarters, Washington, D.C., Project Number SR071401, May 2007.

2. $\quad$ F.C. Raszewski, A.S. Choi, T.B. Edwards, K.M. Fox, and M.E. Smith, "Enhanced DOE High-Level Waste Melter Throughput Studies: Task Plan," Savannah River National Laboratory, Aiken, SC, WSRC-STI-2007-00483, Rev. 0, 2007.

3. D. Kim, "EM-21 HLW Glass Studies Test Plan: Crystal Accumulation Modeling Task Glass Matrix Testing," Pacific Northwest National Laboratory (PNNL), Richland, WA, EMHLW-TP-07-01, Rev. 0,

4. $\quad \mathrm{JMP}^{\mathrm{TM}}$ Version 6.0.3, SAS Institute Inc., Cary, NC, 2005.

5. T.B. Edwards, "Analytical Plans for Measuring the Chemical Compositions of the Glasses of Test Matrix 1 of the Enhanced DOE HLW Melter Throughput Studies," Savannah River National Laboratory, Aiken, SC, SRNL-SCS-2007-00055, Rev. 1, 2007.

6. C.M. Jantzen, J.B. Picket, K.G. Brown, T.B. Edwards, and D.C. Beam, "Process/Product Models for the Defense Waste Processing Facility (DWPF): Part I. Predicting Glass Durability from Composition Using a Thermodynamic Hydration Energy Reaction Model (THERMO)," Westinghouse Savannah River Company, Aiken, SC, WSRC-TR-93-672, Rev. $1,1995$.

7. W.L. Ebert and S.F. Wolf, "Round-Robin Testing of a Reference Glass for Low Activity Waste Forms," Argonne National Laboratory, Argonne, IL, ANL-99/22, 1999.

8. $\quad$ "Standard Test Methods for Determining Chemical Durability of Nuclear, Hazardous, and Mixed Waste Glasses and Multiphase Glass Ceramics: The Product Consistency Test (PCT)," ASTM International, West Conshohocken, PA, ASTM C 1285-02, 2002.

9. C.M. Jantzen, N.E. Bibler, D.C. Beam, C.L. Crawford, and M.A. Pickett, "Characterization of the Defense Waste Processing Facility (DWPF) Environmental Assessment (EA) Glass Standard Reference Material," Westinghouse Savannah River Company, Aiken, SC, WSRCTR-92-346, Rev. 1, 1993.

10. "Nuclear Waste Glass and Glass-Ceramic Product Consistency Test (PCT) Methods (ASTM C1285 Latest Revision)," Savannah River National Laboratory, Aiken, SC, ITS-0009, Rev. 2, 2006.

11. T.B. Edwards, "Analytical Plans for Measuring PCT Solutions for the Test Matrix 1 Glasses of the Enhanced DOE HLW Melter Throughput Studies," Savannah River National Laboratory, Aiken, SC, SRNL-SCS-2007-00059, 2007.

12. T.B. Edwards, R.A. Baker, S.P. Harris, C.D. Harvel, and E.P. Shine, "Software QA Plan and Verification and Validation for Commercial Statistical Packages Utilized by the Statistical Consulting Section of SRTC " Westinghouse Savannah River Company, Aiken, SC, WSRCRP-99-00422, Rev. 2, 2001. 


\section{Appendix A:}

\section{Tables and Figures Supporting the Analysis of the Chemical Composition Measurements of the Test Matrix 1 Glasses}


SRNS-STI-2008-00056

Revision: 0

This page intentionally left blank. 
Table A1. Targeted Oxide Concentrations (in mass fractions) for the Test Matrix 1 Study Glasses (part 1)

\begin{tabular}{|c|c|c|c|c|c|c|c|c|c|c|c|c|c|c|c|}
\hline Glass ID & $\mathrm{Al} 2 \mathrm{O} 3$ & B2O3 & $\mathrm{BaO}$ & $\mathrm{Bi} 2 \mathrm{O} 3$ & $\mathrm{CaO}$ & $\mathrm{CdO}$ & $\mathrm{Ce} 2 \mathrm{O} 3$ & Cr2O3 & $\mathrm{F}$ & Fe2O3 & $\mathrm{K} 2 \mathrm{O}$ & La2O3 & $\mathrm{Li} 2 \mathrm{O}$ & $\mathrm{MgO}$ & $\mathrm{MnO}$ \\
\hline EM07-BL-1 & 0.1 & 0.1 & 0.0005 & 0 & 0 & 0.0014 & 0.0005 & 0.005 & 0.001 & 0.1 & 0 & 0.0007 & 0.0275 & 0.0015 & 0.02 \\
\hline EM07-Al-06 & 0.06 & 0.10444 & 0.00052 & 0 & 0 & 0.00146 & 0.00052 & 0.00522 & 0.00104 & 0.10444 & 0 & 0.00073 & 0.02872 & 0.00157 & 0.02089 \\
\hline EM07-Al-15 & 0.15 & 0.09444 & 0.00047 & 0 & 0 & 0.00132 & 0.00047 & 0.00472 & 0.00094 & 0.09444 & 0 & 0.00066 & 0.02597 & 0.00142 & 0.01889 \\
\hline EM07-Al-20 & 0.2 & 0.08889 & 0.00044 & 0 & 0 & 0.00124 & 0.00044 & 0.00444 & 0.00089 & 0.08889 & 0 & 0.00062 & 0.02444 & 0.00133 & 0.01778 \\
\hline EM07-B-05 & 0.10556 & 0.05 & 0.00053 & 0 & 0 & 0.00148 & 0.00053 & 0.00528 & 0.00106 & 0.10556 & 0 & 0.00074 & 0.02903 & 0.00158 & 0.02111 \\
\hline EM07-B-15 & 0.09444 & 0.15 & 0.00047 & 0 & 0 & 0.00132 & 0.00047 & 0.00472 & 0.00094 & 0.09444 & 0 & 0.00066 & 0.02597 & 0.00142 & 0.01889 \\
\hline EM07-B-20 & 0.08889 & 0.2 & 0.00044 & 0 & 0 & 0.00124 & 0.00044 & 0.00444 & 0.00089 & 0.08889 & 0 & 0.00062 & 0.02444 & 0.00133 & 0.01778 \\
\hline EM07-Bi-025 & 0.0975 & 0.0975 & 0.00049 & 0.025 & 0 & 0.00137 & 0.00049 & 0.00488 & 0.00098 & 0.0975 & 0 & 0.00068 & 0.02681 & 0.00146 & 0.0195 \\
\hline EM07-Bi-05 & 0.095 & 0.095 & 0.00048 & 0.05 & 0 & 0.00133 & 0.00048 & 0.00475 & 0.00095 & 0.095 & 0 & 0.00067 & 0.02613 & 0.00143 & 0.019 \\
\hline EM07-Ca-035 & 0.0965 & 0.0965 & 0.00048 & 0 & 0.035 & 0.00135 & 0.00048 & 0.00483 & 0.00097 & 0.0965 & 0 & 0.00068 & 0.02654 & 0.00145 & 0.0193 \\
\hline EM07-Ca-07 & 0.093 & 0.093 & 0.00047 & 0 & 0.07 & 0.0013 & 0.00047 & 0.00465 & 0.00093 & 0.093 & 0 & 0.00065 & 0.02558 & 0.0014 & 0.0186 \\
\hline EM07-Cr-001 & 0.1004 & 0.1004 & 0.0005 & 0 & 0 & 0.00141 & 0.0005 & 0.001 & 0.001 & 0.1004 & 0 & 0.0007 & 0.02761 & 0.00151 & 0.02008 \\
\hline EM07-Cr-012 & 0.0993 & 0.0993 & 0.0005 & 0 & 0 & 0.00139 & 0.0005 & 0.012 & 0.00099 & 0.0993 & 0 & 0.0007 & 0.02731 & 0.00149 & 0.01986 \\
\hline EM07-Cr-02 & 0.09849 & 0.09849 & 0.00049 & 0 & 0 & 0.00138 & 0.00049 & 0.02 & 0.00098 & 0.09849 & 0 & 0.00069 & 0.02709 & 0.00148 & 0.0197 \\
\hline EM07-F-02 & 0.0981 & 0.0981 & 0.00049 & 0 & 0 & 0.00137 & 0.00049 & 0.0049 & 0.02 & 0.0981 & 0 & 0.00069 & 0.02698 & 0.00147 & 0.01962 \\
\hline EM07-Fe-05 & 0.10556 & 0.10556 & 0.00053 & 0 & 0 & 0.00148 & 0.00053 & 0.00528 & 0.00106 & 0.05 & 0 & 0.00074 & 0.02903 & 0.00158 & 0.02111 \\
\hline EM07-Fe-15 & 0.09444 & 0.09444 & 0.00047 & 0 & 0 & 0.00132 & 0.00047 & 0.00472 & 0.00094 & 0.15 & 0 & 0.00066 & 0.02597 & 0.00142 & 0.01889 \\
\hline EM07-Fe-20 & 0.08889 & 0.08889 & 0.00044 & 0 & 0 & 0.00124 & 0.00044 & 0.00444 & 0.00089 & 0.2 & 0 & 0.00062 & 0.02444 & 0.00133 & 0.01778 \\
\hline EM07-K-03 & 0.097 & 0.097 & 0.00049 & 0 & 0 & 0.00136 & 0.00049 & 0.00485 & 0.00097 & 0.097 & 0.03 & 0.00068 & 0.02668 & 0.00146 & 0.0194 \\
\hline EM07-K-06 & 0.094 & 0.094 & 0.00047 & 0 & 0 & 0.00132 & 0.00047 & 0.0047 & 0.00094 & 0.094 & 0.06 & 0.00066 & 0.02585 & 0.00141 & 0.0188 \\
\hline EM07-Li-015 & 0.10129 & 0.10129 & 0.00051 & 0 & 0 & 0.00142 & 0.00051 & 0.00506 & 0.00101 & 0.10129 & 0 & 0.00071 & 0.015 & 0.00152 & 0.02026 \\
\hline EM07-Li-04 & 0.09871 & 0.09871 & 0.00049 & 0 & 0 & 0.00138 & 0.00049 & 0.00494 & 0.00099 & 0.09871 & 0 & 0.00069 & 0.04 & 0.00148 & 0.01974 \\
\hline EM07-Mn-01 & 0.10194 & 0.10194 & 0.00051 & 0 & 0 & 0.00143 & 0.00051 & 0.0051 & 0.00102 & 0.10194 & 0 & 0.00071 & 0.02803 & 0.00153 & 0.001 \\
\hline EM07-Mn-04 & 0.09796 & 0.09796 & 0.00049 & 0 & 0 & 0.00137 & 0.00049 & 0.0049 & 0.00098 & 0.09796 & 0 & 0.00069 & 0.02694 & 0.00147 & 0.04 \\
\hline EM07-Na-05 & 0.11176 & 0.11176 & 0.00056 & 0 & 0 & 0.00156 & 0.00056 & 0.00559 & 0.00112 & 0.11176 & 0 & 0.00078 & 0.03074 & 0.00168 & 0.02235 \\
\hline EM07-Na-10 & 0.10588 & 0.10588 & 0.00053 & 0 & 0 & 0.00148 & 0.00053 & 0.00529 & 0.00106 & 0.10588 & 0 & 0.00074 & 0.02912 & 0.00159 & 0.02118 \\
\hline EM07-Na-20 & 0.09412 & 0.09412 & 0.00047 & 0 & 0 & 0.00132 & 0.00047 & 0.00471 & 0.00094 & 0.09412 & 0 & 0.00066 & 0.02588 & 0.00141 & 0.01882 \\
\hline EM07-Ni-001 & 0.10091 & 0.10091 & 0.0005 & 0 & 0 & 0.00141 & 0.0005 & 0.00505 & 0.00101 & 0.10091 & 0 & 0.00071 & 0.02775 & 0.00151 & 0.02018 \\
\hline EM07-Ni-02 & 0.09899 & 0.09899 & 0.00049 & 0 & 0 & 0.00139 & 0.00049 & 0.00495 & 0.00099 & 0.09899 & 0 & 0.00069 & 0.02722 & 0.00148 & 0.0198 \\
\hline EM07-P-0 & 0.10127 & 0.10127 & 0.00051 & 0 & 0 & 0.00142 & 0.00051 & 0.00506 & 0.00101 & 0.10127 & 0 & 0.00071 & 0.02785 & 0.00152 & 0.02025 \\
\hline EM07-P-025 & 0.09873 & 0.09873 & 0.00049 & 0 & 0 & 0.00138 & 0.00049 & 0.00494 & 0.00099 & 0.09873 & 0 & 0.00069 & 0.02715 & 0.00148 & 0.01975 \\
\hline EM07-Si-30 & 0.12352 & 0.12352 & 0.00062 & 0 & 0 & 0.00173 & 0.00062 & 0.00618 & 0.00124 & 0.12352 & 0 & 0.00086 & 0.03397 & 0.00185 & 0.0247 \\
\hline EM07-Si-37 & 0.11117 & 0.11117 & 0.00056 & 0 & 0 & 0.00156 & 0.00056 & 0.00556 & 0.00111 & 0.11117 & 0 & 0.00078 & 0.03057 & 0.00167 & 0.02223 \\
\hline EM07-Si-50 & 0.08823 & 0.08823 & 0.00044 & 0 & 0 & 0.00124 & 0.00044 & 0.00441 & 0.00088 & 0.08823 & 0 & 0.00062 & 0.02426 & 0.00132 & 0.01765 \\
\hline EM07-Zr-001 & 0.10246 & 0.10246 & 0.00051 & 0 & 0 & 0.00143 & 0.00051 & 0.00512 & 0.00102 & 0.10246 & 0 & 0.00072 & 0.02818 & 0.00154 & 0.02049 \\
\hline EM07-Zr-05 & 0.09744 & 0.09744 & 0.00049 & 0 & 0 & 0.00136 & 0.00049 & 0.00487 & 0.00097 & 0.09744 & 0 & 0.00068 & 0.02679 & 0.00146 & 0.01949 \\
\hline EM07-NM-0025 & 0.09979 & 0.09979 & 0.0005 & 0 & 0 & 0.0014 & 0.0005 & 0.00499 & 0.001 & 0.09979 & 0 & 0.0007 & 0.02744 & 0.0015 & 0.01996 \\
\hline EM07-BL-2 & 0.1 & 0.1 & 0.0005 & 0 & 0 & 0.0014 & 0.0005 & 0.005 & 0.001 & 0.1 & 0 & 0.0007 & 0.0275 & 0.0015 & 0.02 \\
\hline
\end{tabular}


Table A1. Targeted Oxide Concentrations (in mass fractions) for the Test Matrix 1 Study Glasses (part 2)

\begin{tabular}{|c|c|c|c|c|c|c|c|c|c|c|c|c|c|c|}
\hline "Glass ID & $\mathrm{Na2O}$ & " Nd2O3 & $\mathrm{N \textrm {NiO }}$ & $\begin{array}{l}\mathrm{P} 2 \mathrm{O} 5 \\
\end{array}$ & $\overline{\mathrm{PbbO}}$ & PdO & h2O3 & $\begin{array}{l}\mathrm{RuO} 2 \\
\end{array}$ & $\overline{\mathrm{S \textrm {SiO }} 2}$ & SO3 & $\overline{\mathrm{SrO}}$ & 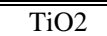 & $\overline{\mathrm{ZnO}}$ & $\overline{\mathrm{ZrO} 2}$ \\
\hline EM07-BL-1 & 0.15 & 0.0007 & 0.01 & 0.0125 & 0.0037 & 0.00009 & 0.00003 & 0.00018 & 0.4333 & 0.003 & 0.0024 & 0.0004 & 0.0006 & 0.025 \\
\hline EM07-Al-06 & .15667 & .00073 & 0.01044 & 0.01306 & 0.00386 & 0.00009 & 0.00003 & 0.00019 & 0.45258 & 0.00313 & 0.00251 & 0.00042 & 0.00063 & 0.02611 \\
\hline EM07-Al-15 & 14167 & .00066 & 0.00944 & 0.01181 & 0.00349 & 0.00009 & 0.00003 & 0.00017 & 0.40924 & 0.00283 & 0.00227 & 0.00038 & 0.00057 & 0.02361 \\
\hline EM07-Al-20 & 13333 & .00062 & 0.00889 & 0.01111 & 0.00329 & 0.00008 & 0.00003 & 0.00016 & 0.38518 & 0.00267 & 0.00213 & 0.00036 & 0.00053 & 0.02222 \\
\hline EM07-B-05 & 15833 & .00074 & 0.01056 & 0.01319 & 0.00391 & 0.0001 & 0.00003 & 0.00019 & 0.45735 & 0.00317 & 0.00253 & 0.00042 & 0.00063 & 0.02639 \\
\hline EM07-B-15 & 14167 & .00066 & 0.00944 & 0.01181 & 0.00349 & 0.00009 & 0.00003 & 0.00017 & 0.40924 & 0.00283 & 0.00227 & 0.00038 & 0.00057 & 0.02361 \\
\hline EM07-B-20 & 13333 & .00062 & 0.00889 & 0.01111 & 0.00329 & 0.00008 & 0.00003 & 0.00016 & 0.38518 & 0.00267 & 0.00213 & 0.00036 & 0.00053 & 0.02222 \\
\hline EM07-Bi-025 & 14625 & .00068 & 0.00975 & 0.01219 & 0.00361 & 0.00009 & 0.00003 & 0.00018 & 0.42243 & 0.00293 & 0.00234 & 0.00039 & 0.00059 & 0.02438 \\
\hline EM07-Bi-05 & 1425 & .00067 & 0.0095 & 0.01188 & 0.00352 & 0.00009 & 0.00003 & 0.00017 & 0.41159 & 0.00285 & 0.00228 & 0.00038 & 0.00057 & 0.02375 \\
\hline EM07-Ca-035 & 14475 & .00068 & 0.00965 & 0.01206 & 0.00357 & 0.00009 & 0.00003 & 0.00017 & 0.4181 & 0.0029 & 0.00232 & 0.00039 & 0.00058 & 0.02413 \\
\hline EM07-Ca-07 & 395 & .00065 & 0.0093 & 0.01163 & 0.00344 & 0.00008 & 0.00003 & 0.0 & 0.40295 & 0.00 & 223 & 0.0 & 056 & 0.1 \\
\hline EM07-Cr-001 & 506 & 0.0 & 0.01004 & 0.0 & 0.00371 & 0.00009 & 0.00 & 0.0 & 0.43507 & 0.00 & 241 & 0.0 & 0.0006 & 51 \\
\hline EM07-Cr-012 & 894 & 0.0007 & 0.00993 & 0.0 & 0.00367 & 0.00009 & 0.0 & 0.0 & 0.43023 & 0.00 & 38 & 0.0 & 0.0006 & 0. \\
\hline EM & 774 & 69 & 0.00985 & 0 & 0.0 & 0.00009 & & & 99 & 0.0 & 36 & 0.0 & 059 & \\
\hline EN & & & & & & & & & & & & & & \\
\hline EM07-Fe-05 & 33 & & 0 & & & & & & & & & & & 639 \\
\hline $\mathrm{EM}$ & 167 & 6 & 0.00 & & 0.0 & 0.0 & & & 24 & 0.0 & & 0.0 & 057 & 361 \\
\hline EM07-Fe-20 & 333 & 0 & 0.00889 & 11 & 0.0 & 0.0 & 0.0 & 0.0 & 0.3 & 0.0 & 13 & 0.0 & 053 & 0.02222 \\
\hline EM & 55 & 8 & 97 & & 0.0 & 0.0 & & 0.0 & 0.4 & 0.0 & & 0.1 & 058 & 425 \\
\hline EM07-K-06 & 41 & 66 & 94 & 75 & 0.00348 & 0.00 & 0.0 & 0.0 & 0.40729 & 0.0 & 226 & 0.0 & 056 & 0.0235 \\
\hline EM07-Li-015 & 193 & 71 & 3 & 6 & 0.0 & 0.0 & 0.0 & 118 & 884 & 0.0 & 243 & 0.0 & 061 & 0.02532 \\
\hline EM07-Li-04 & 807 & 069 & 0.00987 & 34 & 0.00365 & 0.00009 & 0.0 & 018 & .42776 & 0.00 & 237 & 0.00039 & 0.00059 & 0.02468 \\
\hline EM07-Mn-01 & 291 & 71 & 0.01 & 74 & 377 & 0.00009 & 0.0 & 18 & 171 & 0.0 & 245 & 0.00041 & 0.00061 & 0.02548 \\
\hline EM07-Mn-04 & 694 & 00069 & 0.0098 & & 362 & 0.00009 & 0.0 & 0.00018 & 2444 & 0.00 & 235 & 0.00039 & 0.00059 & 0.02449 \\
\hline EM07-Na-05 & 05 & 0.00078 & 0.01118 & 0.01397 & 0.00414 & 0.0001 & 0.00003 & 0.0002 & 0.48429 & 0.00335 & 0.00268 & 0.00045 & 0.00067 & 0.02794 \\
\hline EM07-Na-10 & & 00074 & 0.01059 & 0.01324 & 0.00392 & 0.0001 & 0.00003 & 0.00019 & 0.45878 & 0.00318 & 0.00254 & 0.00042 & 0.00064 & 0.02647 \\
\hline EM07-Na-20 & 0.2 & 0.00066 & 0.00941 & 0.01176 & 0.00348 & 0.00008 & 0.00003 & 0.00017 & 0.40782 & 0.00282 & 0.00226 & 0.00038 & 0.00056 & 0.02353 \\
\hline EM07-Ni-001 & 15136 & 0.00071 & 0.001 & 0.01261 & 0.00373 & 0.00009 & 0.00003 & 0.00018 & 0.43725 & 0.00303 & 0.00242 & 0.0004 & 0.00061 & 0.02523 \\
\hline EM07-Ni-02 & 0.14848 & 0.00069 & 0.02 & 0.01237 & 0.00366 & 0.00009 & 0.00003 & 0.00018 & 0.42894 & 0.00297 & 0.00238 & 0.0004 & 0.00059 & 0.02475 \\
\hline EM07-P-0 & 0.1519 & 0.00071 & 0.01013 & 0 & 0.00375 & 0.00009 & 0.00003 & 0.00018 & 0.43875 & 0.00304 & 0.00243 & 0.00041 & 0.00061 & 0.02532 \\
\hline EM07-P-025 & 0.1481 & 0.00069 & 0.00987 & 0.025 & 0.00365 & 0.00009 & 0.00003 & 0.00018 & 0.42785 & 0.00296 & 0.00237 & 0.00039 & 0.00059 & 0.02468 \\
\hline EM07-Si-30 & 1853 & .00086 & 0.01235 & 0.01544 & 0.00457 & 0.00011 & 0.00004 & 0.00022 & 0.3 & 0.00371 & 0.00296 & 0.00049 & 0.00074 & 0.03088 \\
\hline EM07-Si-37 & 16674 & 0.00078 & 0.01112 & 0.0139 & 0.00411 & 0.0001 & 0.00003 & 0.0002 & 0.37 & 0.00334 & 0.00267 & 0.00044 & 0.00067 & 0.02779 \\
\hline EM07-Si-50 & .13234 & 0.00062 & 0.00882 & 0.01103 & 0.00326 & 0.00008 & 0.00003 & 0.00016 & 0.5 & 0.00265 & 0.00212 & 0.00035 & 0.00053 & 0.02206 \\
\hline EM07-Zr-001 & .15369 & 0.00072 & 0.01025 & 0.01281 & 0.00379 & 0.00009 & 0.00003 & 0.00018 & 0.44399 & 0.00307 & 0.00246 & 0.00041 & 0.00061 & 0.001 \\
\hline EM07-Zr-05 & 0.14615 & 0.00068 & 0.00974 & 0.01218 & 0.00361 & 0.00009 & 0.00003 & 0.00018 & 0.42219 & 0.00292 & 0.00234 & 0.00039 & 0.00058 & 0.05 \\
\hline EM07-NM-0025 & 0.14968 & 0.0007 & 0.00998 & 0.01247 & 0.00369 & 0.00072 & 0.00024 & 0.0 & 0.43239 & 0.00299 & 0.00239 & 0.0004 & 0.0006 & 0.02495 \\
\hline EM07-B & .15 & 07 & 0.01 & 0.0125 & 0.0037 & 0.00009 & 0.00003 & 0.00018 & & 0.003 & 0.0024 & 0.0004 & 0.0006 & 0.025 \\
\hline
\end{tabular}


Table A2. Measured Elemental Concentrations (wt\%) for Samples Prepared Using Lithium Metaborate (part 1)

\begin{tabular}{|c|c|c|c|c|c|c|c|c|c|c|c|c|c|c|c|c|c|}
\hline Set & Glass ID & Block & Sub-Block & Sequence & Lab ID & $\mathrm{Al}(\mathrm{wt} \%)$ & $\mathrm{Ba}$ (wt\%) & $\mathrm{Bi}(\mathrm{wt} \%)$ & \begin{tabular}{|l|l}
$\mathrm{Ca}(\mathrm{wt} \%)$ \\
\end{tabular} & \begin{tabular}{|l|}
$\mathrm{Cd}(\mathrm{wt} \%)$ \\
\end{tabular} & $\mathrm{Ce}(\mathrm{wt} \%)$ & \begin{tabular}{|l|}
$\mathrm{Cr}(\mathrm{wt} \%)$ \\
\end{tabular} & $\mathrm{Fe}(\mathrm{wt} \%)$ & K (wt\%) & \begin{tabular}{|l|l}
$\mathrm{La}(\mathrm{wt} \%)$ \\
\end{tabular} & $\mathrm{Mg}$ (wt\%) & \begin{tabular}{|l|} 
Mn (wt $\%)$ \\
\end{tabular} \\
\hline 1 & Batch 1 & 1 & 1 & 1 & BCHLM1111 & 2.55 & 0.13 & $<0.010$ & \begin{tabular}{|l|}
0.817 \\
\end{tabular} & \begin{tabular}{|c|c|}
$<010$ \\
\end{tabular} & 0.003 & 0.068 & 8.55 & 2.6 & $<0.010$ & 0.815 & 1.28 \\
\hline 1 & EM07-K-06 & 1 & 1 & 2 & A07LM21 & 4.99 & 0.04 & $<0.010$ & 0.013 & 0.109 & 0.04 & 0.323 & 6.39 & 4.16 & 0.044 & 0.074 & 1.45 \\
\hline 1 & EM07-Na-05 & 1 & 1 & 3 & A03LM21 & 6.02 & 0.048 & $<0.010$ & 0.017 & 0.131 & 0.049 & 0.367 & 7.4 & $<0.100$ & 0.053 & 0.09 & 1.71 \\
\hline 1 & EM07-B-15 & 1 & 1 & 4 & A11LM21 & 5.05 & 0.041 & $<0.010$ & 0.015 & 0.109 & 0.041 & 0.307 & 6.55 & $<0.100$ & 0.045 & 0.078 & 1.5 \\
\hline 1 & EM07-F-02 & 1 & 1 & 5 & A13LM11 & 5.34 & 0.042 & $<0.010$ & 0.009 & 0.113 & 0.043 & 0.312 & 6.61 & $<0.100$ & 0.046 & 0.078 & 1.52 \\
\hline 1 & EM07-Сa-035 & 1 & 1 & 6 & A04LM11 & 5.12 & 0.04 & $<0.010$ & 2.68 & 0.106 & 0.041 & 0.295 & 6.37 & $<0.100$ & 0.046 & 0.075 & 1.45 \\
\hline 1 & EM07-K-03 & 1 & 1 & 7 & A06LM21 & 5.2 & 0.042 & $<0.010$ & 0.01 & 0.11 & 0.042 & 0.324 & 6.52 & 2.36 & 0.045 & 0.077 & 1.49 \\
\hline 1 & EM07-Ca-035 & 1 & 1 & 8 & A04LM21 & 5.13 & 0.04 & $<0.010$ & 2.71 & 0.108 & 0.042 & 0.297 & 6.14 & $<0.100$ & 0.046 & 0.077 & 1.4 \\
\hline 1 & EM07-B-05 & 1 & 1 & 9 & A10LM11 & 5.7 & 0.046 & $<0.010$ & 0.012 & 0.121 & 0.045 & 0.353 & 6.65 & $<0.100$ & 0.049 & 0.083 & 1.52 \\
\hline 1 & EM07-Ca-07 & 1 & 1 & 10 & A14LM21 & 4.95 & 0.039 & $<0.010$ & 5.27 & 0.104 & 0.04 & 0.296 & 5.98 & $<0.100$ & 0.044 & 0.096 & 1.36 \\
\hline 1 & EM07-B-15 & 1 & 1 & 11 & A11LM11 & 5.08 & 0.041 & $<0.010$ & 0.011 & 0.108 & 0.042 & 0.304 & 6.19 & $<0.100$ & 0.045 & 0.077 & 1.4 \\
\hline 1 & Batch 1 & 1 & 1 & 12 & BCHLM1112 & 2.6 & 0.128 & $<0.010$ & 0.816 & $<0.010$ & 0.003 & 0.067 & 8.69 & 2.6 & $<0.010$ & 0.807 & 1.3 \\
\hline 1 & EM07-Ca-07 & 1 & 1 & 13 & A14LM11 & 4.92 & 0.038 & $<0.010$ & 5.18 & 0.101 & 0.04 & 0.29 & 6.29 & $<0.100$ & 0.044 & 0.073 & 1.43 \\
\hline 1 & EM07-Bi-05 & 1 & 1 & 14 & A17LM21 & 5.05 & 0.041 & 4.4 & 0.012 & 0.105 & 0.042 & 0.294 & 6.4 & $<0.100$ & 0.044 & 0.074 & 1.46 \\
\hline 1 & EM07-F-02 & 1 & 1 & 15 & A13LM21 & 5.29 & 0.042 & $<0.010$ & 0.012 & 0.111 & 0.043 & 0.308 & 6.63 & $<0.100$ & 0.046 & 0.078 & 1.52 \\
\hline 1 & EM07-Mn-04 & 1 & 1 & 16 & A19LM11 & 5.23 & 0.041 & $<0.010$ & 0.012 & 0.11 & 0.043 & 0.295 & 6.47 & $<0.100$ & 0.046 & 0.078 & 3.01 \\
\hline 1 & EM07-Bi-05 & 1 & 1 & 17 & A17LM11 & 5.06 & 0.041 & 4.38 & 0.011 & 0.106 & 0.042 & 0.298 & 6.23 & $<0.100$ & 0.044 & 0.074 & 1.42 \\
\hline 1 & EM07-K-03 & 1 & 1 & 18 & A06LM11 & 5.15 & 0.042 & $<0.010$ & 0.031 & 0.108 & 0.043 & 0.312 & 6.48 & 2.37 & 0.045 & 0.078 & 1.48 \\
\hline 1 & EM07-K-06 & 1 & 1 & 19 & A07LM11 & 5.07 & 0.04 & $<0.010$ & 0.021 & 0.106 & 0.04 & 0.303 & 6.41 & 4.18 & 0.044 & 0.074 & 1.46 \\
\hline 1 & EM07-B-05 & 1 & 1 & 20 & A10LM21 & 5.66 & 0.045 & $<0.010$ & 0.011 & 0.119 & 0.045 & 0.339 & 7.22 & $<0.100$ & 0.05 & 0.083 & 1.65 \\
\hline 1 & EM07-Mn-04 & 1 & 1 & 21 & A19LM21 & 5.28 & 0.041 & $<0.010$ & 0.017 & 0.11 & 0.042 & 0.292 & 6.34 & $<0.100$ & 0.045 & 0.078 & 2.97 \\
\hline 1 & EM07-Na-05 & 1 & 1 & 22 & A03LM11 & 6.02 & 0.047 & $<0.010$ & 0.017 & 0.127 & 0.048 & 0.363 & 7.12 & $<0.100$ & 0.052 & 0.089 & 1.65 \\
\hline 1 & Batch 1 & 1 & 1 & 23 & BCHLM1113 & 2.62 & 0.127 & $<0.010$ & 0.808 & $<0.010$ & 0.003 & 0.067 & 8.6 & 2.58 & $<0.010$ & 0.805 & 1.29 \\
\hline 1 & Batch 1 & 1 & 2 & 1 & BCHLM1121 & 2.57 & 0.129 & $<0.010$ & 0.806 & $<0.010$ & 0.003 & 0.067 & 9.23 & 2.57 & $<0.010$ & 0.815 & 1.39 \\
\hline 1 & EM07-Na-05 & 1 & 2 & 2 & A03LM12 & 6.06 & 0.047 & $<0.010$ & 0.016 & 0.128 & 0.048 & 0.366 & 8.01 & $<0.100$ & 0.052 & 0.088 & 1.85 \\
\hline 1 & EM07-Ca-07 & 1 & 2 & 3 & A14LM22 & 5.04 & 0.038 & $<0.010$ & 5.29 & 0.102 & 0.04 & 0.296 & 6.6 & $<0.100$ & 0.044 & 0.095 & 1.5 \\
\hline 1 & EM07-Сa-035 & 1 & 2 & 4 & A04LM12 & 5.15 & 0.039 & $<0.010$ & 2.67 & 0.105 & 0.041 & 0.296 & 6.9 & $<0.100$ & 0.047 & 0.074 & 1.56 \\
\hline 1 & EM07-Na-05 & 1 & 2 & 5 & A03LM22 & 6.07 & 0.047 & $<0.010$ & 0.017 & 0.129 & 0.049 & 0.365 & 7.87 & $<0.100$ & 0.053 & 0.088 & 1.79 \\
\hline 1 & EM07-K-03 & 1 & 2 & 6 & A06LM22 & 5.29 & 0.041 & $<0.010$ & 0.009 & 0.108 & 0.042 & 0.324 & 6.47 & 2.36 & 0.045 & 0.076 & 1.47 \\
\hline 1 & EM07-F-02 & 1 & 2 & 7 & A13LM12 & 5.32 & 0.041 & $<0.010$ & 0.009 & 0.11 & 0.043 & 0.311 & 6.73 & $<0.100$ & 0.046 & 0.077 & 1.53 \\
\hline 1 & EM07-Ca-07 & 1 & 2 & 8 & A14LM12 & 5.01 & 0.038 & $<0.010$ & 5.36 & 0.1 & 0.04 & 0.294 & 6.17 & $<0.100$ & 0.044 & 0.073 & 1.39 \\
\hline 1 & EM07-K-03 & 1 & 2 & 9 & A06LM12 & 5.24 & 0.042 & $<0.010$ & 0.03 & 0.108 & 0.042 & 0.313 & 6.65 & 2.34 & 0.045 & 0.077 & 1.51 \\
\hline 1 & EM07-B-05 & 1 & 2 & 10 & A10LM12 & 5.75 & 0.045 & $<0.010$ & 0.011 & 0.119 & 0.045 & 0.353 & 7.18 & $<0.100$ & 0.05 & 0.082 & 1.65 \\
\hline 1 & EM07-B-05 & 1 & 2 & 11 & A10LM22 & 5.73 & 0.045 & $<0.010$ & 0.01 & 0.118 & 0.045 & 0.343 & 6.98 & $<0.100$ & 0.05 & 0.082 & 1.6 \\
\hline 1 & Batch 1 & 1 & 2 & 12 & BCHLM1122 & 2.6 & 0.128 & $<0.010$ & 0.818 & $<0.010$ & 0.003 & 0.067 & 8.85 & 2.61 & $<0.010$ & 0.814 & 1.33 \\
\hline 1 & EM07-Сa-035 & 1 & 2 & 13 & A04LM22 & 5.16 & 0.04 & $<0.010$ & 2.74 & 0.106 & 0.041 & 0.297 & 6.4 & $<0.100$ & 0.046 & 0.075 & 1.45 \\
\hline 1 & EM07-Mn-04 & 1 & 2 & 14 & A19LM22 & 5.31 & 0.041 & $<0.010$ & 0.017 & 0.11 & 0.043 & 0.297 & 6.56 & $<0.100$ & 0.046 & 0.078 & 3.06 \\
\hline 1 & EM07-K-06 & 1 & 2 & 15 & A07LM22 & 5.1 & 0.039 & $<0.010$ & 0.012 & 0.106 & 0.04 & 0.321 & 6.53 & 4.16 & 0.044 & 0.072 & 1.47 \\
\hline 1 & EM07-F-02 & 1 & 2 & 16 & A13LM22 & 5.41 & 0.041 & $<0.010$ & 0.011 & 0.11 & 0.043 & 0.309 & 6.7 & $<0.100$ & 0.047 & 0.077 & 1.52 \\
\hline 1 & EM07-Bi-05 & 1 & 2 & 17 & A17LM22 & 5.16 & 0.04 & 4.46 & 0.012 & 0.105 & 0.042 & 0.294 & 6.4 & $<0.100$ & 0.045 & 0.073 & 1.45 \\
\hline 1 & EM07-Bi-05 & 1 & 2 & 18 & A17LM12 & 5.14 & 0.04 & 4.39 & 0.01 & 0.105 & 0.041 & 0.297 & 6.32 & $<0.100$ & 0.044 & 0.073 & 1.42 \\
\hline 1 & EM07-B-15 & 1 & 2 & 19 & A11LM22 & 5.14 & 0.04 & $<0.010$ & 0.015 & 0.106 & 0.041 & 0.304 & 6.14 & $<0.100$ & 0.045 & 0.075 & 1.39 \\
\hline 1 & EM07-K-06 & 1 & 2 & 20 & A07LM12 & 5.07 & 0.039 & $\begin{array}{l}<0.010 \\
\end{array}$ & 0.021 & 0.105 & 0.04 & 0.305 & 6.13 & 4.17 & 0.044 & 0.073 & 1.39 \\
\hline
\end{tabular}


Table A2. Measured Elemental Concentrations (wt\%) for Samples Prepared Using Lithium Metaborate (part 1)

\begin{tabular}{|c|c|c|c|c|c|c|c|c|c|c|c|c|c|c|c|c|c|}
\hline Set & Glass ID & Block & Sub-Block & Sequence & Lab ID & $\mathrm{Al}(\mathrm{wt} \%)$ & $\mathrm{Ba}$ (wt\%) & $\mathrm{Bi}(\mathrm{wt} \%)$ & \begin{tabular}{|l|l}
$\mathrm{Ca}(\mathrm{wt} \%)$ \\
\end{tabular} & \begin{tabular}{|l|}
$\mathrm{Cd}(\mathrm{wt} \%)$ \\
\end{tabular} & $\mathrm{Ce}(\mathrm{wt} \%)$ & \begin{tabular}{|l|}
$\mathrm{Cr}(\mathrm{wt} \%)$ \\
\end{tabular} & $\mathrm{Fe}(\mathrm{wt} \%)$ & K (wt\%) & $\mathrm{La}(\mathrm{wt} \%)$ & $\mathrm{Mg}$ (wt\%) & Mn (wt\%) \\
\hline 1 & EM07-B-15 & 1 & 2 & 21 & A11LM12 & 5.11 & 0.04 & $<0.010$ & 0.01 & 0.105 & 0.041 & 0.301 & 6.26 & $<0.100$ & 0.045 & 0.074 & 1.42 \\
\hline 1 & EM07-Mn-04 & 1 & 2 & 22 & A19LM12 & 5.29 & 0.04 & $<0.010$ & 0.012 & 0.108 & 0.043 & 0.296 & 6.36 & $<0.100$ & 0.046 & 0.077 & 2.96 \\
\hline 1 & Batch 1 & 1 & 2 & 23 & BCHLM1123 & 2.57 & 0.129 & $<0.010$ & 0.844 & $<0.010$ & 0.003 & 0.067 & 8.71 & 2.67 & $<0.010$ & 0.814 & 1.3 \\
\hline 1 & Batch 1 & 2 & 1 & 1 & BCHLM1211 & 2.59 & 0.129 & $<0.010$ & 0.808 & $<0.010$ & 0.003 & 0.066 & 9.04 & 2.55 & $<0.010$ & 0.811 & 1.36 \\
\hline 1 & EM07-P-0 & 2 & 1 & 2 & A16LM21 & 5.37 & 0.045 & $<0.010$ & 0.011 & 0.113 & 0.045 & 0.311 & 7.1 & $<0.100$ & 0.047 & 0.078 & 1.63 \\
\hline 1 & EM07-Na-20 & 2 & 1 & 3 & A01LM11 & 5.06 & 0.041 & $<0.010$ & 0.015 & 0.103 & 0.04 & 0.313 & 6.61 & $<0.100$ & 0.044 & 0.08 & 1.53 \\
\hline 1 & EM07-Na-10 & 2 & 1 & 4 & A12LM21 & 5.67 & 0.046 & $<0.010$ & 0.011 & 0.116 & 0.046 & 0.309 & 7.23 & $<0.100$ & 0.049 & 0.082 & 1.68 \\
\hline 1 & EM07-B-20 & 2 & 1 & 5 & A18LM21 & 4.76 & 0.038 & $<0.010$ & 0.009 & 0.097 & 0.039 & 0.283 & 6.06 & $<0.100$ & 0.042 & 0.069 & 1.38 \\
\hline 1 & EM07-B-20 & 2 & 1 & 6 & A18LM11 & 4.72 & 0.038 & $<0.010$ & 0.03 & 0.096 & 0.039 & 0.281 & 5.81 & $<0.100$ & 0.042 & 0.069 & 1.36 \\
\hline 1 & EM07-Bi-025 & 2 & 1 & 7 & A05LM21 & 5.22 & 0.043 & 2.16 & 0.041 & 0.108 & 0.043 & 0.338 & 6.38 & $<0.100$ & 0.048 & 0.078 & 1.47 \\
\hline 1 & EM07-Zr-001 & 2 & 1 & 8 & A09LM11 & 5.52 & 0.044 & $<0.010$ & 0.015 & 0.11 & 0.044 & 0.33 & 6.59 & $<0.100$ & 0.048 & 0.08 & 1.51 \\
\hline 1 & EM07-Na-10 & 2 & 1 & 9 & A12LM11 & 5.74 & 0.047 & $<0.010$ & 0.017 & 0.117 & 0.047 & 0.311 & 7.25 & $<0.100$ & 0.05 & 0.083 & 1.67 \\
\hline 1 & EM07-Mn-01 & 2 & 1 & 10 & A02LM21 & 5.51 & 0.045 & $<0.010$ & 0.018 & 0.114 & 0.044 & 0.327 & 6.75 & $<0.100$ & 0.047 & 0.08 & 0.03 \\
\hline 1 & EM07-Na-20 & 2 & 1 & 11 & A01LM21 & 5.09 & 0.041 & $<0.010$ & 0.019 & 0.103 & 0.041 & 0.309 & 6.22 & $<0.100$ & 0.044 & 0.085 & 1.44 \\
\hline 1 & Batch 1 & 2 & 1 & 12 & BCHLM1212 & 2.59 & 0.129 & $<0.010$ & 0.837 & $<0.010$ & 0.004 & 0.066 & 8.46 & 2.63 & $<0.010$ & 0.805 & 1.28 \\
\hline 1 & EM07-Bi-025 & 2 & 1 & 13 & A05LM11 & 5.16 & 0.043 & 2.18 & 0.019 & 0.107 & 0.044 & 0.335 & 6.41 & $<0.100$ & 0.049 & 0.078 & 1.48 \\
\hline 1 & EM07-P-025 & 2 & 1 & 14 & A15LM11 & 5.26 & 0.043 & $<0.010$ & 0.012 & 0.11 & 0.043 & 0.312 & 6.4 & $<0.100$ & 0.047 & 0.108 & 1.48 \\
\hline 1 & EM07-Zr-05 & 2 & 1 & 15 & A08LM21 & 5.19 & 0.043 & $<0.010$ & 0.013 & 0.108 & 0.044 & 0.285 & 6.24 & $<0.100$ & 0.045 & 0.076 & 1.44 \\
\hline 1 & EM07-Zr-001 & 2 & 1 & 16 & A09LM21 & 5.42 & 0.044 & $<0.010$ & 0.018 & 0.11 & 0.044 & 0.327 & 6.54 & $<0.100$ & 0.048 & 0.08 & 1.51 \\
\hline 1 & EM07-Mn-01 & 2 & 1 & 17 & A02LM11 & 5.43 & 0.045 & $<0.010$ & 0.02 & 0.112 & 0.044 & 0.328 & 6.51 & $<0.100$ & 0.048 & 0.08 & 0.03 \\
\hline 1 & EM07-P-0 & 2 & 1 & 18 & A16LM11 & 5.35 & 0.045 & $<0.010$ & 0.016 & 0.112 & 0.046 & 0.312 & 6.48 & $<0.100$ & 0.048 & 0.079 & 1.59 \\
\hline 1 & EM07-Zr-05 & 2 & 1 & 19 & A08LM11 & 5.14 & 0.043 & $<0.010$ & 0.013 & 0.106 & 0.044 & 0.289 & 6.27 & $<0.100$ & 0.045 & 0.075 & 1.43 \\
\hline 1 & EM07-P-025 & 2 & 1 & 20 & A15LM21 & 5.19 & 0.043 & $<0.010$ & 0.013 & 0.107 & 0.043 & 0.299 & 6.35 & $<0.100$ & 0.047 & 0.077 & 1.46 \\
\hline 1 & Batch 1 & 2 & 1 & 21 & BCHLM1213 & 2.58 & 0.131 & $<0.010$ & 0.872 & $<0.010$ & 0.004 & 0.067 & 8.35 & 2.72 & $<0.010$ & 0.813 & 1.25 \\
\hline 1 & Batch 1 & 2 & 2 & 1 & BCHLM1221 & 2.58 & 0.129 & $<0.010$ & 0.81 & $<0.010$ & 0.005 & 0.066 & 8.74 & 2.52 & $<0.010$ & 0.81 & 1.34 \\
\hline 1 & EM07-Na-20 & 2 & 2 & 2 & A01LM22 & 5.03 & 0.04 & $<0.010$ & 0.018 & 0.104 & 0.041 & 0.308 & 6.28 & $<0.100$ & 0.041 & 0.084 & 1.49 \\
\hline 1 & EM07-Zr-05 & 2 & 2 & 3 & A08LM12 & 5.14 & 0.042 & $<0.010$ & 0.012 & 0.106 & 0.042 & 0.286 & 6.36 & $<0.100$ & 0.041 & 0.073 & 1.47 \\
\hline 1 & EM07-Na-20 & 2 & 2 & 4 & A01LM12 & 4.99 & 0.04 & $<0.010$ & 0.015 & 0.103 & 0.041 & 0.311 & 6.29 & $<0.100$ & 0.041 & 0.078 & 1.47 \\
\hline 1 & EM07-P-0 & 2 & 2 & 5 & A16LM22 & 5.39 & 0.044 & $<0.010$ & 0.011 & 0.111 & 0.045 & 0.309 & 6.42 & $<0.100$ & 0.044 & 0.077 & 1.51 \\
\hline 1 & EM07-B-20 & 2 & 2 & 6 & A18LM12 & 4.65 & 0.037 & $<0.010$ & 0.03 & 0.096 & 0.039 & 0.278 & 5.6 & $<0.100$ & 0.038 & 0.067 & 1.34 \\
\hline 1 & EM07-Na-10 & 2 & 2 & 7 & A12LM22 & 5.58 & 0.046 & $<0.010$ & 0.011 & 0.116 & 0.047 & 0.308 & 6.34 & $<0.100$ & 0.047 & 0.081 & 1.51 \\
\hline 1 & EM07-P-025 & 2 & 2 & 8 & A15LM12 & 5.18 & 0.042 & $<0.010$ & 0.011 & 0.109 & 0.043 & 0.307 & 6.22 & $<0.100$ & 0.043 & 0.105 & 1.46 \\
\hline 1 & EM07-Na-10 & 2 & 2 & 9 & A12LM12 & 5.6 & 0.046 & $<0.010$ & 0.016 & 0.116 & 0.047 & 0.308 & 6.89 & $<0.100$ & 0.046 & 0.082 & 1.63 \\
\hline 1 & EM07-Bi-025 & 2 & 2 & 10 & A05LM12 & 5.09 & 0.042 & 2.15 & 0.019 & 0.107 & 0.044 & 0.333 & 6.31 & $<0.100$ & 0.046 & 0.076 & 1.5 \\
\hline 1 & EM07-Mn-01 & 2 & 2 & 11 & A02LM12 & 5.38 & 0.044 & $<0.010$ & 0.019 & 0.112 & 0.045 & 0.324 & 6.49 & $<0.100$ & 0.044 & 0.078 & 0.06 \\
\hline 1 & Batch 1 & 2 & 2 & 12 & BCHLM1222 & 2.64 & 0.129 & $<0.010$ & 0.854 & $<0.010$ & 0.005 & 0.066 & 8.32 & 2.63 & $<0.010$ & 0.805 & 1.28 \\
\hline 1 & EM07-Zr-05 & 2 & 2 & 13 & A08LM22 & 5.1 & 0.042 & $<0.010$ & 0.013 & 0.106 & 0.044 & 0.28 & 6.17 & $<0.100$ & 0.042 & 0.074 & 1.45 \\
\hline 1 & EM07-Bi-025 & 2 & 2 & 14 & A05LM22 & 5.11 & 0.041 & 2.15 & 0.042 & 0.107 & 0.044 & 0.334 & 6.32 & $<0.100$ & 0.045 & 0.076 & 1.49 \\
\hline 1 & EM07-Mn-01 & 2 & 2 & 15 & A02LM22 & 5.38 & 0.044 & $<0.010$ & 0.018 & 0.112 & 0.045 & 0.325 & 6.61 & $<0.100$ & 0.045 & 0.078 & 0.06 \\
\hline 1 & EM07-Zr-001 & 2 & 2 & 16 & A09LM12 & 5.4 & 0.044 & $<0.010$ & 0.015 & 0.109 & 0.046 & 0.33 & 6.55 & $<0.100$ & 0.046 & 0.079 & 1.53 \\
\hline 1 & EM07-P-0 & 2 & 2 & 17 & A16LM12 & 5.33 & 0.044 & $<0.010$ & 0.016 & 0.109 & 0.046 & 0.309 & 6.45 & $<0.100$ & 0.045 & 0.077 & 1.61 \\
\hline 1 & EM07-P-025 & 2 & 2 & 18 & A15LM22 & 5.14 & 0.042 & $<0.010$ & 0.013 & 0.106 & 0.043 & 0.296 & 6.44 & $<0.100$ & 0.045 & 0.076 & 1.51 \\
\hline 1 & EM07-Zr-001 & 2 & 2 & 19 & A09LM22 & 5.31 & 0.043 & $<<0.010$ & 0.019 & 0.107 & 0.045 & 0.324 & 6.77 & \begin{tabular}{|l|}
$<0.100$ \\
\end{tabular} & 0.046 & 0.079 & 1.58 \\
\hline
\end{tabular}


Table A2. Measured Elemental Concentrations (wt\%) for Samples Prepared Using Lithium Metaborate (part 1)

\begin{tabular}{|c|c|c|c|c|c|c|c|c|c|c|c|c|c|c|c|c|c|}
\hline Set & Glass ID & Block & Sub-Block & Sequence & Lab ID & $\mathrm{Al}(\mathrm{wt} \%)$ & $\mathrm{Ba}(\mathrm{wt} \%)$ & $\mathrm{Bi}(\mathrm{wt} \%)$ & \begin{tabular}{|l|l}
$\mathrm{Ca}(\mathrm{wt} \%)$ \\
\end{tabular} & \begin{tabular}{|l|}
$\mathrm{Cd}(\mathrm{wt} \%)$ \\
\end{tabular} & $\mathrm{Ce}(\mathrm{wt} \%)$ & \begin{tabular}{|l|}
$\mathrm{Cr}$ (wt\%) \\
\end{tabular} & $\mathrm{Fe}(\mathrm{wt} \%)$ & K (wt\%) & $\mathrm{La}(\mathrm{wt} \%)$ & $\mathrm{Mg}$ (wt\%) & Mn (wt\%) \\
\hline 1 & EM07-B-20 & 2 & 2 & 20 & A18LM22 & 4.64 & 0.037 & $<0.010$ & \begin{tabular}{|l|}
0.01 \\
\end{tabular} & 0.094 & 0.04 & 0.279 & 5.78 & $<<0.100$ & 0.04 & 0.066 & 1.34 \\
\hline 1 & Batch 1 & 2 & 2 & 21 & BCHLM1223 & 2.63 & 0.127 & $<0.010$ & 0.874 & $<0.010$ & 0.005 & 0.065 & 8.25 & 2.68 & $<0.010$ & 0.792 & 1.27 \\
\hline 2 & Batch 1 & 1 & 1 & 1 & BCHLM2111 & 2.48 & 0.124 & $<0.010$ & 0.821 & $<0.010$ & $<0.010$ & 0.074 & 8.81 & 2.51 & $<0.010$ & 0.808 & 1.32 \\
\hline 2 & EM07-Fe-15 & 1 & 1 & 2 & B01LM11 & 4.86 & 0.039 & $<0.010$ & $<0.010$ & 0.106 & 0.04 & 0.283 & 10.2 & $<0.100$ & 0.048 & 0.074 & 1.5 \\
\hline 2 & EM07-Si-50 & 1 & 1 & 3 & B11LM11 & 4.51 & 0.036 & $<0.010$ & $<0.010$ & 0.097 & 0.037 & 0.288 & 6.08 & $<0.100$ & 0.043 & 0.068 & 1.39 \\
\hline 2 & EM07-Al-15 & 1 & 1 & 4 & B13LM21 & 7.86 & 0.038 & $<0.010$ & $<0.010$ & 0.104 & 0.039 & 0.268 & 6.6 & $<0.100$ & 0.047 & 0.074 & 1.52 \\
\hline 2 & EM07-Cr-02 & 1 & 1 & 5 & B03LM11 & 5.09 & 0.04 & $<0.010$ & 0.012 & 0.107 & 0.041 & 1.04 & 6.63 & $<0.100$ & 0.048 & 0.076 & 1.51 \\
\hline 2 & EM07-Si-30 & 1 & 1 & 6 & B18LM11 & 6.45 & 0.051 & $<0.010$ & $<0.010$ & 0.14 & 0.051 & 0.376 & 8.47 & $<0.100$ & 0.061 & 0.097 & 1.96 \\
\hline 2 & EM07-BL-1 & 1 & 1 & 7 & B07LM21 & 5.25 & 0.041 & $<0.010$ & $<0.010$ & 0.113 & 0.042 & 0.32 & 6.81 & $<0.100$ & 0.05 & 0.079 & 1.56 \\
\hline 2 & EM07-Fe-05 & 1 & 1 & 8 & B14LM21 & 5.42 & 0.044 & $<0.010$ & $<0.010$ & 0.117 & 0.045 & 0.341 & 3.44 & $<0.100$ & 0.052 & 0.083 & 1.61 \\
\hline 2 & EM07-Al-15 & 1 & 1 & 9 & B13LM11 & 7.72 & 0.038 & $<0.010$ & 0.013 & 0.103 & 0.04 & 0.27 & 6.27 & $<0.100$ & 0.048 & 0.074 & 1.45 \\
\hline 2 & EM07-Si-30 & 1 & 1 & 10 & B18LM21 & 6.42 & 0.053 & $<0.010$ & $<0.010$ & 0.138 & 0.051 & 0.373 & 8.75 & $<0.100$ & 0.061 & 0.097 & 1.91 \\
\hline 2 & EM07-Si-37 & 1 & 1 & 11 & B19LM11 & 5.7 & 0.045 & $<0.010$ & $<0.010$ & 0.122 & 0.046 & 0.349 & 7.54 & $<0.100$ & 0.055 & 0.087 & 1.73 \\
\hline 2 & Batch 1 & 1 & 1 & 12 & BCHLM2112 & 2.49 & 0.125 & $<0.010$ & 0.83 & $<0.010$ & $<0.010$ & 0.075 & 9.05 & 2.55 & $<0.010$ & 0.809 & 1.37 \\
\hline 2 & EM07-Al-06 & 1 & 1 & 13 & B17LM21 & 3.17 & 0.042 & $<0.010$ & 0.057 & 0.114 & 0.043 & 0.333 & 7.25 & $<0.100$ & 0.052 & 0.086 & 1.68 \\
\hline 2 & EM07-Fe-20 & 1 & 1 & 14 & B02LM11 & 4.71 & 0.036 & $<0.010$ & 0.028 & 0.095 & 0.038 & 0.27 & 13.5 & $<0.100$ & 0.044 & 0.068 & 1.38 \\
\hline 2 & EM07-Fe-15 & 1 & 1 & 15 & B01LM21 & 4.81 & 0.038 & $<0.010$ & $<0.010$ & 0.101 & 0.04 & 0.273 & 9.62 & $<0.100$ & 0.047 & 0.072 & 1.41 \\
\hline 2 & EM07-Si-50 & 1 & 1 & 16 & B11LM21 & 4.57 & 0.036 & $<0.010$ & $<0.010$ & 0.097 & 0.038 & 0.29 & 5.77 & $<0.100$ & 0.044 & 0.068 & 1.32 \\
\hline 2 & EM07-Cr-02 & 1 & 1 & 17 & B03LM21 & 5.14 & 0.04 & $<0.010$ & 0.011 & 0.107 & 0.042 & 1.057 & 6.41 & $<0.100$ & 0.049 & 0.077 & 1.45 \\
\hline 2 & EM07-Fe-20 & 1 & 1 & 18 & B02LM21 & 4.65 & 0.036 & $<0.010$ & $<0.010$ & 0.097 & 0.038 & 0.271 & 13.3 & $<0.100$ & 0.045 & 0.069 & 1.37 \\
\hline 2 & EM07-Si-37 & 1 & 1 & 19 & B19LM21 & 5.77 & 0.046 & $<0.010$ & $<0.010$ & 0.121 & 0.047 & 0.35 & 7.46 & $<0.100$ & 0.056 & 0.087 & 1.71 \\
\hline 2 & EM07-Al-06 & 1 & 1 & 20 & B17LM11 & 3.2 & 0.043 & $<0.010$ & 0.017 & 0.115 & 0.044 & 0.339 & 7.22 & $<0.100$ & 0.052 & 0.083 & 1.66 \\
\hline 2 & EM07-Fe-05 & 1 & 1 & 21 & B14LM11 & 5.61 & 0.044 & $<0.010$ & 0.014 & 0.115 & 0.046 & 0.343 & 3.47 & $<0.100$ & 0.054 & 0.083 & 1.69 \\
\hline 2 & EM07-BL-1 & 1 & 1 & 22 & B07LM11 & 5.31 & 0.041 & $<0.010$ & 0.01 & 0.113 & 0.044 & 0.32 & 6.84 & $<0.100$ & 0.051 & 0.079 & 1.56 \\
\hline 2 & Batch 1 & 1 & 1 & 23 & BCHLM2113 & 2.51 & 0.125 & $<0.010$ & 0.856 & $<0.010$ & $<0.010$ & 0.075 & 8.68 & 2.64 & $<0.010$ & 0.809 & 1.3 \\
\hline 2 & Batch 1 & 1 & 2 & 1 & BCHLM2121 & 2.5 & 0.127 & $<0.010$ & 0.806 & $<0.010$ & $<0.010$ & 0.076 & 8.94 & 2.63 & $<0.010$ & 0.816 & 1.35 \\
\hline 2 & EM07-Fe-05 & 1 & 2 & 2 & B14LM12 & 5.46 & 0.045 & $<0.010$ & 0.014 & 0.122 & 0.045 & 0.351 & 3.41 & $<0.100$ & 0.054 & 0.086 & 1.65 \\
\hline 2 & EM07-Cr-02 & 1 & 2 & 3 & B03LM12 & 5.12 & 0.041 & $<0.010$ & 0.014 & 0.112 & 0.042 & 1.082 & 6.51 & $<0.100$ & 0.051 & 0.08 & 1.48 \\
\hline 2 & EM07-Al-15 & 1 & 2 & 4 & B13LM12 & 7.69 & 0.039 & $<0.010$ & 0.015 & 0.105 & 0.041 & 0.274 & 6.17 & $<0.100$ & 0.049 & 0.076 & 1.42 \\
\hline 2 & EM07-Si-30 & 1 & 2 & 5 & B18LM12 & 6.42 & 0.052 & $<0.010$ & 0.01 & 0.143 & 0.052 & 0.384 & 8.1 & $<0.100$ & 0.063 & 0.1 & 1.88 \\
\hline 2 & EM07-Fe-05 & 1 & 2 & 6 & B14LM22 & 5.38 & 0.045 & $<0.010$ & 0.011 & 0.121 & 0.046 & 0.348 & 3.32 & $<0.100$ & 0.054 & 0.085 & 1.54 \\
\hline 2 & EM07-Fe-15 & 1 & 2 & 7 & B01LM22 & 4.71 & 0.038 & $<0.010$ & $<0.010$ & 0.104 & 0.04 & 0.275 & 9.36 & $<0.100$ & 0.047 & 0.074 & 1.37 \\
\hline 2 & EM07-Fe-20 & 1 & 2 & 8 & B02LM12 & 4.67 & 0.036 & $<0.010$ & 0.03 & 0.098 & 0.039 & 0.275 & 13.6 & $<0.100$ & 0.046 & 0.071 & 1.38 \\
\hline 2 & EM07-Si-37 & 1 & 2 & 9 & B19LM12 & 5.71 & 0.046 & $<0.010$ & 0.011 & 0.126 & 0.048 & 0.355 & 7.38 & $<0.100$ & 0.057 & 0.089 & 1.71 \\
\hline 2 & EM07-Al-15 & 1 & 2 & 10 & B13LM22 & 7.85 & 0.038 & $<0.010$ & 0.011 & 0.104 & 0.041 & 0.272 & 6.25 & $<0.100$ & 0.049 & 0.076 & 1.44 \\
\hline 2 & EM07-Al-06 & 1 & 2 & 11 & B17LM12 & 3.17 & 0.043 & $<0.010$ & 0.019 & 0.119 & 0.045 & 0.345 & 6.75 & $<0.100$ & 0.054 & 0.086 & 1.56 \\
\hline 2 & Batch 1 & 1 & 2 & 12 & BCHLM2122 & 2.5 & 0.126 & $<0.010$ & 0.868 & $<0.010$ & $<0.010$ & 0.075 & 8.3 & 2.57 & $<0.010$ & 0.801 & 1.26 \\
\hline 2 & EM07-Al-06 & 1 & 2 & 13 & B17LM22 & 3.14 & 0.042 & $<0.010$ & 0.058 & 0.116 & 0.043 & 0.335 & 6.83 & $<0.100$ & 0.052 & 0.087 & 1.58 \\
\hline 2 & EM07-Si-37 & 1 & 2 & 14 & B19LM22 & 5.73 & 0.046 & $<0.010$ & 0.011 & 0.124 & 0.048 & 0.352 & 7.41 & $<0.100$ & 0.056 & 0.089 & 1.71 \\
\hline 2 & EM07-BL-1 & 1 & 2 & 15 & B07LM12 & 5.3 & 0.041 & $<0.010$ & 0.011 & 0.113 & 0.044 & 0.319 & 6.73 & $<0.100$ & 0.052 & 0.08 & 1.55 \\
\hline 2 & EM07-Fe-15 & 1 & 2 & 16 & B01LM12 & 4.94 & 0.038 & $<0.010$ & 0.011 & 0.103 & 0.041 & 0.278 & 9.96 & $<0.100$ & 0.049 & 0.074 & 1.47 \\
\hline 2 & EM07-Fe-20 & 1 & 2 & 17 & B02LM22 & 4.66 & 0.036 & $<0.010$ & 0.01 & 0.096 & 0.039 & 0.269 & 12.9 & $<0.100$ & 0.046 & 0.07 & 1.35 \\
\hline 2 & EM07-Si-50 & 1 & 2 & 18 & B11LM22 & 4.62 & 0.035 & $<0.010$ & 0.011 & 0.097 & 0.039 & 0.291 & 5.66 & \begin{tabular}{|c|}
$<0.100$ \\
\end{tabular} & 0.045 & 0.07 & 1.3 \\
\hline
\end{tabular}


Table A2. Measured Elemental Concentrations (wt\%) for Samples Prepared Using Lithium Metaborate (part 1)

\begin{tabular}{|c|c|c|c|c|c|c|c|c|c|c|c|c|c|c|c|c|c|}
\hline Set & Glass ID & Block & Sub-Block & Sequence & Lab ID & $\mathrm{Al}(\mathrm{wt} \%)$ & $\mathrm{Ba}(\mathrm{wt} \%)$ & $\mathrm{Bi}(\mathrm{wt} \%)$ & Ca (wt\%) & \begin{tabular}{|l|}
$\mathrm{Cd}(\mathrm{wt} \%)$ \\
\end{tabular} & $\mathrm{Ce}(\mathrm{wt} \%)$ & $\mathrm{Cr}(\mathrm{wt} \%)$ & $\mathrm{Fe}(\mathrm{wt} \%)$ & $\mathrm{K}(\mathrm{wt} \%)$ & $\mathrm{La}(\mathrm{wt} \%)$ & $\mathrm{Mg}$ (wt\%) & Mn (wt\%) \\
\hline 2 & EM07-BL-1 & 1 & 2 & 19 & B07LM22 & 5.22 & 0.042 & $<0.010$ & \begin{tabular}{|l|}
0.011 \\
\end{tabular} & 0.115 & 0.044 & 0.326 & 6.44 & $<0.100$ & 0.052 & 0.081 & 1.48 \\
\hline 2 & EM07-Cr-02 & 1 & 2 & 20 & B03LM22 & 5.11 & 0.041 & $<0.010$ & 0.013 & 0.11 & 0.043 & 1.074 & 6.07 & $<0.100$ & 0.051 & 0.079 & 1.38 \\
\hline 2 & EM07-Si-50 & 1 & 2 & 21 & B11LM12 & 4.52 & 0.036 & $<0.010$ & 0.01 & 0.099 & 0.039 & 0.297 & 5.79 & $<0.100$ & 0.046 & 0.071 & 1.32 \\
\hline 2 & EM07-Si-30 & 1 & 2 & 22 & B18LM22 & 6.44 & 0.054 & $<0.010$ & $<0.010$ & 0.143 & 0.053 & 0.385 & 8.73 & $<0.100$ & 0.064 & 0.1 & 1.9 \\
\hline 2 & Batch 1 & 1 & 2 & 23 & BCHLM2123 & 2.52 & 0.129 & $<0.010$ & 0.845 & $<0.010$ & $<0.010$ & 0.077 & 8.84 & 2.43 & $<0.010$ & 0.828 & 1.33 \\
\hline 2 & Batch 1 & 2 & 1 & 1 & BCHLM2211 & 2.51 & 0.125 & $<0.010$ & 0.823 & $<0.010$ & $<0.010$ & 0.074 & 8.81 & 2.48 & $<0.010$ & 0.815 & 1.36 \\
\hline 2 & EM07-Li-015 & 2 & 1 & 2 & B04LM11 & 5.35 & 0.042 & $<0.010$ & $<0.010$ & 0.115 & 0.043 & 0.317 & 7.02 & $<0.100$ & 0.05 & 0.081 & 1.64 \\
\hline 2 & EM07-NM-0025 & 2 & 1 & 3 & B05LM21 & 5.16 & 0.04 & $<0.010$ & 0.012 & 0.112 & 0.042 & 0.318 & 6.64 & $<0.100$ & 0.049 & 0.08 & 1.54 \\
\hline 2 & EM07-BL-2 & 2 & 1 & 4 & B06LM11 & 5.13 & 0.041 & $<0.010$ & 0.013 & 0.113 & 0.042 & 0.325 & 6.35 & $<0.100$ & 0.049 & 0.079 & 1.48 \\
\hline 2 & EM07-Al-20 & 2 & 1 & 5 & B12LM21 & 9.74 & 0.037 & $<0.010$ & $<0.010$ & 0.101 & 0.039 & 0.247 & 5.64 & $<0.100$ & 0.045 & 0.071 & 1.33 \\
\hline 2 & EM07-Ni-001 & 2 & 1 & 6 & B09LM21 & 5.25 & 0.041 & $<0.010$ & $<0.010$ & 0.115 & 0.042 & 0.33 & 6.39 & $<0.100$ & 0.05 & 0.08 & 1.48 \\
\hline 2 & EM07-Cr-001 & 2 & 1 & 7 & B10LM21 & 5.2 & 0.042 & $<0.010$ & $<0.010$ & 0.115 & 0.043 & 0.076 & 6.7 & $<0.100$ & 0.049 & 0.081 & 1.56 \\
\hline 2 & EM07-Al-20 & 2 & 1 & 8 & B12LM11 & 9.69 & 0.037 & $<0.010$ & $<0.010$ & 0.1 & 0.038 & 0.244 & 5.94 & $<0.100$ & 0.045 & 0.071 & 1.39 \\
\hline 2 & EM07-Ni-001 & 2 & 1 & 9 & B09LM11 & 5.26 & 0.041 & $<0.010$ & $<0.010$ & 0.114 & 0.042 & 0.329 & 6.71 & $<0.100$ & 0.05 & 0.079 & 1.56 \\
\hline 2 & EM07-Ni-02 & 2 & 1 & 10 & B15LM11 & 5.13 & 0.04 & $<0.010$ & $<0.010$ & 0.113 & 0.041 & 0.313 & 6.49 & $<0.100$ & 0.049 & 0.079 & 1.52 \\
\hline 2 & EM07-Li-04 & 2 & 1 & 11 & B16LM11 & 5.12 & 0.041 & $<0.010$ & 0.022 & 0.112 & 0.041 & 0.322 & 6.4 & $<0.100$ & 0.049 & 0.079 & 1.49 \\
\hline 2 & Batch 1 & 2 & 1 & 12 & BCHLM2212 & 2.49 & 0.127 & $<0.010$ & 0.855 & $<0.010$ & $<0.010$ & 0.076 & 8.29 & 2.61 & $<0.010$ & 0.825 & 1.29 \\
\hline 2 & EM07-Li-04 & 2 & 1 & 13 & B16LM21 & 5.1 & 0.042 & $<0.010$ & $<0.010$ & 0.114 & 0.042 & 0.32 & 6.32 & $<0.100$ & 0.049 & 0.084 & 1.47 \\
\hline 2 & EM07-BL-2 & 2 & 1 & 14 & B06LM21 & 5.07 & 0.04 & $<0.010$ & $<0.010$ & 0.111 & 0.042 & 0.32 & 6.46 & $<0.100$ & 0.048 & 0.078 & 1.5 \\
\hline 2 & EM07-Cr-012 & 2 & 1 & 15 & B08LM21 & 5.2 & 0.042 & $<0.010$ & $<0.010$ & 0.114 & 0.043 & 0.729 & 6.73 & $<0.100$ & 0.05 & 0.08 & 1.56 \\
\hline 2 & EM07-Cr-012 & 2 & 1 & 16 & B08LM11 & 5.23 & 0.042 & $<0.010$ & $<0.010$ & 0.112 & 0.043 & 0.726 & 6.61 & $<0.100$ & 0.05 & 0.08 & 1.54 \\
\hline 2 & EM07-Cr-001 & 2 & 1 & 17 & B10LM11 & 5.22 & 0.042 & $<0.010$ & $<0.010$ & 0.115 & 0.043 & 0.078 & 6.64 & $<0.100$ & 0.05 & 0.081 & 1.54 \\
\hline 2 & EM07-Li-015 & 2 & 1 & 18 & B04LM21 & 5.34 & 0.042 & $<0.010$ & $<0.010$ & 0.115 & 0.044 & 0.321 & 6.64 & $<0.100$ & 0.05 & 0.082 & 1.54 \\
\hline 2 & EM07-NM-0025 & 2 & 1 & 19 & B05LM11 & 5.24 & 0.041 & $<0.010$ & $<0.010$ & 0.115 & 0.044 & 0.327 & 6.27 & $<0.100$ & 0.05 & 0.081 & 1.47 \\
\hline 2 & EM07-Ni-02 & 2 & 1 & 20 & B15LM21 & 4.94 & 0.039 & $<0.010$ & $<0.010$ & 0.109 & 0.04 & 0.297 & 6.87 & $<0.100$ & 0.047 & 0.075 & 1.39 \\
\hline 2 & Batch 1 & 2 & 1 & 21 & BCHLM2213 & 2.53 & 0.127 & $<0.010$ & 0.873 & $<0.010$ & $<0.010$ & 0.076 & 8.44 & 2.65 & $<0.010$ & 0.826 & 1.3 \\
\hline 2 & Batch 1 & 2 & 2 & 1 & BCHLM2221 & 2.46 & 0.125 & $<0.010$ & 0.821 & $<0.010$ & $<0.010$ & 0.073 & 9.02 & 2.47 & $<0.010$ & 0.812 & 1.33 \\
\hline 2 & EM07-NM-0025 & 2 & 2 & 2 & B05LM12 & 5.14 & 0.039 & $<0.010$ & 0.01 & 0.114 & 0.042 & 0.319 & 7.11 & $<0.100$ & 0.049 & 0.079 & 1.59 \\
\hline 2 & EM07-Ni-02 & 2 & 2 & 3 & B15LM12 & 5.07 & 0.038 & $<0.010$ & $<0.010$ & 0.112 & 0.041 & 0.308 & 7.06 & $<0.100$ & 0.048 & 0.077 & 1.59 \\
\hline 2 & EM07-BL-2 & 2 & 2 & 4 & B06LM22 & 5.05 & 0.038 & $<0.010$ & $<0.010$ & 0.109 & 0.041 & 0.316 & 7.06 & $<0.100$ & 0.048 & 0.077 & 1.58 \\
\hline 2 & EM07-Ni-02 & 2 & 2 & 5 & B15LM22 & 5.05 & 0.038 & $<0.010$ & $<0.010$ & 0.112 & 0.041 & 0.303 & 7.02 & $<0.100$ & 0.047 & 0.077 & 1.58 \\
\hline 2 & EM07-Al-20 & 2 & 2 & 6 & B12LM12 & 9.57 & 0.035 & $<0.010$ & $<0.010$ & 0.1 & 0.038 & 0.243 & 6.09 & $<0.100$ & 0.044 & 0.07 & 1.38 \\
\hline 2 & EM07-Li-015 & 2 & 2 & 7 & B04LM22 & 5.27 & 0.04 & $<0.010$ & $<0.010$ & 0.115 & 0.043 & 0.32 & 6.85 & $<0.100$ & 0.05 & 0.081 & 1.52 \\
\hline 2 & EM07-Li-04 & 2 & 2 & 8 & B16LM12 & 5.04 & 0.039 & $<0.010$ & 0.022 & 0.111 & 0.041 & 0.32 & 6.63 & $<0.100$ & 0.048 & 0.079 & 1.48 \\
\hline 2 & EM07-Cr-012 & 2 & 2 & 9 & B08LM12 & 5.1 & 0.039 & $<0.010$ & $<0.010$ & 0.111 & 0.042 & 0.719 & 6.71 & $<0.100$ & 0.049 & 0.079 & 1.5 \\
\hline 2 & EM07-Al-20 & 2 & 2 & 10 & B12LM22 & 9.65 & 0.035 & $<0.010$ & $<0.010$ & 0.1 & 0.038 & 0.244 & 6.19 & $<0.100$ & 0.044 & 0.071 & 1.38 \\
\hline 2 & EM07-Cr-012 & 2 & 2 & 11 & B08LM22 & 5.17 & 0.04 & $<0.010$ & $<0.010$ & 0.113 & 0.043 & 0.726 & 6.91 & $<0.100$ & 0.049 & 0.08 & 1.54 \\
\hline 2 & Batch 1 & 2 & 2 & 12 & BCHLM2222 & 2.5 & 0.126 & $<0.010$ & 0.834 & $<0.010$ & $<0.010$ & 0.075 & 9.14 & 2.52 & $<0.010$ & 0.82 & 1.35 \\
\hline 2 & EM07-BL-2 & 2 & 2 & 13 & B06LM12 & 5.08 & 0.04 & $<0.010$ & 0.014 & 0.113 & 0.042 & 0.324 & 6.67 & $<0.100$ & 0.049 & 0.079 & 1.49 \\
\hline 2 & EM07-Li-015 & 2 & 2 & 14 & B04LM12 & 5.26 & 0.04 & $<0.010$ & $<0.010$ & 0.117 & 0.043 & 0.319 & 6.77 & $<0.100$ & 0.05 & 0.082 & 1.52 \\
\hline 2 & EM07-Li-04 & 2 & 2 & 15 & B16LM22 & 5.07 & 0.039 & $<0.010$ & $<0.010$ & 0.113 & 0.041 & 0.318 & 6.74 & $<0.100$ & 0.048 & 0.083 & 1.51 \\
\hline 2 & EM07-Ni-001 & 2 & 2 & 16 & B09LM22 & 5.25 & 0.04 & $<0.010$ & 0.01 & 0.116 & 0.043 & 0.334 & 6.92 & $<0.100$ & 0.05 & 0.08 & 1.54 \\
\hline 2 & EM07-Cr-001 & 2 & 2 & 17 & B10LM12 & 5.14 & 0.039 & $<0.010$ & $<0.010$ & 0.113 & 0.042 & 0.075 & 6.96 & $<0.100$ & 0.049 & 0.08 & 1.53 \\
\hline
\end{tabular}


Table A2. Measured Elemental Concentrations (wt\%) for Samples Prepared Using Lithium Metaborate (part 1)

\begin{tabular}{|c|c|c|c|c|c|c|c|c|c|c|c|c|c|c|c|c|c|}
\hline Set & Glass ID & Block & Sub-Block & Sequence & Lab ID & $\mathrm{Al}(\mathrm{wt} \%)$ & Ba (wt\%) & $\mathrm{Bi}(\mathrm{wt} \%)$ & Ca (wt\%) & \begin{tabular}{|l|}
$\mathrm{Cd}(\mathrm{wt} \%)$ \\
\end{tabular} & $\mathrm{Ce}(\mathrm{wt} \%)$ & $\mathrm{Cr}(\mathrm{wt} \%)$ & $\mathrm{Fe}(\mathrm{wt} \%)$ & $\mathrm{K}(\mathrm{wt} \%)$ & $\mathrm{La}(\mathrm{wt} \%)$ & $\mathrm{Mg}(\mathrm{wt} \%)$ & Mn (wt\%) \\
\hline 2 & EM07-Cr-001 & 2 & 2 & 18 & B10LM22 & 5.24 & 0.04 & $<0.010$ & $<0.010$ & 0.115 & 0.043 & 0.074 & 6.96 & $<0.100$ & 0.049 & 0.08 & 1.55 \\
\hline 2 & EM07-Ni-001 & 2 & 2 & 19 & B09LM12 & 5.19 & 0.039 & $<0.010$ & $<0.010$ & 0.112 & 0.042 & 0.326 & 6.93 & $<0.100$ & 0.049 & 0.078 & 1.54 \\
\hline 2 & EM07-NM-0025 & 2 & 2 & 20 & B05LM22 & 5.14 & 0.038 & $<0.010$ & 0.014 & 0.111 & 0.042 & 0.315 & 6.81 & $<0.100$ & 0.049 & 0.079 & 1.51 \\
\hline 2 & Batch 1 & 2 & 2 & 21 & BCHLM2223 & 2.52 & 0.123 & $<0.010$ & 0.847 & $<0.010$ & $<0.010$ & 0.073 & 9.14 & 2.55 & $<0.010$ & 0.805 & 1.34 \\
\hline
\end{tabular}


Table A2. Measured Elemental Concentrations (wt\%) for Samples Prepared Using Lithium Metaborate (part 2)

\begin{tabular}{|c|c|c|c|c|c|c|c|c|c|c|c|c|c|}
\hline Set & Glass ID & Block & Sub-Block & Sequence & Lab ID & $\mathrm{Na}$ (wt\%) & $\mathrm{Nd}(\mathrm{wt} \%)$ & $\mathrm{Ni}$ (wt\%) & $\mathrm{P}(\mathrm{wt} \%)$ & $\mathrm{Pb}(\mathrm{wt} \%)$ & Ti (wt\%) & Zn (wt\%) & $\mathrm{Zr}(\mathrm{wt} \%)$ \\
\hline 1 & Batch 1 & 1 & 1 & 1 & BCHLM1111 & 6.75 & 0.109 & 0.547 & $<0.100$ & $<0.100$ & 0.389 & $<0.010$ & 0.068 \\
\hline 1 & EM07-K-06 & 1 & 1 & 2 & A07LM21 & 10.5 & 0.052 & 0.688 & 0.294 & 0.298 & 0.025 & 0.046 & 1.07 \\
\hline 1 & EM07-Na-05 & 1 & 1 & 3 & A03LM21 & 4.01 & 0.063 & 0.818 & 0.443 & 0.355 & 0.031 & 0.056 & 1.72 \\
\hline 1 & EM07-B-15 & 1 & 1 & 4 & A11LM21 & 10.6 & 0.054 & 0.694 & 0.314 & 0.296 & 0.025 & 0.048 & 1.17 \\
\hline 1 & EM07-F-02 & 1 & 1 & 5 & A13LM11 & 11.3 & 0.056 & 0.722 & 0.311 & 0.313 & 0.027 & 0.049 & 1.26 \\
\hline 1 & EM07-Ca-035 & 1 & 1 & 6 & A04LM11 & 10.8 & 0.054 & 0.675 & 0.378 & 0.295 & 0.026 & 0.046 & 1.26 \\
\hline 1 & EM07-K-03 & 1 & 1 & 7 & A06LM21 & 11 & 0.054 & 0.708 & 0.334 & 0.304 & 0.026 & 0.047 & 1.23 \\
\hline 1 & EM07-Сa-035 & 1 & 1 & 8 & A04LM21 & 10.9 & 0.054 & 0.688 & 0.37 & 0.3 & 0.026 & 0.047 & 1.17 \\
\hline 1 & EM07-B-05 & 1 & 1 & 9 & A10LM11 & 12.3 & 0.059 & 0.758 & 0.352 & 0.326 & 0.028 & 0.052 & 1.28 \\
\hline 1 & EM07-Сa-07 & 1 & 1 & 10 & A14LM21 & 10.8 & 0.052 & 0.671 & 0.334 & 0.289 & 0.025 & 0.046 & 1.25 \\
\hline 1 & EM07-B-15 & 1 & 1 & 11 & A11LM11 & 10.8 & 0.055 & 0.689 & 0.385 & 0.296 & 0.025 & 0.047 & 1.38 \\
\hline 1 & Batch 1 & 1 & 1 & 12 & BCHLM1112 & 6.89 & 0.108 & 0.543 & $<0.100$ & $<0.100$ & 0.383 & $<0.010$ & 0.072 \\
\hline 1 & EM07-Сa-07 & 1 & 1 & 13 & A14LM11 & 10.4 & 0.051 & 0.658 & 0.323 & 0.283 & 0.025 & 0.046 & 0.998 \\
\hline 1 & EM07-Bi-05 & 1 & 1 & 14 & A17LM21 & 10.7 & 0.054 & 0.678 & 0.33 & 0.295 & 0.027 & 0.044 & 1.34 \\
\hline 1 & EM07-F-02 & 1 & 1 & 15 & A13LM21 & 11.1 & 0.056 & 0.715 & 0.363 & 0.308 & 0.027 & 0.05 & 1.3 \\
\hline 1 & EM07-Mn-04 & 1 & 1 & 16 & A19LM11 & 11.2 & 0.055 & 0.69 & 0.354 & 0.304 & 0.026 & 0.047 & 1.24 \\
\hline 1 & EM07-Bi-05 & 1 & 1 & 17 & A17LM11 & 10.7 & 0.054 & 0.675 & 0.355 & 0.294 & 0.027 & 0.045 & 1.26 \\
\hline 1 & EM07-K-03 & 1 & 1 & 18 & A06LM11 & 11 & 0.055 & 0.704 & 0.407 & 0.303 & 0.026 & 0.047 & 1.41 \\
\hline 1 & EM07-K-06 & 1 & 1 & 19 & A07LM11 & 10.8 & 0.052 & 0.681 & 0.393 & 0.292 & 0.025 & 0.045 & 1.36 \\
\hline 1 & EM07-B-05 & 1 & 1 & 20 & A10LM21 & 12 & 0.059 & 0.758 & 0.37 & 0.326 & 0.028 & 0.051 & 1.34 \\
\hline 1 & EM07-Mn-04 & 1 & 1 & 21 & A19LM21 & 11.3 & 0.055 & 0.689 & 0.391 & 0.302 & 0.026 & 0.049 & 1.35 \\
\hline 1 & EM07-Na-05 & 1 & 1 & 22 & A03LM11 & 4.05 & 0.062 & 0.806 & 0.461 & 0.349 & 0.03 & 0.056 & 1.63 \\
\hline 1 & Batch 1 & 1 & 1 & 23 & BCHLM1113 & 7.06 & 0.107 & 0.539 & $<0.100$ & $<0.100$ & 0.383 & $<0.010$ & 0.072 \\
\hline 1 & Batch 1 & 1 & 2 & 1 & BCHLM1121 & 6.78 & 0.108 & 0.544 & $<0.100$ & $<0.100$ & 0.382 & $<0.010$ & 0.06 \\
\hline 1 & EM07-Na-05 & 1 & 2 & 2 & A03LM12 & 3.97 & 0.062 & 0.812 & 0.449 & 0.349 & 0.03 & 0.055 & 1.71 \\
\hline 1 & EM07-Ca-07 & 1 & 2 & 3 & A14LM22 & 10.8 & 0.052 & 0.672 & 0.33 & 0.289 & 0.024 & 0.043 & 1.29 \\
\hline 1 & EM07-Ca-035 & 1 & 2 & 4 & A04LM12 & 10.9 & 0.055 & 0.679 & 0.36 & 0.295 & 0.025 & 0.044 & 1.28 \\
\hline 1 & EM07-Na-05 & 1 & 2 & 5 & A03LM22 & 4.02 & 0.063 & 0.815 & 0.435 & 0.354 & 0.03 & 0.054 & 1.72 \\
\hline 1 & EM07-K-03 & 1 & 2 & 6 & A06LM22 & 11.3 & 0.054 & 0.708 & 0.324 & 0.304 & 0.025 & 0.045 & 1.19 \\
\hline 1 & EM07-F-02 & 1 & 2 & 7 & A13LM12 & 11.4 & 0.055 & 0.718 & 0.247 & 0.309 & 0.026 & 0.047 & 1.23 \\
\hline 1 & EM07-Ca-07 & 1 & 2 & 8 & A14LM12 & 10.7 & 0.052 & 0.66 & 0.291 & 0.285 & 0.024 & 0.044 & 0.992 \\
\hline 1 & EM07-K-03 & 1 & 2 & 9 & A06LM12 & 11.1 & 0.054 & 0.708 & 0.4 & 0.305 & 0.025 & 0.045 & 1.41 \\
\hline 1 & EM07-B-05 & 1 & 2 & 10 & A10LM12 & 12.2 & 0.059 & 0.756 & 0.342 & 0.324 & 0.027 & 0.05 & 1.34 \\
\hline 1 & EM07-B-05 & 1 & 2 & 11 & A10LM22 & 12.2 & 0.06 & 0.766 & 0.362 & 0.329 & 0.027 & 0.05 & 1.28 \\
\hline 1 & Batch 1 & 1 & 2 & 12 & BCHLM1122 & 6.84 & 0.108 & 0.543 & $<0.100$ & $<0.100$ & 0.384 & $<0.010$ & 0.065 \\
\hline 1 & EM07-Ca-035 & 1 & 2 & 13 & A04LM22 & 11 & 0.054 & 0.686 & 0.351 & 0.299 & 0.026 & 0.045 & 1.17 \\
\hline 1 & EM07-Mn-04 & 1 & 2 & 14 & A19LM22 & 11.4 & 0.055 & 0.701 & 0.388 & 0.308 & 0.026 & 0.047 & 1.34 \\
\hline 1 & EM07-K-06 & 1 & 2 & 15 & A07LM22 & 10.7 & 0.052 & 0.683 & 0.272 & 0.292 & 0.024 & 0.043 & 1.07 \\
\hline 1 & EM07-F-02 & 1 & 2 & 16 & A13LM22 & 11.5 & 0.056 & 0.715 & 0.305 & 0.308 & 0.026 & 0.048 & 1.28 \\
\hline 1 & EM07-Bi-05 & 1 & 2 & 17 & A17LM22 & 11 & 0.054 & 0.68 & 0.316 & 0.295 & 0.026 & 0.042 & 1.29 \\
\hline 1 & EM07-Bi-05 & 1 & 2 & 18 & A17LM12 & 10.9 & 0.053 & 0.671 & 0.342 & 0.293 & 0.026 & 0.043 & 1.26 \\
\hline 1 & EM07-B-15 & 1 & 2 & 19 & A11LM22 & 11 & 0.054 & 0.686 & 0.306 & 0.292 & 0.024 & 0.046 & 1.09 \\
\hline 1 & EM07-K-06 & 1 & 2 & 20 & A07LM12 & 10.8 & 0.052 & 0.684 & 0.375 & 0.293 & 0.024 & 0.043 & 1.26 \\
\hline
\end{tabular}


Table A2. Measured Elemental Concentrations (wt\%) for Samples Prepared Using Lithium Metaborate (part 2)

\begin{tabular}{|c|c|c|c|c|c|c|c|c|c|c|c|c|c|}
\hline Set & Glass ID & Block & Sub-Block & Sequence & Lab ID & $\mathrm{Na}(\mathrm{wt} \%)$ & $\mathrm{Nd}(\mathrm{wt} \%)$ & $\mathrm{Ni}$ (wt\%) & $\mathrm{P}(\mathrm{wt} \%)$ & $\mathrm{Pb}(\mathrm{wt} \%)$ & Ti (wt\%) & Zn (wt\%) & $\mathrm{Zr}(\mathrm{wt} \%)$ \\
\hline 1 & EM07-B-15 & 1 & 2 & 21 & A11LM12 & 10.8 & 0.054 & 0.679 & 0.37 & 0.292 & 0.024 & 0.045 & 1.36 \\
\hline 1 & EM07-Mn-04 & 1 & 2 & 22 & A19LM12 & 11.4 & 0.055 & 0.693 & 0.334 & 0.302 & 0.026 & 0.045 & 1.19 \\
\hline 1 & Batch 1 & 1 & 2 & 23 & BCHLM1123 & 6.89 & 0.11 & 0.545 & $<0.100$ & $<0.100$ & 0.388 & $<0.010$ & 0.067 \\
\hline 1 & Batch 1 & 2 & 1 & 1 & BCHLM1211 & 6.86 & 0.108 & 0.538 & $<0.100$ & $<0.100$ & 0.382 & $<0.010$ & 0.06 \\
\hline 1 & EM07-P-0 & 2 & 1 & 2 & A16LM21 & 11.3 & 0.057 & 0.72 & $<0.100$ & 0.315 & 0.028 & 0.049 & 1.75 \\
\hline 1 & EM07-Na-20 & 2 & 1 & 3 & A01LM11 & 14.9 & 0.053 & 0.689 & 0.319 & 0.291 & 0.025 & 0.048 & 1.34 \\
\hline 1 & EM07-Na-10 & 2 & 1 & 4 & A12LM21 & 7.7 & 0.059 & 0.73 & 0.416 & 0.327 & 0.028 & 0.05 & 1.54 \\
\hline 1 & EM07-B-20 & 2 & 1 & 5 & A18LM21 & 10.2 & 0.051 & 0.643 & 0.285 & 0.274 & 0.023 & 0.041 & 1.27 \\
\hline 1 & EM07-B-20 & 2 & 1 & 6 & A18LM11 & 10.2 & 0.05 & 0.642 & 0.267 & 0.271 & 0.023 & 0.042 & 0.971 \\
\hline 1 & EM07-Bi-025 & 2 & 1 & 7 & A05LM21 & 11.1 & 0.057 & 0.724 & 0.305 & 0.304 & 0.025 & 0.052 & 1.14 \\
\hline 1 & EM07-Zr-001 & 2 & 1 & 8 & A09LM11 & 11.7 & 0.101 & 0.74 & 0.483 & 0.318 & 0.027 & 0.049 & 0.049 \\
\hline 1 & EM07-Na-10 & 2 & 1 & 9 & A12LM11 & 7.89 & 0.06 & 0.738 & 0.408 & 0.331 & 0.028 & 0.052 & 1.46 \\
\hline 1 & EM07-Mn-01 & 2 & 1 & 10 & A02LM21 & 11.7 & 0.058 & 0.732 & 0.32 & 0.31 & 0.027 & 0.049 & 1.72 \\
\hline 1 & EM07-Na-20 & 2 & 1 & 11 & A01LM21 & 15.1 & 0.053 & 0.692 & 0.303 & 0.29 & 0.025 & 0.045 & 1.11 \\
\hline 1 & Batch 1 & 2 & 1 & 12 & BCHLM1212 & 6.9 & 0.109 & 0.54 & $<0.100$ & $<0.100$ & 0.385 & $<0.010$ & 0.066 \\
\hline 1 & EM07-Bi-025 & 2 & 1 & 13 & A05LM11 & 11 & 0.058 & 0.721 & 0.336 & 0.301 & 0.026 & 0.049 & 1.16 \\
\hline 1 & EM07-P-025 & 2 & 1 & 14 & A15LM11 & 11.5 & 0.055 & 0.713 & 0.368 & 0.308 & 0.022 & 0.047 & 0.33 \\
\hline 1 & EM07-Zr-05 & 2 & 1 & 15 & A08LM21 & 11.1 & 0.061 & 0.689 & 0.467 & 0.307 & 0.026 & 0.046 & 3.17 \\
\hline 1 & EM07-Zr-001 & 2 & 1 & 16 & A09LM21 & 11.5 & 0.102 & 0.737 & 0.492 & 0.315 & 0.027 & 0.049 & 0.043 \\
\hline 1 & EM07-Mn-01 & 2 & 1 & 17 & A02LM11 & 11.5 & 0.059 & 0.728 & 0.362 & 0.311 & 0.028 & 0.05 & 1.2 \\
\hline 1 & EM07-P-0 & 2 & 1 & 18 & A16LM11 & 11.4 & 0.06 & 0.724 & $<0.100$ & 0.315 & 0.028 & 0.049 & 1.66 \\
\hline 1 & EM07-Zr-05 & 2 & 1 & 19 & A08LM11 & 11 & 0.061 & 0.685 & 0.427 & 0.303 & 0.026 & 0.045 & 2.81 \\
\hline 1 & EM07-P-025 & 2 & 1 & 20 & A15LM21 & 11.1 & 0.055 & 0.705 & 0.374 & 0.304 & 0.022 & 0.047 & 0.391 \\
\hline 1 & Batch 1 & 2 & 1 & 21 & BCHLM1213 & 6.86 & 0.114 & 0.544 & $<0.100$ & $<0.100$ & 0.395 & $<0.010$ & 0.066 \\
\hline 1 & Batch 1 & 2 & 2 & 1 & BCHLM1221 & 6.9 & 0.105 & 0.541 & $<0.100$ & $<0.100$ & 0.384 & $<0.010$ & 0.064 \\
\hline 1 & EM07-Na-20 & 2 & 2 & 2 & A01LM22 & 14.8 & 0.051 & 0.69 & 0.316 & 0.293 & 0.024 & 0.045 & 2.75 \\
\hline 1 & EM07-Zr-05 & 2 & 2 & 3 & A08LM12 & 11.1 & 0.056 & 0.678 & 0.416 & 0.302 & 0.024 & 0.044 & 1.29 \\
\hline 1 & EM07-Na-20 & 2 & 2 & 4 & A01LM12 & 14.8 & 0.051 & 0.686 & 0.318 & 0.288 & 0.024 & 0.047 & 1.67 \\
\hline 1 & EM07-P-0 & 2 & 2 & 5 & A16LM22 & 11.5 & 0.055 & 0.715 & 0.01 & 0.314 & 0.027 & 0.048 & 0.971 \\
\hline 1 & EM07-B-20 & 2 & 2 & 6 & A18LM12 & 10 & 0.048 & 0.638 & 0.284 & 0.272 & 0.022 & 0.042 & 1.4 \\
\hline 1 & EM07-Na-10 & 2 & 2 & 7 & A12LM22 & 7.66 & 0.058 & 0.73 & 0.422 & 0.329 & 0.027 & 0.05 & 1.4 \\
\hline 1 & EM07-P-025 & 2 & 2 & 8 & A15LM12 & 11.2 & 0.051 & 0.704 & 0.415 & 0.307 & 0.022 & 0.046 & 0.439 \\
\hline 1 & EM07-Na-10 & 2 & 2 & 9 & A12LM12 & 7.67 & 0.057 & 0.735 & 0.406 & 0.331 & 0.027 & 0.051 & 1.39 \\
\hline 1 & EM07-Bi-025 & 2 & 2 & 10 & A05LM12 & 10.8 & 0.056 & 0.716 & 0.341 & 0.299 & 0.025 & 0.049 & 1.14 \\
\hline 1 & EM07-Mn-01 & 2 & 2 & 11 & A02LM12 & 11.4 & 0.056 & 0.721 & 0.387 & 0.309 & 0.027 & 0.049 & 1.2 \\
\hline 1 & Batch 1 & 2 & 2 & 12 & BCHLM1222 & 6.88 & 0.109 & 0.538 & $<0.100$ & $<0.100$ & 0.388 & $<0.010$ & 0.072 \\
\hline 1 & EM07-Zr-05 & 2 & 2 & 13 & A08LM22 & 10.9 & 0.058 & 0.679 & 0.461 & 0.304 & 0.025 & 0.044 & 3.1 \\
\hline 1 & EM07-Bi-025 & 2 & 2 & 14 & A05LM22 & 10.7 & 0.055 & 0.714 & 0.3 & 0.3 & 0.025 & 0.062 & 1.13 \\
\hline 1 & EM07-Mn-01 & 2 & 2 & 15 & A02LM22 & 11.3 & 0.056 & 0.725 & 0.317 & 0.311 & 0.027 & 0.048 & 1.59 \\
\hline 1 & EM07-Zr-001 & 2 & 2 & 16 & A09LM12 & 11.4 & 0.101 & 0.736 & 0.48 & 0.315 & 0.027 & 0.049 & 0.043 \\
\hline 1 & EM07-P-0 & 2 & 2 & 17 & A16LM12 & 11.3 & 0.058 & 0.715 & $<0.100$ & 0.311 & 0.027 & 0.048 & 1.64 \\
\hline 1 & EM07-P-025 & 2 & 2 & 18 & A15LM22 & 10.9 & 0.053 & 0.698 & 0.313 & 0.302 & 0.021 & 0.046 & 0.26 \\
\hline 1 & EM07-Zr-001 & 2 & 2 & 19 & A09LM22 & 11.3 & 0.102 & 0.726 & 0.492 & 0.31 & 0.027 & 0.048 & 0.033 \\
\hline
\end{tabular}


Table A2. Measured Elemental Concentrations (wt\%) for Samples Prepared Using Lithium Metaborate (part 2)

\begin{tabular}{|c|c|c|c|c|c|c|c|c|c|c|c|c|c|}
\hline Set & Glass ID & Block & Sub-Block & Sequence & Lab ID & $\mathrm{Na}(\mathrm{wt} \%)$ & $\mathrm{Nd}$ (wt\%) & Ni (wt\%) & $\mathrm{P}(\mathrm{wt} \%)$ & $\mathrm{Pb}(\mathrm{wt} \%)$ & Ti (wt\%) & Zn (wt\%) & $\mathrm{Zr}$ (wt\%) \\
\hline 1 & EM07-B-20 & 2 & 2 & 20 & A18LM22 & 9.91 & 0.05 & 0.634 & 0.284 & 0.27 & 0.023 & 0.041 & 1.16 \\
\hline 1 & Batch 1 & 2 & 2 & 21 & BCHLM1223 & 6.86 & 0.11 & 0.529 & $<0.100$ & $<0.100$ & 0.385 & $<0.010$ & 0.071 \\
\hline 2 & Batch 1 & 1 & 1 & 1 & BCHLM2111 & 6.54 & 0.117 & 0.535 & $<0.100$ & $<0.100$ & 0.382 & $<0.010$ & 0.06 \\
\hline 2 & EM07-Fe-15 & 1 & 1 & 2 & B01LM11 & 10.4 & 0.051 & 0.642 & 0.312 & 0.285 & 0.027 & 0.046 & 1.21 \\
\hline 2 & EM07-Si-50 & 1 & 1 & 3 & B11LM11 & 9.67 & 0.047 & 0.63 & 0.39 & 0.27 & 0.025 & 0.043 & 1.38 \\
\hline 2 & EM07-Al-15 & 1 & 1 & 4 & B13LM21 & 10.4 & 0.05 & 0.63 & 0.283 & 0.29 & 0.026 & 0.045 & 1.11 \\
\hline 2 & EM07-Cr-02 & 1 & 1 & 5 & B03LM11 & 10.8 & 0.053 & 0.645 & 0.351 & 0.302 & 0.027 & 0.044 & 1.31 \\
\hline 2 & EM07-Si-30 & 1 & 1 & 6 & $\begin{array}{l}\text { B18LM11 } \\
\end{array}$ & 13.7 & 0.067 & 0.86 & 0.252 & 0.383 & 0.03 & 0.061 & 1.16 \\
\hline 2 & EM07-BL-1 & 1 & 1 & 7 & B07LM21 & 11.2 & 0.055 & 0.718 & 0.378 & 0.312 & 0.026 & 0.048 & 1.38 \\
\hline 2 & EM07-Fe-05 & 1 & 1 & 8 & B14LM21 & 11.6 & 0.057 & 0.762 & 0.442 & 0.323 & 0.03 & 0.05 & 1.59 \\
\hline 2 & EM07-Al-15 & 1 & 1 & 9 & B13LM11 & 10.3 & 0.051 & 0.637 & 0.297 & 0.292 & 0.026 & 0.045 & 1.16 \\
\hline 2 & EM07-Si-30 & 1 & 1 & 10 & B18LM21 & 13.7 & 0.067 & 0.853 & 0.141 & 0.381 & 0.031 & 0.06 & 0.846 \\
\hline 2 & EM07-Si-37 & 1 & 1 & 11 & B19LM11 & 12.2 & 0.06 & 0.78 & 0.175 & 0.341 & 0.029 & 0.055 & 0.887 \\
\hline 2 & Batch 1 & 1 & 1 & 12 & BCHLM2112 & 6.59 & 0.118 & 0.536 & $<0.100$ & $<0.100$ & 0.383 & $<0.010$ & 0.065 \\
\hline 2 & EM07-Al-06 & 1 & 1 & 13 & B17LM21 & 11.7 & 0.056 & 0.735 & 0.426 & 0.32 & 0.029 & 0.055 & 1.52 \\
\hline 2 & EM07-Fe-20 & 1 & 1 & 14 & B02LM11 & 10.1 & 0.049 & 0.594 & 0.295 & 0.268 & 0.025 & 0.044 & 1.14 \\
\hline 2 & EM07-Fe-15 & 1 & 1 & 15 & B01LM21 & 10.4 & 0.051 & 0.622 & 0.322 & 0.275 & 0.026 & 0.045 & 1.24 \\
\hline 2 & EM07-Si-50 & 1 & 1 & 16 & B11LM21 & 10 & 0.048 & 0.635 & 0.414 & 0.27 & 0.026 & 0.042 & 1.46 \\
\hline 2 & EM07-Cr-02 & 1 & 1 & 17 & B03LM21 & 11.2 & 0.055 & 0.652 & 0.4 & 0.305 & 0.028 & 0.044 & 1.47 \\
\hline 2 & EM07-Fe-20 & 1 & 1 & 18 & B02LM21 & 10 & 0.05 & 0.597 & 0.347 & 0.272 & 0.025 & 0.043 & 1.29 \\
\hline 2 & EM07-Si-37 & 1 & 1 & 19 & B19LM21 & 12.3 & 0.061 & 0.779 & 0.222 & 0.341 & 0.03 & 0.055 & 1.02 \\
\hline 2 & EM07-Al-06 & 1 & 1 & 20 & B17LM11 & 11.7 & 0.058 & 0.742 & 0.385 & 0.324 & 0.029 & 0.051 & 1.41 \\
\hline 2 & EM07-Fe-05 & 1 & 1 & 21 & B14LM11 & 12.1 & 0.059 & 0.76 & 0.404 & 0.322 & 0.03 & 0.05 & 1.49 \\
\hline 2 & EM07-BL-1 & 1 & 1 & 22 & B07LM11 & 11.5 & 0.057 & 0.714 & 0.408 & 0.309 & 0.027 & 0.048 & 1.5 \\
\hline 2 & Batch 1 & 1 & 1 & 23 & BCHLM2113 & 6.78 & 0.121 & 0.534 & $<0.100$ & $<0.100$ & 0.39 & $<0.010$ & 0.066 \\
\hline 2 & Batch 1 & 1 & 2 & 1 & BCHLM2121 & 6.51 & 0.118 & 0.543 & $<0.100$ & $<0.100$ & 0.382 & $<0.010$ & 0.054 \\
\hline 2 & EM07-Fe-05 & 1 & 2 & 2 & B14LM12 & 11.5 & 0.057 & 0.782 & 0.389 & 0.334 & 0.03 & 0.052 & 1.42 \\
\hline 2 & EM07-Cr-02 & 1 & 2 & 3 & B03LM12 & 11 & 0.054 & 0.67 & 0.355 & 0.313 & 0.028 & 0.045 & 1.33 \\
\hline 2 & EM07-Al-15 & 1 & 2 & 4 & B13LM12 & 10.3 & 0.052 & 0.647 & 0.304 & 0.295 & 0.026 & 0.045 & 1.17 \\
\hline 2 & EM07-Si-30 & 1 & 2 & 5 & B18LM12 & 13.8 & 0.068 & 0.876 & 0.21 & 0.391 & 0.031 & 0.062 & 1.04 \\
\hline 2 & EM07-Fe-05 & 1 & 2 & 6 & B14LM22 & 11.6 & 0.057 & 0.774 & 0.446 & 0.331 & 0.031 & 0.051 & 1.59 \\
\hline 2 & EM07-Fe-15 & 1 & 2 & 7 & B01LM22 & 10.3 & 0.051 & 0.626 & 0.316 & 0.277 & 0.026 & 0.045 & 1.22 \\
\hline 2 & EM07-Fe-20 & 1 & 2 & 8 & B02LM12 & 9.85 & 0.05 & 0.605 & 0.298 & 0.275 & 0.025 & 0.045 & 1.14 \\
\hline 2 & EM07-Si-37 & 1 & 2 & 9 & B19LM12 & 12.2 & 0.062 & 0.791 & 0.174 & 0.345 & 0.029 & 0.056 & 0.883 \\
\hline 2 & EM07-Al-15 & 1 & 2 & 10 & B13LM22 & 10.5 & 0.052 & 0.639 & 0.286 & 0.293 & 0.026 & 0.046 & 1.12 \\
\hline 2 & EM07-Al-06 & 1 & 2 & 11 & B17LM12 & 11.8 & 0.059 & 0.76 & 0.391 & 0.331 & 0.03 & 0.052 & 1.41 \\
\hline 2 & Batch 1 & 1 & 2 & 12 & BCHLM2122 & 6.81 & 0.123 & 0.539 & $<0.100$ & $<0.100$ & 0.392 & $<0.010$ & 0.06 \\
\hline 2 & EM07-Al-06 & 1 & 2 & 13 & B17LM22 & 11.7 & 0.056 & 0.739 & 0.424 & 0.321 & 0.029 & 0.055 & 1.5 \\
\hline 2 & EM07-Si-37 & 1 & 2 & 14 & B19LM22 & 12.2 & 0.061 & 0.785 & 0.213 & 0.344 & 0.03 & 0.055 & 0.987 \\
\hline 2 & EM07-BL-1 & 1 & 2 & 15 & B07LM12 & 11.5 & 0.056 & 0.72 & 0.372 & 0.311 & 0.026 & 0.048 & 1.39 \\
\hline 2 & EM07-Fe-15 & 1 & 2 & 16 & B01LM12 & 10.7 & 0.052 & 0.632 & 0.313 & 0.276 & 0.027 & 0.045 & 1.21 \\
\hline 2 & EM07-Fe-20 & 1 & 2 & 17 & B02LM22 & 10.1 & 0.051 & 0.596 & 0.342 & 0.271 & 0.025 & 0.043 & 1.29 \\
\hline 2 & EM07-Si-50 & 1 & 2 & 18 & B11LM22 & 10.1 & 0.048 & 0.635 & 0.415 & 0.269 & 0.026 & 0.042 & 1.45 \\
\hline
\end{tabular}


Table A2. Measured Elemental Concentrations (wt\%) for Samples Prepared Using Lithium Metaborate (part 2)

\begin{tabular}{|c|c|c|c|c|c|c|c|c|c|c|c|c|c|}
\hline Set & Glass ID & Block & Sub-Block & Sequence & Lab ID & $\mathrm{Na}(\mathrm{wt} \%)$ & $\mathrm{Nd}(\mathrm{wt} \%)$ & Ni (wt\%) & $\mathrm{P}(\mathrm{wt} \%)$ & $\mathrm{Pb}(\mathrm{wt} \%)$ & Ti (wt\%) & $\mathrm{Zn}(\mathrm{wt} \%)$ & $\mathrm{Zr}(\mathrm{wt} \%)$ \\
\hline 2 & EM07-BL-1 & 1 & 2 & 19 & B07LM22 & 11.4 & 0.056 & 0.727 & 0.382 & 0.314 & 0.027 & 0.049 & 1.4 \\
\hline 2 & EM07-Cr-02 & 1 & 2 & 20 & B03LM22 & 11.1 & 0.055 & 0.662 & 0.403 & 0.31 & 0.028 & 0.045 & 1.47 \\
\hline 2 & EM07-Si-50 & 1 & 2 & 21 & B11LM12 & 10 & 0.049 & 0.642 & 0.402 & 0.273 & 0.026 & 0.044 & 1.42 \\
\hline 2 & EM07-Si-30 & 1 & 2 & 22 & B18LM22 & 13.7 & 0.069 & 0.873 & 0.141 & 0.391 & 0.032 & 0.062 & 0.852 \\
\hline 2 & Batch 1 & 1 & 2 & 23 & BCHLM2123 & 6.76 & 0.121 & 0.549 & $<0.100$ & $<0.100$ & 0.393 & $<0.010$ & 0.061 \\
\hline 2 & Batch 1 & 2 & 1 & 1 & BCHLM2211 & 6.6 & 0.116 & 0.539 & $<0.100$ & $<0.100$ & 0.385 & $<0.010$ & 0.054 \\
\hline 2 & EM07-Li-015 & 2 & 1 & 2 & B04LM11 & 11.2 & 0.054 & 0.723 & 0.388 & 0.316 & 0.026 & 0.049 & 1.41 \\
\hline 2 & EM07-NM-0025 & 2 & 1 & 3 & B05LM21 & 10.9 & 0.052 & 0.712 & 0.317 & 0.305 & 0.025 & 0.049 & 1.23 \\
\hline 2 & EM07-BL-2 & 2 & 1 & 4 & B06LM11 & 10.9 & 0.052 & 0.724 & 0.456 & 0.312 & 0.025 & 0.049 & 1.59 \\
\hline 2 & EM07-Al-20 & 2 & 1 & 5 & B12LM21 & 10.2 & 0.048 & 0.597 & 0.249 & 0.282 & 0.023 & 0.043 & 0.986 \\
\hline 2 & EM07-Ni-001 & 2 & 1 & 6 & B09LM21 & 11.3 & 0.053 & 0.073 & 0.327 & 0.314 & 0.026 & 0.046 & 1.27 \\
\hline 2 & EM07-Cr-001 & 2 & 1 & 7 & B10LM21 & 11.2 & 0.053 & 0.739 & 0.368 & 0.315 & 0.026 & 0.049 & 1.37 \\
\hline 2 & EM07-Al-20 & 2 & 1 & 8 & B12LM11 & 10 & 0.048 & 0.593 & 0.258 & 0.279 & 0.023 & 0.042 & 1.02 \\
\hline 2 & EM07-Ni-001 & 2 & 1 & 9 & B09LM11 & 11.2 & 0.053 & 0.073 & 0.35 & 0.313 & 0.026 & 0.046 & 1.33 \\
\hline 2 & EM07-Ni-02 & 2 & 1 & 10 & B15LM11 & 11.1 & 0.053 & 1.436 & 0.344 & 0.31 & 0.026 & 0.051 & 1.26 \\
\hline 2 & EM07-Li-04 & 2 & 1 & 11 & B16LM11 & 10.9 & 0.052 & 0.719 & 0.362 & 0.308 & 0.025 & 0.048 & 1.31 \\
\hline 2 & Batch 1 & 2 & 1 & 12 & BCHLM2212 & 6.89 & 0.119 & 0.549 & $<0.100$ & $<0.100$ & 0.395 & $<0.010$ & 0.061 \\
\hline 2 & EM07-Li-04 & 2 & 1 & 13 & B16LM21 & 11.1 & 0.053 & 0.719 & 0.37 & 0.311 & 0.026 & 0.048 & 1.39 \\
\hline 2 & EM07-BL-2 & 2 & 1 & 14 & B06LM21 & 10.8 & 0.052 & 0.711 & 0.358 & 0.307 & 0.025 & 0.048 & 1.33 \\
\hline 2 & EM07-Cr-012 & 2 & 1 & 15 & B08LM21 & 11.3 & 0.054 & 0.714 & 0.38 & 0.313 & 0.026 & 0.048 & 1.4 \\
\hline 2 & EM07-Cr-012 & 2 & 1 & 16 & B08LM11 & 11.2 & 0.053 & 0.706 & 0.329 & 0.311 & 0.026 & 0.048 & 1.27 \\
\hline 2 & EM07-Cr-001 & 2 & 1 & 17 & B10LM11 & 11.2 & 0.053 & 0.737 & 0.415 & 0.314 & 0.027 & 0.049 & 1.5 \\
\hline 2 & EM07-Li-015 & 2 & 1 & 18 & B04LM21 & 11.4 & 0.055 & 0.724 & 0.346 & 0.314 & 0.027 & 0.05 & 1.33 \\
\hline 2 & EM07-NM-0025 & 2 & 1 & 19 & B05LM11 & 11.3 & 0.054 & 0.734 & 0.362 & 0.317 & 0.026 & 0.049 & 1.37 \\
\hline 2 & EM07-Ni-02 & 2 & 1 & 20 & B15LM21 & 10.7 & 0.051 & 1.361 & 0.384 & 0.298 & 0.025 & 0.049 & 1.4 \\
\hline 2 & Batch 1 & 2 & 1 & 21 & BCHLM2213 & 6.84 & 0.121 & 0.548 & $<0.100$ & $<0.100$ & 0.399 & $<0.010$ & 0.062 \\
\hline 2 & Batch 1 & 2 & 2 & 1 & BCHLM2221 & 6.43 & 0.117 & 0.542 & $<0.100$ & $<0.100$ & 0.384 & $<0.010$ & 0.055 \\
\hline 2 & EM07-NM-0025 & 2 & 2 & 2 & B05LM12 & 10.7 & 0.053 & 0.722 & 0.346 & 0.311 & 0.025 & 0.048 & 1.29 \\
\hline 2 & EM07-Ni-02 & 2 & 2 & 3 & B15LM12 & 10.7 & 0.052 & 1.412 & 0.331 & 0.308 & 0.025 & 0.05 & 1.21 \\
\hline 2 & EM07-BL-2 & 2 & 2 & 4 & B06LM22 & 10.5 & 0.052 & 0.705 & 0.349 & 0.303 & 0.025 & 0.047 & 1.3 \\
\hline 2 & EM07-Ni-02 & 2 & 2 & 5 & B15LM22 & 10.7 & 0.052 & 1.392 & 0.385 & 0.305 & 0.025 & 0.049 & 1.39 \\
\hline 2 & EM07-Al-20 & 2 & 2 & 6 & B12LM12 & 9.8 & 0.048 & 0.589 & 0.251 & 0.279 & 0.023 & 0.041 & 0.992 \\
\hline 2 & EM07-Li-015 & 2 & 2 & 7 & B04LM22 & 11.1 & 0.054 & 0.722 & 0.336 & 0.313 & 0.026 & 0.048 & 1.29 \\
\hline 2 & EM07-Li-04 & 2 & 2 & 8 & B16LM12 & 10.7 & 0.052 & 0.714 & 0.353 & 0.308 & 0.025 & 0.047 & 1.29 \\
\hline 2 & EM07-Cr-012 & 2 & 2 & 9 & B08LM12 & 10.8 & 0.053 & 0.698 & 0.321 & 0.31 & 0.026 & 0.046 & 1.24 \\
\hline 2 & EM07-Al-20 & 2 & 2 & 10 & B12LM22 & 10 & 0.048 & 0.592 & 0.244 & 0.28 & 0.023 & 0.042 & 0.967 \\
\hline 2 & EM07-Cr-012 & 2 & 2 & 11 & B08LM22 & 11 & 0.054 & 0.708 & 0.37 & 0.314 & 0.026 & 0.047 & 1.37 \\
\hline 2 & Batch 1 & 2 & 2 & 12 & BCHLM2222 & 6.88 & 0.119 & 0.545 & $<0.100$ & $<0.100$ & 0.389 & $<0.010$ & 0.062 \\
\hline 2 & EM07-BL-2 & 2 & 2 & 13 & B06LM12 & 10.8 & 0.053 & 0.723 & 0.452 & 0.313 & 0.026 & 0.049 & 1.58 \\
\hline 2 & EM07-Li-015 & 2 & 2 & 14 & B04LM12 & 11.2 & 0.055 & 0.728 & 0.39 & 0.319 & 0.026 & 0.049 & 1.41 \\
\hline 2 & EM07-Li-04 & 2 & 2 & 15 & B16LM22 & 10.7 & 0.053 & 0.712 & 0.363 & 0.311 & 0.025 & 0.047 & 1.36 \\
\hline 2 & EM07-Ni-001 & 2 & 2 & 16 & B09LM22 & 11.2 & 0.054 & 0.074 & 0.325 & 0.318 & 0.026 & 0.046 & 1.27 \\
\hline 2 & EM07-Cr-001 & 2 & 2 & 17 & B10LM12 & 10.9 & 0.053 & 0.726 & 0.4 & 0.311 & 0.027 & 0.047 & 1.45 \\
\hline
\end{tabular}


Table A2. Measured Elemental Concentrations (wt\%) for Samples Prepared Using Lithium Metaborate (part 2)

\begin{tabular}{|c|c|c|c|c|c|c|c|c|c|c|c|c|c|}
\hline Set & Glass ID & Block & Sub-Block & Sequence & Lab ID & $\mathrm{Na}(\mathrm{wt} \%)$ & $\mathrm{Nd}(\mathrm{wt} \%)$ & $\mathrm{Ni}(\mathrm{wt} \%)$ & $\mathrm{P}$ (wt\%) & $\mathrm{Pb}(\mathrm{wt} \%)$ & Ti (wt\%) & $\mathrm{Zn}(\mathrm{wt} \%)$ & $\mathrm{Zr}(\mathrm{wt} \%)$ \\
\hline 2 & EM07-Cr-001 & 2 & 2 & 18 & B10LM22 & 11.2 & 0.053 & 0.733 & 0.361 & 0.314 & 0.026 & 0.047 & 1.35 \\
\hline 2 & EM07-Ni-001 & 2 & 2 & 19 & B09LM12 & 11.2 & 0.053 & 0.072 & 0.34 & 0.311 & 0.026 & 0.045 & 1.31 \\
\hline 2 & EM07-NM-0025 & 2 & 2 & 20 & B05LM22 & 11.1 & 0.053 & 0.703 & 0.314 & 0.303 & 0.025 & 0.047 & 1.23 \\
\hline 2 & Batch 1 & 2 & 2 & 21 & BCHLM2223 & 6.62 & 0.119 & 0.536 & $<0.100$ & $<0.100$ & 0.386 & $<0.010$ & 0.062 \\
\hline
\end{tabular}




\section{Table A3. Measured Elemental Concentrations (wt\%)} for Samples Prepared Using Peroxide Fusion

\begin{tabular}{|c|c|c|c|c|c|c|c|c|}
\hline Set & Glass ID & Block & Sub-Block & Sequence & Lab ID & $\mathrm{B}(\mathrm{wt} \%)$ & $\mathrm{Li}(\mathrm{wt} \%)$ & Si (wt\%) \\
\hline 1 & Batch 1 & 1 & 1 & 1 & BCHPF1111 & 2.39 & 2.15 & 23.6 \\
\hline 1 & EM07-Zr-05 & 1 & 1 & 2 & A08PF11 & 3.08 & 1.3 & 20 \\
\hline 1 & EM07-Zr-001 & 1 & 1 & 3 & A09PF11 & 3.34 & 1.34 & 20.9 \\
\hline 1 & EM07-K-06 & 1 & 1 & 4 & A07PF21 & 2.95 & 1.22 & 18.9 \\
\hline 1 & EM07-Mn-04 & 1 & 1 & 5 & A19PF21 & 3.04 & 1.26 & 19.7 \\
\hline 1 & EM07-Zr-001 & 1 & 1 & 6 & A09PF21 & 3.27 & 1.31 & 20.7 \\
\hline 1 & EM07-Zr-05 & 1 & 1 & 7 & A08PF21 & 3.09 & 1.26 & 19.7 \\
\hline 1 & EM07-Bi-025 & 1 & 1 & 8 & A05PF21 & 3.11 & 1.27 & 19.9 \\
\hline 1 & EM07-F-02 & 1 & 1 & 9 & A13PF21 & 3.2 & 1.29 & 20.2 \\
\hline 1 & EM07-P-025 & 1 & 1 & 10 & A15PF11 & 3.25 & 1.29 & 20.5 \\
\hline 1 & EM07-Na-10 & 1 & 1 & 11 & A12PF11 & 3.42 & 1.37 & 21.7 \\
\hline 1 & Batch 1 & 1 & 1 & 12 & BCHPF1112 & 2.35 & 2.06 & 22.9 \\
\hline 1 & EM07-K-06 & 1 & 1 & 13 & A07PF11 & 3.03 & 1.22 & 19 \\
\hline 1 & EM07-Bi-05 & 1 & 1 & 14 & A17PF21 & 3.07 & 1.24 & 19.4 \\
\hline 1 & EM07-Mn-04 & 1 & 1 & 15 & A19PF11 & 3.15 & 1.28 & 20 \\
\hline 1 & EM07-F-02 & 1 & 1 & 16 & A13PF11 & 3.2 & 1.29 & 20.2 \\
\hline 1 & EM07-Na-10 & 1 & 1 & 17 & A12PF21 & 3.39 & 1.37 & 21.3 \\
\hline 1 & EM07-P-025 & 1 & 1 & 18 & A15PF21 & 3.09 & 1.27 & 19.6 \\
\hline 1 & EM07-Bi-05 & 1 & 1 & 19 & A17PF11 & 3 & 1.22 & 19 \\
\hline 1 & EM07-Ca-07 & 1 & 1 & 20 & A14PF21 & 3.01 & 1.22 & 19.1 \\
\hline 1 & EM07-Ca-07 & 1 & 1 & 21 & A14PF11 & 2.98 & 1.2 & 18.5 \\
\hline 1 & EM07-Bi-025 & 1 & 1 & 22 & A05PF11 & 3.07 & 1.23 & 19.5 \\
\hline 1 & Batch 1 & 1 & 1 & 23 & BCHPF1113 & 2.45 & 2.1 & 23.4 \\
\hline 1 & Batch 1 & 1 & 2 & 1 & BCHPF1121 & 2.62 & 2.1 & 23.6 \\
\hline 1 & EM07-Bi-05 & 1 & 2 & 2 & A17PF22 & 3.19 & 1.26 & 19.7 \\
\hline 1 & EM07-Bi-05 & 1 & 2 & 3 & A17PF12 & 3.17 & 1.25 & 19.7 \\
\hline 1 & EM07-F-02 & 1 & 2 & 4 & A13PF22 & 3.24 & 1.29 & 20 \\
\hline 1 & EM07-Zr-05 & 1 & 2 & 5 & A08PF12 & 3.13 & 1.26 & 19.5 \\
\hline 1 & EM07-Ca-07 & 1 & 2 & 6 & A14PF12 & 3.03 & 1.21 & 18.6 \\
\hline 1 & EM07-Ca-07 & 1 & 2 & 7 & A14PF22 & 3.11 & 1.23 & 19.5 \\
\hline 1 & EM07-Na-10 & 1 & 2 & 8 & A12PF22 & 3.44 & 1.38 & 21.5 \\
\hline 1 & EM07-Bi-025 & 1 & 2 & 9 & A05PF22 & 3.24 & 1.28 & 20.2 \\
\hline 1 & EM07-Zr-001 & 1 & 2 & 10 & A09PF12 & 3.44 & 1.35 & 21.2 \\
\hline 1 & EM07-Mn-04 & 1 & 2 & 11 & A19PF12 & 3.13 & 1.26 & 19.6 \\
\hline 1 & Batch 1 & 1 & 2 & 12 & BCHPF1122 & 2.47 & 2.07 & 23.2 \\
\hline 1 & EM07-F-02 & 1 & 2 & 13 & A13PF12 & 3.24 & 1.29 & 20.1 \\
\hline 1 & EM07-Zr-05 & 1 & 2 & 14 & A08PF22 & 3.22 & 1.28 & 19.8 \\
\hline 1 & EM07-Bi-025 & 1 & 2 & 15 & A05PF12 & 3.15 & 1.25 & 19.7 \\
\hline 1 & EM07-K-06 & 1 & 2 & 16 & A07PF12 & 3.13 & 1.25 & 19.5 \\
\hline 1 & EM07-Mn-04 & 1 & 2 & 17 & A19PF22 & 3.24 & 1.28 & 20.3 \\
\hline 1 & EM07-Na-10 & 1 & 2 & 18 & A12PF12 & 3.4 & 1.36 & 21.3 \\
\hline 1 & EM07-P-025 & 1 & 2 & 19 & A15PF12 & 3.23 & 1.29 & 20 \\
\hline 1 & EM07-Zr-001 & 1 & 2 & 20 & A09PF22 & 3.45 & 1.33 & 21 \\
\hline 1 & EM07-K-06 & 1 & 2 & 21 & A07PF22 & 3.1 & 1.23 & 19.3 \\
\hline 1 & EM07-P-025 & 1 & 2 & 22 & A15PF22 & 3.25 & 1.3 & 20.1 \\
\hline 1 & Batch 1 & 1 & 2 & 23 & BCHPF1123 & 2.53 & 2.08 & 23.3 \\
\hline 1 & Batch 1 & 2 & 1 & 1 & BCHPF1211 & 2.56 & 2.09 & 23 \\
\hline 1 & EM07-B-05 & 2 & 1 & 2 & A10PF11 & 1.69 & 1.36 & 21.2 \\
\hline 1 & EM07-K-03 & 2 & 1 & 3 & A06PF11 & 3.2 & 1.26 & 19.5 \\
\hline 1 & EM07-Na-20 & 2 & 1 & 4 & A01PF21 & 2.96 & 1.22 & 20 \\
\hline 1 & EM07-Na-20 & 2 & 1 & 5 & A01PF11 & 2.94 & 1.21 & 18.7 \\
\hline 1 & EM07-B-20 & 2 & 1 & 6 & A18PF21 & 6.24 & 1.15 & 17.8 \\
\hline 1 & EM07-Mn-01 & 2 & 1 & 7 & A02PF21 & 3.19 & 1.34 & 20 \\
\hline 1 & EM07-B-05 & 2 & 1 & 8 & A10PF21 & 1.68 & 1.37 & 21 \\
\hline 1 & EM07-B-15 & 2 & 1 & 9 & A11PF21 & 4.76 & 1.23 & 19.1 \\
\hline 1 & EM07-Ca-035 & 2 & 1 & 10 & A04PF21 & 3.21 & 1.25 & 19.7 \\
\hline 1 & EM07-B-15 & 2 & 1 & 11 & A11PF11 & 4.69 & 1.22 & 18.8 \\
\hline 1 & Batch 1 & 2 & 1 & 12 & BCHPF1212 & 2.39 & 2.07 & 22.7 \\
\hline 1 & EM07-Ca-035 & 2 & 1 & 13 & A04PF11 & 3.12 & 1.22 & 19 \\
\hline 1 & EM07-P-0 & 2 & 1 & 14 & A16PF21 & 3.18 & 1.29 & 20 \\
\hline 1 & EM07-K-03 & 2 & 1 & 15 & A06PF21 & 3.21 & 1.25 & 19.5 \\
\hline
\end{tabular}




\section{Table A3. Measured Elemental Concentrations (wt\%) for Samples Prepared Using Peroxide Fusion}

\begin{tabular}{|c|c|c|c|c|c|c|c|c|}
\hline Set & Glass ID & Block & Sub-Block & Sequence & Lab ID & $\mathrm{B}(\mathrm{wt} \%)$ & $\mathrm{Li}(\mathrm{wt} \%)$ & Si (wt\%) \\
\hline 1 & EM07-B-20 & 2 & 1 & 16 & A18PF11 & 6.4 & 1.18 & 18.1 \\
\hline 1 & EM07-Na-05 & 2 & 1 & 17 & A03PF21 & 3.5 & 1.43 & 21.5 \\
\hline 1 & EM07-Mn-01 & 2 & 1 & 18 & A02PF11 & 3.25 & 1.32 & 20.3 \\
\hline 1 & EM07-P-0 & 2 & 1 & 19 & A16PF11 & 3.13 & 1.28 & 19.7 \\
\hline 1 & EM07-Na-05 & 2 & 1 & 20 & A03PF11 & 3.52 & 1.43 & 21.8 \\
\hline 1 & Batch 1 & 2 & 1 & 21 & BCHPF1213 & 2.44 & 2.1 & 23 \\
\hline 1 & Batch 1 & 2 & 2 & 1 & BCHPF1221 & 2.45 & 2.03 & 22.5 \\
\hline 1 & EM07-B-20 & 2 & 2 & 2 & A18PF22 & 6.05 & 1.13 & 17.4 \\
\hline 1 & EM07-P-0 & 2 & 2 & 3 & A16PF12 & 3.11 & 1.26 & 19.6 \\
\hline 1 & EM07-Na-05 & 2 & 2 & 4 & A03PF22 & 3.5 & 1.42 & 21.8 \\
\hline 1 & EM07-K-03 & 2 & 2 & 5 & A06PF22 & 3.16 & 1.25 & 19.4 \\
\hline 1 & EM07-B-05 & 2 & 2 & 6 & A10PF22 & 1.61 & 1.35 & 21.1 \\
\hline 1 & EM07-Na-20 & 2 & 2 & 7 & A01PF22 & 3.03 & 1.23 & 19.3 \\
\hline 1 & EM07-K-03 & 2 & 2 & 8 & A06PF12 & 3.13 & 1.24 & 19.3 \\
\hline 1 & EM07-B-05 & 2 & 2 & 9 & A10PF12 & 1.55 & 1.33 & 20.7 \\
\hline 1 & EM07-B-15 & 2 & 2 & 10 & A11PF22 & 4.54 & 1.2 & 18.6 \\
\hline 1 & EM07-P-0 & 2 & 2 & 11 & A16PF22 & 3.04 & 1.27 & 19.7 \\
\hline 1 & Batch 1 & 2 & 2 & 12 & BCHPF1222 & 2.4 & 2.08 & 23.1 \\
\hline 1 & EM07-Ca-035 & 2 & 2 & 13 & A04PF12 & 3.12 & 1.23 & 19.3 \\
\hline 1 & EM07-Na-05 & 2 & 2 & 14 & A03PF12 & 3.42 & 1.42 & 21.4 \\
\hline 1 & EM07-Ca-035 & 2 & 2 & 15 & A04PF22 & 3.02 & 1.22 & 18.9 \\
\hline 1 & EM07-Mn-01 & 2 & 2 & 16 & A02PF22 & 3.14 & 1.3 & 19.7 \\
\hline 1 & EM07-Mn-01 & 2 & 2 & 17 & A02PF12 & 3.18 & 1.31 & 20.3 \\
\hline 1 & EM07-Na-20 & 2 & 2 & 18 & A01PF12 & 2.94 & 1.21 & 18.8 \\
\hline 1 & EM07-B-20 & 2 & 2 & 19 & A18PF12 & 6.22 & 1.16 & 18.1 \\
\hline 1 & EM07-B-15 & 2 & 2 & 20 & A11PF12 & 4.72 & 1.23 & 19 \\
\hline 1 & Batch 1 & 2 & 2 & 21 & BCHPF1223 & 2.41 & 2.08 & 22.9 \\
\hline 2 & Batch 1 & 1 & 1 & 1 & BCHPF2111 & 2.6 & 2.08 & 22.1 \\
\hline 2 & EM07-Si-37 & 1 & 1 & 2 & B19PF21 & 3.43 & 1.35 & 17 \\
\hline 2 & EM07-Al-06 & 1 & 1 & 3 & B17PF11 & 3.29 & 1.28 & 19.8 \\
\hline 2 & EM07-Si-50 & 1 & 1 & 4 & B11PF11 & 2.78 & 1.09 & 22.4 \\
\hline 2 & EM07-Si-37 & 1 & 1 & 5 & B19PF11 & 3.54 & 1.37 & 16.9 \\
\hline 2 & EM07-BL-1 & 1 & 1 & 6 & B07PF11 & 3.12 & 1.25 & 19.8 \\
\hline 2 & EM07-Fe-05 & 1 & 1 & 7 & B14PF11 & 3.43 & 1.31 & 20.5 \\
\hline 2 & EM07-Li-015 & 1 & 1 & 8 & B04PF21 & 3.28 & 0.69 & 20.1 \\
\hline 2 & EM07-Cr-02 & 1 & 1 & 9 & B03PF21 & 3.22 & 1.23 & 19.6 \\
\hline 2 & EM07-BL-1 & 1 & 1 & 10 & B07PF21 & 3.18 & 1.25 & 19.7 \\
\hline 2 & EM07-Al-15 & 1 & 1 & 11 & B13PF11 & 3.06 & 1.18 & 18.9 \\
\hline 2 & Batch 1 & 1 & 1 & 12 & BCHPF2112 & 2.41 & 2.05 & 21.6 \\
\hline 2 & EM07-Fe-20 & 1 & 1 & 13 & B02PF11 & 2.83 & 1.1 & 17.7 \\
\hline 2 & EM07-Al-15 & 1 & 1 & 14 & B13PF21 & 3 & 1.17 & 18.9 \\
\hline 2 & EM07-Al-06 & 1 & 1 & 15 & B17PF21 & 3.25 & 1.28 & 20.2 \\
\hline 2 & EM07-Li-015 & 1 & 1 & 16 & B04PF11 & 3.2 & 0.69 & 20 \\
\hline 2 & EM07-Ni-001 & 1 & 1 & 17 & B09PF21 & 3.15 & 1.23 & 19.9 \\
\hline 2 & EM07-Fe-05 & 1 & 1 & 18 & B14PF21 & 3.26 & 1.3 & 20.6 \\
\hline 2 & EM07-Fe-20 & 1 & 1 & 19 & B02PF21 & 2.78 & 1.09 & 17.6 \\
\hline 2 & EM07-Ni-001 & 1 & 1 & 20 & B09PF11 & 3.14 & 1.23 & 19.8 \\
\hline 2 & EM07-Si-50 & 1 & 1 & 21 & B11PF21 & 2.73 & 1.08 & 21.7 \\
\hline 2 & EM07-Cr-02 & 1 & 1 & 22 & B03PF11 & 3.06 & 1.21 & 19.2 \\
\hline 2 & Batch 1 & 1 & 1 & 23 & BCHPF2113 & 2.32 & 2.01 & 21.6 \\
\hline 2 & Batch 1 & 1 & 2 & 1 & BCHPF2121 & 2.54 & 2.08 & 22.2 \\
\hline 2 & EM07-Si-37 & 1 & 2 & 2 & B19PF22 & 3.49 & 1.36 & 17 \\
\hline 2 & EM07-Fe-20 & 1 & 2 & 3 & B02PF12 & 2.73 & 1.1 & 17.8 \\
\hline 2 & EM07-Al-15 & 1 & 2 & 4 & B13PF12 & 2.91 & 1.17 & 18.9 \\
\hline 2 & EM07-Al-15 & 1 & 2 & 5 & B13PF22 & 3.01 & 1.18 & 19.2 \\
\hline 2 & EM07-BL-1 & 1 & 2 & 6 & B07PF22 & 3.2 & 1.26 & 20.1 \\
\hline 2 & EM07-Fe-05 & 1 & 2 & 7 & B14PF12 & 3.42 & 1.31 & 20.8 \\
\hline 2 & EM07-Al-06 & 1 & 2 & 8 & B17PF22 & 3.36 & 1.3 & 20.5 \\
\hline 2 & EM07-Ni-001 & 1 & 2 & 9 & B09PF22 & 3.19 & 1.26 & 20 \\
\hline 2 & EM07-Al-06 & 1 & 2 & 10 & B17PF12 & 3.26 & 1.28 & 20.1 \\
\hline 2 & EM07-BL-1 & 1 & 2 & 11 & B07PF12 & 3.06 & 1.25 & 19.7 \\
\hline
\end{tabular}




\section{Table A3. Measured Elemental Concentrations (wt\%)} for Samples Prepared Using Peroxide Fusion

\begin{tabular}{|c|c|c|c|c|c|c|c|c|}
\hline Set & Glass ID & Block & Sub-Block & Sequence & Lab ID & $\mathrm{B}(\mathrm{wt} \%)$ & $\mathrm{Li}(\mathrm{wt} \%)$ & $\mathrm{Si}(\mathrm{wt} \%)$ \\
\hline 2 & Batch 1 & 1 & 2 & 12 & BCHPF2122 & 2.37 & 2.06 & 21.8 \\
\hline 2 & EM07-Si-50 & 1 & 2 & 13 & B11PF22 & 2.81 & 1.1 & 21.9 \\
\hline 2 & EM07-Ni-001 & 1 & 2 & 14 & B09PF12 & 3.22 & 1.25 & 20.1 \\
\hline 2 & EM07-Li-015 & 1 & 2 & 15 & B04PF22 & 3.2 & 0.69 & 19.9 \\
\hline 2 & EM07-Cr-02 & 1 & 2 & 16 & B03PF12 & 3.11 & 1.22 & 19.3 \\
\hline 2 & EM07-Cr-02 & 1 & 2 & 17 & B03PF22 & 3.02 & 1.2 & 18.8 \\
\hline 2 & EM07-Fe-05 & 1 & 2 & 18 & B14PF22 & 3.22 & 1.3 & 20.4 \\
\hline 2 & EM07-Si-50 & 1 & 2 & 19 & B11PF12 & 2.76 & 1.08 & 22.2 \\
\hline 2 & EM07-Li-015 & 1 & 2 & 20 & B04PF12 & 3.22 & 0.69 & 20 \\
\hline 2 & EM07-Fe-20 & 1 & 2 & 21 & B02PF22 & 2.8 & 1.11 & 17.7 \\
\hline 2 & EM07-Si-37 & 1 & 2 & 22 & B19PF12 & 3.37 & 1.35 & 16.6 \\
\hline 2 & Batch 1 & 1 & 2 & 23 & BCHPF2123 & 2.29 & 2.03 & 21.7 \\
\hline 2 & Batch 1 & 2 & 1 & 1 & BCHPF2211 & 2.55 & 2.08 & 21.9 \\
\hline 2 & EM07-Al-20 & 2 & 1 & 2 & B12PF11 & 2.91 & 1.14 & 18 \\
\hline 2 & EM07-NM-0025 & 2 & 1 & 3 & B05PF11 & 3.18 & 1.25 & 19.4 \\
\hline 2 & EM07-Ni-02 & 2 & 1 & 4 & B15PF11 & 3.11 & 1.24 & 19.3 \\
\hline 2 & EM07-Li-04 & 2 & 1 & 5 & B16PF11 & 3.09 & 1.79 & 19.1 \\
\hline 2 & EM07-Si-30 & 2 & 1 & 6 & B18PF11 & 3.91 & 1.55 & 13.7 \\
\hline 2 & EM07-Fe-15 & 2 & 1 & 7 & B01PF11 & 3 & 1.2 & 18.6 \\
\hline 2 & EM07-Si-30 & 2 & 1 & 8 & B18PF21 & 3.85 & 1.53 & 13.7 \\
\hline 2 & EM07-Cr-012 & 2 & 1 & 9 & B08PF21 & 3.08 & 1.23 & 19.3 \\
\hline 2 & EM07-Cr-012 & 2 & 1 & 10 & B08PF11 & 3.12 & 1.25 & 19.4 \\
\hline 2 & EM07-Ni-02 & 2 & 1 & 11 & B15PF21 & 3.07 & 1.23 & 19.2 \\
\hline 2 & Batch 1 & 2 & 1 & 12 & BCHPF2212 & 2.32 & 2.04 & 21.4 \\
\hline 2 & EM07-Cr-001 & 2 & 1 & 13 & B10PF11 & 3.2 & 1.26 & 19.4 \\
\hline 2 & EM07-Al-20 & 2 & 1 & 14 & B12PF21 & 2.84 & 1.14 & 17.9 \\
\hline 2 & EM07-BL-2 & 2 & 1 & 15 & B06PF21 & 3.1 & 1.26 & 19.5 \\
\hline 2 & EM07-Cr-001 & 2 & 1 & 16 & B10PF21 & 3.08 & 1.25 & 19.3 \\
\hline 2 & EM07-NM-0025 & 2 & 1 & 17 & B05PF21 & 3.13 & 1.24 & 19.4 \\
\hline 2 & EM07-Fe-15 & 2 & 1 & 18 & B01PF21 & 2.91 & 1.18 & 18.2 \\
\hline 2 & EM07-BL-2 & 2 & 1 & 19 & B06PF11 & 3.09 & 1.26 & 19.3 \\
\hline 2 & EM07-Li-04 & 2 & 1 & 20 & B16PF21 & 3.04 & 1.8 & 18.8 \\
\hline 2 & Batch 1 & 2 & 1 & 21 & BCHPF2213 & 2.37 & 2.06 & 21.9 \\
\hline 2 & Batch 1 & 2 & 2 & 1 & BCHPF2221 & 2.57 & 2.04 & 21.8 \\
\hline 2 & EM07-Si-30 & 2 & 2 & 2 & B18PF22 & 4.08 & 1.52 & 14.1 \\
\hline 2 & EM07-Cr-001 & 2 & 2 & 3 & B10PF12 & 3.33 & 1.23 & 19.7 \\
\hline 2 & EM07-Ni-02 & 2 & 2 & 4 & B15PF22 & 3.27 & 1.22 & 19.6 \\
\hline 2 & EM07-BL-2 & 2 & 2 & 5 & B06PF22 & 3.23 & 1.24 & 19.7 \\
\hline 2 & EM07-Al-20 & 2 & 2 & 6 & B12PF22 & 2.96 & 1.12 & 18.2 \\
\hline 2 & EM07-Li-04 & 2 & 2 & 7 & B16PF12 & 3.24 & 1.79 & 19.7 \\
\hline 2 & EM07-Ni-02 & 2 & 2 & 8 & B15PF12 & 3.22 & 1.22 & 19.7 \\
\hline 2 & EM07-Cr-001 & 2 & 2 & 9 & B10PF22 & 3.22 & 1.24 & 19.7 \\
\hline 2 & EM07-BL-2 & 2 & 2 & 10 & B06PF12 & 3.24 & 1.24 & 20.1 \\
\hline 2 & EM07-Li-04 & 2 & 2 & 11 & B16PF22 & 3.16 & 1.78 & 19.1 \\
\hline 2 & Batch 1 & 2 & 2 & 12 & BCHPF2222 & 2.43 & 2.03 & 22.2 \\
\hline 2 & EM07-Cr-012 & 2 & 2 & 13 & B08PF12 & 3.21 & 1.22 & 19.5 \\
\hline 2 & EM07-Fe-15 & 2 & 2 & 14 & B01PF22 & 3.05 & 1.15 & 18.6 \\
\hline 2 & EM07-Fe-15 & 2 & 2 & 15 & B01PF12 & 3 & 1.15 & 18.4 \\
\hline 2 & EM07-Si-30 & 2 & 2 & 16 & B18PF12 & 3.98 & 1.52 & 13.9 \\
\hline 2 & EM07-Al-20 & 2 & 2 & 17 & B12PF12 & 2.91 & 1.11 & 17.8 \\
\hline 2 & EM07-NM-0025 & 2 & 2 & 18 & B05PF22 & 3.21 & 1.22 & 19.7 \\
\hline 2 & EM07-Cr-012 & 2 & 2 & 19 & B08PF22 & 3.24 & 1.24 & 19.6 \\
\hline 2 & EM07-NM-0025 & 2 & 2 & 20 & B05PF12 & 3.21 & 1.23 & 19.4 \\
\hline 2 & Batch 1 & 2 & 2 & 21 & BCHPF2223 & 2.46 & 2.05 & 21.8 \\
\hline
\end{tabular}


Table A4. Measured Elemental Concentrations (wt\%) for Samples Prepared Using Potassium Hydroxide

\begin{tabular}{||c|c|c|c|c|c|c||}
\hline Set & Glass ID & Block & Sub Block & Seq. & ID Check & F (wt\%) \\
\hline 1 & LRM & 1 & 1 & 1 & LRMKH11 & 0.77 \\
\hline 1 & EM07-F-02 & 1 & 1 & 2 & a13KH21 & 1.58 \\
\hline 1 & EM07-F-02 & 1 & 1 & 3 & a13KH11 & 1.61 \\
\hline 1 & LRM & 1 & 1 & 4 & LRMKH12 & 0.77 \\
\hline 1 & LRM & 2 & 1 & 1 & LRMKH21 & 0.79 \\
\hline 1 & EM07-F-02 & 2 & 1 & 2 & a13KH22 & 1.64 \\
\hline 1 & EM07-F-02 & 2 & 1 & 3 & a13KH12 & 1.66 \\
\hline 1 & LRM & 2 & 1 & 4 & LRMKH22 & 0.78 \\
\hline \hline
\end{tabular}


Table A5. Average Measured and Bias-Corrected Chemical Compositions Versus Targeted Compositions for the Test Matrix 1 Study Glasses

\begin{tabular}{|c|c|c|c|c|c|c|c|c|c|}
\hline & & & & Measured & & & & & \\
\hline & & & Measured & Bias-Corrected & Targeted & Diff of & Diff of & $\%$ Diff of & $\%$ Diff of \\
\hline Set & Glass ID & Oxide & (wt\%) & (wt\%) & (wt\%) & Measured & Meas BC & Measured & Meas BC \\
\hline 1 & Batch 1 & $\mathrm{Al} 2 \mathrm{O} 3$ & 4.9001 & 4.8770 & 4.8770 & 0.0231 & 0.0000 & $0.5 \%$ & $0.0 \%$ \\
\hline 1 & Batch 1 & $\mathrm{~B} 2 \mathrm{O} 3$ & 7.9049 & 7.7770 & 7.7770 & 0.1279 & 0.0000 & $1.6 \%$ & $0.0 \%$ \\
\hline 1 & Batch 1 & $\mathrm{BaO}$ & 0.1437 & 0.1510 & 0.1510 & -0.0073 & 0.0000 & $-4.8 \%$ & $0.0 \%$ \\
\hline 1 & Batch 1 & Bi2O3 & 0.0056 & 0.0056 & 0.0000 & 0.0056 & 0.0056 & & \\
\hline 1 & Batch 1 & $\mathrm{CaO}$ & 1.1618 & 1.2200 & 1.2200 & -0.0582 & 0.0000 & $-4.8 \%$ & $0.0 \%$ \\
\hline 1 & Batch 1 & $\mathrm{CdO}$ & 0.0057 & 0.0057 & 0.0000 & 0.0057 & 0.0057 & & \\
\hline 1 & Batch 1 & Ce2O3 & 0.0043 & 0.0043 & 0.0000 & 0.0043 & 0.0043 & & \\
\hline 1 & Batch 1 & Cr2O3 & 0.0973 & 0.1070 & 0.1070 & -0.0097 & 0.0000 & $-9.0 \%$ & $0.0 \%$ \\
\hline 1 & Batch 1 & Fe2O3 & 12.3657 & 12.8390 & 12.8390 & -0.4733 & 0.0000 & $-3.7 \%$ & $0.0 \%$ \\
\hline 1 & Batch 1 & $\mathrm{~K} 2 \mathrm{O}$ & 3.1480 & 3.3270 & 3.3270 & -0.1790 & 0.0000 & $-5.4 \%$ & $0.0 \%$ \\
\hline 1 & Batch 1 & La2O3 & 0.0059 & 0.0059 & 0.0000 & 0.0059 & 0.0059 & & \\
\hline 1 & Batch 1 & $\mathrm{Li} 2 \mathrm{O}$ & 4.4870 & 4.4290 & 4.4290 & 0.0580 & 0.0000 & $1.3 \%$ & $0.0 \%$ \\
\hline 1 & Batch 1 & $\mathrm{MgO}$ & 1.3413 & 1.4190 & 1.4190 & -0.0777 & 0.0000 & $-5.5 \%$ & $0.0 \%$ \\
\hline 1 & Batch 1 & $\mathrm{MnO}$ & 1.6861 & 1.7260 & 1.7260 & -0.0399 & 0.0000 & $-2.3 \%$ & $0.0 \%$ \\
\hline 1 & Batch 1 & $\mathrm{Na} 2 \mathrm{O}$ & 9.2641 & 9.0030 & 9.0030 & 0.2611 & 0.0000 & $2.9 \%$ & $0.0 \%$ \\
\hline 1 & Batch 1 & Nd2O3 & 0.1268 & 0.1470 & 0.1470 & -0.0202 & 0.0000 & $-13.7 \%$ & $0.0 \%$ \\
\hline 1 & Batch 1 & $\mathrm{NiO}$ & 0.6883 & 0.7510 & 0.7510 & -0.0627 & 0.0000 & $-8.3 \%$ & $0.0 \%$ \\
\hline 1 & Batch 1 & P2O5 & 0.1146 & 0.1146 & 0.0000 & 0.1146 & 0.1146 & & \\
\hline 1 & Batch 1 & $\mathrm{PbO}$ & 0.0539 & 0.0539 & 0.0000 & 0.0539 & 0.0539 & & \\
\hline 1 & Batch 1 & $\mathrm{SiO} 2$ & 49.4178 & 50.2200 & 50.2200 & -0.8022 & 0.0000 & $-1.6 \%$ & $0.0 \%$ \\
\hline 1 & Batch 1 & TiO2 & 0.6433 & 0.6770 & 0.6770 & -0.0337 & 0.0000 & $-5.0 \%$ & $0.0 \%$ \\
\hline 1 & Batch 1 & $\mathrm{ZnO}$ & 0.0062 & 0.0062 & 0.0000 & 0.0062 & 0.0062 & & \\
\hline 1 & Batch 1 & $\mathrm{ZrO} 2$ & 0.0904 & 0.0904 & 0.0980 & -0.0076 & -0.0076 & $-7.8 \%$ & $-7.8 \%$ \\
\hline 1 & Batch 1 & Sum & 97.6628 & 98.9565 & 98.7680 & -1.1052 & 0.1885 & $-1.1 \%$ & $0.2 \%$ \\
\hline 1 & EM07-B-05 & $\mathrm{Al2O} 3$ & 10.7890 & 10.7729 & 10.5560 & 0.2330 & 0.2169 & $2.2 \%$ & $2.1 \%$ \\
\hline 1 & EM07-B-05 & $\mathrm{B} 2 \mathrm{O} 3$ & 5.2565 & 5.1986 & 5.0000 & 0.2565 & 0.1986 & $5.1 \%$ & $4.0 \%$ \\
\hline 1 & EM07-B-05 & $\mathrm{BaO}$ & 0.0505 & 0.0532 & 0.0530 & -0.0025 & 0.0002 & $-4.7 \%$ & $0.3 \%$ \\
\hline 1 & EM07-B-05 & $\mathrm{Bi} 2 \mathrm{O} 3$ & 0.0056 & 0.0056 & 0.0000 & 0.0056 & 0.0056 & & \\
\hline 1 & EM07-B-05 & $\mathrm{CaO}$ & 0.0154 & 0.0164 & 0.0000 & 0.0154 & 0.0164 & & \\
\hline 1 & EM07-B-05 & $\mathrm{CdO}$ & 0.1362 & 0.1362 & 0.1480 & -0.0118 & -0.0118 & $-8.0 \%$ & $-8.0 \%$ \\
\hline 1 & EM07-B-05 & $\mathrm{Ce} 2 \mathrm{O} 3$ & 0.0527 & 0.0527 & 0.0530 & -0.0003 & -0.0003 & $-0.6 \%$ & $-0.6 \%$ \\
\hline 1 & EM07-B-05 & Cr2O3 & 0.5072 & 0.5528 & 0.5280 & -0.0208 & 0.0248 & $-3.9 \%$ & $4.7 \%$ \\
\hline 1 & EM07-B-05 & Fe2O3 & 10.0186 & 10.2582 & 10.5560 & -0.5374 & -0.2978 & $-5.1 \%$ & $-2.8 \%$ \\
\hline 1 & EM07-B-05 & $\mathrm{K} 2 \mathrm{O}$ & 0.0602 & 0.0639 & 0.0000 & 0.0602 & 0.0639 & & \\
\hline 1 & EM07-B-05 & La2O3 & 0.0583 & 0.0583 & 0.0740 & -0.0157 & -0.0157 & $-21.2 \%$ & $-21.2 \%$ \\
\hline 1 & EM07-B-05 & $\mathrm{Li} 2 \mathrm{O}$ & 2.9118 & 2.8868 & 2.9030 & 0.0088 & -0.0162 & $0.3 \%$ & $-0.6 \%$ \\
\hline 1 & EM07-B-05 & $\mathrm{MgO}$ & 0.1368 & 0.1442 & 0.1580 & -0.0212 & -0.0138 & $-13.4 \%$ & $-8.7 \%$ \\
\hline 1 & EM07-B-05 & $\mathrm{MnO}$ & 2.0724 & 2.1069 & 2.1110 & -0.0386 & -0.0041 & $-1.8 \%$ & $-0.2 \%$ \\
\hline$\frac{1}{1}$ & EM07-B-05 & $\mathrm{Na} 2 \mathrm{O}$ & 16.4119 & 15.9595 & 15.8330 & 0.5789 & 0.1265 & $3.7 \%$ & $0.8 \%$ \\
\hline 1 & EM07-B-05 & Nd2O3 & 0.0691 & 0.0804 & 0.0740 & -0.0049 & 0.0064 & $-6.6 \%$ & $8.6 \%$ \\
\hline 1 & EM07-B-05 & $\mathrm{NiO}$ & 0.9665 & 1.0495 & 1.0560 & -0.0895 & -0.0065 & $-8.5 \%$ & $-0.6 \%$ \\
\hline 1 & EM07-B-05 & $\mathrm{P} 2 \mathrm{O} 5$ & 0.8169 & 0.8169 & 1.3190 & -0.5021 & -0.5021 & $-38.1 \%$ & $-38.1 \%$ \\
\hline 1 & EM07-B-05 & $\mathrm{PbO}$ & 0.3514 & 0.3514 & 0.3910 & -0.0396 & -0.0396 & $-10.1 \%$ & $-10.1 \%$ \\
\hline 1 & EM07-B-05 & $\mathrm{SiO} 2$ & 44.9253 & 46.1202 & 45.7350 & -0.8097 & 0.3852 & $-1.8 \%$ & $0.8 \%$ \\
\hline 1 & EM07-B-05 & $\mathrm{TiO} 2$ & 0.0459 & 0.0484 & 0.0420 & 0.0039 & 0.0064 & $9.2 \%$ & $15.2 \%$ \\
\hline 1 & EM07-B-05 & $\mathrm{ZnO}$ & 0.0632 & 0.0632 & 0.0630 & 0.0002 & 0.0002 & $0.3 \%$ & $0.3 \%$ \\
\hline 1 & EM07-B-05 & $\mathrm{ZrO} 2$ & 1.7695 & 1.7695 & 2.6390 & -0.8695 & -0.8695 & $-32.9 \%$ & $-32.9 \%$ \\
\hline 1 & EM07-B-05 & Sum & 97.4910 & 98.5658 & 99.2920 & -1.8010 & -0.7262 & $-1.8 \%$ & $-0.7 \%$ \\
\hline 1 & EM07-B-15 & $\mathrm{Al} 2 \mathrm{O} 3$ & 9.6270 & 9.6126 & 9.4440 & 0.1830 & 0.1686 & $1.9 \%$ & $1.8 \%$ \\
\hline 1 & EM07-B-15 & $\mathrm{B} 2 \mathrm{O} 3$ & 15.0611 & 14.8982 & 15.0000 & 0.0611 & -0.1018 & $0.4 \%$ & $-0.7 \%$ \\
\hline 1 & EM07-B-15 & $\mathrm{BaO}$ & 0.0452 & 0.0476 & 0.0470 & -0.0018 & 0.0006 & $-3.8 \%$ & $1.3 \%$ \\
\hline 1 & EM07-B-15 & Bi2O3 & 0.0056 & 0.0056 & 0.0000 & 0.0056 & 0.0056 & & \\
\hline 1 & EM07-B-15 & $\mathrm{CaO}$ & 0.0178 & 0.0190 & 0.0000 & 0.0178 & 0.0190 & & \\
\hline 1 & EM07-B-15 & $\mathrm{CdO}$ & 0.1222 & 0.1222 & 0.1320 & -0.0098 & -0.0098 & $-7.4 \%$ & $-7.4 \%$ \\
\hline 1 & EM07-B-15 & Ce2O3 & 0.0483 & 0.0483 & 0.0470 & 0.0013 & 0.0013 & $2.8 \%$ & $2.8 \%$ \\
\hline 1 & EM07-B-15 & Cr2O3 & 0.4443 & 0.4843 & 0.4720 & -0.0277 & 0.0123 & $-5.9 \%$ & $2.6 \%$ \\
\hline
\end{tabular}




\section{Table A5. Average Measured and Bias-Corrected Chemical Compositions Versus} Targeted Compositions for the Test Matrix 1 Study Glasses

\begin{tabular}{|c|c|c|c|c|c|c|c|c|c|}
\hline & & & & "Measured & & & & & \\
\hline & & & Measured & Bias-Corrected & Targeted & Diff of & Diff of & $\%$ Diff of & \% Diff of \\
\hline Set & Glass ID & Oxide & (wt\%) & (wt\%) & $(w t \%)$ & Measured & Meas BC & Measured & Meas BC \\
\hline 1 & EM07-B-15 & $\mathrm{Fe} 2 \mathrm{O} 3$ & 8.9857 & 9.2045 & 9.4440 & -0.4583 & -0.2395 & $-4.9 \%$ & $-2.5 \%$ \\
\hline 1 & EM07-B-15 & K2O & 0.0602 & 0.0639 & 0.0000 & 0.0602 & 0.0639 & & \\
\hline 1 & EM07-B-15 & La2O3 & 0.0528 & 0.0528 & 0.0660 & -0.0132 & -0.0132 & $-20.0 \%$ & $-20.0 \%$ \\
\hline 1 & EM07-B-15 & $\mathrm{Li} 2 \mathrm{O}$ & 2.6265 & 2.6041 & 2.5970 & 0.0295 & 0.0071 & $1.1 \%$ & $0.3 \%$ \\
\hline 1 & EM07-B-15 & $\mathrm{MgO}$ & 0.1260 & 0.1329 & 0.1420 & -0.0160 & -0.0091 & $-11.2 \%$ & $-6.4 \%$ \\
\hline 1 & EM07-B-15 & $\mathrm{MnO}$ & 1.8432 & 1.8749 & 1.8890 & -0.0458 & -0.0141 & $-2.4 \%$ & $-0.7 \%$ \\
\hline 1 & EM07-B-15 & $\mathrm{Na} 2 \mathrm{O}$ & 14.5584 & 14.1575 & 14.1670 & 0.3914 & -0.0095 & $2.8 \%$ & $-0.1 \%$ \\
\hline 1 & EM07-B-15 & $\mathrm{Nd} 2 \mathrm{O} 3$ & 0.0633 & 0.0736 & 0.0660 & -0.0027 & 0.0076 & $-4.1 \%$ & $11.5 \%$ \\
\hline 1 & EM07-B-15 & $\mathrm{NiO}$ & 0.8742 & 0.9493 & 0.9440 & -0.0698 & 0.0053 & $-7.4 \%$ & $0.6 \%$ \\
\hline 1 & EM07-B-15 & $\mathrm{P} 2 \mathrm{O} 5$ & 0.7877 & 0.7877 & 1.1810 & -0.3933 & -0.3933 & $-33.3 \%$ & $-33.3 \%$ \\
\hline 1 & EM07-B-15 & $\mathrm{PbO}$ & 0.3167 & 0.3167 & 0.3490 & -0.0323 & -0.0323 & $-9.3 \%$ & $-9.3 \%$ \\
\hline 1 & EM07-B-15 & $\mathrm{SiO} 2$ & 40.3793 & 41.4533 & 40.9240 & -0.5447 & 0.5293 & $-1.3 \%$ & $1.3 \%$ \\
\hline 1 & EM07-B-15 & $\mathrm{TiO} 2$ & 0.0409 & 0.0431 & 0.0380 & 0.0029 & 0.0051 & $7.5 \%$ & $13.4 \%$ \\
\hline 1 & EM07-B-15 & $\mathrm{ZnO}$ & 0.0579 & 0.0579 & 0.0570 & 0.0009 & 0.0009 & $1.5 \%$ & $1.5 \%$ \\
\hline 1 & EM07-B-15 & $\mathrm{ZrO} 2$ & 1.6885 & 1.6885 & 2.3610 & -0.6725 & -0.6725 & $-28.5 \%$ & $-28.5 \%$ \\
\hline 1 & EM07-B-15 & Sum & 97.8328 & 98.6985 & 99.3670 & -1.5342 & -0.6685 & $-1.5 \%$ & $-0.7 \%$ \\
\hline 1 & EM07-B-20 & $\mathrm{Al} 2 \mathrm{O} 3$ & 8.8665 & 8.7972 & 8.8890 & -0.0225 & -0.0918 & $-0.3 \%$ & $-1.0 \%$ \\
\hline 1 & EM07-B-20 & $\mathrm{B} 2 \mathrm{O} 3$ & 20.0519 & 19.8343 & 20.0000 & 0.0519 & -0.1657 & $0.3 \%$ & $-0.8 \%$ \\
\hline 1 & EM07-B-20 & $\mathrm{BaO}$ & 0.0419 & 0.0439 & 0.0440 & -0.0021 & -0.0001 & $-4.8 \%$ & $-0.2 \%$ \\
\hline 1 & EM07-B-20 & $\mathrm{Bi} 2 \mathrm{O} 3$ & 0.0056 & 0.0056 & 0.0000 & 0.0056 & 0.0056 & & \\
\hline 1 & EM07-B-20 & $\mathrm{CaO}$ & 0.0276 & 0.0286 & 0.0000 & 0.0276 & 0.0286 & & \\
\hline 1 & EM07-B-20 & $\mathrm{CdO}$ & 0.1094 & 0.1094 & 0.1240 & -0.0146 & -0.0146 & $-11.8 \%$ & $-11.8 \%$ \\
\hline 1 & EM07-B-20 & Ce2O3 & 0.0460 & 0.0460 & 0.0440 & 0.0020 & 0.0020 & $4.5 \%$ & $4.5 \%$ \\
\hline 1 & EM07-B-20 & Cr2O3 & 0.4096 & 0.4543 & 0.4440 & -0.0344 & 0.0103 & $-7.7 \%$ & $2.3 \%$ \\
\hline 1 & EM07-B-20 & $\mathrm{Fe} 2 \mathrm{O} 3$ & 8.3101 & 8.7512 & 8.8890 & -0.5789 & -0.1378 & $-6.5 \%$ & $-1.6 \%$ \\
\hline 1 & EM07-B-20 & $\mathrm{K} 2 \mathrm{O}$ & 0.0602 & 0.0635 & 0.0000 & 0.0602 & 0.0635 & & \\
\hline 1 & EM07-B-20 & $\mathrm{La} 2 \mathrm{O} 3$ & 0.0475 & 0.0475 & 0.0620 & -0.0145 & -0.0145 & $-23.4 \%$ & $-23.4 \%$ \\
\hline 1 & EM07-B-20 & Li2O & 2.4866 & 2.4653 & 2.4440 & 0.0426 & 0.0213 & $1.7 \%$ & $0.9 \%$ \\
\hline 1 & EM07-B-20 & $\mathrm{MgO}$ & 0.1123 & 0.1193 & 0.1330 & -0.0207 & -0.0137 & $-15.5 \%$ & $-10.3 \%$ \\
\hline 1 & EM07-B-20 & $\mathrm{MnO}$ & 1.7496 & 1.8036 & 1.7780 & -0.0284 & 0.0256 & $-1.6 \%$ & $1.4 \%$ \\
\hline 1 & EM07-B-20 & $\mathrm{Na} 2 \mathrm{O}$ & 13.5845 & 13.1936 & 13.3330 & 0.2515 & -0.1394 & $1.9 \%$ & $-1.0 \%$ \\
\hline 1 & EM07-B-20 & $\mathrm{Nd} 2 \mathrm{O} 3$ & 0.0580 & 0.0670 & 0.0620 & -0.0040 & 0.0050 & $-6.4 \%$ & $8.0 \%$ \\
\hline 1 & EM07-B-20 & $\mathrm{NiO}$ & 0.8134 & 0.8918 & 0.8890 & -0.0756 & 0.0028 & $-8.5 \%$ & $0.3 \%$ \\
\hline 1 & EM07-B-20 & P2O5 & 0.6416 & 0.6416 & 1.1110 & -0.4694 & -0.4694 & $-42.3 \%$ & $-42.3 \%$ \\
\hline 1 & EM07-B-20 & $\mathrm{PbO}$ & 0.2927 & 0.2927 & 0.3290 & -0.0363 & -0.0363 & $-11.0 \%$ & $-11.0 \%$ \\
\hline 1 & EM07-B-20 & $\mathrm{SiO} 2$ & 38.1865 & 39.2021 & 38.5180 & -0.3315 & 0.6841 & $-0.9 \%$ & $1.8 \%$ \\
\hline 1 & EM07-B-20 & $\mathrm{TiO} 2$ & 0.0379 & 0.0398 & 0.0360 & 0.0019 & 0.0038 & $5.4 \%$ & $10.7 \%$ \\
\hline 1 & EM07-B-20 & $\mathrm{ZnO}$ & 0.0517 & 0.0517 & 0.0530 & -0.0013 & -0.0013 & $-2.5 \%$ & $-2.5 \%$ \\
\hline 1 & EM07-B-20 & $\mathrm{ZrO} 2$ & 1.6213 & 1.6213 & 2.2220 & -0.6007 & -0.6007 & $-27.0 \%$ & $-27.0 \%$ \\
\hline 1 & EM07-B-20 & Sum & 97.6125 & 98.5712 & 99.4040 & -1.7915 & -0.8328 & $-1.8 \%$ & $-0.8 \%$ \\
\hline 1 & EM07-Bi-025 & $\mathrm{Al} 2 \mathrm{O} 3$ & 9.7215 & 9.6455 & 9.7500 & -0.0285 & -0.1045 & $-0.3 \%$ & $-1.1 \%$ \\
\hline 1 & EM07-Bi-025 & $\mathrm{B} 2 \mathrm{O} 3$ & 10.1185 & 9.9046 & 9.7500 & 0.3685 & 0.1546 & $3.8 \%$ & $1.6 \%$ \\
\hline 1 & EM07-Bi-025 & $\mathrm{BaO}$ & 0.0472 & 0.0495 & 0.0490 & -0.0018 & 0.0005 & $-3.7 \%$ & $0.9 \%$ \\
\hline 1 & EM07-Bi-025 & $\mathrm{Bi} 2 \mathrm{O} 3$ & 2.4080 & 2.4080 & 2.5000 & -0.0920 & -0.0920 & $-3.7 \%$ & $-3.7 \%$ \\
\hline 1 & EM07-Bi-025 & $\mathrm{CaO}$ & 0.0423 & 0.0438 & 0.0000 & 0.0423 & 0.0438 & & \\
\hline 1 & EM07-Bi-025 & $\mathrm{CdO}$ & 0.1225 & 0.1225 & 0.1370 & -0.0145 & -0.0145 & $-10.6 \%$ & $-10.6 \%$ \\
\hline 1 & EM07-Bi-025 & Ce2O3 & 0.0512 & 0.0512 & 0.0490 & 0.0022 & 0.0022 & $4.6 \%$ & $4.6 \%$ \\
\hline 1 & EM07-Bi-025 & Cr2O3 & 0.4896 & 0.5431 & 0.4880 & 0.0016 & 0.0551 & $0.3 \%$ & $11.3 \%$ \\
\hline 1 & EM07-Bi-025 & $\mathrm{Fe} 2 \mathrm{O} 3$ & 9.0857 & 9.5695 & 9.7500 & -0.6643 & -0.1805 & $-6.8 \%$ & $-1.9 \%$ \\
\hline 1 & EM07-Bi-025 & $\mathrm{K} 2 \mathrm{O}$ & 0.0602 & 0.0635 & 0.0000 & 0.0602 & 0.0635 & & \\
\hline 1 & EM07-Bi-025 & $\mathrm{La} 2 \mathrm{O} 3$ & 0.0551 & 0.0551 & 0.0680 & -0.0129 & -0.0129 & $-18.9 \%$ & $-18.9 \%$ \\
\hline 1 & EM07-Bi-025 & $\mathrm{Li} 2 \mathrm{O}$ & 2.7073 & 2.6607 & 2.6810 & 0.0263 & -0.0203 & $1.0 \%$ & $-0.8 \%$ \\
\hline 1 & EM07-Bi-025 & $\mathrm{MgO}$ & 0.1277 & 0.1356 & 0.1460 & -0.0183 & -0.0104 & $-12.5 \%$ & $-7.2 \%$ \\
\hline 1 & EM07-Bi-025 & $\mathrm{MnO}$ & 1.9174 & 1.9767 & 1.9500 & -0.0326 & 0.0267 & $-1.7 \%$ & $1.4 \%$ \\
\hline 1 & EM07-Bi-025 & $\mathrm{Na} 2 \mathrm{O}$ & 14.6932 & 14.2705 & 14.6250 & 0.0682 & -0.3545 & $0.5 \%$ & $-2.4 \%$ \\
\hline 1 & EM07-Bi-025 & $\mathrm{Nd} 2 \mathrm{O} 3$ & 0.0659 & 0.0761 & 0.0680 & -0.0021 & 0.0081 & $-3.1 \%$ & $11.9 \%$ \\
\hline
\end{tabular}




\section{Table A5. Average Measured and Bias-Corrected Chemical Compositions Versus} Targeted Compositions for the Test Matrix 1 Study Glasses

\begin{tabular}{|c|c|c|c|c|c|c|c|c|c|}
\hline & & & & Measured & & & & & \\
\hline & & & Measured & Bias-Corrected & Targeted & Diff of & Diff of & $\%$ Diff of & $\%$ Diff of \\
\hline Set & Glass ID & Oxide & $(\mathrm{wt} \%)$ & (wt\%) & (wt\%) & Measured & Meas BC & Measured & Meas BC \\
\hline 1 & EM07-Bi-025 & $\mathrm{NiO}$ & 0.9146 & 1.0027 & 0.9750 & $\begin{array}{l}-0.0604 \\
\end{array}$ & 0.0277 & $-6.2 \%$ & $2.8 \%$ \\
\hline 1 & EM07-Bi-025 & P2O5 & 0.7344 & 0.7344 & 1.2190 & -0.4846 & -0.4846 & $-39.8 \%$ & $-39.8 \%$ \\
\hline 1 & EM07-Bi-025 & $\mathrm{PbO}$ & 0.3242 & 0.3242 & 0.3610 & -0.0368 & -0.0368 & $-10.2 \%$ & $-10.2 \%$ \\
\hline 1 & EM07-Bi-025 & $\mathrm{SiO} 2$ & 42.4116 & 42.6688 & 42.2430 & 0.1686 & 0.4258 & $0.4 \%$ & $1.0 \%$ \\
\hline 1 & EM07-Bi-025 & $\mathrm{TiO} 2$ & 0.0421 & 0.0442 & 0.0390 & 0.0031 & 0.0052 & $8.0 \%$ & $13.4 \%$ \\
\hline 1 & EM07-Bi-025 & $\mathrm{ZnO}$ & 0.0660 & 0.0660 & 0.0590 & 0.0070 & 0.0070 & $11.8 \%$ & $11.8 \%$ \\
\hline 1 & EM07-Bi-025 & $\mathrm{ZrO} 2$ & 1.5433 & 1.5433 & 2.4380 & -0.8947 & -0.8947 & $-36.7 \%$ & $-36.7 \%$ \\
\hline 1 & EM07-Bi-025 & Sum & 97.7497 & 97.9593 & 99.3450 & -1.5953 & -1.3857 & $-1.6 \%$ & $-1.4 \%$ \\
\hline 1 & EM07-Bi-05 & $\mathrm{Al} 2 \mathrm{O} 3$ & 9.6412 & 9.6269 & 9.5000 & 0.1412 & 0.1269 & $1.5 \%$ & $1.3 \%$ \\
\hline 1 & EM07-Bi-05 & B2O3 & 10.0058 & 9.7925 & 9.5000 & 0.5058 & 0.2925 & $5.3 \%$ & $3.1 \%$ \\
\hline 1 & EM07-Bi-05 & $\mathrm{BaO}$ & 0.0452 & 0.0476 & 0.0480 & -0.0028 & -0.0004 & $-5.8 \%$ & $-0.8 \%$ \\
\hline 1 & EM07-Bi-05 & $\mathrm{Bi} 2 \mathrm{O} 3$ & 4.9135 & 4.9135 & 5.0000 & -0.0865 & -0.0865 & $-1.7 \%$ & $-1.7 \%$ \\
\hline 1 & EM07-Bi-05 & $\mathrm{CaO}$ & 0.0157 & 0.0168 & 0.0000 & 0.0157 & 0.0168 & & \\
\hline 1 & EM07-Bi-05 & $\mathrm{CdO}$ & 0.1202 & 0.1202 & 0.1330 & -0.0128 & -0.0128 & $-9.6 \%$ & $-9.6 \%$ \\
\hline 1 & EM07-Bi-05 & Ce2O3 & 0.0489 & 0.0489 & 0.0480 & 0.0009 & 0.0009 & $1.9 \%$ & $1.9 \%$ \\
\hline 1 & EM07-Bi-05 & $\mathrm{Cr} 2 \mathrm{O} 3$ & 0.4323 & 0.4711 & 0.4750 & -0.0427 & -0.0039 & $-9.0 \%$ & $-0.8 \%$ \\
\hline 1 & EM07-Bi-05 & $\mathrm{Fe} 2 \mathrm{O} 3$ & 9.0607 & 9.2786 & 9.5000 & -0.4393 & -0.2214 & $-4.6 \%$ & $-2.3 \%$ \\
\hline 1 & EM07-Bi-05 & $\mathrm{K} 2 \mathrm{O}$ & 0.0602 & 0.0639 & 0.0000 & 0.0602 & 0.0639 & & \\
\hline 1 & EM07-Bi-05 & $\mathrm{La} 2 \mathrm{O} 3$ & 0.0519 & 0.0519 & 0.0670 & -0.0151 & -0.0151 & $-22.5 \%$ & $-22.5 \%$ \\
\hline 1 & EM07-Bi-05 & $\mathrm{Li} 2 \mathrm{O}$ & 2.6750 & 2.6290 & 2.6130 & 0.0620 & 0.0160 & $2.4 \%$ & $0.6 \%$ \\
\hline 1 & EM07-Bi-05 & $\mathrm{MgO}$ & 0.1219 & 0.1285 & 0.1430 & -0.0211 & -0.0145 & $-14.8 \%$ & $-10.1 \%$ \\
\hline 1 & EM07-Bi-05 & $\mathrm{MnO}$ & 1.8561 & 1.8875 & 1.9000 & -0.0439 & -0.0125 & $-2.3 \%$ & $-0.7 \%$ \\
\hline 1 & EM07-Bi-05 & $\mathrm{Na} 2 \mathrm{O}$ & 14.5921 & 14.1904 & 14.2500 & 0.3421 & $\begin{array}{l}-0.0596 \\
\end{array}$ & $2.4 \%$ & $-0.4 \%$ \\
\hline 1 & EM07-Bi-05 & $\mathrm{Nd} 2 \mathrm{O} 3$ & 0.0627 & 0.0729 & 0.0670 & -0.0043 & 0.0059 & $-6.4 \%$ & $8.9 \%$ \\
\hline 1 & EM07-Bi-05 & $\mathrm{NiO}$ & 0.8602 & 0.9341 & 0.9500 & -0.0898 & -0.0159 & $-9.5 \%$ & $-1.7 \%$ \\
\hline 1 & EM07-Bi-05 & P2O5 & 0.7693 & 0.7693 & 1.1880 & -0.4187 & -0.4187 & $-35.2 \%$ & $-35.2 \%$ \\
\hline$\frac{1}{1}$ & EM07-Bi-05 & $\mathrm{PbO}$ & 0.3170 & 0.3170 & 0.3520 & -0.0350 & -0.0350 & $-10.0 \%$ & $-10.0 \%$ \\
\hline 1 & EM07-Bi-05 & $\mathrm{SiO} 2$ & 41.6094 & 41.8613 & 41.1590 & 0.4504 & 0.7023 & $1.1 \%$ & $1.7 \%$ \\
\hline 1 & EM07-Bi-05 & $\mathrm{TiO} 2$ & 0.0442 & 0.0466 & 0.0380 & 0.0062 & 0.0086 & $16.3 \%$ & $22.7 \%$ \\
\hline 1 & EM07-Bi-05 & $\mathrm{ZnO}$ & 0.0541 & 0.0541 & 0.0570 & -0.0029 & -0.0029 & $-5.0 \%$ & $-5.0 \%$ \\
\hline 1 & EM07-Bi-05 & $\mathrm{ZrO} 2$ & 1.7392 & 1.7392 & 2.3750 & -0.6358 & -0.6358 & $-26.8 \%$ & $-26.8 \%$ \\
\hline 1 & EM07-Bi-05 & Sum & 99.0969 & 99.0618 & 99.3630 & -0.2661 & -0.3012 & $-0.3 \%$ & $-0.3 \%$ \\
\hline 1 & EM07-Ca-035 & $\mathrm{Al} 2 \mathrm{O} 3$ & 9.7120 & 9.6975 & 9.6500 & 0.0620 & 0.0475 & $0.6 \%$ & $0.5 \%$ \\
\hline 1 & EM07-Ca-035 & $\mathrm{B} 2 \mathrm{O} 3$ & 10.0380 & 9.9290 & 9.6500 & 0.3880 & 0.2790 & $4.0 \%$ & $2.9 \%$ \\
\hline 1 & EM07-Ca-035 & $\mathrm{BaO}$ & 0.0444 & 0.0467 & 0.0480 & $\begin{array}{l}-0.0036 \\
\end{array}$ & -0.0013 & $-7.5 \%$ & $-2.7 \%$ \\
\hline 1 & EM07-Ca-035 & $\mathrm{Bi} 2 \mathrm{O} 3$ & 0.0056 & 0.0056 & 0.0000 & 0.0056 & 0.0056 & & \\
\hline 1 & EM07-Ca-035 & $\mathrm{CaO}$ & 3.7778 & 4.0262 & 3.5000 & 0.2778 & 0.5262 & $7.9 \%$ & $15.0 \%$ \\
\hline 1 & EM07-Ca-035 & $\mathrm{CdO}$ & 0.1214 & 0.1214 & 0.1350 & -0.0136 & -0.0136 & $-10.1 \%$ & $-10.1 \%$ \\
\hline 1 & EM07-Ca-035 & Ce2O3 & 0.0483 & 0.0483 & 0.0480 & 0.0003 & 0.0003 & $0.7 \%$ & $0.7 \%$ \\
\hline 1 & EM07-Ca-035 & $\mathrm{Cr} 2 \mathrm{O} 3$ & 0.4330 & 0.4719 & 0.4830 & -0.0500 & -0.0111 & $-10.4 \%$ & $-2.3 \%$ \\
\hline 1 & EM07-Ca-035 & $\mathrm{Fe} 2 \mathrm{O} 3$ & 9.2251 & 9.4423 & 9.6500 & -0.4249 & -0.2077 & $-4.4 \%$ & $-2.2 \%$ \\
\hline 1 & EM07-Ca-035 & $\mathrm{K} 2 \mathrm{O}$ & 0.0602 & 0.0639 & 0.0000 & 0.0602 & 0.0639 & & \\
\hline 1 & EM07-Ca-035 & $\mathrm{La} 2 \mathrm{O} 3$ & 0.0542 & 0.0542 & 0.0680 & -0.0138 & -0.0138 & $-20.2 \%$ & $-20.2 \%$ \\
\hline 1 & EM07-Ca-035 & $\mathrm{Li} 2 \mathrm{O}$ & 2.6481 & 2.6254 & 2.6540 & -0.0059 & -0.0286 & $-0.2 \%$ & $-1.1 \%$ \\
\hline 1 & EM07-Ca-035 & $\mathrm{MgO}$ & 0.1248 & 0.1316 & 0.1450 & -0.0202 & -0.0134 & $-13.9 \%$ & $-9.3 \%$ \\
\hline 1 & EM07-Ca-035 & $\mathrm{MnO}$ & 1.8916 & 1.9226 & 1.9300 & -0.0384 & -0.0074 & $-2.0 \%$ & $-0.4 \%$ \\
\hline 1 & EM07-Ca-035 & $\mathrm{Na} 2 \mathrm{O}$ & 14.6932 & 14.2883 & 14.4750 & 0.2182 & -0.1867 & $1.5 \%$ & $-1.3 \%$ \\
\hline 1 & EM07-Ca-035 & $\mathrm{Nd} 2 \mathrm{O} 3$ & 0.0633 & 0.0736 & 0.0680 & -0.0047 & 0.0056 & $-6.9 \%$ & $8.3 \%$ \\
\hline 1 & EM07-Ca-035 & $\mathrm{NiO}$ & 0.8678 & 0.9424 & 0.9650 & -0.0972 & -0.0226 & $-10.1 \%$ & $-2.3 \%$ \\
\hline 1 & EM07-Ca-035 & $\mathrm{P} 2 \mathrm{O} 5$ & 0.8358 & 0.8358 & 1.2060 & -0.3702 & -0.3702 & $-30.7 \%$ & $-30.7 \%$ \\
\hline 1 & EM07-Ca-035 & $\mathrm{PbO}$ & 0.3202 & 0.3202 & 0.3570 & -0.0368 & -0.0368 & $-10.3 \%$ & $-10.3 \%$ \\
\hline 1 & EM07-Ca-035 & $\mathrm{SiO} 2$ & 41.1280 & 42.2218 & 41.8100 & -0.6820 & 0.4118 & $-1.6 \%$ & $1.0 \%$ \\
\hline 1 & EM07-Ca-035 & $\mathrm{TiO} 2$ & 0.0430 & 0.0453 & 0.0390 & 0.0040 & 0.0063 & $10.1 \%$ & $16.2 \%$ \\
\hline 1 & EM07-Ca-035 & $\mathrm{ZnO}$ & 0.0566 & 0.0566 & 0.0580 & -0.0014 & -0.0014 & $-2.3 \%$ & $-2.3 \%$ \\
\hline 1 & EM07-Ca-035 & $\mathrm{ZrO} 2$ & 1.6480 & 1.6480 & 2.4130 & -0.7650 & -0.7650 & $-31.7 \%$ & $-31.7 \%$ \\
\hline 1 & EM07-Ca-035 & Sum & 97.8405 & 99.0186 & 99.3520 & -1.5115 & -0.3334 & $-1.5 \%$ & $-0.3 \%$ \\
\hline
\end{tabular}


Table A5. Average Measured and Bias-Corrected Chemical Compositions Versus Targeted Compositions for the Test Matrix 1 Study Glasses

\begin{tabular}{|c|c|c|c|c|c|c|c|c|c|}
\hline & & & & Measured & & & & & \\
\hline & & & Measured & Bias-Corrected & Targeted & Diff of & Diff of & $\%$ Diff of & $\%$ Diff of \\
\hline Set & Glass ID & Oxide & (wt\%) & (wt\%) & (wt\%) & Measured & Meas BC & Measured & Meas BC \\
\hline 1 & EM07-Ca-07 & $\mathrm{Al} 2 \mathrm{O} 3$ & 9.4097 & 9.3957 & 9.3000 & 0.1097 & 0.0957 & $1.2 \%$ & $1.0 \%$ \\
\hline 1 & EM07-Ca-07 & $\mathrm{B} 2 \mathrm{O} 3$ & 9.7643 & 9.5592 & 9.3000 & 0.4643 & 0.2592 & $5.0 \%$ & $2.8 \%$ \\
\hline 1 & EM07-Ca-07 & $\mathrm{BaO}$ & 0.0427 & 0.0449 & 0.0470 & -0.0043 & -0.0021 & $-9.1 \%$ & $-4.4 \%$ \\
\hline 1 & EM07-Ca-07 & Bi2O3 & 0.0056 & 0.0056 & 0.0000 & 0.0056 & 0.0056 & & \\
\hline 1 & EM07-Ca-07 & $\mathrm{CaO}$ & 7.3808 & 7.8656 & 7.0000 & 0.3808 & 0.8656 & $5.4 \%$ & $12.4 \%$ \\
\hline 1 & EM07-Ca-07 & $\mathrm{CdO}$ & 0.1162 & 0.1162 & 0.1300 & -0.0138 & -0.0138 & $-10.6 \%$ & $-10.6 \%$ \\
\hline 1 & EM07-Ca-07 & Ce2O3 & 0.0469 & 0.0469 & 0.0470 & -0.0001 & -0.0001 & $-0.3 \%$ & $-0.3 \%$ \\
\hline 1 & EM07-Ca-07 & Cr2O3 & 0.4297 & 0.4684 & 0.4650 & -0.0353 & 0.0034 & $-7.6 \%$ & $0.7 \%$ \\
\hline 1 & EM07-Ca-07 & Fe2O3 & 8.9499 & 9.1624 & 9.3000 & -0.3501 & -0.1376 & $-3.8 \%$ & $-1.5 \%$ \\
\hline 1 & EM07-Ca-07 & $\mathrm{K} 2 \mathrm{O}$ & 0.0602 & 0.0639 & 0.0000 & 0.0602 & 0.0639 & & \\
\hline 1 & EM07-Ca-07 & La2O3 & 0.0516 & 0.0516 & 0.0650 & -0.0134 & -0.0134 & $-20.6 \%$ & $-20.6 \%$ \\
\hline 1 & EM07-Ca-07 & $\mathrm{Li} 2 \mathrm{O}$ & 2.6158 & 2.5708 & 2.5580 & 0.0578 & 0.0128 & $2.3 \%$ & $0.5 \%$ \\
\hline 1 & EM07-Ca-07 & $\mathrm{MgO}$ & 0.1397 & 0.1473 & 0.1400 & -0.0003 & 0.0073 & $-0.2 \%$ & $5.2 \%$ \\
\hline 1 & EM07-Ca-07 & $\mathrm{MnO}$ & 1.8335 & 1.8639 & 1.8600 & -0.0265 & 0.0039 & $-1.4 \%$ & $0.2 \%$ \\
\hline 1 & EM07-Ca-07 & $\mathrm{Na} 2 \mathrm{O}$ & 14.3899 & 13.9935 & 13.9500 & 0.4399 & 0.0435 & $3.2 \%$ & $0.3 \%$ \\
\hline 1 & EM07-Ca-07 & Nd2O3 & 0.0604 & 0.0702 & 0.0650 & -0.0046 & 0.0052 & $-7.1 \%$ & $8.0 \%$ \\
\hline 1 & EM07-Ca-07 & $\mathrm{NiO}$ & 0.8465 & 0.9192 & 0.9300 & -0.0835 & -0.0108 & $-9.0 \%$ & $-1.2 \%$ \\
\hline 1 & EM07-Ca-07 & P2O5 & 0.7321 & 0.7321 & 1.1630 & -0.4309 & -0.4309 & $-37.1 \%$ & $-37.1 \%$ \\
\hline 1 & EM07-Ca-07 & $\mathrm{PbO}$ & 0.3086 & 0.3086 & 0.3440 & -0.0354 & -0.0354 & $-10.3 \%$ & $-10.3 \%$ \\
\hline 1 & EM07-Ca-07 & $\mathrm{SiO} 2$ & 40.4863 & 40.7317 & 40.2950 & 0.1913 & 0.4367 & $0.5 \%$ & $1.1 \%$ \\
\hline 1 & EM07-Ca-07 & $\mathrm{TiO} 2$ & 0.0409 & 0.0431 & 0.0370 & 0.0039 & 0.0061 & $10.4 \%$ & $16.5 \%$ \\
\hline 1 & EM07-Ca-07 & $\mathrm{ZnO}$ & 0.0557 & 0.0557 & 0.0560 & -0.0003 & -0.0003 & $-0.5 \%$ & $-0.5 \%$ \\
\hline 1 & EM07-Ca-07 & $\mathrm{ZrO} 2$ & 1.5298 & 1.5298 & 2.3250 & -0.7952 & -0.7952 & $-34.2 \%$ & $-34.2 \%$ \\
\hline 1 & EM07-Ca-07 & Sum & 99.2968 & 99.7462 & 99.3770 & -0.0802 & 0.3692 & $-0.1 \%$ & $0.4 \%$ \\
\hline 1 & EM07-F-02 & $\mathrm{Al2O} 3$ & 10.0899 & 10.0749 & 9.8100 & 0.2799 & 0.2649 & $2.9 \%$ & $2.7 \%$ \\
\hline 1 & EM07-F-02 & $\mathrm{B} 2 \mathrm{O} 3$ & 10.3681 & 10.1520 & 9.8100 & 0.5581 & 0.3420 & $5.7 \%$ & $3.5 \%$ \\
\hline 1 & EM07-F-02 & $\mathrm{BaO}$ & 0.0463 & 0.0488 & 0.0490 & -0.0027 & -0.0002 & $-5.4 \%$ & $-0.5 \%$ \\
\hline 1 & EM07-F-02 & $\mathrm{Bi} 2 \mathrm{O} 3$ & 0.0056 & 0.0056 & 0.0000 & 0.0056 & 0.0056 & & \\
\hline 1 & EM07-F-02 & $\mathrm{CaO}$ & 0.0143 & 0.0153 & 0.0000 & 0.0143 & 0.0153 & & \\
\hline 1 & EM07-F-02 & $\mathrm{CdO}$ & 0.1268 & 0.1268 & 0.1370 & -0.0102 & -0.0102 & $-7.4 \%$ & $-7.4 \%$ \\
\hline 1 & EM07-F-02 & $\mathrm{Ce} 2 \mathrm{O} 3$ & 0.0504 & 0.0504 & 0.0490 & 0.0014 & 0.0014 & $2.8 \%$ & $2.8 \%$ \\
\hline 1 & EM07-F-02 & Cr2O3 & 0.4531 & 0.4938 & 0.4900 & -0.0369 & 0.0038 & $-7.5 \%$ & $0.8 \%$ \\
\hline 1 & EM07-F-02 & $\mathrm{F}$ & 1.6225 & 2.0867 & 2.0000 & -0.3775 & 0.0867 & $-18.9 \%$ & $4.3 \%$ \\
\hline 1 & EM07-F-02 & Fe2O3 & 9.5325 & 9.7611 & 9.8100 & -0.2775 & -0.0489 & $-2.8 \%$ & $-0.5 \%$ \\
\hline 1 & EM07-F-02 & K2O & 0.0602 & 0.0639 & 0.0000 & 0.0602 & 0.0639 & & \\
\hline 1 & EM07-F-02 & La2O3 & 0.0542 & 0.0542 & 0.0690 & -0.0148 & -0.0148 & $-21.4 \%$ & $-21.4 \%$ \\
\hline 1 & EM07-F-02 & $\mathrm{Li} 2 \mathrm{O}$ & 2.7772 & 2.7294 & 2.6980 & 0.0792 & 0.0314 & $2.9 \%$ & $1.2 \%$ \\
\hline 1 & EM07-F-02 & $\mathrm{MgO}$ & 0.1285 & 0.1355 & 0.1470 & -0.0185 & -0.0115 & $-12.6 \%$ & $-7.8 \%$ \\
\hline$\frac{1}{1}$ & EM07-F-02 & $\mathrm{MnO}$ & 1.9659 & 1.9990 & 1.9620 & 0.0039 & 0.0370 & $0.2 \%$ & $1.9 \%$ \\
\hline 1 & EM07-F-02 & $\mathrm{Na} 2 \mathrm{O}$ & 15.2661 & 14.8459 & 14.7150 & 0.5511 & 0.1309 & $3.7 \%$ & $0.9 \%$ \\
\hline 1 & EM07-F-02 & Nd2O3 & 0.0650 & 0.0757 & 0.0690 & -0.0040 & 0.0067 & $-5.8 \%$ & $9.6 \%$ \\
\hline 1 & EM07-F-02 & $\mathrm{NiO}$ & 0.9130 & 0.9914 & 0.9810 & -0.0680 & 0.0104 & $-6.9 \%$ & $1.1 \%$ \\
\hline 1 & EM07-F-02 & $\mathrm{P} 2 \mathrm{O} 5$ & 0.7023 & 0.7023 & 1.2260 & -0.5237 & -0.5237 & $-42.7 \%$ & $-42.7 \%$ \\
\hline 1 & EM07-F-02 & $\mathrm{PbO}$ & 0.3334 & 0.3334 & 0.3630 & -0.0296 & -0.0296 & $-8.2 \%$ & $-8.2 \%$ \\
\hline 1 & EM07-F-02 & $\mathrm{SiO} 2$ & 43.0534 & 43.3151 & 42.5060 & 0.5474 & 0.8091 & $1.3 \%$ & $1.9 \%$ \\
\hline 1 & EM07-F-02 & $\mathrm{TiO} 2$ & 0.0442 & 0.0466 & 0.0390 & 0.0052 & 0.0076 & $13.3 \%$ & $19.5 \%$ \\
\hline 1 & EM07-F-02 & $\mathrm{ZnO}$ & 0.0604 & 0.0604 & 0.0590 & 0.0014 & 0.0014 & $2.3 \%$ & $2.3 \%$ \\
\hline 1 & EM07-F-02 & $\mathrm{ZrO} 2$ & 1.7121 & 1.7121 & 2.4520 & -0.7399 & -0.7399 & $-30.2 \%$ & $-30.2 \%$ \\
\hline 1 & EM07-F-02 & Sum & 99.4456 & 99.8801 & 99.4410 & 0.0046 & 0.4391 & $0.0 \%$ & $0.4 \%$ \\
\hline 1 & EM07-K-03 & $\mathrm{Al} 2 \mathrm{O} 3$ & 9.8632 & 9.8485 & 9.7000 & 0.1632 & 0.1485 & $1.7 \%$ & $1.5 \%$ \\
\hline 1 & EM07-K-03 & $\mathrm{B} 2 \mathrm{O} 3$ & 10.2232 & 10.1127 & 9.7000 & 0.5232 & 0.4127 & $5.4 \%$ & $4.3 \%$ \\
\hline 1 & EM07-K-03 & $\mathrm{BaO}$ & 0.0466 & 0.0491 & 0.0490 & -0.0024 & 0.0001 & $-4.9 \%$ & $0.1 \%$ \\
\hline 1 & EM07-K-03 & $\mathrm{Bi} 2 \mathrm{O} 3$ & 0.0056 & 0.0056 & 0.0000 & 0.0056 & 0.0056 & & \\
\hline 1 & EM07-K-03 & $\mathrm{CaO}$ & 0.0280 & 0.0298 & 0.0000 & 0.0280 & 0.0298 & & \\
\hline 1 & EM07-K-03 & $\mathrm{CdO}$ & 0.1239 & 0.1239 & 0.1360 & -0.0121 & -0.0121 & $-8.9 \%$ & $-8.9 \%$ \\
\hline 1 & EM07-K-03 & Ce2O3 & 0.0495 & 0.0495 & 0.0490 & 0.0005 & 0.0005 & $1.0 \%$ & $1.0 \%$ \\
\hline
\end{tabular}




\section{Table A5. Average Measured and Bias-Corrected Chemical Compositions Versus} Targeted Compositions for the Test Matrix 1 Study Glasses

\begin{tabular}{|c|c|c|c|c|c|c|c|c|c|}
\hline & & & & "Measured & & & & & \\
\hline & & & Measured & Bias-Corrected & Targeted & Diff of & Diff of & $\%$ Diff of & \% Diff of \\
\hline Set & Glass ID & Oxide & (wt\%) & (wt\%) & (wt\%) & Measured & Meas BC & Measured & Meas BC \\
\hline 1 & EM07-K-03 & $\mathrm{Cr} 2 \mathrm{O} 3$ & 0.4652 & 0.5070 & 0.4850 & -0.0198 & 0.0220 & $-4.1 \%$ & $4.5 \%$ \\
\hline 1 & EM07-K-03 & $\mathrm{Fe} 2 \mathrm{O} 3$ & 9.3359 & 9.5602 & 9.7000 & -0.3641 & -0.1398 & $-3.8 \%$ & $-1.4 \%$ \\
\hline 1 & EM07-K-03 & $\mathrm{K} 2 \mathrm{O}$ & 2.8398 & 3.0110 & 3.0000 & -0.1602 & 0.0110 & $-5.3 \%$ & $0.4 \%$ \\
\hline 1 & EM07-K-03 & $\mathrm{La} 2 \mathrm{O} 3$ & 0.0528 & 0.0528 & 0.0680 & -0.0152 & -0.0152 & $-22.4 \%$ & $-22.4 \%$ \\
\hline 1 & EM07-K-03 & $\mathrm{Li} 2 \mathrm{O}$ & 2.6911 & 2.6681 & 2.6680 & 0.0231 & 0.0001 & $0.9 \%$ & $0.0 \%$ \\
\hline 1 & EM07-K-03 & $\mathrm{MgO}$ & 0.1277 & 0.1346 & 0.1460 & -0.0183 & -0.0114 & $-12.5 \%$ & $-7.8 \%$ \\
\hline 1 & EM07-K-03 & $\mathrm{MnO}$ & 1.9207 & 1.9531 & 1.9400 & -0.0193 & 0.0131 & $-1.0 \%$ & $0.7 \%$ \\
\hline 1 & EM07-K-03 & $\mathrm{Na} 2 \mathrm{O}$ & 14.9628 & 14.5508 & 14.5500 & 0.4128 & 0.0008 & $2.8 \%$ & $0.0 \%$ \\
\hline 1 & EM07-K-03 & $\mathrm{Nd} 2 \mathrm{O} 3$ & 0.0633 & 0.0736 & 0.0680 & -0.0047 & 0.0056 & $-6.9 \%$ & $8.3 \%$ \\
\hline 1 & EM07-K-03 & $\mathrm{NiO}$ & 0.8997 & 0.9769 & 0.9700 & -0.0703 & 0.0069 & $-7.3 \%$ & $0.7 \%$ \\
\hline 1 & EM07-K-03 & P2O5 & 0.8392 & 0.8392 & 1.2130 & -0.3738 & -0.3738 & $-30.8 \%$ & $-30.8 \%$ \\
\hline 1 & EM07-K-03 & $\mathrm{PbO}$ & 0.3275 & 0.3275 & 0.3590 & -0.0315 & -0.0315 & $-8.8 \%$ & $-8.8 \%$ \\
\hline 1 & EM07-K-03 & $\mathrm{SiO} 2$ & 41.5559 & 42.6612 & 42.0270 & -0.4711 & 0.6342 & $-1.1 \%$ & $1.5 \%$ \\
\hline 1 & EM07-K-03 & $\mathrm{TiO} 2$ & 0.0425 & 0.0449 & 0.0390 & 0.0035 & 0.0059 & $9.1 \%$ & $15.0 \%$ \\
\hline 1 & EM07-K-03 & $\mathrm{ZnO}$ & 0.0573 & 0.0573 & 0.0580 & -0.0007 & -0.0007 & $-1.3 \%$ & $-1.3 \%$ \\
\hline 1 & EM07-K-03 & ZrO2 & 1.7695 & 1.7695 & 2.4250 & -0.6555 & -0.6555 & $-27.0 \%$ & $-27.0 \%$ \\
\hline 1 & EM07-K-03 & Sum & 98.2908 & 99.4068 & 99.3500 & -1.0592 & 0.0568 & $-1.1 \%$ & $0.1 \%$ \\
\hline 1 & EM07-K-06 & $\mathrm{Al} 2 \mathrm{O} 3$ & 9.5561 & 9.5419 & 9.4000 & 0.1561 & 0.1419 & $1.7 \%$ & $1.5 \%$ \\
\hline 1 & EM07-K-06 & $\mathrm{B} 2 \mathrm{O} 3$ & 9.8287 & 9.6199 & 9.4000 & 0.4287 & 0.2199 & $4.6 \%$ & $2.3 \%$ \\
\hline 1 & EM07-K-06 & $\mathrm{BaO}$ & 0.0441 & 0.0464 & 0.0470 & -0.0029 & -0.0006 & $-6.2 \%$ & $-1.2 \%$ \\
\hline 1 & EM07-K-06 & $\mathrm{Bi} 2 \mathrm{O} 3$ & 0.0056 & 0.0056 & 0.0000 & 0.0056 & 0.0056 & & \\
\hline 1 & EM07-K-06 & $\mathrm{CaO}$ & 0.0234 & 0.0250 & 0.0000 & 0.0234 & 0.0250 & & \\
\hline 1 & EM07-K-06 & $\mathrm{CdO}$ & 0.1217 & 0.1217 & 0.1320 & -0.0103 & -0.0103 & $-7.8 \%$ & $-7.8 \%$ \\
\hline 1 & EM07-K-06 & $\mathrm{Ce} 2 \mathrm{O} 3$ & 0.0469 & 0.0469 & 0.0470 & -0.0001 & -0.0001 & $-0.3 \%$ & $-0.3 \%$ \\
\hline 1 & EM07-K-06 & $\mathrm{Cr} 2 \mathrm{O} 3$ & 0.4575 & 0.4986 & 0.4700 & -0.0125 & 0.0286 & $-2.7 \%$ & $6.1 \%$ \\
\hline 1 & EM07-K-06 & $\mathrm{Fe} 2 \mathrm{O} 3$ & 9.1000 & 9.3203 & 9.4000 & -0.3000 & -0.0797 & $-3.2 \%$ & $-0.8 \%$ \\
\hline 1 & EM07-K-06 & $\mathrm{K} 2 \mathrm{O}$ & 5.0202 & 5.3227 & 6.0000 & -0.9798 & -0.6773 & $-16.3 \%$ & $-11.3 \%$ \\
\hline 1 & EM07-K-06 & La2O3 & 0.0516 & 0.0516 & 0.0660 & -0.0144 & -0.0144 & $-21.8 \%$ & $-21.8 \%$ \\
\hline 1 & EM07-K-06 & $\mathrm{Li} 2 \mathrm{O}$ & 2.6481 & 2.6026 & 2.5850 & 0.0631 & 0.0176 & $2.4 \%$ & $0.7 \%$ \\
\hline 1 & EM07-K-06 & $\mathrm{MgO}$ & 0.1215 & 0.1281 & 0.1410 & -0.0195 & -0.0129 & $-13.9 \%$ & $-9.2 \%$ \\
\hline 1 & EM07-K-06 & $\mathrm{MnO}$ & 1.8626 & 1.8943 & 1.8800 & -0.0174 & 0.0143 & $-0.9 \%$ & $0.8 \%$ \\
\hline 1 & EM07-K-06 & $\mathrm{Na} 2 \mathrm{O}$ & 14.4236 & 14.0261 & 14.1000 & 0.3236 & -0.0739 & $2.3 \%$ & $-0.5 \%$ \\
\hline 1 & EM07-K-06 & $\mathrm{Nd} 2 \mathrm{O} 3$ & 0.0607 & 0.0706 & 0.0660 & -0.0053 & 0.0046 & $-8.1 \%$ & $6.9 \%$ \\
\hline 1 & EM07-K-06 & $\mathrm{NiO}$ & 0.8704 & 0.9451 & 0.9400 & -0.0696 & 0.0051 & $-7.4 \%$ & $0.5 \%$ \\
\hline 1 & EM07-K-06 & P2O5 & 0.7642 & 0.7642 & 1.1750 & -0.4108 & -0.4108 & $-35.0 \%$ & $-35.0 \%$ \\
\hline 1 & EM07-K-06 & $\mathrm{PbO}$ & 0.3164 & 0.3164 & 0.3480 & -0.0316 & -0.0316 & $-9.1 \%$ & $-9.1 \%$ \\
\hline 1 & EM07-K-06 & $\mathrm{SiO} 2$ & 41.0211 & 41.2695 & 40.7290 & 0.2921 & 0.5405 & $0.7 \%$ & $1.3 \%$ \\
\hline 1 & EM07-K-06 & $\mathrm{TiO} 2$ & 0.0409 & 0.0431 & 0.0380 & 0.0029 & 0.0051 & $7.5 \%$ & $13.4 \%$ \\
\hline 1 & EM07-K-06 & $\mathrm{ZnO}$ & 0.0551 & 0.0551 & 0.0560 & -0.0009 & -0.0009 & $-1.6 \%$ & $-1.6 \%$ \\
\hline 1 & EM07-K-06 & ZrO2 & 1.6075 & 1.6075 & 2.3500 & -0.7425 & -0.7425 & $-31.6 \%$ & $-31.6 \%$ \\
\hline 1 & EM07-K-06 & Sum & 98.0476 & 98.3231 & 99.3700 & -1.3224 & -1.0469 & $-1.3 \%$ & $-1.1 \%$ \\
\hline 1 & EM07-Mn-01 & $\mathrm{Al} 2 \mathrm{O} 3$ & 10.2505 & 10.1704 & 10.1940 & 0.0565 & -0.0236 & $0.6 \%$ & $-0.2 \%$ \\
\hline 1 & EM07-Mn-01 & $\mathrm{B} 2 \mathrm{O} 3$ & 10.2715 & 10.1605 & 10.1940 & 0.0775 & -0.0335 & $0.8 \%$ & $-0.3 \%$ \\
\hline 1 & EM07-Mn-01 & $\mathrm{BaO}$ & 0.0497 & 0.0521 & 0.0510 & -0.0013 & 0.0011 & $-2.6 \%$ & $2.1 \%$ \\
\hline 1 & EM07-Mn-01 & $\mathrm{Bi} 2 \mathrm{O} 3$ & 0.0056 & 0.0056 & 0.0000 & 0.0056 & 0.0056 & & \\
\hline 1 & EM07-Mn-01 & $\mathrm{CaO}$ & 0.0262 & 0.0272 & 0.0000 & 0.0262 & 0.0272 & & \\
\hline 1 & EM07-Mn-01 & $\mathrm{CdO}$ & 0.1285 & 0.1285 & 0.1430 & -0.0145 & -0.0145 & $-10.1 \%$ & $-10.1 \%$ \\
\hline 1 & EM07-Mn-01 & Ce2O3 & 0.0521 & 0.0521 & 0.0510 & 0.0011 & 0.0011 & $2.2 \%$ & $2.2 \%$ \\
\hline 1 & EM07-Mn-01 & $\mathrm{Cr} 2 \mathrm{O} 3$ & 0.4765 & 0.5285 & 0.5100 & -0.0335 & 0.0185 & $-6.6 \%$ & $3.6 \%$ \\
\hline 1 & EM07-Mn-01 & $\mathrm{Fe} 2 \mathrm{O} 3$ & 9.4217 & 9.9233 & 10.1940 & -0.7723 & -0.2707 & $-7.6 \%$ & $-2.7 \%$ \\
\hline 1 & EM07-Mn-01 & $\mathrm{K} 2 \mathrm{O}$ & 0.0602 & 0.0635 & 0.0000 & 0.0602 & 0.0635 & & \\
\hline 1 & EM07-Mn-01 & $\mathrm{La} 2 \mathrm{O} 3$ & 0.0539 & 0.0539 & 0.0710 & -0.0171 & -0.0171 & $-24.0 \%$ & $-24.0 \%$ \\
\hline 1 & EM07-Mn-01 & $\mathrm{Li} 2 \mathrm{O}$ & 2.8364 & 2.8121 & 2.8030 & 0.0334 & 0.0091 & $1.2 \%$ & $0.3 \%$ \\
\hline 1 & EM07-Mn-01 & $\mathrm{MgO}$ & 0.1310 & 0.1391 & 0.1530 & -0.0220 & -0.0139 & $-14.4 \%$ & $-9.1 \%$ \\
\hline 1 & EM07-Mn-01 & $\mathrm{MnO}$ & 0.0581 & 0.0599 & 0.1000 & -0.0419 & -0.0401 & $-41.9 \%$ & $-40.1 \%$ \\
\hline 1 & EM07-Mn-01 & $\mathrm{Na} 2 \mathrm{O}$ & 15.4683 & 15.0233 & 15.2910 & 0.1773 & -0.2677 & $1.2 \%$ & $-1.8 \%$ \\
\hline
\end{tabular}




\section{Table A5. Average Measured and Bias-Corrected Chemical Compositions Versus} Targeted Compositions for the Test Matrix 1 Study Glasses

\begin{tabular}{|c|c|c|c|c|c|c|c|c|c|}
\hline & & & & Measured & & & & & \\
\hline & & & Measured & Bias-Corrected & Targeted & Diff of & Diff of & \% Diff of & $\%$ Diff of \\
\hline Set & Glass ID & Oxide & (wt\%) & (wt\%) & $(\mathrm{wt} \%)$ & Measured & Meas BC & Measured & Meas BC \\
\hline 1 & EM07-Mn-01 & $\mathrm{Nd} 2 \mathrm{O} 3$ & 0.0668 & 0.0771 & 0.0710 & -0.0042 & 0.0061 & $-5.9 \%$ & $8.6 \%$ \\
\hline 1 & EM07-Mn-01 & $\mathrm{NiO}$ & 0.9245 & 1.0135 & 1.0190 & -0.0945 & -0.0055 & $-9.3 \%$ & $-0.5 \%$ \\
\hline 1 & EM07-Mn-01 & $\mathrm{P} 2 \mathrm{O} 5$ & 0.7940 & 0.7940 & 1.2740 & -0.4800 & -0.4800 & $-37.7 \%$ & $-37.7 \%$ \\
\hline 1 & EM07-Mn-01 & $\mathrm{PbO}$ & 0.3342 & 0.3342 & 0.3770 & -0.0428 & -0.0428 & $-11.4 \%$ & $-11.4 \%$ \\
\hline 1 & EM07-Mn-01 & $\mathrm{SiO} 2$ & 42.9464 & 44.0888 & 44.1710 & -1.2246 & -0.0822 & $-2.8 \%$ & $-0.2 \%$ \\
\hline 1 & EM07-Mn-01 & $\mathrm{TiO} 2$ & 0.0455 & 0.0477 & 0.0410 & 0.0045 & 0.0067 & $10.9 \%$ & $16.4 \%$ \\
\hline 1 & EM07-Mn-01 & $\mathrm{ZnO}$ & 0.0610 & 0.0610 & 0.0610 & 0.0000 & 0.0000 & $0.0 \%$ & $0.0 \%$ \\
\hline 1 & EM07-Mn-01 & $\mathrm{ZrO} 2$ & 1.9283 & 1.9283 & 2.5480 & -0.6197 & -0.6197 & $-24.3 \%$ & $-24.3 \%$ \\
\hline 1 & EM07-Mn-01 & Sum & 96.3910 & 97.5444 & 99.3170 & -2.9260 & -1.7726 & $-2.9 \%$ & $-1.8 \%$ \\
\hline 1 & EM07-Mn-04 & $\mathrm{Al} 2 \mathrm{O} 3$ & 9.9718 & 9.9569 & 9.7960 & 0.1758 & 0.1609 & $1.8 \%$ & $1.6 \%$ \\
\hline 1 & EM07-Mn-04 & B2O3 & 10.1105 & 9.8975 & 9.7960 & 0.3145 & 0.1015 & $3.2 \%$ & $1.0 \%$ \\
\hline 1 & EM07-Mn-04 & $\mathrm{BaO}$ & 0.0455 & 0.0479 & 0.0490 & -0.0035 & -0.0011 & $-7.1 \%$ & $-2.3 \%$ \\
\hline 1 & EM07-Mn-04 & $\mathrm{Bi} 2 \mathrm{O} 3$ & 0.0056 & 0.0056 & 0.0000 & 0.0056 & 0.0056 & & \\
\hline 1 & EM07-Mn-04 & $\mathrm{CaO}$ & 0.0203 & 0.0216 & 0.0000 & 0.0203 & 0.0216 & & \\
\hline 1 & EM07-Mn-04 & $\mathrm{CdO}$ & 0.1251 & 0.1251 & 0.1370 & -0.0119 & -0.0119 & $-8.7 \%$ & $-8.7 \%$ \\
\hline 1 & EM07-Mn-04 & $\mathrm{Ce} 2 \mathrm{O} 3$ & 0.0501 & 0.0501 & 0.0490 & 0.0011 & 0.0011 & $2.2 \%$ & $2.2 \%$ \\
\hline 1 & EM07-Mn-04 & Cr2O3 & 0.4312 & 0.4700 & 0.4900 & -0.0588 & -0.0200 & $-12.0 \%$ & $-4.1 \%$ \\
\hline 1 & EM07-Mn-04 & $\mathrm{Fe} 2 \mathrm{O} 3$ & 9.1965 & 9.4175 & 9.7960 & -0.5995 & -0.3785 & $-6.1 \%$ & $-3.9 \%$ \\
\hline 1 & EM07-Mn-04 & $\mathrm{K} 2 \mathrm{O}$ & 0.0602 & 0.0639 & 0.0000 & 0.0602 & 0.0639 & & \\
\hline 1 & EM07-Mn-04 & $\mathrm{La} 2 \mathrm{O} 3$ & 0.0537 & 0.0537 & 0.0690 & -0.0153 & -0.0153 & $-22.2 \%$ & $-22.2 \%$ \\
\hline 1 & EM07-Mn-04 & $\mathrm{Li} 2 \mathrm{O}$ & 2.7342 & 2.6871 & 2.6940 & 0.0402 & -0.0069 & $1.5 \%$ & $-0.3 \%$ \\
\hline 1 & EM07-Mn-04 & $\mathrm{MgO}$ & 0.1289 & 0.1359 & 0.1470 & -0.0181 & -0.0111 & $-12.3 \%$ & $-7.5 \%$ \\
\hline 1 & EM07-Mn-04 & $\mathrm{MnO}$ & 3.8736 & 3.9388 & 4.0000 & -0.1264 & -0.0612 & $-3.2 \%$ & $-1.5 \%$ \\
\hline 1 & EM07-Mn-04 & $\mathrm{Na} 2 \mathrm{O}$ & 15.2661 & 14.8456 & 14.6940 & 0.5721 & 0.1516 & $3.9 \%$ & $1.0 \%$ \\
\hline 1 & EM07-Mn-04 & $\mathrm{Nd} 2 \mathrm{O} 3$ & 0.0642 & 0.0746 & 0.0690 & -0.0048 & 0.0056 & $-7.0 \%$ & $8.2 \%$ \\
\hline 1 & EM07-Mn-04 & $\mathrm{NiO}$ & 0.8822 & 0.9579 & 0.9800 & -0.0978 & -0.0221 & $-10.0 \%$ & $-2.3 \%$ \\
\hline 1 & EM07-Mn-04 & $\mathrm{P} 2 \mathrm{O} 5$ & 0.8404 & 0.8404 & 1.2240 & -0.3836 & -0.3836 & $-31.3 \%$ & $-31.3 \%$ \\
\hline 1 & EM07-Mn-04 & $\mathrm{PbO}$ & 0.3275 & 0.3275 & 0.3620 & -0.0345 & -0.0345 & $-9.5 \%$ & $-9.5 \%$ \\
\hline 1 & EM07-Mn-04 & $\mathrm{SiO} 2$ & 42.5721 & 42.8304 & 42.4440 & 0.1281 & 0.3864 & $0.3 \%$ & $0.9 \%$ \\
\hline 1 & EM07-Mn-04 & $\mathrm{TiO} 2$ & 0.0434 & 0.0457 & 0.0390 & 0.0044 & 0.0067 & $11.2 \%$ & $17.3 \%$ \\
\hline 1 & EM07-Mn-04 & $\mathrm{ZnO}$ & 0.0585 & 0.0585 & 0.0590 & -0.0005 & -0.0005 & $-0.8 \%$ & $-0.8 \%$ \\
\hline 1 & EM07-Mn-04 & $\mathrm{ZrO} 2$ & 1.7290 & 1.7290 & 2.4490 & -0.7200 & -0.7200 & $-29.4 \%$ & $-29.4 \%$ \\
\hline 1 & EM07-Mn-04 & Sum & 98.5904 & 98.5811 & 99.3430 & -0.7526 & -0.7619 & $-0.8 \%$ & $-0.8 \%$ \\
\hline 1 & EM07-Na-05 & $\mathrm{Al} 2 \mathrm{O} 3$ & 11.4173 & 11.4002 & 11.1760 & 0.2413 & 0.2242 & $2.2 \%$ & $2.0 \%$ \\
\hline 1 & EM07-Na-05 & $\mathrm{B} 2 \mathrm{O} 3$ & 11.2214 & 11.1003 & 11.1760 & 0.0454 & -0.0757 & $0.4 \%$ & $-0.7 \%$ \\
\hline 1 & EM07-Na-05 & $\mathrm{BaO}$ & 0.0528 & 0.0555 & 0.0560 & -0.0032 & -0.0005 & $-5.8 \%$ & $-0.9 \%$ \\
\hline 1 & EM07-Na-05 & $\mathrm{Bi} 2 \mathrm{O} 3$ & 0.0056 & 0.0056 & 0.0000 & 0.0056 & 0.0056 & & \\
\hline 1 & EM07-Na-05 & $\mathrm{CaO}$ & 0.0234 & 0.0250 & 0.0000 & 0.0234 & 0.0250 & & \\
\hline 1 & EM07-Na-05 & $\mathrm{CdO}$ & 0.1471 & 0.1471 & 0.1560 & -0.0089 & -0.0089 & $-5.7 \%$ & $-5.7 \%$ \\
\hline 1 & EM07-Na-05 & $\mathrm{Ce} 2 \mathrm{O} 3$ & 0.0568 & 0.0568 & 0.0560 & 0.0008 & 0.0008 & $1.4 \%$ & $1.4 \%$ \\
\hline 1 & EM07-Na-05 & Cr2O3 & 0.5338 & 0.5819 & 0.5590 & -0.0252 & 0.0229 & $-4.5 \%$ & $4.1 \%$ \\
\hline 1 & EM07-Na-05 & $\mathrm{Fe} 2 \mathrm{O} 3$ & 10.8657 & 11.1187 & 11.1760 & -0.3103 & -0.0573 & $-2.8 \%$ & $-0.5 \%$ \\
\hline 1 & EM07-Na-05 & $\mathrm{K} 2 \mathrm{O}$ & 0.0602 & 0.0639 & 0.0000 & 0.0602 & 0.0639 & & \\
\hline 1 & EM07-Na-05 & $\mathrm{La} 2 \mathrm{O} 3$ & 0.0616 & 0.0616 & 0.0780 & -0.0164 & -0.0164 & $-21.1 \%$ & $-21.1 \%$ \\
\hline 1 & EM07-Na-05 & $\mathrm{Li} 2 \mathrm{O}$ & 3.0679 & 3.0416 & 3.0740 & -0.0061 & -0.0324 & $-0.2 \%$ & $-1.1 \%$ \\
\hline 1 & EM07-Na-05 & $\mathrm{MgO}$ & 0.1472 & 0.1552 & 0.1680 & -0.0208 & -0.0128 & $-12.4 \%$ & $-7.6 \%$ \\
\hline 1 & EM07-Na-05 & $\mathrm{MnO}$ & 2.2596 & 2.2960 & 2.2350 & 0.0246 & 0.0610 & $1.1 \%$ & $2.7 \%$ \\
\hline 1 & EM07-Na-05 & $\mathrm{Na} 2 \mathrm{O}$ & 5.4089 & 5.2596 & 5.0000 & 0.4089 & 0.2596 & $8.2 \%$ & $5.2 \%$ \\
\hline 1 & EM07-Na-05 & $\mathrm{Nd} 2 \mathrm{O} 3$ & 0.0729 & 0.0848 & 0.0780 & -0.0051 & 0.0068 & $-6.5 \%$ & $8.7 \%$ \\
\hline 1 & EM07-Na-05 & $\mathrm{NiO}$ & 1.0342 & 1.1230 & 1.1180 & -0.0838 & 0.0050 & $-7.5 \%$ & $0.5 \%$ \\
\hline 1 & EM07-Na-05 & $\mathrm{P} 2 \mathrm{O} 5$ & 1.0243 & 1.0243 & 1.3970 & -0.3727 & -0.3727 & $-26.7 \%$ & $-26.7 \%$ \\
\hline 1 & EM07-Na-05 & $\mathrm{PbO}$ & 0.3789 & 0.3789 & 0.4140 & -0.0351 & -0.0351 & $-8.5 \%$ & $-8.5 \%$ \\
\hline 1 & EM07-Na-05 & $\mathrm{SiO} 2$ & 46.2624 & 47.4931 & 48.4290 & -2.1666 & -0.9359 & $-4.5 \%$ & $-1.9 \%$ \\
\hline 1 & EM07-Na-05 & $\mathrm{TiO} 2$ & 0.0505 & 0.0532 & 0.0450 & 0.0055 & 0.0082 & $12.1 \%$ & $18.3 \%$ \\
\hline 1 & EM07-Na-05 & $\mathrm{ZnO}$ & 0.0688 & 0.0688 & 0.0670 & 0.0018 & 0.0018 & $2.6 \%$ & $2.6 \%$ \\
\hline 1 & EM07-Na-05 & $\mathrm{ZrO} 2$ & 2.2896 & 2.2896 & 2.7940 & -0.5044 & -0.5044 & $-18.1 \%$ & $-18.1 \%$ \\
\hline
\end{tabular}




\section{Table A5. Average Measured and Bias-Corrected Chemical Compositions Versus} Targeted Compositions for the Test Matrix 1 Study Glasses

\begin{tabular}{|c|c|c|c|c|c|c|c|c|c|}
\hline & & & & Measured & & & & & \\
\hline & & & Measured & Bias-Corrected & Targeted & Diff of & Diff of & \% Diff of & $\%$ Diff of \\
\hline Set & Glass ID & Oxide & (wt\%) & (wt\%) & (wt\%) & Measured & Meas BC & Measured & Meas BC \\
\hline 1 & EM07-Na-05 & Sum & 96.5107 & 97.8846 & 99.2520 & -2.7413 & -1.3674 & $-2.8 \%$ & $-1.4 \%$ \\
\hline 1 & EM07-Na-10 & $\mathrm{Al} 2 \mathrm{O} 3$ & 10.6710 & 10.5876 & 10.5880 & 0.0830 & -0.0004 & $0.8 \%$ & $0.0 \%$ \\
\hline 1 & EM07-Na-10 & $\mathrm{B} 2 \mathrm{O} 3$ & 10.9879 & 10.7602 & 10.5880 & 0.3999 & 0.1722 & $3.8 \%$ & $1.6 \%$ \\
\hline 1 & EM07-Na-10 & $\mathrm{BaO}$ & 0.0516 & 0.0541 & 0.0530 & -0.0014 & 0.0011 & $-2.6 \%$ & $2.1 \%$ \\
\hline 1 & EM07-Na-10 & Bi2O3 & 0.0056 & 0.0056 & 0.0000 & 0.0056 & 0.0056 & & \\
\hline 1 & EM07-Na-10 & $\mathrm{CaO}$ & 0.0192 & 0.0199 & 0.0000 & 0.0192 & 0.0199 & & \\
\hline 1 & EM07-Na-10 & $\mathrm{CdO}$ & 0.1328 & 0.1328 & 0.1480 & -0.0152 & -0.0152 & $-10.3 \%$ & $-10.3 \%$ \\
\hline 1 & EM07-Na-10 & $\mathrm{Ce} 2 \mathrm{O} 3$ & 0.0548 & 0.0548 & 0.0530 & 0.0018 & 0.0018 & $3.3 \%$ & $3.3 \%$ \\
\hline 1 & EM07-Na-10 & Cr2O3 & 0.4516 & 0.5010 & 0.5290 & -0.0774 & -0.0280 & $-14.6 \%$ & $-5.3 \%$ \\
\hline 1 & EM07-Na-10 & $\mathrm{Fe} 2 \mathrm{O} 3$ & 9.9042 & 10.4273 & 10.5880 & -0.6838 & -0.1607 & $-6.5 \%$ & $-1.5 \%$ \\
\hline 1 & EM07-Na-10 & $\mathrm{K} 2 \mathrm{O}$ & 0.0602 & 0.0635 & 0.0000 & 0.0602 & 0.0635 & & \\
\hline 1 & EM07-Na-10 & La2O3 & 0.0563 & 0.0563 & 0.0740 & -0.0177 & -0.0177 & $-23.9 \%$ & $-23.9 \%$ \\
\hline 1 & EM07-Na-10 & Li2O & 2.9495 & 2.8987 & 2.9120 & 0.0375 & -0.0133 & $1.3 \%$ & $-0.5 \%$ \\
\hline 1 & EM07-Na-10 & $\mathrm{MgO}$ & 0.1360 & 0.1444 & 0.1590 & -0.0230 & -0.0146 & $-14.5 \%$ & $-9.2 \%$ \\
\hline 1 & EM07-Na-10 & $\mathrm{MnO}$ & 2.0950 & 2.1597 & 2.1180 & -0.0230 & 0.0417 & $-1.1 \%$ & $2.0 \%$ \\
\hline 1 & EM07-Na-10 & $\mathrm{Na} 2 \mathrm{O}$ & 10.4200 & 10.1202 & 10.0000 & 0.4200 & 0.1202 & $4.2 \%$ & $1.2 \%$ \\
\hline 1 & EM07-Na-10 & $\mathrm{Nd} 2 \mathrm{O} 3$ & 0.0682 & 0.0788 & 0.0740 & -0.0058 & 0.0048 & $-7.8 \%$ & $6.4 \%$ \\
\hline 1 & EM07-Na-10 & $\mathrm{NiO}$ & 0.9331 & 1.0229 & 1.0590 & -0.1259 & -0.0361 & $-11.9 \%$ & $-3.4 \%$ \\
\hline 1 & EM07-Na-10 & P2O5 & 0.9463 & 0.9463 & 1.3240 & -0.3777 & -0.3777 & $-28.5 \%$ & $-28.5 \%$ \\
\hline 1 & EM07-Na-10 & $\mathrm{PbO}$ & 0.3549 & 0.3549 & 0.3920 & -0.0371 & -0.0371 & $-9.5 \%$ & $-9.5 \%$ \\
\hline 1 & EM07-Na-10 & $\mathrm{SiO} 2$ & 45.8880 & 46.1668 & 45.8780 & 0.0100 & 0.2888 & $0.0 \%$ & $0.6 \%$ \\
\hline 1 & EM07-Na-10 & $\mathrm{TiO} 2$ & 0.0459 & 0.0482 & 0.0420 & 0.0039 & 0.0062 & $9.2 \%$ & $14.7 \%$ \\
\hline 1 & EM07-Na-10 & $\mathrm{ZnO}$ & 0.0632 & 0.0632 & 0.0640 & -0.0008 & -0.0008 & $-1.3 \%$ & $-1.3 \%$ \\
\hline 1 & EM07-Na-10 & $\mathrm{ZrO} 2$ & 1.9553 & 1.9553 & 2.6470 & -0.6917 & -0.6917 & $-26.1 \%$ & $-26.1 \%$ \\
\hline 1 & EM07-Na-10 & Sum & 98.2506 & 98.6223 & 99.2900 & -1.0394 & -0.6677 & $-1.0 \%$ & $-0.7 \%$ \\
\hline 1 & EM07-Na-20 & $\mathrm{Al} 2 \mathrm{O} 3$ & 9.5278 & 9.4532 & 9.4120 & 0.1158 & 0.0412 & $1.2 \%$ & $0.4 \%$ \\
\hline 1 & EM07-Na-20 & B2O3 & 9.5551 & 9.4531 & 9.4120 & 0.1431 & 0.0411 & $1.5 \%$ & $0.4 \%$ \\
\hline 1 & EM07-Na-20 & $\mathrm{BaO}$ & 0.0452 & 0.0474 & 0.0470 & -0.0018 & 0.0004 & $-3.8 \%$ & $0.9 \%$ \\
\hline 1 & EM07-Na-20 & Bi2O3 & 0.0056 & 0.0056 & 0.0000 & 0.0056 & 0.0056 & & \\
\hline 1 & EM07-Na-20 & $\mathrm{CaO}$ & 0.0234 & 0.0243 & 0.0000 & 0.0234 & 0.0243 & & \\
\hline 1 & EM07-Na-20 & $\mathrm{CdO}$ & 0.1179 & 0.1179 & 0.1320 & -0.0141 & -0.0141 & $-10.6 \%$ & $-10.6 \%$ \\
\hline 1 & EM07-Na-20 & $\mathrm{Ce} 2 \mathrm{O} 3$ & 0.0477 & 0.0477 & 0.0470 & 0.0007 & 0.0007 & $1.6 \%$ & $1.6 \%$ \\
\hline 1 & EM07-Na-20 & Cr2O3 & 0.4535 & 0.5030 & 0.4710 & -0.0175 & 0.0320 & $-3.7 \%$ & $6.8 \%$ \\
\hline 1 & EM07-Na-20 & Fe2O3 & 9.0786 & 9.5615 & 9.4120 & -0.3334 & 0.1495 & $-3.5 \%$ & $1.6 \%$ \\
\hline 1 & EM07-Na-20 & $\mathrm{K} 2 \mathrm{O}$ & 0.0602 & 0.0635 & 0.0000 & 0.0602 & 0.0635 & & \\
\hline 1 & EM07-Na-20 & $\mathrm{La} 2 \mathrm{O} 3$ & 0.0498 & 0.0498 & 0.0660 & -0.0162 & -0.0162 & $-24.5 \%$ & $-24.5 \%$ \\
\hline 1 & EM07-Na-20 & Li2O & 2.6212 & 2.5988 & 2.5880 & 0.0332 & 0.0108 & $1.3 \%$ & $0.4 \%$ \\
\hline 1 & EM07-Na-20 & $\mathrm{MgO}$ & 0.1356 & 0.1439 & 0.1410 & -0.0054 & 0.0029 & $-3.9 \%$ & $2.1 \%$ \\
\hline 1 & EM07-Na-20 & $\mathrm{MnO}$ & 1.9142 & 1.9734 & 1.8820 & 0.0322 & 0.0914 & $1.7 \%$ & $4.9 \%$ \\
\hline 1 & EM07-Na-20 & $\mathrm{Na} 2 \mathrm{O}$ & 20.0852 & 19.5073 & 20.0000 & 0.0852 & -0.4927 & $0.4 \%$ & $-2.5 \%$ \\
\hline 1 & EM07-Na-20 & $\mathrm{Nd} 2 \mathrm{O} 3$ & 0.0607 & 0.0700 & 0.0660 & -0.0053 & 0.0040 & $-8.1 \%$ & $6.1 \%$ \\
\hline 1 & EM07-Na-20 & $\mathrm{NiO}$ & 0.8771 & 0.9615 & 0.9410 & -0.0639 & 0.0205 & $-6.8 \%$ & $2.2 \%$ \\
\hline 1 & EM07-Na-20 & P2O5 & 0.7195 & 0.7195 & 1.1760 & -0.4565 & -0.4565 & $-38.8 \%$ & $-38.8 \%$ \\
\hline 1 & EM07-Na-20 & $\mathrm{PbO}$ & 0.3129 & 0.3129 & 0.3480 & -0.0351 & -0.0351 & $-10.1 \%$ & $-10.1 \%$ \\
\hline 1 & EM07-Na-20 & $\mathrm{SiO} 2$ & 41.0746 & 42.1668 & 40.7820 & 0.2926 & 1.3848 & $0.7 \%$ & $3.4 \%$ \\
\hline 1 & EM07-Na-20 & $\mathrm{TiO} 2$ & 0.0409 & 0.0429 & 0.0380 & 0.0029 & 0.0049 & $7.5 \%$ & $12.9 \%$ \\
\hline 1 & EM07-Na-20 & $\mathrm{ZnO}$ & 0.0576 & 0.0576 & 0.0560 & 0.0016 & 0.0016 & $2.8 \%$ & $2.8 \%$ \\
\hline 1 & EM07-Na-20 & $\mathrm{ZrO} 2$ & 2.3200 & 2.3200 & 2.3530 & -0.0330 & -0.0330 & $-1.4 \%$ & $-1.4 \%$ \\
\hline 1 & EM07-Na-20 & Sum & 99.1842 & 100.2017 & 99.3700 & -0.1858 & 0.8317 & $-0.2 \%$ & $0.8 \%$ \\
\hline 1 & EM07-P-0 & $\mathrm{Al} 2 \mathrm{O} 3$ & 10.1277 & 10.0480 & 10.1270 & 0.0007 & -0.0790 & $0.0 \%$ & $-0.8 \%$ \\
\hline 1 & EM07-P-0 & B2O3 & 10.0300 & 9.9213 & 10.1270 & -0.0970 & -0.2057 & $-1.0 \%$ & $-2.0 \%$ \\
\hline 1 & EM07-P-0 & $\mathrm{BaO}$ & 0.0497 & 0.0521 & 0.0510 & -0.0013 & 0.0011 & $-2.6 \%$ & $2.1 \%$ \\
\hline 1 & EM07-P-0 & $\mathrm{Bi2O} 3$ & 0.0056 & 0.0056 & 0.0000 & 0.0056 & 0.0056 & & \\
\hline 1 & EM07-P-0 & $\mathrm{CaO}$ & 0.0189 & 0.0195 & 0.0000 & 0.0189 & 0.0195 & & \\
\hline 1 & EM07-P-0 & $\mathrm{CdO}$ & 0.1271 & 0.1271 & 0.1420 & -0.0149 & -0.0149 & $-10.5 \%$ & $-10.5 \%$ \\
\hline 1 & EM07-P-0 & $\mathrm{Ce} 2 \mathrm{O} 3$ & 0.0533 & 0.0533 & 0.0510 & 0.0023 & 0.0023 & $4.5 \%$ & $4.5 \%$ \\
\hline
\end{tabular}




\section{Table A5. Average Measured and Bias-Corrected Chemical Compositions Versus} Targeted Compositions for the Test Matrix 1 Study Glasses

\begin{tabular}{|c|c|c|c|c|c|c|c|c|c|}
\hline & & & & Measured & & & & & \\
\hline & & & Measured & Bias-Corrected & Targeted & Diff of & Diff of & \% Diff of & $\%$ Diff of \\
\hline Set & Glass ID & Oxide & $(\mathrm{wt} \%)$ & (wt\%) & $(\mathrm{wt} \%)$ & Measured & Meas BC & Measured & Meas BC \\
\hline 1 & EM07-P-0 & Cr2O3 & 0.4535 & 0.5030 & 0.5060 & -0.0525 & -0.0030 & $-10.4 \%$ & $-0.6 \%$ \\
\hline 1 & EM07-P-0 & $\mathrm{Fe} 2 \mathrm{O} 3$ & 9.4539 & 9.9550 & 10.1270 & -0.6731 & -0.1720 & $-6.6 \%$ & $-1.7 \%$ \\
\hline 1 & EM07-P-0 & $\mathrm{K} 2 \mathrm{O}$ & 0.0602 & 0.0635 & 0.0000 & 0.0602 & 0.0635 & & \\
\hline 1 & EM07-P-0 & $\mathrm{La} 2 \mathrm{O} 3$ & 0.0539 & 0.0539 & 0.0710 & -0.0171 & -0.0171 & $-24.0 \%$ & $-24.0 \%$ \\
\hline 1 & EM07-P-0 & $\mathrm{Li} 2 \mathrm{O}$ & 2.7449 & 2.7214 & 2.7850 & -0.0401 & -0.0636 & $-1.4 \%$ & $-2.3 \%$ \\
\hline 1 & EM07-P-0 & $\mathrm{MgO}$ & 0.1289 & 0.1369 & 0.1520 & -0.0231 & -0.0151 & $-15.2 \%$ & $-9.9 \%$ \\
\hline 1 & EM07-P-0 & $\mathrm{MnO}$ & 2.0466 & 2.1098 & 2.0250 & 0.0216 & 0.0848 & $1.1 \%$ & $4.2 \%$ \\
\hline 1 & EM07-P-0 & $\mathrm{Na} 2 \mathrm{O}$ & 15.3335 & 14.8923 & 15.1900 & 0.1435 & -0.2977 & $0.9 \%$ & $-2.0 \%$ \\
\hline 1 & EM07-P-0 & $\mathrm{Nd} 2 \mathrm{O} 3$ & 0.0671 & 0.0774 & 0.0710 & -0.0039 & 0.0064 & $-5.5 \%$ & $9.0 \%$ \\
\hline 1 & EM07-P-0 & $\mathrm{NiO}$ & 0.9143 & 1.0023 & 1.0130 & -0.0987 & -0.0107 & $-9.7 \%$ & $-1.1 \%$ \\
\hline 1 & EM07-P-0 & $\mathrm{P} 2 \mathrm{O} 5$ & 0.0917 & 0.0917 & 0.0000 & 0.0917 & 0.0917 & & \\
\hline 1 & EM07-P-0 & $\mathrm{PbO}$ & 0.3380 & 0.3380 & 0.3750 & -0.0370 & -0.0370 & $-9.9 \%$ & $-9.9 \%$ \\
\hline 1 & EM07-P-0 & $\mathrm{SiO} 2$ & 42.2512 & 43.3749 & 43.8750 & -1.6238 & -0.5001 & $-3.7 \%$ & $-1.1 \%$ \\
\hline 1 & EM07-P-0 & $\mathrm{TiO} 2$ & 0.0459 & 0.0482 & 0.0410 & 0.0049 & 0.0072 & $11.9 \%$ & $17.5 \%$ \\
\hline 1 & EM07-P-0 & $\mathrm{ZnO}$ & 0.0604 & 0.0604 & 0.0610 & -0.0006 & -0.0006 & $-1.0 \%$ & $-1.0 \%$ \\
\hline 1 & EM07-P-0 & $\mathrm{ZrO} 2$ & 2.0333 & 2.0333 & 2.5320 & -0.4987 & -0.4987 & $-19.7 \%$ & $-19.7 \%$ \\
\hline 1 & EM07-P-0 & Sum & 96.4894 & 97.6888 & 99.3220 & -2.8326 & -1.6332 & $-2.9 \%$ & $-1.6 \%$ \\
\hline 1 & EM07-P-025 & $\mathrm{Al} 2 \mathrm{O} 3$ & 9.8112 & 9.7344 & 9.8730 & -0.0618 & -0.1386 & $-0.6 \%$ & $-1.4 \%$ \\
\hline 1 & EM07-P-025 & $\mathrm{B} 2 \mathrm{O} 3$ & 10.3198 & 10.1033 & 9.8730 & 0.4468 & 0.2303 & $4.5 \%$ & $2.3 \%$ \\
\hline 1 & EM07-P-025 & $\mathrm{BaO}$ & 0.0475 & 0.0497 & 0.0490 & -0.0015 & 0.0007 & $-3.2 \%$ & $1.5 \%$ \\
\hline 1 & EM07-P-025 & $\mathrm{Bi} 2 \mathrm{O} 3$ & 0.0056 & 0.0056 & 0.0000 & 0.0056 & 0.0056 & & \\
\hline 1 & EM07-P-025 & $\mathrm{CaO}$ & 0.0171 & 0.0177 & 0.0000 & 0.0171 & 0.0177 & & \\
\hline 1 & EM07-P-025 & $\mathrm{CdO}$ & 0.1234 & 0.1234 & 0.1380 & -0.0146 & -0.0146 & $-10.6 \%$ & $-10.6 \%$ \\
\hline 1 & EM07-P-025 & $\mathrm{Ce} 2 \mathrm{O} 3$ & 0.0504 & 0.0504 & 0.0490 & 0.0014 & 0.0014 & $2.8 \%$ & $2.8 \%$ \\
\hline 1 & EM07-P-025 & Cr2O3 & 0.4436 & 0.4920 & 0.4940 & -0.0504 & -0.0020 & $-10.2 \%$ & $-0.4 \%$ \\
\hline 1 & EM07-P-025 & $\mathrm{Fe} 2 \mathrm{O} 3$ & 9.0822 & 9.5660 & 9.8730 & -0.7908 & -0.3070 & $-8.0 \%$ & $-3.1 \%$ \\
\hline 1 & EM07-P-025 & $\mathrm{K} 2 \mathrm{O}$ & 0.0602 & 0.0635 & 0.0000 & 0.0602 & 0.0635 & & \\
\hline 1 & EM07-P-025 & $\mathrm{La} 2 \mathrm{O} 3$ & 0.0534 & 0.0534 & 0.0690 & -0.0156 & -0.0156 & $-22.7 \%$ & $-22.7 \%$ \\
\hline 1 & EM07-P-025 & $\mathrm{Li} 2 \mathrm{O}$ & 2.7719 & 2.7242 & 2.7150 & 0.0569 & 0.0092 & $2.1 \%$ & $0.3 \%$ \\
\hline 1 & EM07-P-025 & $\mathrm{MgO}$ & 0.1517 & 0.1611 & 0.1480 & 0.0037 & 0.0131 & $2.5 \%$ & $8.8 \%$ \\
\hline 1 & EM07-P-025 & $\mathrm{MnO}$ & 1.9077 & 1.9667 & 1.9750 & -0.0673 & -0.0083 & $-3.4 \%$ & $-0.4 \%$ \\
\hline 1 & EM07-P-025 & $\mathrm{Na} 2 \mathrm{O}$ & 15.0639 & 14.6305 & 14.8100 & 0.2539 & -0.1795 & $1.7 \%$ & $-1.2 \%$ \\
\hline 1 & EM07-P-025 & $\mathrm{Nd} 2 \mathrm{O} 3$ & 0.0624 & 0.0720 & 0.0690 & -0.0066 & 0.0030 & $-9.6 \%$ & $4.4 \%$ \\
\hline 1 & EM07-P-025 & $\mathrm{NiO}$ & 0.8971 & 0.9835 & 0.9870 & -0.0899 & -0.0035 & $-9.1 \%$ & $-0.4 \%$ \\
\hline 1 & EM07-P-025 & $\mathrm{P} 2 \mathrm{O} 5$ & 0.8421 & 0.8421 & 2.5000 & -1.6579 & -1.6579 & $-66.3 \%$ & $-66.3 \%$ \\
\hline 1 & EM07-P-025 & $\mathrm{PbO}$ & 0.3288 & 0.3288 & 0.3650 & -0.0362 & -0.0362 & $-9.9 \%$ & $-9.9 \%$ \\
\hline 1 & EM07-P-025 & $\mathrm{SiO} 2$ & 42.8930 & 43.1534 & 42.7850 & 0.1080 & 0.3684 & $0.3 \%$ & $0.9 \%$ \\
\hline 1 & EM07-P-025 & $\mathrm{TiO} 2$ & 0.0363 & 0.0381 & 0.0390 & -0.0027 & -0.0009 & $-7.0 \%$ & $-2.3 \%$ \\
\hline 1 & EM07-P-025 & $\mathrm{ZnO}$ & 0.0579 & 0.0579 & 0.0590 & -0.0011 & -0.0011 & $-1.9 \%$ & $-1.9 \%$ \\
\hline 1 & EM07-P-025 & $\mathrm{ZrO} 2$ & 0.4795 & 0.4795 & 2.4680 & -1.9885 & -1.9885 & $-80.6 \%$ & $-80.6 \%$ \\
\hline 1 & EM07-P-025 & Sum & 95.5066 & 95.6972 & 99.3380 & -3.8314 & -3.6408 & $-3.9 \%$ & $-3.7 \%$ \\
\hline 1 & EM07-Zr-001 & $\mathrm{Al} 2 \mathrm{O} 3$ & 10.2269 & 10.1471 & 10.2460 & -0.0191 & -0.0989 & $-0.2 \%$ & $-1.0 \%$ \\
\hline 1 & EM07-Zr-001 & $\mathrm{B} 2 \mathrm{O} 3$ & 10.8672 & 10.6362 & 10.2460 & 0.6212 & 0.3902 & $6.1 \%$ & $3.8 \%$ \\
\hline 1 & EM07-Zr-001 & $\mathrm{BaO}$ & 0.0488 & 0.0512 & 0.0510 & -0.0022 & 0.0002 & $-4.2 \%$ & $0.4 \%$ \\
\hline 1 & EM07-Zr-001 & $\mathrm{Bi} 2 \mathrm{O} 3$ & 0.0056 & 0.0056 & 0.0000 & 0.0056 & 0.0056 & & \\
\hline 1 & EM07-Zr-001 & $\mathrm{CaO}$ & 0.0234 & 0.0243 & 0.0000 & 0.0234 & 0.0243 & & \\
\hline 1 & EM07-Zr-001 & $\mathrm{CdO}$ & 0.1245 & 0.1245 & 0.1430 & -0.0185 & -0.0185 & $-12.9 \%$ & $-12.9 \%$ \\
\hline 1 & EM07-Zr-001 & $\mathrm{Ce} 2 \mathrm{O} 3$ & 0.0524 & 0.0524 & 0.0510 & 0.0014 & 0.0014 & $2.8 \%$ & $2.8 \%$ \\
\hline 1 & EM07-Zr-001 & Cr2O3 & 0.4790 & 0.5314 & 0.5120 & -0.0330 & 0.0194 & $-6.4 \%$ & $3.8 \%$ \\
\hline 1 & EM07-Zr-001 & $\mathrm{Fe} 2 \mathrm{O} 3$ & 9.4539 & 9.9586 & 10.2460 & -0.7921 & -0.2874 & $-7.7 \%$ & $-2.8 \%$ \\
\hline 1 & EM07-Zr-001 & $\mathrm{K} 2 \mathrm{O}$ & 0.0602 & 0.0635 & 0.0000 & 0.0602 & 0.0635 & & \\
\hline 1 & EM07-Zr-001 & $\mathrm{La} 2 \mathrm{O} 3$ & 0.0551 & 0.0551 & 0.0720 & -0.0169 & -0.0169 & $-23.4 \%$ & $-23.4 \%$ \\
\hline 1 & EM07-Zr-001 & $\mathrm{Li2O}$ & 2.8687 & 2.8194 & 2.8180 & 0.0507 & 0.0014 & $1.8 \%$ & $0.0 \%$ \\
\hline 1 & EM07-Zr-001 & $\mathrm{MgO}$ & 0.1318 & 0.1400 & 0.1540 & -0.0222 & -0.0140 & $-14.4 \%$ & $-9.1 \%$ \\
\hline 1 & EM07-Zr-001 & $\mathrm{MnO}$ & 1.9788 & 2.0399 & 2.0490 & -0.0702 & -0.0091 & $-3.4 \%$ & $-0.4 \%$ \\
\hline 1 & EM07-Zr-001 & $\mathrm{Na} 2 \mathrm{O}$ & 15.4683 & 15.0233 & 15.3690 & 0.0993 & -0.3457 & $0.6 \%$ & $-2.2 \%$ \\
\hline
\end{tabular}


Table A5. Average Measured and Bias-Corrected Chemical Compositions Versus Targeted Compositions for the Test Matrix 1 Study Glasses

\begin{tabular}{|c|c|c|c|c|c|c|c|c|c|}
\hline & & & & Measured & & & & & \\
\hline & & & Measured & Bias-Corrected & Targeted & Diff of & Diff of & $\%$ Diff of & \% Diff of \\
\hline Set & Glass ID & Oxide & (wt\%) & (wt\%) & (wt\%) & Measured & Meas BC & Measured & Meas BC \\
\hline 1 & EM07-Zr-001 & $\mathrm{Nd2O} 3$ & 0.1184 & 0.1367 & 0.0720 & 0.0464 & 0.0647 & $64.4 \%$ & $89.8 \%$ \\
\hline 1 & EM07-Zr-001 & $\mathrm{NiO}$ & 0.9350 & 1.0250 & 1.0250 & -0.0900 & 0.0000 & $-8.8 \%$ & $0.0 \%$ \\
\hline 1 & EM07-Zr-001 & $\mathrm{P} 2 \mathrm{O} 5$ & 1.1153 & 1.1153 & 1.2810 & -0.1657 & -0.1657 & $-12.9 \%$ & $-12.9 \%$ \\
\hline 1 & EM07-Zr-001 & $\mathrm{PbO}$ & 0.3388 & 0.3388 & 0.3790 & -0.0402 & -0.0402 & $-10.6 \%$ & $-10.6 \%$ \\
\hline 1 & EM07-Zr-001 & SiO2 & 44.8183 & 45.0900 & 44.3990 & 0.4193 & 0.6910 & $0.9 \%$ & $1.6 \%$ \\
\hline 1 & EM07-Zr-001 & $\mathrm{TiO} 2$ & 0.0450 & 0.0473 & 0.0410 & 0.0040 & 0.0063 & $9.8 \%$ & $15.4 \%$ \\
\hline 1 & EM07-Zr-001 & $\mathrm{ZnO}$ & 0.0607 & 0.0607 & 0.0610 & -0.0003 & -0.0003 & $-0.5 \%$ & $-0.5 \%$ \\
\hline 1 & EM07-Zr-001 & $\mathrm{ZrO} 2$ & 0.0567 & 0.0567 & 0.1000 & -0.0433 & -0.0433 & $-43.3 \%$ & $-43.3 \%$ \\
\hline 1 & EM07-Zr-001 & Sum & 99.3331 & 99.5429 & 99.3150 & 0.0181 & 0.2279 & $0.0 \%$ & $0.2 \%$ \\
\hline 1 & EM07-Zr-05 & $\mathrm{Al} 2 \mathrm{O} 3$ & 9.7168 & 9.6405 & 9.7440 & -0.0272 & -0.1035 & $-0.3 \%$ & $-1.1 \%$ \\
\hline 1 & EM07-Zr-05 & B2O3 & 10.0783 & 9.8659 & 9.7440 & 0.3343 & 0.1219 & $3.4 \%$ & $1.3 \%$ \\
\hline 1 & EM07-Zr-05 & $\mathrm{BaO}$ & 0.0475 & 0.0497 & 0.0490 & -0.0015 & 0.0007 & $-3.2 \%$ & $1.5 \%$ \\
\hline 1 & EM07-Zr-05 & $\mathrm{Bi} 2 \mathrm{O} 3$ & 0.0056 & 0.0056 & 0.0000 & 0.0056 & 0.0056 & & \\
\hline 1 & EM07-Zr-05 & $\mathrm{CaO}$ & 0.0178 & 0.0185 & 0.0000 & 0.0178 & 0.0185 & & \\
\hline 1 & EM07-Zr-05 & $\mathrm{CdO}$ & 0.1217 & 0.1217 & 0.1360 & -0.0143 & -0.0143 & $-10.5 \%$ & $-10.5 \%$ \\
\hline 1 & EM07-Zr-05 & $\mathrm{Ce} 2 \mathrm{O} 3$ & 0.0510 & 0.0510 & 0.0490 & 0.0020 & 0.0020 & $4.0 \%$ & $4.0 \%$ \\
\hline 1 & EM07-Zr-05 & $\mathrm{Cr} 2 \mathrm{O} 3$ & 0.4166 & 0.4620 & 0.4870 & -0.0704 & -0.0250 & $-14.5 \%$ & $-5.1 \%$ \\
\hline 1 & EM07-Zr-05 & $\mathrm{Fe} 2 \mathrm{O} 3$ & 8.9499 & 9.4271 & 9.7440 & -0.7941 & -0.3169 & $-8.1 \%$ & $-3.3 \%$ \\
\hline 1 & EM07-Zr-05 & $\mathrm{K} 2 \mathrm{O}$ & 0.0602 & 0.0635 & 0.0000 & 0.0602 & 0.0635 & & \\
\hline 1 & EM07-Zr-05 & La2O3 & 0.0507 & 0.0507 & 0.0680 & -0.0173 & -0.0173 & $-25.4 \%$ & $-25.4 \%$ \\
\hline 1 & EM07-Zr-05 & $\mathrm{Li} 2 \mathrm{O}$ & 2.7449 & 2.6976 & 2.6790 & 0.0659 & 0.0186 & $2.5 \%$ & $0.7 \%$ \\
\hline 1 & EM07-Zr-05 & $\mathrm{MgO}$ & 0.1235 & 0.1312 & 0.1460 & -0.0225 & -0.0148 & $-15.4 \%$ & $-10.2 \%$ \\
\hline 1 & EM07-Zr-05 & $\mathrm{MnO}$ & 1.8690 & 1.9268 & 1.9490 & -0.0800 & -0.0222 & $-4.1 \%$ & $-1.1 \%$ \\
\hline 1 & EM07-Zr-05 & $\mathrm{Na} 2 \mathrm{O}$ & 14.8617 & 14.4341 & 14.6150 & 0.2467 & -0.1809 & $1.7 \%$ & $-1.2 \%$ \\
\hline 1 & EM07-Zr-05 & $\mathrm{Nd2O3}$ & 0.0688 & 0.0794 & 0.0680 & 0.0008 & 0.0114 & $1.2 \%$ & $16.8 \%$ \\
\hline 1 & EM07-Zr-05 & $\mathrm{NiO}$ & 0.8688 & 0.9525 & 0.9740 & -0.1052 & -0.0215 & $-10.8 \%$ & $-2.2 \%$ \\
\hline 1 & EM07-Zr-05 & $\mathrm{P} 2 \mathrm{O} 5$ & 1.0145 & 1.0145 & 1.2180 & -0.2035 & -0.2035 & $-16.7 \%$ & $-16.7 \%$ \\
\hline 1 & EM07-Zr-05 & $\mathrm{PbO}$ & 0.3275 & 0.3275 & 0.3610 & -0.0335 & -0.0335 & $-9.3 \%$ & $-9.3 \%$ \\
\hline 1 & EM07-Zr-05 & $\mathrm{SiO} 2$ & 42.2512 & 42.5080 & 42.2190 & 0.0322 & 0.2890 & $0.1 \%$ & $0.7 \%$ \\
\hline 1 & EM07-Zr-05 & TiO2 & 0.0421 & 0.0442 & 0.0390 & 0.0031 & 0.0052 & $8.0 \%$ & $13.4 \%$ \\
\hline 1 & EM07-Zr-05 & $\mathrm{ZnO}$ & 0.0557 & 0.0557 & 0.0580 & -0.0023 & -0.0023 & $-4.0 \%$ & $-4.0 \%$ \\
\hline 1 & EM07-Zr-05 & $\mathrm{ZrO} 2$ & 3.5019 & 3.5019 & 5.0000 & $\begin{array}{l}-1.4981 \\
\end{array}$ & -1.4981 & $-30.0 \%$ & $-30.0 \%$ \\
\hline 1 & EM07-Zr-05 & Sum & 97.2457 & 97.4296 & 99.3470 & -2.1013 & -1.9174 & $-2.1 \%$ & $-1.9 \%$ \\
\hline 1 & LRM & $\mathrm{F}$ & 0.7775 & 1.0000 & 1.0000 & -0.2225 & 0.0000 & $-22.3 \%$ & $0.0 \%$ \\
\hline 2 & Batch 1 & $\mathrm{Al} 2 \mathrm{O} 3$ & 4.7253 & 4.8770 & 4.8770 & -0.1517 & 0.0000 & $-3.1 \%$ & $0.0 \%$ \\
\hline 2 & Batch 1 & $\mathrm{~B} 2 \mathrm{O} 3$ & 7.8431 & 7.7770 & 7.7770 & 0.0661 & 0.0000 & $0.9 \%$ & $0.0 \%$ \\
\hline 2 & Batch 1 & $\mathrm{BaO}$ & 0.1404 & 0.1510 & 0.1510 & -0.0106 & 0.0000 & $-7.0 \%$ & $0.0 \%$ \\
\hline 2 & Batch 1 & Bi2O3 & 0.0056 & 0.0056 & 0.0000 & 0.0056 & 0.0056 & & \\
\hline 2 & Batch 1 & $\mathrm{CaO}$ & 1.1752 & 1.2200 & 1.2200 & -0.0448 & 0.0000 & $-3.7 \%$ & $0.0 \%$ \\
\hline 2 & Batch 1 & $\mathrm{CdO}$ & 0.0057 & 0.0057 & 0.0000 & 0.0057 & 0.0057 & & \\
\hline 2 & Batch 1 & $\mathrm{Ce} 2 \mathrm{O} 3$ & 0.0059 & 0.0059 & 0.0000 & 0.0059 & 0.0059 & & \\
\hline 2 & Batch 1 & Cr2O3 & 0.1095 & 0.1070 & 0.1070 & 0.0025 & 0.0000 & $2.3 \%$ & $0.0 \%$ \\
\hline 2 & Batch 1 & $\mathrm{Fe} 2 \mathrm{O} 3$ & 12.5647 & 12.8390 & 12.8390 & -0.2743 & 0.0000 & $-2.1 \%$ & $0.0 \%$ \\
\hline 2 & Batch 1 & $\mathrm{~K} 2 \mathrm{O}$ & 3.0727 & 3.3270 & 3.3270 & -0.2543 & 0.0000 & $-7.6 \%$ & $0.0 \%$ \\
\hline 2 & Batch 1 & La2O3 & 0.0059 & 0.0059 & 0.0000 & 0.0059 & 0.0059 & & \\
\hline 2 & Batch 1 & $\mathrm{Li} 2 \mathrm{O}$ & 4.4152 & 4.4290 & 4.4290 & -0.0138 & 0.0000 & $-0.3 \%$ & $0.0 \%$ \\
\hline 2 & Batch 1 & $\mathrm{MgO}$ & 1.3507 & 1.4190 & 1.4190 & -0.0683 & 0.0000 & $-4.8 \%$ & $0.0 \%$ \\
\hline 2 & Batch 1 & $\mathrm{MnO}$ & 1.7108 & 1.7260 & 1.7260 & -0.0152 & 0.0000 & $-0.9 \%$ & $0.0 \%$ \\
\hline 2 & Batch 1 & $\mathrm{Na} 2 \mathrm{O}$ & 9.0148 & 9.0030 & 9.0030 & 0.0117 & 0.0000 & $0.1 \%$ & $0.0 \%$ \\
\hline 2 & Batch 1 & $\mathrm{Nd} 2 \mathrm{O} 3$ & 0.1389 & 0.1470 & 0.1470 & -0.0081 & 0.0000 & $-5.5 \%$ & $0.0 \%$ \\
\hline 2 & Batch 1 & $\mathrm{NiO}$ & 0.6887 & 0.7510 & 0.7510 & -0.0623 & 0.0000 & $-8.3 \%$ & $0.0 \%$ \\
\hline 2 & Batch 1 & $\mathrm{P} 2 \mathrm{O} 5$ & 0.1146 & 0.1146 & 0.0000 & 0.1146 & 0.1146 & & \\
\hline 2 & Batch 1 & $\mathrm{PbO}$ & 0.0539 & 0.0539 & 0.0000 & 0.0539 & 0.0539 & & \\
\hline 2 & Batch 1 & $\mathrm{SiO} 2$ & 46.7081 & 50.2200 & 50.2200 & -3.5120 & 0.0000 & $-7.0 \%$ & $0.0 \%$ \\
\hline 2 & Batch 1 & $\mathrm{TiO} 2$ & 0.6477 & 0.6770 & 0.6770 & -0.0293 & 0.0000 & $-4.3 \%$ & $0.0 \%$ \\
\hline 2 & Batch 1 & $\mathrm{ZnO}$ & 0.0062 & 0.0062 & 0.0000 & 0.0062 & 0.0062 & & \\
\hline
\end{tabular}




\section{Table A5. Average Measured and Bias-Corrected Chemical Compositions Versus} Targeted Compositions for the Test Matrix 1 Study Glasses

\begin{tabular}{|c|c|c|c|c|c|c|c|c|c|}
\hline & & & & "Measured & & & & & \\
\hline & & & Measured & Bias-Corrected & Targeted & Diff of & Diff of & $\%$ Diff of & \% Diff of \\
\hline Set & Glass ID & Oxide & (wt\%) & (wt\%) & $(w t \%)$ & Measured & Meas BC & Measured & Meas BC \\
\hline 2 & Batch 1 & $\mathrm{ZrO} 2$ & 0.0813 & 0.0813 & 0.0980 & -0.0167 & -0.0167 & $-17.1 \%$ & $-17.1 \%$ \\
\hline 2 & Batch 1 & Sum & 94.5849 & 98.9489 & 98.7680 & -4.1831 & 0.1809 & $-4.2 \%$ & $0.2 \%$ \\
\hline 2 & EM07-Al-06 & $\mathrm{Al} 2 \mathrm{O} 3$ & 5.9897 & 6.1842 & 6.0000 & -0.0103 & 0.1842 & $-0.2 \%$ & $3.1 \%$ \\
\hline 2 & EM07-Al-06 & $\mathrm{B} 2 \mathrm{O} 3$ & 10.5935 & 10.5670 & 10.4440 & 0.1495 & 0.1230 & $1.4 \%$ & $1.2 \%$ \\
\hline 2 & EM07-Al-06 & $\mathrm{BaO}$ & 0.0475 & 0.0509 & 0.0520 & -0.0045 & -0.0011 & $-8.7 \%$ & $-2.0 \%$ \\
\hline 2 & EM07-Al-06 & $\mathrm{Bi} 2 \mathrm{O} 3$ & 0.0056 & 0.0056 & 0.0000 & 0.0056 & 0.0056 & & \\
\hline 2 & EM07-Al-06 & $\mathrm{CaO}$ & 0.0528 & 0.0550 & 0.0000 & 0.0528 & 0.0550 & & \\
\hline 2 & EM07-Al-06 & $\mathrm{CdO}$ & 0.1325 & 0.1325 & 0.1460 & -0.0135 & -0.0135 & $-9.2 \%$ & $-9.2 \%$ \\
\hline 2 & EM07-Al-06 & $\mathrm{Ce} 2 \mathrm{O} 3$ & 0.0512 & 0.0512 & 0.0520 & -0.0008 & -0.0008 & $-1.5 \%$ & $-1.5 \%$ \\
\hline 2 & EM07-Al-06 & Cr2O3 & 0.4940 & 0.4801 & 0.5220 & -0.0280 & -0.0419 & $-5.4 \%$ & $-8.0 \%$ \\
\hline 2 & EM07-Al-06 & $\mathrm{Fe} 2 \mathrm{O} 3$ & 10.0258 & 10.2640 & 10.4440 & -0.4182 & -0.1800 & $-4.0 \%$ & $-1.7 \%$ \\
\hline 2 & EM07-Al-06 & $\mathrm{K} 2 \mathrm{O}$ & 0.0602 & 0.0651 & 0.0000 & 0.0602 & 0.0651 & & \\
\hline 2 & EM07-Al-06 & $\mathrm{La} 2 \mathrm{O} 3$ & 0.0616 & 0.0616 & 0.0730 & -0.0114 & -0.0114 & $-15.7 \%$ & $-15.7 \%$ \\
\hline 2 & EM07-Al-06 & $\mathrm{Li} 2 \mathrm{O}$ & 2.7665 & 2.7740 & 2.8720 & -0.1055 & -0.0980 & $-3.7 \%$ & $-3.4 \%$ \\
\hline 2 & EM07-Al-06 & $\mathrm{MgO}$ & 0.1418 & 0.1494 & 0.1570 & -0.0152 & -0.0076 & $-9.7 \%$ & $-4.8 \%$ \\
\hline 2 & EM07-Al-06 & $\mathrm{MnO}$ & 2.0917 & 2.1153 & 2.0890 & 0.0027 & 0.0263 & $0.1 \%$ & $1.3 \%$ \\
\hline 2 & EM07-Al-06 & $\mathrm{Na} 2 \mathrm{O}$ & 15.8053 & 15.8381 & 15.6670 & 0.1383 & 0.1711 & $0.9 \%$ & $1.1 \%$ \\
\hline 2 & EM07-Al-06 & $\mathrm{Nd} 2 \mathrm{O} 3$ & 0.0668 & 0.0703 & 0.0730 & -0.0062 & -0.0027 & $-8.5 \%$ & $-3.7 \%$ \\
\hline 2 & EM07-Al-06 & $\mathrm{NiO}$ & 0.9467 & 1.0360 & 1.0440 & -0.0973 & -0.0080 & $-9.3 \%$ & $-0.8 \%$ \\
\hline 2 & EM07-Al-06 & P2O5 & 0.9315 & 0.9315 & 1.3060 & -0.3745 & -0.3745 & $-28.7 \%$ & $-28.7 \%$ \\
\hline 2 & EM07-Al-06 & $\mathrm{PbO}$ & 0.3490 & 0.3490 & 0.3860 & -0.0370 & -0.0370 & $-9.6 \%$ & $-9.6 \%$ \\
\hline 2 & EM07-Al-06 & $\mathrm{SiO} 2$ & 43.1069 & 46.3475 & 45.2580 & -2.1511 & 1.0895 & $-4.8 \%$ & $2.4 \%$ \\
\hline 2 & EM07-Al-06 & $\mathrm{TiO} 2$ & 0.0488 & 0.0512 & 0.0420 & 0.0068 & 0.0092 & $16.2 \%$ & $21.8 \%$ \\
\hline 2 & EM07-Al-06 & $\mathrm{ZnO}$ & 0.0663 & 0.0663 & 0.0630 & 0.0033 & 0.0033 & $5.2 \%$ & $5.2 \%$ \\
\hline 2 & EM07-Al-06 & $\mathrm{ZrO} 2$ & 1.9722 & 1.9722 & 2.6110 & -0.6388 & -0.6388 & $-24.5 \%$ & $-24.5 \%$ \\
\hline 2 & EM07-Al-06 & Sum & 95.8078 & 99.6179 & 99.3010 & -3.4932 & 0.3169 & $-3.5 \%$ & $0.3 \%$ \\
\hline 2 & EM07-Al-15 & $\mathrm{Al} 2 \mathrm{O} 3$ & 14.7003 & 15.1774 & 15.0000 & -0.2997 & 0.1774 & $-2.0 \%$ & $1.2 \%$ \\
\hline 2 & EM07-Al-15 & $\mathrm{B} 2 \mathrm{O} 3$ & 9.6436 & 9.6180 & 9.4440 & 0.1996 & 0.1740 & $2.1 \%$ & $1.8 \%$ \\
\hline 2 & EM07-Al-15 & $\mathrm{BaO}$ & 0.0427 & 0.0458 & 0.0470 & -0.0043 & -0.0012 & $-9.1 \%$ & $-2.5 \%$ \\
\hline 2 & EM07-Al-15 & $\mathrm{Bi} 2 \mathrm{O} 3$ & 0.0056 & 0.0056 & 0.0000 & 0.0056 & 0.0056 & & \\
\hline 2 & EM07-Al-15 & $\mathrm{CaO}$ & 0.0154 & 0.0160 & 0.0000 & 0.0154 & 0.0160 & & \\
\hline 2 & EM07-Al-15 & $\mathrm{CdO}$ & 0.1188 & 0.1188 & 0.1320 & -0.0132 & -0.0132 & $-10.0 \%$ & $-10.0 \%$ \\
\hline 2 & EM07-Al-15 & $\mathrm{Ce} 2 \mathrm{O} 3$ & 0.0471 & 0.0471 & 0.0470 & 0.0001 & 0.0001 & $0.3 \%$ & $0.3 \%$ \\
\hline 2 & EM07-Al-15 & $\mathrm{Cr} 2 \mathrm{O} 3$ & 0.3961 & 0.3849 & 0.4720 & -0.0759 & -0.0871 & $-16.1 \%$ & $-18.4 \%$ \\
\hline 2 & EM07-Al-15 & $\mathrm{Fe} 2 \mathrm{O} 3$ & 9.0393 & 9.2552 & 9.4440 & -0.4047 & -0.1888 & $-4.3 \%$ & $-2.0 \%$ \\
\hline 2 & EM07-Al-15 & $\mathrm{K} 2 \mathrm{O}$ & 0.0602 & 0.0651 & 0.0000 & 0.0602 & 0.0651 & & \\
\hline 2 & EM07-Al-15 & $\mathrm{La} 2 \mathrm{O} 3$ & 0.0566 & 0.0566 & 0.0660 & -0.0094 & -0.0094 & $-14.3 \%$ & $-14.3 \%$ \\
\hline 2 & EM07-Al-15 & $\mathrm{Li} 2 \mathrm{O}$ & 2.5297 & 2.5365 & 2.5970 & -0.0673 & -0.0605 & $-2.6 \%$ & $-2.3 \%$ \\
\hline 2 & EM07-Al-15 & $\mathrm{MgO}$ & 0.1244 & 0.1311 & 0.1420 & -0.0176 & -0.0109 & $-12.4 \%$ & $-7.7 \%$ \\
\hline 2 & EM07-Al-15 & $\mathrm{MnO}$ & 1.8819 & 1.9032 & 1.8890 & -0.0071 & 0.0142 & $-0.4 \%$ & $0.8 \%$ \\
\hline 2 & EM07-Al-15 & $\mathrm{Na} 2 \mathrm{O}$ & 13.9855 & 14.0145 & 14.1670 & -0.1815 & -0.1525 & $-1.3 \%$ & $-1.1 \%$ \\
\hline 2 & EM07-Al-15 & $\mathrm{Nd} 2 \mathrm{O} 3$ & 0.0598 & 0.0630 & 0.0660 & -0.0062 & -0.0030 & $-9.4 \%$ & $-4.6 \%$ \\
\hline 2 & EM07-Al-15 & $\mathrm{NiO}$ & 0.8122 & 0.8887 & 0.9440 & -0.1318 & -0.0553 & $-14.0 \%$ & $-5.9 \%$ \\
\hline 2 & EM07-Al-15 & P2O5 & 0.6702 & 0.6702 & 1.1810 & -0.5108 & -0.5108 & $-43.2 \%$ & $-43.2 \%$ \\
\hline 2 & EM07-Al-15 & $\mathrm{PbO}$ & 0.3151 & 0.3151 & 0.3490 & -0.0339 & -0.0339 & $-9.7 \%$ & $-9.7 \%$ \\
\hline 2 & EM07-Al-15 & $\mathrm{SiO} 2$ & 40.5932 & 43.6453 & 40.9240 & -0.3308 & 2.7213 & $-0.8 \%$ & $6.6 \%$ \\
\hline 2 & EM07-Al-15 & $\overline{\mathrm{TiO} 2}$ & 0.0434 & 0.0455 & 0.0380 & 0.0054 & 0.0075 & $14.1 \%$ & $19.7 \%$ \\
\hline 2 & EM07-Al-15 & $\mathrm{ZnO}$ & 0.0563 & 0.0563 & 0.0570 & -0.0007 & -0.0007 & $-1.2 \%$ & $-1.2 \%$ \\
\hline 2 & EM07-Al-15 & $\mathrm{ZrO} 2$ & 1.5399 & 1.5399 & 2.3610 & -0.8211 & -0.8211 & $-34.8 \%$ & $-34.8 \%$ \\
\hline 2 & EM07-Al-15 & Sum & 96.7373 & 100.6000 & 99.3670 & -2.6297 & 1.2330 & $-2.6 \%$ & $1.2 \%$ \\
\hline 2 & EM07-Al-20 & $\mathrm{Al} 2 \mathrm{O} 3$ & 18.2573 & 18.8369 & 20.0000 & -1.7427 & -1.1631 & $-8.7 \%$ & $-5.8 \%$ \\
\hline 2 & EM07-Al-20 & $\mathrm{B} 2 \mathrm{O} 3$ & 9.3538 & 9.2219 & 8.8890 & 0.4648 & 0.3329 & $5.2 \%$ & $3.7 \%$ \\
\hline 2 & EM07-Al-20 & $\mathrm{BaO}$ & 0.0402 & 0.0433 & 0.0440 & -0.0038 & -0.0007 & $-8.6 \%$ & $-1.6 \%$ \\
\hline 2 & EM07-Al-20 & $\mathrm{Bi} 2 \mathrm{O} 3$ & 0.0056 & 0.0056 & 0.0000 & 0.0056 & 0.0056 & & \\
\hline 2 & EM07-Al-20 & $\mathrm{CaO}$ & 0.0070 & 0.0072 & 0.0000 & 0.0070 & 0.0072 & & \\
\hline 2 & EM07-Al-20 & $\mathrm{CdO}$ & 0.1145 & 0.1145 & 0.1240 & -0.0095 & -0.0095 & $-7.6 \%$ & $-7.6 \%$ \\
\hline
\end{tabular}




\section{Table A5. Average Measured and Bias-Corrected Chemical Compositions Versus} Targeted Compositions for the Test Matrix 1 Study Glasses

\begin{tabular}{|c|c|c|c|c|c|c|c|c|c|}
\hline & & & & "Measured & & & & & \\
\hline & & & Measured & Bias-Corrected & Targeted & Diff of & Diff of & $\%$ Diff of & $\%$ Diff of \\
\hline Set & Glass ID & Oxide & (wt\%) & $(\mathrm{wt} \%)$ & (wt\%) & Measured & Meas BC & Measured & Meas BC \\
\hline 2 & EM07-Al-20 & Ce2O3 & 0.0448 & 0.0448 & 0.0440 & 0.0008 & 0.0008 & $1.8 \%$ & $1.8 \%$ \\
\hline 2 & EM07-Al-20 & Cr2O3 & 0.3574 & 0.3512 & 0.4440 & $\begin{array}{l}-0.0866 \\
\end{array}$ & -0.0928 & $-19.5 \%$ & $-20.9 \%$ \\
\hline 2 & EM07-Al-20 & $\mathrm{Fe} 2 \mathrm{O} 3$ & 8.5282 & 8.6974 & 8.8890 & -0.3608 & -0.1916 & $-4.1 \%$ & $-2.2 \%$ \\
\hline 2 & EM07-Al-20 & $\mathrm{K} 2 \mathrm{O}$ & 0.0602 & 0.0653 & 0.0000 & 0.0602 & 0.0653 & & \\
\hline 2 & EM07-Al-20 & $\mathrm{La} 2 \mathrm{O} 3$ & 0.0522 & 0.0522 & 0.0620 & -0.0098 & -0.0098 & $-15.8 \%$ & $-15.8 \%$ \\
\hline 2 & EM07-Al-20 & $\mathrm{Li} 2 \mathrm{O}$ & 2.4274 & 2.4359 & 2.4440 & -0.0166 & -0.0081 & $-0.7 \%$ & $-0.3 \%$ \\
\hline 2 & EM07-Al-20 & $\mathrm{MgO}$ & 0.1173 & 0.1229 & 0.1330 & -0.0157 & -0.0101 & $-11.8 \%$ & $-7.6 \%$ \\
\hline 2 & EM07-Al-20 & $\mathrm{MnO}$ & 1.7689 & 1.7802 & 1.7780 & -0.0091 & 0.0022 & $-0.5 \%$ & $0.1 \%$ \\
\hline 2 & EM07-Al-20 & $\mathrm{Na} 2 \mathrm{O}$ & 13.4800 & 13.4173 & 13.3330 & 0.1470 & 0.0843 & $1.1 \%$ & $0.6 \%$ \\
\hline 2 & EM07-Al-20 & $\mathrm{Nd} 2 \mathrm{O} 3$ & 0.0560 & 0.0595 & 0.0620 & $\begin{array}{l}-0.0060 \\
\end{array}$ & -0.0025 & $-9.7 \%$ & $-4.0 \%$ \\
\hline 2 & EM07-Al-20 & $\mathrm{NiO}$ & 0.7543 & 0.8196 & 0.8890 & -0.1347 & -0.0694 & $-15.2 \%$ & $-7.8 \%$ \\
\hline 2 & EM07-Al-20 & $\mathrm{P} 2 \mathrm{O} 5$ & 0.5740 & 0.5740 & 1.1110 & -0.5370 & -0.5370 & $-48.3 \%$ & $-48.3 \%$ \\
\hline 2 & EM07-Al-20 & $\mathrm{PbO}$ & 0.3016 & 0.3016 & 0.3290 & -0.0274 & -0.0274 & $-8.3 \%$ & $-8.3 \%$ \\
\hline 2 & EM07-Al-20 & $\mathrm{SiO} 2$ & 38.4539 & 41.3458 & 38.5180 & -0.0641 & 2.8278 & $-0.2 \%$ & $7.3 \%$ \\
\hline 2 & EM07-Al-20 & TiO2 & 0.0384 & 0.0400 & 0.0360 & 0.0024 & 0.0040 & $6.6 \%$ & $11.0 \%$ \\
\hline 2 & EM07-Al-20 & $\mathrm{ZnO}$ & 0.0523 & 0.0523 & 0.0530 & -0.0007 & -0.0007 & $-1.4 \%$ & $-1.4 \%$ \\
\hline 2 & EM07-Al-20 & $\mathrm{ZrO} 2$ & 1.3390 & 1.3390 & 2.2220 & -0.8830 & -0.8830 & $-39.7 \%$ & $-39.7 \%$ \\
\hline 2 & EM07-Al-20 & Sum & 96.1842 & 99.7283 & 99.4040 & -3.2198 & 0.3243 & $-3.2 \%$ & $0.3 \%$ \\
\hline 2 & EM07-BL-1 & $\mathrm{Al} 2 \mathrm{O} 3$ & 9.9577 & 10.2808 & 10.0000 & -0.0423 & 0.2808 & $-0.4 \%$ & $2.8 \%$ \\
\hline 2 & EM07-BL-1 & B2O3 & 10.1105 & 10.0844 & 10.0000 & 0.1105 & 0.0844 & $1.1 \%$ & $0.8 \%$ \\
\hline 2 & EM07-BL-1 & $\mathrm{BaO}$ & 0.0461 & 0.0494 & 0.0500 & -0.0039 & -0.0006 & $-7.9 \%$ & $-1.1 \%$ \\
\hline 2 & EM07-BL-1 & $\mathrm{Bi} 2 \mathrm{O} 3$ & 0.0056 & 0.0056 & 0.0000 & 0.0056 & 0.0056 & & \\
\hline 2 & EM07-BL-1 & $\mathrm{CaO}$ & 0.0129 & 0.0135 & 0.0000 & 0.0129 & 0.0135 & & \\
\hline 2 & EM07-BL-1 & $\mathrm{CdO}$ & 0.1297 & 0.1297 & 0.1400 & -0.0103 & -0.0103 & $-7.4 \%$ & $-7.4 \%$ \\
\hline 2 & EM07-BL-1 & Ce2O3 & 0.0510 & 0.0510 & 0.0500 & 0.0010 & 0.0010 & $1.9 \%$ & $1.9 \%$ \\
\hline 2 & EM07-BL-1 & Cr2O3 & 0.4695 & 0.4563 & 0.5000 & -0.0305 & -0.0437 & $-6.1 \%$ & $-8.7 \%$ \\
\hline 2 & EM07-BL-1 & $\mathrm{Fe} 2 \mathrm{O} 3$ & 9.5861 & 9.8151 & 10.0000 & -0.4139 & -0.1849 & $-4.1 \%$ & $-1.8 \%$ \\
\hline 2 & EM07-BL-1 & $\mathrm{K} 2 \mathrm{O}$ & 0.0602 & 0.0651 & 0.0000 & 0.0602 & 0.0651 & & \\
\hline 2 & EM07-BL-1 & La2O3 & 0.0601 & 0.0601 & 0.0700 & -0.0099 & -0.0099 & $-14.1 \%$ & $-14.1 \%$ \\
\hline 2 & EM07-BL-1 & $\mathrm{Li} 2 \mathrm{O}$ & 2.6965 & 2.7038 & 2.7500 & -0.0535 & -0.0462 & $-1.9 \%$ & $-1.7 \%$ \\
\hline 2 & EM07-BL-1 & $\mathrm{MgO}$ & 0.1322 & 0.1394 & 0.1500 & -0.0178 & -0.0106 & $-11.8 \%$ & $-7.1 \%$ \\
\hline 2 & EM07-BL-1 & $\mathrm{MnO}$ & 1.9852 & 2.0078 & 2.0000 & -0.0148 & 0.0078 & $-0.7 \%$ & $0.4 \%$ \\
\hline 2 & EM07-BL-1 & $\mathrm{Na} 2 \mathrm{O}$ & 15.3672 & 15.3990 & 15.0000 & 0.3672 & 0.3990 & $2.4 \%$ & $2.7 \%$ \\
\hline 2 & EM07-BL-1 & Nd2O3 & 0.0653 & 0.0688 & 0.0700 & -0.0047 & -0.0012 & $-6.7 \%$ & $-1.7 \%$ \\
\hline 2 & EM07-BL-1 & $\mathrm{NiO}$ & 0.9159 & 1.0022 & 1.0000 & -0.0841 & 0.0022 & $-8.4 \%$ & $0.2 \%$ \\
\hline 2 & EM07-BL-1 & P2O5 & 0.8822 & 0.8822 & 1.2500 & -0.3678 & -0.3678 & $-29.4 \%$ & $-29.4 \%$ \\
\hline 2 & EM07-BL-1 & $\mathrm{PbO}$ & 0.3355 & 0.3355 & 0.3700 & -0.0345 & -0.0345 & $-9.3 \%$ & $-9.3 \%$ \\
\hline 2 & EM07-BL-1 & $\mathrm{SiO} 2$ & 42.4116 & 45.6004 & 43.3300 & -0.9184 & 2.2704 & $-2.1 \%$ & $5.2 \%$ \\
\hline 2 & EM07-BL-1 & $\mathrm{TiO} 2$ & 0.0442 & 0.0464 & 0.0400 & 0.0042 & 0.0064 & $10.5 \%$ & $15.9 \%$ \\
\hline 2 & EM07-BL-1 & $\mathrm{ZnO}$ & 0.0601 & 0.0601 & 0.0600 & 0.0001 & 0.0001 & $0.1 \%$ & $0.1 \%$ \\
\hline 2 & EM07-BL-1 & $\mathrm{ZrO} 2$ & 1.9148 & 1.9148 & 2.5000 & -0.5852 & -0.5852 & $-23.4 \%$ & $-23.4 \%$ \\
\hline 2 & EM07-BL-1 & Sum & 97.3001 & 101.1713 & 99.3300 & -2.0299 & 1.8413 & $-2.0 \%$ & $1.9 \%$ \\
\hline 2 & EM07-BL-2 & $\mathrm{Al} 2 \mathrm{O} 3$ & 9.6034 & 9.9083 & 10.0000 & -0.3966 & -0.0917 & $-4.0 \%$ & $-0.9 \%$ \\
\hline 2 & EM07-BL-2 & B2O3 & 10.1910 & 10.0455 & 10.0000 & 0.1910 & 0.0455 & $1.9 \%$ & $0.5 \%$ \\
\hline 2 & EM07-BL-2 & $\mathrm{BaO}$ & 0.0444 & 0.0478 & 0.0500 & -0.0056 & -0.0022 & $-11.2 \%$ & $-4.4 \%$ \\
\hline 2 & EM07-BL-2 & $\mathrm{Bi} 2 \mathrm{O} 3$ & 0.0056 & 0.0056 & 0.0000 & 0.0056 & 0.0056 & & \\
\hline 2 & EM07-BL-2 & $\mathrm{CaO}$ & 0.0129 & 0.0134 & 0.0000 & 0.0129 & 0.0134 & & \\
\hline 2 & EM07-BL-2 & $\mathrm{CdO}$ & 0.1274 & 0.1274 & 0.1400 & $\begin{array}{l}-0.0126 \\
\end{array}$ & -0.0126 & $-9.0 \%$ & $-9.0 \%$ \\
\hline 2 & EM07-BL-2 & Ce2O3 & 0.0489 & 0.0489 & 0.0500 & -0.0011 & -0.0011 & $-2.2 \%$ & $-2.2 \%$ \\
\hline 2 & EM07-BL-2 & Cr2O3 & 0.4695 & 0.4614 & 0.5000 & -0.0305 & -0.0386 & $-6.1 \%$ & $-7.7 \%$ \\
\hline 2 & EM07-BL-2 & $\mathrm{Fe} 2 \mathrm{O} 3$ & 9.4861 & 9.6725 & 10.0000 & -0.5139 & -0.3275 & $-5.1 \%$ & $-3.3 \%$ \\
\hline 2 & EM07-BL-2 & $\mathrm{K} 2 \mathrm{O}$ & 0.0602 & 0.0653 & 0.0000 & 0.0602 & 0.0653 & & \\
\hline 2 & EM07-BL-2 & La2O3 & 0.0569 & 0.0569 & 0.0700 & -0.0131 & -0.0131 & $-18.7 \%$ & $-18.7 \%$ \\
\hline 2 & EM07-BL-2 & $\mathrm{Li} 2 \mathrm{O}$ & 2.6911 & 2.7006 & 2.7500 & -0.0589 & -0.0494 & $-2.1 \%$ & $-1.8 \%$ \\
\hline 2 & EM07-BL-2 & $\mathrm{MgO}$ & 0.1298 & 0.1359 & 0.1500 & -0.0202 & -0.0141 & $-13.5 \%$ & $-9.4 \%$ \\
\hline 2 & EM07-BL-2 & $\mathrm{MnO}$ & 1.9529 & 1.9652 & 2.0000 & -0.0471 & -0.0348 & $-2.4 \%$ & $-1.7 \%$ \\
\hline
\end{tabular}




\section{Table A5. Average Measured and Bias-Corrected Chemical Compositions Versus} Targeted Compositions for the Test Matrix 1 Study Glasses

\begin{tabular}{|c|c|c|c|c|c|c|c|c|c|}
\hline & & & & "Measured & & & & & \\
\hline & & & Measured & Bias-Corrected & Targeted & Diff of & Diff of & $\%$ Diff of & $\%$ Diff of \\
\hline Set & Glass ID & Oxide & $(\mathrm{wt} \%)$ & (wt\%) & (wt\%) & Measured & Meas BC & Measured & Meas BC \\
\hline 2 & EM07-BL-2 & $\mathrm{Na} 2 \mathrm{O}$ & 14.4910 & 14.4237 & 15.0000 & $\begin{array}{l}-0.5090 \\
\end{array}$ & $\begin{array}{l}-0.5763 \\
\end{array}$ & $-3.4 \%$ & $-3.8 \%$ \\
\hline 2 & EM07-BL-2 & $\mathrm{Nd} 2 \mathrm{O} 3$ & 0.0609 & 0.0648 & 0.0700 & -0.0091 & -0.0052 & $-12.9 \%$ & $-7.4 \%$ \\
\hline 2 & EM07-BL-2 & $\mathrm{NiO}$ & 0.9108 & 0.9896 & 1.0000 & -0.0892 & -0.0104 & $-8.9 \%$ & $-1.0 \%$ \\
\hline 2 & EM07-BL-2 & P2O5 & 0.9252 & 0.9252 & 1.2500 & -0.3248 & -0.3248 & $-26.0 \%$ & $-26.0 \%$ \\
\hline 2 & EM07-BL-2 & $\mathrm{PbO}$ & 0.3326 & 0.3326 & 0.3700 & -0.0374 & -0.0374 & $-10.1 \%$ & $-10.1 \%$ \\
\hline 2 & EM07-BL-2 & $\mathrm{SiO} 2$ & 42.0372 & 45.1963 & 43.3300 & -1.2928 & 1.8663 & $-3.0 \%$ & $4.3 \%$ \\
\hline 2 & EM07-BL-2 & $\mathrm{TiO} 2$ & 0.0421 & 0.0439 & 0.0400 & 0.0021 & 0.0039 & $5.3 \%$ & $9.7 \%$ \\
\hline 2 & EM07-BL-2 & $\mathrm{ZnO}$ & 0.0601 & 0.0601 & 0.0600 & 0.0001 & 0.0001 & $0.1 \%$ & $0.1 \%$ \\
\hline 2 & EM07-BL-2 & $\mathrm{ZrO} 2$ & 1.9587 & 1.9587 & 2.5000 & -0.5413 & -0.5413 & $-21.7 \%$ & $-21.7 \%$ \\
\hline 2 & EM07-BL-2 & Sum & 95.6986 & 99.2495 & 99.3300 & -3.6314 & -0.0805 & $-3.7 \%$ & $-0.1 \%$ \\
\hline 2 & EM07-Cr-001 & $\mathrm{Al} 2 \mathrm{O} 3$ & 9.8254 & 10.1374 & 10.0400 & $\begin{array}{l}-0.2146 \\
\end{array}$ & 0.0974 & $-2.1 \%$ & $1.0 \%$ \\
\hline 2 & EM07-Cr-001 & $\mathrm{B} 2 \mathrm{O} 3$ & 10.3278 & 10.1806 & 10.0400 & 0.2878 & 0.1406 & $2.9 \%$ & $1.4 \%$ \\
\hline 2 & EM07-Cr-001 & $\mathrm{BaO}$ & 0.0455 & 0.0490 & 0.0500 & -0.0045 & -0.0010 & $-9.0 \%$ & $-2.0 \%$ \\
\hline 2 & EM07-Cr-001 & $\mathrm{Bi} 2 \mathrm{O} 3$ & 0.0056 & 0.0056 & 0.0000 & 0.0056 & 0.0056 & & \\
\hline 2 & EM07-Cr-001 & $\mathrm{CaO}$ & 0.0070 & 0.0072 & 0.0000 & 0.0070 & 0.0072 & & \\
\hline 2 & EM07-Cr-001 & $\mathrm{CdO}$ & 0.1308 & 0.1308 & 0.1410 & -0.0102 & -0.0102 & $-7.2 \%$ & $-7.2 \%$ \\
\hline 2 & EM07-Cr-001 & Ce2O3 & 0.0501 & 0.0501 & 0.0500 & 0.0001 & 0.0001 & $0.1 \%$ & $0.1 \%$ \\
\hline 2 & EM07-Cr-001 & $\mathrm{Cr} 2 \mathrm{O} 3$ & 0.1107 & 0.1088 & 0.1000 & 0.0107 & 0.0088 & $10.7 \%$ & $8.8 \%$ \\
\hline 2 & EM07-Cr-001 & $\mathrm{Fe} 2 \mathrm{O} 3$ & 9.7434 & 9.9394 & 10.0400 & -0.2966 & -0.1006 & $-3.0 \%$ & $-1.0 \%$ \\
\hline 2 & EM07-Cr-001 & $\mathrm{K} 2 \mathrm{O}$ & 0.0602 & 0.0653 & 0.0000 & 0.0602 & 0.0653 & & \\
\hline 2 & EM07-Cr-001 & $\mathrm{La} 2 \mathrm{O} 3$ & 0.0578 & 0.0578 & 0.0700 & -0.0122 & -0.0122 & $-17.5 \%$ & $-17.5 \%$ \\
\hline 2 & EM07-Cr-001 & $\mathrm{Li} 2 \mathrm{O}$ & 2.6804 & 2.6898 & 2.7610 & -0.0806 & -0.0712 & $-2.9 \%$ & $-2.6 \%$ \\
\hline 2 & EM07-Cr-001 & $\mathrm{MgO}$ & 0.1335 & 0.1398 & 0.1510 & -0.0175 & -0.0112 & $-11.6 \%$ & $-7.4 \%$ \\
\hline 2 & EM07-Cr-001 & $\mathrm{MnO}$ & 1.9949 & 2.0077 & 2.0080 & -0.0131 & -0.0003 & $-0.7 \%$ & $0.0 \%$ \\
\hline 2 & EM07-Cr-001 & $\mathrm{Na} 2 \mathrm{O}$ & 14.9965 & 14.9272 & 15.0600 & -0.0635 & -0.1328 & $-0.4 \%$ & $-0.9 \%$ \\
\hline 2 & EM07-Cr-001 & $\mathrm{Nd} 2 \mathrm{O} 3$ & 0.0618 & 0.0657 & 0.0700 & -0.0082 & -0.0043 & $-11.7 \%$ & $-6.1 \%$ \\
\hline 2 & EM07-Cr-001 & $\mathrm{NiO}$ & 0.9337 & 1.0145 & 1.0040 & -0.0703 & 0.0105 & $-7.0 \%$ & $1.0 \%$ \\
\hline 2 & EM07-Cr-001 & P2O5 & 0.8845 & 0.8845 & 1.2550 & -0.3705 & -0.3705 & $-29.5 \%$ & $-29.5 \%$ \\
\hline 2 & EM07-Cr-001 & $\mathrm{PbO}$ & 0.3377 & 0.3377 & 0.3710 & -0.0333 & -0.0333 & $-9.0 \%$ & $-9.0 \%$ \\
\hline 2 & EM07-Cr-001 & $\mathrm{SiO} 2$ & 41.7698 & 44.9096 & 43.5070 & -1.7372 & 1.4026 & $-4.0 \%$ & $3.2 \%$ \\
\hline 2 & EM07-Cr-001 & $\mathrm{TiO} 2$ & 0.0442 & 0.0460 & 0.0400 & 0.0042 & 0.0060 & $10.5 \%$ & $15.1 \%$ \\
\hline 2 & EM07-Cr-001 & $\mathrm{ZnO}$ & 0.0598 & 0.0598 & 0.0600 & -0.0002 & -0.0002 & $-0.4 \%$ & $-0.4 \%$ \\
\hline 2 & EM07-Cr-001 & $\mathrm{ZrO} 2$ & 1.9148 & 1.9148 & 2.5100 & -0.5952 & -0.5952 & $-23.7 \%$ & $-23.7 \%$ \\
\hline 2 & EM07-Cr-001 & Sum & 96.1758 & 99.7291 & 99.3280 & -3.1522 & 0.4011 & $-3.2 \%$ & $0.4 \%$ \\
\hline 2 & EM07-Cr-012 & $\mathrm{Al} 2 \mathrm{O} 3$ & 9.7782 & 10.0885 & 9.9300 & -0.1518 & 0.1585 & $-1.5 \%$ & $1.6 \%$ \\
\hline 2 & EM07-Cr-012 & $\mathrm{B} 2 \mathrm{O} 3$ & 10.1829 & 10.0380 & 9.9300 & 0.2529 & 0.1080 & $2.5 \%$ & $1.1 \%$ \\
\hline 2 & EM07-Cr-012 & $\mathrm{BaO}$ & 0.0455 & 0.0490 & 0.0500 & -0.0045 & -0.0010 & $-9.0 \%$ & $-2.0 \%$ \\
\hline 2 & EM07-Cr-012 & $\mathrm{Bi} 2 \mathrm{O} 3$ & 0.0056 & 0.0056 & 0.0000 & 0.0056 & 0.0056 & & \\
\hline 2 & EM07-Cr-012 & $\mathrm{CaO}$ & 0.0070 & 0.0072 & 0.0000 & 0.0070 & 0.0072 & & \\
\hline 2 & EM07-Cr-012 & $\mathrm{CdO}$ & 0.1285 & 0.1285 & 0.1390 & -0.0105 & -0.0105 & $-7.5 \%$ & $-7.5 \%$ \\
\hline 2 & EM07-Cr-012 & $\mathrm{Ce} 2 \mathrm{O} 3$ & 0.0501 & 0.0501 & 0.0500 & 0.0001 & 0.0001 & $0.1 \%$ & $0.1 \%$ \\
\hline 2 & EM07-Cr-012 & $\mathrm{Cr} 2 \mathrm{O} 3$ & 1.0597 & 1.0414 & 1.2000 & -0.1403 & -0.1586 & $-11.7 \%$ & $-13.2 \%$ \\
\hline 2 & EM07-Cr-012 & $\mathrm{Fe} 2 \mathrm{O} 3$ & 9.6362 & 9.8336 & 9.9300 & -0.2938 & -0.0964 & $-3.0 \%$ & $-1.0 \%$ \\
\hline 2 & EM07-Cr-012 & $\mathrm{K} 2 \mathrm{O}$ & 0.0602 & 0.0653 & 0.0000 & 0.0602 & 0.0653 & & \\
\hline 2 & EM07-Cr-012 & $\mathrm{La} 2 \mathrm{O} 3$ & 0.0581 & 0.0581 & 0.0700 & -0.0119 & -0.0119 & $-17.1 \%$ & $-17.1 \%$ \\
\hline 2 & EM07-Cr-012 & $\mathrm{Li} 2 \mathrm{O}$ & 2.6588 & 2.6682 & 2.7310 & -0.0722 & -0.0628 & $-2.6 \%$ & $-2.3 \%$ \\
\hline 2 & EM07-Cr-012 & $\mathrm{MgO}$ & 0.1322 & 0.1385 & 0.1490 & -0.0168 & -0.0105 & $-11.2 \%$ & $-7.1 \%$ \\
\hline 2 & EM07-Cr-012 & $\mathrm{MnO}$ & 1.9820 & 1.9949 & 1.9860 & -0.0040 & 0.0089 & $-0.2 \%$ & $0.4 \%$ \\
\hline 2 & EM07-Cr-012 & $\mathrm{Na} 2 \mathrm{O}$ & 14.9291 & 14.8588 & 14.8940 & 0.0351 & -0.0352 & $0.2 \%$ & $-0.2 \%$ \\
\hline 2 & EM07-Cr-012 & $\mathrm{Nd} 2 \mathrm{O} 3$ & 0.0624 & 0.0664 & 0.0700 & -0.0076 & -0.0036 & $-10.9 \%$ & $-5.2 \%$ \\
\hline 2 & EM07-Cr-012 & $\mathrm{NiO}$ & 0.8990 & 0.9768 & 0.9930 & -0.0940 & -0.0162 & $-9.5 \%$ & $-1.6 \%$ \\
\hline 2 & EM07-Cr-012 & P2O5 & 0.8020 & 0.8020 & 1.2410 & -0.4390 & -0.4390 & $-35.4 \%$ & $-35.4 \%$ \\
\hline 2 & EM07-Cr-012 & $\mathrm{PbO}$ & 0.3361 & 0.3361 & 0.3670 & -0.0309 & -0.0309 & $-8.4 \%$ & $-8.4 \%$ \\
\hline 2 & EM07-Cr-012 & $\mathrm{SiO} 2$ & 41.6094 & 44.7379 & 43.0230 & -1.4136 & 1.7149 & $-3.3 \%$ & $4.0 \%$ \\
\hline 2 & EM07-Cr-012 & $\mathrm{TiO} 2$ & 0.0434 & 0.0452 & 0.0400 & 0.0034 & 0.0052 & $8.4 \%$ & $12.9 \%$ \\
\hline 2 & EM07-Cr-012 & $\mathrm{ZnO}$ & 0.0588 & 0.0588 & 0.0600 & -0.0012 & -0.0012 & $-2.0 \%$ & $-2.0 \%$ \\
\hline
\end{tabular}




\section{Table A5. Average Measured and Bias-Corrected Chemical Compositions Versus} Targeted Compositions for the Test Matrix 1 Study Glasses

\begin{tabular}{|c|c|c|c|c|c|c|c|c|c|}
\hline & & & & "Measured & & & & & \\
\hline & & & Measured & Bias-Corrected & Targeted & Diff of & Diff of & $\%$ Diff of & \% Diff of \\
\hline Set & Glass ID & Oxide & (wt\%) & (wt\%) & $(w t \%)$ & Measured & Meas BC & Measured & Meas BC \\
\hline 2 & EM07-Cr-012 & $\mathrm{ZrO} 2$ & 1.7831 & 1.7831 & 2.4820 & -0.6989 & -0.6989 & $-28.2 \%$ & $-28.2 \%$ \\
\hline 2 & EM07-Cr-012 & Sum & 96.3082 & 99.8317 & 99.3350 & -3.0268 & 0.4967 & $-3.0 \%$ & $0.5 \%$ \\
\hline 2 & EM07-Cr-02 & $\mathrm{Al} 2 \mathrm{O} 3$ & 9.6648 & 9.9784 & 9.8490 & -0.1842 & 0.1294 & $-1.9 \%$ & $1.3 \%$ \\
\hline 2 & EM07-Cr-02 & $\mathrm{B} 2 \mathrm{O} 3$ & 9.9897 & 9.9632 & 9.8490 & 0.1407 & 0.1142 & $1.4 \%$ & $1.2 \%$ \\
\hline 2 & EM07-Cr-02 & $\mathrm{BaO}$ & 0.0452 & 0.0485 & 0.0490 & -0.0038 & -0.0005 & $-7.7 \%$ & $-0.9 \%$ \\
\hline 2 & EM07-Cr-02 & $\mathrm{Bi} 2 \mathrm{O} 3$ & 0.0056 & 0.0056 & 0.0000 & 0.0056 & 0.0056 & & \\
\hline 2 & EM07-Cr-02 & $\mathrm{CaO}$ & 0.0175 & 0.0182 & 0.0000 & 0.0175 & 0.0182 & & \\
\hline 2 & EM07-Cr-02 & $\mathrm{CdO}$ & 0.1245 & 0.1245 & 0.1380 & -0.0135 & -0.0135 & $-9.8 \%$ & $-9.8 \%$ \\
\hline 2 & EM07-Cr-02 & Ce2O3 & 0.0492 & 0.0492 & 0.0490 & 0.0002 & 0.0002 & $0.4 \%$ & $0.4 \%$ \\
\hline 2 & EM07-Cr-02 & Cr2O3 & 1.5540 & 1.5101 & 2.0000 & -0.4460 & -0.4899 & $-22.3 \%$ & $-24.5 \%$ \\
\hline 2 & EM07-Cr-02 & $\mathrm{Fe} 2 \mathrm{O} 3$ & 9.1572 & 9.3760 & 9.8490 & -0.6918 & -0.4730 & $-7.0 \%$ & $-4.8 \%$ \\
\hline 2 & EM07-Cr-02 & $\mathrm{K} 2 \mathrm{O}$ & 0.0602 & 0.0651 & 0.0000 & 0.0602 & 0.0651 & & \\
\hline 2 & EM07-Cr-02 & $\mathrm{La} 2 \mathrm{O} 3$ & 0.0583 & 0.0583 & 0.0690 & -0.0107 & -0.0107 & $-15.4 \%$ & $-15.4 \%$ \\
\hline 2 & EM07-Cr-02 & $\mathrm{Li} 2 \mathrm{O}$ & 2.6158 & 2.6229 & 2.7090 & -0.0932 & -0.0861 & $-3.4 \%$ & $-3.2 \%$ \\
\hline 2 & EM07-Cr-02 & $\mathrm{MgO}$ & 0.1293 & 0.1363 & 0.1480 & -0.0187 & -0.0117 & $-12.6 \%$ & $-7.9 \%$ \\
\hline 2 & EM07-Cr-02 & $\mathrm{MnO}$ & 1.8787 & 1.9000 & 1.9700 & -0.0913 & -0.0700 & $-4.6 \%$ & $-3.6 \%$ \\
\hline 2 & EM07-Cr-02 & $\mathrm{Na} 2 \mathrm{O}$ & 14.8617 & 14.8926 & 14.7740 & 0.0877 & 0.1186 & $0.6 \%$ & $0.8 \%$ \\
\hline 2 & EM07-Cr-02 & $\mathrm{Nd} 2 \mathrm{O} 3$ & 0.0633 & 0.0666 & 0.0690 & -0.0057 & -0.0024 & $-8.3 \%$ & $-3.4 \%$ \\
\hline 2 & EM07-Cr-02 & $\mathrm{NiO}$ & 0.8364 & 0.9152 & 0.9850 & -0.1486 & -0.0698 & $-15.1 \%$ & $-7.1 \%$ \\
\hline 2 & EM07-Cr-02 & P2O5 & 0.8644 & 0.8644 & 1.2310 & -0.3666 & -0.3666 & $-29.8 \%$ & $-29.8 \%$ \\
\hline 2 & EM07-Cr-02 & $\mathrm{PbO}$ & 0.3312 & 0.3312 & 0.3640 & -0.0328 & -0.0328 & $-9.0 \%$ & $-9.0 \%$ \\
\hline 2 & EM07-Cr-02 & $\mathrm{SiO} 2$ & 41.1280 & 44.2221 & 42.6790 & -1.5510 & 1.5431 & $-3.6 \%$ & $3.6 \%$ \\
\hline 2 & EM07-Cr-02 & $\mathrm{TiO} 2$ & 0.0463 & 0.0485 & 0.0390 & 0.0073 & 0.0095 & $18.7 \%$ & $24.5 \%$ \\
\hline 2 & EM07-Cr-02 & $\mathrm{ZnO}$ & 0.0554 & 0.0554 & 0.0590 & -0.0036 & -0.0036 & $-6.1 \%$ & $-6.1 \%$ \\
\hline 2 & EM07-Cr-02 & $\mathrm{ZrO} 2$ & 1.8844 & 1.8844 & 2.4620 & -0.5776 & -0.5776 & $-23.5 \%$ & $-23.5 \%$ \\
\hline 2 & EM07-Cr-02 & Sum & 95.4213 & 99.1368 & 99.3410 & -3.9197 & -0.2042 & $-3.9 \%$ & $-0.2 \%$ \\
\hline 2 & EM07-Fe-05 & $\mathrm{Al} 2 \mathrm{O} 3$ & 10.3308 & 10.6663 & 10.5560 & -0.2252 & 0.1103 & $-2.1 \%$ & $1.0 \%$ \\
\hline 2 & EM07-Fe-05 & $\mathrm{B} 2 \mathrm{O} 3$ & 10.7303 & 10.7026 & 10.5560 & 0.1743 & 0.1466 & $1.7 \%$ & $1.4 \%$ \\
\hline 2 & EM07-Fe-05 & $\mathrm{BaO}$ & 0.0497 & 0.0533 & 0.0530 & -0.0033 & 0.0003 & $-6.3 \%$ & $0.6 \%$ \\
\hline 2 & EM07-Fe-05 & $\mathrm{Bi} 2 \mathrm{O} 3$ & 0.0056 & 0.0056 & 0.0000 & 0.0056 & 0.0056 & & \\
\hline 2 & EM07-Fe-05 & $\mathrm{CaO}$ & 0.0154 & 0.0160 & 0.0000 & 0.0154 & 0.0160 & & \\
\hline 2 & EM07-Fe-05 & $\mathrm{CdO}$ & 0.1356 & 0.1356 & 0.1480 & -0.0124 & -0.0124 & $-8.3 \%$ & $-8.3 \%$ \\
\hline 2 & EM07-Fe-05 & $\mathrm{Ce} 2 \mathrm{O} 3$ & 0.0533 & 0.0533 & 0.0530 & 0.0003 & 0.0003 & $0.6 \%$ & $0.6 \%$ \\
\hline 2 & EM07-Fe-05 & $\mathrm{Cr} 2 \mathrm{O} 3$ & 0.5053 & 0.4911 & 0.5280 & -0.0227 & -0.0369 & $-4.3 \%$ & $-7.0 \%$ \\
\hline 2 & EM07-Fe-05 & $\mathrm{Fe} 2 \mathrm{O} 3$ & 4.8753 & 4.9919 & 5.0000 & -0.1247 & -0.0081 & $-2.5 \%$ & $-0.2 \%$ \\
\hline 2 & EM07-Fe-05 & $\mathrm{K} 2 \mathrm{O}$ & 0.0602 & 0.0651 & 0.0000 & 0.0602 & 0.0651 & & \\
\hline 2 & EM07-Fe-05 & $\mathrm{La} 2 \mathrm{O} 3$ & 0.0627 & 0.0627 & 0.0740 & -0.0113 & -0.0113 & $-15.2 \%$ & $-15.2 \%$ \\
\hline 2 & EM07-Fe-05 & $\mathrm{Li} 2 \mathrm{O}$ & 2.8095 & 2.8172 & 2.9030 & -0.0935 & -0.0858 & $-3.2 \%$ & $-3.0 \%$ \\
\hline 2 & EM07-Fe-05 & $\mathrm{MgO}$ & 0.1397 & 0.1473 & 0.1580 & -0.0183 & -0.0107 & $-11.6 \%$ & $-6.8 \%$ \\
\hline 2 & EM07-Fe-05 & $\mathrm{MnO}$ & 2.0950 & 2.1187 & 2.1110 & -0.0160 & 0.0077 & $-0.8 \%$ & $0.4 \%$ \\
\hline 2 & EM07-Fe-05 & $\mathrm{Na} 2 \mathrm{O}$ & 15.7716 & 15.8054 & 15.8330 & -0.0614 & -0.0276 & $-0.4 \%$ & $-0.2 \%$ \\
\hline 2 & EM07-Fe-05 & $\mathrm{Nd} 2 \mathrm{O} 3$ & 0.0671 & 0.0706 & 0.0740 & -0.0069 & -0.0034 & $-9.4 \%$ & $-4.5 \%$ \\
\hline 2 & EM07-Fe-05 & $\mathrm{NiO}$ & 0.9792 & 1.0715 & 1.0560 & -0.0768 & 0.0155 & $-7.3 \%$ & $1.5 \%$ \\
\hline 2 & EM07-Fe-05 & $\mathrm{P} 2 \mathrm{O} 5$ & 0.9630 & 0.9630 & 1.3190 & -0.3560 & -0.3560 & $-27.0 \%$ & $-27.0 \%$ \\
\hline 2 & EM07-Fe-05 & $\mathrm{PbO}$ & 0.3528 & 0.3528 & 0.3910 & -0.0382 & -0.0382 & $-9.8 \%$ & $-9.8 \%$ \\
\hline 2 & EM07-Fe-05 & $\mathrm{SiO} 2$ & 44.0161 & 47.3259 & 45.7350 & -1.7189 & 1.5909 & $-3.8 \%$ & $3.5 \%$ \\
\hline 2 & EM07-Fe-05 & $\overline{\mathrm{TiO} 2}$ & 0.0505 & 0.0529 & 0.0420 & 0.0085 & 0.0109 & $20.1 \%$ & $26.0 \%$ \\
\hline 2 & EM07-Fe-05 & $\mathrm{ZnO}$ & 0.0632 & 0.0632 & 0.0630 & 0.0002 & 0.0002 & $0.3 \%$ & $0.3 \%$ \\
\hline 2 & EM07-Fe-05 & $\mathrm{ZrO} 2$ & 2.0566 & 2.0566 & 2.6390 & -0.5824 & -0.5824 & $-22.1 \%$ & $-22.1 \%$ \\
\hline 2 & EM07-Fe-05 & Sum & 96.1885 & 100.0886 & 99.2920 & -3.1035 & 0.7966 & $-3.1 \%$ & $0.8 \%$ \\
\hline 2 & EM07-Fe-15 & $\mathrm{Al} 2 \mathrm{O} 3$ & 9.1263 & 9.4225 & 9.4440 & -0.3177 & -0.0215 & $-3.4 \%$ & $-0.2 \%$ \\
\hline 2 & EM07-Fe-15 & $\mathrm{B} 2 \mathrm{O} 3$ & 9.6275 & 9.4916 & 9.4440 & 0.1835 & 0.0476 & $1.9 \%$ & $0.5 \%$ \\
\hline 2 & EM07-Fe-15 & $\mathrm{BaO}$ & 0.0427 & 0.0458 & 0.0470 & -0.0043 & -0.0012 & $-9.1 \%$ & $-2.5 \%$ \\
\hline 2 & EM07-Fe-15 & $\mathrm{Bi} 2 \mathrm{O} 3$ & 0.0056 & 0.0056 & 0.0000 & 0.0056 & 0.0056 & & \\
\hline 2 & EM07-Fe-15 & $\mathrm{CaO}$ & 0.0091 & 0.0095 & 0.0000 & 0.0091 & 0.0095 & & \\
\hline 2 & EM07-Fe-15 & $\mathrm{CdO}$ & 0.1182 & 0.1182 & 0.1320 & -0.0138 & -0.0138 & $-10.4 \%$ & $-10.4 \%$ \\
\hline
\end{tabular}




\section{Table A5. Average Measured and Bias-Corrected Chemical Compositions Versus} Targeted Compositions for the Test Matrix 1 Study Glasses

\begin{tabular}{|c|c|c|c|c|c|c|c|c|c|}
\hline & & & & "Measured & & & & & \\
\hline & & & Measured & Bias-Corrected & Targeted & Diff of & Diff of & $\%$ Diff of & $\%$ Diff of \\
\hline Set & Glass ID & Oxide & $(\mathrm{wt} \%)$ & (wt\%) & (wt\%) & Measured & Meas BC & Measured & Meas BC \\
\hline 2 & EM07-Fe-15 & Ce2O3 & 0.0471 & 0.0471 & 0.0470 & 0.0001 & 0.0001 & $0.3 \%$ & $0.3 \%$ \\
\hline 2 & EM07-Fe-15 & $\mathrm{Cr} 2 \mathrm{O} 3$ & 0.4052 & 0.3938 & 0.4720 & -0.0668 & -0.0782 & $-14.1 \%$ & $-16.6 \%$ \\
\hline 2 & EM07-Fe-15 & $\mathrm{Fe} 2 \mathrm{O} 3$ & 13.9896 & 14.3244 & 15.0000 & -1.0104 & -0.6756 & $-6.7 \%$ & $-4.5 \%$ \\
\hline 2 & EM07-Fe-15 & $\mathrm{K} 2 \mathrm{O}$ & 0.0602 & 0.0651 & 0.0000 & 0.0602 & 0.0651 & & \\
\hline 2 & EM07-Fe-15 & $\mathrm{La} 2 \mathrm{O} 3$ & 0.0560 & 0.0560 & 0.0660 & -0.0100 & -0.0100 & $-15.1 \%$ & $-15.1 \%$ \\
\hline 2 & EM07-Fe-15 & $\mathrm{Li} 2 \mathrm{O}$ & 2.5189 & 2.5276 & 2.5970 & -0.0781 & -0.0694 & $-3.0 \%$ & $-2.7 \%$ \\
\hline 2 & EM07-Fe-15 & $\mathrm{MgO}$ & 0.1219 & 0.1285 & 0.1420 & -0.0201 & -0.0135 & $-14.2 \%$ & $-9.5 \%$ \\
\hline 2 & EM07-Fe-15 & $\mathrm{MnO}$ & 1.8561 & 1.8772 & 1.8890 & -0.0329 & -0.0118 & $-1.7 \%$ & $-0.6 \%$ \\
\hline 2 & EM07-Fe-15 & $\mathrm{Na} 2 \mathrm{O}$ & 14.0866 & 14.1157 & 14.1670 & -0.0804 & -0.0513 & $-0.6 \%$ & $-0.4 \%$ \\
\hline 2 & EM07-Fe-15 & $\mathrm{Nd} 2 \mathrm{O} 3$ & 0.0598 & 0.0630 & 0.0660 & -0.0062 & -0.0030 & $-9.4 \%$ & $-4.6 \%$ \\
\hline 2 & EM07-Fe-15 & $\mathrm{NiO}$ & 0.8023 & 0.8780 & 0.9440 & -0.1417 & -0.0660 & $-15.0 \%$ & $-7.0 \%$ \\
\hline 2 & EM07-Fe-15 & $\mathrm{P} 2 \mathrm{O} 5$ & 0.7235 & 0.7235 & 1.1810 & -0.4575 & -0.4575 & $-38.7 \%$ & $-38.7 \%$ \\
\hline 2 & EM07-Fe-15 & $\mathrm{PbO}$ & 0.2997 & 0.2997 & 0.3490 & -0.0493 & -0.0493 & $-14.1 \%$ & $-14.1 \%$ \\
\hline 2 & EM07-Fe-15 & $\mathrm{SiO} 2$ & 39.4701 & 42.4382 & 40.9240 & -1.4539 & 1.5142 & $-3.6 \%$ & $3.7 \%$ \\
\hline 2 & EM07-Fe-15 & $\mathrm{TiO} 2$ & 0.0442 & 0.0464 & 0.0380 & 0.0062 & 0.0084 & $16.3 \%$ & $22.0 \%$ \\
\hline 2 & EM07-Fe-15 & $\mathrm{ZnO}$ & 0.0563 & 0.0563 & 0.0570 & -0.0007 & -0.0007 & $-1.2 \%$ & $-1.2 \%$ \\
\hline 2 & EM07-Fe-15 & $\mathrm{ZrO} 2$ & 1.6480 & 1.6480 & 2.3610 & -0.7130 & -0.7130 & $-30.2 \%$ & $-30.2 \%$ \\
\hline 2 & EM07-Fe-15 & Sum & 95.1750 & 98.7817 & 99.3670 & -4.1920 & -0.5853 & $-4.2 \%$ & $-0.6 \%$ \\
\hline 2 & EM07-Fe-20 & $\mathrm{Al} 2 \mathrm{O} 3$ & 8.8287 & 9.1152 & 8.8890 & -0.0603 & 0.2262 & $-0.7 \%$ & $2.5 \%$ \\
\hline 2 & EM07-Fe-20 & $\mathrm{B} 2 \mathrm{O} 3$ & 8.9674 & 8.9440 & 8.8890 & 0.0784 & 0.0550 & $0.9 \%$ & $0.6 \%$ \\
\hline 2 & EM07-Fe-20 & $\mathrm{BaO}$ & 0.0402 & 0.0431 & 0.0440 & -0.0038 & -0.0009 & $-8.6 \%$ & $-1.9 \%$ \\
\hline 2 & EM07-Fe-20 & $\mathrm{Bi} 2 \mathrm{O} 3$ & 0.0056 & 0.0056 & 0.0000 & 0.0056 & 0.0056 & & \\
\hline 2 & EM07-Fe-20 & $\mathrm{CaO}$ & 0.0255 & 0.0266 & 0.0000 & 0.0255 & 0.0266 & & \\
\hline 2 & EM07-Fe-20 & $\mathrm{CdO}$ & 0.1102 & 0.1102 & 0.1240 & -0.0138 & -0.0138 & $-11.1 \%$ & $-11.1 \%$ \\
\hline 2 & EM07-Fe-20 & Ce2O3 & 0.0451 & 0.0451 & 0.0440 & 0.0011 & 0.0011 & $2.5 \%$ & $2.5 \%$ \\
\hline 2 & EM07-Fe-20 & $\mathrm{Cr} 2 \mathrm{O} 3$ & 0.3965 & 0.3853 & 0.4440 & -0.0475 & -0.0587 & $-10.7 \%$ & $-13.2 \%$ \\
\hline 2 & EM07-Fe-20 & $\mathrm{Fe} 2 \mathrm{O} 3$ & 19.0508 & 19.5079 & 20.0000 & -0.9492 & -0.4921 & $-4.7 \%$ & $-2.5 \%$ \\
\hline 2 & EM07-Fe-20 & $\mathrm{K} 2 \mathrm{O}$ & 0.0602 & 0.0651 & 0.0000 & 0.0602 & 0.0651 & & \\
\hline 2 & EM07-Fe-20 & $\mathrm{La} 2 \mathrm{O} 3$ & 0.0531 & 0.0531 & 0.0620 & -0.0089 & -0.0089 & $-14.4 \%$ & $-14.4 \%$ \\
\hline 2 & EM07-Fe-20 & $\mathrm{Li} 2 \mathrm{O}$ & 2.3682 & 2.3746 & 2.4440 & -0.0758 & -0.0694 & $-3.1 \%$ & $-2.8 \%$ \\
\hline 2 & EM07-Fe-20 & $\mathrm{MgO}$ & 0.1153 & 0.1215 & 0.1330 & -0.0177 & -0.0115 & $-13.3 \%$ & $-8.7 \%$ \\
\hline 2 & EM07-Fe-20 & $\mathrm{MnO}$ & 1.7689 & 1.7891 & 1.7780 & -0.0091 & 0.0111 & $-0.5 \%$ & $0.6 \%$ \\
\hline 2 & EM07-Fe-20 & $\mathrm{Na} 2 \mathrm{O}$ & 13.4969 & 13.5252 & 13.3330 & 0.1639 & 0.1922 & $1.2 \%$ & $1.4 \%$ \\
\hline 2 & EM07-Fe-20 & $\mathrm{Nd} 2 \mathrm{O} 3$ & 0.0583 & 0.0614 & 0.0620 & -0.0037 & -0.0006 & $-5.9 \%$ & $-0.9 \%$ \\
\hline 2 & EM07-Fe-20 & $\mathrm{NiO}$ & 0.7610 & 0.8327 & 0.8890 & -0.1280 & -0.0563 & $-14.4 \%$ & $-6.3 \%$ \\
\hline 2 & EM07-Fe-20 & P2O5 & 0.7344 & 0.7344 & 1.1110 & -0.3766 & -0.3766 & $-33.9 \%$ & $-33.9 \%$ \\
\hline 2 & EM07-Fe-20 & $\mathrm{PbO}$ & 0.2925 & 0.2925 & 0.3290 & -0.0365 & -0.0365 & $-11.1 \%$ & $-11.1 \%$ \\
\hline 2 & EM07-Fe-20 & $\mathrm{SiO} 2$ & 37.8656 & 40.7127 & 38.5180 & -0.6524 & 2.1947 & $-1.7 \%$ & $5.7 \%$ \\
\hline 2 & EM07-Fe-20 & $\mathrm{TiO} 2$ & 0.0417 & 0.0437 & 0.0360 & 0.0057 & 0.0077 & $15.8 \%$ & $21.5 \%$ \\
\hline 2 & EM07-Fe-20 & $\mathrm{ZnO}$ & 0.0545 & 0.0545 & 0.0530 & 0.0015 & 0.0015 & $2.8 \%$ & $2.8 \%$ \\
\hline 2 & EM07-Fe-20 & $\mathrm{ZrO} 2$ & 1.6412 & 1.6412 & 2.2220 & -0.5808 & -0.5808 & $-26.1 \%$ & $-26.1 \%$ \\
\hline 2 & EM07-Fe-20 & Sum & 96.7816 & 100.4848 & 99.4040 & -2.6224 & 1.0808 & $-2.6 \%$ & $1.1 \%$ \\
\hline 2 & EM07-Li-015 & $\mathrm{Al} 2 \mathrm{O} 3$ & 10.0238 & 10.3420 & 10.1290 & -0.1052 & 0.2130 & $-1.0 \%$ & $2.1 \%$ \\
\hline 2 & EM07-Li-015 & $\mathrm{B} 2 \mathrm{O} 3$ & 10.3842 & 10.3572 & 10.1290 & 0.2552 & 0.2282 & $2.5 \%$ & $2.3 \%$ \\
\hline 2 & EM07-Li-015 & $\mathrm{BaO}$ & 0.0458 & 0.0493 & 0.0510 & -0.0052 & -0.0017 & $-10.2 \%$ & $-3.3 \%$ \\
\hline 2 & EM07-Li-015 & $\mathrm{Bi} 2 \mathrm{O} 3$ & 0.0056 & 0.0056 & 0.0000 & 0.0056 & 0.0056 & & \\
\hline 2 & EM07-Li-015 & $\mathrm{CaO}$ & 0.0070 & 0.0072 & 0.0000 & 0.0070 & 0.0072 & & \\
\hline 2 & EM07-Li-015 & $\mathrm{CdO}$ & 0.1319 & 0.1319 & 0.1420 & -0.0101 & -0.0101 & $-7.1 \%$ & $-7.1 \%$ \\
\hline 2 & EM07-Li-015 & $\mathrm{Ce} 2 \mathrm{O} 3$ & 0.0507 & 0.0507 & 0.0510 & -0.0003 & -0.0003 & $-0.7 \%$ & $-0.7 \%$ \\
\hline 2 & EM07-Li-015 & $\mathrm{Cr} 2 \mathrm{O} 3$ & 0.4666 & 0.4586 & 0.5060 & -0.0394 & -0.0474 & $-7.8 \%$ & $-9.4 \%$ \\
\hline 2 & EM07-Li-015 & $\mathrm{Fe} 2 \mathrm{O} 3$ & 9.7506 & 9.9542 & 10.1290 & -0.3784 & -0.1748 & $-3.7 \%$ & $-1.7 \%$ \\
\hline 2 & EM07-Li-015 & $\mathrm{K} 2 \mathrm{O}$ & 0.0602 & 0.0653 & 0.0000 & 0.0602 & 0.0653 & & \\
\hline 2 & EM07-Li-015 & $\mathrm{La} 2 \mathrm{O} 3$ & 0.0586 & 0.0586 & 0.0710 & -0.0124 & -0.0124 & $-17.4 \%$ & $-17.4 \%$ \\
\hline 2 & EM07-Li-015 & $\mathrm{Li} 2 \mathrm{O}$ & 1.4855 & 1.4895 & 1.5000 & -0.0145 & -0.0105 & $-1.0 \%$ & $-0.7 \%$ \\
\hline 2 & EM07-Li-015 & $\mathrm{MgO}$ & 0.1352 & 0.1415 & 0.1520 & -0.0168 & -0.0105 & $-11.1 \%$ & $-6.9 \%$ \\
\hline 2 & EM07-Li-015 & $\mathrm{MnO}$ & 2.0078 & 2.0211 & 2.0260 & -0.0182 & -0.0049 & $-0.9 \%$ & $-0.2 \%$ \\
\hline
\end{tabular}




\section{Table A5. Average Measured and Bias-Corrected Chemical Compositions Versus} Targeted Compositions for the Test Matrix 1 Study Glasses

\begin{tabular}{|c|c|c|c|c|c|c|c|c|c|}
\hline & & & & Measured & & & & & \\
\hline & & & Measured & Bias-Corrected & Targeted & Diff of & Diff of & \% Diff of & $\%$ Diff of \\
\hline Set & Glass ID & Oxide & (wt\%) & (wt\%) & $(w t \%)$ & Measured & Meas BC & Measured & Meas BC \\
\hline 2 & EM07-Li-015 & $\mathrm{Na} 2 \mathrm{O}$ & 15.1313 & 15.0614 & 15.1930 & -0.0617 & -0.1316 & $-0.4 \%$ & $-0.9 \%$ \\
\hline 2 & EM07-Li-015 & $\mathrm{Nd} 2 \mathrm{O} 3$ & 0.0636 & 0.0676 & 0.0710 & -0.0074 & -0.0034 & $-10.5 \%$ & $-4.8 \%$ \\
\hline 2 & EM07-Li-015 & $\mathrm{NiO}$ & 0.9216 & 1.0014 & 1.0130 & -0.0914 & -0.0116 & $-9.0 \%$ & $-1.1 \%$ \\
\hline 2 & EM07-Li-015 & P2O5 & 0.8364 & 0.8364 & 1.2660 & -0.4296 & -0.4296 & $-33.9 \%$ & $-33.9 \%$ \\
\hline 2 & EM07-Li-015 & $\mathrm{PbO}$ & 0.3399 & 0.3399 & 0.3750 & -0.0351 & -0.0351 & $-9.4 \%$ & $-9.4 \%$ \\
\hline 2 & EM07-Li-015 & $\mathrm{SiO} 2$ & 42.7860 & 46.0038 & 43.8840 & -1.0980 & 2.1198 & $-2.5 \%$ & $4.8 \%$ \\
\hline 2 & EM07-Li-015 & $\mathrm{TiO} 2$ & 0.0438 & 0.0456 & 0.0410 & 0.0028 & 0.0046 & $6.8 \%$ & $11.2 \%$ \\
\hline 2 & EM07-Li-015 & $\mathrm{ZnO}$ & 0.0610 & 0.0610 & 0.0610 & 0.0000 & 0.0000 & $0.0 \%$ & $0.0 \%$ \\
\hline 2 & EM07-Li-015 & $\mathrm{ZrO} 2$ & 1.8371 & 1.8371 & 2.5320 & -0.6949 & -0.6949 & $-27.4 \%$ & $-27.4 \%$ \\
\hline 2 & EM07-Li-015 & Sum & 96.6340 & 100.3870 & 99.3220 & -2.6880 & 1.0650 & $-2.7 \%$ & $1.1 \%$ \\
\hline 2 & EM07-Li-04 & $\mathrm{Al} 2 \mathrm{O} 3$ & 9.6034 & 9.9083 & 9.8710 & -0.2676 & 0.0373 & $-2.7 \%$ & $0.4 \%$ \\
\hline 2 & EM07-Li-04 & $\mathrm{B} 2 \mathrm{O} 3$ & 10.0863 & 9.9425 & 9.8710 & 0.2153 & 0.0715 & $2.2 \%$ & $0.7 \%$ \\
\hline 2 & EM07-Li-04 & $\mathrm{BaO}$ & 0.0449 & 0.0484 & 0.0490 & -0.0041 & -0.0006 & $-8.3 \%$ & $-1.2 \%$ \\
\hline 2 & EM07-Li-04 & $\mathrm{Bi} 2 \mathrm{O} 3$ & 0.0056 & 0.0056 & 0.0000 & 0.0056 & 0.0056 & & \\
\hline 2 & EM07-Li-04 & $\mathrm{CaO}$ & 0.0189 & 0.0196 & 0.0000 & 0.0189 & 0.0196 & & \\
\hline 2 & EM07-Li-04 & $\mathrm{CdO}$ & 0.1285 & 0.1285 & 0.1380 & -0.0095 & -0.0095 & $-6.9 \%$ & $-6.9 \%$ \\
\hline 2 & EM07-Li-04 & Ce2O3 & 0.0483 & 0.0483 & 0.0490 & -0.0007 & -0.0007 & $-1.4 \%$ & $-1.4 \%$ \\
\hline 2 & EM07-Li-04 & $\mathrm{Cr} 2 \mathrm{O} 3$ & 0.4677 & 0.4596 & 0.4940 & -0.0263 & -0.0344 & $-5.3 \%$ & $-7.0 \%$ \\
\hline 2 & EM07-Li-04 & $\mathrm{Fe} 2 \mathrm{O} 3$ & 9.3252 & 9.5116 & 9.8710 & -0.5458 & -0.3594 & $-5.5 \%$ & $-3.6 \%$ \\
\hline 2 & EM07-Li-04 & $\mathrm{K} 2 \mathrm{O}$ & 0.0602 & 0.0653 & 0.0000 & 0.0602 & 0.0653 & & \\
\hline 2 & EM07-Li-04 & La2O3 & 0.0569 & 0.0569 & 0.0690 & -0.0121 & -0.0121 & $-17.6 \%$ & $-17.6 \%$ \\
\hline 2 & EM07-Li-04 & Li2O & 3.8537 & 3.8673 & 4.0000 & -0.1463 & -0.1327 & $-3.7 \%$ & $-3.3 \%$ \\
\hline 2 & EM07-Li-04 & $\mathrm{MgO}$ & 0.1347 & 0.1411 & 0.1480 & -0.0133 & -0.0069 & $-9.0 \%$ & $-4.7 \%$ \\
\hline 2 & EM07-Li-04 & $\mathrm{MnO}$ & 1.9207 & 1.9329 & 1.9740 & -0.0533 & -0.0411 & $-2.7 \%$ & $-2.1 \%$ \\
\hline 2 & EM07-Li-04 & $\mathrm{Na} 2 \mathrm{O}$ & 14.6258 & 14.5572 & 14.8070 & -0.1812 & -0.2498 & $-1.2 \%$ & $-1.7 \%$ \\
\hline 2 & EM07-Li-04 & $\mathrm{Nd} 2 \mathrm{O} 3$ & 0.0612 & 0.0651 & 0.0690 & -0.0078 & -0.0039 & $-11.3 \%$ & $-5.6 \%$ \\
\hline 2 & EM07-Li-04 & $\mathrm{NiO}$ & 0.9111 & 0.9900 & 0.9870 & -0.0759 & 0.0030 & $-7.7 \%$ & $0.3 \%$ \\
\hline 2 & EM07-Li-04 & P2O5 & 0.8295 & 0.8295 & 1.2340 & -0.4045 & -0.4045 & $-32.8 \%$ & $-32.8 \%$ \\
\hline 2 & EM07-Li-04 & $\mathrm{PbO}$ & 0.3334 & 0.3334 & 0.3650 & -0.0316 & -0.0316 & $-8.7 \%$ & $-8.7 \%$ \\
\hline 2 & EM07-Li-04 & SiO2 & 41.0211 & 44.1040 & 42.7760 & -1.7549 & 1.3280 & $-4.1 \%$ & $3.1 \%$ \\
\hline 2 & EM07-Li-04 & TiO2 & 0.0421 & 0.0439 & 0.0390 & 0.0031 & 0.0049 & $8.0 \%$ & $12.5 \%$ \\
\hline 2 & EM07-Li-04 & $\mathrm{ZnO}$ & 0.0591 & 0.0591 & 0.0590 & 0.0001 & 0.0001 & $0.2 \%$ & $0.2 \%$ \\
\hline 2 & EM07-Li-04 & $\mathrm{ZrO} 2$ & 1.8067 & 1.8067 & 2.4680 & -0.6613 & -0.6613 & $-26.8 \%$ & $-26.8 \%$ \\
\hline 2 & EM07-Li-04 & Sum & 95.4451 & 98.9247 & 99.3380 & -3.8929 & -0.4133 & $-3.9 \%$ & $-0.4 \%$ \\
\hline 2 & EM07-Ni-001 & $\mathrm{Al} 2 \mathrm{O} 3$ & 9.8963 & 10.2105 & 10.0910 & -0.1947 & 0.1195 & $-1.9 \%$ & $1.2 \%$ \\
\hline 2 & EM07-Ni-001 & $\mathrm{B} 2 \mathrm{O} 3$ & 10.2232 & 10.1980 & 10.0910 & 0.1322 & 0.1070 & $1.3 \%$ & $1.1 \%$ \\
\hline 2 & EM07-Ni-001 & $\mathrm{BaO}$ & 0.0449 & 0.0484 & 0.0500 & -0.0051 & -0.0016 & $-10.1 \%$ & $-3.2 \%$ \\
\hline 2 & EM07-Ni-001 & $\mathrm{Bi} 2 \mathrm{O} 3$ & 0.0056 & 0.0056 & 0.0000 & 0.0056 & 0.0056 & & \\
\hline 2 & EM07-Ni-001 & $\mathrm{CaO}$ & 0.0087 & 0.0091 & 0.0000 & 0.0087 & 0.0091 & & \\
\hline 2 & EM07-Ni-001 & $\mathrm{CdO}$ & 0.1305 & 0.1305 & 0.1410 & -0.0105 & -0.0105 & $-7.4 \%$ & $-7.4 \%$ \\
\hline 2 & EM07-Ni-001 & Ce2O3 & 0.0495 & 0.0495 & 0.0500 & -0.0005 & -0.0005 & $-1.0 \%$ & $-1.0 \%$ \\
\hline 2 & EM07-Ni-001 & $\mathrm{Cr} 2 \mathrm{O} 3$ & 0.4820 & 0.4737 & 0.5050 & -0.0230 & -0.0313 & $-4.6 \%$ & $-6.2 \%$ \\
\hline 2 & EM07-Ni-001 & $\mathrm{Fe} 2 \mathrm{O} 3$ & 9.6326 & 9.8242 & 10.0910 & -0.4584 & -0.2668 & $-4.5 \%$ & $-2.6 \%$ \\
\hline 2 & EM07-Ni-001 & $\mathrm{K} 2 \mathrm{O}$ & 0.0602 & 0.0653 & 0.0000 & 0.0602 & 0.0653 & & \\
\hline 2 & EM07-Ni-001 & $\mathrm{La} 2 \mathrm{O} 3$ & 0.0583 & 0.0583 & 0.0710 & -0.0127 & -0.0127 & $-17.8 \%$ & $-17.8 \%$ \\
\hline 2 & EM07-Ni-001 & Li2O & 2.6750 & 2.6822 & 2.7750 & -0.1000 & -0.0928 & $-3.6 \%$ & $-3.3 \%$ \\
\hline 2 & EM07-Ni-001 & $\mathrm{MgO}$ & 0.1314 & 0.1376 & 0.1510 & -0.0196 & -0.0134 & $-13.0 \%$ & $-8.9 \%$ \\
\hline 2 & EM07-Ni-001 & $\mathrm{MnO}$ & 1.9755 & 1.9881 & 2.0180 & -0.0425 & -0.0299 & $-2.1 \%$ & $-1.5 \%$ \\
\hline 2 & EM07-Ni-001 & $\mathrm{Na} 2 \mathrm{O}$ & 15.1313 & 15.0621 & 15.1360 & -0.0047 & -0.0739 & $0.0 \%$ & $-0.5 \%$ \\
\hline 2 & EM07-Ni-001 & $\mathrm{Nd} 2 \mathrm{O} 3$ & 0.0621 & 0.0661 & 0.0710 & -0.0089 & -0.0049 & $-12.5 \%$ & $-7.0 \%$ \\
\hline 2 & EM07-Ni-001 & $\mathrm{NiO}$ & 0.0929 & 0.1009 & 0.1000 & -0.0071 & 0.0009 & $-7.1 \%$ & $0.9 \%$ \\
\hline 2 & EM07-Ni-001 & P2O5 & 0.7688 & 0.7688 & 1.2610 & -0.4922 & -0.4922 & $-39.0 \%$ & $-39.0 \%$ \\
\hline 2 & EM07-Ni-001 & $\mathrm{PbO}$ & 0.3382 & 0.3382 & 0.3730 & -0.0348 & -0.0348 & $-9.3 \%$ & $-9.3 \%$ \\
\hline 2 & EM07-Ni-001 & $\mathrm{SiO} 2$ & 42.6790 & 45.8878 & 43.7250 & -1.0460 & 2.1628 & $-2.4 \%$ & $4.9 \%$ \\
\hline 2 & EM07-Ni-001 & $\mathrm{TiO} 2$ & 0.0434 & 0.0452 & 0.0400 & 0.0034 & 0.0052 & $8.4 \%$ & $12.9 \%$ \\
\hline 2 & EM07-Ni-001 & $\mathrm{ZnO}$ & 0.0569 & 0.0569 & 0.0610 & -0.0041 & -0.0041 & $-6.6 \%$ & $-6.6 \%$ \\
\hline
\end{tabular}




\section{Table A5. Average Measured and Bias-Corrected Chemical Compositions Versus} Targeted Compositions for the Test Matrix 1 Study Glasses

\begin{tabular}{|c|c|c|c|c|c|c|c|c|c|}
\hline & & & & Measured & & & & & \\
\hline & & & Measured & Bias-Corrected & Targeted & Diff of & Diff of & \% Diff of & \% Diff of \\
\hline Set & Glass ID & Oxide & (wt\%) & (wt\%) & (wt\%) & Measured & Meas BC & Measured & Meas BC \\
\hline 2 & EM07-Ni-001 & $\mathrm{ZrO} 2$ & 1.7493 & 1.7493 & 2.5230 & -0.7737 & -0.7737 & $-30.7 \%$ & $-30.7 \%$ \\
\hline 2 & EM07-Ni-001 & Sum & 96.2957 & 99.9562 & 99.3240 & -3.0283 & 0.6322 & $-3.0 \%$ & $0.6 \%$ \\
\hline 2 & EM07-Ni-02 & $\mathrm{Al} 2 \mathrm{O} 3$ & 9.5373 & 9.8403 & 9.8990 & -0.3617 & -0.0587 & $-3.7 \%$ & $-0.6 \%$ \\
\hline 2 & EM07-Ni-02 & $\mathrm{B} 2 \mathrm{O} 3$ & 10.1990 & 10.0531 & 9.8990 & 0.3000 & 0.1541 & $3.0 \%$ & $1.6 \%$ \\
\hline 2 & EM07-Ni-02 & $\mathrm{BaO}$ & 0.0433 & 0.0466 & 0.0490 & -0.0057 & -0.0024 & $-11.7 \%$ & $-4.9 \%$ \\
\hline 2 & EM07-Ni-02 & Bi2O3 & 0.0056 & 0.0056 & 0.0000 & 0.0056 & 0.0056 & & \\
\hline 2 & EM07-Ni-02 & $\mathrm{CaO}$ & 0.0070 & 0.0072 & 0.0000 & 0.0070 & 0.0072 & & \\
\hline 2 & EM07-Ni-02 & $\mathrm{CdO}$ & 0.1274 & 0.1274 & 0.1390 & -0.0116 & -0.0116 & $-8.4 \%$ & $-8.4 \%$ \\
\hline 2 & EM07-Ni-02 & $\mathrm{Ce} 2 \mathrm{O} 3$ & 0.0477 & 0.0477 & 0.0490 & -0.0013 & -0.0013 & $-2.6 \%$ & $-2.6 \%$ \\
\hline 2 & EM07-Ni-02 & Cr2O3 & 0.4462 & 0.4385 & 0.4950 & -0.0488 & -0.0565 & $-9.9 \%$ & $-11.4 \%$ \\
\hline 2 & EM07-Ni-02 & $\mathrm{Fe} 2 \mathrm{O} 3$ & 9.8077 & 10.0034 & 9.8990 & -0.0913 & 0.1044 & $-0.9 \%$ & $1.1 \%$ \\
\hline 2 & EM07-Ni-02 & $\mathrm{K} 2 \mathrm{O}$ & 0.0602 & 0.0653 & 0.0000 & 0.0602 & 0.0653 & & \\
\hline 2 & EM07-Ni-02 & La2O3 & 0.0560 & 0.0560 & 0.0690 & -0.0130 & -0.0130 & $-18.8 \%$ & $-18.8 \%$ \\
\hline 2 & EM07-Ni-02 & $\mathrm{Li} 2 \mathrm{O}$ & 2.6427 & 2.6520 & 2.7220 & -0.0793 & -0.0700 & $-2.9 \%$ & $-2.6 \%$ \\
\hline 2 & EM07-Ni-02 & $\mathrm{MgO}$ & 0.1277 & 0.1337 & 0.1480 & -0.0203 & -0.0143 & $-13.7 \%$ & $-9.7 \%$ \\
\hline 2 & EM07-Ni-02 & $\mathrm{MnO}$ & 1.9626 & 1.9745 & 1.9800 & -0.0174 & -0.0055 & $-0.9 \%$ & $-0.3 \%$ \\
\hline 2 & EM07-Ni-02 & $\mathrm{Na} 2 \mathrm{O}$ & 14.5584 & 14.4908 & 14.8480 & -0.2896 & -0.3572 & $-2.0 \%$ & $-2.4 \%$ \\
\hline 2 & EM07-Ni-02 & $\mathrm{Nd} 2 \mathrm{O} 3$ & 0.0607 & 0.0645 & 0.0690 & -0.0083 & -0.0045 & $-12.1 \%$ & $-6.5 \%$ \\
\hline 2 & EM07-Ni-02 & $\mathrm{NiO}$ & 1.7818 & 1.9361 & 2.0000 & -0.2182 & -0.0639 & $-10.9 \%$ & $-3.2 \%$ \\
\hline 2 & EM07-Ni-02 & $\mathrm{P} 2 \mathrm{O} 5$ & 0.8272 & 0.8272 & 1.2370 & -0.4098 & -0.4098 & $-33.1 \%$ & $-33.1 \%$ \\
\hline 2 & EM07-Ni-02 & $\mathrm{PbO}$ & 0.3288 & 0.3288 & 0.3660 & -0.0372 & -0.0372 & $-10.2 \%$ & $-10.2 \%$ \\
\hline 2 & EM07-Ni-02 & $\mathrm{SiO} 2$ & 41.6094 & 44.7368 & 42.8940 & -1.2846 & 1.8428 & $-3.0 \%$ & $4.3 \%$ \\
\hline 2 & EM07-Ni-02 & $\mathrm{TiO} 2$ & 0.0421 & 0.0439 & 0.0400 & 0.0021 & 0.0039 & $5.3 \%$ & $9.7 \%$ \\
\hline 2 & EM07-Ni-02 & $\mathrm{ZnO}$ & 0.0619 & 0.0619 & 0.0590 & 0.0029 & 0.0029 & $5.0 \%$ & $5.0 \%$ \\
\hline 2 & EM07-Ni-02 & $\mathrm{ZrO} 2$ & 1.7763 & 1.7763 & 2.4750 & -0.6987 & -0.6987 & $-28.2 \%$ & $-28.2 \%$ \\
\hline 2 & EM07-Ni-02 & Sum & 96.1170 & 99.7175 & 99.3360 & -3.2190 & 0.3815 & $-3.2 \%$ & $0.4 \%$ \\
\hline 2 & EM07-NM-0025 & $\mathrm{Al2O} 3$ & 9.7687 & 10.0788 & 9.9790 & -0.2103 & 0.0998 & $-2.1 \%$ & $1.0 \%$ \\
\hline 2 & EM07-NM-0025 & B2O3 & 10.2473 & 10.1031 & 9.9790 & 0.2683 & 0.1241 & $2.7 \%$ & $1.2 \%$ \\
\hline 2 & EM07-NM-0025 & $\mathrm{BaO}$ & 0.0441 & 0.0475 & 0.0500 & -0.0059 & -0.0025 & $-11.8 \%$ & $-5.0 \%$ \\
\hline 2 & EM07-NM-0025 & $\mathrm{Bi} 2 \mathrm{O} 3$ & 0.0056 & 0.0056 & 0.0000 & 0.0056 & 0.0056 & & \\
\hline 2 & EM07-NM-0025 & $\mathrm{CaO}$ & 0.0143 & 0.0149 & 0.0000 & 0.0143 & 0.0149 & & \\
\hline 2 & EM07-NM-0025 & $\mathrm{CdO}$ & 0.1291 & 0.1291 & 0.1400 & -0.0109 & -0.0109 & $-7.8 \%$ & $-7.8 \%$ \\
\hline 2 & EM07-NM-0025 & $\mathrm{Ce} 2 \mathrm{O} 3$ & 0.0498 & 0.0498 & 0.0500 & -0.0002 & -0.0002 & $-0.4 \%$ & $-0.4 \%$ \\
\hline 2 & EM07-NM-0025 & Cr2O3 & 0.4673 & 0.4593 & 0.4990 & -0.0317 & -0.0397 & $-6.3 \%$ & $-8.0 \%$ \\
\hline 2 & EM07-NM-0025 & $\mathrm{Fe} 2 \mathrm{O} 3$ & 9.5897 & 9.7773 & 9.9790 & -0.3893 & -0.2017 & $-3.9 \%$ & $-2.0 \%$ \\
\hline 2 & EM07-NM-0025 & $\mathrm{K} 2 \mathrm{O}$ & 0.0602 & 0.0653 & 0.0000 & 0.0602 & 0.0653 & & \\
\hline 2 & EM07-NM-0025 & La2O3 & 0.0578 & 0.0578 & 0.0700 & -0.0122 & -0.0122 & $-17.5 \%$ & $-17.5 \%$ \\
\hline 2 & EM07-NM-0025 & $\mathrm{Li} 2 \mathrm{O}$ & 2.6588 & 2.6682 & 2.7440 & -0.0852 & -0.0758 & $-3.1 \%$ & $-2.8 \%$ \\
\hline 2 & EM07-NM-0025 & $\mathrm{MgO}$ & 0.1322 & 0.1385 & 0.1500 & -0.0178 & -0.0115 & $-11.8 \%$ & $-7.7 \%$ \\
\hline 2 & EM07-NM-0025 & $\mathrm{MnO}$ & 1.9723 & 1.9847 & 1.9960 & -0.0237 & -0.0113 & $-1.2 \%$ & $-0.6 \%$ \\
\hline 2 & EM07-NM-0025 & $\mathrm{Na} 2 \mathrm{O}$ & 14.8280 & 14.7591 & 14.9680 & -0.1400 & -0.2089 & $-0.9 \%$ & $-1.4 \%$ \\
\hline 2 & EM07-NM-0025 & $\mathrm{Nd} 2 \mathrm{O} 3$ & 0.0618 & 0.0657 & 0.0700 & -0.0082 & -0.0043 & $-11.7 \%$ & $-6.1 \%$ \\
\hline 2 & EM07-NM-0025 & $\mathrm{NiO}$ & 0.9133 & 0.9924 & 0.9980 & -0.0847 & -0.0056 & $-8.5 \%$ & $-0.6 \%$ \\
\hline 2 & EM07-NM-0025 & $\mathrm{P} 2 \mathrm{O} 5$ & 0.7670 & 0.7670 & 1.2470 & -0.4800 & -0.4800 & $-38.5 \%$ & $-38.5 \%$ \\
\hline 2 & EM07-NM-0025 & $\mathrm{PbO}$ & 0.3329 & 0.3329 & 0.3690 & -0.0361 & -0.0361 & $-9.8 \%$ & $-9.8 \%$ \\
\hline 2 & EM07-NM-0025 & $\mathrm{SiO} 2$ & 41.6629 & 44.7956 & 43.2390 & -1.5761 & 1.5566 & $-3.6 \%$ & $3.6 \%$ \\
\hline 2 & EM07-NM-0025 & $\mathrm{TiO} 2$ & 0.0421 & 0.0439 & 0.0400 & 0.0021 & 0.0039 & $5.3 \%$ & $9.7 \%$ \\
\hline 2 & EM07-NM-0025 & $\mathrm{ZnO}$ & 0.0601 & 0.0601 & 0.0600 & 0.0001 & 0.0001 & $0.1 \%$ & $0.1 \%$ \\
\hline 2 & EM07-NM-0025 & $\mathrm{ZrO} 2$ & 1.7290 & 1.7290 & 2.4950 & -0.7660 & -0.7660 & $-30.7 \%$ & $-30.7 \%$ \\
\hline 2 & EM07-NM-0025 & Sum & 95.5945 & 99.1255 & 99.1220 & -3.5275 & 0.0035 & $-3.6 \%$ & $0.0 \%$ \\
\hline 2 & EM07-Si-30 & $\mathrm{Al} 2 \mathrm{O} 3$ & 12.1542 & 12.5486 & 12.3520 & -0.1978 & 0.1966 & $-1.6 \%$ & $1.6 \%$ \\
\hline 2 & EM07-Si-30 & $\mathrm{B} 2 \mathrm{O} 3$ & 12.7347 & 12.5535 & 12.3520 & 0.3827 & 0.2015 & $3.1 \%$ & $1.6 \%$ \\
\hline 2 & EM07-Si-30 & $\mathrm{BaO}$ & 0.0586 & 0.0629 & 0.0620 & -0.0034 & 0.0009 & $-5.5 \%$ & $1.5 \%$ \\
\hline 2 & EM07-Si-30 & $\mathrm{Bi} 2 \mathrm{O} 3$ & 0.0056 & 0.0056 & 0.0000 & 0.0056 & 0.0056 & & \\
\hline 2 & EM07-Si-30 & $\mathrm{CaO}$ & 0.0087 & 0.0091 & 0.0000 & 0.0087 & 0.0091 & & \\
\hline 2 & EM07-Si-30 & $\mathrm{CdO}$ & 0.1611 & 0.1611 & 0.1730 & -0.0119 & -0.0119 & $-6.9 \%$ & $-6.9 \%$ \\
\hline
\end{tabular}




\section{Table A5. Average Measured and Bias-Corrected Chemical Compositions Versus} Targeted Compositions for the Test Matrix 1 Study Glasses

\begin{tabular}{|c|c|c|c|c|c|c|c|c|c|}
\hline & & & & "Measured & & & & & \\
\hline & & & Measured & Bias-Corrected & Targeted & Diff of & Diff of & $\%$ Diff of & $\%$ Diff of \\
\hline Set & Glass ID & Oxide & $(\mathrm{wt} \%)$ & (wt\%) & (wt\%) & Measured & Meas BC & Measured & Meas BC \\
\hline 2 & EM07-Si-30 & Ce2O3 & 0.0606 & 0.0606 & 0.0620 & -0.0014 & $\begin{array}{l}-0.0014 \\
\end{array}$ & $-2.2 \%$ & $-2.2 \%$ \\
\hline 2 & EM07-Si-30 & $\mathrm{Cr} 2 \mathrm{O} 3$ & 0.5547 & 0.5390 & 0.6180 & -0.0633 & -0.0790 & $-10.2 \%$ & $-12.8 \%$ \\
\hline 2 & EM07-Si-30 & $\mathrm{Fe} 2 \mathrm{O} 3$ & 12.1703 & 12.4617 & 12.3520 & -0.1817 & 0.1097 & $-1.5 \%$ & $0.9 \%$ \\
\hline 2 & EM07-Si-30 & $\mathrm{K} 2 \mathrm{O}$ & 0.0602 & 0.0651 & 0.0000 & 0.0602 & 0.0651 & & \\
\hline 2 & EM07-Si-30 & $\mathrm{La} 2 \mathrm{O} 3$ & 0.0730 & 0.0730 & 0.0860 & -0.0130 & -0.0130 & $-15.1 \%$ & $-15.1 \%$ \\
\hline 2 & EM07-Si-30 & $\mathrm{Li} 2 \mathrm{O}$ & 3.2939 & 3.3055 & 3.3970 & -0.1031 & -0.0915 & $-3.0 \%$ & $-2.7 \%$ \\
\hline 2 & EM07-Si-30 & $\mathrm{MgO}$ & 0.1633 & 0.1722 & 0.1850 & -0.0217 & -0.0128 & $-11.7 \%$ & $-6.9 \%$ \\
\hline 2 & EM07-Si-30 & $\mathrm{MnO}$ & 2.4694 & 2.4975 & 2.4700 & -0.0006 & 0.0275 & $0.0 \%$ & $1.1 \%$ \\
\hline 2 & EM07-Si-30 & $\mathrm{Na} 2 \mathrm{O}$ & 18.5013 & 18.5398 & 18.5300 & -0.0287 & 0.0098 & $-0.2 \%$ & $0.1 \%$ \\
\hline 2 & EM07-Si-30 & Nd2O3 & 0.0790 & 0.0832 & 0.0860 & $\begin{array}{l}-0.0070 \\
\end{array}$ & $\begin{array}{l}-0.0028 \\
\end{array}$ & $-8.1 \%$ & $-3.2 \%$ \\
\hline 2 & EM07-Si-30 & $\mathrm{NiO}$ & 1.1013 & 1.2052 & 1.2350 & -0.1337 & $\begin{array}{l}-0.0298 \\
\end{array}$ & $-10.8 \%$ & $-2.4 \%$ \\
\hline 2 & EM07-Si-30 & $\mathrm{P} 2 \mathrm{O} 5$ & 0.4262 & 0.4262 & 1.5440 & -1.1178 & -1.1178 & $-72.4 \%$ & $-72.4 \%$ \\
\hline 2 & EM07-Si-30 & $\mathrm{PbO}$ & 0.4163 & 0.4163 & 0.4570 & -0.0407 & -0.0407 & $-8.9 \%$ & $-8.9 \%$ \\
\hline 2 & EM07-Si-30 & $\mathrm{SiO} 2$ & 29.6293 & 31.8562 & 30.0000 & -0.3707 & 1.8562 & $-1.2 \%$ & $6.2 \%$ \\
\hline 2 & EM07-Si-30 & $\mathrm{TiO} 2$ & 0.0517 & 0.0542 & 0.0490 & 0.0027 & 0.0052 & $5.5 \%$ & $10.7 \%$ \\
\hline 2 & EM07-Si-30 & $\mathrm{ZnO}$ & 0.0762 & 0.0762 & 0.0740 & 0.0022 & 0.0022 & $3.0 \%$ & $3.0 \%$ \\
\hline 2 & EM07-Si-30 & $\mathrm{ZrO} 2$ & 1.3164 & 1.3164 & 3.0880 & -1.7716 & -1.7716 & $-57.4 \%$ & $-57.4 \%$ \\
\hline 2 & EM07-Si-30 & Sum & 95.5663 & 98.4892 & 99.1720 & -3.6057 & -0.6828 & $-3.6 \%$ & $-0.7 \%$ \\
\hline 2 & EM07-Si-37 & $\mathrm{Al} 2 \mathrm{O} 3$ & 10.8221 & 11.1733 & 11.1170 & -0.2949 & 0.0563 & $-2.7 \%$ & $0.5 \%$ \\
\hline 2 & EM07-Si-37 & B2O3 & 11.1328 & 11.1036 & 11.1170 & 0.0158 & -0.0134 & $0.1 \%$ & $-0.1 \%$ \\
\hline 2 & EM07-Si-37 & $\mathrm{BaO}$ & 0.0511 & 0.0548 & 0.0560 & -0.0049 & -0.0012 & $-8.8 \%$ & $-2.1 \%$ \\
\hline 2 & EM07-Si-37 & $\mathrm{Bi} 2 \mathrm{O} 3$ & 0.0056 & 0.0056 & 0.0000 & 0.0056 & 0.0056 & & \\
\hline 2 & EM07-Si-37 & $\mathrm{CaO}$ & 0.0112 & 0.0116 & 0.0000 & 0.0112 & 0.0116 & & \\
\hline 2 & EM07-Si-37 & $\mathrm{CdO}$ & 0.1408 & 0.1408 & 0.1560 & -0.0152 & -0.0152 & $-9.8 \%$ & $-9.8 \%$ \\
\hline 2 & EM07-Si-37 & Ce2O3 & 0.0553 & 0.0553 & 0.0560 & -0.0007 & -0.0007 & $-1.2 \%$ & $-1.2 \%$ \\
\hline 2 & EM07-Si-37 & $\mathrm{Cr} 2 \mathrm{O} 3$ & 0.5138 & 0.4993 & 0.5560 & -0.0422 & -0.0567 & $-7.6 \%$ & $-10.2 \%$ \\
\hline 2 & EM07-Si-37 & $\mathrm{Fe} 2 \mathrm{O} 3$ & 10.6477 & 10.9031 & 11.1170 & -0.4693 & -0.2139 & $-4.2 \%$ & $-1.9 \%$ \\
\hline 2 & EM07-Si-37 & $\mathrm{K} 2 \mathrm{O}$ & 0.0602 & 0.0651 & 0.0000 & 0.0602 & 0.0651 & & \\
\hline 2 & EM07-Si-37 & $\mathrm{La} 2 \mathrm{O} 3$ & 0.0657 & 0.0657 & 0.0780 & -0.0123 & -0.0123 & $-15.8 \%$ & $-15.8 \%$ \\
\hline 2 & EM07-Si-37 & $\mathrm{Li} 2 \mathrm{O}$ & 2.9226 & 2.9305 & 3.0570 & -0.1344 & -0.1265 & $-4.4 \%$ & $-4.1 \%$ \\
\hline 2 & EM07-Si-37 & $\mathrm{MgO}$ & 0.1459 & 0.1538 & 0.1670 & -0.0211 & -0.0132 & $-12.6 \%$ & $-7.9 \%$ \\
\hline 2 & EM07-Si-37 & $\mathrm{MnO}$ & 2.2144 & 2.2397 & 2.2230 & -0.0086 & 0.0167 & $-0.4 \%$ & $0.8 \%$ \\
\hline 2 & EM07-Si-37 & $\mathrm{Na} 2 \mathrm{O}$ & 16.4793 & 16.5138 & 16.6740 & -0.1947 & -0.1602 & $-1.2 \%$ & $-1.0 \%$ \\
\hline 2 & EM07-Si-37 & Nd2O3 & 0.0712 & 0.0749 & 0.0780 & -0.0068 & -0.0031 & $-8.8 \%$ & $-3.9 \%$ \\
\hline 2 & EM07-Si-37 & $\mathrm{NiO}$ & 0.9973 & 1.0914 & 1.1120 & -0.1147 & -0.0206 & $-10.3 \%$ & $-1.9 \%$ \\
\hline 2 & EM07-Si-37 & P2O5 & 0.4491 & 0.4491 & 1.3900 & -0.9409 & -0.9409 & $-67.7 \%$ & $-67.7 \%$ \\
\hline 2 & EM07-Si-37 & $\mathrm{PbO}$ & 0.3692 & 0.3692 & 0.4110 & -0.0418 & -0.0418 & $-10.2 \%$ & $-10.2 \%$ \\
\hline 2 & EM07-Si-37 & $\mathrm{SiO} 2$ & 36.1007 & 38.8160 & 37.0000 & -0.8993 & 1.8160 & $-2.4 \%$ & $4.9 \%$ \\
\hline 2 & EM07-Si-37 & $\mathrm{TiO} 2$ & 0.0492 & 0.0516 & 0.0440 & 0.0052 & 0.0076 & $11.8 \%$ & $17.3 \%$ \\
\hline 2 & EM07-Si-37 & $\mathrm{ZnO}$ & 0.0688 & 0.0688 & 0.0670 & 0.0018 & 0.0018 & $2.6 \%$ & $2.6 \%$ \\
\hline 2 & EM07-Si-37 & $\mathrm{ZrO} 2$ & 1.2755 & 1.2755 & 2.7790 & -1.5035 & -1.5035 & $-54.1 \%$ & $-54.1 \%$ \\
\hline 2 & EM07-Si-37 & Sum & 94.6494 & 98.1125 & 99.2550 & -4.6056 & -1.1425 & $-4.6 \%$ & $-1.2 \%$ \\
\hline 2 & EM07-Si-50 & $\mathrm{Al} 2 \mathrm{O} 3$ & 8.6067 & 8.8859 & 8.8230 & -0.2163 & 0.0629 & $-2.5 \%$ & $0.7 \%$ \\
\hline 2 & EM07-Si-50 & $\mathrm{B} 2 \mathrm{O} 3$ & 8.9191 & 8.8968 & 8.8230 & 0.0961 & 0.0738 & $1.1 \%$ & $0.8 \%$ \\
\hline 2 & EM07-Si-50 & $\mathrm{BaO}$ & 0.0399 & 0.0429 & 0.0440 & -0.0041 & -0.0011 & $-9.3 \%$ & $-2.6 \%$ \\
\hline 2 & EM07-Si-50 & $\mathrm{Bi} 2 \mathrm{O} 3$ & 0.0056 & 0.0056 & 0.0000 & 0.0056 & 0.0056 & & \\
\hline 2 & EM07-Si-50 & $\mathrm{CaO}$ & 0.0108 & 0.0113 & 0.0000 & 0.0108 & 0.0113 & & \\
\hline 2 & EM07-Si-50 & $\mathrm{CdO}$ & 0.1114 & 0.1114 & 0.1240 & -0.0126 & -0.0126 & $-10.2 \%$ & $-10.2 \%$ \\
\hline 2 & EM07-Si-50 & $\mathrm{Ce} 2 \mathrm{O} 3$ & 0.0448 & 0.0448 & 0.0440 & 0.0008 & 0.0008 & $1.8 \%$ & $1.8 \%$ \\
\hline 2 & EM07-Si-50 & $\mathrm{Cr} 2 \mathrm{O} 3$ & 0.4261 & 0.4140 & 0.4410 & -0.0149 & -0.0270 & $-3.4 \%$ & $-6.1 \%$ \\
\hline 2 & EM07-Si-50 & $\mathrm{Fe} 2 \mathrm{O} 3$ & 8.3280 & 8.5270 & 8.8230 & -0.4950 & -0.2960 & $-5.6 \%$ & $-3.4 \%$ \\
\hline 2 & EM07-Si-50 & $\mathrm{K} 2 \mathrm{O}$ & 0.0602 & 0.0651 & 0.0000 & 0.0602 & 0.0651 & & \\
\hline 2 & EM07-Si-50 & La2O3 & 0.0522 & 0.0522 & 0.0620 & -0.0098 & -0.0098 & $-15.8 \%$ & $-15.8 \%$ \\
\hline 2 & EM07-Si-50 & $\mathrm{Li} 2 \mathrm{O}$ & 2.3413 & 2.3476 & 2.4260 & -0.0847 & -0.0784 & $-3.5 \%$ & $-3.2 \%$ \\
\hline 2 & EM07-Si-50 & $\mathrm{MgO}$ & 0.1148 & 0.1210 & 0.1320 & -0.0172 & -0.0110 & $-13.0 \%$ & $-8.3 \%$ \\
\hline 2 & EM07-Si-50 & $\mathrm{MnO}$ & 1.7205 & 1.7400 & 1.7650 & -0.0445 & -0.0250 & $-2.5 \%$ & $-1.4 \%$ \\
\hline
\end{tabular}


Table A5. Average Measured and Bias-Corrected Chemical Compositions Versus Targeted Compositions for the Test Matrix 1 Study Glasses

\begin{tabular}{||c|c|c|c|c|c|c|c|c|c||}
\hline \hline & & & & Measured & & & & & \\
\hline & & & Measured & Bias-Corrected & Targeted & Diff of & Diff of & \% Diff of & $\%$ Diff of \\
\hline Set & Glass ID & Oxide & $($ wt\%) & $($ wt $\%)$ & wt\%) & Measured & Meas BC & Measured & Meas BC \\
\hline 2 & EM07-Si-50 & Na2O & 13.4025 & 13.4298 & 13.2340 & 0.1685 & 0.1958 & $1.3 \%$ & $1.5 \%$ \\
\hline 2 & EM07-Si-50 & Nd2O3 & 0.0560 & 0.0590 & 0.0620 & -0.0060 & -0.0030 & $-9.7 \%$ & $-4.9 \%$ \\
\hline 2 & EM07-Si-50 & NiO & 0.8087 & 0.8849 & 0.8820 & -0.0733 & 0.0029 & $-8.3 \%$ & $0.3 \%$ \\
\hline 2 & EM07-Si-50 & P2O5 & 0.9286 & 0.9286 & 1.1030 & -0.1744 & -0.1744 & $-15.8 \%$ & $-15.8 \%$ \\
\hline 2 & EM07-Si-50 & PbO & 0.2914 & 0.2914 & 0.3260 & -0.0346 & -0.0346 & $-10.6 \%$ & $-10.6 \%$ \\
\hline 2 & EM07-Si-50 & SiO2 & 47.1716 & 50.7188 & 50.0000 & -2.8284 & 0.7188 & $-5.7 \%$ & $1.4 \%$ \\
\hline 2 & EM07-Si-50 & TiO2 & 0.0430 & 0.0450 & 0.0350 & 0.0080 & 0.0100 & $22.7 \%$ & $28.7 \%$ \\
\hline 2 & EM07-Si-50 & ZnO & 0.0532 & 0.0532 & 0.0530 & 0.0002 & 0.0002 & $0.4 \%$ & $0.4 \%$ \\
\hline 2 & EM07-Si-50 & ZrO2 & 1.9283 & 1.9283 & 2.2060 & -0.2777 & -0.2777 & $-12.6 \%$ & $-12.6 \%$ \\
\hline 2 & EM07-Si-50 & Sum & 95.4645 & 99.6046 & 99.4080 & -3.9435 & 0.1966 & $-4.0 \%$ & $0.2 \%$ \\
\hline
\end{tabular}




\section{Figure A1. Measurements in Analytical Sequence by Analyte Grouped by Preparation Method}

Measured By Analytical Sequence Prep=KH, Analyte=F (wt\%)

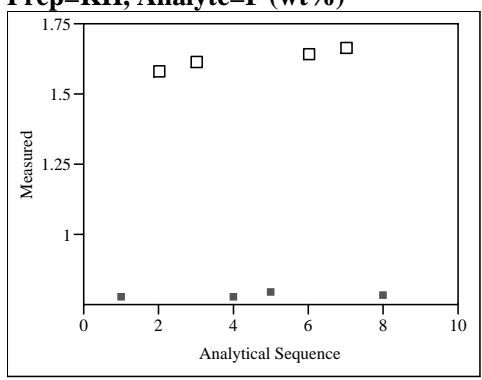

Measured By Analytical Sequence Prep=LM, Analyte=AI2O3 (wt\%)

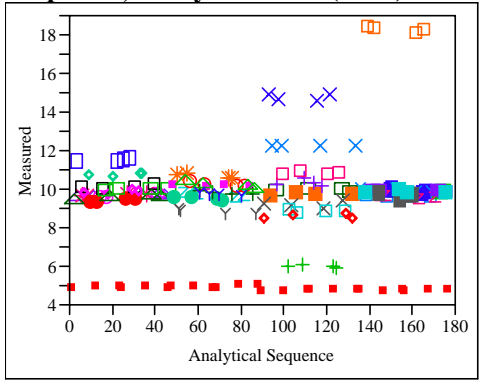

Measured By Analytical Sequence

Prep $=$ LM, Analyte $=$ BaO (wt \%)

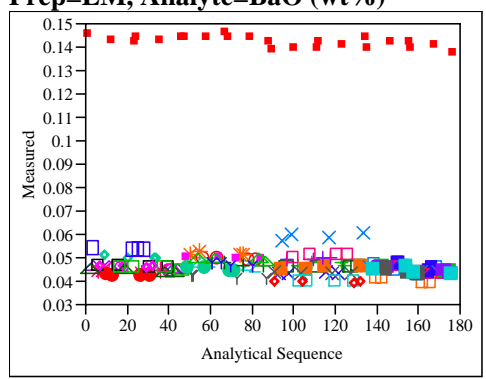

Measured By Analytical Sequence Prep $=$ LM, Analyte $=$ Bi2O3 (wt\%)

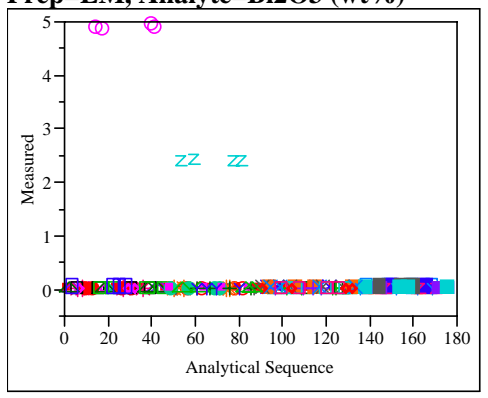

Measured By Analytical Sequence Prep=LM, Analyte $=\mathrm{CaO}(\mathrm{wt} \%)$

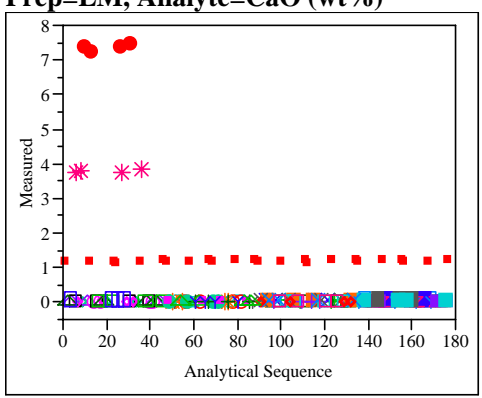

Measured By Analytical Sequence Prep=LM, Analyte=CdO (wt\%)

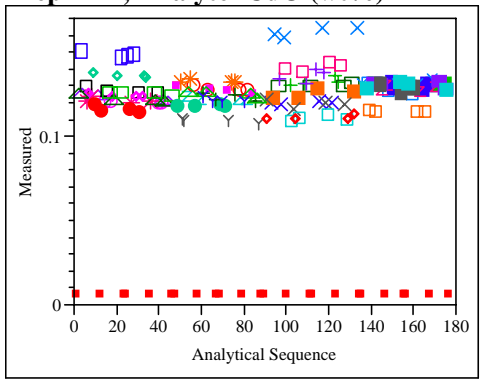

Measured By Analytical Sequence Prep=LM, Analyte=Ce2O3 (wt\%)

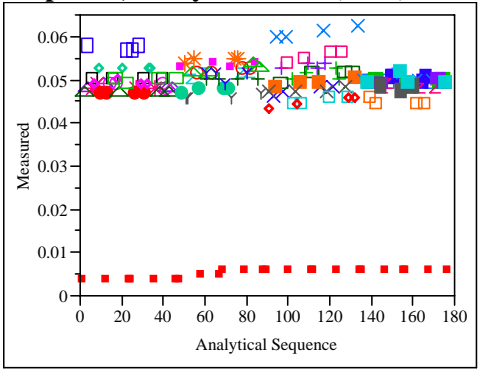

Measured By Analytical Sequence Prep=LM, Analyte=Cr2O3 (wt \%)

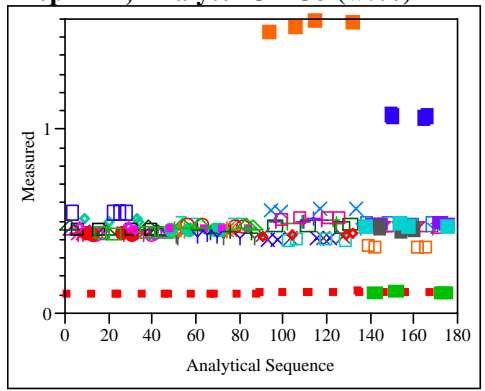

Measured By Analytical Sequence Prep=LM, Analyte=Fe2O3 (wt\%)

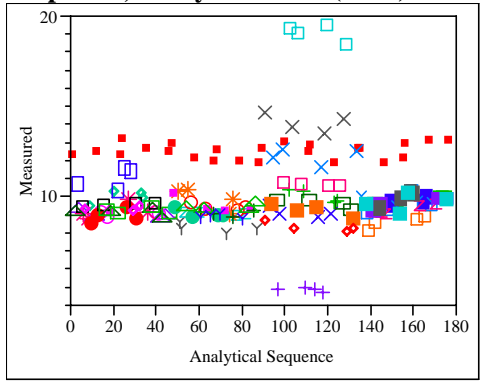

Measured By Analytical Sequence Prep=LM, Analyte=K2O (wt\%)

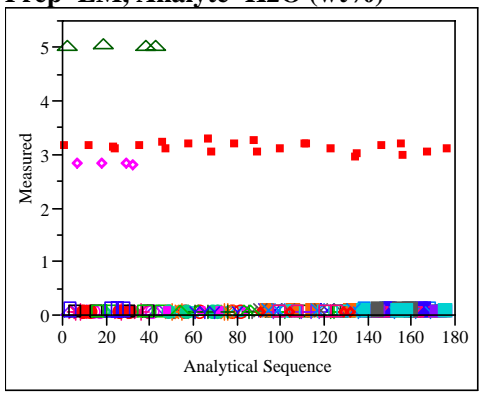

Measured By Analytical Sequence Prep=LM, Analyte=La2O3 (wt\%)

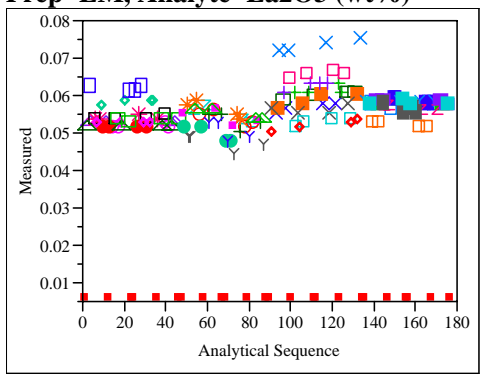

Measured By Analytical Sequence Prep $=$ LM, Analyte $=$ MgO (wt\%)

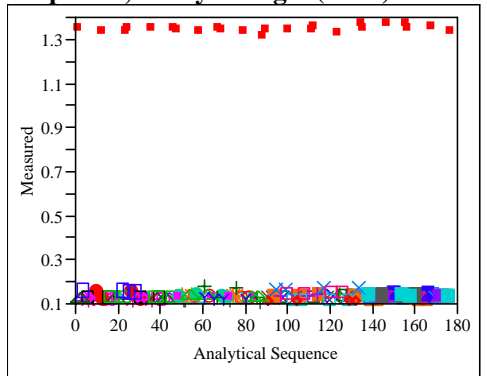




\section{Figure A1. Measurements in Analytical Sequence by Analyte} Grouped by Preparation Method

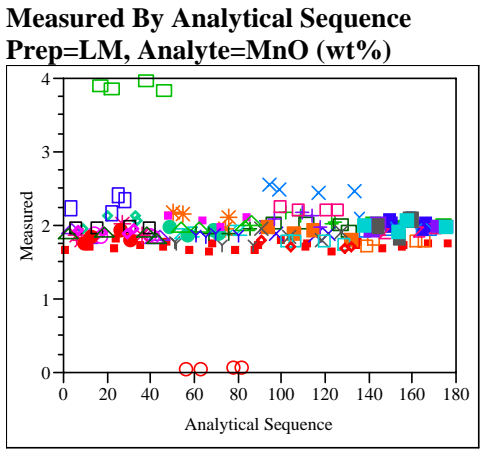

Measured By Analytical Sequence Prep $=$ LM, Analyte $=\mathrm{Na} 2 \mathrm{O}(\mathrm{wt} \%)$

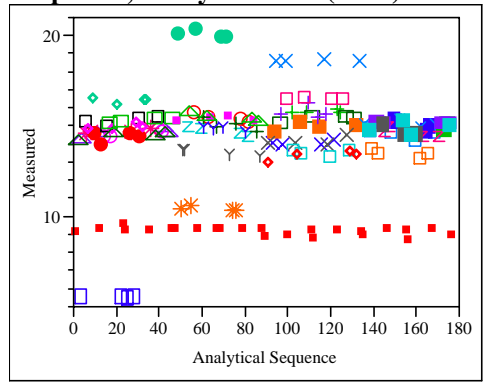

Measured By Analytical Sequence Prep=LM, Analyte=Nd2O3 (wt\%)

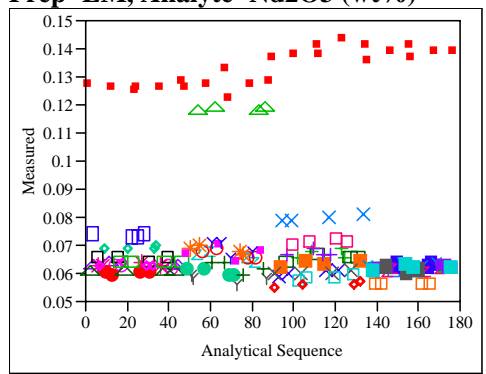

Measured By Analytical Sequence Prep $=$ LM, Analyte $=\mathrm{NiO}$ (wt\%)

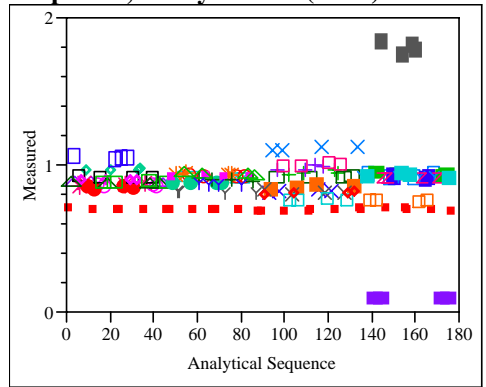

Measured By Analytical Sequence Prep $=$ LM, Analyte $=$ P2O5 (wt \%)

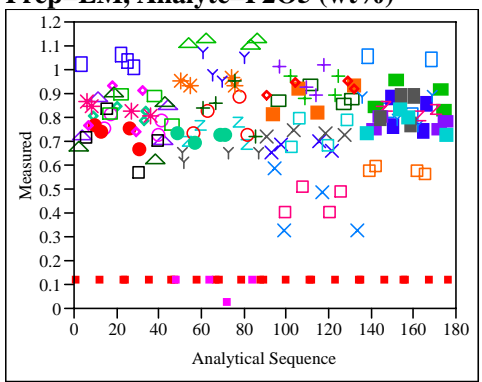

Measured By Analytical Sequence Prep $=$ LM, Analyte $=\mathbf{P b O}$ (wt\%)

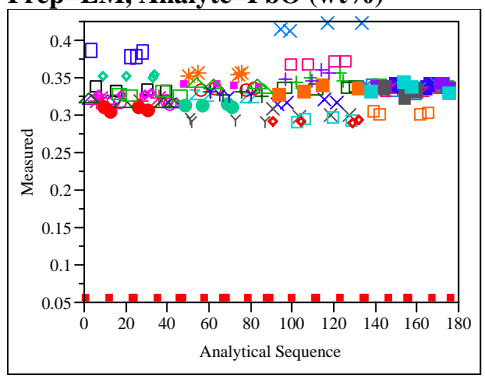

Measured By Analytical Sequence Prep=LM, Analyte=TiO2 (wt\%)

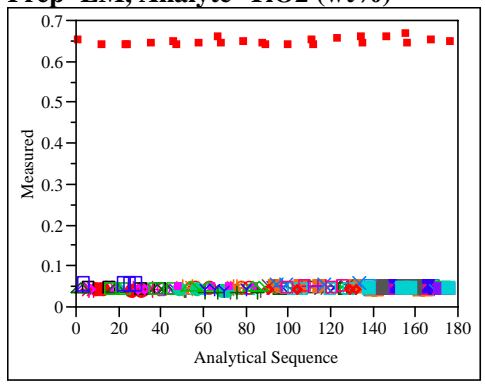

Measured By Analytical Sequence Prep $=\mathrm{LM}$, Analyte $=\mathrm{ZnO}($ wt $\%)$

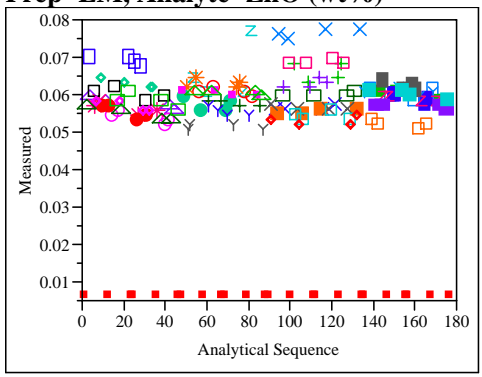

Measured By Analytical Sequence Prep=LM, Analyte $=\mathrm{ZrO} 2$ (wt\%)

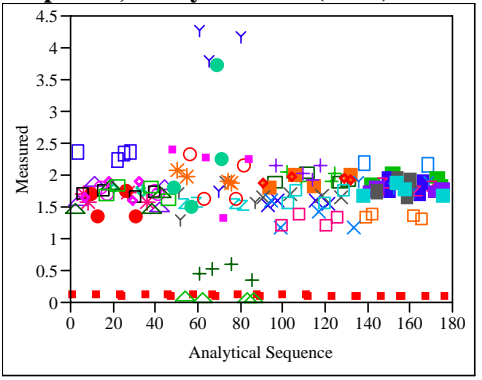

Measured By Analytical Sequence Prep=PF, Analyte $=$ B2O3 (wt \%)

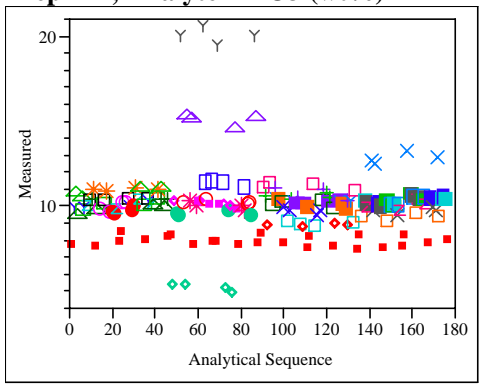

Measured By Analytical Sequence Prep=PF, Analyte $=\mathrm{Li} 2 O(w \mathrm{t} \%)$

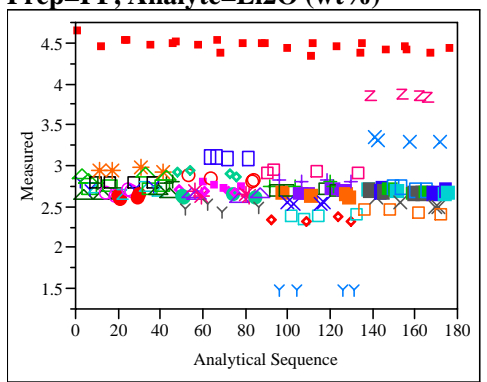

Measured By Analytical Sequence Prep=PF, Analyte $=\mathrm{SiO} 2$ (wt \%)

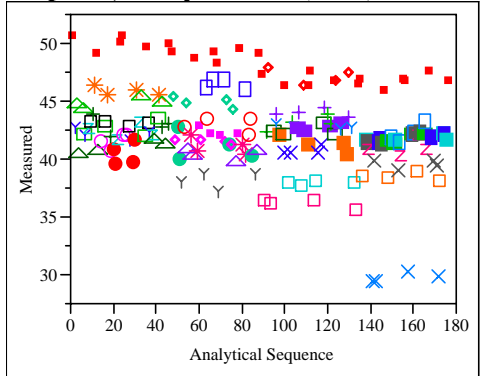


Figure A2. PSAL Measurements for Each Analyte by Analytical Block for Samples of the Batch 1 and LRM Standards Grouped by Preparation Method

Oneway Analysis of Measured By Set/Block/Sub-Block Glass ID=Batch 1, Prep=LM, Analyte=Al2O3 (wt \%)

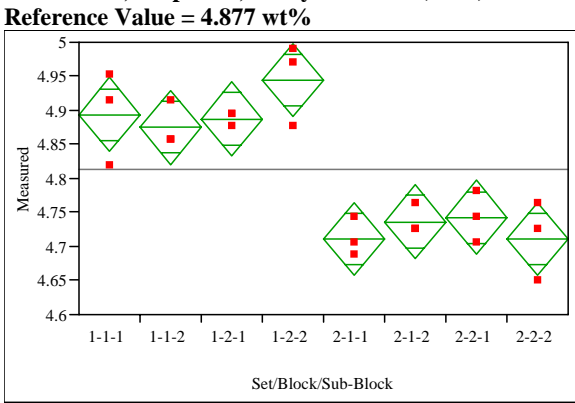

Oneway Anova
Summary of Fit

$\begin{array}{lr}\text { Rsquare } & 0.861586 \\ \text { Adj Rsquare } & 0.80103 \\ \text { Root Mean Square Error } & 0.044145 \\ \text { Mean of Response } & 4.812714 \\ \text { Observations (or Sum Wgts) } & 24\end{array}$

Observations (or Sum Wgts) 4.812714

$\begin{array}{lrrrrr}\text { Analysis of Variance } & & & & \\ \text { Source } & \text { DF } & \text { Sum of Squares } & \text { Mean Square } & \text { F Ratio } & \text { Prob }>\text { F } \\ \text { Set/Block/Sub-Block } & 7 & 0.19408555 & 0.027727 & 14.2279 & <.0001 \\ \text { Error } & 16 & 0.03117984 & 0.001949 & & \\ \text { C. Total } & 23 & 0.22526539 & & & \end{array}$

Means for Oneway Anova

Level Number Mean Std Error Lower 95\% Upper 95\%

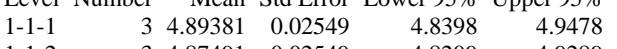

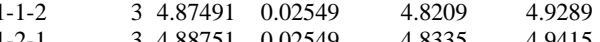

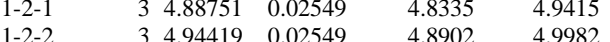

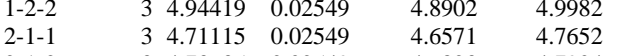

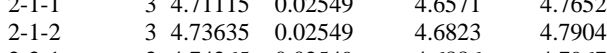

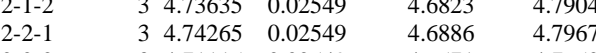

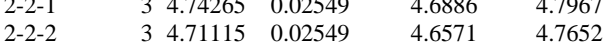

Std Error uses a pooled estimate of error variance
Oneway Analysis of Measured By Set/Block/Sub-Block Glass ID=Batch 1, Prep=LM, Analyte=BaO (wt \%)

Reference Value $=0.151 \mathrm{wt} \%$

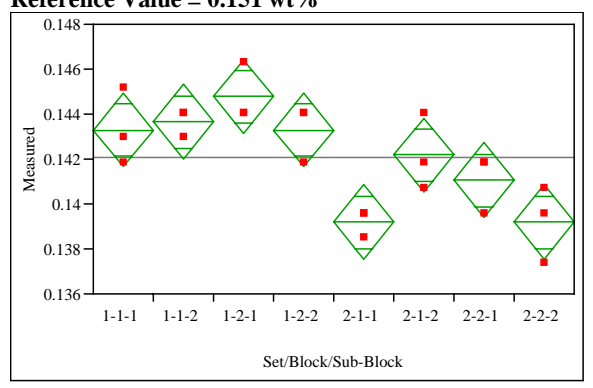

\section{Oneway Anova}

Summary of Fit

$\begin{array}{lr}\text { Rsquare } & 0.758204 \\ \text { Adj Rsquare } & 0.652418 \\ \text { Root Mean Square Error } & 0.001348 \\ \text { Mean of Response } & 0.142075 \\ \text { Observations (or Sum Wgts) } & 24\end{array}$

Analysis of Variance $\begin{array}{lrrrrr}\text { Analysis of Variance } & & & & \\ \text { Source } & \text { DF } & \text { Sum of Squares } & \text { Mean Square } & \text { F Ratio } & \text { Prob > F } \\ \text { Set/Block/Sub-Block } & 7 & 0.00009121 & 0.000013 & 7.1673 & 0.0006 \\ \text { Error } & 16 & 0.00002909 & 1.818 \mathrm{e}-6 & & \\ \text { C. Total } & 23 & 0.00012029 & & & \end{array}$ 0.00012029

\section{Means for Oneway Anova}

Level Number Mean Std Error Lower 95\% Upper 95\%

$\begin{array}{lllllll}1-1-1 & & 3 & 0.143284 & 0.00078 & 0.14163 & 0.14493\end{array}$

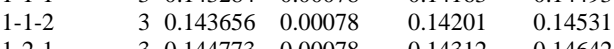

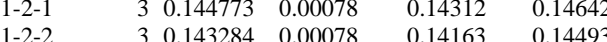

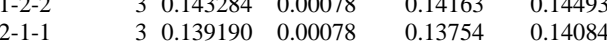

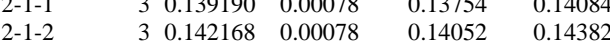

$\begin{array}{lllllll}2-1-2 & & 3 & 0.142168 & 0.00078 & 0.14052 & 0.14382 \\ 2-2-1 & & 3 & 0.141051 & 0.00078 & 0.13940 & 0.14270\end{array}$

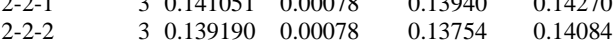

Std Error uses a pooled estimate of error variance
Oneway Analysis of Measured By Set/Block/Sub-Block Glass ID=Batch 1, Prep=LM, Analyte=Bi2O3 (wt\%) Reference Value $=0.0 \mathrm{w} \%$

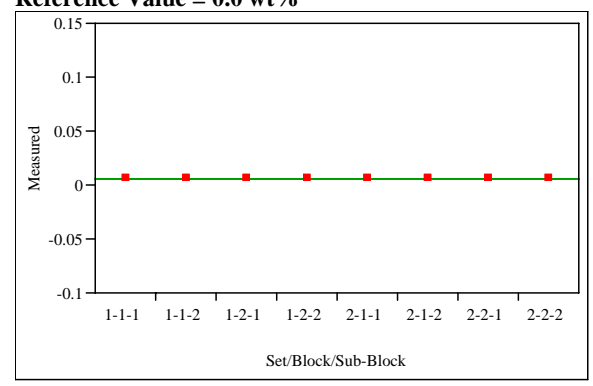

Oneway Anova

Summary of Fit

Rsquare

Adj Rsquare

$\begin{array}{lr}\text { Root Mean Square Error } & 0 \\ \text { Mean of Response } & 0.005574\end{array}$

Observations (or Sum Wgts) $\quad 24$

Analysis of Variance

Source DF Sum of Squares Mean Square F Ratio Prob > F $\begin{array}{lrrr}\text { Set/Block/Sub-Block } & 7 & 0 & 0 \\ \text { Error } & 16 & 0 & 0\end{array}$

Means for Oneway Anova

Level Number Mean Std Error Lower 95\% Upper 95\%

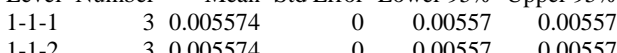

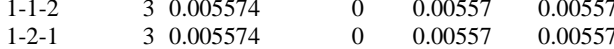

$\begin{array}{llllll}1-2-1 & 3 & 0.005574 & 0 & 0.00557 & 0.00557 \\ 1-2-2 & 3 & 0.005574 & 0 & 0.00557 & 0.00557\end{array}$

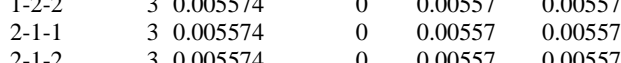

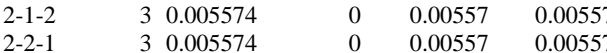

$\begin{array}{lllllll}2-2-1 & & 3 & 0.005574 & 0 & 0.00557 & 0.00557 \\ 2-2-2 & & 3 & 0.005574 & 0 & 0.00557 & 0.00557\end{array}$

Std Error uses a pooled estimate of error variance 
Figure A2. PSAL Measurements for Each Analyte by Analytical Block for Samples of the Batch 1 and LRM Standards Grouped by Preparation Method

Oneway Analysis of Measured By Set/Block/Sub-Block Glass ID=Batch 1, Prep=LM, Analyte=CaO $(w t \%)$

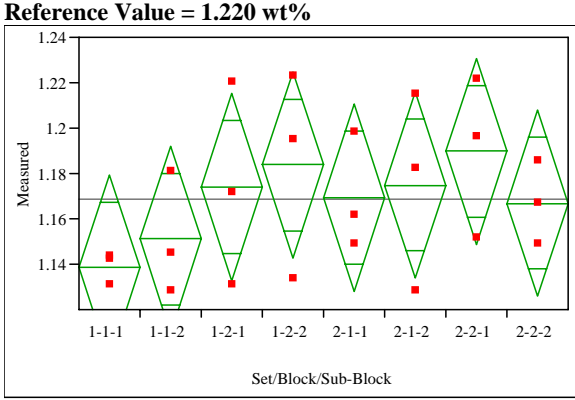

Oneway Anova

Summary of Fit

$\begin{array}{lr}\text { Rsquare } & 0.245401 \\ \text { Adj Rsquare } & -0.08474 \\ \text { Root Mean Square Error } & 0.033638 \\ \text { Mean of Response } & 1.168507 \\ \text { Observations (or Sum Wgts) } & 24\end{array}$

Observations (or Sum Wgts)

$\begin{array}{lrrrrr}\text { Analysis of Variance } & & & & \\ \text { Source } & \text { DF } & \text { Sum of Squares } & \text { Mean Square } & \text { F Ratio } & \text { Prob }>\text { F } \\ \text { Set/Block/Sub-Block } & 7 & 0.00588756 & 0.000841 & 0.7433 & 0.6400 \\ \text { Error } & 16 & 0.01810407 & 0.001132 & & \\ \text { C. Total } & 23 & 0.02399162 & & & \end{array}$

Means for Oneway Anova

Level Number Mean Std Error Lower 95\% Upper 95\%

$\begin{array}{llllll}1-1-1 & 3 & 1.13848 & 0.01942 & 1.0973 & 1.1797\end{array}$

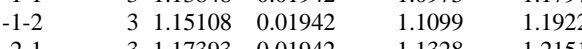

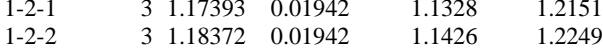

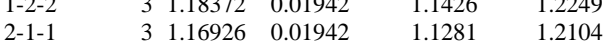

$\begin{array}{llllll}2-1-2 & 3 & 1.17486 & 0.01942 & 1.1337 & 1.2160\end{array}$

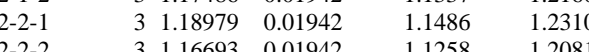

Std Error uses a pooled estimate of error variance
Oneway Analysis of Measured By Set/Block/Sub-Block Glass ID=Batch 1, Prep=LM, Analyte=CdO $(w t \%)$ Reference Value $=0.0 \mathrm{wt} \%$

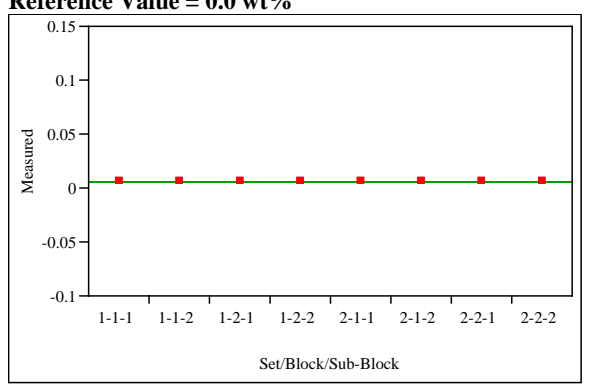

\section{Oneway Anova}

Summary of Fit

\section{Rsquare}

Adj Rsquare

Root Mean Square Error

Mean of Response

0
0.005712

Observations (or Sum Wgts) $\quad 24$

Analysis of Variance

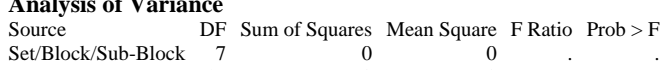

Error $\quad 16$

Means for Oneway Anova

Level Number Mean Std Error Lower 95\% Upper 95\%

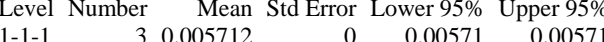

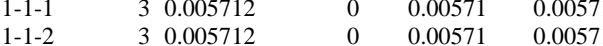

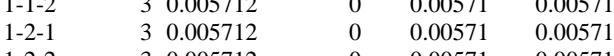

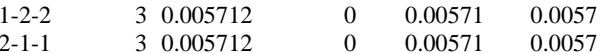

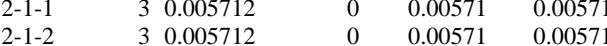

$\begin{array}{llllll}2-1-2 & 3 & 0.005712 & 0 & 0.00571 & 0.00571 \\ 2-2-1 & 3 & 0.005712 & 0 & 0.00571 & 0.00571\end{array}$

$\begin{array}{llllll}2-2-1 & 3 & 0.005712 & 0 & 0.00571 & 0.00571 \\ 2-2-2 & 3 & 0.005712 & 0 & 0.00571 & 0.00571\end{array}$

Std Error uses a pooled estimate of error variance
Oneway Analysis of Measured By Set/Block/Sub-Block Glass ID=Batch 1, Prep=LM, Analyte $=\mathrm{Ce} 2 \mathrm{O} 3(\mathrm{wt} \%)$

$$
\text { Reference Value }=0.0 \mathrm{wt} \%
$$

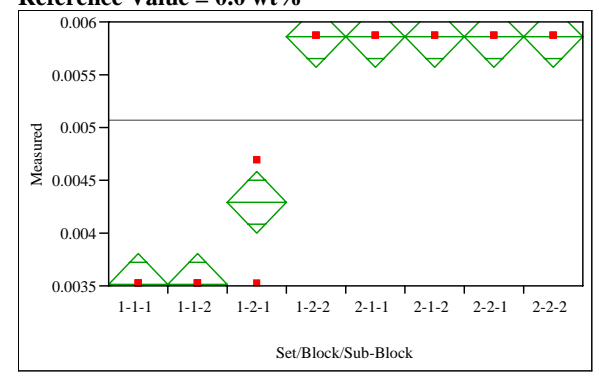

Oneway Anova

Summary of Fit

$\begin{array}{lr}\text { Rsquare } & 0.965517 \\ \text { Adj Rsquare } & 0.950431 \\ \text { Root Mean Square Error } & 0.000239 \\ \text { Mean of Response } & 0.005076 \\ \text { Observations (or Sum Wgts) } & 24\end{array}$

Observations (or Sum Wgts)

Analysis of Variance

Source DF Sum of Squares Mean Square F Ratio Prob > F $\begin{array}{lrllll}\text { Set/Block/Sub-Block } & 7 & 0.00002561 & 3.6585 \mathrm{e}-6 & 64.0000 & <.000\end{array}$ C. Total $\begin{array}{ll}16 & 0.00000091 \\ 23 & 0.00002652\end{array}$

Means for Oneway Anova

Level Number Mean Std Error Lower 95\% Upper 95\% $\begin{array}{llllll}1-1-1 & 3 & 0.003514 & 0.00014 & 0.00322 & 0.00381\end{array}$ $\begin{array}{llllll}1-1-2 & 3 & 0.003514 & 0.00014 & 0.00322 & 0.00381\end{array}$ $\begin{array}{llllll}1-2-1 & 3 & 0.004295 & 0.00014 & 0.00400 & 0.00459\end{array}$ $\begin{array}{llllll}1-2-2 & 3 & 0.005857 & 0.00014 & 0.00556 & 0.00615\end{array}$

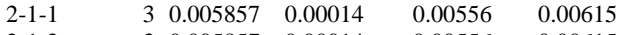
$\begin{array}{llllll}2-1-2 & 3 & 0.005857 & 0.00014 & 0.00556 & 0.00615\end{array}$ $\begin{array}{lllllll}2-2-1 & & 3 & 0.005857 & 0.00014 & 0.00556 & 0.00615\end{array}$ $\begin{array}{llllll}2-2-2 & 3 & 0.005857 & 0.00014 & 0.00556 & 0.00615\end{array}$

Std Error uses a pooled estimate of error variance 
Figure A2. PSAL Measurements for Each Analyte by Analytical Block for Samples of the Batch 1 and LRM Standards Grouped by Preparation Method

Oneway Analysis of Measured By Set/Block/Sub-Block Glass ID=Batch 1, Prep=LM, Analyte=Cr2O3 (wt \%)

$$
\text { Reference Value = } 0.107 \text { wt\% }
$$

\section{Oneway Anova}

Summary of Fit

$\begin{array}{lr}\text { Rsquare } & 0.977299 \\ \text { Adj Rsquare } & 0.967367 \\ \text { Root Mean Square Error } & 0.001155 \\ \text { Mean of Response } & 0.103408 \\ \text { Observations (or Sum Wgts) } & 24\end{array}$

\section{Analysis of Variance}

Source DF Sum of Squares Mean Square F Ratio Prob > F $\begin{array}{lrrrrr}\text { Set/Block/Sub-Block } & 7 & 0.00091967 & 0.000131 & 98.4000 & <.000\end{array}$ Error
C. Total $16 \quad 0.00002136$

Means for Oneway Anova

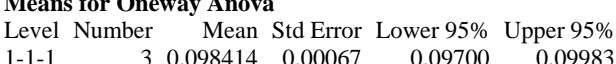

$\begin{array}{lllllll}1-1-1 & & 3 & 0.098414 & 0.00067 & 0.09700 & 0.09983 \\ 1-1-2 & & 3 & 0.097927 & 0.00067 & 0.09651 & 0.09934\end{array}$

$\begin{array}{lllllll}1-1-2 & & 3 & 0.097927 & 0.00067 & 0.09651 & 0.09934 \\ 1-2-1 & & 3 & 0.096953 & 0.00067 & 0.09554 & 0.09837 \\ 1 & & 3 & 0.095978 & 0.00067 & 0.0945 & 0.09739\end{array}$

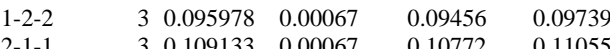

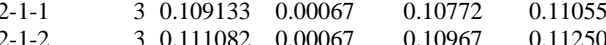

$\begin{array}{lllllll}2-1-2 & & 3 & 0.111082 & 0.00067 & 0.10967 & 0.11250 \\ 2-2-1 & & 3 & 0.110107 & 0.00067 & 0.10869 & 0.11152\end{array}$

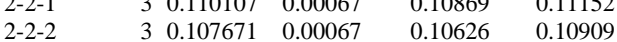

Std Error uses a pooled estimate of error variance
Oneway Analysis of Measured By Set/Block/Sub-Block Glass ID=Batch 1, Prep=LM, Analyte=Fe2O3 (wt \%)

Reference Value $=12.839 \mathrm{wt} \%$

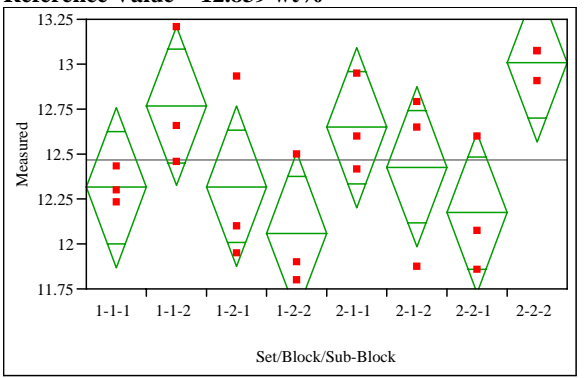

Oneway Anova

Summary of Fit

$\begin{array}{lr}\text { Rsquare } & 0.504867 \\ \text { Adj Rsquare } & 0.288247 \\ \text { Root Mean Square Error } & 0.362852 \\ \text { Mean of Response } & 12.4652 \\ \text { Observations (or Sum Wgts) } & 24\end{array}$

Observations (or Sum Wgts) $\quad \begin{array}{r}12.4652 \\ 24\end{array}$

Analysis of Variance

Source DF Sum of Squares Mean Square F Ratio Prob > F

$\begin{array}{lrrrrr}\text { Set/Block/Sub-Block } & 7 & 2.1480072 & 0.306858 & 2.3307 & 0.0764\end{array}$

C. Total

2.1065898
4.2545970

Means for Oneway Anova

Level Number Mean Std Error Lower 95\% Upper 95\%

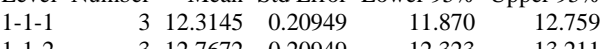

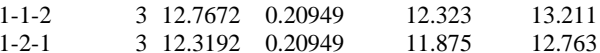

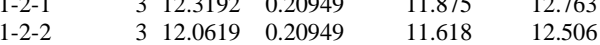

$\begin{array}{lllllll}1-2-2 & & 3 & 12.0619 & 0.20949 & 11.618 & 12.506 \\ 2-1-1 & & 3 & 12.6481 & 0.20949 & 12.204 & 13.092 \\ 2-1-2 & & 3 & 12.4289 & 0.20949 & 11.985 & 12.873\end{array}$

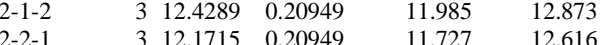

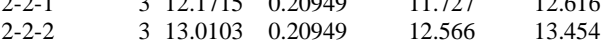

Std Error uses a pooled estimate of error variance
Oneway Analysis of Measured By Set/Block/Sub-Block Glass ID=Batch 1, Prep=LM, Analyte=K2O (wt\%)

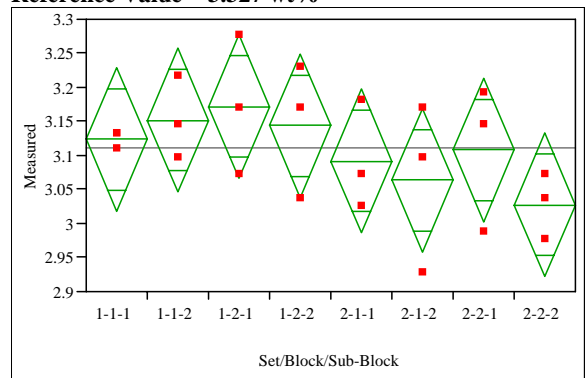

\section{Oneway Anova}

$\begin{array}{lr}\text { Rsquare } & 0.29013 \\ \text { Adj Rsquare } & -0.02044 \\ \text { Root Mean Square Error } & 0.086341 \\ \text { Mean of Response } & 3.110378 \\ \text { Observations (or Sum Wgts) } & 24\end{array}$

Observations (or Sum Wgts) 3.110378

Analysis of Variance

Source $/$ DF Sum of Squares Mean Square F Ratio Prob > F $\begin{array}{lrrrrr}\text { Set/Block/Sub-Block } & 7 & 0.04874961 & 0.006964 & 0.9342 & 0.5074\end{array}$ Error $\begin{array}{ll}16 & 0.11927723 \\ 23 & 0.16802684\end{array}$

0.007455

Means for Oneway Anova

Level Number Mean Std Error Lower 95\% Upper 95\% $\begin{array}{rrrrrr}1-1-1 & 3 & 3.12393 & 0.04985 & 3.0183 & 3.2296\end{array}$

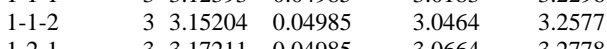

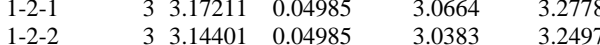

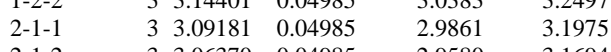

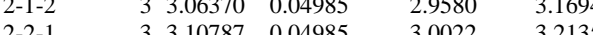

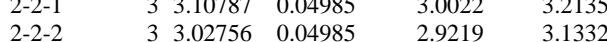

Std Error uses a pooled estimate of error variance 
Figure A2. PSAL Measurements for Each Analyte by Analytical Block for Samples of the Batch 1 and LRM Standards Grouped by Preparation Method

Oneway Analysis of Measured By Set/Block/Sub-Block Glass ID=Batch 1, Prep=LM, Analyte $=$ La2O3 $(w t \%)$

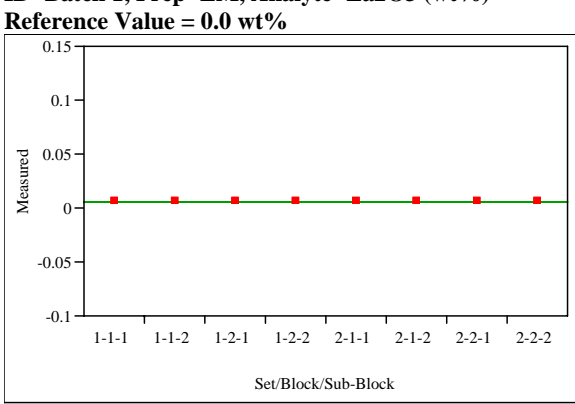

Oneway Anova

Summary of Fit

Rsquare

Adj Rsquare

Root Mean Square Erro

Mean of Response

0.005864

Observations (or Sum Wgts) $\quad 24$

Analysis of Variance

Source DF Sum of Squares Mean Square F Ratio Prob > F

$\begin{array}{lr}\text { Set/Block/Sub-Block } & 7 \\ \text { Error } & 16\end{array}$

C. Tota

16
23

Means for Oneway Anova

Level Number Mean Std Error Lower 95\% Upper 95\%

$\begin{array}{llllll}1-1-1 & 3 & 0.005864 & 0 & 0.00586 & 0.00586\end{array}$

$\begin{array}{llllll}1-1-2 & 3 & 0.005864 & 0 & 0.00586 & 0.00586\end{array}$

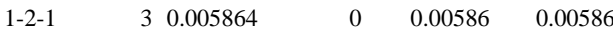

$\begin{array}{llllll}1-2-2 & 3 & 0.005864 & 0 & 0.00586 & 0.00586\end{array}$

$\begin{array}{llllll}2-1-1 & 3 & 0.005864 & 0 & 0.00586 & 0.00586\end{array}$

$\begin{array}{llllll}2-1-2 & 3 & 0.005864 & 0 & 0.00586 & 0.00586\end{array}$

$\begin{array}{llllll}2-2-1 & 3 & 0.005864 & 0 & 0.00586 & 0.00586\end{array}$

$\begin{array}{llllll}2-2-2 & 3 & 0.005864 & 0 & 0.00586 & 0.00586\end{array}$

Std Error uses a pooled estimate of error variance
Oneway Analysis of Measured By Set/Block/Sub-Block Glass ID=Batch 1, Prep=LM, Analyte=MgO (wt\%)

Reference Value $=1.419 \mathrm{wt} \%$

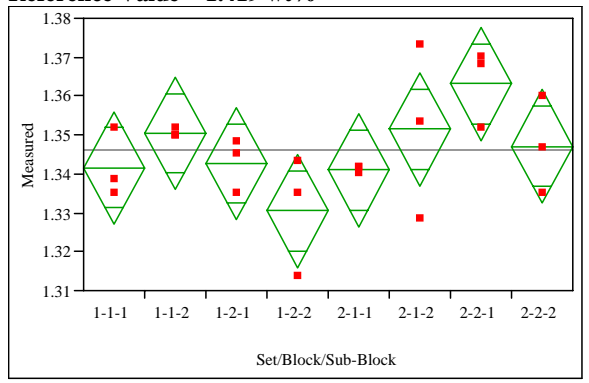

Oneway Anova

Summary of Fit

$\begin{array}{lr}\text { Rsquare } & 0.460625 \\ \text { Adj Rsquare } & 0.224648 \\ \text { Root Mean Square Error } & 0.011852 \\ \text { Mean of Response } & 1.345987 \\ \text { Observations (or Sum Wgts) } & 24\end{array}$

Observations (or Sum Wgts) 1.345987

Analysis of Variance

$\begin{array}{llrrrr}\text { Source } & \text { DF } & \text { Sum of Squares } & \text { Mean Square } & \text { F Ratio } & \text { Prob > F } \\ \text { Set/Block/Sub-Block } & 7 & 0.00191947 & 0.000274 & 1.9520 & 0.1269\end{array}$ Set/Bloc

C. Total

$23 \quad 0.00416710$

Means for Oneway Anova

Level Number Mean Std Error Lower 95\% Upper 95\%

$\begin{array}{rrrrrr}1-1-1 & 3 & 1.34156 & 0.00684 & 1.3271 & 1.3561\end{array}$

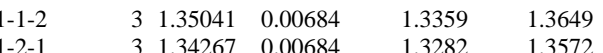

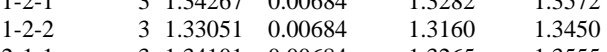

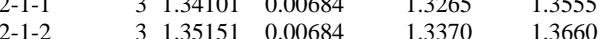

$\begin{array}{llllll}2-2-1 & 3 & 1.36312 & 0.00684 & 1.33786 & 1.3660\end{array}$

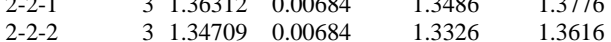

Std Error uses a pooled estimate of error variance
Oneway Analysis of Measured By Set/Block/Sub-Block Glass ID=Batch 1, Prep=LM, Analyte $=\mathrm{MnO}(\mathrm{wt} \%)$

Reference Value $=1.726 \mathrm{wt} \%$

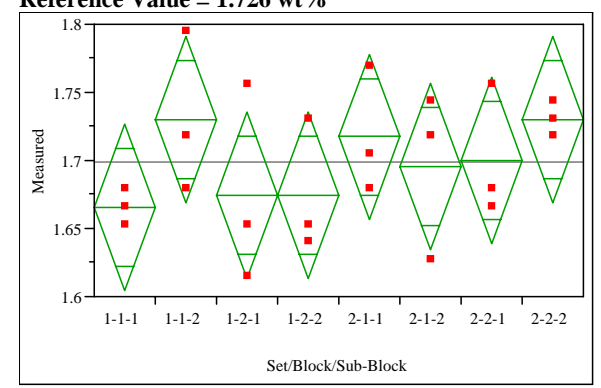

Oneway Anova

Summary of Fit

Adj Rsquare $\quad-0.06561$

Root Mean Square Error $\quad 0.049869$

Mean of Response 1.698466

Observations (or Sum Wgts)

Analysis of Variance

Source DF Sum of Squares Mean Square F Ratio Prob > F $\begin{array}{lrlllll}\text { Set/Block/Sub-Block } & 7 & 0.01388637 & 0.001984 & 0.7977 & 0.6005\end{array}$ Error $\begin{array}{ll}16 & 0.03979045 \\ 23 & 0.05367681\end{array}$

Means for Oneway Anova

Level Number Mean Std Error Lower 95\% Upper 95\% $\begin{array}{rrrrrr}\text { Level Number } & \text { Mean } & \text { Std Error } & & & \\ 1-1-1 & 3 & 1.66565 & 0.02879 & 1.6046 & 1.7267\end{array}$

$\begin{array}{lllllll}1-1-1 & & 3 & 1.66565 & 0.02879 & 1.6046 & 1.7267 \\ 1-1-2 & & 3 & 1.73021 & 0.02879 & 1.6692 & 1.7912 \\ 1-2-1 & & 3 & 1.67426 & 0.02879 & 1.6132 & 1.7353\end{array}$

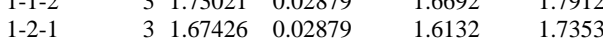

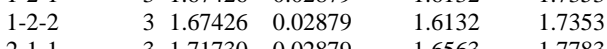

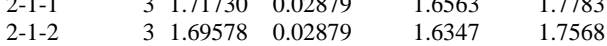

$\begin{array}{llllll}2-2-1 & 3 & 1.70008 & 0.02879 & 1.6390 & 1.7611\end{array}$

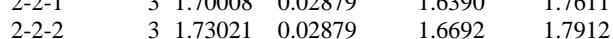

Std Error uses a pooled estimate of error variance 
Figure A2. PSAL Measurements for Each Analyte by Analytical Block for Samples of the Batch 1 and LRM Standards Grouped by Preparation Method

Oneway Analysis of Measured By Set/Block/Sub-Block Glass ID=Batch 1, Prep $=$ LM, Analyte $=$ Na2O $(w t \%)$

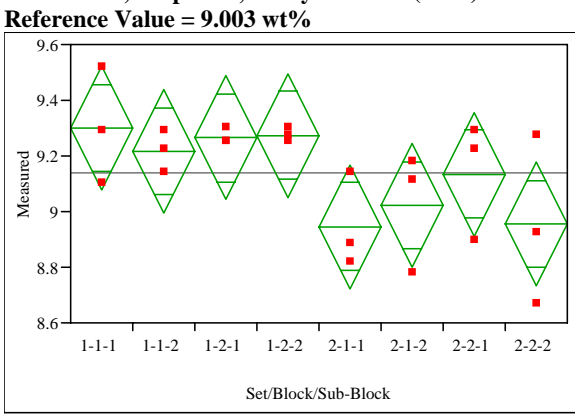

Oneway Anova

Summary of Fit

$\begin{array}{lr}\text { Rsquare } & 0.462166 \\ \text { Adj Rsquare } & 0.226864 \\ \text { Root Mean Square Error } & 0.18148 \\ \text { Mean of Response } & 9.13944 \\ \text { Observations (or Sum Wgts) } & 24\end{array}$

Analysis of Variance

$\begin{array}{lrrrrr}\text { Source } & \text { DF } & \text { Sum of Squares } & \text { Mean Square } & \text { F Ratio } & \text { Prob }>\text { F } \\ \text { Set/Block/Sub-Block } & 7 & 0.45282232 & 0.064689 & 1.9641 & 0.1248\end{array}$

Error

$\begin{array}{ll}16 & 0.52696016 \\ 23 & 0.97978248\end{array}$

Means for Oneway Anova

evel Number Mean Std Error Lower 95\% Upper 95\%

$\begin{array}{llllll}1-1-1 & 3 & 9.30120 & 0.10478 & 9.0791 & 9.5233\end{array}$

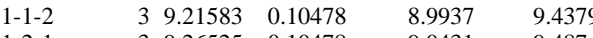

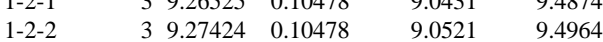

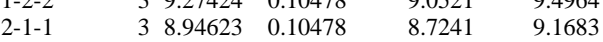

$\begin{array}{llllll}2-1-2 & 3 & 9.02261 & 0.10478 & 8.8005 & 9.2447\end{array}$

$\begin{array}{llllll}2-2-1 & 3 & 9.13495 & 0.10478 & 8.9128 & 9.357\end{array}$

Std Error uses a pooled estimate of error variance
Oneway Analysis of Measured By Set/Block/Sub-Block Glass ID=Batch 1, Prep=LM, Analyte=Nd2O3 (wt\%)

Reference Value $=0.147 \mathrm{wt} \%$

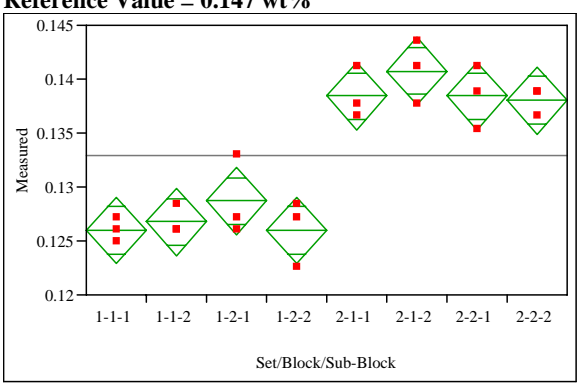

Oneway Anova

Summary of Fit

$\begin{array}{lr}\text { Rsquare } & 0.896996 \\ \text { Adj Rsquare } & 0.851931 \\ \text { Root Mean Square Error } & 0.002542 \\ \text { Mean of Response } & 0.132872 \\ \text { Observations (or Sum Wgts) } & 24\end{array}$

Analysis of Variance

$\begin{array}{lrrrrr}\text { Source } & \text { DF } & \text { Sum of Squares } & \text { Mean Square } & \text { F Ratio } & \text { Prob }>\text { F } \\ \text { Set/Block/Sub-Block } & 7 & 0.00090042 & 0.000129 & 19.9048 & <.0001\end{array}$ $\begin{array}{llll}\text { Error } & 16 & 0.00010340 & 6.462 \mathrm{e}-6 \\ \text { C. Total } & 23 & 0.00100381 & \end{array}$

Means for Oneway Anova

Level Number Mean Std Error Lower 95\% Upper 95\%

$\begin{array}{lrrrrr}\text { Level Number } & \text { Mean } & \text { Std Error } & \text { Lower 95\% } & \text { Uper 95\% } \\ 1-1-1 & 3 & 0.125971 & 0.00147 & 0.12286 & 0.12908 \\ 1-1-2 & 3 & 0.126749 & 0.00147 & 0.12364 & 0.12986 \\ 1-2-1 & 3 & 0.128693 & 0.00147 & 0.12558 & 0.13180\end{array}$

$\begin{array}{lllllll}1-1-2 & & 3 & 0.126749 & 0.00147 & 0.12364 & 0.12986 \\ 1-2-1 & & 3 & 0.128693 & 0.00147 & 0.12558 & 0.13180 \\ 1-2-2 & & 3 & 0.125971 & 0.00147 & 0.12286 & 0.12908\end{array}$

$\begin{array}{lllllll}1-2-1 & & 3 & 0.128693 & 0.00147 & 0.12558 & 0.13180 \\ 1-2-2 & & 3 & 0.125971 & 0.00147 & 0.12286 & 0.12908\end{array}$

$\begin{array}{lllllll}2-1-1 & & 3 & 0.138413 & 0.00147 & 0.13530 & 0.12908 \\ 2-1-2 & & 3 & 0.140746 & 0.00147 & 0.13763 & 0.14386\end{array}$

$\begin{array}{lllllll}2-1-1 & & 3 & 0.138413 & 0.00147 & 0.13530 & 0.14152 \\ 2-1-2 & & 3 & 0.140746 & 0.00147 & 0.13763 & 0.14386\end{array}$

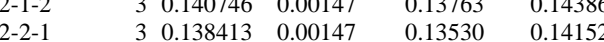

$\begin{array}{lllllll}2-2-2 & & 3 & 0.138024 & 0.00147 & 0.13491 & 0.14114\end{array}$

Std Error uses a pooled estimate of error variance
Oneway Analysis of Measured By Set/Block/Sub-Block Glass ID=Batch 1, Prep $=$ LM, Analyte $=\mathrm{NiO}(w t \%)$$$
\text { SetBlocksub-Block }
$$

Oneway Anova

Summary of Fit

Root Mean Square Error $\quad 0.00538$

$\begin{array}{lr}\text { Mean of Response } & 0.688529 \\ \text { Observations (or Sum Wgts) } & 24\end{array}$

Analysis of Variance

Source DF Sum of Squares Mean Square F Ratio Prob $>$ F $\begin{array}{lrllll}\text { Set/Block/Sub-Block } & 7 & 0.00048551 & 0.000069 & 2.3963 & 0.0700\end{array}$ $\begin{array}{lll}\text { Error } & 16 & 0.00046311 \\ \text { C. Total } & 23 & 0.00094861\end{array}$

Means for Oneway Anova

Level Number Mean Std Error Lower 95\% Upper 95\%

$\begin{array}{lrrrrr}1-1-1 & 3 & 0.690968 & 0.00311 & 0.68438 & 0.69755 \\ 1 & 3 & 0.692240 & 0.00311 & 0.68566 & 0.6982\end{array}$

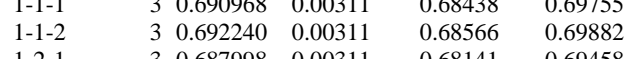

$\begin{array}{lllllll}1-2-1 & & 3 & 0.687998 & 0.00311 & 0.68141 & 0.69458 \\ 1-2-2 & & 3 & 0.682060 & 0.00311 & 0.67548 & 0.68864\end{array}$

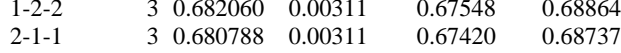

$\begin{array}{lllllll}2-1-1 & & 3 & 0.680788 & 0.00311 & 0.67420 & 0.68737 \\ 2-1-2 & & 3 & 0.691816 & 0.00311 & 0.68523 & 0.69840\end{array}$

$\begin{array}{lllllll}2-2-1 & & 3 & 0.691816 & 0.00311 & 0.68523 & 0.69840 \\ 2-2-1 & & & 0.693937 & 0.00311 & 0.68735 & 0.70052\end{array}$

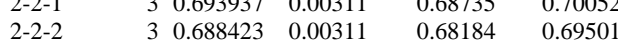

Std Error uses a pooled estimate of error variance 
Figure A2. PSAL Measurements for Each Analyte by Analytical Block for Samples of the Batch 1 and LRM Standards Grouped by Preparation Method

Oneway Analysis of Measured By Set/Block/Sub-Block Glass ID=Batch 1, Prep=LM, Analyte=P2O5 (wt \%)

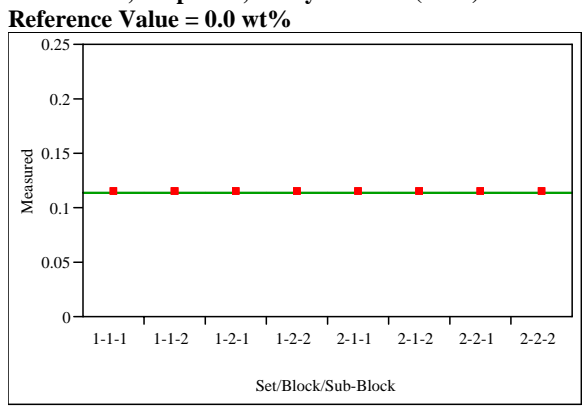

Oneway Anova

Summary of Fit

\section{Rsquare}

Adj Rsquare

Root Mean Square Error

$\begin{array}{lr}\text { Mean of Response } & 0.11457 \\ \text { Observations (or Sum Wgts) } & 24\end{array}$

$\begin{array}{lrrrr}\text { Analysis of Variance } & & & \\ \text { Source } & \text { DF } & \text { Sum of Squares } & \text { Mean Square } & \text { F Ratio Prob > F } \\ \text { Set/Block/Sub-Block } & 7 & 4.6222 \mathrm{e}-33 & 6.603 \mathrm{e}-34 & \\ \text { Error } & 16 & 0 & 0 & \\ \text { C. Total } & 23 & 4.6222 \mathrm{e}-33 & & \end{array}$

Means for Oneway Anova

Level Number Mean Std Error Lower 95\% Upper 95\%

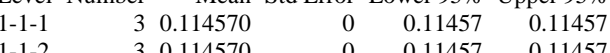

$\begin{array}{llllll}1-1-2 & 3 & 0.114570 & 0 & 0.11457 & 0.11457 \\ 1-2-1 & 3 & 0.114570 & 0 & 0.11457 & 0.11457\end{array}$

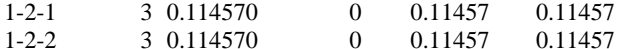

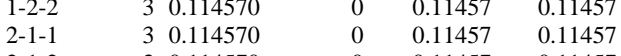

$\begin{array}{llllll}2-1-2 & 3 & 0.114570 & 0 & 0.11457 & 0.11457\end{array}$

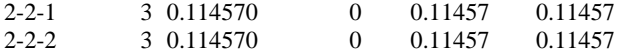

Std Error uses a pooled estimate of error variance
Oneway Analysis of Measured By Set/Block/Sub-Block Glass ID=Batch 1, Prep=LM, Analyte=PbO (wt \%) Reference Value $=0.0 \mathrm{wt} \%$

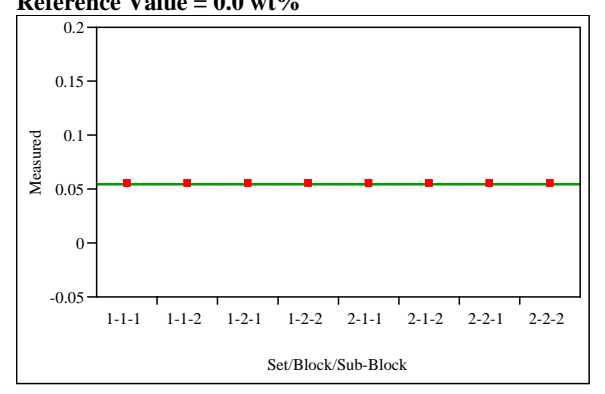

\section{Oneway Anova}

Summary of Fit

\section{Rsquare}

Adj Rsquare

Root Mean Square Error

$\begin{array}{lr}\text { Mean of Response } & 0.05386 \\ \text { Observations (or Sum Wgts) } & 24\end{array}$

Analysis of Variance

Source DF Sum of Squares Mean Square F Ratio Prob > F

$\begin{array}{lrrr}\text { Set/Block/Sub-Block } & 7 & 4.6222 \mathrm{e}-33 & 6.603 \mathrm{e}-34 \\ \text { Error } & 16 & 0 & 0 \\ \text { C. Total } & 23 & 4.6222 \mathrm{e}-33 & \end{array}$

Means for Oneway Anova

Level Number Mean Std Error Lower 95\% Upper 95\%

$\begin{array}{rrrrrr}\text { Level } & \text { Number } & \text { Mean } & \text { Std Error } & \text { Lower 95\% } & \text { Upper 95\% } \\ 1-1-1 & 3 & 0.053860 & 0 & 0.05386 & 0.05386 \\ 1-1-2 & 3 & 0.053860 & 0 & 0.05386 & 0.05386\end{array}$

$\begin{array}{llllll}1-1-2 & 3 & 0.053860 & 0 & 0.05386 & 0.05386 \\ 1-2-1 & 3 & 0.053860 & 0 & 0.05386 & 0.05386\end{array}$

$\begin{array}{llllll}1-2-2 & 3 & 0.053860 & 0 & 0.05386 & 0.05386 \\ 1-2 & & 3 & 0.053860 & 0 & 0.05386\end{array}$

$\begin{array}{llllll}2-1-1 & 3 & 0.053860 & 0 & 0.05386 & 0.05386 \\ 2-1-2 & & 3 & 0.053860 & 0 & 0.05386\end{array}$

$\begin{array}{lllllll}2-1-1 & 3 & 0.053860 & 0 & 0.05386 & 0.05386 \\ 2-1-2 & 3 & 0.053860 & 0 & 0.05386 & 0.05386\end{array}$

$\begin{array}{llllll}2-1-2 & 3 & 0.053860 & 0 & 0.05386 & 0.05386 \\ 2-2-1 & 3 & 0.053860 & 0 & 0.05386 & 0.05386\end{array}$

$\begin{array}{llllll}2-2-1 & 3 & 0.053860 & 0 & 0.05386 & 0.05386 \\ 2-2-2 & 3 & 0.053860 & 0 & 0.05386 & 0.05386\end{array}$

Std Error uses a pooled estimate of error variance
Oneway Analysis of Measured By Set/Block/Sub-Block Glass ID=Batch 1, Prep=LM, Analyte=TiO2 (wt \%)

Reference Value $=0.677 \mathrm{w} \%$

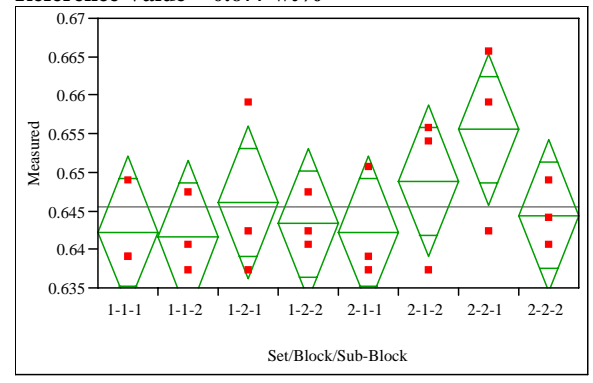

Oneway Anova

Summary of Fit

Root Mean Square Error $\quad 0.00805$

$\begin{array}{lr}\text { Mean of Response } & 0.645516 \\ \text { Observations (or Sum Wgts) } & 24\end{array}$

Analysis of Variance

Source DF Sum of Squares Mean Square F Ratio Prob $>$ F $\begin{array}{lrrrrr}\text { Set/Block/Sub-Block } & 7 & 0.00046556 & 0.000067 & 1.0263 & 0.4505\end{array}$ $\begin{array}{llll}\text { Error } & 16 & 0.00103684 & 0.000065 \\ \text { C. Total } & 23 & 0.00150240 & \end{array}$

Means for Oneway Anova

Level Number Mean Std Error Lower 95\% Upper 95\%

$\begin{array}{rrrrrr}1-1-1 & 3 & 0.642180 & 0.00465 & 0.63233 & 0.65203 \\ 1-2 & 3 & 0.64624\end{array}$

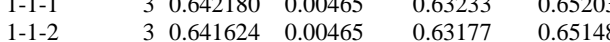

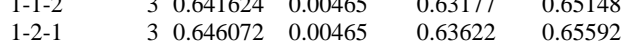

$\begin{array}{llllll}1-2-2 & 3 & 0.643292 & 0.00465 & 0.63344 & 0.65314\end{array}$

$\begin{array}{llllll}1-1-1 & 3 & 0.642180 & 0.00465 & 0.63233 & 0.65203\end{array}$

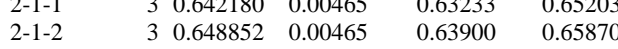

$\begin{array}{lllllll}2-2-1 & & 3 & 0.655524 & 0.00465 & 0.64567 & 0.658538\end{array}$

$\begin{array}{llllll}2-2-2 & 3 & 0.644404 & 0.00465 & 0.63455 & 0.65426\end{array}$

Std Error uses a pooled estimate of error variance 
Figure A2. PSAL Measurements for Each Analyte by Analytical Block for Samples of the Batch 1 and LRM Standards Grouped by Preparation Method

Oneway Analysis of Measured By Set/Block/Sub-Block Glass ID=Batch 1, Prep=LM, Analyte $=\mathrm{ZnO}(\mathrm{wt} \%)$

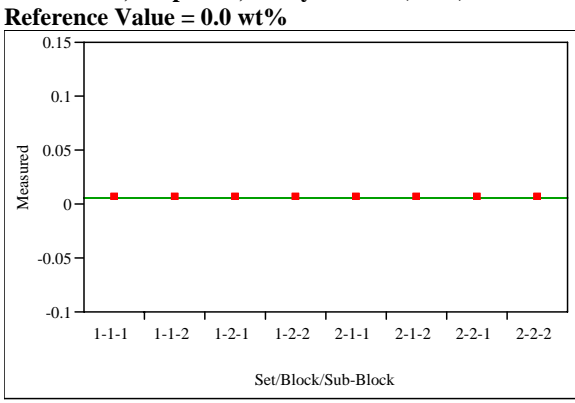

Oneway Anova

Summary of Fit

Rsquare

Adj Rsquare

Root Mean Square Erro

Mean of Response

0
0.006224
24

Analysis of Variance

Source DF Sum of Squares Mean Square F Ratio Prob $>F$ $\begin{array}{lr}\text { Set/Block/Sub-Block } & 7 \\ \text { Error } & 16\end{array}$

C. Tota 16
23

Means for Oneway Anova

Level Number Mean Std Error Lower 95\% Upper 95\%

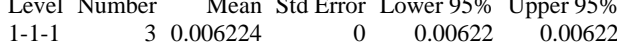

$\begin{array}{llllll}1-1-2 & 3 & 0.006224 & 0 & 0.00622 & 0.00622\end{array}$

$\begin{array}{llllll}1-2-1 & 3 & 0.006224 & 0 & 0.00622 & 0.00622\end{array}$

$\begin{array}{llllll}1-2-2 & 3 & 0.006224 & 0 & 0.00622 & 0.00622\end{array}$

$\begin{array}{llllll}2-1-1 & 3 & 0.006224 & 0 & 0.00622 & 0.00622\end{array}$

$\begin{array}{llllll}2-1-2 & 3 & 0.006224 & 0 & 0.00622 & 0.00622\end{array}$

$\begin{array}{llllll}2-2-1 & 3 & 0.006224 & 0 & 0.00622 & 0.00622\end{array}$

Std Error uses a pooled estimate of error variance
Oneway Analysis of Measured By Set/Block/Sub-Block Glass ID=Batch 1, Prep=LM, Analyte=ZrO2 (wt \%)

Reference Value $=0.098 \mathrm{wt} \%$

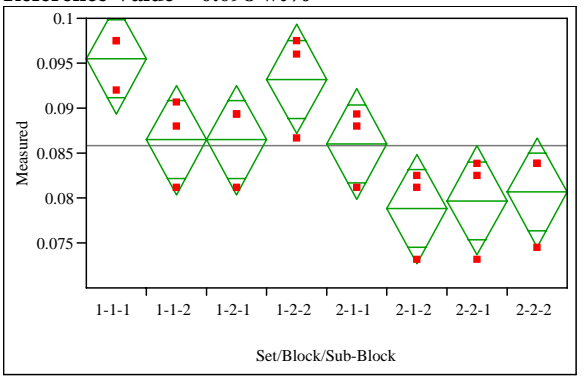

Oneway Anova

Summary of Fit

Rsquare

$\begin{array}{ll} & 0.663568 \\ \text { Adj Rsquare } & 0.516379\end{array}$

Root Mean Square Error $\quad 0.004994$

Mean of Response $\quad 0.085832$

Observations (or Sum Wgts) 24

Analysis of Variance

Source DF Sum of Squares Mean Square F Ratio Prob > F $\begin{array}{lrllll}\text { Set/Block/Sub-Block } & 7 & 0.00078696 & 0.000112 & 4.5083 & 0.0060\end{array}$ Error $\begin{array}{ll}16 & 0.00039899 \\ 23 & 0.00118595\end{array}$

\section{Means for Oneway Anova}

Level Number Mean Std Error Lower 95\% Upper 95\%

$\begin{array}{llllll}1-1-1 & 3 & 0.095457 & 0.00288 & 0.08934 & 0.10157\end{array}$

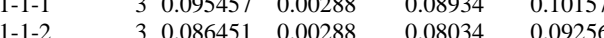

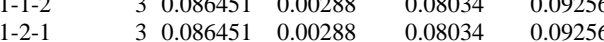

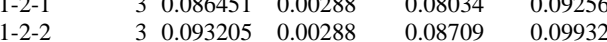

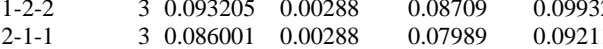

$\begin{array}{lllllll}2-1-2 & & 3 & 0.078797 & 0.00288 & 0.07268 & 0.08491 \\ 2-2-1 & & 3 & 0.079697 & 0.0288 & 0.07359 & 0.08581\end{array}$

$\begin{array}{llllll}2-2-1 & 3 & 0.079697 & 0.00288 & 0.07359 & 0.08491 \\ 2-2-2 & 3 & 0.080598 & 0.00288 & 0.07469 & 0.08671\end{array}$

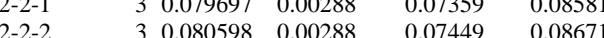

Std Error uses a pooled estimate of error variance
Oneway Analysis of Measured By Set/Block/Sub-Block Glass ID=Batch 1, Prep=PF, Analyte=B2O3 (wt\%)$$
\text { Reference Value }=7.777 \mathrm{wt} \%
$$

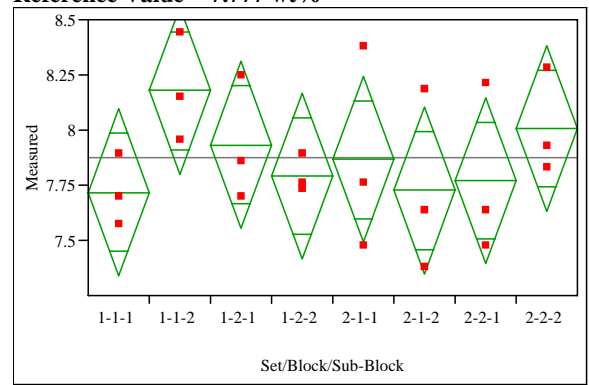

\section{Oneway Anova}

Summary of Fit

$\begin{array}{lr}\text { Rsquare } & 0.258907 \\ \text { Adj Rsquare } & -0.06532 \\ \text { Root Mean Square Error } & 0.308352 \\ \text { Mean of Response } & 7.873997 \\ \text { Observations (or Sum Wgts) } & 24\end{array}$

Observations (or Sum Wgts)

Analysis of Variance

Source DF Sum of Squares Mean Square F Ratio Prob $>$ F
Set/Block/Sub-Block Set/Blocks \begin{tabular}{crrrr}
7 & 0.5314771 & 0.075925 & 0.7985 & 0.5999 \\
\hline & 1.5212954 & 0.095081 & &
\end{tabular}

Total $\begin{array}{ll}16 & 1.5212954 \\ 23 & 2.0527725\end{array}$

0.095081

Means for Oneway Anova

Level Number Mean Std Error Lower 95\% Upper 95\%

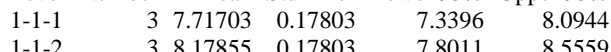

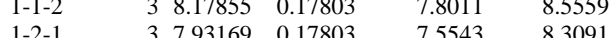

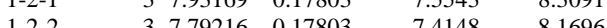

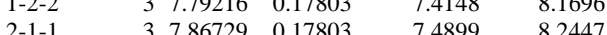

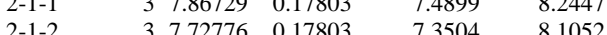

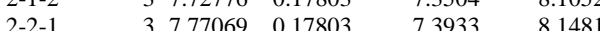
$\begin{array}{llllll}2-2-2 & 3 & 8.00682 & 0.17803 & 7.6294 & 8.3842\end{array}$

Std Error uses a pooled estimate of error variance 
Figure A2. PSAL Measurements for Each Analyte by Analytical Block for Samples of the Batch 1 and LRM Standards Grouped by Preparation Method

Oneway Analysis of Measured By Set/Block/Sub-Block Glass ID=Batch 1, Prep=PF, Analyte=Li2O (wt \%)

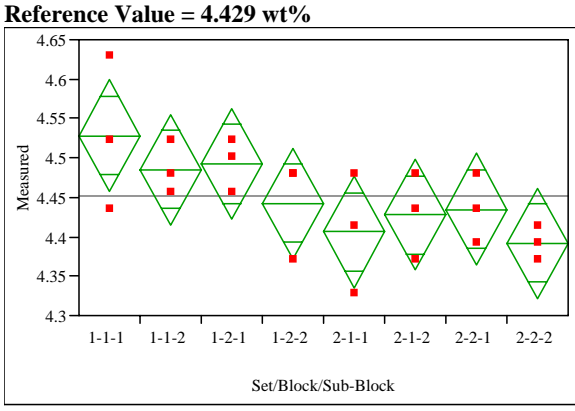

\section{Oneway Anova}

Summary of Fit

$\begin{array}{lr}\text { Rsquare } & 0.463529 \\ \text { Adj Rsquare } & 0.228824 \\ \text { Root Mean Square Error } & 0.057467 \\ \text { Mean of Response } & 4.451121 \\ \text { Observations (or Sum Wgts) } & 24\end{array}$

Analysis of Variance

Source $\quad$ DF Sum of Squares Mean Square F Ratio Prob > F

$\begin{array}{llllll} & & 0.006522 & 1.9749 & 0.1230\end{array}$

C. Total

$16 \quad 0.05283875$

0.003302

Means for Oneway Anova

Level Number Mean Std Error Lower 95\% Upper 95\%

$\begin{array}{llllll}1-1-1 & 3 & 4.52827 & 0.03318 & 4.4579 & 4.5986\end{array}$

$\begin{array}{llllll}1-1-2 & 3 & 4.48521 & 0.03318 & 4.4149 & 4.5555 \\ 1-2-1 & 3 & 4.49238 & 0.03318 & 4.4220 & 4.5627\end{array}$

$\begin{array}{llllll}1-2-1 & 3 & 4.49238 & 0.03318 & 4.4220 & 4.5627\end{array}$

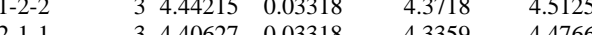

$\begin{array}{llllll}2-1-1 & 3 & 4.40627 & 0.03318 & 4.3359 & 4.4766\end{array}$

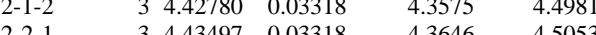

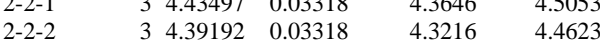

Std Error uses a pooled estimate of error variance
Oneway Analysis of Measured By Set/Block/Sub-Block Glass ID=Batch 1, Prep=PF, Analyte=SiO2 (wt \%)

Reference Value $=50.22 \mathrm{wt} \%$

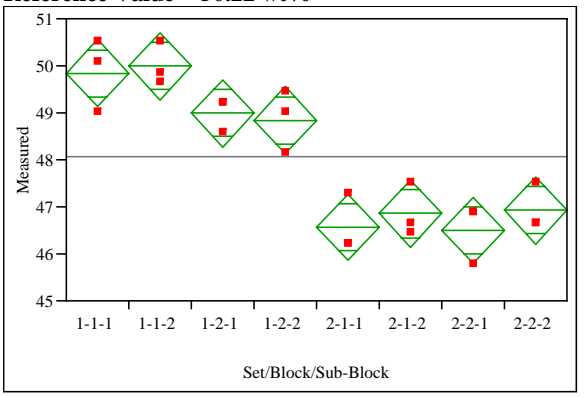

Oneway Anova

Summary of Fit

Rsquare

Adj Rsquare $\quad 0.898442$

\begin{tabular}{l}
0.879325 \\
\hline
\end{tabular}

Mean of Response

48.06294

Analysis of Variance

Source $\quad$ DF Sum of Squares Mean Square F Ratio Prob $>$ F

Set/Block/Sub-Block $\quad 7 \quad 47.505155$

$\begin{array}{llr}\text { Error } & 16 & 5.369883 \\ \text { C. Total } & 23 & 52.875037\end{array}$

Means for Oneway Anova

Level Number Mean Std Error Lower 95\% Upper 95\%

$\begin{array}{llllll}1-1-1 & 3 & 49.8457 & 0.33447 & 49.137 & 50.555\end{array}$

$\begin{array}{llllll}1-1-2 & 3 & 49.9883 & 0.33447 & 49.279 & 50.697\end{array}$

$\begin{array}{llllll}1-2-1 & 3 & 48.9900 & 0.33447 & 48.281 & 49.699\end{array}$

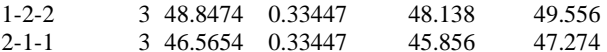

$\begin{array}{llllll}2-1-2 & 3 & 46.8507 & 0.33447 & 46.142 & 47.560\end{array}$

$\begin{array}{llllll}2-2-1 & 3 & 46.4941 & 0.33447 & 45.785 & 47.203\end{array}$

$\begin{array}{llllll}2-2-2 & 3 & 46.9220 & 0.33447 & 46.213 & 47.631\end{array}$

Std Error uses a pooled estimate of error variance
Oneway Analysis of Measured By Set/Block/Sub-Block Glass ID $=$ LRM, Prep $=K H$, Analyte $=F(w t \%)$

Reference Value $=1.0 \mathrm{wt} \%$

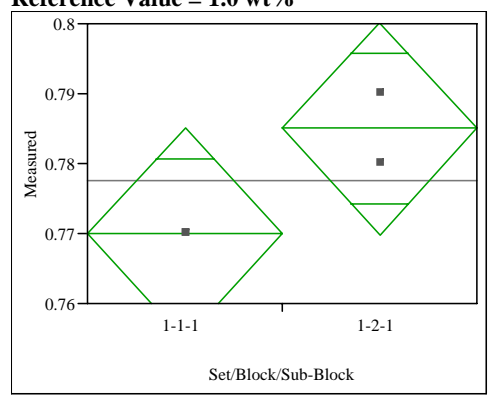

Oneway Anova

Summary of Fit

$\begin{array}{lr}\text { Rsquare } & 0.818182 \\ \text { Adj Rsquare } & 0.727273 \\ \text { Root Mean Square Error } & 0.005 \\ \text { Mean of Response } & 0.7775 \\ \text { Observations (or Sum Wgts) } & 4\end{array}$

t Test

1-2-1-1-1-1

Assuming equal variances

Difference $\quad 0.01500 \mathrm{t}$ Ratio

Std Err Dif $\quad 0.00500$ DF

Upper CL Dif 0.03651 Prob $>|t| \begin{array}{r}2 \\ 0.0955\end{array}$

Lower CL Dif -0.00651 Prob $>t \quad 0.0477$

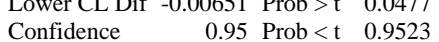

Analysis of Variance

Source DF Sum of Squares Mean Square F Ratio Prob $>$ F $\begin{array}{lrrrrr}\text { Sot/Block/Sub-Block } & 1 & 0.00022500 & 0.000225 & 9.0000 & 0.0955\end{array}$ $\begin{array}{llll}\text { Error } & 2 & 0.00005000 & 0.000025 \\ C \text { Total } & 3 & 0.00027500 & \end{array}$

Means for Oneway Anova

Level Number Mean Std Error Lower 95\% Upper 95\%

$\begin{array}{llllll}1-1-1 & 2 & 0.770000 & 0.00354 & 0.75479 & 0.7852\end{array}$

$\begin{array}{llllll}1-2-1 & 2 & 0.785000 & 0.00354 & 0.76979 & 0.8002\end{array}$

Std Error uses a pooled estimate of error variance 
Figure A3. Measured and Measured Bias-Corrected Weight Percents for Each Analyte Grouped by Preparation Method by Lab ID within Glass ID

Variability Gage Prep=KH, Analyte=F (wt \%)

Variability Chart for Measured

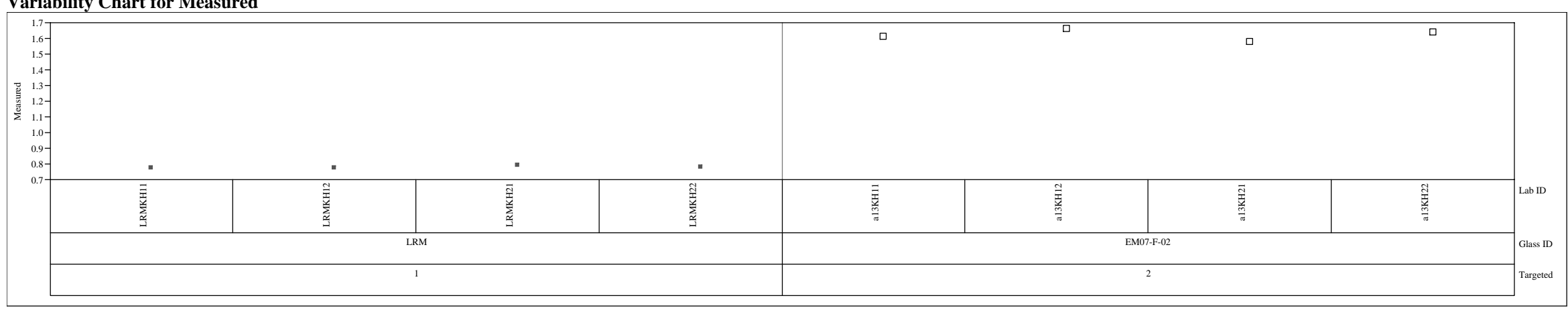

Variability Gage Prep=KH, Analyte=F (wt\%)

Variability Chart for Measured bc

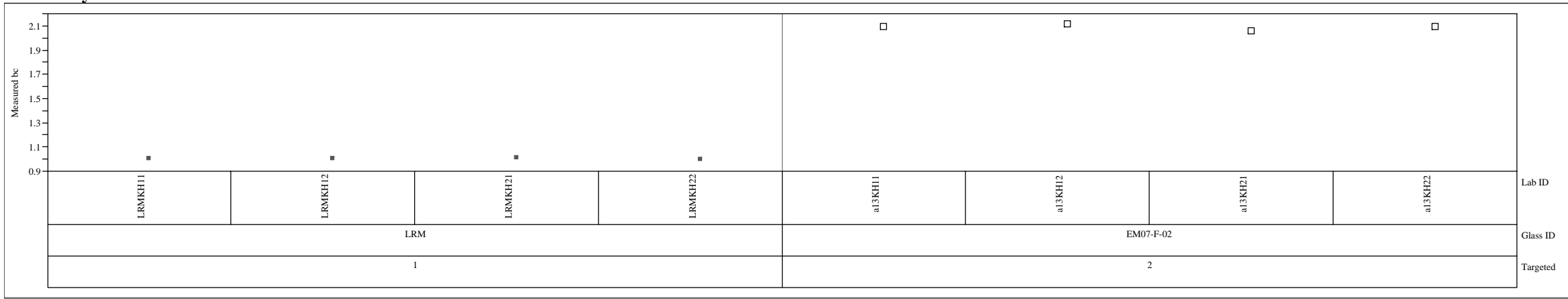


Figure A3. Measured and Measured Bias-Corrected Weight Percents for Each Analyte Grouped by Preparation Method by Lab ID within Glass ID

Variability Gage Prep=LM, Analyte=Al2O3 (wt \%)

Variability Chart for Measured

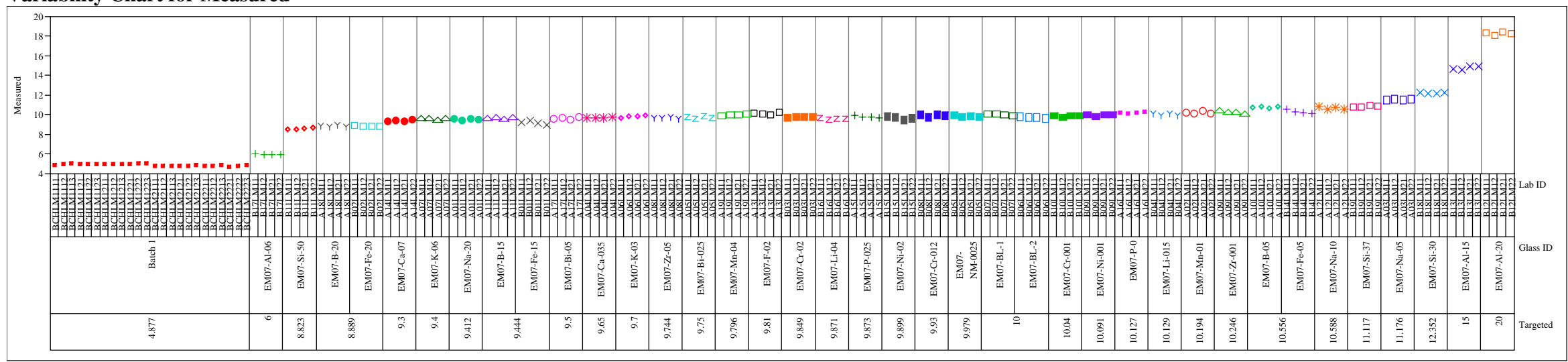

Variability Gage Prep=LM, Analyte=Al2O3 (wt\%)

Variability Chart for Measured bc

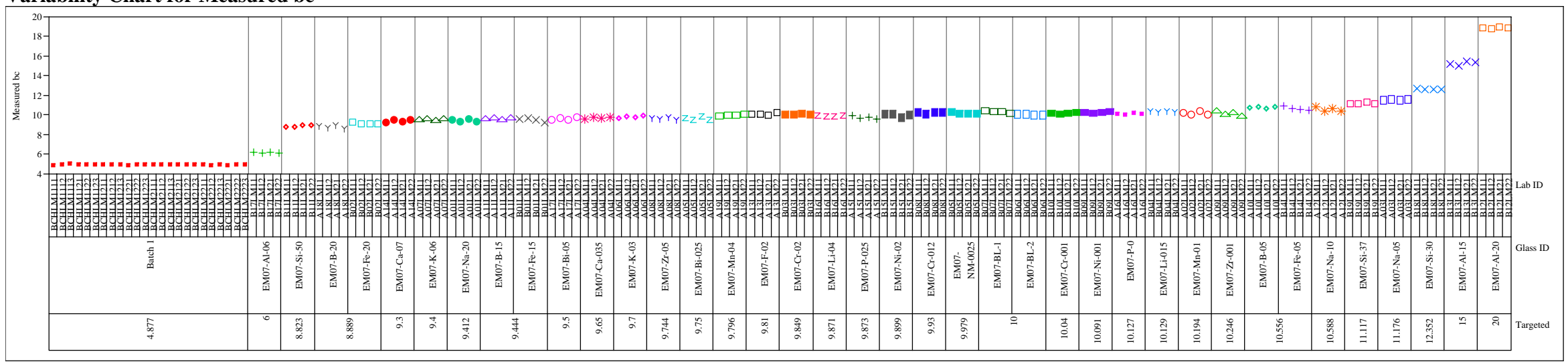


Figure A3. Measured and Measured Bias-Corrected Weight Percents for Each Analyte Grouped by Preparation Method by Lab ID within Glass ID

Variability Gage Prep=LM, Analyte=BaO (wt \%)

Variability Chart for Measured

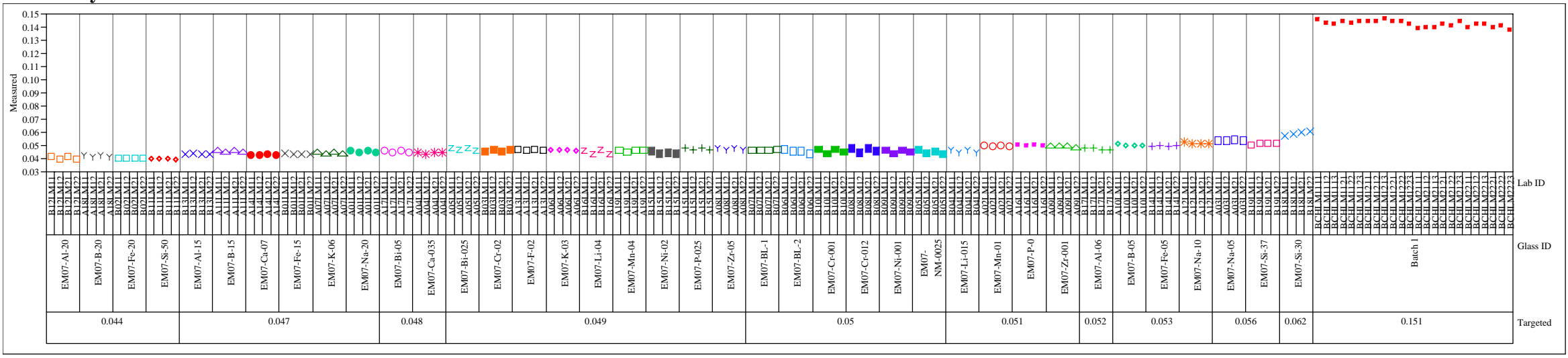

Variability Gage Prep=LM, Analyte=BaO (wt\%)

Variability Chart for Measured bc

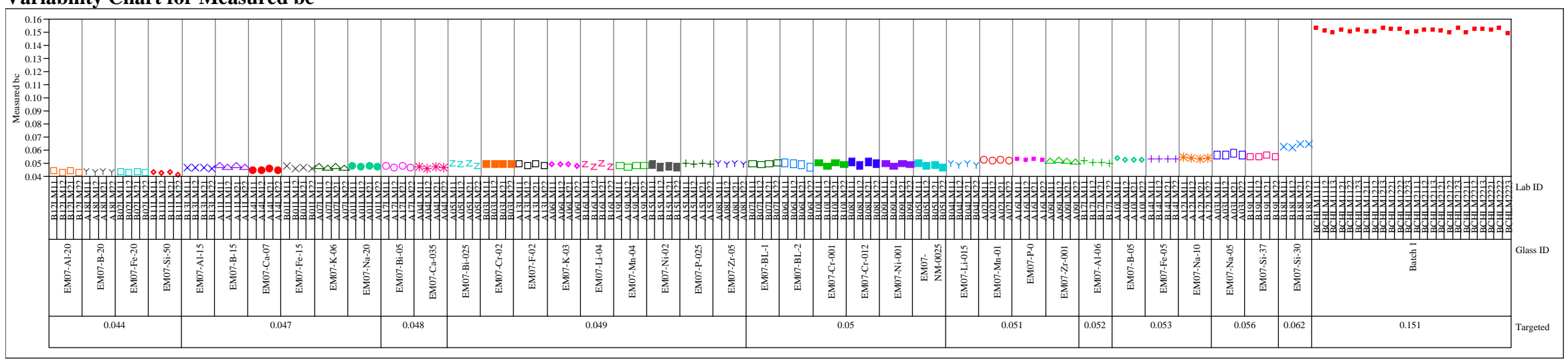


Figure A3. Measured and Measured Bias-Corrected Weight Percents for Each Analyte Grouped by Preparation Method by Lab ID within Glass ID

Variability Gage Prep=LM, Analyte=Bi2O3 (wt\%)

Variability Chart for Measured

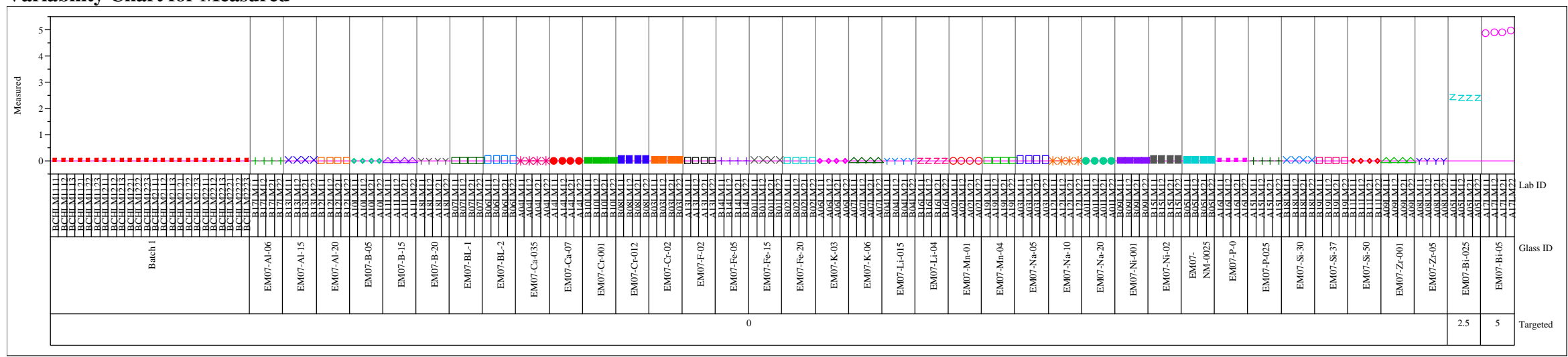

Variability Gage Prep=LM, Analyte=Bi2O3 (wt\%)

Variability Chart for Measured bc

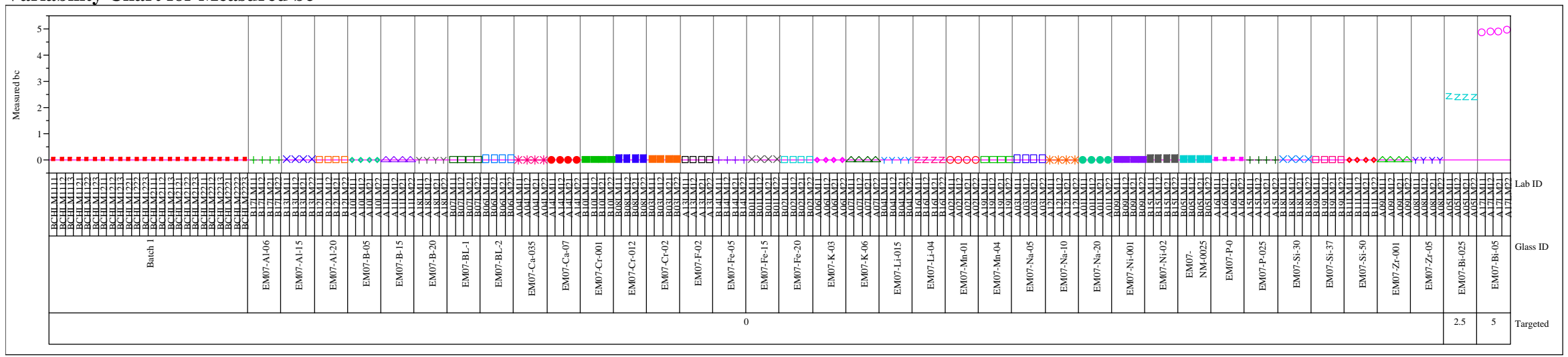


Figure A3. Measured and Measured Bias-Corrected Weight Percents for Each Analyte Grouped by Preparation Method by Lab ID within Glass ID

Variability Gage Prep=LM, Analyte=CaO (wt \%)

Variability Chart for Measured

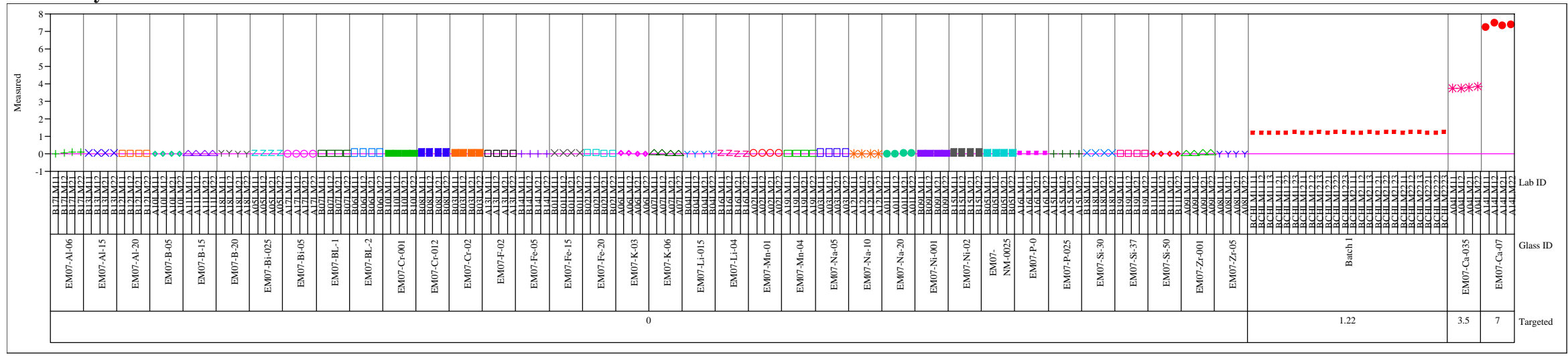

Variability Gage Prep=LM, Analyte=CaO (wt\%)

Variability Chart for Measured bc

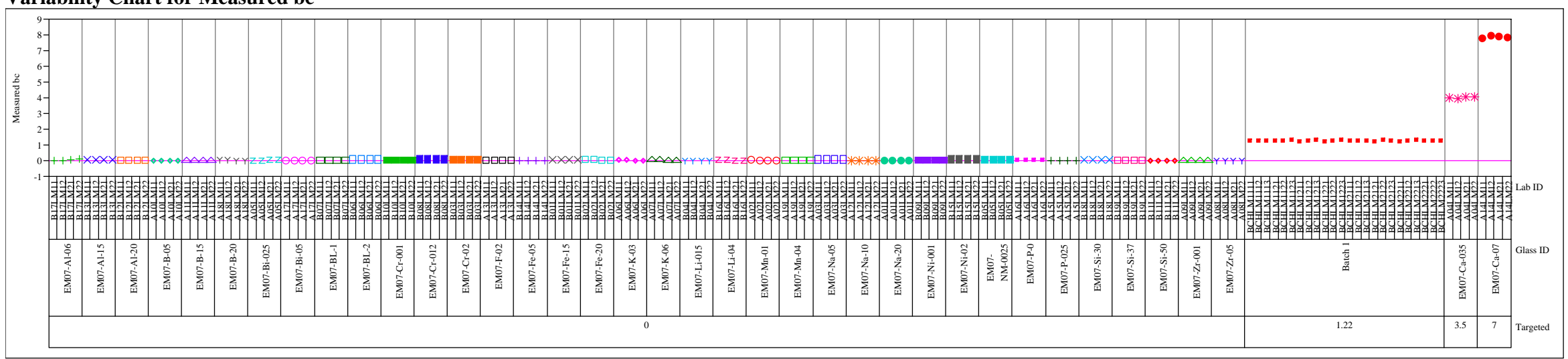


Figure A3. Measured and Measured Bias-Corrected Weight Percents for Each Analyte Grouped by Preparation Method by Lab ID within Glass ID

Variability Gage Prep=LM, Analyte=CdO (wt \%)

Variability Chart for Measured

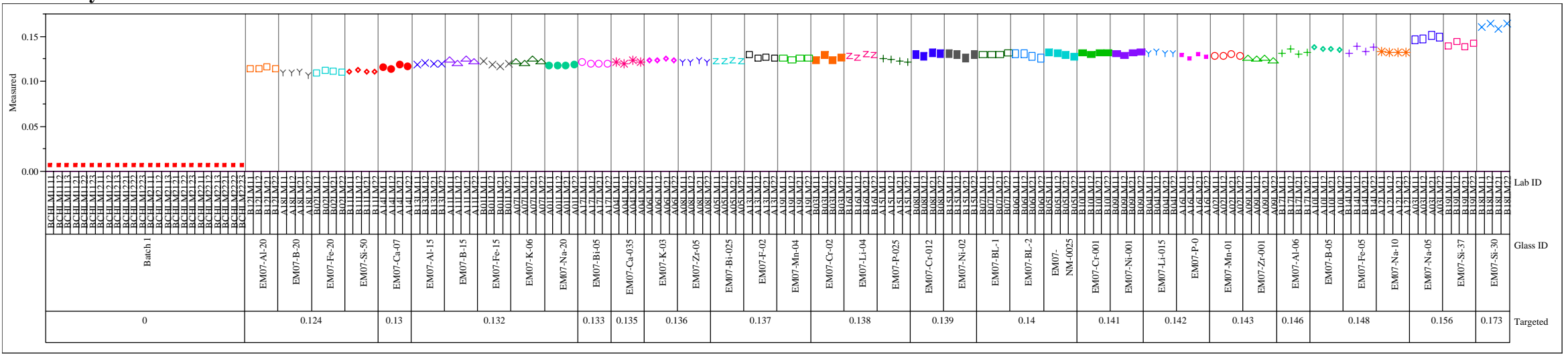

Variability Gage Prep=LM, Analyte $=$ CdO (wt\%)

Variability Chart for Measured bc

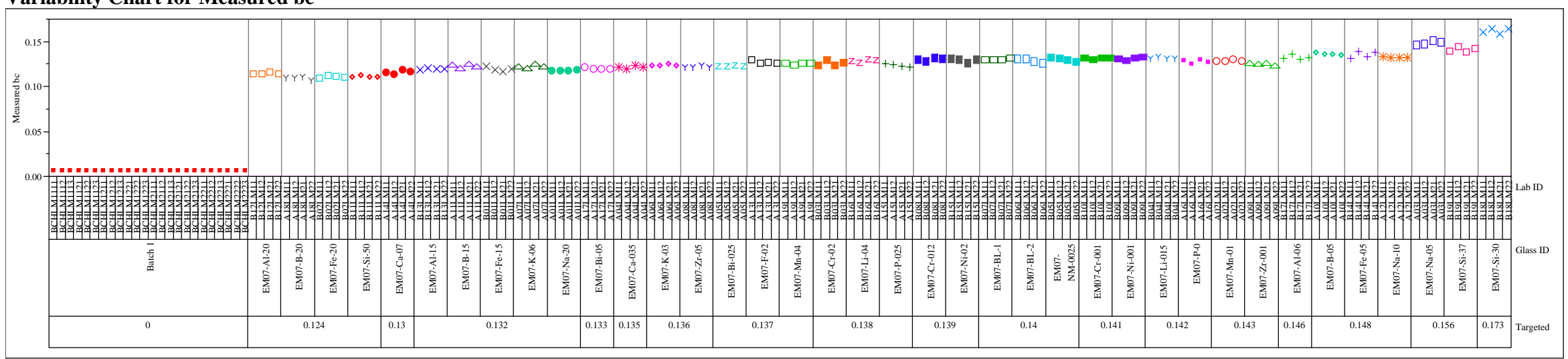


Figure A3. Measured and Measured Bias-Corrected Weight Percents for Each Analyte Grouped by Preparation Method by Lab ID within Glass ID

Variability Gage Prep=LM, Analyte=Ce2O3 (wt \%)

Variability Chart for Measured

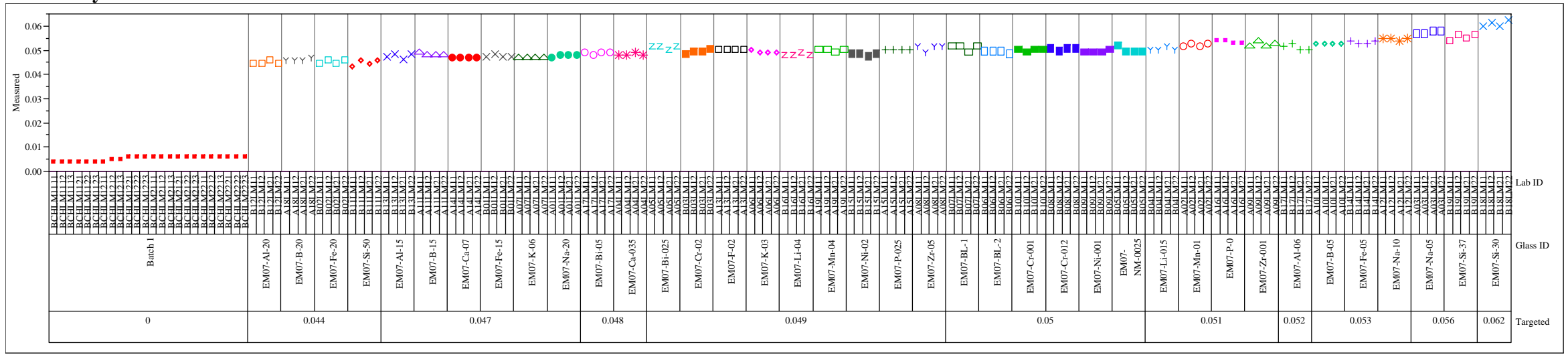

Variability Gage Prep=LM, Analyte=Ce2O3 (wt\%)

Variability Chart for Measured bc

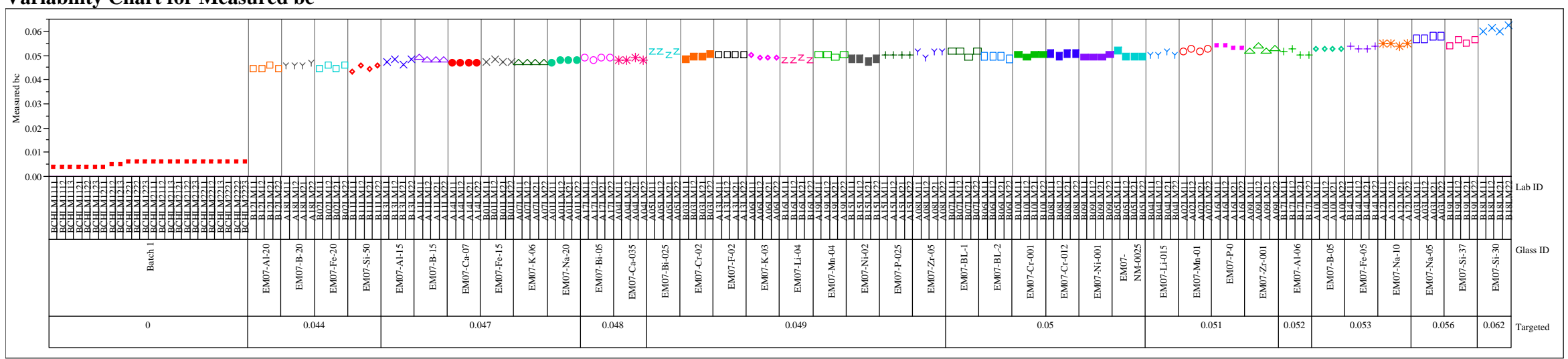


Figure A3. Measured and Measured Bias-Corrected Weight Percents for Each Analyte Grouped by Preparation Method by Lab ID within Glass ID

Variability Gage Prep=LM, Analyte=Cr2O3 (wt \%)

Variability Chart for Measured

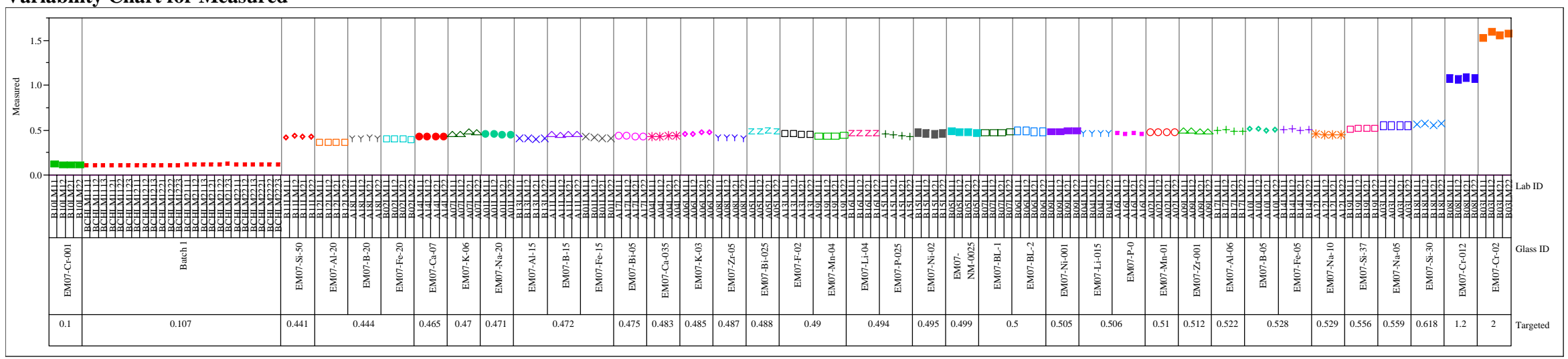

Variability Gage Prep=LM, Analyte=Cr2O3 (wt\%)

Variability Chart for Measured bc

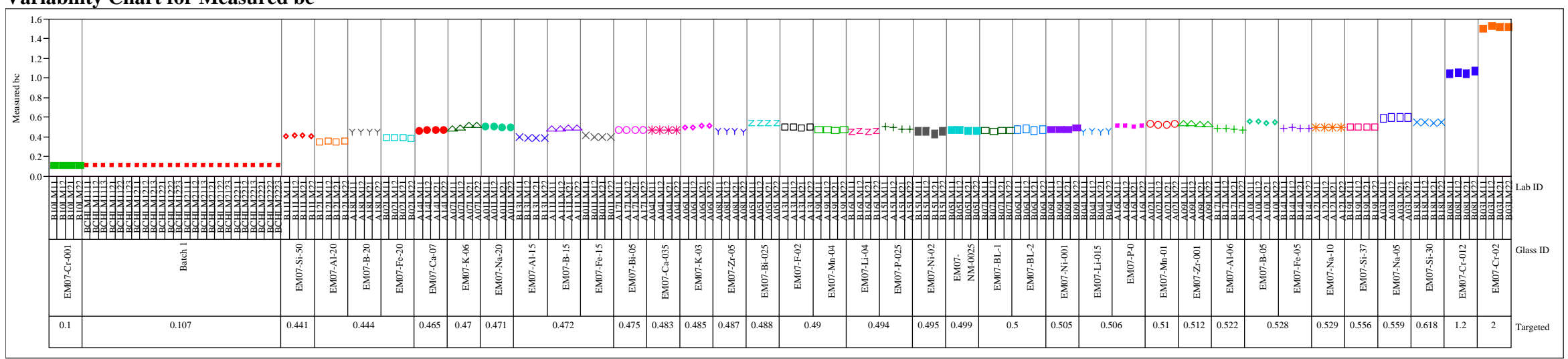


Figure A3. Measured and Measured Bias-Corrected Weight Percents for Each Analyte Grouped by Preparation Method by Lab ID within Glass ID

Variability Gage Prep=LM, Analyte=Fe2O3 (wt\%)

Variability Chart for Measured

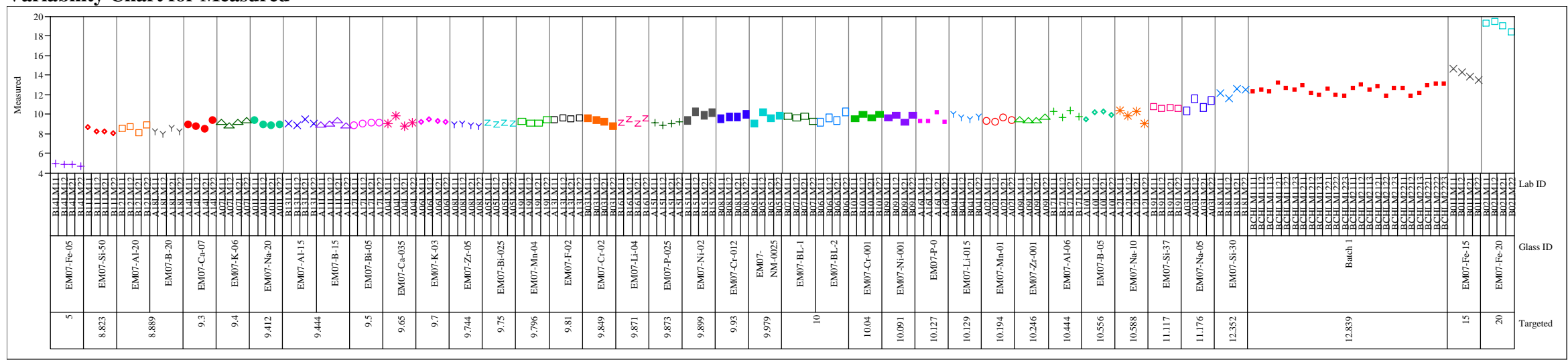

Variability Gage Prep=LM, Analyte=Fe2O3 (wt \%)

Variability Chart for Measured bc

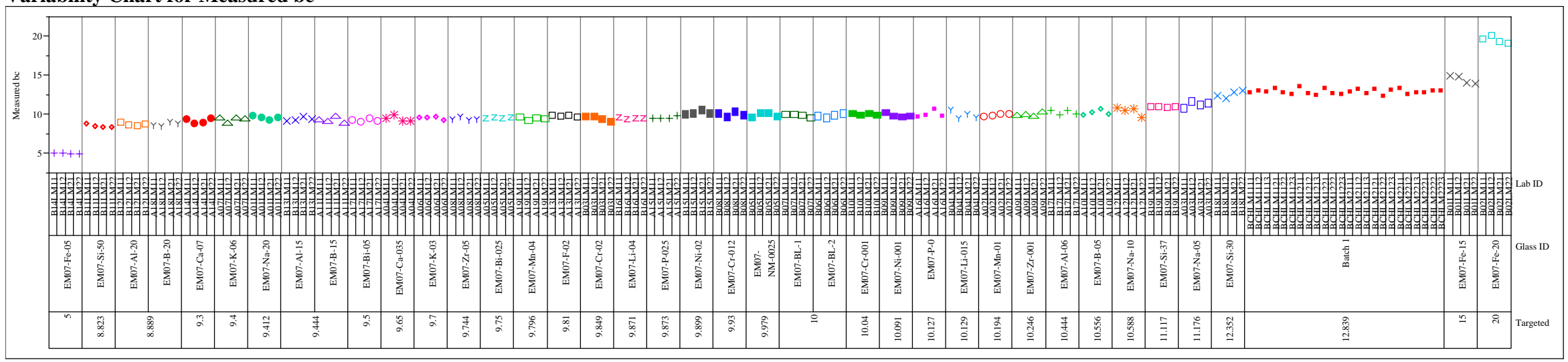


Figure A3. Measured and Measured Bias-Corrected Weight Percents for Each Analyte Grouped by Preparation Method by Lab ID within Glass ID

Variability Gage Prep=LM, Analyte=K2O (wt \%)

Variability Chart for Measured

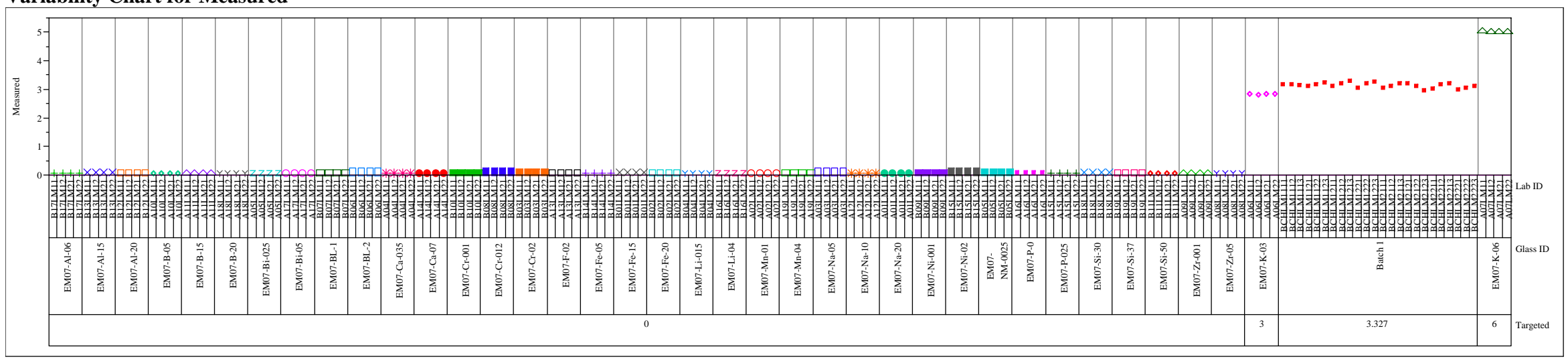

Variability Gage Prep=LM, Analyte=K2O (wt\%)

Variability Chart for Measured bc

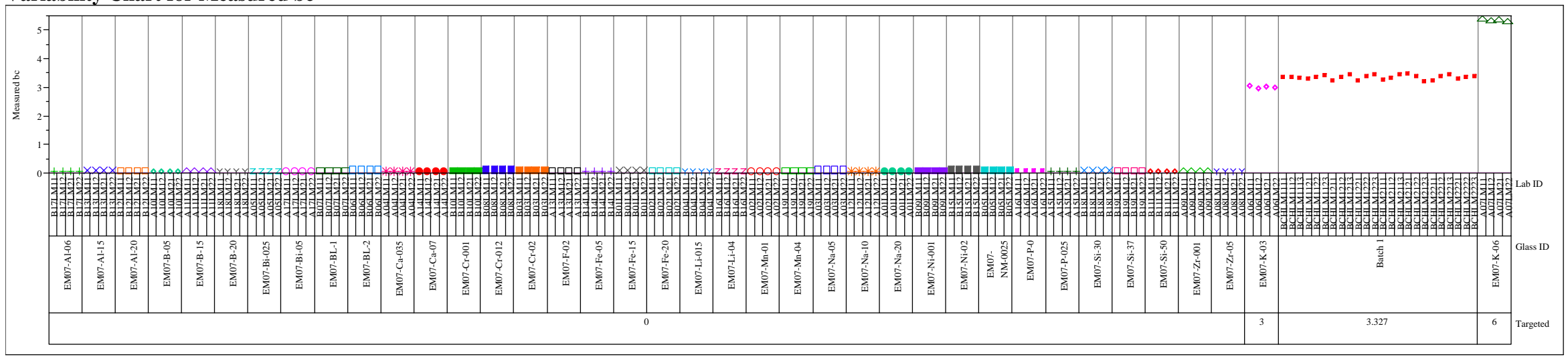


Figure A3. Measured and Measured Bias-Corrected Weight Percents for Each Analyte Grouped by Preparation Method by Lab ID within Glass ID

Variability Gage Prep=LM, Analyte=La2O3 (wt\%)

Variability Chart for Measured

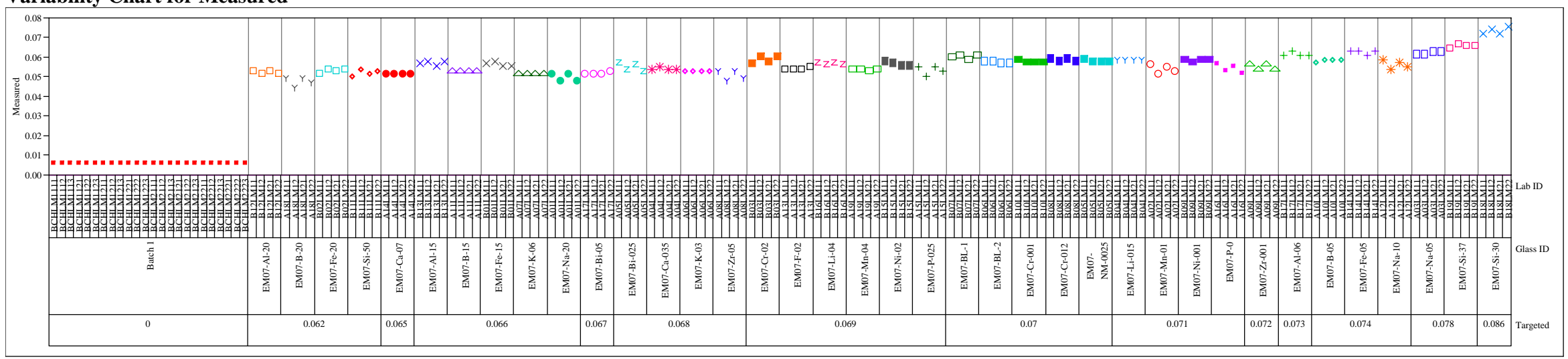

Variability Gage Prep=LM, Analyte=La2O3 (wt\%)

Variability Chart for Measured bc

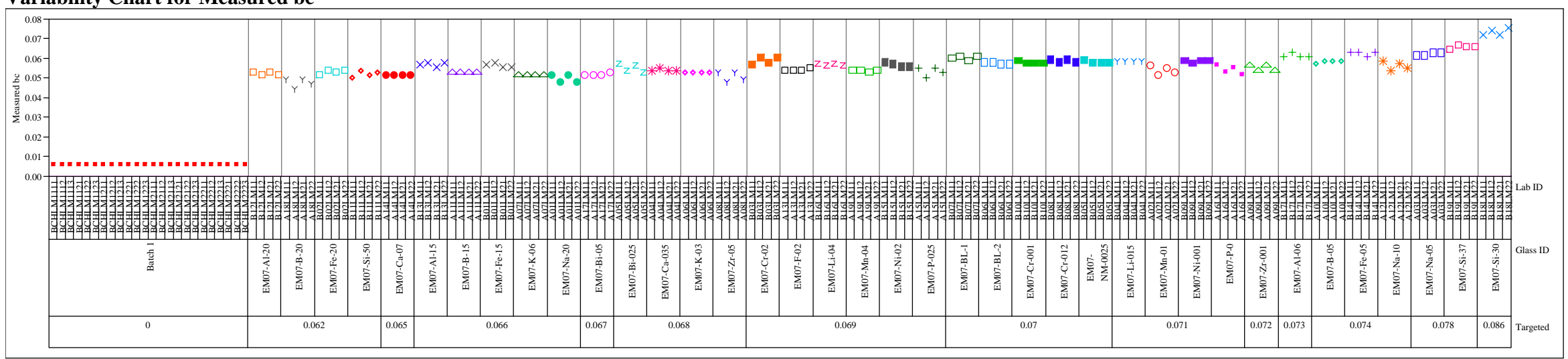


Figure A3. Measured and Measured Bias-Corrected Weight Percents for Each Analyte Grouped by Preparation Method by Lab ID within Glass ID

Variability Gage Prep=LM, Analyte=MgO (wt \%)

Variability Chart for Measured

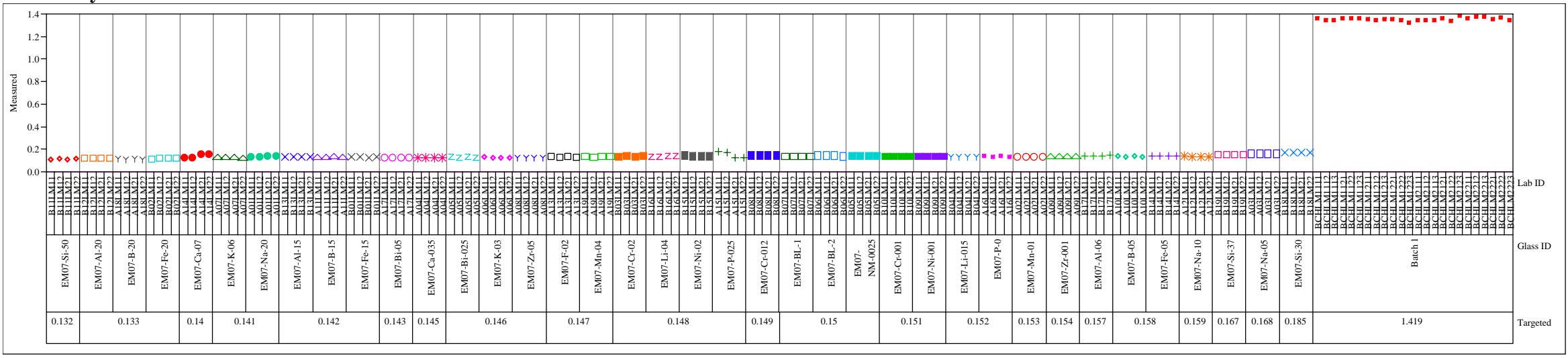

Variability Gage Prep=LM, Analyte $=\mathrm{MgO}(\mathrm{wt} \%)$

Variability Chart for Measured bc

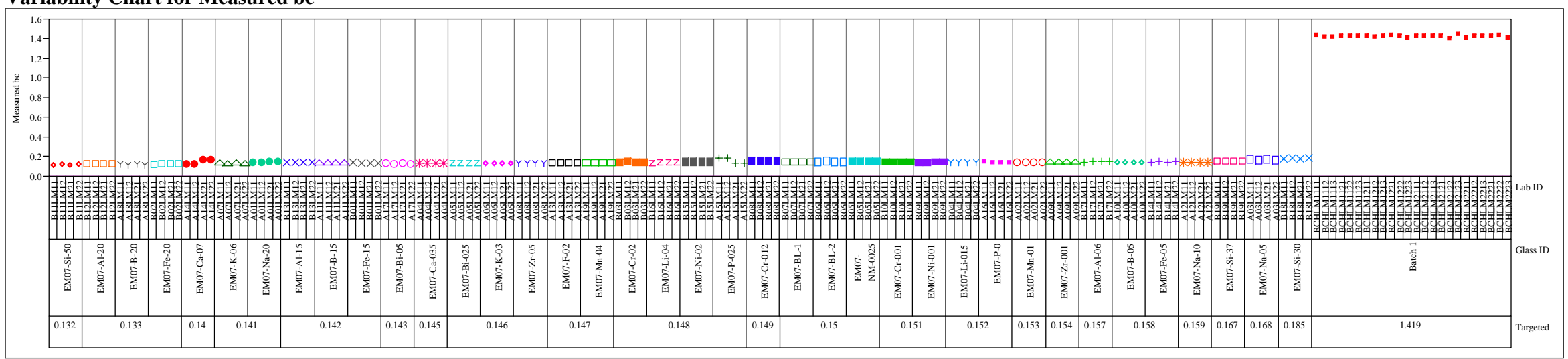


Figure A3. Measured and Measured Bias-Corrected Weight Percents for Each Analyte Grouped by Preparation Method by Lab ID within Glass ID

Variability Gage Prep=LM, Analyte=MnO (wt\%)

Variability Chart for Measured

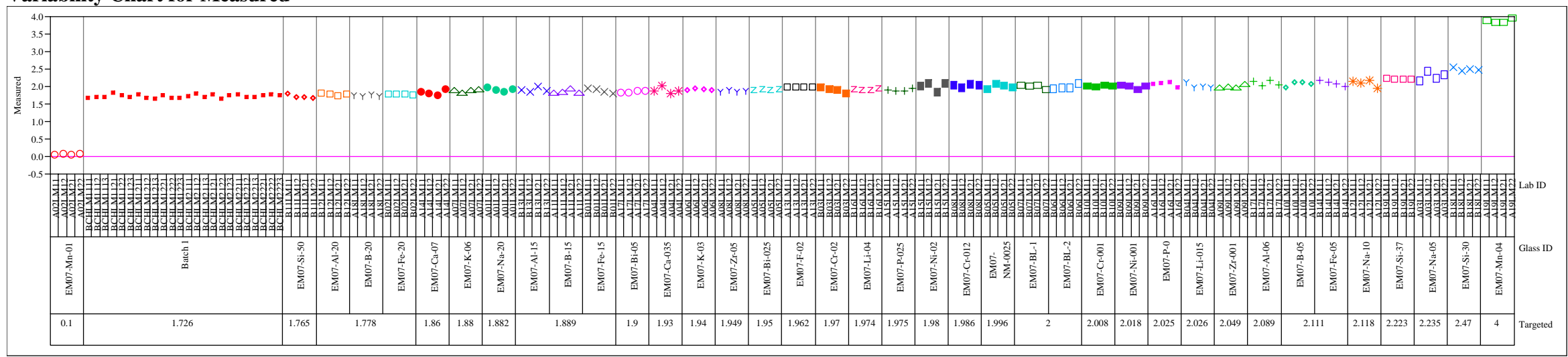

Variability Gage Prep=LM, Analyte $=\mathrm{MnO}$ (wt\%)

Variability Chart for Measured bc

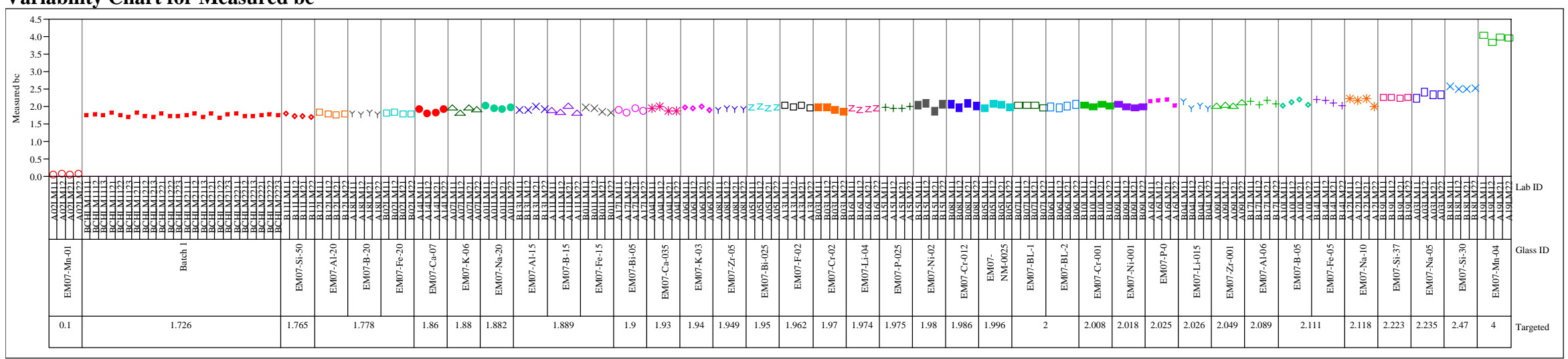


Figure A3. Measured and Measured Bias-Corrected Weight Percents for Each Analyte Grouped by Preparation Method by Lab ID within Glass ID

Variability Gage Prep=LM, Analyte=Na2O (wt\%)

Variability Chart for Measured

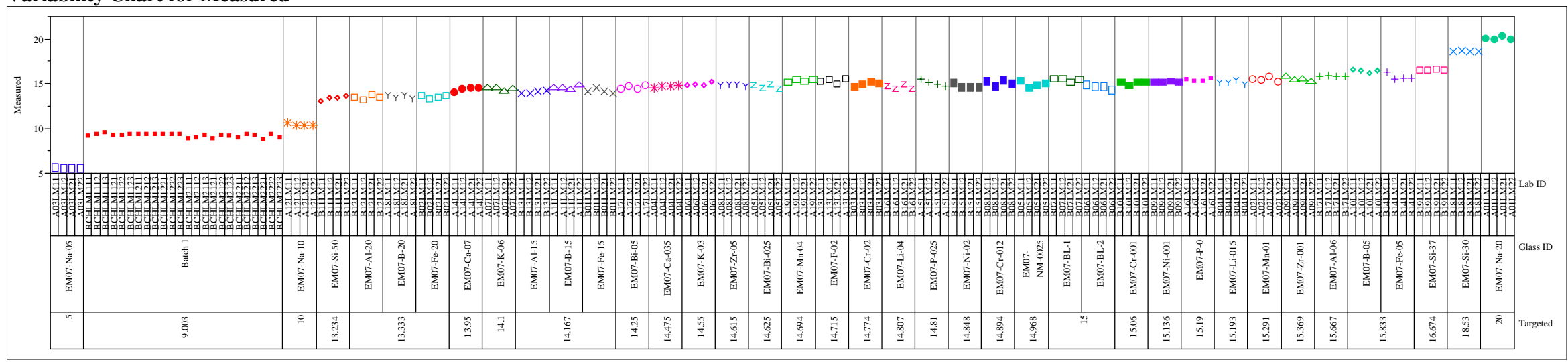

Variability Gage Prep=LM, Analyte=Na2O (wt\%)

Variability Chart for Measured bc

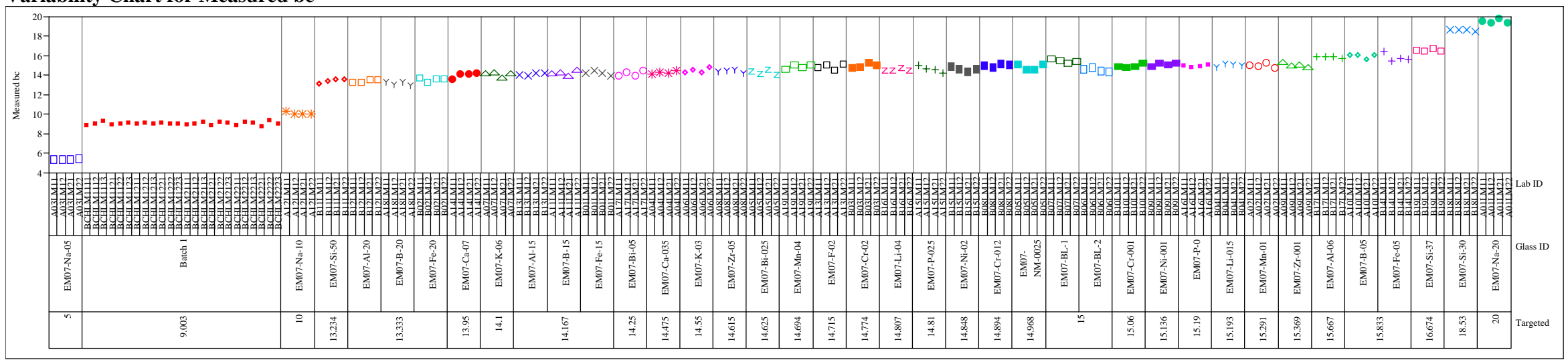


Figure A3. Measured and Measured Bias-Corrected Weight Percents for Each Analyte Grouped by Preparation Method by Lab ID within Glass ID

Variability Gage Prep=LM, Analyte=Nd2O3 (wt\%)

Variability Chart for Measured

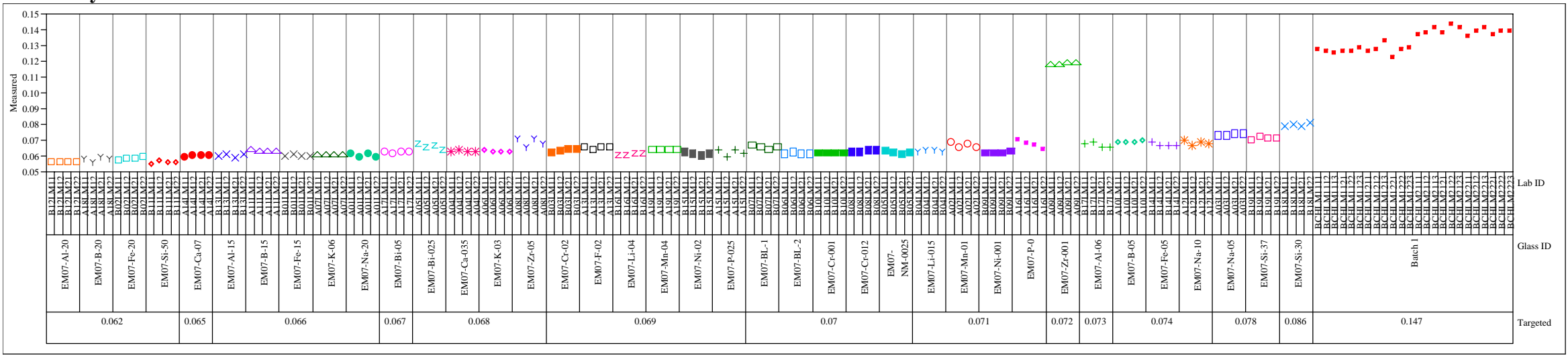

Variability Gage Prep=LM, Analyte=Nd2O3 (wt\%)

Variability Chart for Measured bc

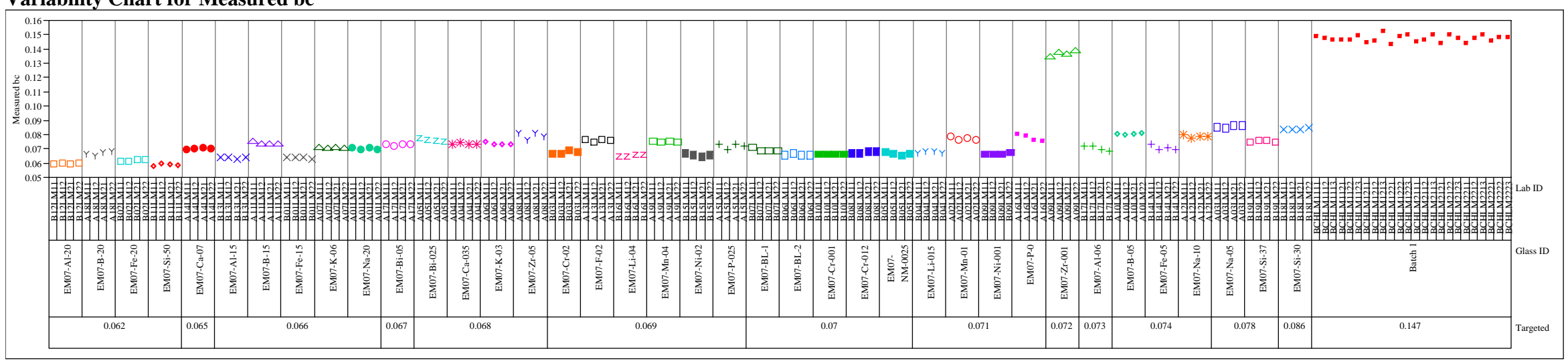


Figure A3. Measured and Measured Bias-Corrected Weight Percents for Each Analyte Grouped by Preparation Method by Lab ID within Glass ID

Variability Gage Prep=LM, Analyte=NiO (wt\%)

Variability Chart for Measured

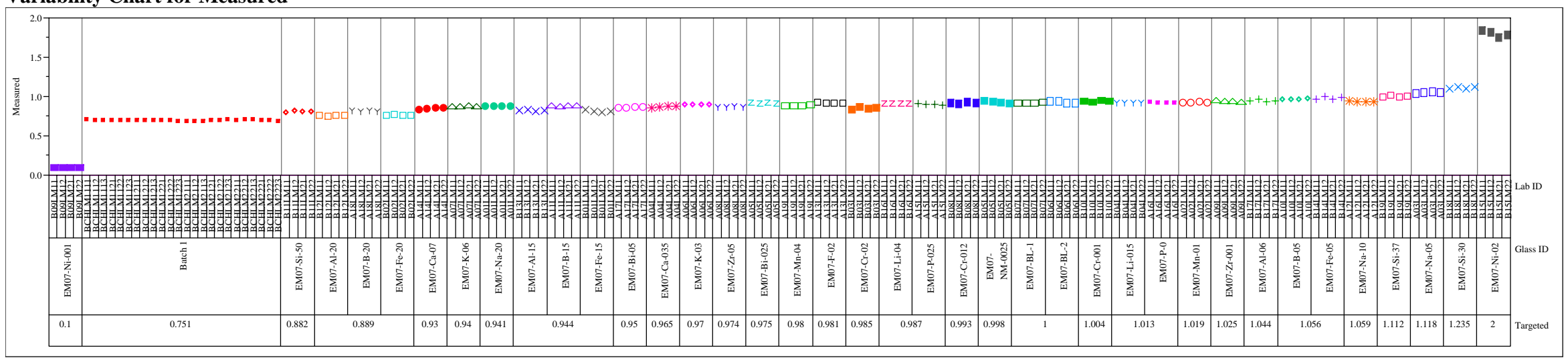

Variability Gage Prep=LM, Analyte $=\mathrm{NiO}(\mathrm{wt} \%)$

Variability Chart for Measured bc

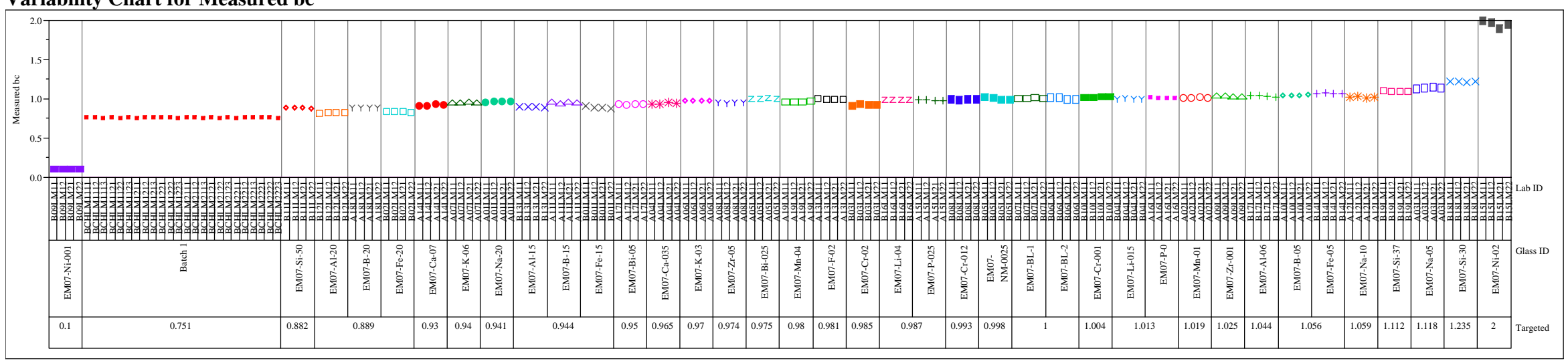


Figure A3. Measured and Measured Bias-Corrected Weight Percents for Each Analyte Grouped by Preparation Method by Lab ID within Glass ID

Variability Gage Prep=LM, Analyte=P2O5 (wt \%)

Variability Chart for Measured

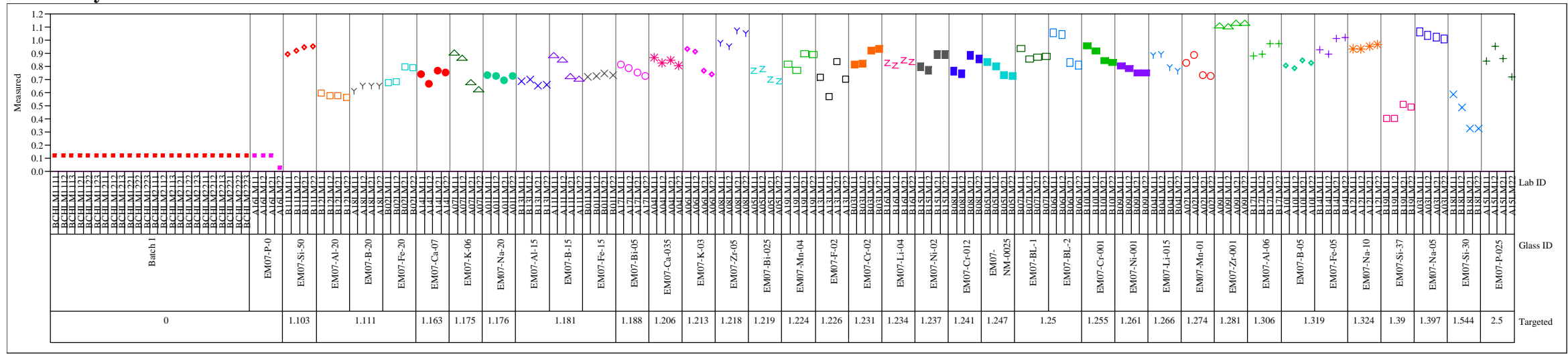

Variability Gage Prep=LM, Analyte=P2O5 (wt\%)

Variability Chart for Measured bc

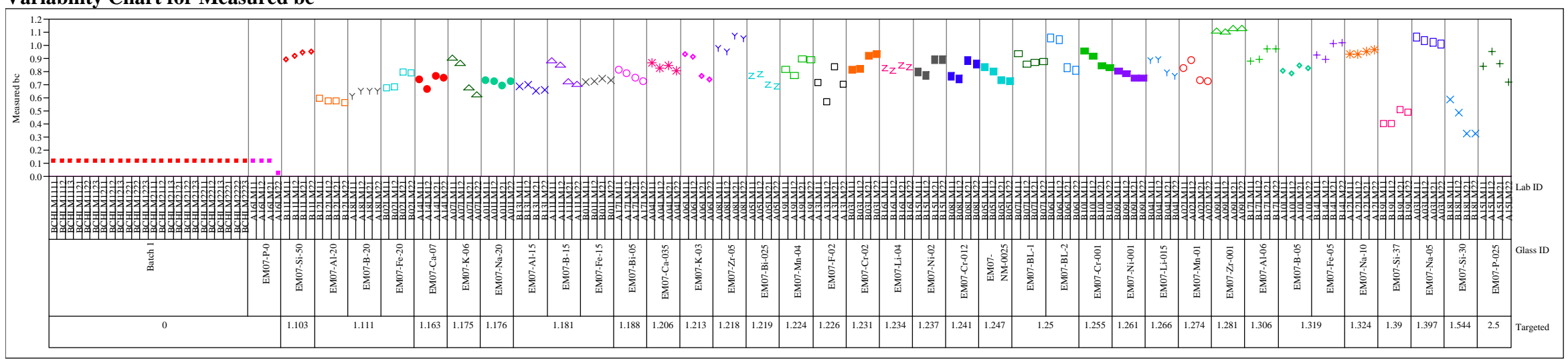


Figure A3. Measured and Measured Bias-Corrected Weight Percents for Each Analyte Grouped by Preparation Method by Lab ID within Glass ID

Variability Gage Prep=LM, Analyte=PbO (wt\%)

Variability Chart for Measured

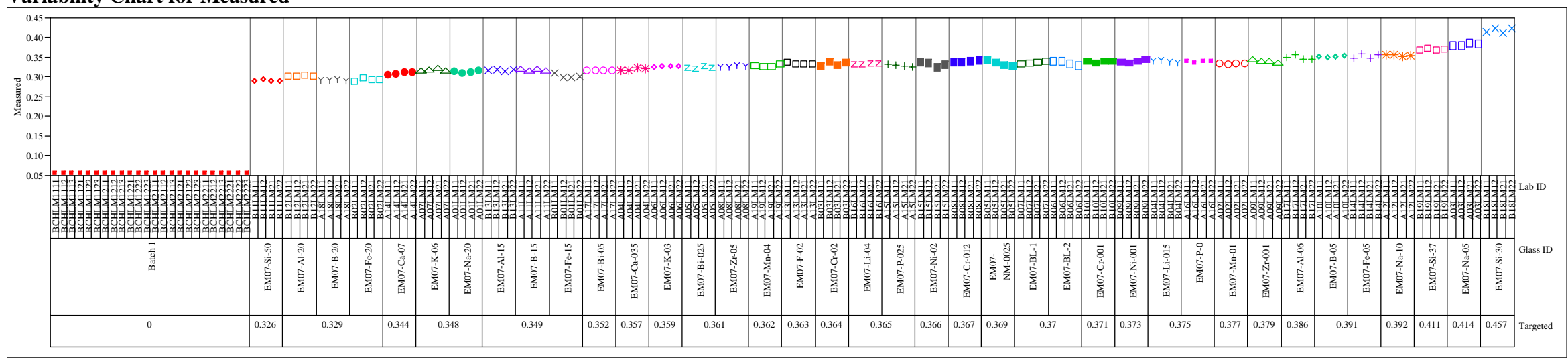

Variability Gage Prep=LM, Analyte=PbO (wt\%)

Variability Chart for Measured bc

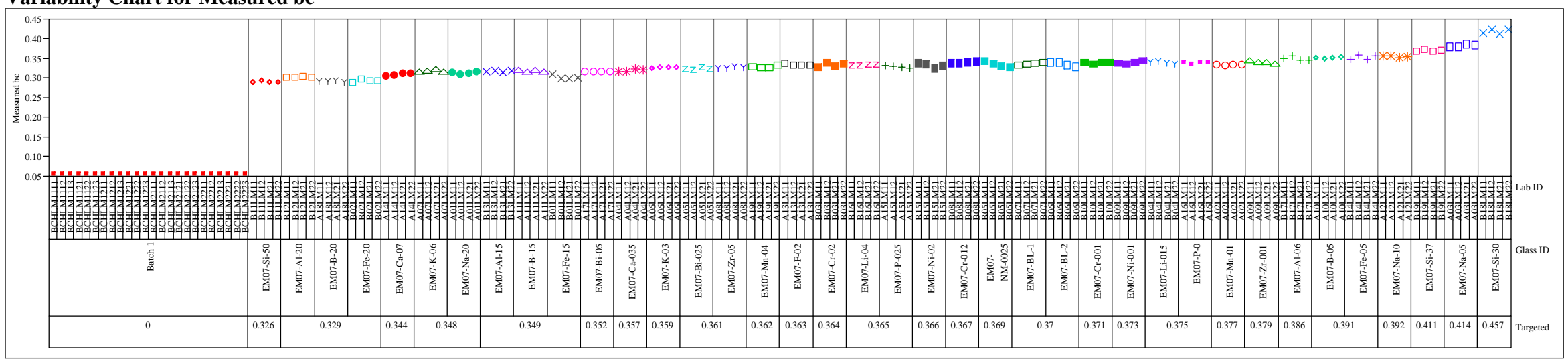


Figure A3. Measured and Measured Bias-Corrected Weight Percents for Each Analyte Grouped by Preparation Method by Lab ID within Glass ID

Variability Gage Prep=LM, Analyte=TiO2 (wt\%)

Variability Chart for Measured

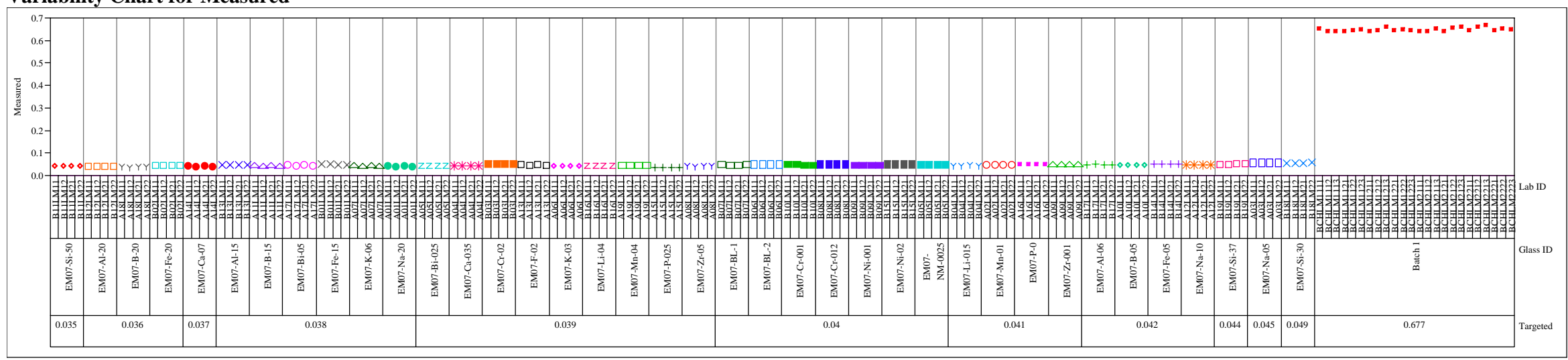

Variability Gage Prep=LM, Analyte=TiO2 (wt\%)

Variability Chart for Measured bc

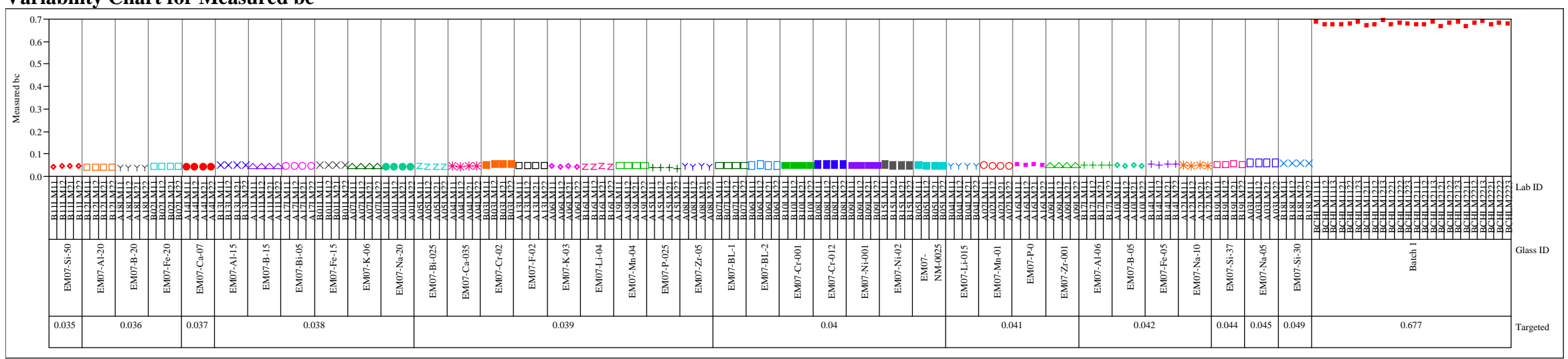


Figure A3. Measured and Measured Bias-Corrected Weight Percents for Each Analyte Grouped by Preparation Method by Lab ID within Glass ID

Variability Gage Prep=LM, Analyte=ZnO (wt\%)

Variability Chart for Measured

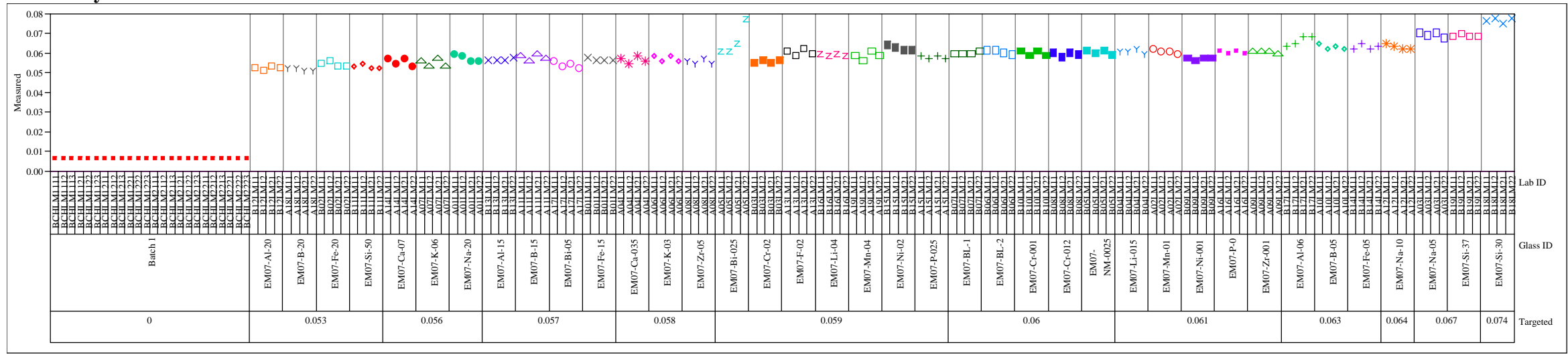

Variability Gage Prep=LM, Analyte=ZnO (wt\%)

Variability Chart for Measured bc

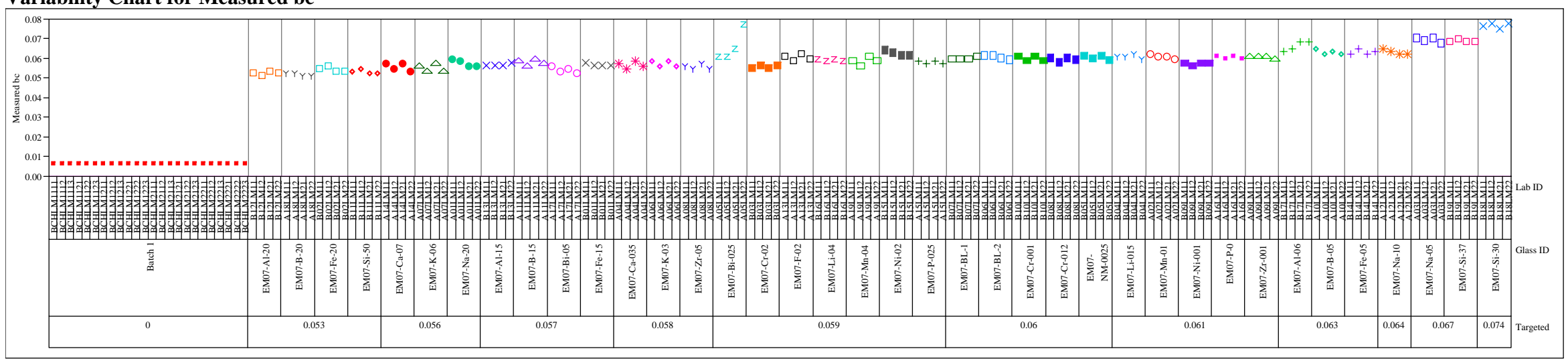


Figure A3. Measured and Measured Bias-Corrected Weight Percents for Each Analyte Grouped by Preparation Method by Lab ID within Glass ID

Variability Gage Prep=LM, Analyte=ZrO2 (wt\%)

Variability Chart for Measured

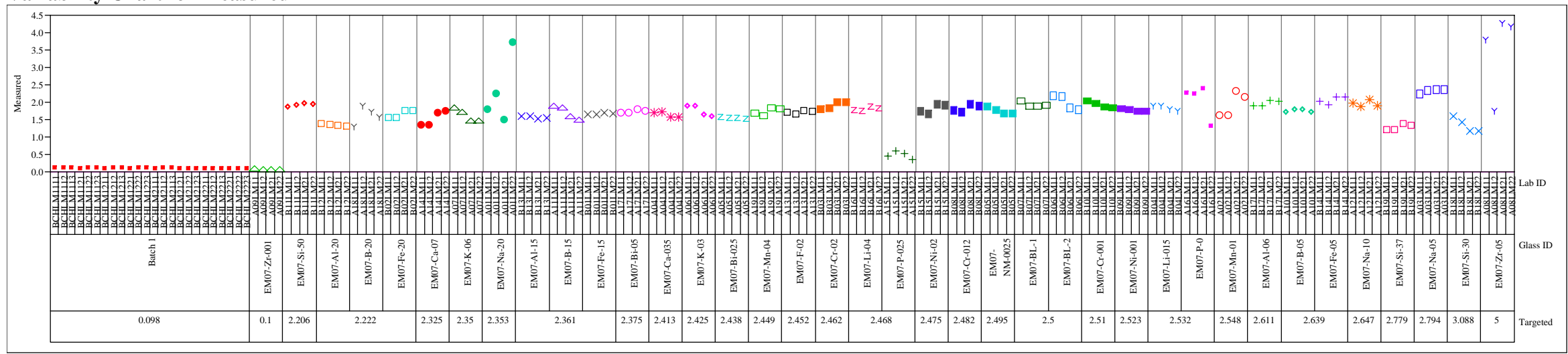

Variability Gage Prep=LM, Analyte=ZrO2 (wt\%)

Variability Chart for Measured bc

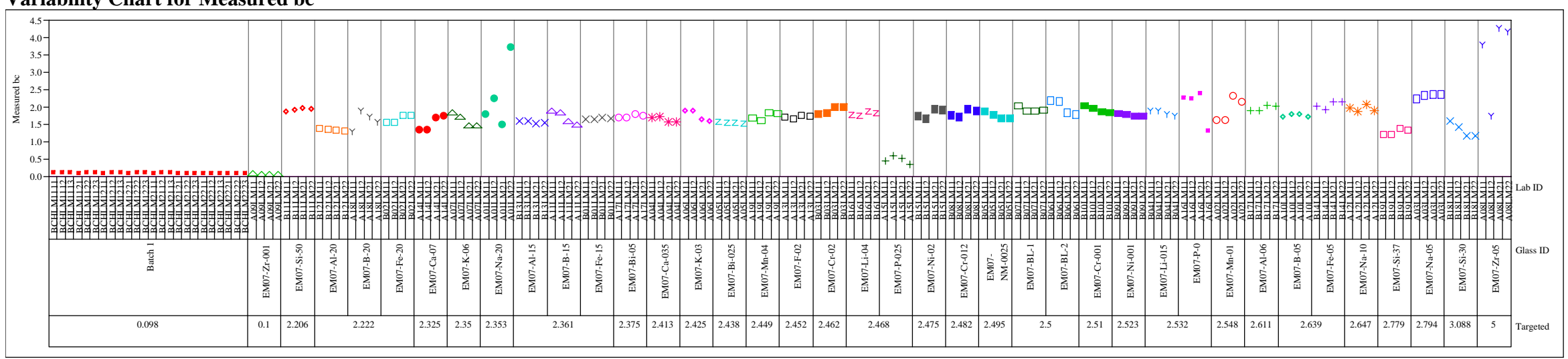


Figure A3. Measured and Measured Bias-Corrected Weight Percents for Each Analyte Grouped by Preparation Method by Lab ID within Glass ID

Variability Gage Prep=PF, Analyte=B2O3 (wt \%)

Variability Chart for Measured

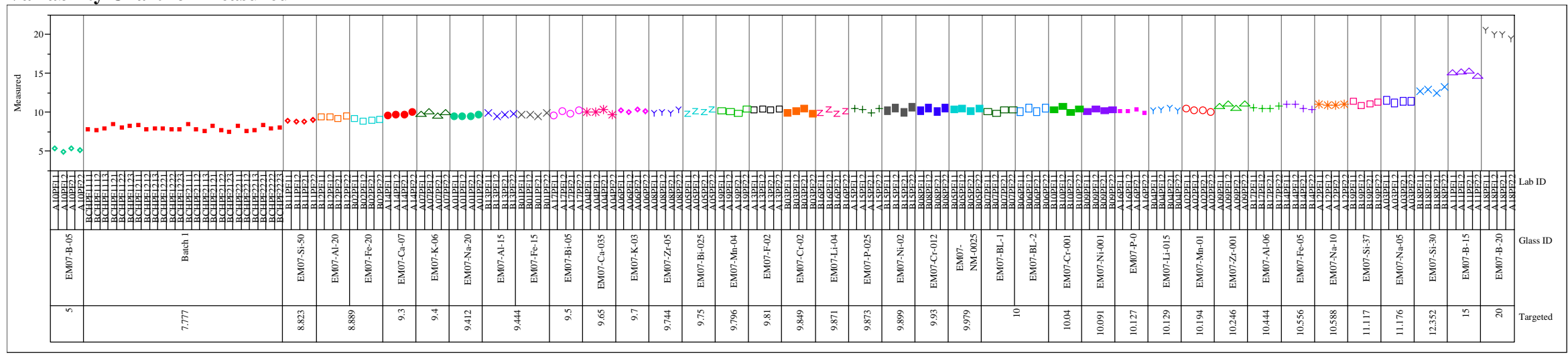

Variability Gage Prep=PF, Analyte=B2O3 (wt \%)

Variability Chart for Measured bc

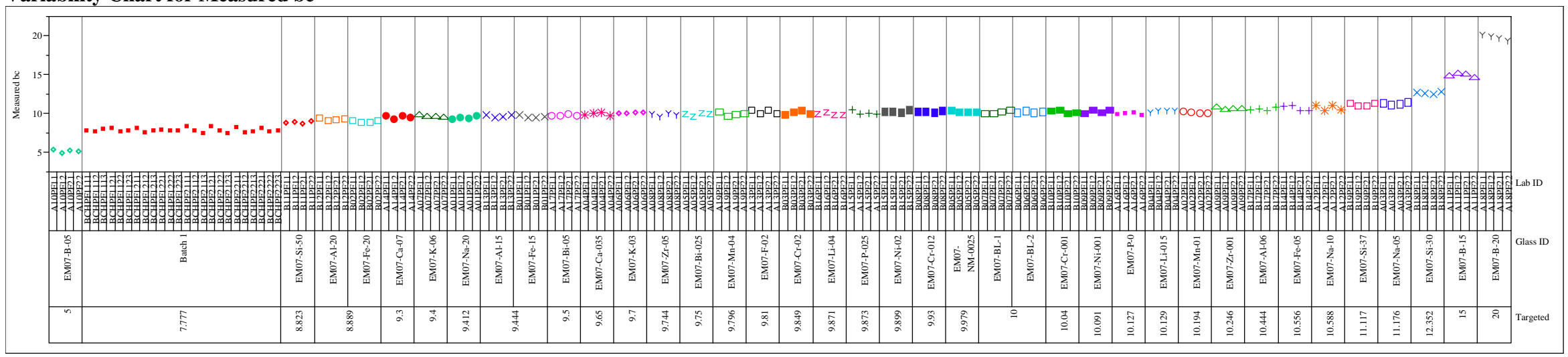


Figure A3. Measured and Measured Bias-Corrected Weight Percents for Each Analyte Grouped by Preparation Method by Lab ID within Glass ID

Variability Gage Prep=PF, Analyte=Li2O (wt \%)

Variability Chart for Measured

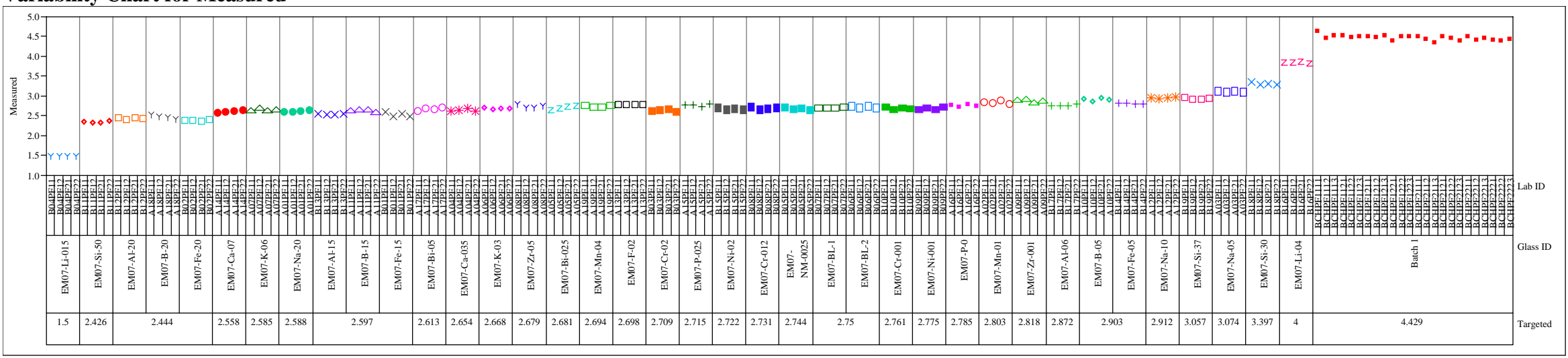

Variability Gage Prep=PF, Analyte $=$ Li2O (wt \%)

Variability Chart for Measured bc

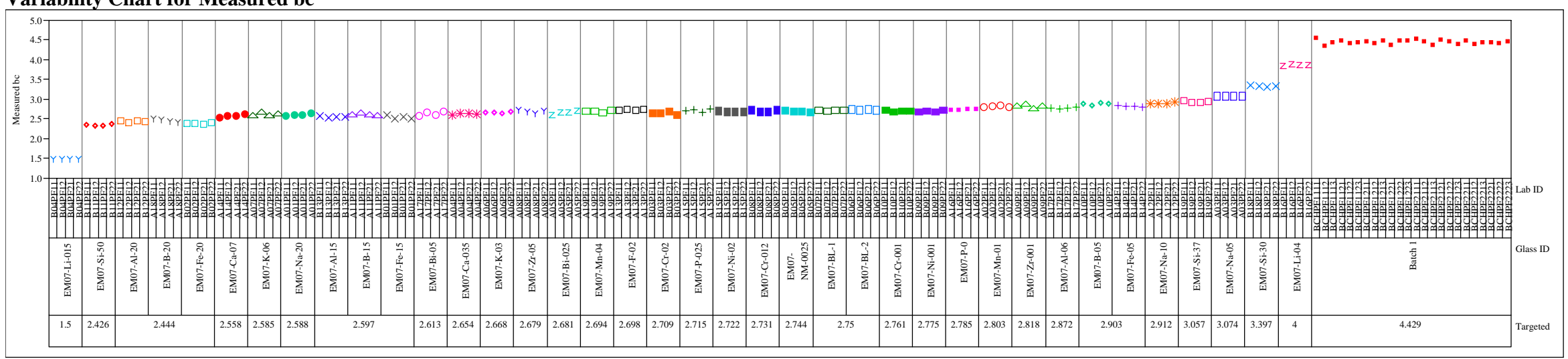


Figure A3. Measured and Measured Bias-Corrected Weight Percents for Each Analyte Grouped by Preparation Method by Lab ID within Glass ID

Variability Gage Prep=PF, Analyte=SiO2 (wt \%)

Variability Chart for Measured

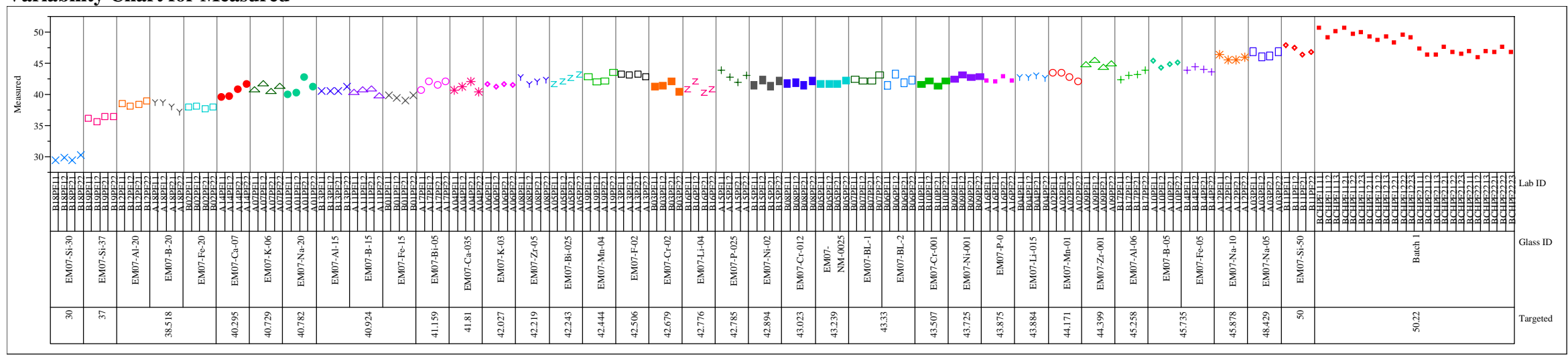

Variability Gage Prep=PF, Analyte=SiO2 (wt\%)

Variability Chart for Measured bc

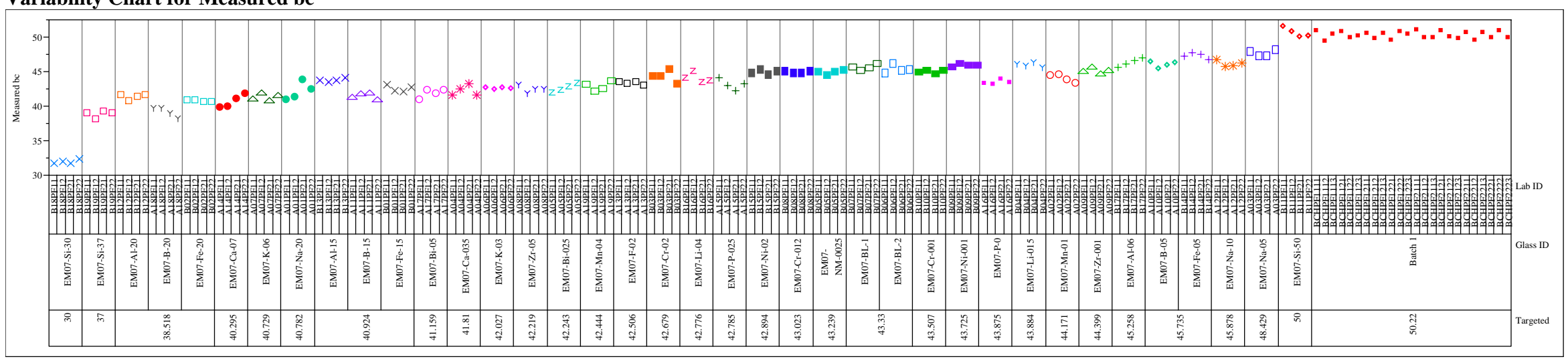


Figure A4. Average Measured and Bias-Corrected (bc) Versus Targeted Compositions by Glass \# by Analyte

Overlay Plot Analyte=Al2O3 (wt \%)

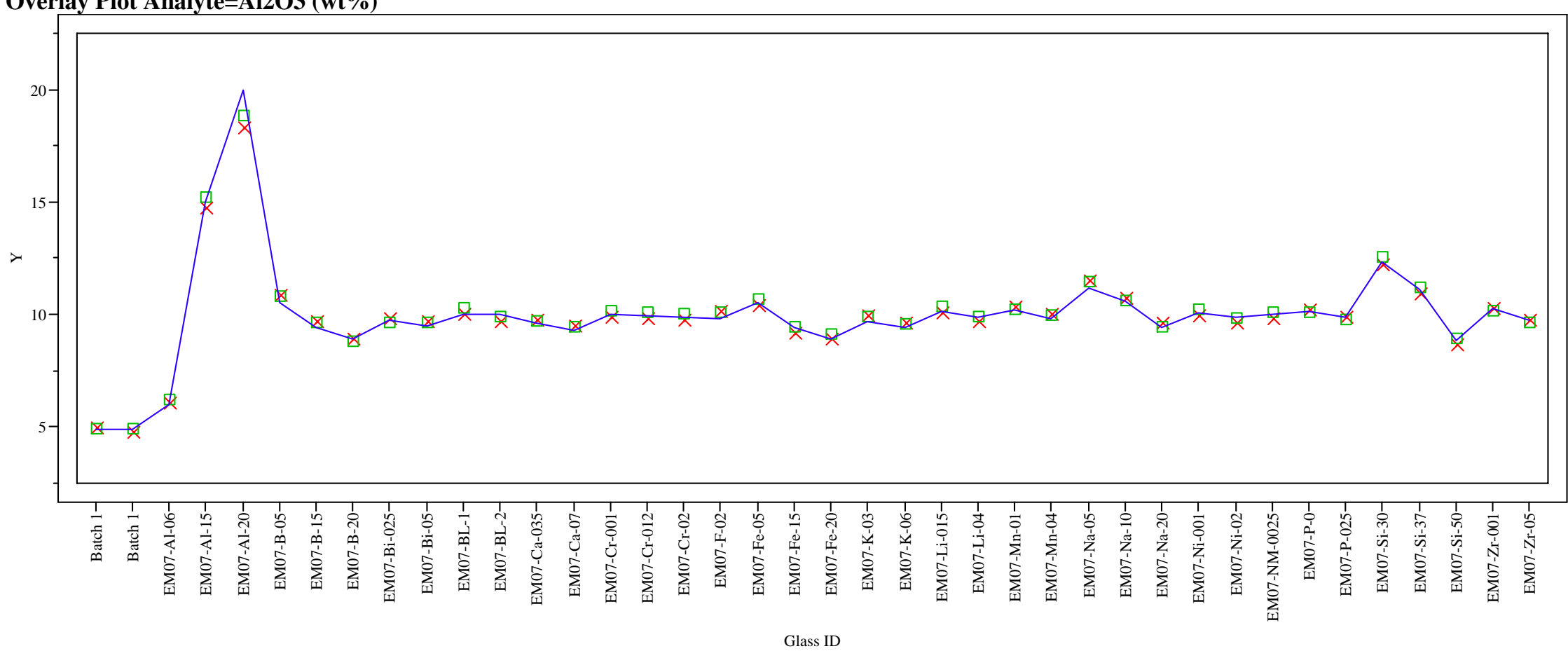

Y $\times$ Measured

- Measured bc — Targeted 
Figure A4. Average Measured and Bias-Corrected (bc) Versus Targeted Compositions by Glass \# by Analyte

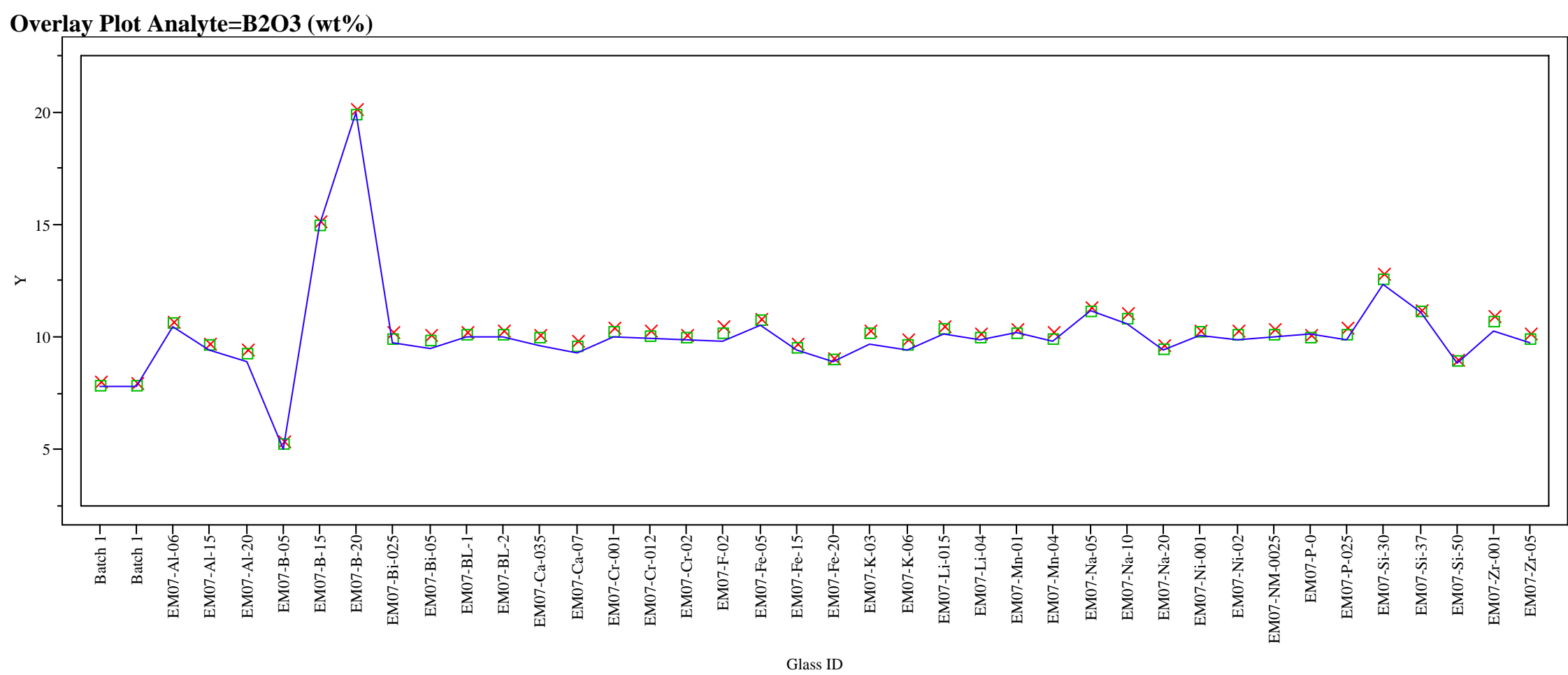

Y X Measured $\square$ Measured bc $\quad$ Targeted 
Figure A4. Average Measured and Bias-Corrected (bc) Versus Targeted Compositions by Glass \# by Analyte

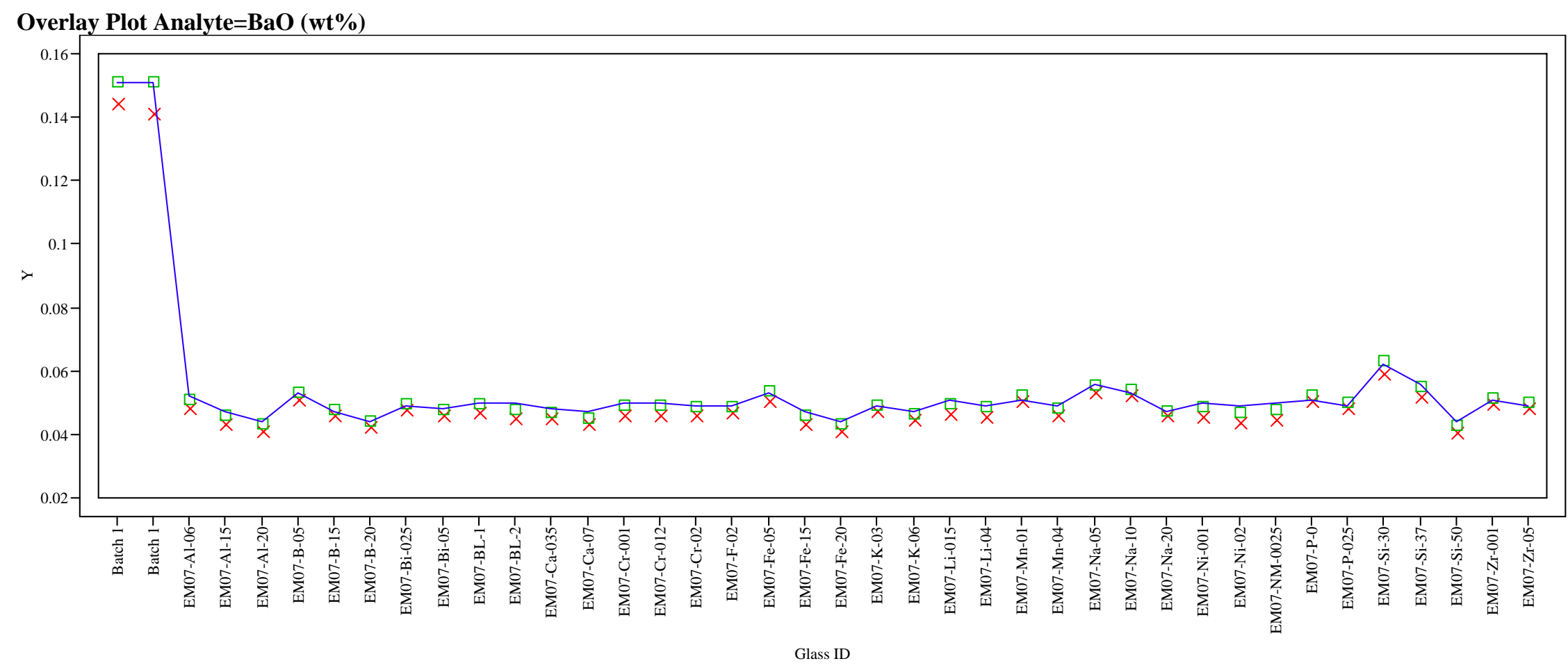

Y $\times$ Measured

- Measured bc

- Targeted 
Figure A4. Average Measured and Bias-Corrected (bc) Versus Targeted Compositions by Glass \# by Analyte

Overlay Plot Analyte=Bi2O3 (wt \%)

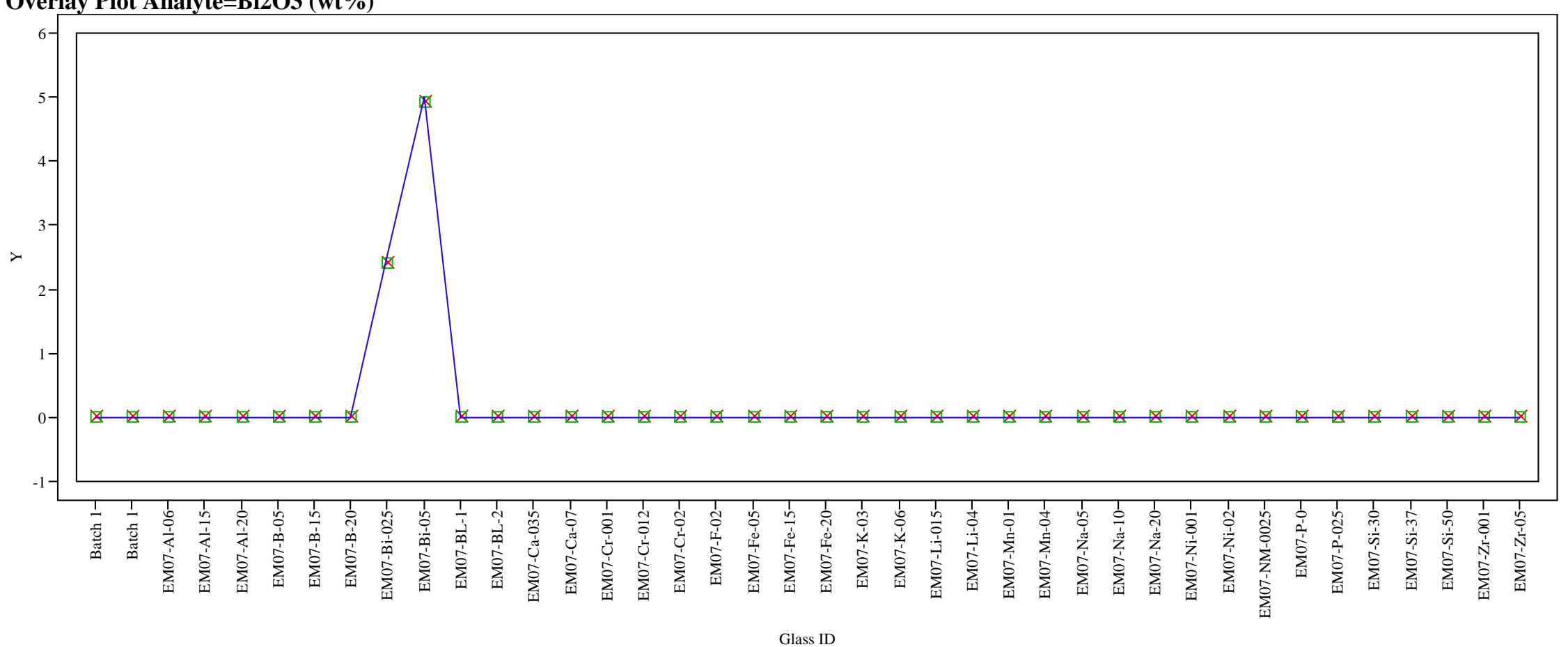

$\mathrm{Y} \times$ Measured $\square$ Measured bc $\quad$ Targeted 
Figure A4. Average Measured and Bias-Corrected (bc) Versus Targeted Compositions by Glass \# by Analyte

Overlay Plot Analyte $=\mathrm{CaO}($ wt $\%)$

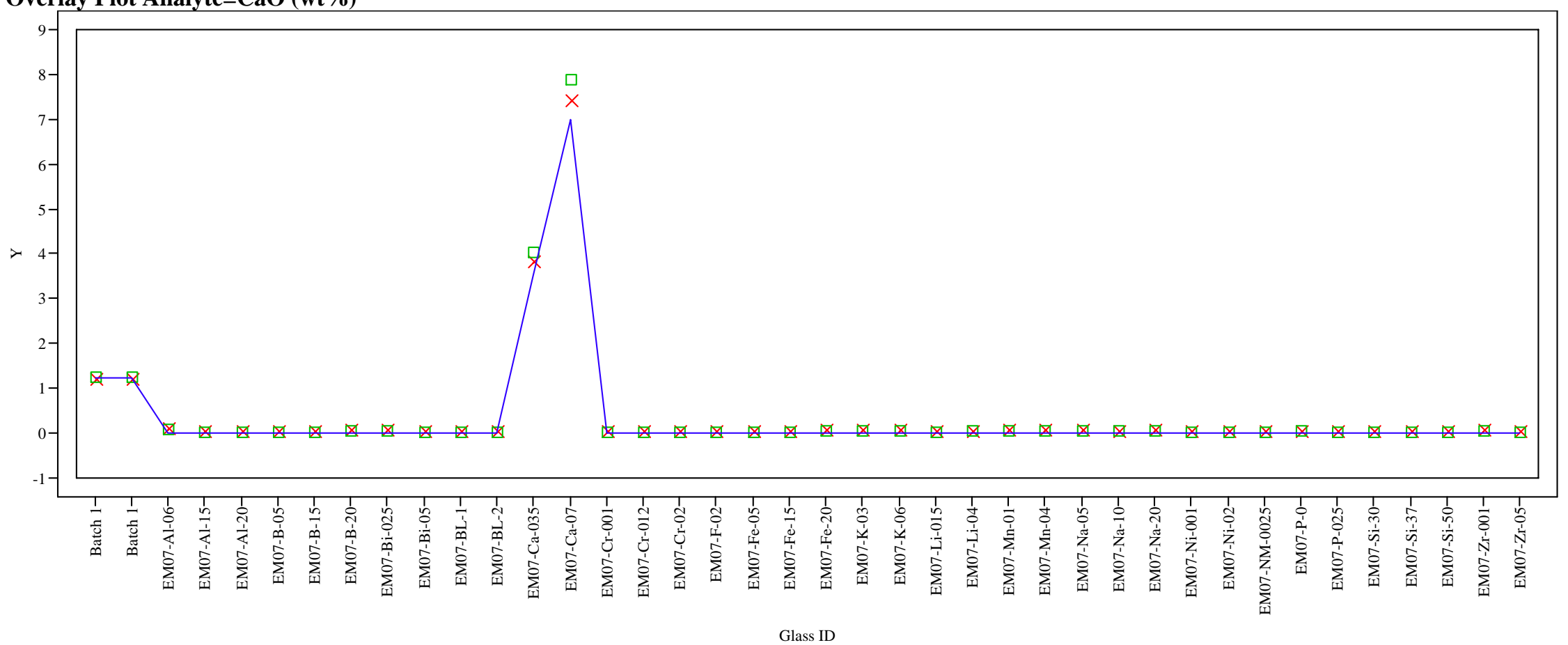

Y X Measured $\square$ Measured bc — Targeted 
Figure A4. Average Measured and Bias-Corrected (bc) Versus Targeted Compositions by Glass \# by Analyte

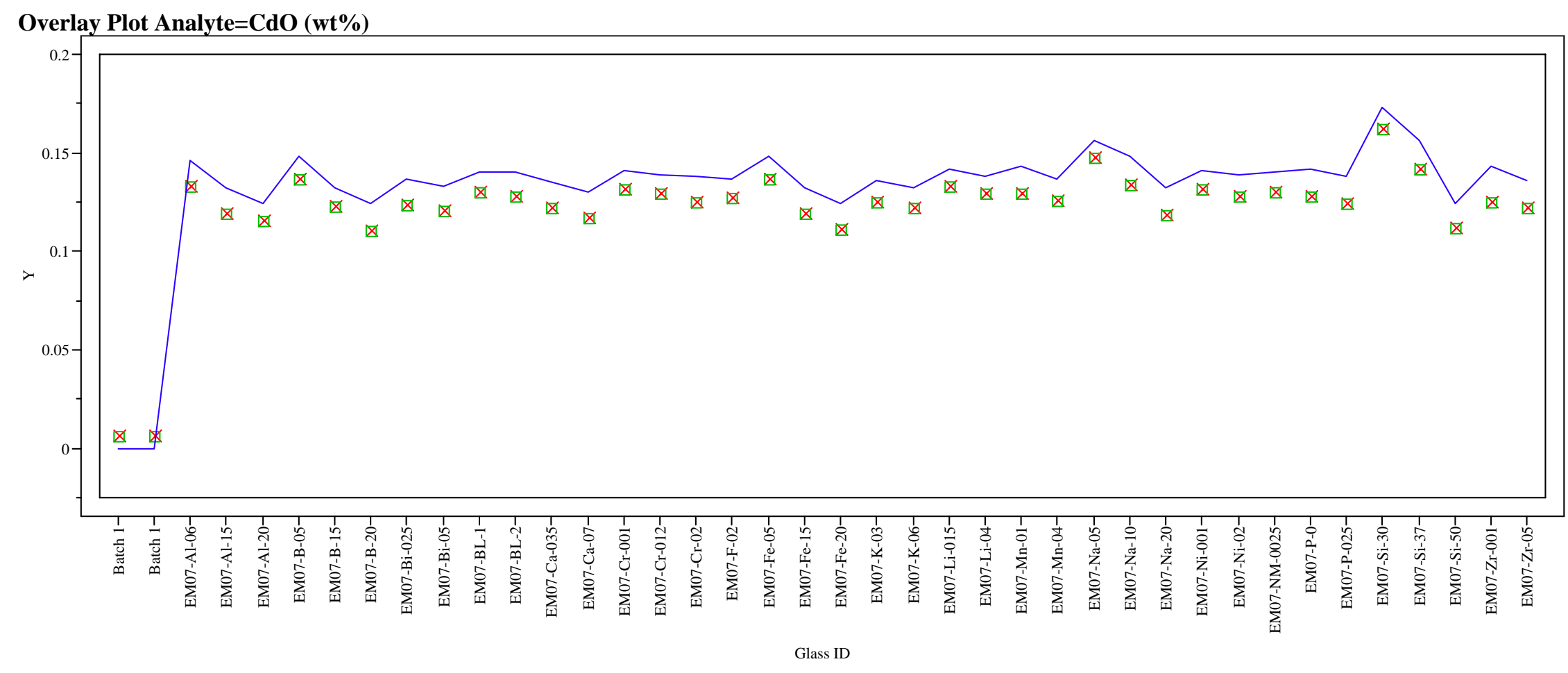

$\mathrm{Y} \times$ Measured

a Measured bc - Targeted 
Figure A4. Average Measured and Bias-Corrected (bc) Versus Targeted Compositions by Glass \# by Analyte

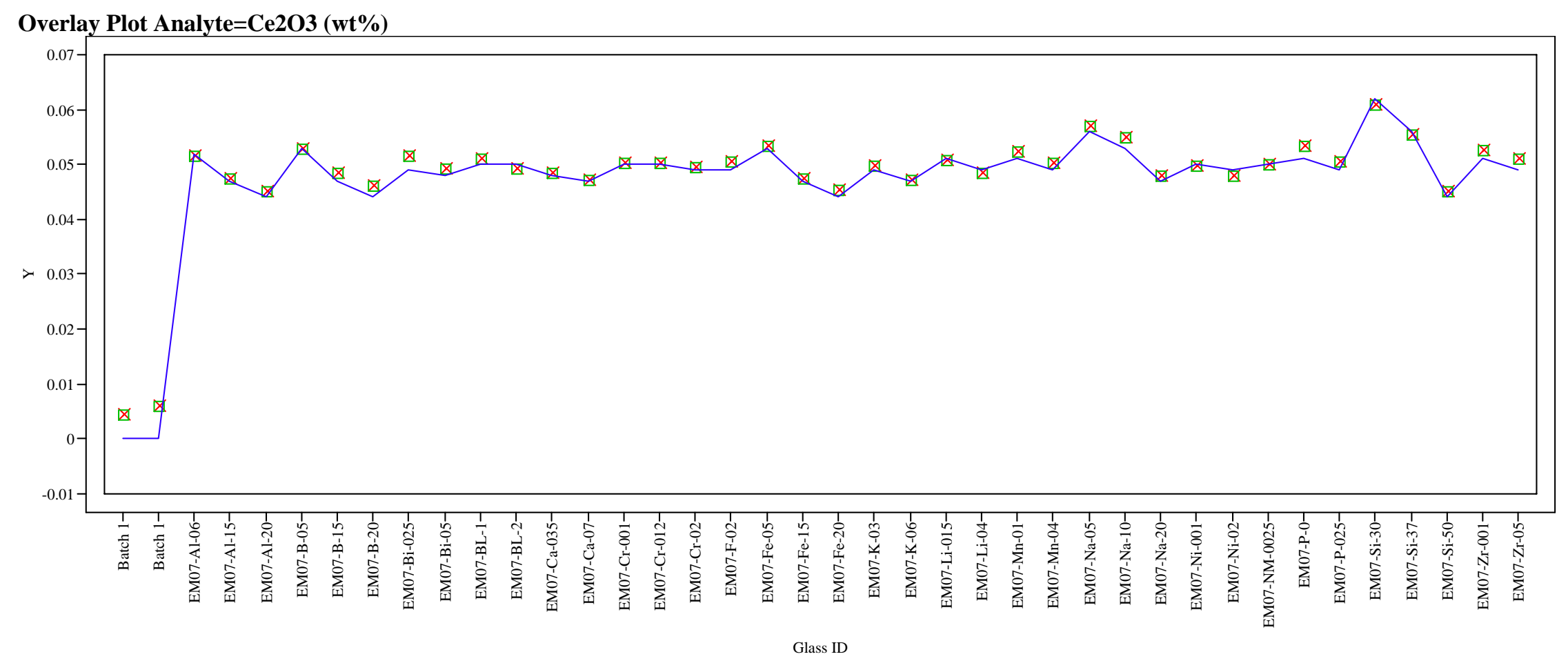


Figure A4. Average Measured and Bias-Corrected (bc) Versus Targeted Compositions by Glass \# by Analyte

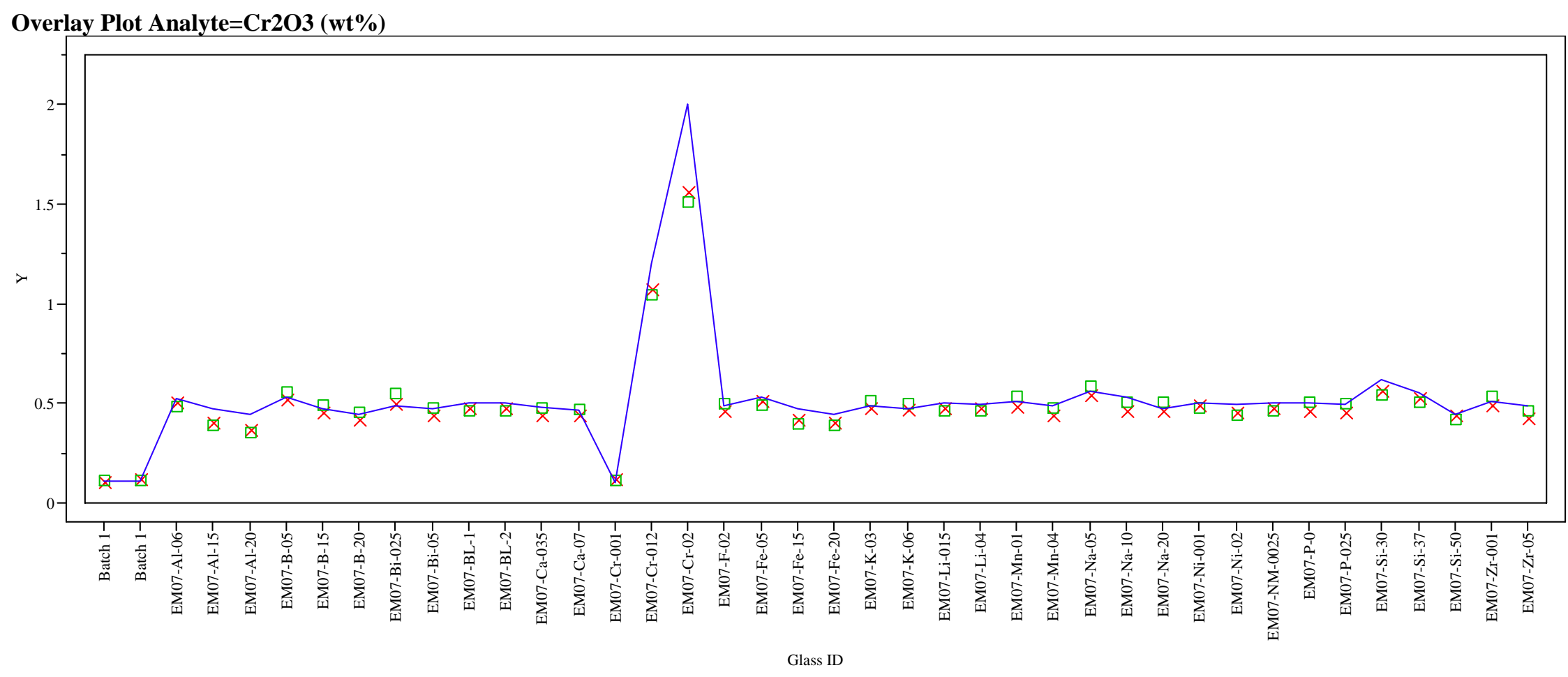

Y $\times$ Measured

- Measured bc - Targeted 
Figure A4. Average Measured and Bias-Corrected (bc) Versus Targeted Compositions by Glass \# by Analyte

Overlay Plot Analyte=F (wt \%)

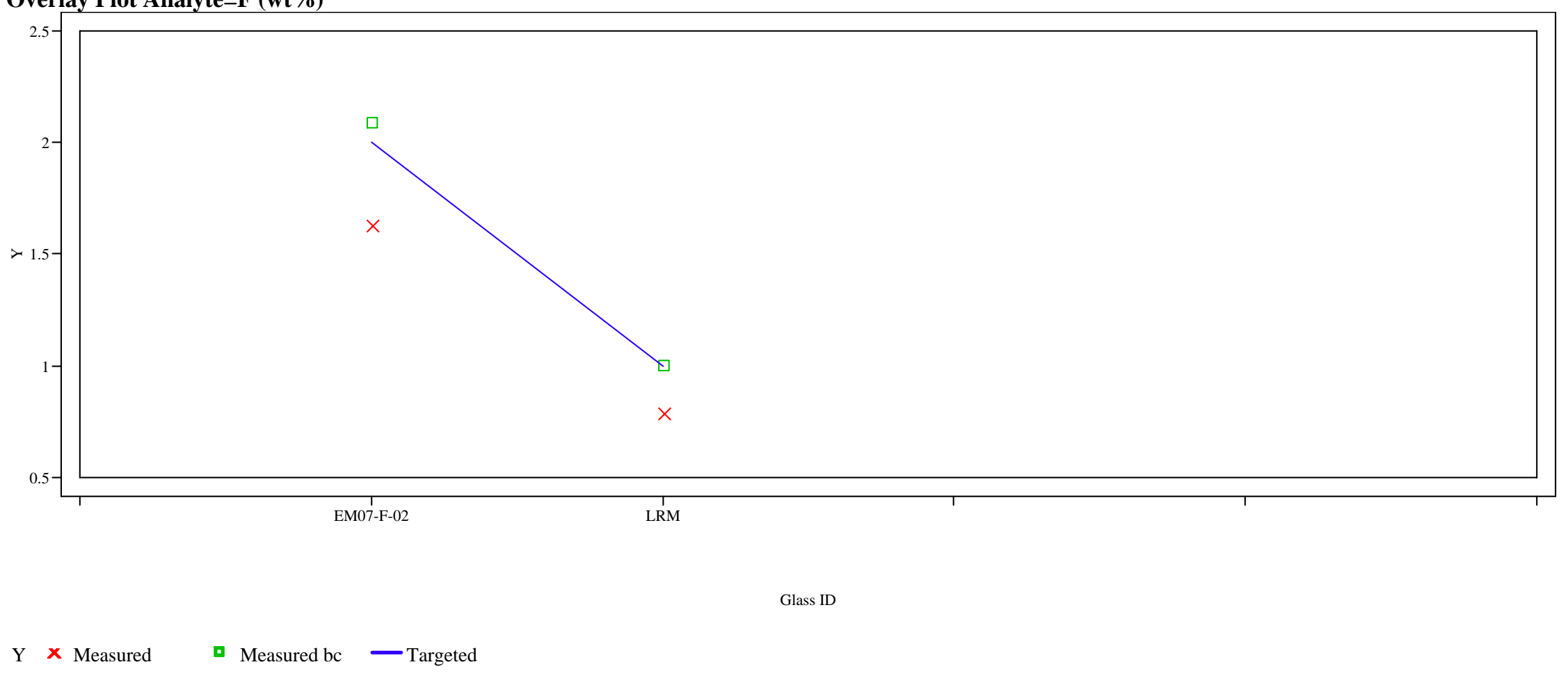


Figure A4. Average Measured and Bias-Corrected (bc) Versus Targeted Compositions by Glass \# by Analyte

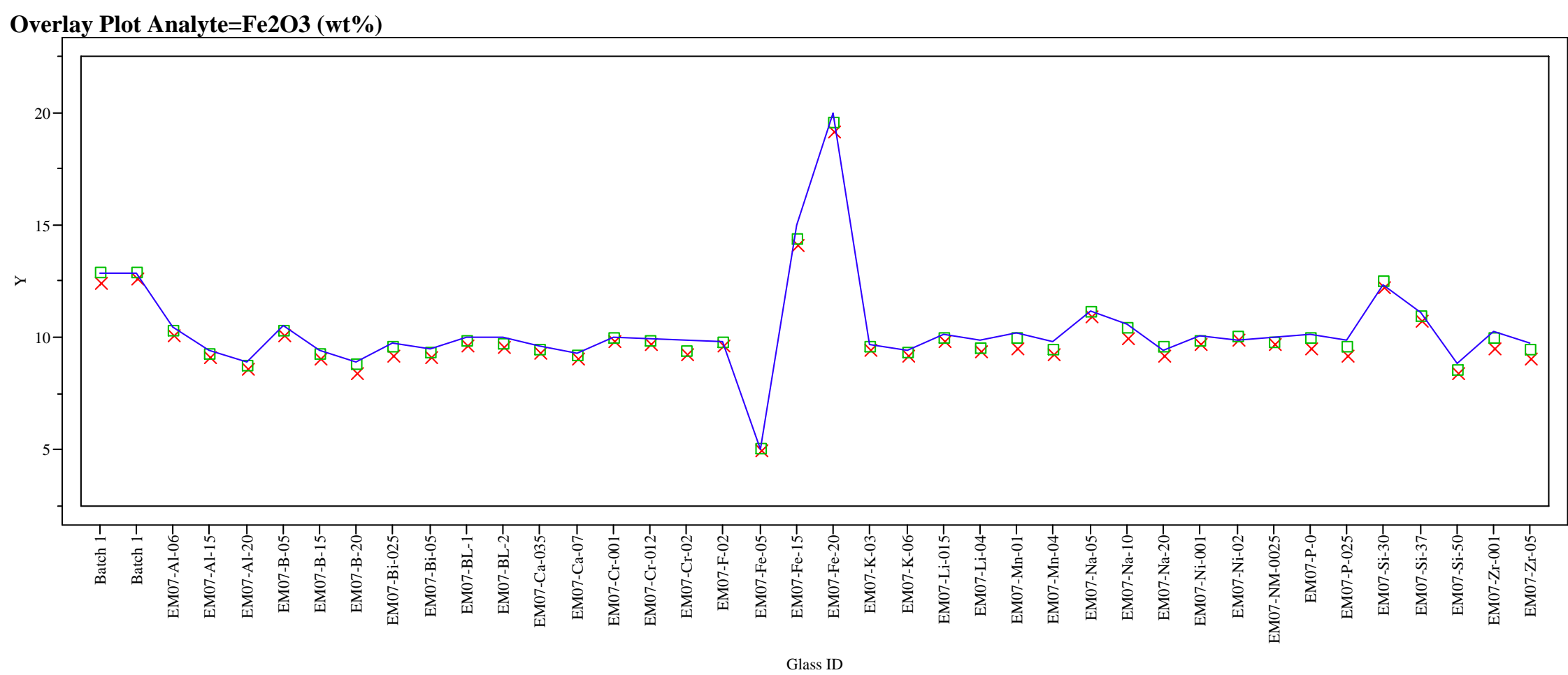

Y X Measured $\square$ Measured bc $\quad$ Targeted 
Figure A4. Average Measured and Bias-Corrected (bc) Versus Targeted Compositions by Glass \# by Analyte

Overlay Plot Analyte=K2O (wt\%)

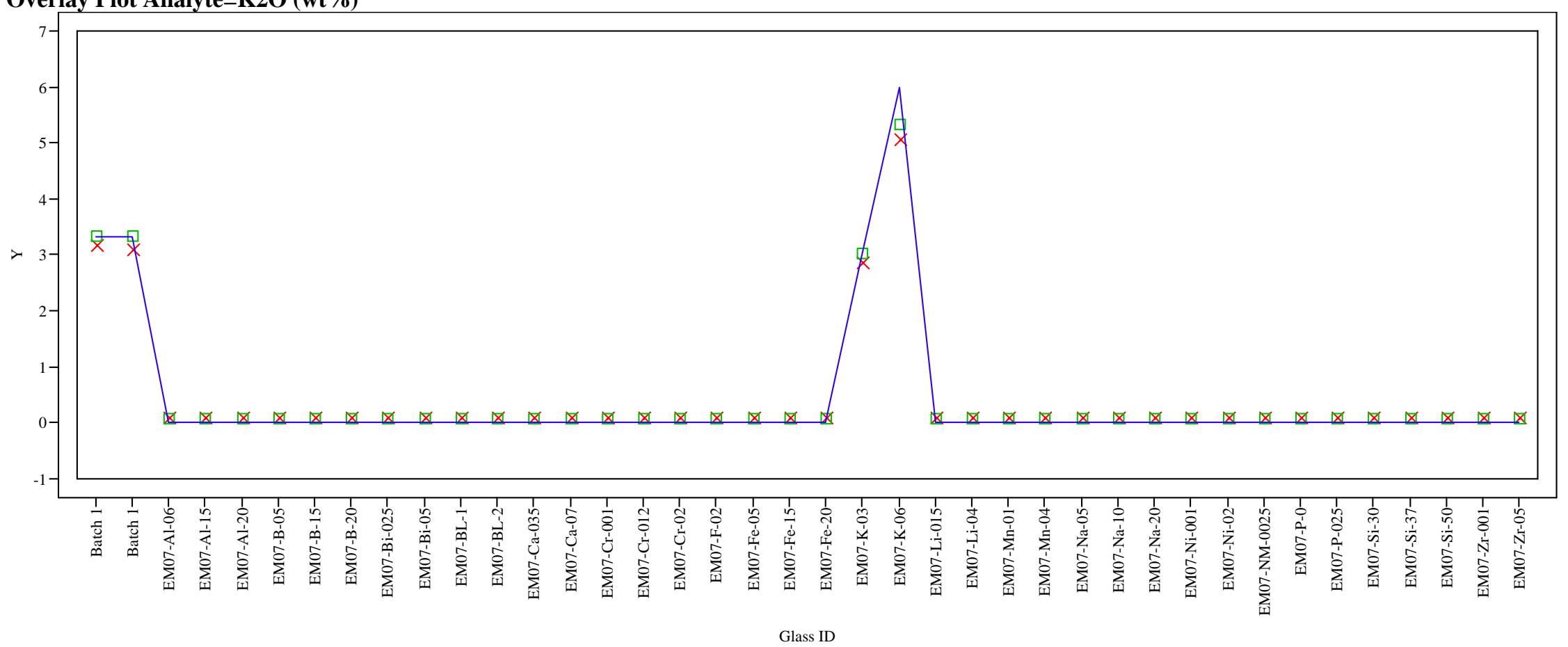

Y X Measured $\square$ Measured bc — Targeted 
Figure A4. Average Measured and Bias-Corrected (bc) Versus Targeted Compositions by Glass \# by Analyte

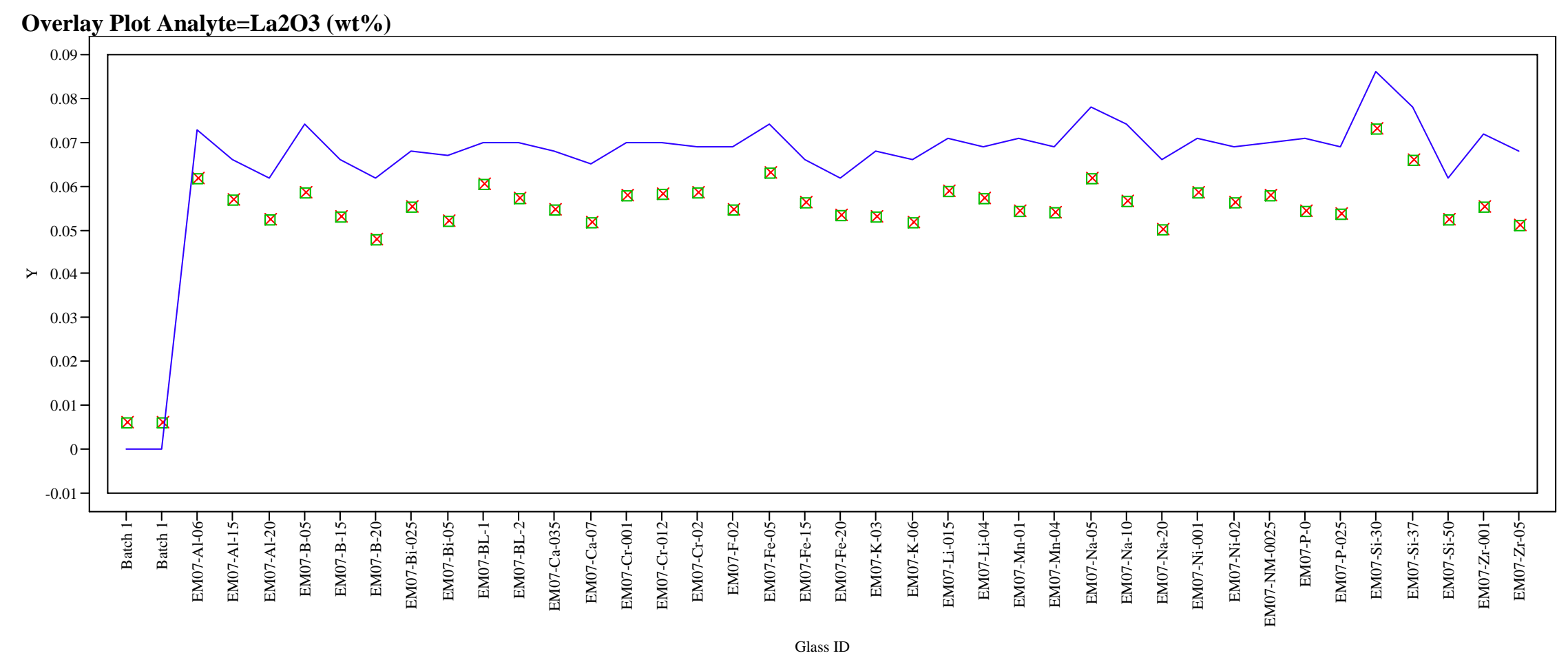

Y $\times$ Measured

- Measured bc - Targeted 
Figure A4. Average Measured and Bias-Corrected (bc) Versus Targeted Compositions by Glass \# by Analyte

Overlay Plot Analyte $=\mathrm{Li2O}(\mathrm{wt} \%)$

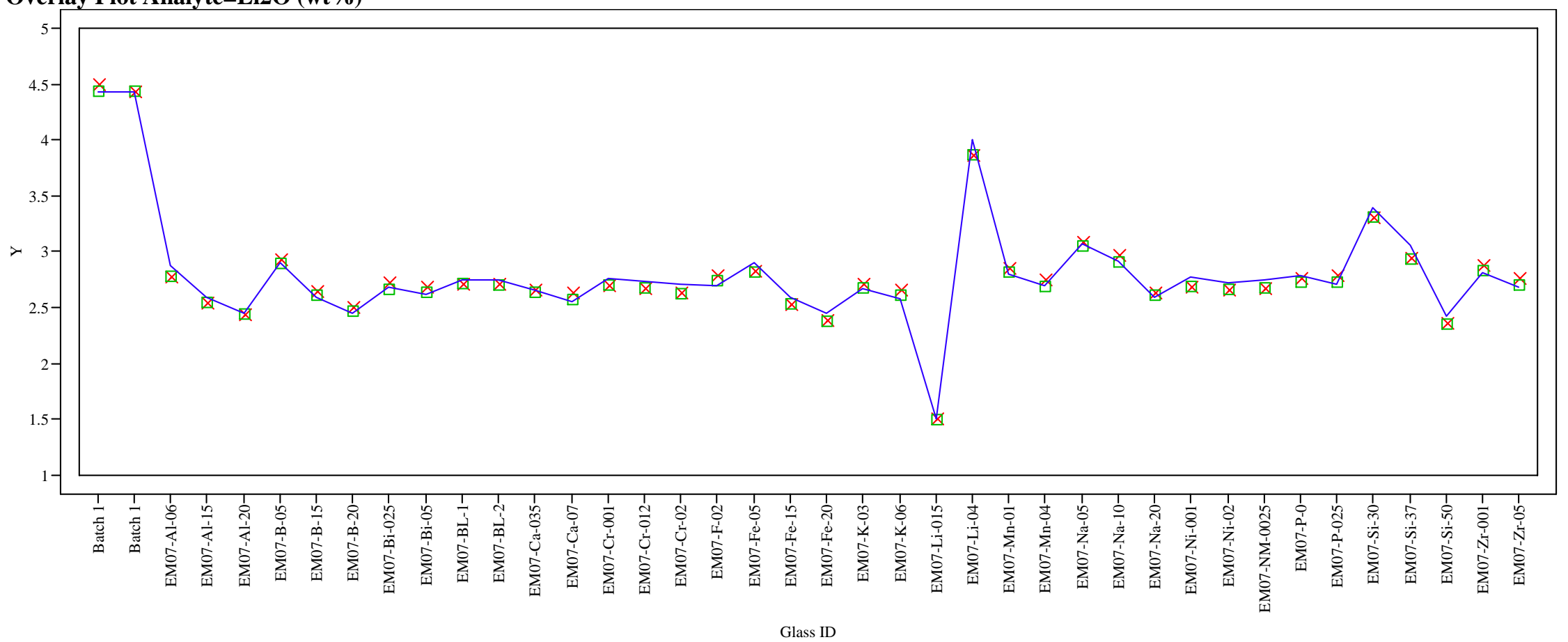

Y X Measured $\square$ Measured bc - Targeted 
Figure A4. Average Measured and Bias-Corrected (bc) Versus Targeted Compositions by Glass \# by Analyte

Overlay Plot Analyte=MgO (wt\%)

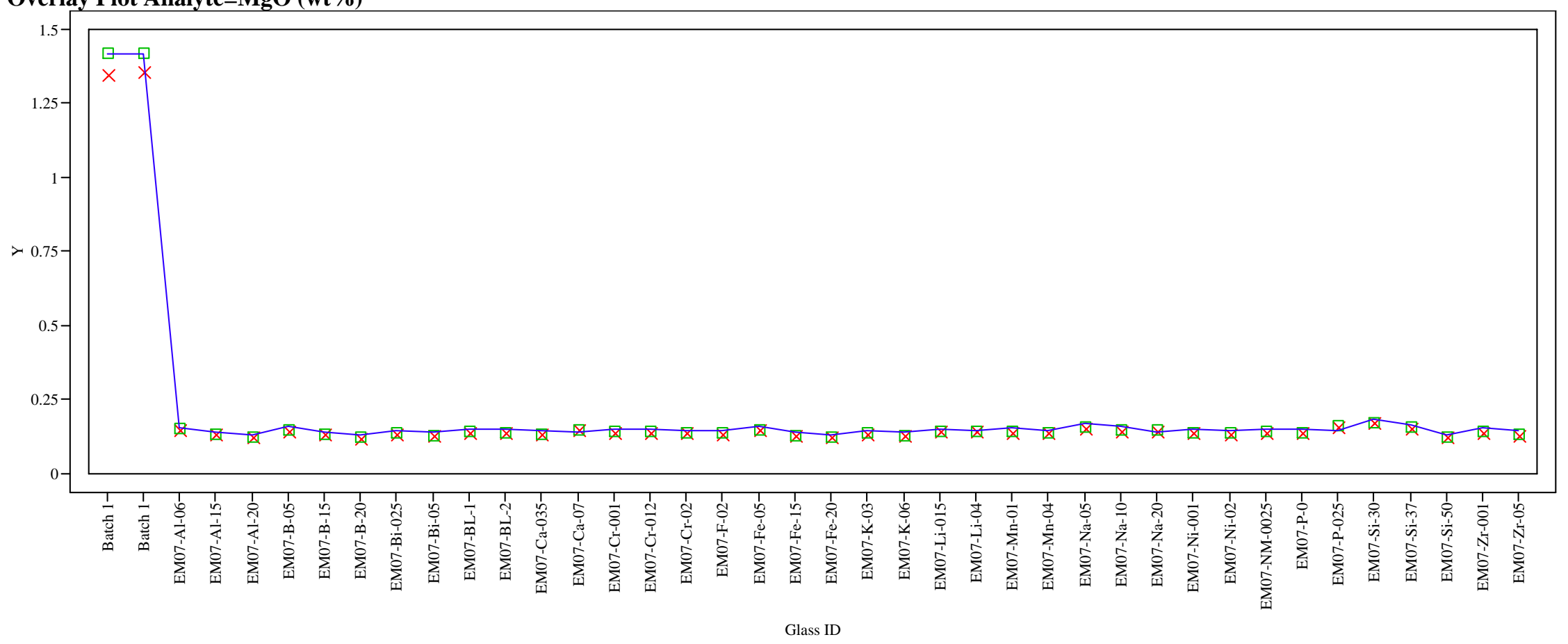

$\mathrm{Y} \times$ Measured

- Measured bc

- Targeted 
Figure A4. Average Measured and Bias-Corrected (bc) Versus Targeted Compositions by Glass \# by Analyte

Overlay Plot Analyte=MnO (wt \%)

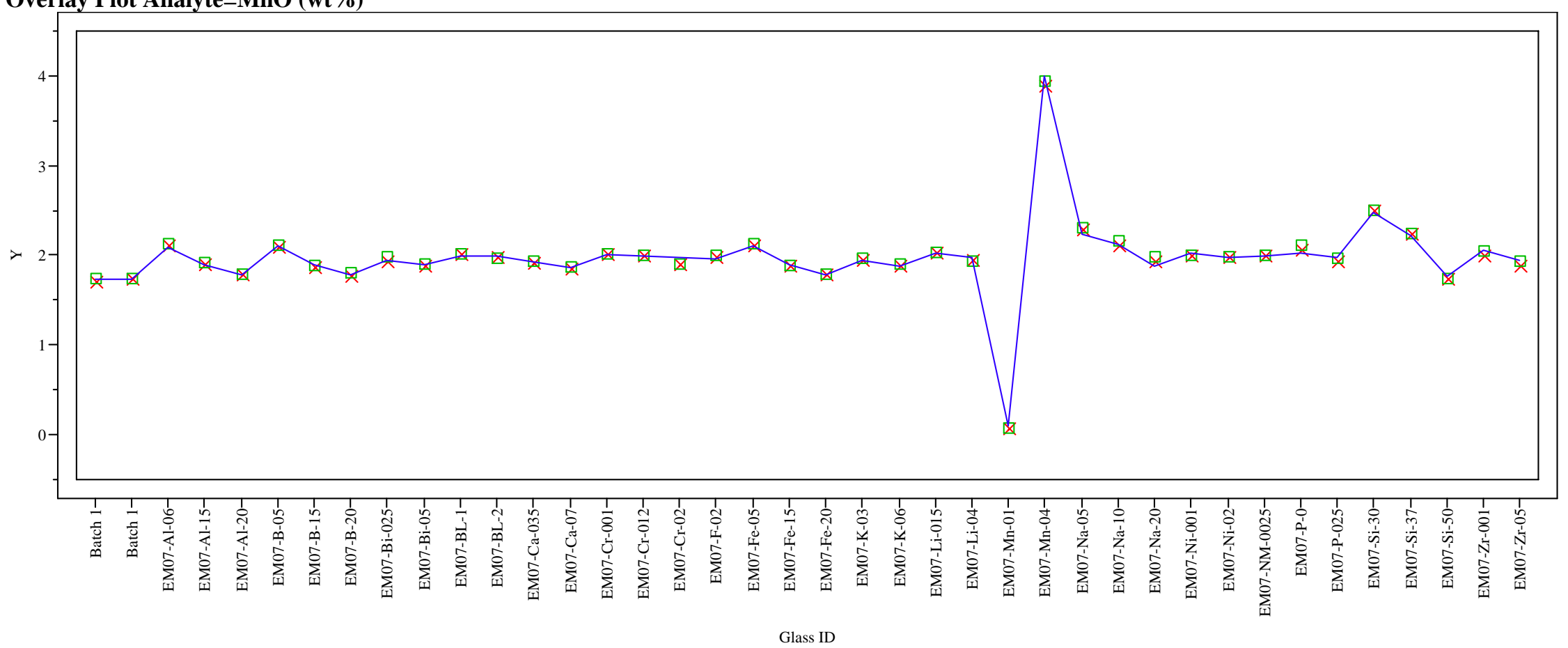

Y X Measured $\square$ Measured bc — Targeted 
Figure A4. Average Measured and Bias-Corrected (bc) Versus Targeted Compositions by Glass \# by Analyte

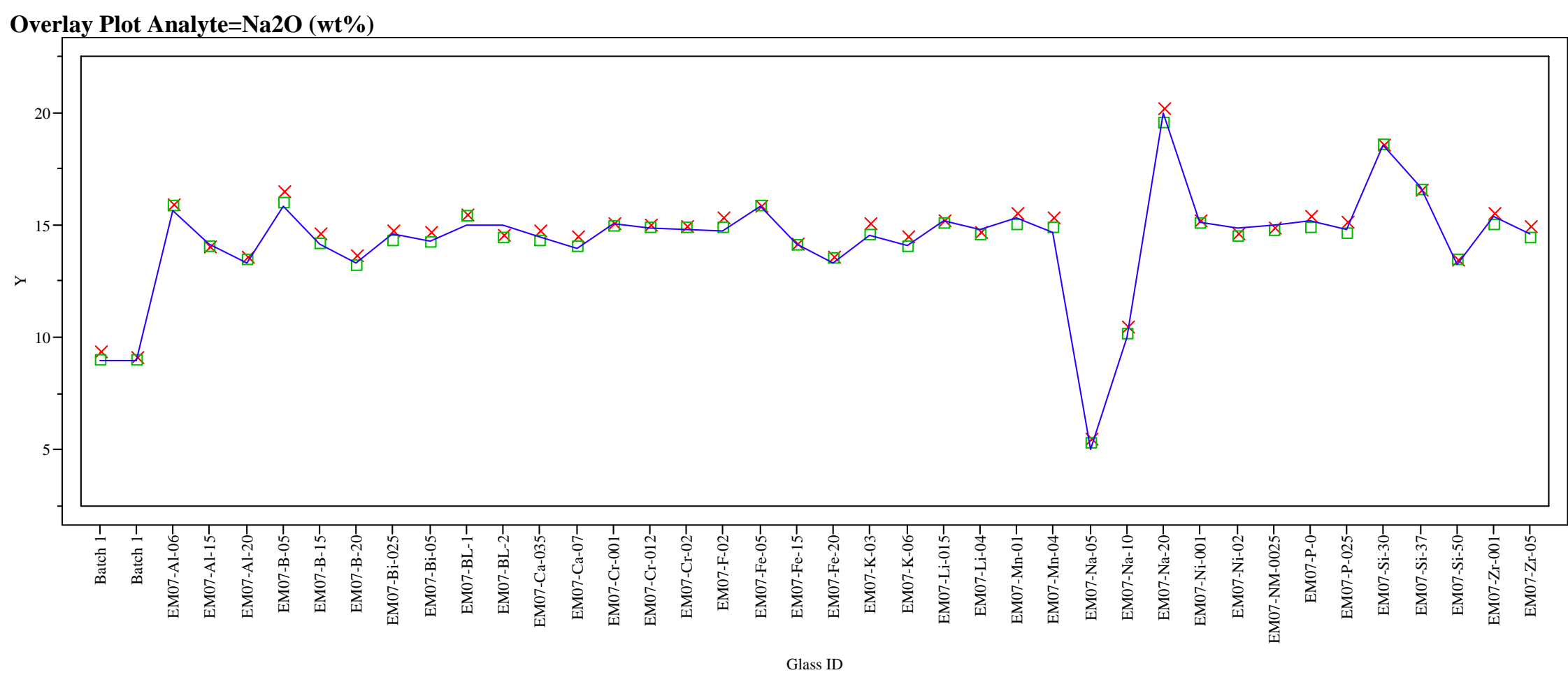

Y X Measured $\square$ Measured bc — Targeted 
Figure A4. Average Measured and Bias-Corrected (bc) Versus Targeted Compositions by Glass \# by Analyte

Overlay Plot Analyte=Nd2O3 (wt\%)

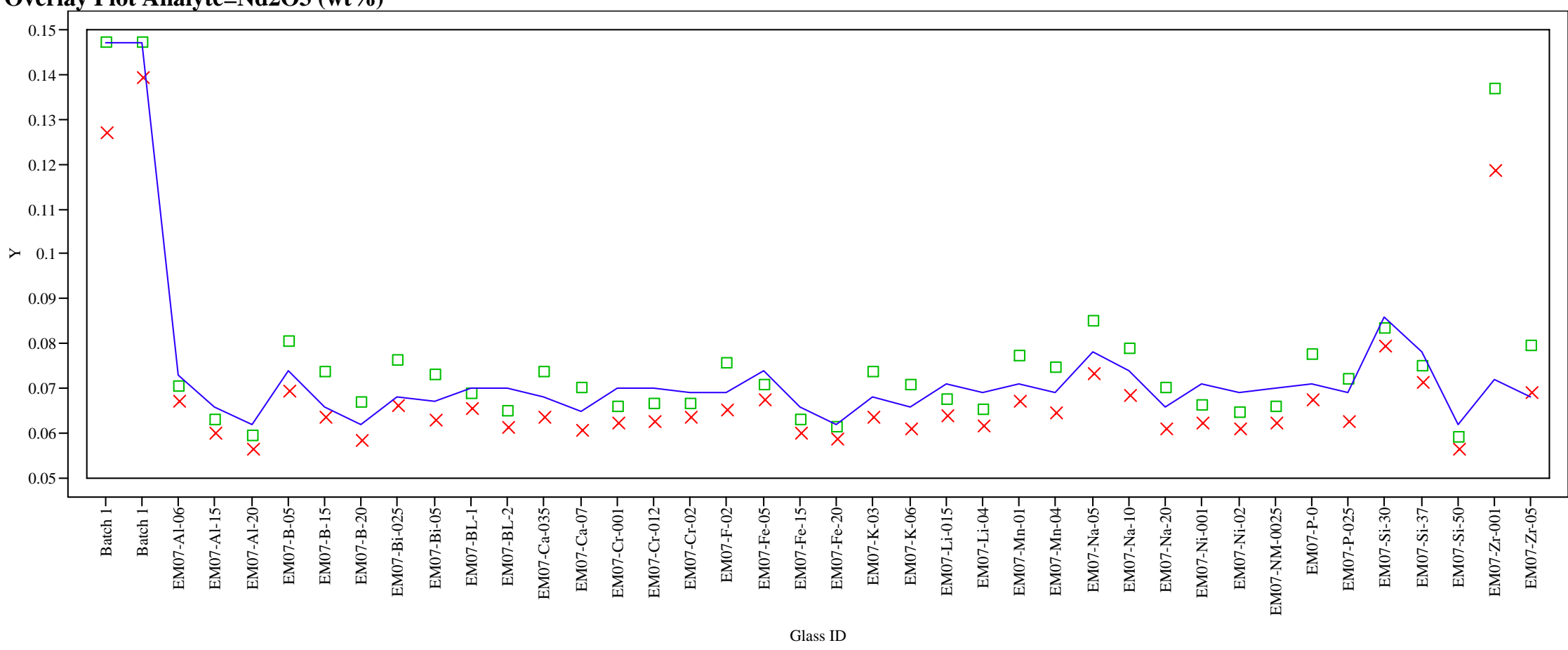

$\mathrm{Y} \times$ Measured $\quad$ Measured bc Targeted 
Figure A4. Average Measured and Bias-Corrected (bc) Versus Targeted Compositions by Glass \# by Analyte

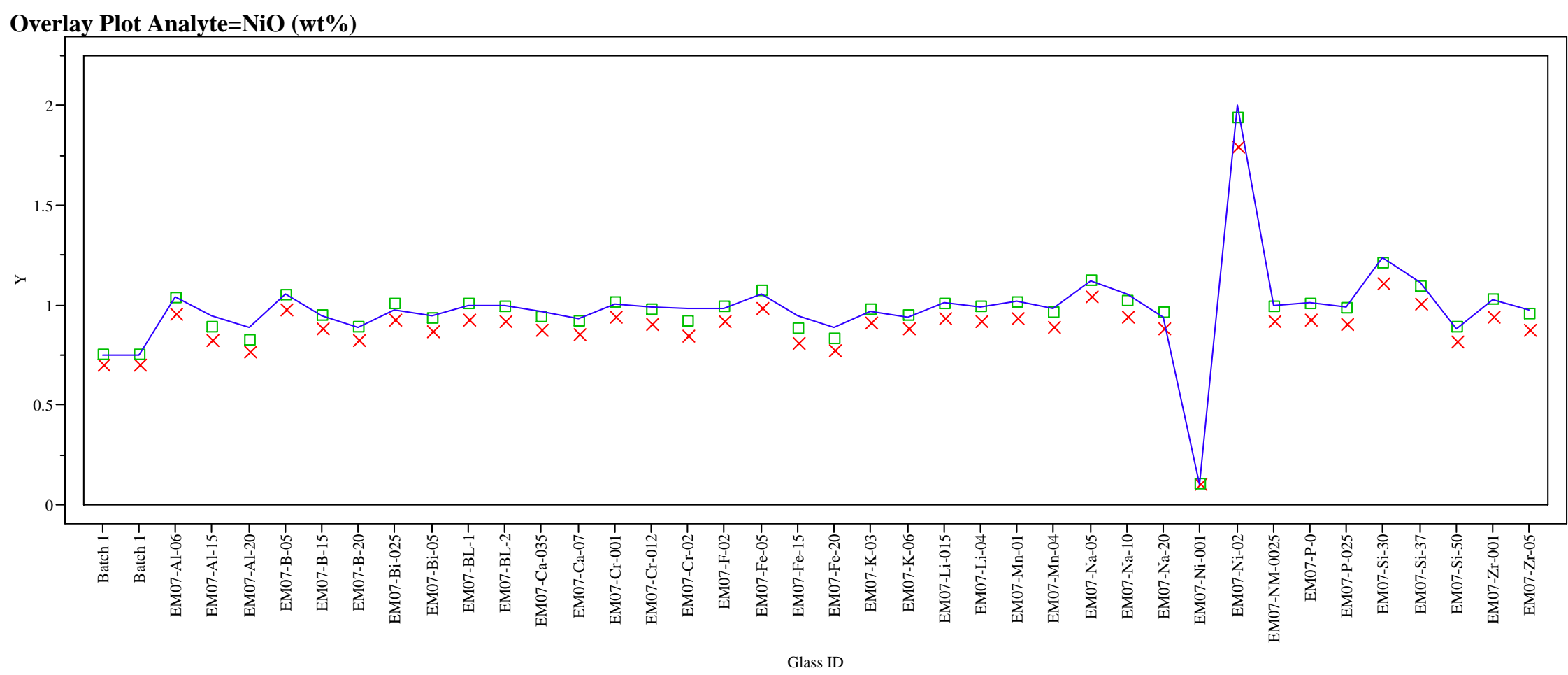

Y X Measured $\square$ Measured bc - Targeted 
Figure A4. Average Measured and Bias-Corrected (bc) Versus Targeted Compositions by Glass \# by Analyte

Overlay Plot Analyte=P2O5 (wt \%)

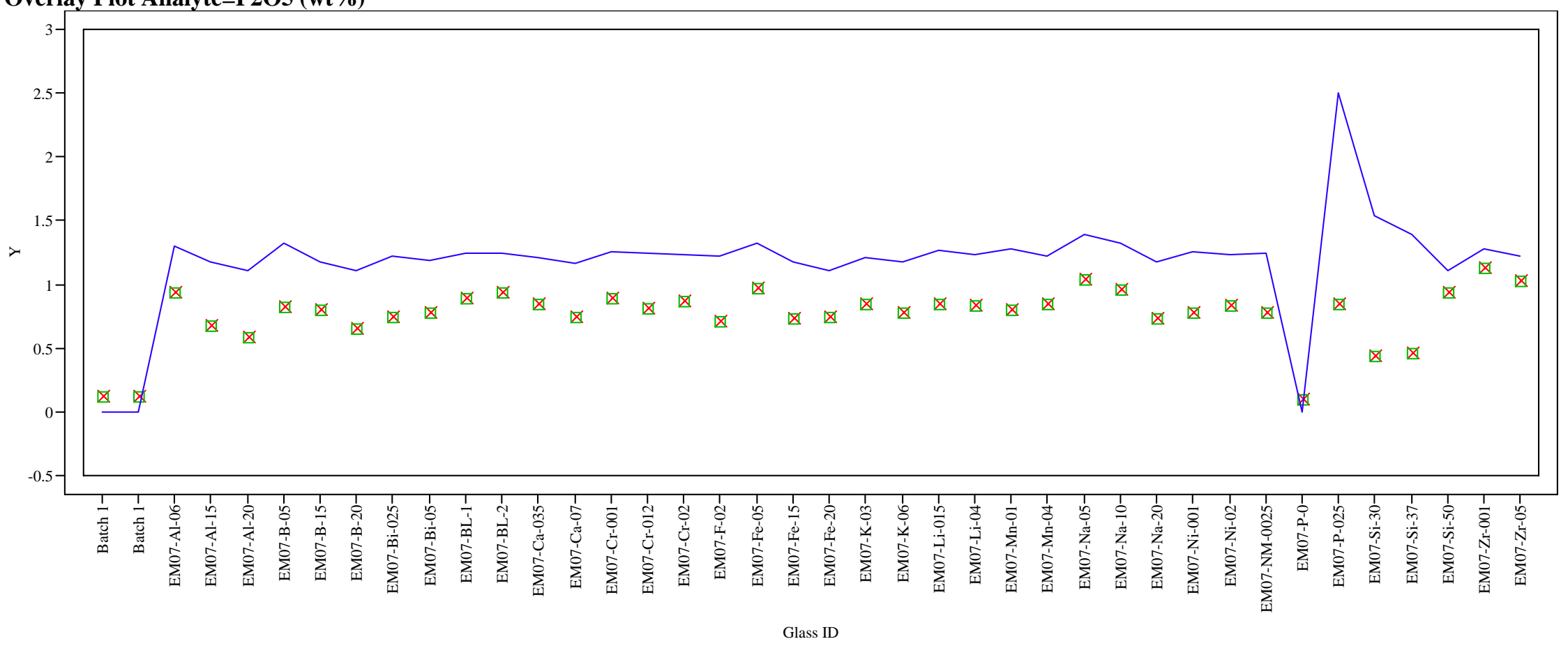

Y $\times$ Measured

Measured bc

Targeted 
Figure A4. Average Measured and Bias-Corrected (bc) Versus Targeted Compositions by Glass \# by Analyte

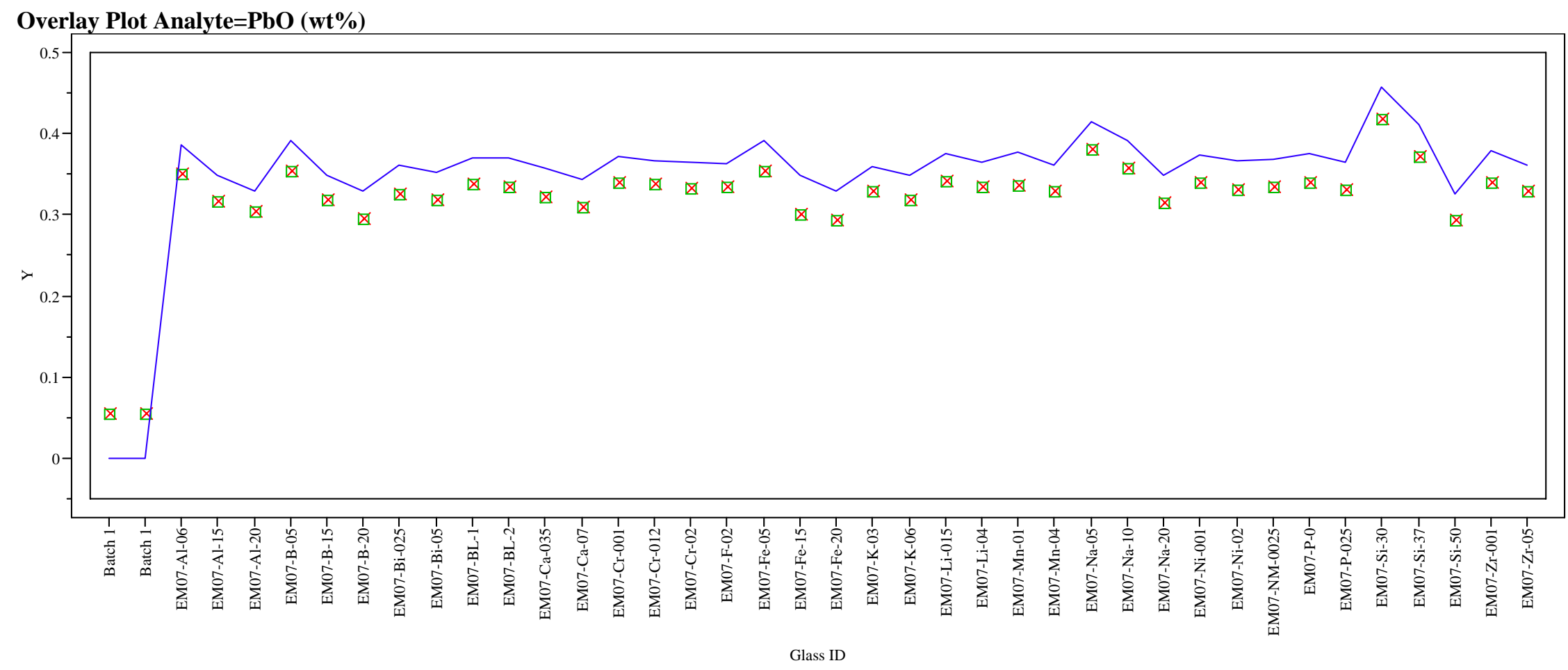

Y $\times$ Measured $\square$ Measured bc - Targeted 
Figure A4. Average Measured and Bias-Corrected (bc) Versus Targeted Compositions by Glass \# by Analyte

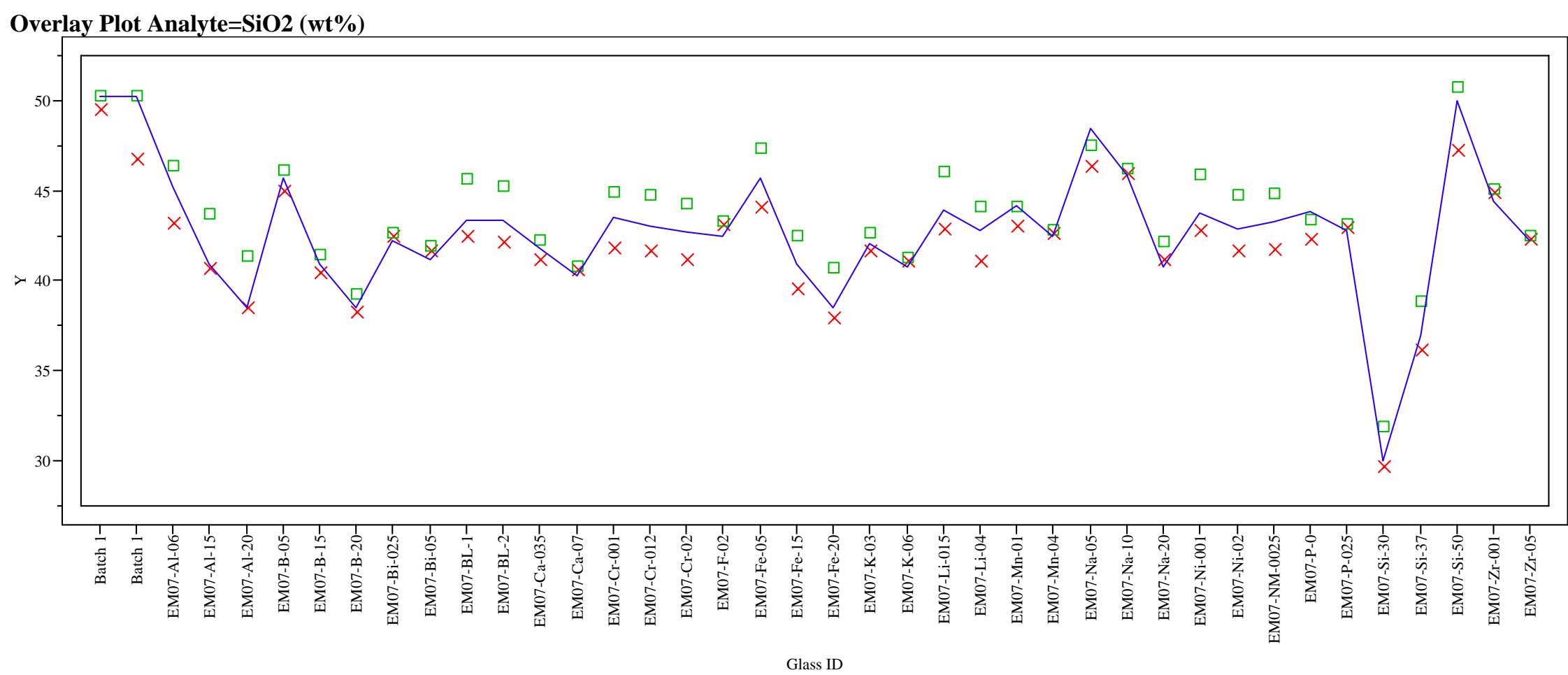

Y X Measured $\square$ Measured bc $\quad$ Targeted 
Figure A4. Average Measured and Bias-Corrected (bc) Versus Targeted Compositions by Glass \# by Analyte

Overlay Plot Analyte=TiO2 (wt\%)

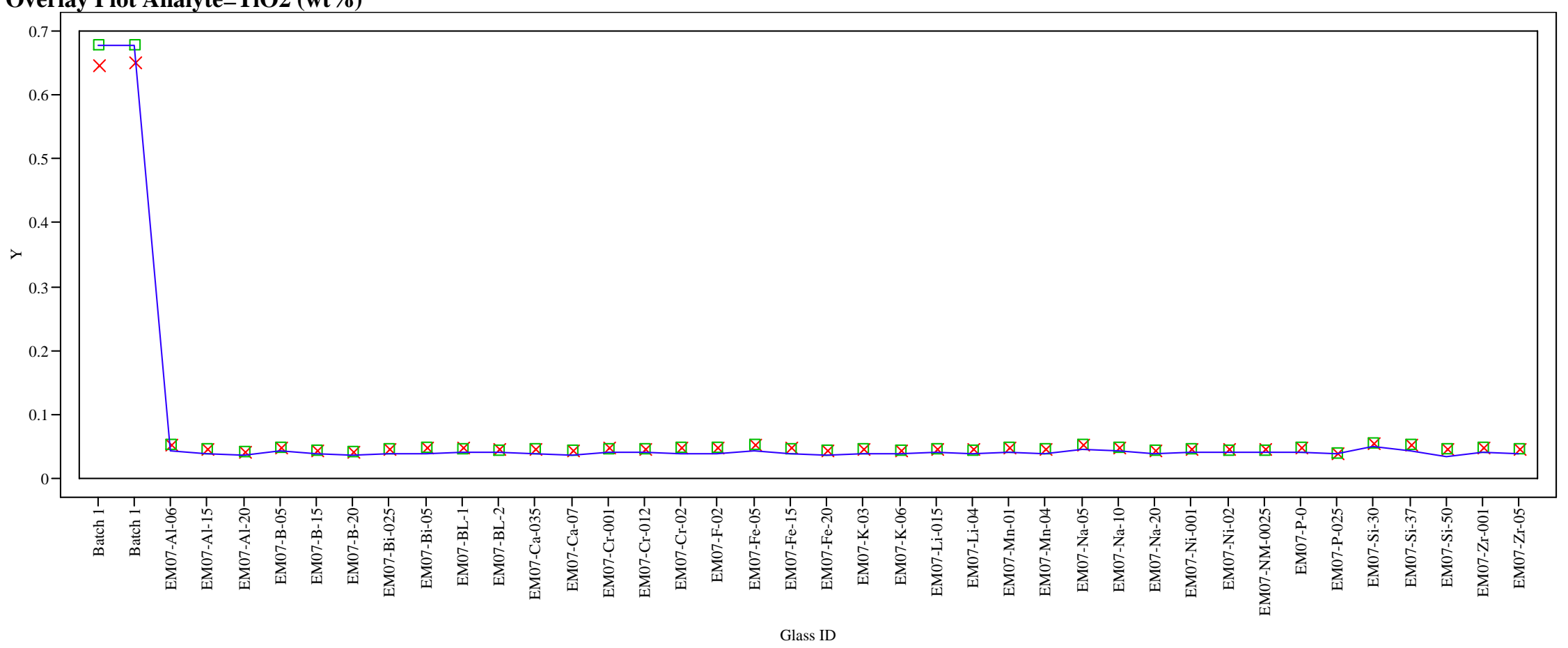

Y X Measured $\square$ Measured bc - Targeted 
Figure A4. Average Measured and Bias-Corrected (bc) Versus Targeted Compositions by Glass \# by Analyte

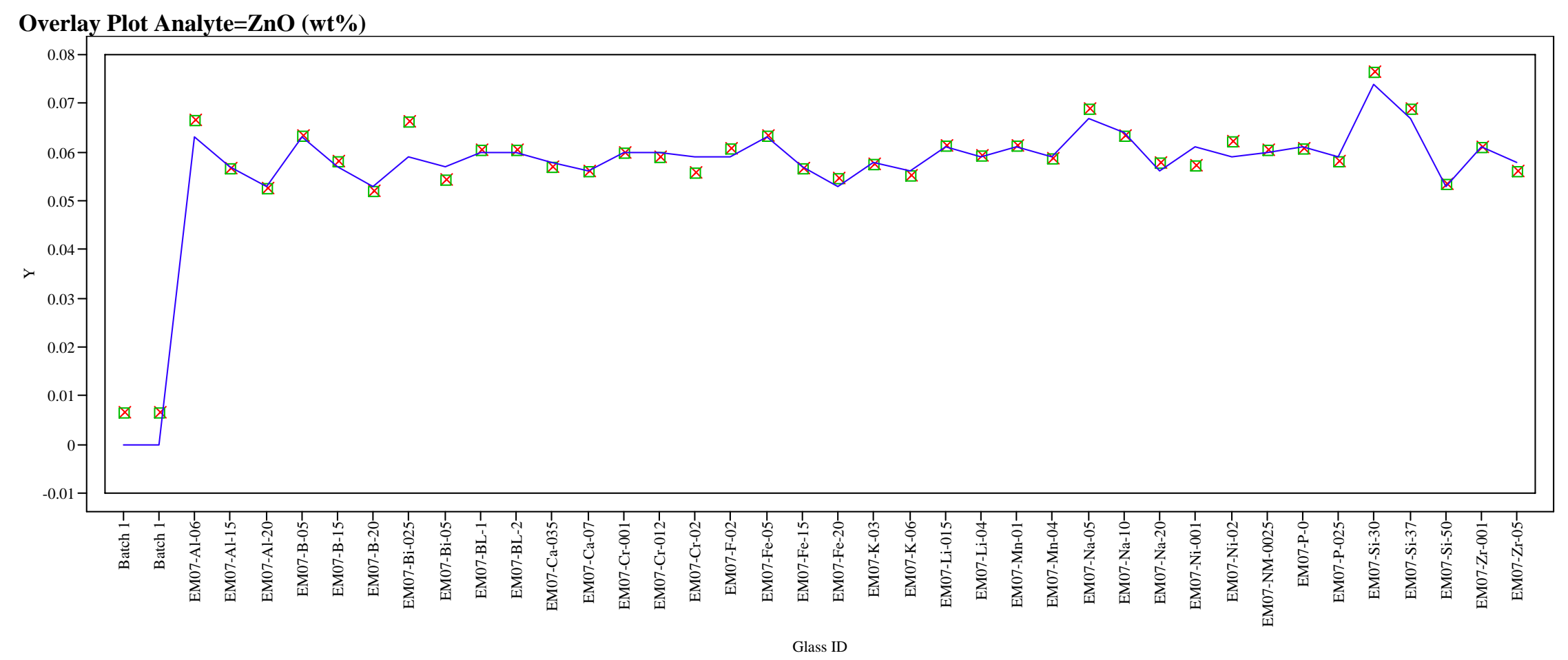

Y $\times$ Measured

- Measured bc - Targeted 
Figure A4. Average Measured and Bias-Corrected (bc) Versus Targeted Compositions by Glass \# by Analyte

Overlay Plot Analyte=ZrO2 (wt \%)

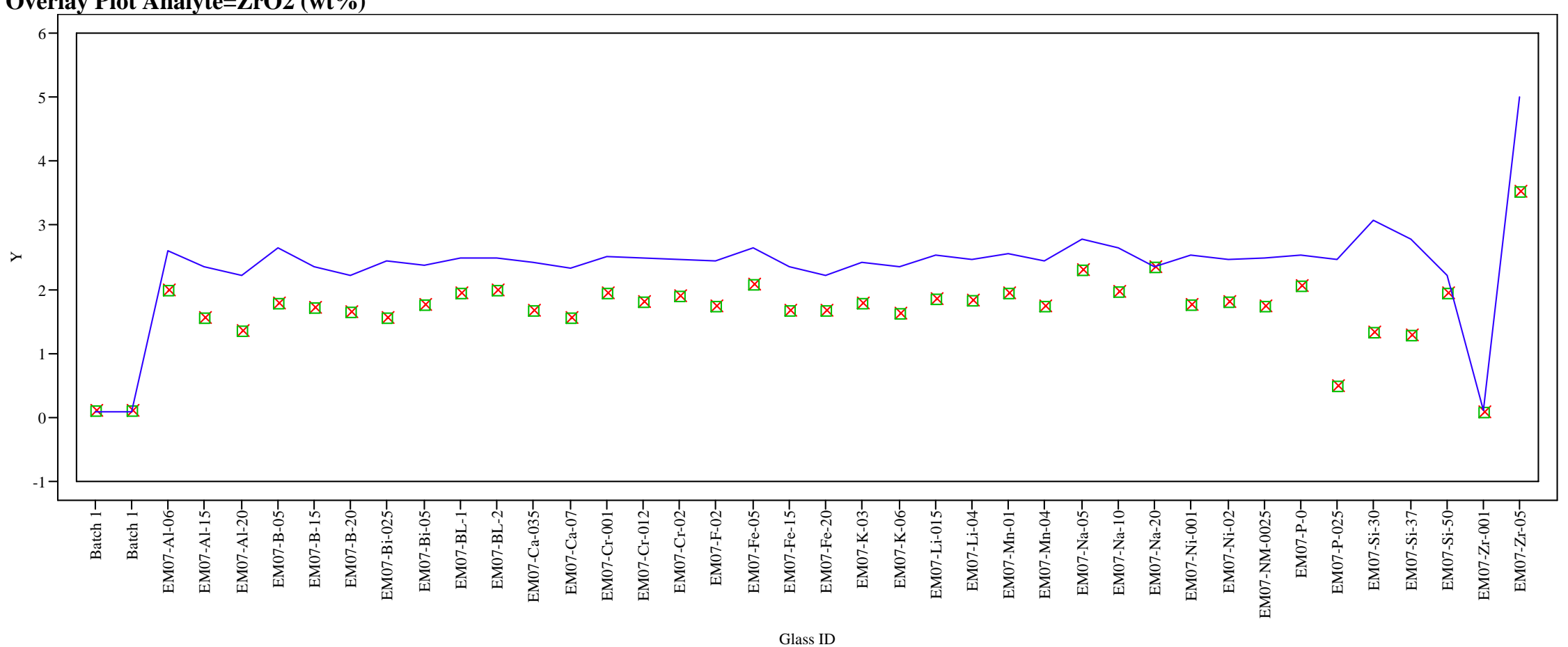

Y X Measured $\square$ Measured bc — Targeted 
Figure A4. Average Measured and Bias-Corrected (bc) Versus Targeted Compositions by Glass \# by Analyte

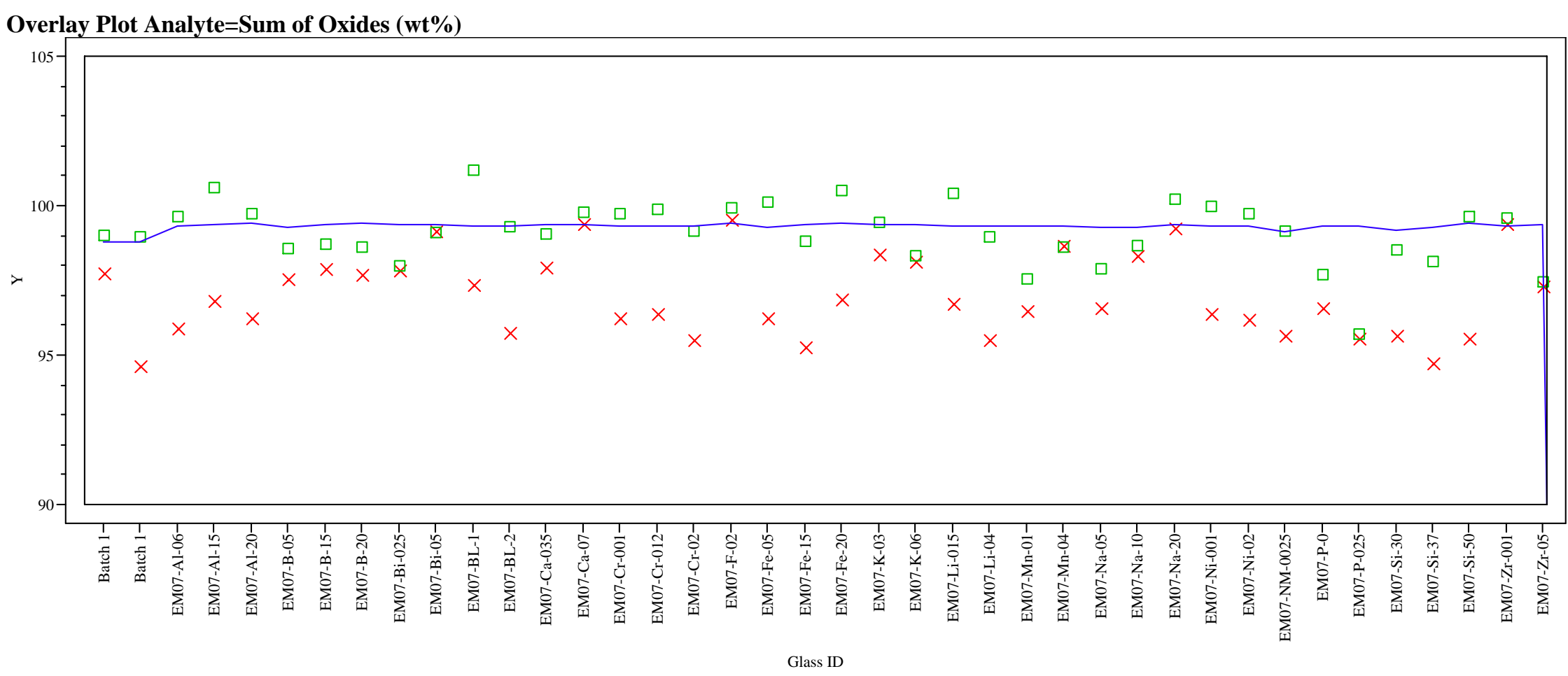
Y x Measured
- Measured bc
- Targeted 


\section{Appendix B:}

\section{Tables and Figures Supporting the Analysis of the PCT Results for the Test Matrix 1 Study Glasses}


SRNS-STI-2008-00056

Revision: 0

This page intentionally left blank. 
Table B1. PSAL Measurements of the Test Matrix 1 PCT Solutions

\begin{tabular}{|c|c|c|c|c|c|c|c|c|c|}
\hline Set & Glass ID & Heat Treatment & Block & Seq & Lab ID & $\mathrm{B}$ ar & $\mathrm{Li}$ ar & $\mathrm{Na}$ ar & Si ar \\
\hline 1 & Soln Std & ref & 1 & 1 & STD-11-1 & 20.3 & 9.73 & 82 & 49.2 \\
\hline 1 & EM07-Na-05 & CCC & 1 & 2 & C01 & 6.43 & 5.54 & 0.861 & 35.2 \\
\hline 1 & EM07-Zr-05 & CCC & 1 & 3 & $\mathrm{C} 10$ & 6.9 & 3.42 & 26.7 & 30.5 \\
\hline 1 & blank & ref & 1 & 4 & $\mathrm{C} 08$ & $<0.100$ & $<1.00$ & $<0.100$ & $<0.100$ \\
\hline 1 & EM07-Li-015 & quenched & 1 & 5 & C58 & 7.1 & 1.97 & 28.6 & 30.9 \\
\hline 1 & EM07-F-02 & CCC & 1 & 6 & C62 & 7.2 & 3.23 & 27.7 & 33.1 \\
\hline 1 & EM07-Ni-001 & quenched & 1 & 7 & C32 & 7.61 & 3.62 & 32.1 & 33.5 \\
\hline 1 & EM07-Zr-001 & CCC & 1 & 8 & $\mathrm{C} 50$ & 8.7 & 3.82 & 32.7 & 42.6 \\
\hline 1 & EM07-Li-04 & quenched & 1 & 9 & C06 & 9.28 & 5.78 & 36.7 & 37.5 \\
\hline 1 & EM07-Ni-001 & CCC & 1 & 10 & $\mathrm{C} 22$ & 7.58 & 3.62 & 30.7 & 36.2 \\
\hline 1 & EM07-Ni-02 & CCC & 1 & 11 & C35 & 7.9 & 3.69 & 30.6 & 36.3 \\
\hline 1 & EM07-F-02 & quenched & 1 & 12 & C17 & 7.19 & 3.45 & 29.6 & 31.2 \\
\hline 1 & Soln Std & ref & 1 & 13 & STD-11-2 & 19.7 & 10 & 82.6 & 50.7 \\
\hline 1 & EM07-Na-20 & quenched & 1 & 14 & C36 & 15.2 & 3.55 & 84.7 & 42.8 \\
\hline 1 & EM07-Zr-001 & quenched & 1 & 15 & C47 & 9.16 & 3.79 & 36 & 40.7 \\
\hline 1 & EM07-Li-04 & CCC & 1 & 16 & C18 & 9.09 & 5.8 & 35.4 & 39.8 \\
\hline 1 & EM07-Na-10 & quenched & 1 & 17 & C54 & 7.05 & 5.5 & 10 & 35.3 \\
\hline 1 & ARM-1 & ref & 1 & 18 & $\mathrm{C} 40$ & 9.52 & 7.93 & 22.7 & 35.3 \\
\hline 1 & EM07-Zr-05 & quenched & 1 & 19 & C04 & 7.1 & 3.65 & 30.6 & 30.4 \\
\hline 1 & EM07-Na-05 & quenched & 1 & 20 & C11 & 6.24 & 5.47 & 1.22 & 36.9 \\
\hline 1 & EM07-Na-20 & CCC & 1 & 21 & C21 & 17.9 & 4.69 & 89 & 46.5 \\
\hline 1 & EM07-Ni-02 & quenched & 1 & 22 & C46 & 8.25 & 3.78 & 33.2 & 35.5 \\
\hline 1 & EA & ref & 1 & 23 & C33 & 38.3 & 11.7 & 102.6 & 55.3 \\
\hline 1 & EM07-Li-015 & CCC & 1 & 24 & $\mathrm{C} 45$ & 7.33 & 2.1 & 28.4 & 35.6 \\
\hline 1 & EM07-Na-10 & CCC & 1 & 25 & C65 & 6.24 & 4.62 & 10.5 & 36.9 \\
\hline 1 & Soln Std & ref & 1 & 26 & STD-11-3 & 19.8 & 10.1 & 83.6 & 50.8 \\
\hline 1 & Soln Std & ref & 2 & 1 & STD-12-1 & 20.7 & 9.78 & 81.6 & 49.7 \\
\hline 1 & EM07-Ni-001 & quenched & 2 & 2 & C34 & 8.58 & 3.59 & 30.9 & 33.2 \\
\hline 1 & EA & ref & 2 & 3 & C68 & 38.1 & 11.3 & 103 & 52.7 \\
\hline 1 & EM07-Na-05 & quenched & 2 & 4 & C57 & 6.46 & 4.97 & $<0.100$ & 32.2 \\
\hline 1 & ARM-1 & ref & 2 & 5 & C56 & 10.4 & 7.93 & 22.2 & 35 \\
\hline 1 & EM07-Li-015 & $\mathrm{CCC}$ & 2 & 6 & $\mathrm{C} 20$ & 7.85 & 2.02 & 30.3 & 34.8 \\
\hline 1 & EM07-Na-05 & CCC & 2 & 7 & C41 & 5.98 & 5.18 & 0.778 & 32.7 \\
\hline 1 & EM07-Na-10 & quenched & 2 & 8 & $\mathrm{C} 23$ & 7.59 & 5.41 & 10.6 & 35 \\
\hline 1 & EM07-Na-10 & CCC & 2 & 9 & C53 & 6.68 & 4.5 & 10.7 & 36.1 \\
\hline 1 & EM07-F-02 & quenched & 2 & 10 & C63 & 8.23 & 3.63 & 31.9 & 32.9 \\
\hline 1 & EM07-Li-015 & quenched & 2 & 11 & $\mathrm{C} 24$ & 7.62 & 1.93 & 29 & 31.6 \\
\hline 1 & EM07-Li-04 & quenched & 2 & 12 & C52 & 9.81 & 5.71 & 37.2 & 37.5 \\
\hline 1 & Soln Std & ref & 2 & 13 & STD-12-2 & 20.3 & 9.89 & 81.8 & 50.2 \\
\hline 1 & EM07-Zr-05 & quenched & 2 & 14 & C14 & 7.73 & 3.51 & 28.4 & 29.2 \\
\hline 1 & EM07-Zr-001 & CCC & 2 & 15 & C12 & 9.25 & 3.78 & 34.6 & 42.2 \\
\hline 1 & EM07-F-02 & CCC & 2 & 16 & C07 & 7.64 & 3.19 & 28.3 & 33.6 \\
\hline 1 & EM07-Na-20 & quenched & 2 & 17 & C60 & 15.8 & 3.54 & 88.4 & 43.1 \\
\hline 1 & EM07-Ni-02 & CCC & 2 & 18 & C38 & 8.29 & 3.6 & 31.5 & 35.8 \\
\hline 1 & EM07-Ni-001 & CCC & 2 & 19 & $\mathrm{C} 25$ & 8.05 & 3.56 & 31 & 36.1 \\
\hline 1 & EM07-Li-04 & CCC & 2 & 20 & C59 & 9.42 & 5.71 & 35.6 & 39.5 \\
\hline 1 & EM07-Na-20 & CCC & 2 & 21 & C15 & 17.8 & 4.53 & 89.8 & 44.8 \\
\hline 1 & EM07-Zr-001 & quenched & 2 & 22 & C13 & 9.28 & 3.71 & 35.4 & 39.3 \\
\hline 1 & EM07-Ni-02 & quenched & 2 & 23 & C31 & 8.44 & 3.65 & 35.7 & 34.1 \\
\hline 1 & EM07-Zr-05 & CCC & 2 & 24 & C48 & 6.83 & 3.36 & 26.9 & 30.3 \\
\hline 1 & Soln Std & ref & 2 & 25 & STD-12-3 & 19.9 & 9.86 & 82.5 & 49.9 \\
\hline 1 & Soln Std & ref & 3 & 1 & STD-13-1 & 19.8 & 9.67 & 82.8 & 48.1 \\
\hline 1 & EM07-Li-015 & quenched & 3 & 2 & C39 & 7.73 & 1.94 & 31.9 & 30.4 \\
\hline 1 & EM07-F-02 & quenched & 3 & 3 & C44 & 8.5 & 3.66 & 31.8 & 33 \\
\hline 1 & blank & ref & 3 & 4 & $\mathrm{C} 26$ & $<0.100$ & $<1.00$ & $<0.100$ & $<0.100$ \\
\hline 1 & EM07-Li-04 & quenched & 3 & 5 & C64 & 9.32 & 5.65 & 37.9 & 36 \\
\hline 1 & EM07-Na-10 & quenched & 3 & 6 & C49 & 7.28 & 5.44 & 10.7 & 34.7 \\
\hline 1 & EM07-Ni-02 & CCC & 3 & 7 & $\mathrm{C} 30$ & 8.02 & 3.62 & 33.9 & 35.5 \\
\hline 1 & ARM-1 & ref & 3 & 8 & C43 & 9.56 & 7.75 & 22.7 & 34 \\
\hline 1 & EM07-Li-04 & CCC & 3 & 9 & C09 & 9.15 & 5.71 & 35.6 & 39.1 \\
\hline
\end{tabular}


Table B1. PSAL Measurements of the Test Matrix 1 PCT Solutions

\begin{tabular}{|c|c|c|c|c|c|c|c|c|c|}
\hline Set & Glass ID & Heat Treatment & Block & Seq & Lab ID & $\mathrm{B}$ ar & $\mathrm{Li}$ ar & $\mathrm{Na}$ ar & Si ar \\
\hline 1 & EM07-Zr-05 & quenched & 3 & 10 & C51 & 7.18 & 3.5 & 28.7 & 28.9 \\
\hline 1 & EM07-Ni-001 & quenched & 3 & 11 & $\mathrm{C03}$ & 7.5 & 3.56 & 31 & 32.6 \\
\hline 1 & EM07-F-02 & CCC & 3 & 12 & C19 & 7.09 & 3.2 & 28.5 & 32.7 \\
\hline 1 & Soln Std & ref & 3 & 13 & STD-13-2 & 19.5 & 9.72 & 81.9 & 49 \\
\hline 1 & EM07-Na-20 & quenched & 3 & 14 & C67 & 15.5 & 3.52 & 88.9 & 42 \\
\hline 1 & EA & ref & 3 & 15 & $\mathrm{C} 28$ & 37.3 & 11.3 & 105 & 52.4 \\
\hline 1 & EM07-Zr-001 & CCC & 3 & 16 & C37 & 9.41 & 3.89 & 35.2 & 42.8 \\
\hline 1 & EM07-Zr-05 & CCC & 3 & 17 & C66 & 6.9 & 3.39 & 28.4 & 30.2 \\
\hline 1 & EM07-Na-10 & $\mathrm{CCC}$ & 3 & 18 & $\mathrm{C} 27$ & 6.51 & 4.55 & 10.7 & 36 \\
\hline 1 & EM07-Li-015 & CCC & 3 & 19 & C61 & 6.89 & 1.91 & 28.3 & 32.5 \\
\hline 1 & EM07-Na-05 & CCC & 3 & 20 & $\mathrm{C} 05$ & 5.8 & 5.28 & 0.141 & 33.3 \\
\hline 1 & EM07-Na-20 & CCC & 3 & 21 & C16 & 17.5 & 4.54 & 90.2 & 44.3 \\
\hline 1 & EM07-Na-05 & quenched & 3 & 22 & C42 & 6.25 & 5.33 & $<0.100$ & 34.8 \\
\hline 1 & EM07-Ni-02 & quenched & 3 & 23 & $\mathrm{C} 29$ & 7.93 & 3.57 & 31.8 & 33.2 \\
\hline 1 & EM07-Zr-001 & quenched & 3 & 24 & $\mathrm{C} 55$ & 8.99 & 3.69 & 34.4 & 39.1 \\
\hline 1 & EM07-Ni-001 & CCC & 3 & 25 & $\mathrm{C} 02$ & 7.57 & 3.52 & 29.3 & 35.1 \\
\hline 1 & Soln Std & ref & 3 & 26 & STD-13-3 & 19.6 & 9.83 & 82.3 & 49.4 \\
\hline 2 & Soln Std & ref & 1 & 1 & STD-21-1 & 20.3 & 9.7 & 77.8 & 49.3 \\
\hline 2 & EM07-B-05 & quenched & 1 & 2 & D32 & 4.14 & 3.78 & 40.5 & 42.1 \\
\hline 2 & EM07-Bi-05 & quenched & 1 & 3 & D34 & 8.86 & 3.85 & 32.5 & 34.4 \\
\hline 2 & EM07-Bi-025 & quenched & 1 & 4 & D41 & 9.1 & 4.05 & 34 & 36.3 \\
\hline 2 & EM07-Si-50 & quenched & 1 & 5 & D51 & 5.99 & 3.48 & 21.7 & 37.5 \\
\hline 2 & EM07-P-0 & CCC & 1 & 6 & D05 & 8.38 & 3.72 & 31.1 & 38.5 \\
\hline 2 & EM07-Bi-05 & CCC & 1 & 7 & D37 & 8.83 & 3.98 & 32.2 & 36.5 \\
\hline 2 & ARM-1 & ref & 1 & 8 & D43 & 9.8 & 7.92 & 19.8 & 35.3 \\
\hline 2 & EM07-Si-37 & quenched & 1 & 9 & D24 & 15.4 & 5.02 & 52.5 & 32.1 \\
\hline 2 & EM07-P-025 & $\mathrm{CCC}$ & 1 & 10 & D56 & 8.87 & 7.78 & 38.1 & 39 \\
\hline 2 & EA & ref & 1 & 11 & D29 & 36.8 & 11.2 & 101 & 53 \\
\hline 2 & EM07-B-20 & quenched & 1 & 12 & D10 & 243 & 39.4 & 217 & 20.3 \\
\hline 2 & Soln Std & ref & 1 & 13 & STD-21-2 & 20.8 & 9.68 & 80.9 & 48.3 \\
\hline 2 & EM07-P-0 & quenched & 1 & 14 & D13 & 9.32 & 3.71 & 32.4 & 35 \\
\hline 2 & blank & ref & 1 & 15 & D48 & 0.557 & $<1.00$ & $<0.100$ & $<0.100$ \\
\hline 2 & EM07-Si-30 & quenched & 1 & 16 & D20 & 63.2 & 16.8 & 154 & 36.1 \\
\hline 2 & EM07-Si-37 & CCC & 1 & 17 & D16 & 25 & 7.27 & 66.8 & 36.8 \\
\hline 2 & EM07-Si-50 & CCC & 1 & 18 & D01 & 6.27 & 3.37 & 20.2 & 40.2 \\
\hline 2 & EM07-B-20 & CCC & 1 & 19 & D63 & 368 & 58.6 & 322 & 20.2 \\
\hline 2 & EM07-B-05 & CCC & 1 & 20 & D38 & 5.68 & 3.79 & 39 & 42.4 \\
\hline 2 & EM07-Si-30 & CCC & 1 & 21 & D31 & 1310 & 270 & 2170 & 92.1 \\
\hline 2 & EM07-B-15 & CCC & 1 & 22 & D33 & 55.2 & 10.5 & 65.6 & 31.6 \\
\hline 2 & EM07-Bi-025 & CCC & 1 & 23 & D28 & 14.4 & 3.88 & 30.7 & 37.2 \\
\hline 2 & EM07-B-15 & quenched & 1 & 24 & D58 & 41.2 & 8.48 & 56 & 29.4 \\
\hline 2 & EM07-P-025 & quenched & 1 & 25 & D53 & 11.1 & 3.81 & 32 & 34 \\
\hline 2 & Soln Std & ref & 1 & 26 & STD-21-3 & 21.8 & 9.66 & 81.3 & 47.9 \\
\hline 2 & Soln Std & ref & 2 & 1 & STD-22-1 & 19.7 & 9.82 & 79.5 & 48.4 \\
\hline 2 & ARM-1 & ref & 2 & 2 & D42 & 9.3 & 7.93 & 19.9 & 34.2 \\
\hline 2 & EM07-Bi-05 & quenched & 2 & 3 & D04 & 8.05 & 4.01 & 33.6 & 33.6 \\
\hline 2 & EM07-Bi-025 & CCC & 2 & 4 & D44 & 7.32 & 3.89 & 31.2 & 35.1 \\
\hline 2 & EM07-P-0 & CCC & 2 & 5 & D25 & 7.47 & 3.83 & 31.6 & 36.8 \\
\hline 2 & EM07-B-20 & CCC & 2 & 6 & D21 & 384 & 58.9 & 317 & 20.8 \\
\hline 2 & EM07-Si-50 & $\mathrm{CCC}$ & 2 & 7 & D55 & 6.69 & 3.47 & 20.8 & 38.9 \\
\hline 2 & EM07-B-20 & quenched & 2 & 8 & $\mathrm{D} 22$ & 255 & 39.5 & 219 & 20.6 \\
\hline 2 & EM07-Si-37 & CCC & 2 & 9 & D23 & 24.9 & 7.28 & 66.8 & 35.4 \\
\hline 2 & EM07-Si-30 & $\mathrm{CCC}$ & 2 & 10 & D39 & 1300 & 306 & 2110 & 90.8 \\
\hline 2 & EM07-Si-30 & quenched & 2 & 11 & D03 & 66.1 & 16.4 & 155 & 34.5 \\
\hline 2 & EM07-Si-37 & quenched & 2 & 12 & D19 & 20.6 & 5.07 & 52.1 & 31.3 \\
\hline 2 & Soln Std & ref & 2 & 13 & STD-22-2 & 21.2 & 9.7 & 80.4 & 47.6 \\
\hline 2 & EM07-P-0 & quenched & 2 & 14 & D09 & 11.3 & 3.88 & 32.2 & 35.3 \\
\hline 2 & EM07-B-05 & CCC & 2 & 15 & D35 & 6.23 & 4.03 & 39.5 & 43 \\
\hline 2 & EM07-P-025 & quenched & 2 & 16 & D06 & 9.54 & 3.98 & 32.6 & 34.2 \\
\hline 2 & EM07-B-15 & CCC & 2 & 17 & D64 & 45.4 & 10.3 & 65.3 & 30.7 \\
\hline
\end{tabular}


Table B1. PSAL Measurements of the Test Matrix 1 PCT Solutions

\begin{tabular}{|c|c|c|c|c|c|c|c|c|c|}
\hline Set & Glass ID & Heat Treatment & Block & Seq & Lab ID & $\mathrm{B}$ ar & $\mathrm{Li}$ ar & $\mathrm{Na}$ ar & Si ar \\
\hline 2 & EM07-B-15 & quenched & 2 & 18 & D36 & 35.7 & 8.37 & 54.7 & 28.4 \\
\hline 2 & EA & ref & 2 & 19 & D57 & 35.9 & 11.2 & 101 & 51.4 \\
\hline 2 & EM07-Bi-025 & quenched & 2 & 20 & D67 & 9.12 & 4.04 & 35.2 & 34.2 \\
\hline 2 & EM07-Si-50 & quenched & 2 & 21 & D49 & 6.32 & 3.62 & 20.6 & 35.9 \\
\hline 2 & EM07-Bi-05 & $\mathrm{CCC}$ & 2 & 22 & D26 & 8.93 & 3.97 & 31 & 34.7 \\
\hline 2 & EM07-B-05 & quenched & 2 & 23 & D18 & 3.94 & 3.96 & 42.5 & 40.8 \\
\hline 2 & EM07-P-025 & CCC & 2 & 24 & D62 & 8.81 & 7.8 & 38.3 & 38.5 \\
\hline 2 & Soln Std & ref & 2 & 25 & STD-22-3 & 19.1 & 9.71 & 79 & 47.6 \\
\hline 2 & Soln Std & ref & 3 & 1 & STD-23-1 & 19.4 & 9.58 & 78.2 & 48.1 \\
\hline 2 & blank & ref & 3 & 2 & D02 & $<0.100$ & $<1.00$ & 0.72 & $<0.100$ \\
\hline 2 & EM07-P-025 & CCC & 3 & 3 & D59 & 8.32 & 7.63 & 37.8 & 37.9 \\
\hline 2 & EM07-Bi-05 & quenched & 3 & 4 & D45 & 7.81 & 3.75 & 32.9 & 32.4 \\
\hline 2 & ARM-1 & ref & 3 & 5 & D66 & 9.59 & 8.02 & 20.5 & 35.4 \\
\hline 2 & EM07-Si-37 & CCC & 3 & 6 & D46 & 23.7 & 7.27 & 66.9 & 36.7 \\
\hline 2 & EM07-Si-30 & CCC & 3 & 7 & D68 & 1290 & 307 & 2310 & 92.7 \\
\hline 2 & EM07-Si-30 & quenched & 3 & 8 & D54 & 64.8 & 16.5 & 153 & 34.8 \\
\hline 2 & EM07-B-05 & quenched & 3 & 9 & D15 & 8.05 & 3.78 & 41.4 & 40.9 \\
\hline 2 & EM07-P-0 & CCC & 3 & 10 & D30 & 11.1 & 3.68 & 31.6 & 36.4 \\
\hline 2 & EM07-P-0 & quenched & 3 & 11 & D50 & 10.6 & 3.67 & 33.3 & 34 \\
\hline 2 & EM07-B-15 & quenched & 3 & 12 & D08 & 37.4 & 8.23 & 56.8 & 28.7 \\
\hline 2 & Soln Std & ref & 3 & 13 & STD-23-2 & 21.4 & 9.69 & 77.6 & 48.9 \\
\hline 2 & EM07-Si-50 & quenched & 3 & 14 & D40 & 7.36 & 3.49 & 20.1 & 36.6 \\
\hline 2 & EM07-B-20 & quenched & 3 & 15 & D12 & 246 & 38.7 & 228 & 20.3 \\
\hline 2 & EA & ref & 3 & 16 & D27 & 37.3 & 10.8 & 95.4 & 51.3 \\
\hline 2 & EM07-Si-37 & quenched & 3 & 17 & D65 & 16.1 & 4.9 & 51.8 & 31 \\
\hline 2 & EM07-B-20 & CCC & 3 & 18 & D61 & 361 & 56.7 & 330 & 19.7 \\
\hline 2 & EM07-Bi-025 & quenched & 3 & 19 & D11 & 11 & 3.86 & 33.2 & 34.4 \\
\hline 2 & EM07-P-025 & quenched & 3 & 20 & D14 & 10.7 & 4.01 & 33.9 & 36.4 \\
\hline 2 & EM07-Si-50 & CCC & 3 & 21 & D52 & 7.3 & 3.33 & 19.6 & 39.5 \\
\hline 2 & EM07-B-15 & CCC & 3 & 22 & D47 & 46.4 & 10.1 & 62.7 & 31.1 \\
\hline 2 & EM07-B-05 & CCC & 3 & 23 & D17 & 4.51 & 3.81 & 39.1 & 43 \\
\hline 2 & EM07-Bi-025 & CCC & 3 & 24 & D60 & 8.74 & 3.75 & 30.5 & 36 \\
\hline 2 & EM07-Bi-05 & CCC & 3 & 25 & D07 & 8.56 & 3.75 & 31.7 & 34.1 \\
\hline 2 & Soln Std & ref & 3 & 26 & STD-23-3 & 19.4 & 9.52 & 77.4 & 47.9 \\
\hline 3 & Soln Std & ref & 1 & 1 & STD-31-1 & 21 & 9.93 & 82.2 & 51.4 \\
\hline 3 & EM07-Ca-07 & CCC & 1 & 2 & E35 & 8.52 & 4.17 & 36.9 & 24.1 \\
\hline 3 & EM07-Mn-04 & CCC & 1 & 3 & E23 & 9 & 3.74 & 31.7 & 36.6 \\
\hline 3 & EM07-BL-2 & quenched & 1 & 4 & E52 & 9.05 & 3.74 & 31.6 & 35.4 \\
\hline 3 & EM07-Mn-01 & CCC & 1 & 5 & E45 & 8.58 & 3.73 & 31 & 38.2 \\
\hline 3 & EM07-Ca-07 & quenched & 1 & 6 & E34 & 12.2 & 5.06 & 47 & 28.7 \\
\hline 3 & EA & ref & 1 & 7 & E30 & 40.3 & 12 & 103 & 57.2 \\
\hline 3 & EM07-BL-2 & $\mathrm{CCC}$ & 1 & 8 & E14 & 9.03 & 3.59 & 28.9 & 36.4 \\
\hline 3 & ARM-1 & ref & 1 & 9 & E26 & 13.9 & 10 & 25.7 & 42.9 \\
\hline 3 & EM07-Mn-01 & quenched & 1 & 10 & E32 & 8.85 & 3.7 & 31.9 & 36.7 \\
\hline 3 & EM07-K-06 & quenched & 1 & 11 & E21 & 17.7 & 6.37 & 53 & 37 \\
\hline 3 & EM07-BL-1 & quenched & 1 & 12 & E06 & 9.3 & 3.78 & 32.9 & 36.7 \\
\hline 3 & Soln Std & ref & 1 & 13 & STD-31-2 & 21.4 & 10.2 & 80.8 & 51.8 \\
\hline 3 & EM07-K-03 & quenched & 1 & 14 & E13 & 9.91 & 3.66 & 34 & 33.1 \\
\hline 3 & blank & ref & 1 & 15 & E60 & 0.496 & $<1.00$ & $<0.100$ & $<0.100$ \\
\hline 3 & EM07-Mn-04 & quenched & 1 & 16 & E56 & 9.62 & 3.91 & 33 & 36.8 \\
\hline 3 & EM07-NM-0025 & CCC & 1 & 17 & E43 & 8.92 & 3.65 & 29.9 & 38.9 \\
\hline 3 & EM07-K-03 & CCC & 1 & 18 & E01 & 11.2 & 4.13 & 35.3 & 35.9 \\
\hline 3 & EM07-Ca-035 & quenched & 1 & 19 & E59 & 0.399 & $<1.00$ & $<0.100$ & $<0.100$ \\
\hline 3 & EM07-K-06 & CCC & 1 & 20 & E09 & 27.9 & 10.2 & 84.3 & 64.6 \\
\hline 3 & EM07-Ca-035 & CCC & 1 & 21 & E04 & 8.79 & 3.77 & 31.7 & 30.2 \\
\hline 3 & EM07-NM-0025 & quenched & 1 & 22 & E27 & 9.57 & 3.89 & 31.2 & 36.9 \\
\hline 3 & EM07-BL-1 & CCC & 1 & 23 & E31 & 8.77 & 3.7 & 29.5 & 39.1 \\
\hline 3 & Soln Std & ref & 1 & 24 & STD-31-3 & 21.6 & 10.3 & 79.5 & 53.4 \\
\hline 3 & Soln Std & ref & 2 & 1 & STD-32-1 & 21.1 & 9.9 & 83 & 51.9 \\
\hline 3 & EM07-BL-2 & CCC & 2 & 2 & E16 & 8.72 & 3.46 & 30 & 36.7 \\
\hline
\end{tabular}


Table B1. PSAL Measurements of the Test Matrix 1 PCT Solutions

\begin{tabular}{|c|c|c|c|c|c|c|c|c|c|}
\hline Set & Glass ID & Heat Treatment & Block & Seq & Lab ID & $\mathrm{B}$ ar & $\mathrm{Li}$ ar & $\mathrm{Na}$ ar & Si ar \\
\hline 3 & EM07-K-06 & CCC & 2 & 3 & E33 & 20 & 7.11 & 61.2 & 36.7 \\
\hline 3 & EM07-Ca-07 & quenched & 2 & 4 & E57 & 11.8 & 4.9 & 48.7 & 28.8 \\
\hline 3 & EM07-Ca-035 & CCC & 2 & 5 & E49 & 8.04 & 3.57 & 33.7 & 28.9 \\
\hline 3 & EM07-K-03 & CCC & 2 & 6 & E42 & 11.1 & 4.24 & 39 & 36.3 \\
\hline 3 & EM07-Ca-035 & quenched & 2 & 7 & E17 & 9.66 & 3.86 & 37.9 & 30.1 \\
\hline 3 & ARM-1 & ref & 2 & 8 & E50 & 13.1 & 9.88 & 26.8 & 42 \\
\hline 3 & EM07-Mn-01 & CCC & 2 & 9 & E24 & 7.98 & 3.53 & 31 & 37.3 \\
\hline 3 & EA & ref & 2 & 10 & E11 & 40.1 & 11.7 & 107 & 56.8 \\
\hline 3 & EM07-BL-2 & quenched & 2 & 11 & E02 & 8.73 & 3.59 & 32.3 & 35.2 \\
\hline 3 & EM07-K-06 & quenched & 2 & 12 & E28 & 16 & 5.85 & 52 & 34.8 \\
\hline 3 & Soln Std & ref & 2 & 13 & STD-32-2 & 20.3 & 9.86 & 82.6 & 50.7 \\
\hline 3 & EM07-NM-0025 & quenched & 2 & 14 & E40 & 8.89 & 3.62 & 32.1 & 34.8 \\
\hline 3 & EM07-Ca-07 & CCC & 2 & 15 & E20 & 8.43 & 4.16 & 37.8 & 24.8 \\
\hline 3 & EM07-Mn-04 & quenched & 2 & 16 & E62 & 9.27 & 3.83 & 35.3 & 37 \\
\hline 3 & EM07-Mn-04 & CCC & 2 & 17 & E58 & 8.34 & 3.61 & 32.1 & 36.9 \\
\hline 3 & EM07-K-03 & quenched & 2 & 18 & E36 & 9.01 & 3.46 & 35.3 & 31.3 \\
\hline 3 & EM07-Mn-01 & quenched & 2 & 19 & E08 & 8.04 & 3.57 & 33.3 & 36.5 \\
\hline 3 & EM07-BL-1 & quenched & 2 & 20 & E39 & 8.22 & 3.52 & 33.1 & 35.2 \\
\hline 3 & EM07-NM-0025 & CCC & 2 & 21 & E54 & 8.22 & 3.51 & 30.9 & 38.6 \\
\hline 3 & EM07-BL-1 & CCC & 2 & 22 & E03 & 7.93 & 3.53 & 31.2 & 37.6 \\
\hline 3 & Soln Std & ref & 2 & 23 & STD-32-3 & 20.1 & 9.89 & 83.4 & 50.6 \\
\hline 3 & Soln Std & ref & 3 & 1 & STD-33-1 & 21 & 9.89 & 82.1 & 51.8 \\
\hline 3 & EM07-Ca-07 & quenched & 3 & 2 & E44 & 12.4 & 4.9 & 47.8 & 29.4 \\
\hline 3 & EM07-K-06 & $\mathrm{CCC}$ & 3 & 3 & E15 & 20 & 7.04 & 59.4 & 37.8 \\
\hline 3 & EM07-NM-0025 & quenched & 3 & 4 & E51 & 9.07 & 3.64 & 32.5 & 35.3 \\
\hline 3 & EA & ref & 3 & 5 & E41 & 39.7 & 11.6 & 104 & 56.6 \\
\hline 3 & EM07-Ca-035 & CCC & 3 & 6 & E47 & 8.41 & 3.5 & 32.4 & 29.5 \\
\hline 3 & EM07-Ca-07 & CCC & 3 & 7 & E29 & 8.73 & 4.23 & 37.9 & 25.6 \\
\hline 3 & EM07-BL-2 & CCC & 3 & 8 & E46 & 8.5 & 3.47 & 30.1 & 37 \\
\hline 3 & ARM-1 & ref & 3 & 9 & E19 & 13.3 & 9.68 & 25.9 & 43 \\
\hline 3 & EM07-Mn-01 & quenched & 3 & 10 & E18 & 8.44 & 3.57 & 32.7 & 37.7 \\
\hline 3 & EM07-Ca-035 & quenched & 3 & 11 & E10 & 9.7 & 3.8 & 36.6 & 31.1 \\
\hline 3 & EM07-K-03 & quenched & 3 & 12 & E25 & 9.54 & 3.54 & 35.1 & 33.3 \\
\hline 3 & Soln Std & ref & 3 & 13 & STD-33-2 & 21.5 & 10.2 & 81.4 & 54.8 \\
\hline 3 & blank & ref & 3 & 14 & E22 & 0.54 & $<1.00$ & $<0.100$ & $<0.100$ \\
\hline 3 & EM07-BL-1 & quenched & 3 & 15 & E48 & 8.95 & 3.59 & 32.4 & 38.3 \\
\hline 3 & EM07-BL-1 & CCC & 3 & 16 & E37 & 8.41 & 3.55 & 30.9 & 39.6 \\
\hline 3 & EM07-Mn-04 & CCC & 3 & 17 & E53 & 8.75 & 3.65 & 32 & 38.8 \\
\hline 3 & EM07-K-06 & quenched & 3 & 18 & E61 & 15.7 & 5.74 & 50 & 35.9 \\
\hline 3 & EM07-Mn-01 & CCC & 3 & 19 & E07 & 8.51 & 3.63 & 31.7 & 39.6 \\
\hline 3 & EM07-Mn-04 & quenched & 3 & 20 & E05 & 9.63 & 3.9 & 35 & 39.3 \\
\hline 3 & EM07-BL-2 & quenched & 3 & 21 & E12 & 8.83 & 3.59 & 31.9 & 36.4 \\
\hline 3 & EM07-K-03 & CCC & 3 & 22 & E38 & 10.6 & 3.97 & 36 & 36.3 \\
\hline 3 & EM07-NM-0025 & CCC & 3 & 23 & E55 & 8.43 & 3.5 & 30.4 & 39.4 \\
\hline 3 & Soln Std & ref & 3 & 24 & STD-23-2 & 20.4 & 9.89 & 80.9 & 52.6 \\
\hline 4 & Soln Std & ref & 1 & 1 & STD-31-1 & 21.1 & 10 & 82.4 & 52.1 \\
\hline 4 & EM07-Fe-15 & CCC & 1 & 2 & F18 & 9.07 & 3.62 & 30.1 & 39.7 \\
\hline 4 & EM07-Al-20 & CCC & 1 & 3 & F03 & 8.34 & 4.44 & 22.9 & 37.5 \\
\hline 4 & EM07-Fe-05 & $\mathrm{CCC}$ & 1 & 4 & F33 & 9.18 & 3.66 & 32.2 & 38.8 \\
\hline 4 & EM07-Fe-20 & CCC & 1 & 5 & F34 & 8.05 & 3.14 & 28.1 & 37.8 \\
\hline 4 & EM07-Cr-012 & quenched & 1 & 6 & F52 & 9.17 & 3.88 & 33.5 & 37.5 \\
\hline 4 & blank & ref & 1 & 7 & F32 & 0.289 & $<1.00$ & $<0.100$ & 0.916 \\
\hline 4 & ARM-1 & ref & 1 & 8 & F04 & 12.8 & 9.49 & 25.5 & 41.6 \\
\hline 4 & EM07-Cr-001 & quenched & 1 & 9 & F02 & 9.49 & 3.92 & 33.3 & 37.7 \\
\hline 4 & EM07-Al-15 & $\mathrm{CCC}$ & 1 & 10 & F24 & 7.18 & 3.51 & 24.9 & 37.2 \\
\hline 4 & EM07-Cr-012 & CCC & 1 & 11 & F44 & 9.64 & 4.05 & 33.2 & 42.4 \\
\hline 4 & EM07-Fe-15 & quenched & 1 & 12 & F56 & 9.26 & 4.08 & 31.1 & 36.2 \\
\hline 4 & Soln Std & ref & 1 & 13 & STD-31-2 & 20.8 & 10 & 80.7 & 52.6 \\
\hline 4 & EM07-Fe-20 & quenched & 1 & 14 & F62 & 9.51 & 4.1 & 29.1 & 34.7 \\
\hline 4 & EM07-Al-06 & quenched & 1 & 15 & F37 & 13.6 & 4.65 & 44.9 & 41.4 \\
\hline
\end{tabular}


Table B1. PSAL Measurements of the Test Matrix 1 PCT Solutions

\begin{tabular}{|c|c|c|c|c|c|c|c|c|c|}
\hline Set & Glass ID & Heat Treatment & Block & Seq & Lab ID & $\mathrm{B}$ ar & $\mathrm{Li}$ ar & $\mathrm{Na}$ ar & Si ar \\
\hline 4 & EM07-Al-06 & CCC & 1 & 16 & F11 & 11.8 & 4.2 & 35.6 & 32.2 \\
\hline 4 & EA & ref & 1 & 17 & F31 & 33 & 10 & 87.7 & 50.4 \\
\hline 4 & EM07-Cr-02 & quenched & 1 & 18 & F14 & 9.51 & 3.86 & 32.7 & 39.2 \\
\hline 4 & EM07-Cr-001 & CCC & 1 & 19 & F50 & 9.85 & 4.02 & 31.8 & 41.8 \\
\hline 4 & EM07-Al-20 & quenched & 1 & 20 & F05 & 7.62 & 4.5 & 22.2 & 38.3 \\
\hline 4 & EM07-Cr-02 & CCC & 1 & 21 & F13 & 8.71 & 3.6 & 29 & 39.8 \\
\hline 4 & EM07-Al-15 & quenched & 1 & 22 & F40 & 7.91 & 3.9 & 25.5 & 34.6 \\
\hline 4 & EM07-Fe-05 & quenched & 1 & 23 & F51 & 9.68 & 3.6 & 34.6 & 37.1 \\
\hline 4 & Soln Std & ref & 1 & 24 & STD-31-3 & 20.5 & 9.89 & 79.8 & 52.4 \\
\hline 4 & Soln Std & ref & 2 & 1 & STD-32-1 & 20.7 & 9.8 & 83.3 & 51.3 \\
\hline 4 & EM07-Fe-15 & quenched & 2 & 2 & F10 & 9.06 & 3.96 & 31.7 & 34.5 \\
\hline 4 & EM07-Al-06 & CCC & 2 & 3 & F16 & 11.5 & 4.21 & 36.5 & 31.9 \\
\hline 4 & EM07-Al-20 & quenched & 2 & 4 & F57 & 11.8 & 7.22 & 38.1 & 60.6 \\
\hline 4 & EM07-Cr-001 & quenched & 2 & 5 & F01 & 9.12 & 3.87 & 32.8 & 37.7 \\
\hline 4 & EM07-Al-20 & CCC & 2 & 6 & F17 & 7.85 & 4.43 & 23.4 & 37.5 \\
\hline 4 & EM07-Fe-05 & CCC & 2 & 7 & F47 & 8.91 & 3.72 & 32.8 & 39.3 \\
\hline 4 & EM07-Cr-02 & quenched & 2 & 8 & F42 & 8.47 & 3.69 & 32.4 & 36.6 \\
\hline 4 & EM07-Cr-012 & quenched & 2 & 9 & F08 & 8.31 & 3.63 & 32 & 34.8 \\
\hline 4 & EM07-Cr-012 & CCC & 2 & 10 & F43 & 8.56 & 3.78 & 32 & 39.6 \\
\hline 4 & ARM-1 & ref & 2 & 11 & F61 & 12.5 & 9.46 & 26.2 & 41.2 \\
\hline 4 & EM07-Cr-02 & CCC & 2 & 12 & F21 & 7.98 & 3.53 & 29.6 & 38.1 \\
\hline 4 & Soln Std & ref & 2 & 13 & STD-32-2 & 20.3 & 9.92 & 81.7 & 52 \\
\hline 4 & EM07-Al-15 & quenched & 2 & 14 & F53 & 8.04 & 3.96 & 32.5 & 35 \\
\hline 4 & EM07-Al-15 & CCC & 2 & 15 & F29 & 7.11 & 3.53 & 25 & 37.4 \\
\hline 4 & EM07-Fe-20 & quenched & 2 & 16 & F49 & 9.16 & 4.24 & 30.6 & 35.5 \\
\hline 4 & EM07-Fe-05 & quenched & 2 & 17 & F06 & 9.27 & 3.55 & 33.6 & 36.5 \\
\hline 4 & EA & ref & 2 & 18 & F35 & 32.3 & 9.95 & 88.9 & 50.5 \\
\hline 4 & EM07-Fe-15 & CCC & 2 & 19 & F38 & 9.51 & 3.98 & 33 & 43.4 \\
\hline 4 & EM07-Al-06 & quenched & 2 & 20 & F23 & 13.6 & 4.77 & 44.5 & 43.1 \\
\hline 4 & EM07-Cr-001 & CCC & 2 & 21 & F45 & 9.7 & 4.14 & 32.5 & 41.7 \\
\hline 4 & EM07-Fe-20 & CCC & 2 & 22 & F07 & 7.85 & 3.17 & 28.2 & 38.2 \\
\hline 4 & Soln Std & ref & 2 & 23 & STD-32-3 & 20.1 & 9.84 & 80.4 & 51.9 \\
\hline 4 & Soln Std & ref & 3 & 1 & STD-33-1 & 20.5 & 9.8 & 83.4 & 51 \\
\hline 4 & EM07-Fe-05 & quenched & 3 & 2 & F39 & 9.41 & 3.42 & 35 & 35.8 \\
\hline 4 & EM07-Cr-012 & CCC & 3 & 3 & F26 & 9.09 & 3.83 & 33.6 & 40.2 \\
\hline 4 & EM07-Al-15 & CCC & 3 & 4 & F09 & 6.99 & 3.38 & 25 & 36.5 \\
\hline 4 & EM07-Al-20 & quenched & 3 & 5 & F59 & 6.89 & 4.27 & 22.9 & 35.5 \\
\hline 4 & EM07-Cr-02 & quenched & 3 & 6 & F46 & 8.66 & 3.69 & 33.1 & 37.7 \\
\hline 4 & EM07-Fe-20 & CCC & 3 & 7 & F30 & 7.79 & 3.08 & 29.4 & 37.7 \\
\hline 4 & EM07-Fe-20 & quenched & 3 & 8 & F55 & 9 & 4.1 & 31.5 & 34.8 \\
\hline 4 & EM07-Al-06 & quenched & 3 & 9 & F15 & 12.9 & 4.5 & 44.8 & 40.4 \\
\hline 4 & EM07-Fe-05 & $\mathrm{CCC}$ & 3 & 10 & F48 & 8.7 & 3.52 & 31.9 & 38.1 \\
\hline 4 & EA & ref & 3 & 11 & F58 & 29.9 & 9.22 & 83.7 & 47.6 \\
\hline 4 & EM07-Cr-02 & CCC & 3 & 12 & F25 & 8.25 & 3.4 & 29.3 & 38.1 \\
\hline 4 & Soln Std & ref & 3 & 13 & STD-33-2 & 20.3 & 10 & 81.6 & 53.5 \\
\hline 4 & blank & ref & 3 & 14 & F19 & 0.477 & $<1.00$ & $<0.100$ & $<0.100$ \\
\hline 4 & EM07-Fe-15 & CCC & 3 & 15 & F54 & 8.34 & 3.46 & 29.4 & 38.5 \\
\hline 4 & EM07-Al-06 & CCC & 3 & 16 & F36 & 11.3 & 4.05 & 35.1 & 31.4 \\
\hline 4 & EM07-Fe-15 & quenched & 3 & 17 & F12 & 8.93 & 3.9 & 32.2 & 35.5 \\
\hline 4 & ARM-1 & ref & 3 & 18 & F28 & 12.5 & 9.32 & 26.1 & 42.6 \\
\hline 4 & EM07-Cr-001 & quenched & 3 & 19 & F41 & 9.19 & 3.81 & 32.7 & 37.9 \\
\hline 4 & EM07-Al-20 & CCC & 3 & 20 & F60 & 7.93 & 4.3 & 22 & 37.9 \\
\hline 4 & EM07-Cr-001 & $\mathrm{CCC}$ & 3 & 21 & F22 & 9.45 & 4 & 32.3 & 41.3 \\
\hline 4 & EM07-Cr-012 & quenched & 3 & 22 & F20 & 8.87 & 3.67 & 31.5 & 37.2 \\
\hline 4 & EM07-Al-15 & quenched & 3 & 23 & F27 & 7.63 & 3.83 & 25.9 & 34.6 \\
\hline 4 & Soln Std & ref & 3 & 24 & STD-23-2 & 20.1 & 9.72 & 79.7 & 52.2 \\
\hline
\end{tabular}


Table B2. PSAL Measurements of the Test Matrix 1 PCT Solutions After Appropriate Adjustments

\begin{tabular}{|c|c|c|c|c|c|c|c|c|c|}
\hline Set & Glass ID & Heat Treatment & Block & Seq & Lab ID & $\mathrm{B}(\mathrm{ppm})$ & Li (ppm) & $\mathrm{Na}(\mathrm{ppm})$ & Si (ppm) \\
\hline 1 & Soln Std & ref & 1 & 1 & STD-11-1 & 20.3 & 9.73 & 82 & 49.2 \\
\hline 1 & EM07-Na-05 & CCC & 1 & 2 & C01 & 6.43 & 5.54 & 0.861 & 35.2 \\
\hline 1 & EM07-Zr-05 & CCC & 1 & 3 & $\mathrm{C} 10$ & 6.9 & 3.42 & 26.7 & 30.5 \\
\hline 1 & blank & ref & 1 & 4 & $\mathrm{C08}$ & $<0.100$ & $<1.00$ & $<0.100$ & $<0.100$ \\
\hline 1 & EM07-Li-015 & quenched & 1 & 5 & C58 & 7.1 & 1.97 & 28.6 & 30.9 \\
\hline 1 & EM07-F-02 & CCC & 1 & 6 & C62 & 7.2 & 3.23 & 27.7 & 33.1 \\
\hline 1 & EM07-Ni-001 & quenched & 1 & 7 & C32 & 7.61 & 3.62 & 32.1 & 33.5 \\
\hline 1 & EM07-Zr-001 & CCC & 1 & 8 & $\mathrm{C} 50$ & 8.7 & 3.82 & 32.7 & 42.6 \\
\hline 1 & EM07-Li-04 & quenched & 1 & 9 & C06 & 9.28 & 5.78 & 36.7 & 37.5 \\
\hline 1 & EM07-Ni-001 & CCC & 1 & 10 & $\mathrm{C} 22$ & 7.58 & 3.62 & 30.7 & 36.2 \\
\hline 1 & EM07-Ni-02 & $\mathrm{CCC}$ & 1 & 11 & C35 & 7.9 & 3.69 & 30.6 & 36.3 \\
\hline 1 & EM07-F-02 & quenched & 1 & 12 & C17 & 7.19 & 3.45 & 29.6 & 31.2 \\
\hline 1 & Soln Std & ref & 1 & 13 & STD-11-2 & 19.7 & 10 & 82.6 & 50.7 \\
\hline 1 & EM07-Na-20 & quenched & 1 & 14 & C36 & 15.2 & 3.55 & 84.7 & 42.8 \\
\hline 1 & EM07-Zr-001 & quenched & 1 & 15 & C47 & 9.16 & 3.79 & 36 & 40.7 \\
\hline 1 & EM07-Li-04 & CCC & 1 & 16 & C18 & 9.09 & 5.8 & 35.4 & 39.8 \\
\hline 1 & EM07-Na-10 & quenched & 1 & 17 & C54 & 7.05 & 5.5 & 10 & 35.3 \\
\hline 1 & ARM-1 & ref & 1 & 18 & $\mathrm{C} 40$ & 9.52 & 7.93 & 22.7 & 35.3 \\
\hline 1 & EM07-Zr-05 & quenched & 1 & 19 & $\mathrm{C04}$ & 7.1 & 3.65 & 30.6 & 30.4 \\
\hline 1 & EM07-Na-05 & quenched & 1 & 20 & C11 & 6.24 & 5.47 & 1.22 & 36.9 \\
\hline 1 & EM07-Na-20 & CCC & 1 & 21 & $\mathrm{C} 21$ & 17.9 & 4.69 & 89 & 46.5 \\
\hline 1 & EM07-Ni-02 & quenched & 1 & 22 & C46 & 8.25 & 3.78 & 33.2 & 35.5 \\
\hline 1 & EA & ref & 1 & 23 & C33 & 38.3 & 11.7 & 102.6 & 55.3 \\
\hline 1 & EM07-Li-015 & $\mathrm{CCC}$ & 1 & 24 & $\mathrm{C} 45$ & 7.33 & 2.1 & 28.4 & 35.6 \\
\hline 1 & EM07-Na-10 & CCC & 1 & 25 & C65 & 6.24 & 4.62 & 10.5 & 36.9 \\
\hline 1 & Soln Std & ref & 1 & 26 & STD-11-3 & 19.8 & 10.1 & 83.6 & 50.8 \\
\hline 1 & Soln Std & ref & 2 & 1 & STD-12-1 & 20.7 & 9.78 & 81.6 & 49.7 \\
\hline 1 & EM07-Ni-001 & quenched & 2 & 2 & C34 & 8.58 & 3.59 & 30.9 & 33.2 \\
\hline 1 & EA & ref & 2 & 3 & C68 & 38.1 & 11.3 & 103 & 52.7 \\
\hline 1 & EM07-Na-05 & quenched & 2 & 4 & C57 & 6.46 & 4.97 & $<0.100$ & 32.2 \\
\hline 1 & ARM-1 & ref & 2 & 5 & C56 & 10.4 & 7.93 & 22.2 & 35 \\
\hline 1 & EM07-Li-015 & CCC & 2 & 6 & $\mathrm{C} 20$ & 7.85 & 2.02 & 30.3 & 34.8 \\
\hline 1 & EM07-Na-05 & CCC & 2 & 7 & C41 & 5.98 & 5.18 & 0.778 & 32.7 \\
\hline 1 & EM07-Na-10 & quenched & 2 & 8 & $\mathrm{C} 23$ & 7.59 & 5.41 & 10.6 & 35 \\
\hline 1 & EM07-Na-10 & CCC & 2 & 9 & C53 & 6.68 & 4.5 & 10.7 & 36.1 \\
\hline 1 & EM07-F-02 & quenched & 2 & 10 & C63 & 8.23 & 3.63 & 31.9 & 32.9 \\
\hline 1 & EM07-Li-015 & quenched & 2 & 11 & $\mathrm{C} 24$ & 7.62 & 1.93 & 29 & 31.6 \\
\hline 1 & EM07-Li-04 & quenched & 2 & 12 & C52 & 9.81 & 5.71 & 37.2 & 37.5 \\
\hline 1 & Soln Std & ref & 2 & 13 & STD-12-2 & 20.3 & 9.89 & 81.8 & 50.2 \\
\hline 1 & EM07-Zr-05 & quenched & 2 & 14 & C14 & 7.73 & 3.51 & 28.4 & 29.2 \\
\hline 1 & EM07-Zr-001 & CCC & 2 & 15 & C12 & 9.25 & 3.78 & 34.6 & 42.2 \\
\hline 1 & EM07-F-02 & $\mathrm{CCC}$ & 2 & 16 & $\mathrm{C07}$ & 7.64 & 3.19 & 28.3 & 33.6 \\
\hline 1 & EM07-Na-20 & quenched & 2 & 17 & C60 & 15.8 & 3.54 & 88.4 & 43.1 \\
\hline 1 & EM07-Ni-02 & CCC & 2 & 18 & C38 & 8.29 & 3.6 & 31.5 & 35.8 \\
\hline 1 & EM07-Ni-001 & $\mathrm{CCC}$ & 2 & 19 & $\mathrm{C} 25$ & 8.05 & 3.56 & 31 & 36.1 \\
\hline 1 & EM07-Li-04 & CCC & 2 & 20 & C59 & 9.42 & 5.71 & 35.6 & 39.5 \\
\hline 1 & EM07-Na-20 & $\mathrm{CCC}$ & 2 & 21 & $\mathrm{C} 15$ & 17.8 & 4.53 & 89.8 & 44.8 \\
\hline 1 & EM07-Zr-001 & quenched & 2 & 22 & $\mathrm{C} 13$ & 9.28 & 3.71 & 35.4 & 39.3 \\
\hline 1 & EM07-Ni-02 & quenched & 2 & 23 & C31 & 8.44 & 3.65 & 35.7 & 34.1 \\
\hline 1 & EM07-Zr-05 & CCC & 2 & 24 & C48 & 6.83 & 3.36 & 26.9 & 30.3 \\
\hline 1 & Soln Std & ref & 2 & 25 & STD-12-3 & 19.9 & 9.86 & 82.5 & 49.9 \\
\hline 1 & Soln Std & ref & 3 & 1 & STD-13-1 & 19.8 & 9.67 & 82.8 & 48.1 \\
\hline 1 & EM07-Li-015 & quenched & 3 & 2 & C39 & 7.73 & 1.94 & 31.9 & 30.4 \\
\hline 1 & EM07-F-02 & quenched & 3 & 3 & $\mathrm{C} 44$ & 8.5 & 3.66 & 31.8 & 33 \\
\hline 1 & blank & ref & 3 & 4 & C26 & $<0.100$ & $<1.00$ & $<0.100$ & $<0.100$ \\
\hline 1 & EM07-Li-04 & quenched & 3 & 5 & C64 & 9.32 & 5.65 & 37.9 & 36 \\
\hline 1 & EM07-Na-10 & quenched & 3 & 6 & C49 & 7.28 & 5.44 & 10.7 & 34.7 \\
\hline 1 & EM07-Ni-02 & $\mathrm{CCC}$ & 3 & 7 & C30 & 8.02 & 3.62 & 33.9 & 35.5 \\
\hline
\end{tabular}


Table B2. PSAL Measurements of the Test Matrix 1 PCT Solutions After Appropriate Adjustments

\begin{tabular}{|c|c|c|c|c|c|c|c|c|c|}
\hline Set & Glass ID & Heat Treatment & Block & Seq & Lab ID & $\mathrm{B}(\mathrm{ppm})$ & Li (ppm) & $\mathrm{Na}(\mathrm{ppm})$ & Si (ppm) \\
\hline 1 & ARM-1 & ref & 3 & 8 & $\mathrm{C} 43$ & 9.56 & 7.75 & 22.7 & 34 \\
\hline 1 & EM07-Li-04 & CCC & 3 & 9 & $\mathrm{C09}$ & 9.15 & 5.71 & 35.6 & 39.1 \\
\hline 1 & EM07-Zr-05 & quenched & 3 & 10 & $\mathrm{C51}$ & 7.18 & 3.5 & 28.7 & 28.9 \\
\hline 1 & EM07-Ni-001 & quenched & 3 & 11 & $\mathrm{C03}$ & 7.5 & 3.56 & 31 & 32.6 \\
\hline 1 & EM07-F-02 & CCC & 3 & 12 & C19 & 7.09 & 3.2 & 28.5 & 32.7 \\
\hline 1 & Soln Std & ref & 3 & 13 & STD-13-2 & 19.5 & 9.72 & 81.9 & 49 \\
\hline 1 & EM07-Na-20 & quenched & 3 & 14 & C67 & 15.5 & 3.52 & 88.9 & 42 \\
\hline 1 & EA & ref & 3 & 15 & $\mathrm{C} 28$ & 37.3 & 11.3 & 105 & 52.4 \\
\hline 1 & EM07-Zr-001 & CCC & 3 & 16 & C37 & 9.41 & 3.89 & 35.2 & 42.8 \\
\hline 1 & EM07-Zr-05 & CCC & 3 & 17 & C66 & 6.9 & 3.39 & 28.4 & 30.2 \\
\hline 1 & EM07-Na-10 & $\mathrm{CCC}$ & 3 & 18 & $\mathrm{C} 27$ & 6.51 & 4.55 & 10.7 & 36 \\
\hline 1 & EM07-Li-015 & CCC & 3 & 19 & C61 & 6.89 & 1.91 & 28.3 & 32.5 \\
\hline 1 & EM07-Na-05 & CCC & 3 & 20 & $\mathrm{C05}$ & 5.8 & 5.28 & 0.141 & 33.3 \\
\hline 1 & EM07-Na-20 & CCC & 3 & 21 & C16 & 17.5 & 4.54 & 90.2 & 44.3 \\
\hline 1 & EM07-Na-05 & quenched & 3 & 22 & C42 & 6.25 & 5.33 & $<0.100$ & 34.8 \\
\hline 1 & EM07-Ni-02 & quenched & 3 & 23 & C29 & 7.93 & 3.57 & 31.8 & 33.2 \\
\hline 1 & EM07-Zr-001 & quenched & 3 & 24 & C55 & 8.99 & 3.69 & 34.4 & 39.1 \\
\hline 1 & EM07-Ni-001 & $\mathrm{CCC}$ & 3 & 25 & $\mathrm{C} 02$ & 7.57 & 3.52 & 29.3 & 35.1 \\
\hline 1 & Soln Std & ref & 3 & 26 & STD-13-3 & 19.6 & 9.83 & 82.3 & 49.4 \\
\hline 2 & Soln Std & ref & 1 & 1 & STD-21-1 & 20.3 & 9.7 & 77.8 & 49.3 \\
\hline 2 & EM07-B-05 & quenched & 1 & 2 & D32 & 4.14 & 3.78 & 40.5 & 42.1 \\
\hline 2 & EM07-Bi-05 & quenched & 1 & 3 & D34 & 8.86 & 3.85 & 32.5 & 34.4 \\
\hline 2 & EM07-Bi-025 & quenched & 1 & 4 & D41 & 9.1 & 4.05 & 34 & 36.3 \\
\hline 2 & EM07-Si-50 & quenched & 1 & 5 & D51 & 5.99 & 3.48 & 21.7 & 37.5 \\
\hline 2 & EM07-P-0 & CCC & 1 & 6 & D05 & 8.38 & 3.72 & 31.1 & 38.5 \\
\hline 2 & EM07-Bi-05 & CCC & 1 & 7 & D37 & 8.83 & 3.98 & 32.2 & 36.5 \\
\hline 2 & ARM-1 & ref & 1 & 8 & D43 & 9.8 & 7.92 & 19.8 & 35.3 \\
\hline 2 & EM07-Si-37 & quenched & 1 & 9 & D24 & 15.4 & 5.02 & 52.5 & 32.1 \\
\hline 2 & EM07-P-025 & CCC & 1 & 10 & D56 & 8.87 & 7.78 & 38.1 & 39 \\
\hline 2 & EA & ref & 1 & 11 & D29 & 36.8 & 11.2 & 101 & 53 \\
\hline 2 & EM07-B-20 & quenched & 1 & 12 & D10 & 243 & 39.4 & 217 & 20.3 \\
\hline 2 & Soln Std & ref & 1 & 13 & STD-21-2 & 20.8 & 9.68 & 80.9 & 48.3 \\
\hline 2 & EM07-P-0 & quenched & 1 & 14 & D13 & 9.32 & 3.71 & 32.4 & 35 \\
\hline 2 & blank & ref & 1 & 15 & D48 & 0.557 & $<1.00$ & $<0.100$ & $<0.100$ \\
\hline 2 & EM07-Si-30 & quenched & 1 & 16 & D20 & 63.2 & 16.8 & 154 & 36.1 \\
\hline 2 & EM07-Si-37 & $\mathrm{CCC}$ & 1 & 17 & D16 & 25 & 7.27 & 66.8 & 36.8 \\
\hline 2 & EM07-Si-50 & CCC & 1 & 18 & D01 & 6.27 & 3.37 & 20.2 & 40.2 \\
\hline 2 & EM07-B-20 & CCC & 1 & 19 & D63 & 368 & 58.6 & 322 & 20.2 \\
\hline 2 & EM07-B-05 & CCC & 1 & 20 & D38 & 5.68 & 3.79 & 39 & 42.4 \\
\hline 2 & EM07-Si-30 & CCC & 1 & 21 & D31 & 1310 & 270 & 2170 & 92.1 \\
\hline 2 & EM07-B-15 & CCC & 1 & 22 & D33 & 55.2 & 10.5 & 65.6 & 31.6 \\
\hline 2 & EM07-Bi-025 & $\mathrm{CCC}$ & 1 & 23 & D28 & 14.4 & 3.88 & 30.7 & 37.2 \\
\hline 2 & EM07-B-15 & quenched & 1 & 24 & D58 & 41.2 & 8.48 & 56 & 29.4 \\
\hline 2 & EM07-P-025 & quenched & 1 & 25 & D53 & 11.1 & 3.81 & 32 & 34 \\
\hline 2 & Soln Std & ref & 1 & 26 & STD-21-3 & 21.8 & 9.66 & 81.3 & 47.9 \\
\hline 2 & Soln Std & ref & 2 & 1 & STD-22-1 & 19.7 & 9.82 & 79.5 & 48.4 \\
\hline 2 & ARM-1 & ref & 2 & 2 & $\mathrm{D} 42$ & 9.3 & 7.93 & 19.9 & 34.2 \\
\hline 2 & EM07-Bi-05 & quenched & 2 & 3 & D04 & 8.05 & 4.01 & 33.6 & 33.6 \\
\hline 2 & EM07-Bi-025 & CCC & 2 & 4 & D44 & 7.32 & 3.89 & 31.2 & 35.1 \\
\hline 2 & EM07-P-0 & CCC & 2 & 5 & D25 & 7.47 & 3.83 & 31.6 & 36.8 \\
\hline 2 & EM07-B-20 & $\mathrm{CCC}$ & 2 & 6 & D21 & 384 & 58.9 & 317 & 20.8 \\
\hline 2 & EM07-Si-50 & $\mathrm{CCC}$ & 2 & 7 & D55 & 6.69 & 3.47 & 20.8 & 38.9 \\
\hline 2 & EM07-B-20 & quenched & 2 & 8 & D22 & 255 & 39.5 & 219 & 20.6 \\
\hline 2 & EM07-Si-37 & CCC & 2 & 9 & D23 & 24.9 & 7.28 & 66.8 & 35.4 \\
\hline 2 & EM07-Si-30 & CCC & 2 & 10 & D39 & 1300 & 306 & 2110 & 90.8 \\
\hline 2 & EM07-Si-30 & quenched & 2 & 11 & D03 & 66.1 & 16.4 & 155 & 34.5 \\
\hline 2 & EM07-Si-37 & quenched & 2 & 12 & D19 & 20.6 & 5.07 & 52.1 & 31.3 \\
\hline 2 & Soln Std & ref & 2 & 13 & STD-22-2 & 21.2 & 9.7 & 80.4 & 47.6 \\
\hline
\end{tabular}


Table B2. PSAL Measurements of the Test Matrix 1 PCT Solutions After Appropriate Adjustments

\begin{tabular}{|c|c|c|c|c|c|c|c|c|c|}
\hline Set & Glass ID & Heat Treatment & Block & Seq & Lab ID & B (ppm) & Li (ppm) & $\mathrm{Na}(\mathrm{ppm})$ & Si (ppm) \\
\hline 2 & EM07-P-0 & quenched & 2 & 14 & D09 & 11.3 & 3.88 & 32.2 & 35.3 \\
\hline 2 & EM07-B-05 & CCC & 2 & 15 & D35 & 6.23 & 4.03 & 39.5 & 43 \\
\hline 2 & EM07-P-025 & quenched & 2 & 16 & D06 & 9.54 & 3.98 & 32.6 & 34.2 \\
\hline 2 & EM07-B-15 & CCC & 2 & 17 & D64 & 45.4 & 10.3 & 65.3 & 30.7 \\
\hline 2 & EM07-B-15 & quenched & 2 & 18 & D36 & 35.7 & 8.37 & 54.7 & 28.4 \\
\hline 2 & EA & ref & 2 & 19 & D57 & 35.9 & 11.2 & 101 & 51.4 \\
\hline 2 & EM07-Bi-025 & quenched & 2 & 20 & D67 & 9.12 & 4.04 & 35.2 & 34.2 \\
\hline 2 & EM07-Si-50 & quenched & 2 & 21 & D49 & 6.32 & 3.62 & 20.6 & 35.9 \\
\hline 2 & EM07-Bi-05 & CCC & 2 & 22 & D26 & 8.93 & 3.97 & 31 & 34.7 \\
\hline 2 & EM07-B-05 & quenched & 2 & 23 & D18 & 3.94 & 3.96 & 42.5 & 40.8 \\
\hline 2 & EM07-P-025 & CCC & 2 & 24 & D62 & 8.81 & 7.8 & 38.3 & 38.5 \\
\hline 2 & Soln Std & ref & 2 & 25 & STD-22-3 & 19.1 & 9.71 & 79 & 47.6 \\
\hline 2 & Soln Std & ref & 3 & 1 & STD-23-1 & 19.4 & 9.58 & 78.2 & 48.1 \\
\hline 2 & blank & ref & 3 & 2 & D02 & $<0.100$ & $<1.00$ & 0.72 & $<0.100$ \\
\hline 2 & EM07-P-025 & CCC & 3 & 3 & D59 & 8.32 & 7.63 & 37.8 & 37.9 \\
\hline 2 & EM07-Bi-05 & quenched & 3 & 4 & D45 & 7.81 & 3.75 & 32.9 & 32.4 \\
\hline 2 & ARM-1 & ref & 3 & 5 & D66 & 9.59 & 8.02 & 20.5 & 35.4 \\
\hline 2 & EM07-Si-37 & CCC & 3 & 6 & D46 & 23.7 & 7.27 & 66.9 & 36.7 \\
\hline 2 & EM07-Si-30 & $\mathrm{CCC}$ & 3 & 7 & D68 & 1290 & 307 & 2310 & 92.7 \\
\hline 2 & EM07-Si-30 & quenched & 3 & 8 & D54 & 64.8 & 16.5 & 153 & 34.8 \\
\hline 2 & EM07-B-05 & quenched & 3 & 9 & D15 & 8.05 & 3.78 & 41.4 & 40.9 \\
\hline 2 & EM07-P-0 & CCC & 3 & 10 & D30 & 11.1 & 3.68 & 31.6 & 36.4 \\
\hline 2 & EM07-P-0 & quenched & 3 & 11 & D50 & 10.6 & 3.67 & 33.3 & 34 \\
\hline 2 & EM07-B-15 & quenched & 3 & 12 & D08 & 37.4 & 8.23 & 56.8 & 28.7 \\
\hline 2 & Soln Std & ref & 3 & 13 & STD-23-2 & 21.4 & 9.69 & 77.6 & 48.9 \\
\hline 2 & EM07-Si-50 & quenched & 3 & 14 & D40 & 7.36 & 3.49 & 20.1 & 36.6 \\
\hline 2 & EM07-B-20 & quenched & 3 & 15 & D12 & 246 & 38.7 & 228 & 20.3 \\
\hline 2 & EA & ref & 3 & 16 & D27 & 37.3 & 10.8 & 95.4 & 51.3 \\
\hline 2 & EM07-Si-37 & quenched & 3 & 17 & D65 & 16.1 & 4.9 & 51.8 & 31 \\
\hline 2 & EM07-B-20 & CCC & 3 & 18 & D61 & 361 & 56.7 & 330 & 19.7 \\
\hline 2 & EM07-Bi-025 & quenched & 3 & 19 & D11 & 11 & 3.86 & 33.2 & 34.4 \\
\hline 2 & EM07-P-025 & quenched & 3 & 20 & D14 & 10.7 & 4.01 & 33.9 & 36.4 \\
\hline 2 & EM07-Si-50 & CCC & 3 & 21 & D52 & 7.3 & 3.33 & 19.6 & 39.5 \\
\hline 2 & EM07-B-15 & CCC & 3 & 22 & D47 & 46.4 & 10.1 & 62.7 & 31.1 \\
\hline 2 & EM07-B-05 & CCC & 3 & 23 & D17 & 4.51 & 3.81 & 39.1 & 43 \\
\hline 2 & EM07-Bi-025 & CCC & 3 & 24 & D60 & 8.74 & 3.75 & 30.5 & 36 \\
\hline 2 & EM07-Bi-05 & CCC & 3 & 25 & D07 & 8.56 & 3.75 & 31.7 & 34.1 \\
\hline 2 & Soln Std & ref & 3 & 26 & STD-23-3 & 19.4 & 9.52 & 77.4 & 47.9 \\
\hline 3 & Soln Std & ref & 1 & 1 & STD-31-1 & 21 & 9.93 & 82.2 & 51.4 \\
\hline 3 & EM07-Ca-07 & CCC & 1 & 2 & E35 & 8.52 & 4.17 & 36.9 & 24.1 \\
\hline 3 & EM07-Mn-04 & CCC & 1 & 3 & E23 & 9 & 3.74 & 31.7 & 36.6 \\
\hline 3 & EM07-BL-2 & quenched & 1 & 4 & E52 & 9.05 & 3.74 & 31.6 & 35.4 \\
\hline 3 & EM07-Mn-01 & CCC & 1 & 5 & E45 & 8.58 & 3.73 & 31 & 38.2 \\
\hline 3 & EM07-Ca-07 & quenched & 1 & 6 & E34 & 12.2 & 5.06 & 47 & 28.7 \\
\hline 3 & EA & ref & 1 & 7 & E30 & 40.3 & 12 & 103 & 57.2 \\
\hline 3 & EM07-BL-2 & CCC & 1 & 8 & E14 & 9.03 & 3.59 & 28.9 & 36.4 \\
\hline 3 & ARM-1 & ref & 1 & 9 & E26 & 13.9 & 10 & 25.7 & 42.9 \\
\hline 3 & EM07-Mn-01 & quenched & 1 & 10 & E32 & 8.85 & 3.7 & 31.9 & 36.7 \\
\hline 3 & EM07-K-06 & quenched & 1 & 11 & E21 & 17.7 & 6.37 & 53 & 37 \\
\hline 3 & EM07-BL-1 & quenched & 1 & 12 & E06 & 9.3 & 3.78 & 32.9 & 36.7 \\
\hline 3 & Soln Std & ref & 1 & 13 & STD-31-2 & 21.4 & 10.2 & 80.8 & 51.8 \\
\hline 3 & EM07-K-03 & quenched & 1 & 14 & E13 & 9.91 & 3.66 & 34 & 33.1 \\
\hline 3 & blank & ref & 1 & 15 & E60 & 0.496 & $<1.00$ & $<0.100$ & $<0.100$ \\
\hline 3 & EM07-Mn-04 & quenched & 1 & 16 & E56 & 9.62 & 3.91 & 33 & 36.8 \\
\hline 3 & EM07-NM-0025 & CCC & 1 & 17 & E43 & 8.92 & 3.65 & 29.9 & 38.9 \\
\hline 3 & EM07-K-03 & CCC & 1 & 18 & E01 & 11.2 & 4.13 & 35.3 & 35.9 \\
\hline 3 & EM07-Ca-035 & quenched & 1 & 19 & E59 & 0.399 & $<1.00$ & $<0.100$ & $<0.100$ \\
\hline 3 & EM07-K-06 & $\mathrm{CCC}$ & 1 & 20 & E09 & 27.9 & 10.2 & 84.3 & 64.6 \\
\hline
\end{tabular}


Table B2. PSAL Measurements of the Test Matrix 1 PCT Solutions After Appropriate Adjustments

\begin{tabular}{|c|c|c|c|c|c|c|c|c|c|}
\hline Set & Glass ID & Heat Treatment & Block & Seq & Lab ID & $\mathrm{B}(\mathrm{ppm})$ & Li (ppm) & $\mathrm{Na}(\mathrm{ppm})$ & Si (ppm) \\
\hline 3 & EM07-Ca-035 & CCC & 1 & 21 & E04 & 8.79 & 3.77 & 31.7 & 30.2 \\
\hline 3 & EM07-NM-0025 & quenched & 1 & 22 & E27 & 9.57 & 3.89 & 31.2 & 36.9 \\
\hline 3 & EM07-BL-1 & CCC & 1 & 23 & E31 & 8.77 & 3.7 & 29.5 & 39.1 \\
\hline 3 & Soln Std & ref & 1 & 24 & STD-31-3 & 21.6 & 10.3 & 79.5 & 53.4 \\
\hline 3 & Soln Std & ref & 2 & 1 & STD-32-1 & 21.1 & 9.9 & 83 & 51.9 \\
\hline 3 & EM07-BL-2 & CCC & 2 & 2 & E16 & 8.72 & 3.46 & 30 & 36.7 \\
\hline 3 & EM07-K-06 & CCC & 2 & 3 & E33 & 20 & 7.11 & 61.2 & 36.7 \\
\hline 3 & EM07-Ca-07 & quenched & 2 & 4 & E57 & 11.8 & 4.9 & 48.7 & 28.8 \\
\hline 3 & EM07-Ca-035 & CCC & 2 & 5 & E49 & 8.04 & 3.57 & 33.7 & 28.9 \\
\hline 3 & EM07-K-03 & CCC & 2 & 6 & E42 & 11.1 & 4.24 & 39 & 36.3 \\
\hline 3 & EM07-Ca-035 & quenched & 2 & 7 & E17 & 9.66 & 3.86 & 37.9 & 30.1 \\
\hline 3 & ARM-1 & ref & 2 & 8 & E50 & 13.1 & 9.88 & 26.8 & 42 \\
\hline 3 & EM07-Mn-01 & CCC & 2 & 9 & E24 & 7.98 & 3.53 & 31 & 37.3 \\
\hline 3 & EA & ref & 2 & 10 & E11 & 40.1 & 11.7 & 107 & 56.8 \\
\hline 3 & EM07-BL-2 & quenched & 2 & 11 & E02 & 8.73 & 3.59 & 32.3 & 35.2 \\
\hline 3 & EM07-K-06 & quenched & 2 & 12 & E28 & 16 & 5.85 & 52 & 34.8 \\
\hline 3 & Soln Std & ref & 2 & 13 & STD-32-2 & 20.3 & 9.86 & 82.6 & 50.7 \\
\hline 3 & EM07-NM-0025 & quenched & 2 & 14 & $\mathrm{E} 40$ & 8.89 & 3.62 & 32.1 & 34.8 \\
\hline 3 & EM07-Ca-07 & CCC & 2 & 15 & E20 & 8.43 & 4.16 & 37.8 & 24.8 \\
\hline 3 & EM07-Mn-04 & quenched & 2 & 16 & E62 & 9.27 & 3.83 & 35.3 & 37 \\
\hline 3 & EM07-Mn-04 & CCC & 2 & 17 & E58 & 8.34 & 3.61 & 32.1 & 36.9 \\
\hline 3 & EM07-K-03 & quenched & 2 & 18 & E36 & 9.01 & 3.46 & 35.3 & 31.3 \\
\hline 3 & EM07-Mn-01 & quenched & 2 & 19 & E08 & 8.04 & 3.57 & 33.3 & 36.5 \\
\hline 3 & EM07-BL-1 & quenched & 2 & 20 & E39 & 8.22 & 3.52 & 33.1 & 35.2 \\
\hline 3 & EM07-NM-0025 & CCC & 2 & 21 & E54 & 8.22 & 3.51 & 30.9 & 38.6 \\
\hline 3 & EM07-BL-1 & CCC & 2 & 22 & E03 & 7.93 & 3.53 & 31.2 & 37.6 \\
\hline 3 & Soln Std & ref & 2 & 23 & STD-32-3 & 20.1 & 9.89 & 83.4 & 50.6 \\
\hline 3 & Soln Std & ref & 3 & 1 & STD-33-1 & 21 & 9.89 & 82.1 & 51.8 \\
\hline 3 & EM07-Ca-07 & quenched & 3 & 2 & E44 & 12.4 & 4.9 & 47.8 & 29.4 \\
\hline 3 & EM07-K-06 & CCC & 3 & 3 & E15 & 20 & 7.04 & 59.4 & 37.8 \\
\hline 3 & EM07-NM-0025 & quenched & 3 & 4 & E51 & 9.07 & 3.64 & 32.5 & 35.3 \\
\hline 3 & EA & ref & 3 & 5 & E41 & 39.7 & 11.6 & 104 & 56.6 \\
\hline 3 & EM07-Ca-035 & CCC & 3 & 6 & E47 & 8.41 & 3.5 & 32.4 & 29.5 \\
\hline 3 & EM07-Ca-07 & CCC & 3 & 7 & E29 & 8.73 & 4.23 & 37.9 & 25.6 \\
\hline 3 & EM07-BL-2 & CCC & 3 & 8 & E46 & 8.5 & 3.47 & 30.1 & 37 \\
\hline 3 & ARM-1 & ref & 3 & 9 & E19 & 13.3 & 9.68 & 25.9 & 43 \\
\hline 3 & EM07-Mn-01 & quenched & 3 & 10 & E18 & 8.44 & 3.57 & 32.7 & 37.7 \\
\hline 3 & EM07-Ca-035 & quenched & 3 & 11 & E10 & 9.7 & 3.8 & 36.6 & 31.1 \\
\hline 3 & EM07-K-03 & quenched & 3 & 12 & E25 & 9.54 & 3.54 & 35.1 & 33.3 \\
\hline 3 & Soln Std & ref & 3 & 13 & STD-33-2 & 21.5 & 10.2 & 81.4 & 54.8 \\
\hline 3 & blank & ref & 3 & 14 & E22 & 0.54 & $<1.00$ & $<0.100$ & $<0.100$ \\
\hline 3 & EM07-BL-1 & quenched & 3 & 15 & E48 & 8.95 & 3.59 & 32.4 & 38.3 \\
\hline 3 & EM07-BL-1 & CCC & 3 & 16 & E37 & 8.41 & 3.55 & 30.9 & 39.6 \\
\hline 3 & EM07-Mn-04 & CCC & 3 & 17 & E53 & 8.75 & 3.65 & 32 & 38.8 \\
\hline 3 & EM07-K-06 & quenched & 3 & 18 & E61 & 15.7 & 5.74 & 50 & 35.9 \\
\hline 3 & EM07-Mn-01 & CCC & 3 & 19 & E07 & 8.51 & 3.63 & 31.7 & 39.6 \\
\hline 3 & EM07-Mn-04 & quenched & 3 & 20 & E05 & 9.63 & 3.9 & 35 & 39.3 \\
\hline 3 & EM07-BL-2 & quenched & 3 & 21 & E12 & 8.83 & 3.59 & 31.9 & 36.4 \\
\hline 3 & EM07-K-03 & CCC & 3 & 22 & E38 & 10.6 & 3.97 & 36 & 36.3 \\
\hline 3 & EM07-NM-0025 & CCC & 3 & 23 & E55 & 8.43 & 3.5 & 30.4 & 39.4 \\
\hline 3 & Soln Std & ref & 3 & 24 & STD-23-2 & 20.4 & 9.89 & 80.9 & 52.6 \\
\hline 4 & Soln Std & ref & 1 & 1 & STD-31-1 & 21.1 & 10 & 82.4 & 52.1 \\
\hline 4 & EM07-Fe-15 & CCC & 1 & 2 & F18 & 9.07 & 3.62 & 30.1 & 39.7 \\
\hline 4 & EM07-Al-20 & $\mathrm{CCC}$ & 1 & 3 & F03 & 8.34 & 4.44 & 22.9 & 37.5 \\
\hline 4 & EM07-Fe-05 & CCC & 1 & 4 & F33 & 9.18 & 3.66 & 32.2 & 38.8 \\
\hline 4 & EM07-Fe-20 & CCC & 1 & 5 & F34 & 8.05 & 3.14 & 28.1 & 37.8 \\
\hline 4 & EM07-Cr-012 & quenched & 1 & 6 & F52 & 9.17 & 3.88 & 33.5 & 37.5 \\
\hline 4 & blank & ref & 1 & 7 & F32 & 0.289 & $<1.00$ & $<0.100$ & 0.916 \\
\hline
\end{tabular}


Table B2. PSAL Measurements of the Test Matrix 1 PCT Solutions After Appropriate Adjustments

\begin{tabular}{|c|c|c|c|c|c|c|c|c|c|}
\hline Set & Glass ID & Heat Treatment & Block & Seq & Lab ID & $\mathrm{B}(\mathrm{ppm})$ & Li (ppm) & $\mathrm{Na}(\mathrm{ppm})$ & Si (ppm) \\
\hline 4 & ARM-1 & ref & 1 & 8 & F04 & 12.8 & 9.49 & 25.5 & 41.6 \\
\hline 4 & EM07-Cr-001 & quenched & 1 & 9 & F02 & 9.49 & 3.92 & 33.3 & 37.7 \\
\hline 4 & EM07-Al-15 & CCC & 1 & 10 & F24 & 7.18 & 3.51 & 24.9 & 37.2 \\
\hline 4 & EM07-Cr-012 & CCC & 1 & 11 & F44 & 9.64 & 4.05 & 33.2 & 42.4 \\
\hline 4 & EM07-Fe-15 & quenched & 1 & 12 & F56 & 9.26 & 4.08 & 31.1 & 36.2 \\
\hline 4 & Soln Std & ref & 1 & 13 & STD-31-2 & 20.8 & 10 & 80.7 & 52.6 \\
\hline 4 & EM07-Fe-20 & quenched & 1 & 14 & F62 & 9.51 & 4.1 & 29.1 & 34.7 \\
\hline 4 & EM07-Al-06 & quenched & 1 & 15 & F37 & 13.6 & 4.65 & 44.9 & 41.4 \\
\hline 4 & EM07-Al-06 & CCC & 1 & 16 & F11 & 11.8 & 4.2 & 35.6 & 32.2 \\
\hline 4 & EA & ref & 1 & 17 & F31 & 33 & 10 & 87.7 & 50.4 \\
\hline 4 & EM07-Cr-02 & quenched & 1 & 18 & F14 & 9.51 & 3.86 & 32.7 & 39.2 \\
\hline 4 & EM07-Cr-001 & CCC & 1 & 19 & F50 & 9.85 & 4.02 & 31.8 & 41.8 \\
\hline 4 & EM07-Al-20 & quenched & 1 & 20 & F05 & 7.62 & 4.5 & 22.2 & 38.3 \\
\hline 4 & EM07-Cr-02 & CCC & 1 & 21 & F13 & 8.71 & 3.6 & 29 & 39.8 \\
\hline 4 & EM07-Al-15 & quenched & 1 & 22 & F40 & 7.91 & 3.9 & 25.5 & 34.6 \\
\hline 4 & EM07-Fe-05 & quenched & 1 & 23 & F51 & 9.68 & 3.6 & 34.6 & 37.1 \\
\hline 4 & Soln Std & ref & 1 & 24 & STD-31-3 & 20.5 & 9.89 & 79.8 & 52.4 \\
\hline 4 & Soln Std & ref & 2 & 1 & STD-32-1 & 20.7 & 9.8 & 83.3 & 51.3 \\
\hline 4 & EM07-Fe-15 & quenched & 2 & 2 & F10 & 9.06 & 3.96 & 31.7 & 34.5 \\
\hline 4 & EM07-Al-06 & CCC & 2 & 3 & F16 & 11.5 & 4.21 & 36.5 & 31.9 \\
\hline 4 & EM07-Al-20 & quenched & 2 & 4 & F57 & 11.8 & 7.22 & 38.1 & 60.6 \\
\hline 4 & EM07-Cr-001 & quenched & 2 & 5 & F01 & 9.12 & 3.87 & 32.8 & 37.7 \\
\hline 4 & EM07-Al-20 & CCC & 2 & 6 & F17 & 7.85 & 4.43 & 23.4 & 37.5 \\
\hline 4 & EM07-Fe-05 & $\mathrm{CCC}$ & 2 & 7 & F47 & 8.91 & 3.72 & 32.8 & 39.3 \\
\hline 4 & EM07-Cr-02 & quenched & 2 & 8 & F42 & 8.47 & 3.69 & 32.4 & 36.6 \\
\hline 4 & EM07-Cr-012 & quenched & 2 & 9 & F08 & 8.31 & 3.63 & 32 & 34.8 \\
\hline 4 & EM07-Cr-012 & CCC & 2 & 10 & F43 & 8.56 & 3.78 & 32 & 39.6 \\
\hline 4 & ARM-1 & ref & 2 & 11 & F61 & 12.5 & 9.46 & 26.2 & 41.2 \\
\hline 4 & EM07-Cr-02 & CCC & 2 & 12 & F21 & 7.98 & 3.53 & 29.6 & 38.1 \\
\hline 4 & Soln Std & ref & 2 & 13 & STD-32-2 & 20.3 & 9.92 & 81.7 & 52 \\
\hline 4 & EM07-Al-15 & quenched & 2 & 14 & F53 & 8.04 & 3.96 & 32.5 & 35 \\
\hline 4 & EM07-Al-15 & CCC & 2 & 15 & F29 & 7.11 & 3.53 & 25 & 37.4 \\
\hline 4 & EM07-Fe-20 & quenched & 2 & 16 & F49 & 9.16 & 4.24 & 30.6 & 35.5 \\
\hline 4 & EM07-Fe-05 & quenched & 2 & 17 & F06 & 9.27 & 3.55 & 33.6 & 36.5 \\
\hline 4 & EA & ref & 2 & 18 & F35 & 32.3 & 9.95 & 88.9 & 50.5 \\
\hline 4 & EM07-Fe-15 & $\mathrm{CCC}$ & 2 & 19 & F38 & 9.51 & 3.98 & 33 & 43.4 \\
\hline 4 & EM07-Al-06 & quenched & 2 & 20 & F23 & 13.6 & 4.77 & 44.5 & 43.1 \\
\hline 4 & EM07-Cr-001 & CCC & 2 & 21 & F45 & 9.7 & 4.14 & 32.5 & 41.7 \\
\hline 4 & EM07-Fe-20 & CCC & 2 & 22 & F07 & 7.85 & 3.17 & 28.2 & 38.2 \\
\hline 4 & Soln Std & ref & 2 & 23 & STD-32-3 & 20.1 & 9.84 & 80.4 & 51.9 \\
\hline 4 & Soln Std & ref & 3 & 1 & STD-33-1 & 20.5 & 9.8 & 83.4 & 51 \\
\hline 4 & EM07-Fe-05 & quenched & 3 & 2 & F39 & 9.41 & 3.42 & 35 & 35.8 \\
\hline 4 & EM07-Cr-012 & CCC & 3 & 3 & F26 & 9.09 & 3.83 & 33.6 & 40.2 \\
\hline 4 & EM07-Al-15 & CCC & 3 & 4 & F09 & 6.99 & 3.38 & 25 & 36.5 \\
\hline 4 & EM07-Al-20 & quenched & 3 & 5 & F59 & 6.89 & 4.27 & 22.9 & 35.5 \\
\hline 4 & EM07-Cr-02 & quenched & 3 & 6 & F46 & 8.66 & 3.69 & 33.1 & 37.7 \\
\hline 4 & EM07-Fe-20 & $\mathrm{CCC}$ & 3 & 7 & F30 & 7.79 & 3.08 & 29.4 & 37.7 \\
\hline 4 & EM07-Fe-20 & quenched & 3 & 8 & F55 & 9 & 4.1 & 31.5 & 34.8 \\
\hline 4 & EM07-Al-06 & quenched & 3 & 9 & F15 & 12.9 & 4.5 & 44.8 & 40.4 \\
\hline 4 & EM07-Fe-05 & CCC & 3 & 10 & F48 & 8.7 & 3.52 & 31.9 & 38.1 \\
\hline 4 & EA & ref & 3 & 11 & F58 & 29.9 & 9.22 & 83.7 & 47.6 \\
\hline 4 & EM07-Cr-02 & $\mathrm{CCC}$ & 3 & 12 & F25 & 8.25 & 3.4 & 29.3 & 38.1 \\
\hline 4 & Soln Std & ref & 3 & 13 & STD-33-2 & 20.3 & 10 & 81.6 & 53.5 \\
\hline 4 & blank & ref & 3 & 14 & F19 & 0.477 & $<1.00$ & $<0.100$ & $<0.100$ \\
\hline 4 & EM07-Fe-15 & CCC & 3 & 15 & F54 & 8.34 & 3.46 & 29.4 & 38.5 \\
\hline 4 & EM07-Al-06 & CCC & 3 & 16 & F36 & 11.3 & 4.05 & 35.1 & 31.4 \\
\hline 4 & EM07-Fe-15 & quenched & 3 & 17 & F12 & 8.93 & 3.9 & 32.2 & 35.5 \\
\hline 4 & ARM-1 & ref & 3 & 18 & F28 & 12.5 & 9.32 & 26.1 & 42.6 \\
\hline
\end{tabular}


SRNS-STI-2008-00056

Revision: 0

Table B2. PSAL Measurements of the Test Matrix 1 PCT Solutions After Appropriate Adjustments

\begin{tabular}{||c|c|c|c|c|c|c|c|c|c||}
\hline Set & Glass ID & Heat Treatment & Block & Seq & Lab ID & B (ppm) & Li (ppm) & Na $(\mathrm{ppm})$ & Si (ppm) \\
\hline 4 & EM07-Cr-001 & quenched & 3 & 19 & F41 & 9.19 & 3.81 & 32.7 & 37.9 \\
\hline 4 & EM07-Al-20 & ccc & 3 & 20 & F60 & 7.93 & 4.3 & 22 & 37.9 \\
\hline 4 & EM07-Cr-001 & ccc & 3 & 21 & F22 & 9.45 & 4 & 32.3 & 41.3 \\
\hline 4 & EM07-Cr-012 & quenched & 3 & 22 & F20 & 8.87 & 3.67 & 31.5 & 37.2 \\
\hline 4 & EM07-Al-15 & quenched & 3 & 23 & F27 & 7.63 & 3.83 & 25.9 & 34.6 \\
\hline 4 & Soln Std & ref & 3 & 24 & STD-23-2 & 20.1 & 9.72 & 79.7 & 52.2 \\
\hline
\end{tabular}


Figure B1. Laboratory PCT Measurements in Analytical Sequence for Test Matrix 1 (TM1) Glasses, EA, ARM, Blanks, and Solution Standards

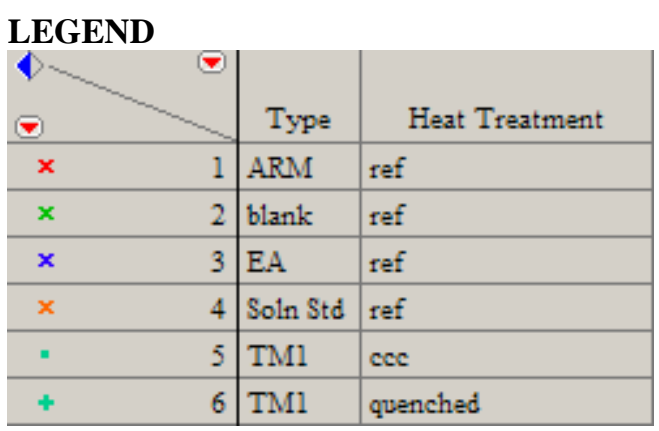

\section{B (ppm) By Analytical Sequence}

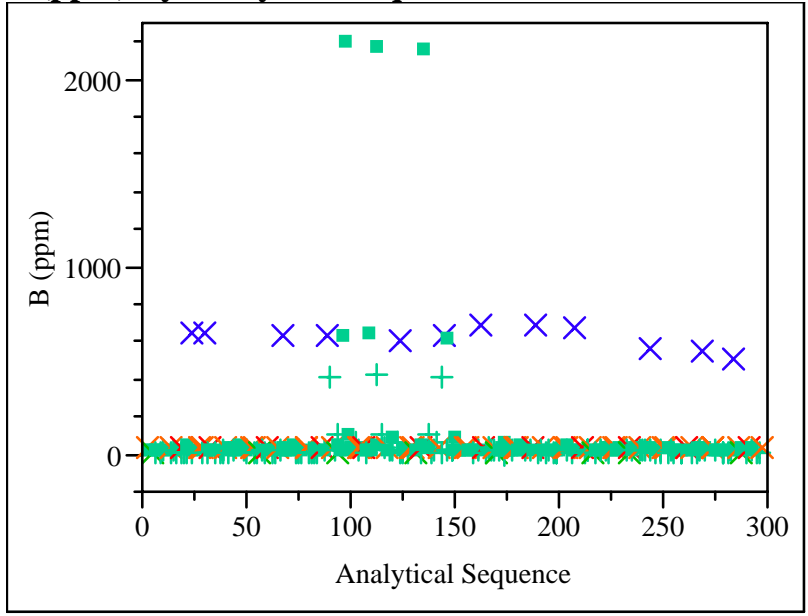

\section{Li (ppm) By Analytical Sequence}

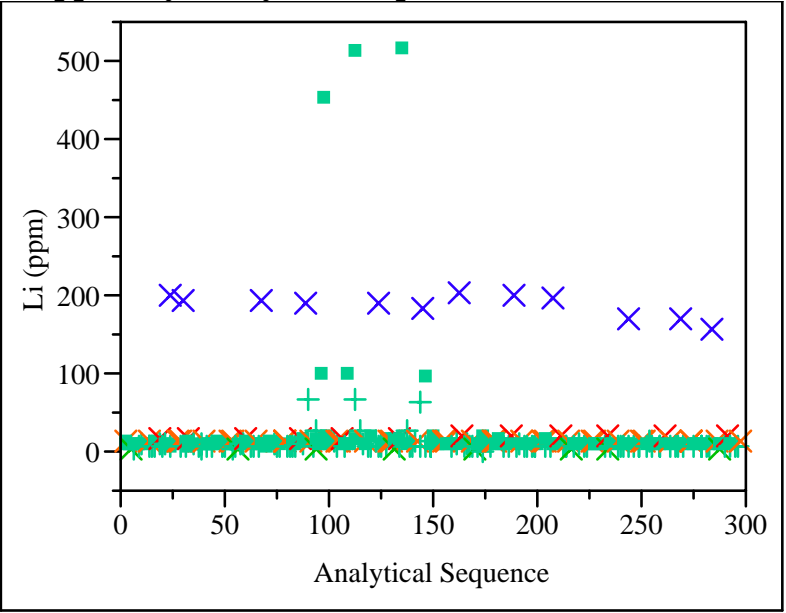

$\mathrm{Na}(\mathrm{ppm})$ By Analytical Sequence

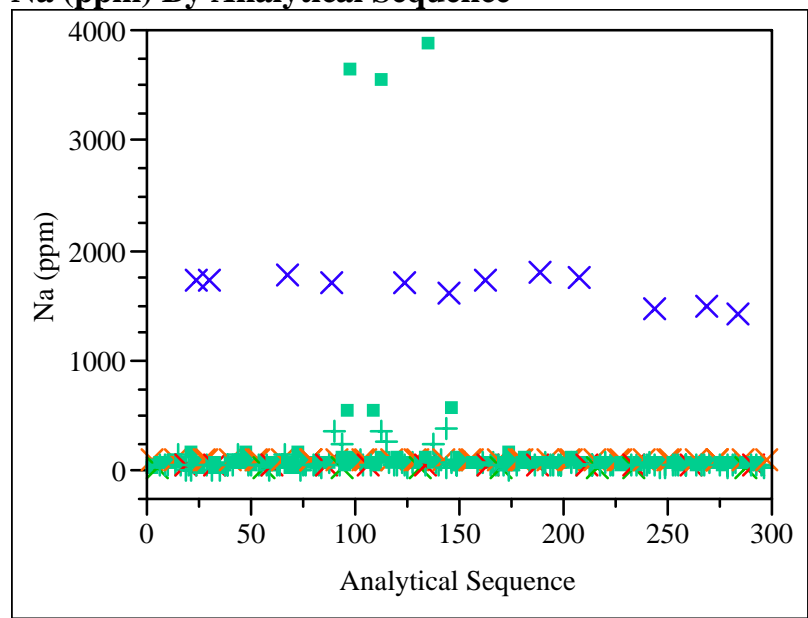

Si (ppm) By Analytical Sequence

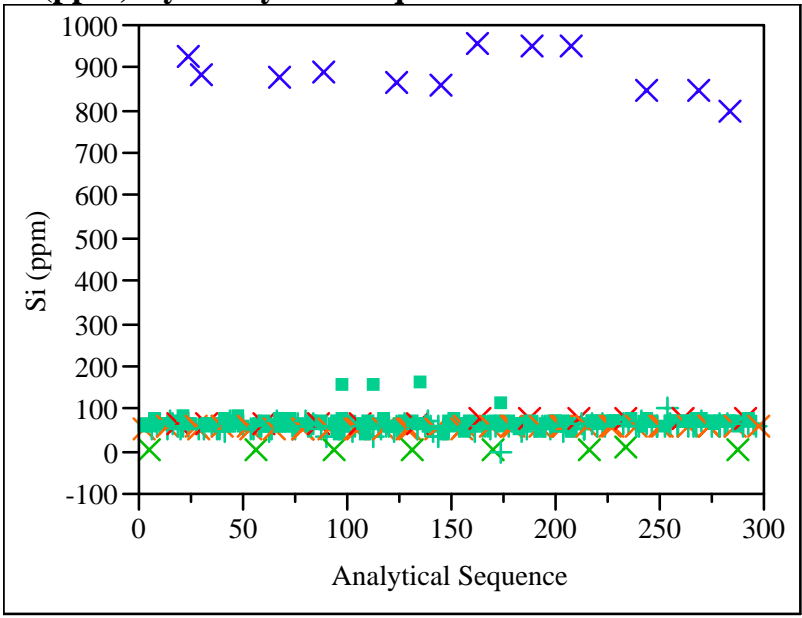


Figure B2. Laboratory PCT Measurements in Analytical Sequence for Test Matrix 1 Glasses

B (ppm) By Analytical Sequence

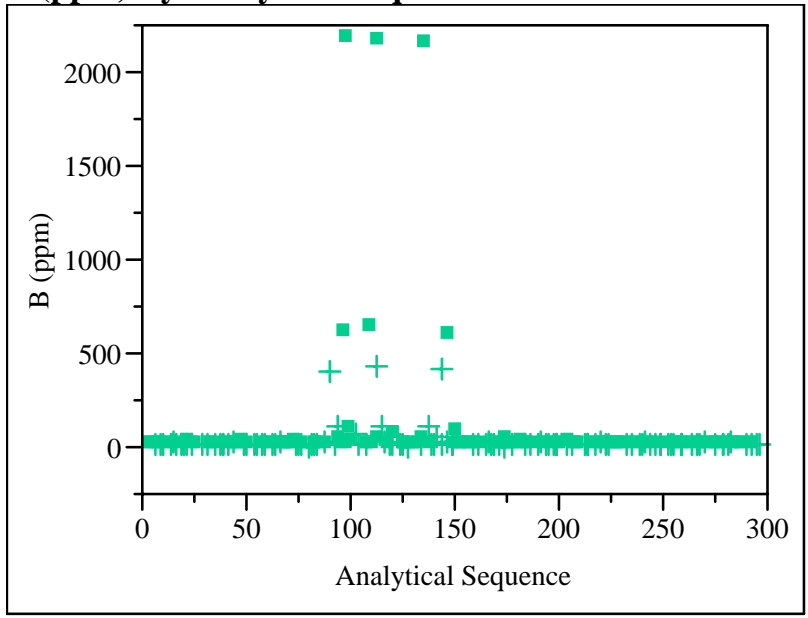

\section{Li (ppm) By Analytical Sequence}

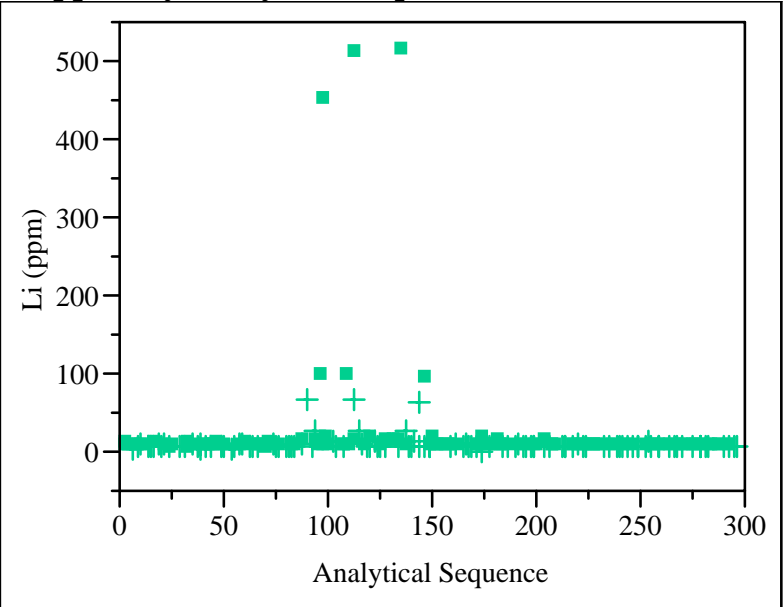

Na (ppm) By Analytical Sequence

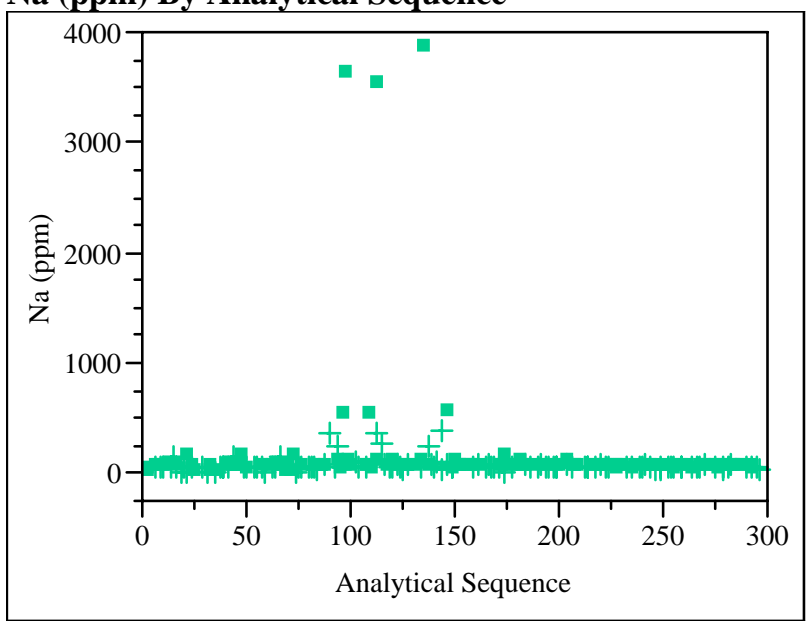

Si (ppm) By Analytical Sequence

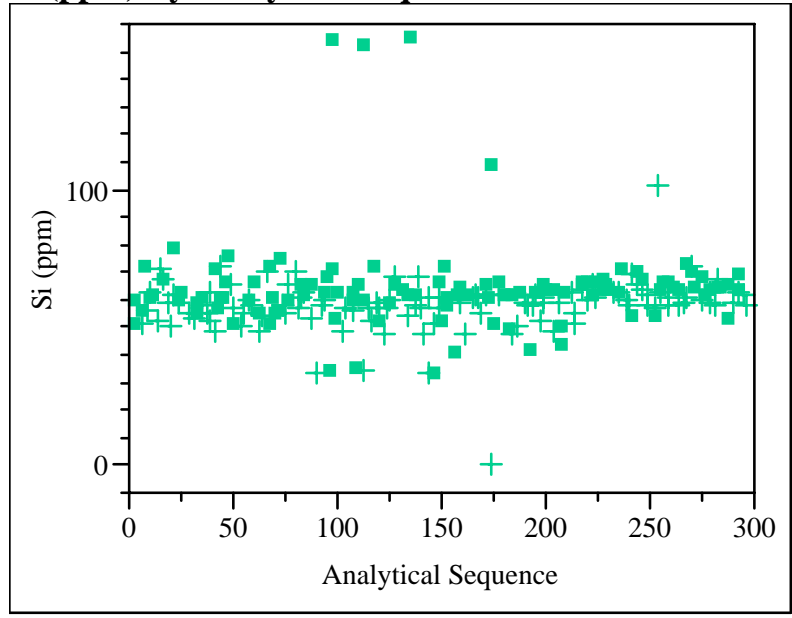




\section{Figure B3. Measurements of the Multi-Element Solution Standard} by ICP Block

Oneway Analysis of B (ppm) By Set/Block Reference Value $=20 \mathrm{ppm}$

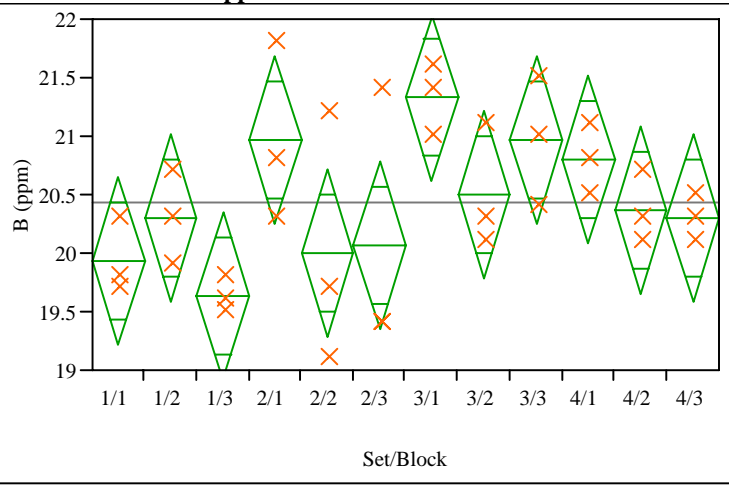

Oneway Anova

Summary of Fit

$\begin{array}{lr}\text { Rsquare } & 0.492972 \\ \text { Adj Rsquare } & 0.260584 \\ \text { Root Mean Square Error } & 0.59675 \\ \text { Mean of Response } & 20.43056 \\ \text { Observations (or Sum Wgts) } & 36\end{array}$

Analysis of Variance

Source DF Sum of Squares Mean Square F Ratio Prob $>$ F

$\begin{array}{llllll}\text { Set/Block } 11 & 8.309722 & 0.755429 & 2.1213 & 0.0599\end{array}$

$\begin{array}{llll}\text { Error } & 24 & 8.546667 & 0.356111\end{array}$

C. Total $35 \quad 16.856389$

Means for Oneway Anova

Level Number Mean Std Error Lower 95\% Upper 95\%

$\begin{array}{llllll}1 / 1 & 3 & 19.9333 & 0.34453 & 19.222 & 20.644\end{array}$

$\begin{array}{llllll}1 / 2 & 3 & 20.3000 & 0.34453 & 19.589 & 21.011\end{array}$

$\begin{array}{llllll}1 / 3 & 3 & 19.6333 & 0.34453 & 18.922 & 20.344\end{array}$

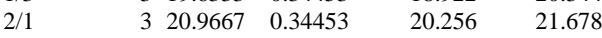

$\begin{array}{llllll}2 / 2 & 3 & 20.0000 & 0.34453 & 19.289 & 20.711\end{array}$

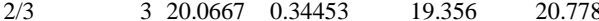

$\begin{array}{llllll}3 / 1 & 3 & 21.3333 & 0.34453 & 20.622 & 22.044\end{array}$

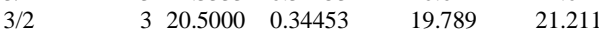

$\begin{array}{llllll}3 / 3 & 3 & 20.9667 & 0.34453 & 20.256 & 21.678\end{array}$

$\begin{array}{llllll}4 / 1 & 3 & 20.8000 & 0.34453 & 20.089 & 21.511\end{array}$

$\begin{array}{llllll}4 / 2 & 3 & 20.3667 & 0.34453 & 19.656 & 21.078\end{array}$

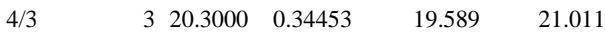

Std Error uses a pooled estimate of error variance
Oneway Analysis of Li (ppm) By Set/Block

Reference Value $=10 \mathrm{ppm}$

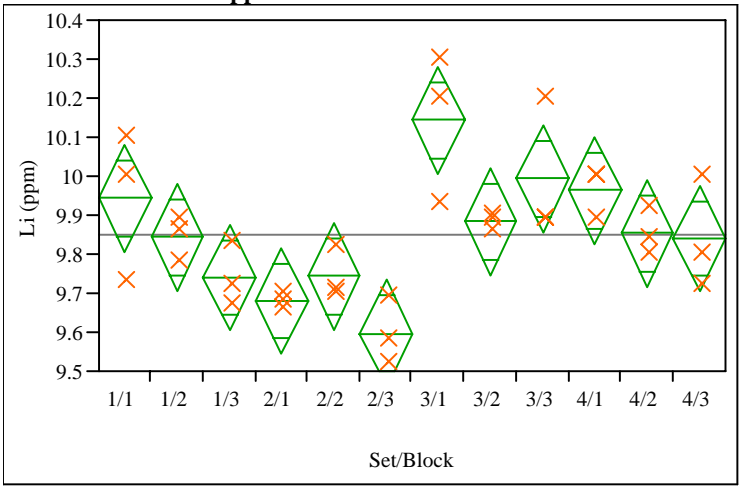

Oneway Anova

Summary of Fit

$\begin{array}{lr}\text { Rsquare } & 0.702121 \\ \text { Adj Rsquare } & 0.565594 \\ \text { Root Mean Square Error } & 0.1142 \\ \text { Mean of Response } & 9.851944 \\ \text { Observations (or Sum Wgts) } & 36\end{array}$

Analysis of Variance

Source DF Sum of Squares Mean Square F Ratio Prob $>$ F

$\begin{array}{llllll}\text { Set/Block } & 11 & 0.7377639 & 0.067069 & 5.1427 & 0.0004\end{array}$

$\begin{array}{llll}\text { Error } & 24 & 0.3130000 & 0.013042\end{array}$

C. Total $35 \quad 1.0507639$

Means for Oneway Anova

Level Number Mean Std Error Lower 95\% Upper 95\%

$\begin{array}{lrrrrr}1 / 1 & 3 & 9.9433 & 0.06593 & 9.807 & 10.079\end{array}$

$\begin{array}{llllll}1 / 2 & 3 & 9.8433 & 0.06593 & 9.707 & 9.979\end{array}$

$\begin{array}{llllll}1 / 3 & 3 & 9.7400 & 0.06593 & 9.604 & 9.876\end{array}$

$\begin{array}{llllll}2 / 1 & 3 & 9.6800 & 0.06593 & 9.544 & 9.816\end{array}$

$\begin{array}{llllll}2 / 2 & 3 & 9.7433 & 0.06593 & 9.607 & 9.879\end{array}$

$\begin{array}{llllll}2 / 3 & 3 & 9.5967 & 0.06593 & 9.461 & 9.733\end{array}$

$\begin{array}{llllll}3 / 1 & 3 & 10.1433 & 0.06593 & 10.007 & 10.279\end{array}$

$\begin{array}{llllrr}3 / 2 & 3 & 9.8833 & 0.06593 & 9.747 & 10.019\end{array}$

$\begin{array}{llllll}3 / 3 & 3 & 9.9933 & 0.06593 & 9.857 & 10.129\end{array}$

$\begin{array}{llllll}4 / 1 & 3 & 9.9633 & 0.06593 & 9.827 & 10.099\end{array}$

$\begin{array}{lllllr}4 / 2 & 3 & 9.8533 & 0.06593 & 9.717 & 9.989\end{array}$

$\begin{array}{llllll}4 / 3 & 3 & 9.8400 & 0.06593 & 9.704 & 9.976\end{array}$

Std Error uses a pooled estimate of error variance 


\section{Figure B3. Measurements of the Multi-Element Solution Standard} by ICP Block

Oneway Analysis of Na (ppm) By Set/Block Reference Value $=81$ ppm

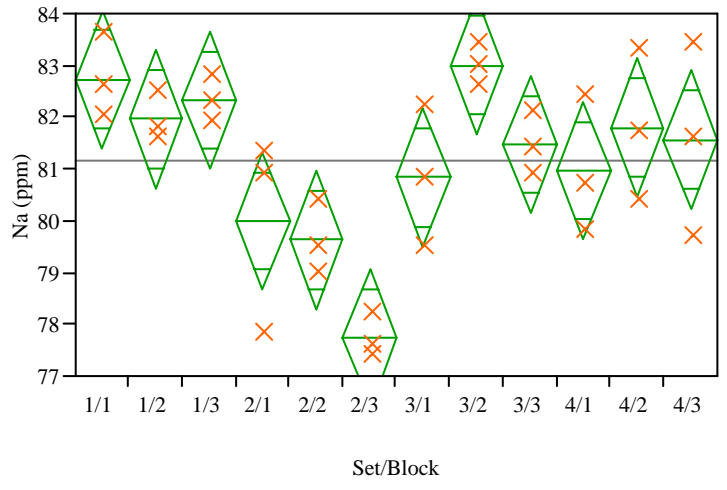

Oneway Anova

Summary of Fit

Rsquare $\quad 0.706216$

Adj Rsquare $\quad 0.571566$

Root Mean Square Error 1.119896

Mean of Response 81.16944

Observations (or Sum Wgts) 36

Analysis of Variance

Source DF Sum of Squares Mean Square F Ratio Prob $>$ F

$\begin{array}{llllll}\text { Set/Block } & 11 & 72.35639 & 6.57785 & 5.2448 & 0.0003\end{array}$

$\begin{array}{llll}\text { Error } & 24 & 30.10000 & 1.25417\end{array}$

C. Total $35 \quad 102.45639$

Means for Oneway Anova

Level Number Mean Std Error Lower 95\% Upper 95\%

$382.7333 \quad 0.64657$

$\begin{array}{llllll}1 / 2 & 3 & 81.9667 & 0.64657 & 80.632 & 83.301\end{array}$

$\begin{array}{llllll}1 / 3 & 3 & 82.3333 & 0.64657 & 80.999 & 83.668\end{array}$

$\begin{array}{llllll}2 / 1 & 3 & 80.0000 & 0.64657 & 78.666 & 81.334\end{array}$

$\begin{array}{llllll}2 / 2 & 3 & 79.6333 & 0.64657 & 78.299 & 80.968\end{array}$

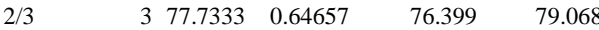

$\begin{array}{llllll}3 / 1 & 3 & 80.8333 & 0.64657 & 79.499 & 82.168\end{array}$

$\begin{array}{llllll}3 / 2 & 383.0000 & 0.64657 & 81.666 & 84.334\end{array}$

$\begin{array}{llllll}3 / 3 & 3 & 81.4667 & 0.64657 & 80.132 & 82.801\end{array}$

$\begin{array}{llllll}4 / 1 & 3 & 80.9667 & 0.64657 & 79.632 & 82.301\end{array}$

$\begin{array}{llllll}4 / 2 & 3 & 81.8000 & 0.64657 & 80.466 & 83.134\end{array}$

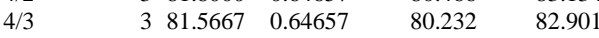

Std Error uses a pooled estimate of error variance
Oneway Analysis of Si (ppm) By Set/Block

Reference Value $=\mathbf{5 0} \mathrm{ppm}$

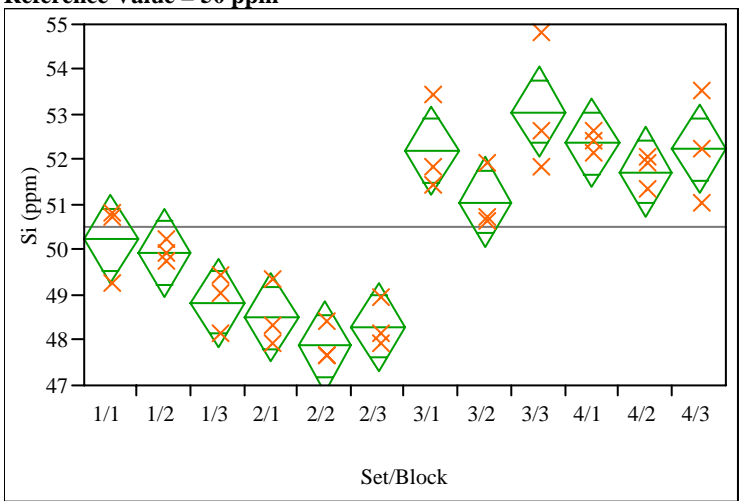

Oneway Anova

Summary of Fit

Rsquare

0.871179

Adj Rsquare

0.812136

Root Mean Square Error $\quad 0.824116$

Mean of Response

50.52778

Observations (or Sum Wgts) 36

Analysis of Variance

Source DF Sum of Squares Mean Square F Ratio Prob $>$ F

$\begin{array}{llllll}\text { Set/Block } & 11 & 110.23222 & 10.0211 & 14.7550 & <.0001\end{array}$

$\begin{array}{llrr}\text { Error } & 24 & 16.30000 & 0.6792\end{array}$

C. Total $35 \quad 126.53222$

Means for Oneway Anova

Level Number Mean Std Error Lower 95\% Upper 95\%

$\begin{array}{llllll}1 / 1 & 3 & 50.2333 & 0.47580 & 49.251 & 51.215 \\ 1 / 2 & 3 & 49.9333 & 0.47580 & 48.951 & 50.915\end{array}$

$\begin{array}{llllll}1 / 3 & 3 & 48.8333 & 0.47580 & 47.851 & 49.815\end{array}$

$\begin{array}{llllll}2 / 1 & 3 & 48.5000 & 0.47580 & 47.518 & 49.482\end{array}$

$\begin{array}{llllll}2 / 2 & 3 & 47.8667 & 0.47580 & 46.885 & 48.849\end{array}$

$\begin{array}{llllll}2 / 3 & 3 & 48.3000 & 0.47580 & 47.318 & 49.282\end{array}$

$\begin{array}{llllll}3 / 1 & 3 & 52.2000 & 0.47580 & 51.218 & 53.182\end{array}$

$\begin{array}{llllll}3 / 2 & 3 & 51.0667 & 0.47580 & 50.085 & 52.049\end{array}$

$\begin{array}{llllll}3 / 3 & 3 & 53.0667 & 0.47580 & 52.085 & 54.049\end{array}$

$\begin{array}{llllll}4 / 1 & 3 & 52.3667 & 0.47580 & 51.385 & 53.349\end{array}$

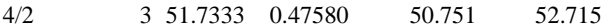

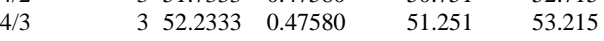

Std Error uses a pooled estimate of error variance 
Figure B4. Laboratory PCT Measurements by Glass Number for Study Glasses and Standards (ppm and $\log (\mathrm{ppm})$ )

Variability Gage Set $=1$

Variability Chart for B (ppm)

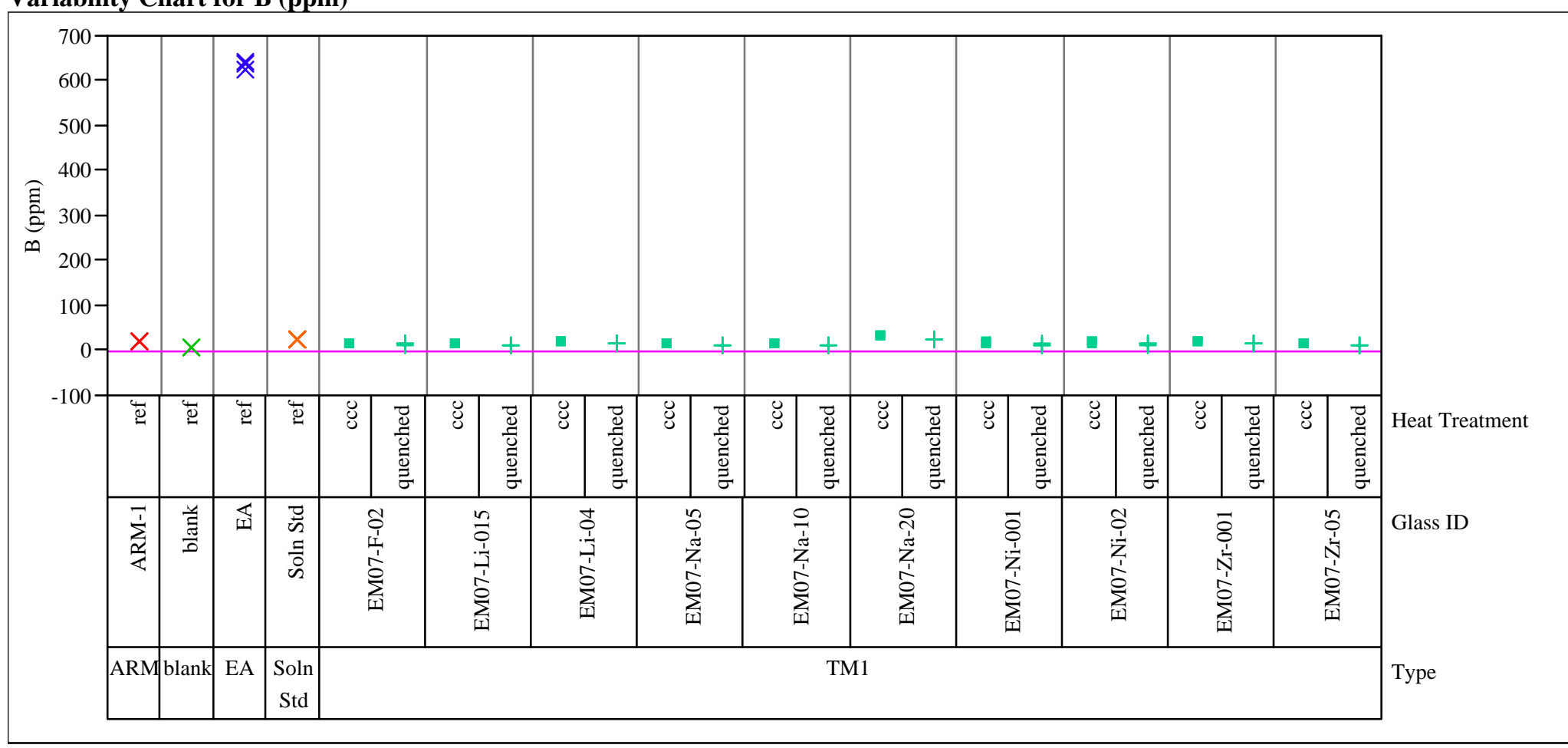


Figure B4. Laboratory PCT Measurements by Glass Number for Study Glasses and Standards (ppm and $\log (\mathrm{ppm})$ )

Variability Gage Set $=1$

Variability Chart for Li (ppm)

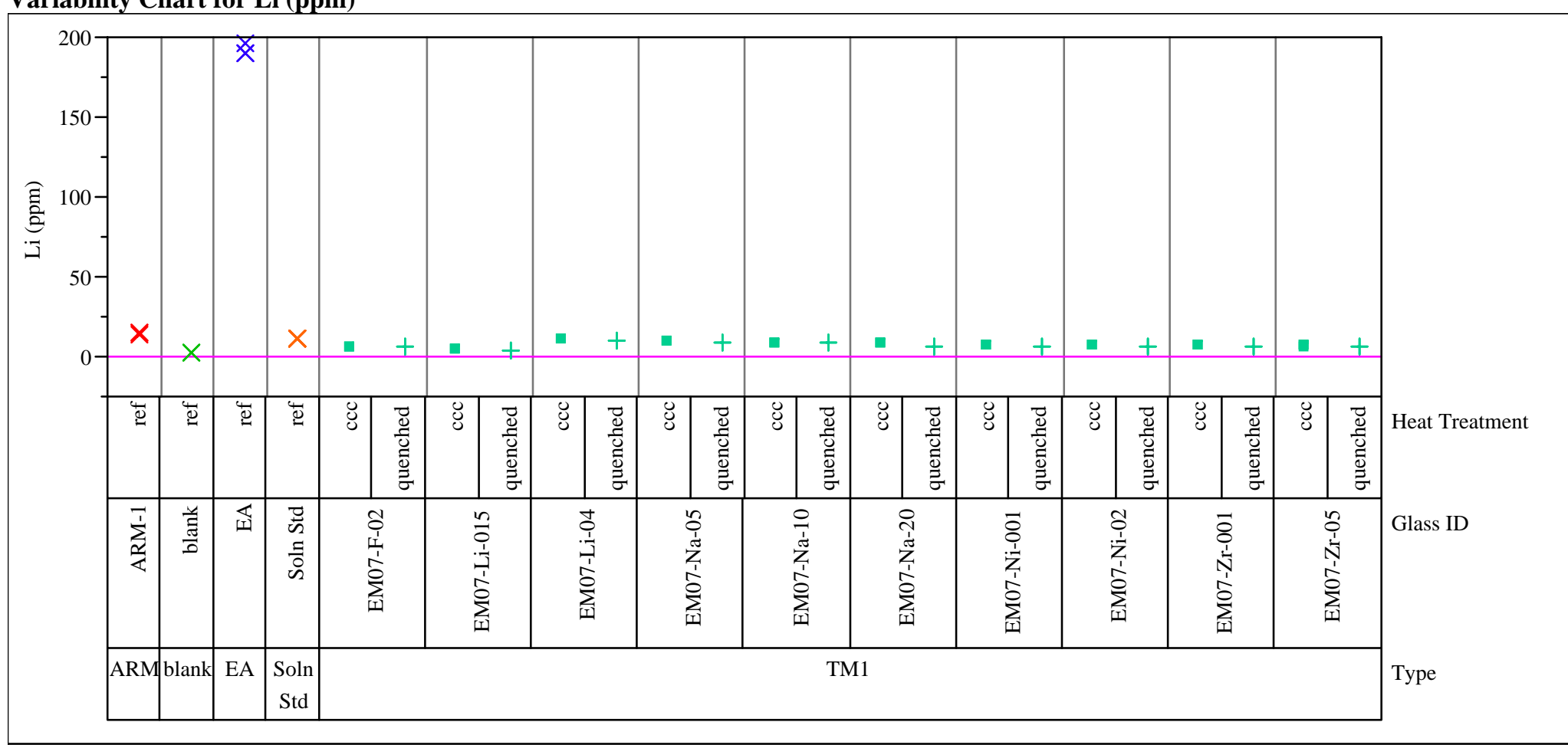


Figure B4. Laboratory PCT Measurements by Glass Number for Study Glasses and Standards (ppm and $\log (\mathrm{ppm})$ )

Variability Gage Set $=1$

Variability Chart for Na (ppm)

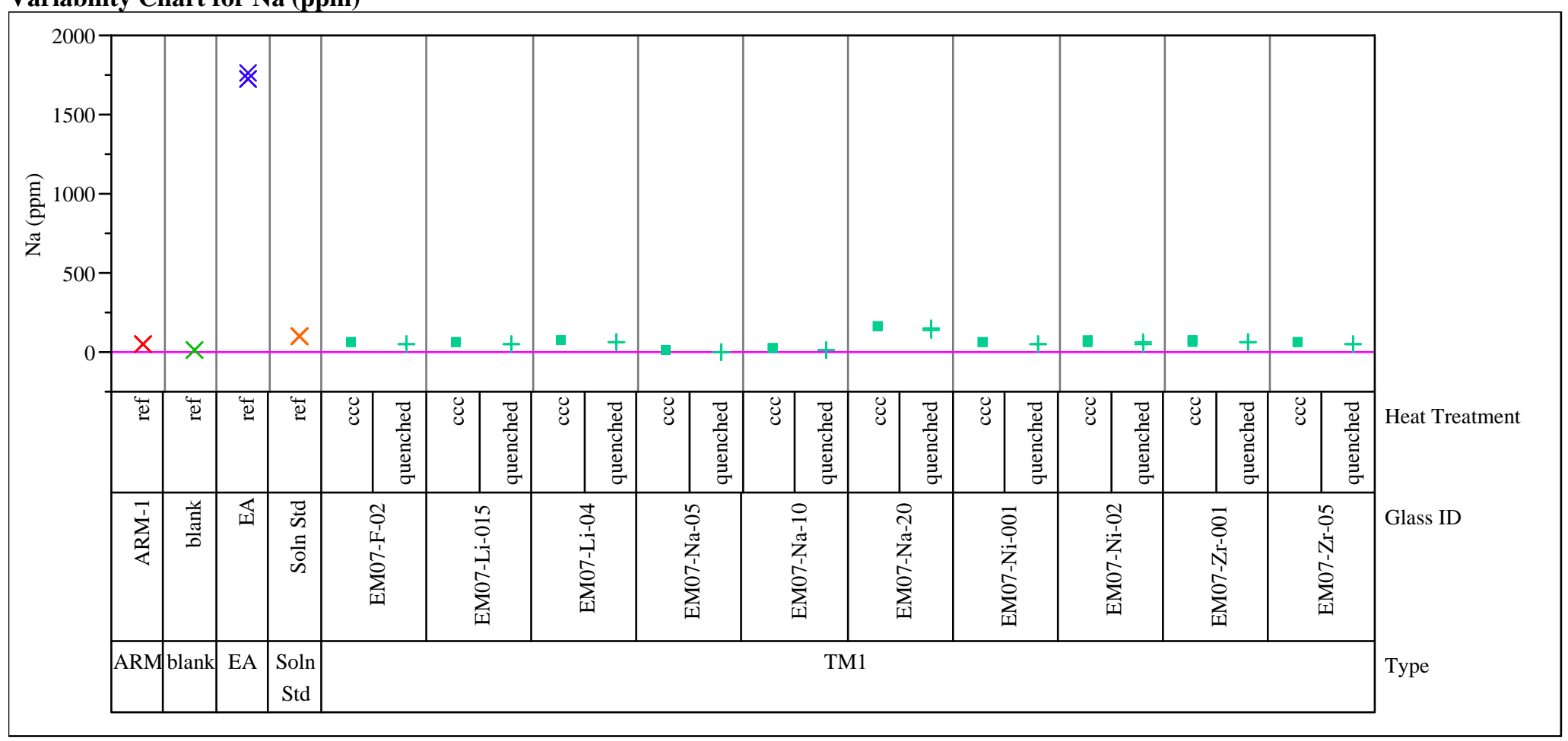


Figure B4. Laboratory PCT Measurements by Glass Number for Study Glasses and Standards (ppm and $\log (\mathrm{ppm})$ )

Variability Gage Set $=1$

Variability Chart for Si (ppm)

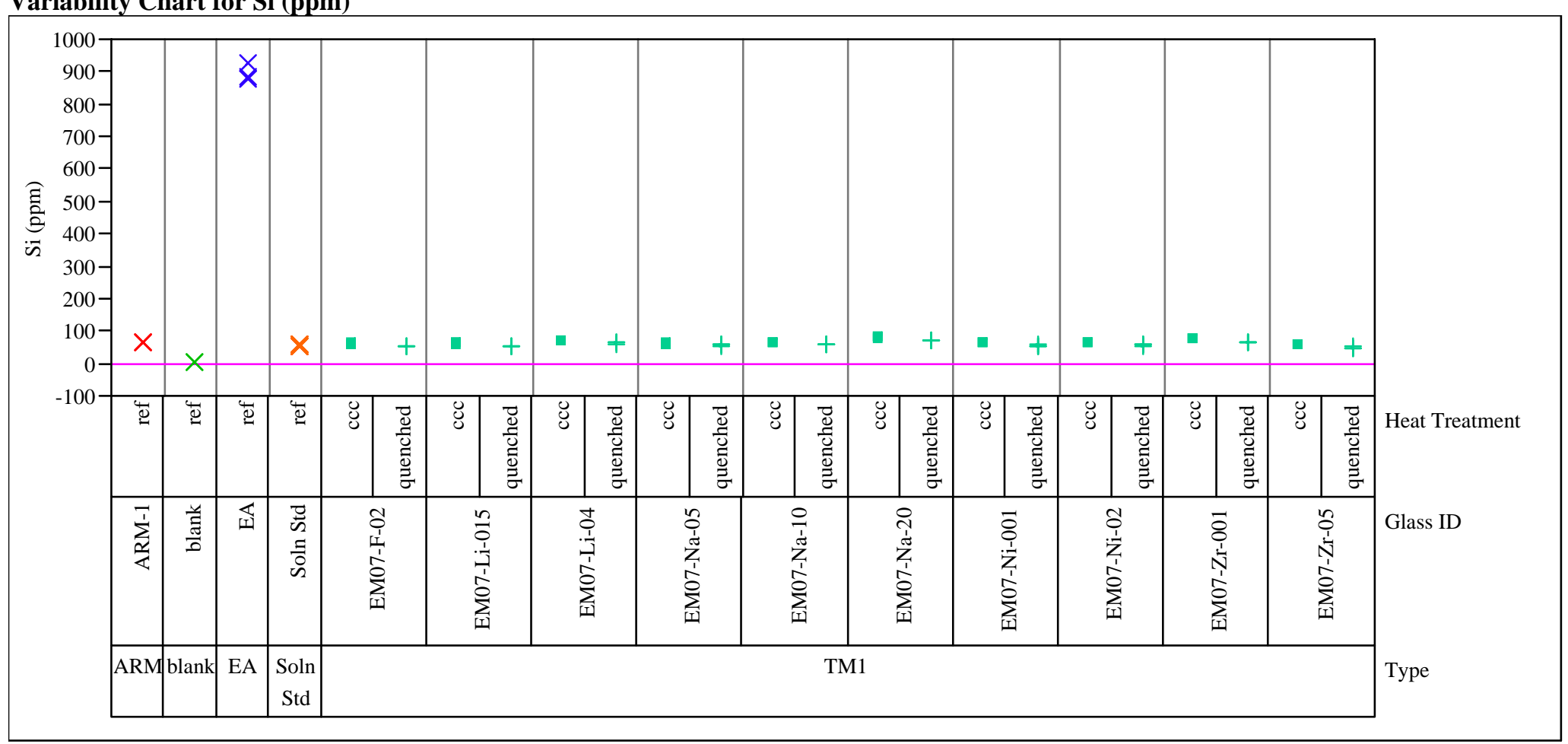


Figure B4. Laboratory PCT Measurements by Glass Number for Study Glasses and Standards (ppm and $\log (\mathrm{ppm})$ )

Variability Gage Set $=1$

Variability Chart for $\log [\mathrm{B}$ ppm]

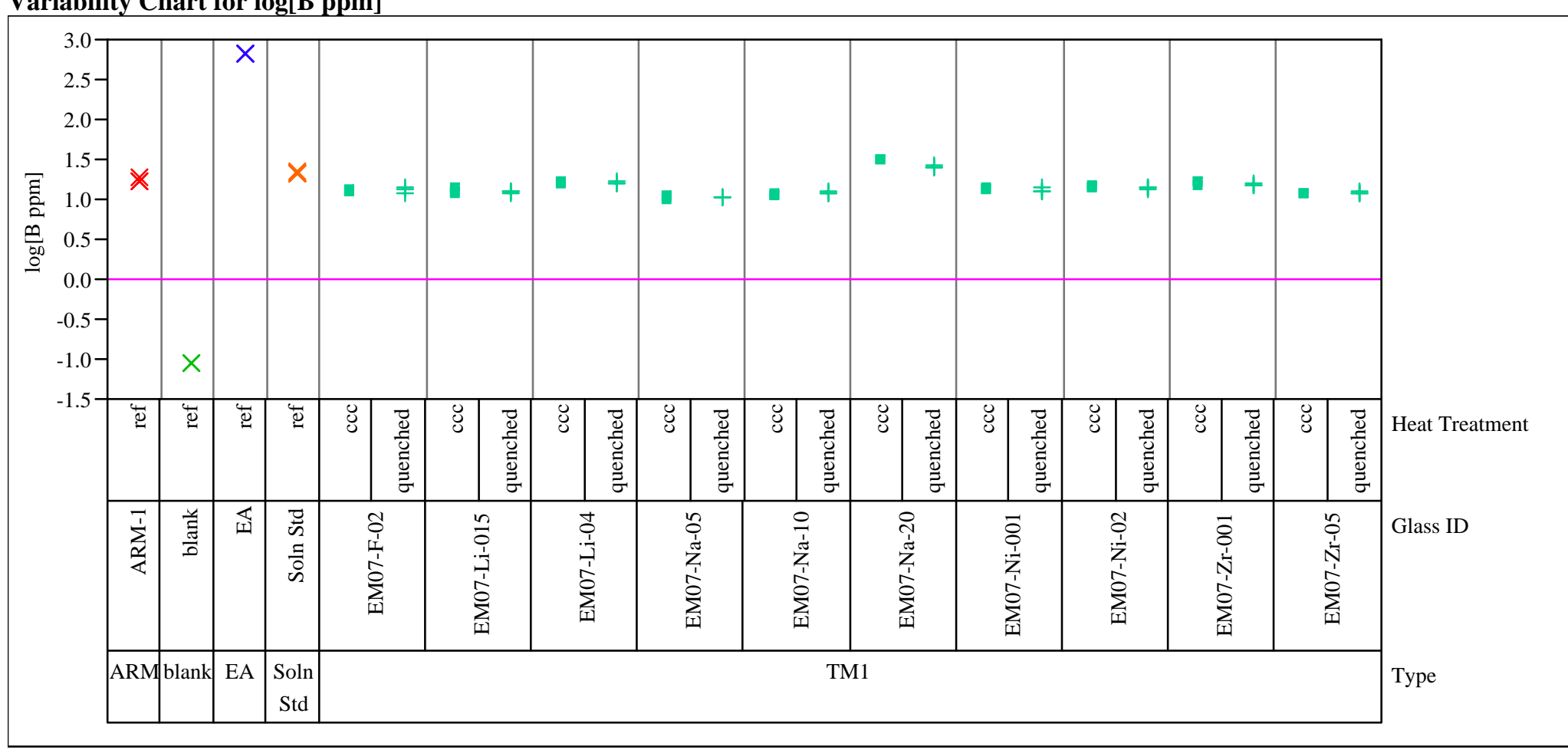


Figure B4. Laboratory PCT Measurements by Glass Number for Study Glasses and Standards (ppm and $\log (\mathrm{ppm})$ )

Variability Gage Set $=1$

Variability Chart for $\log [\mathrm{Li} \mathrm{ppm}]$

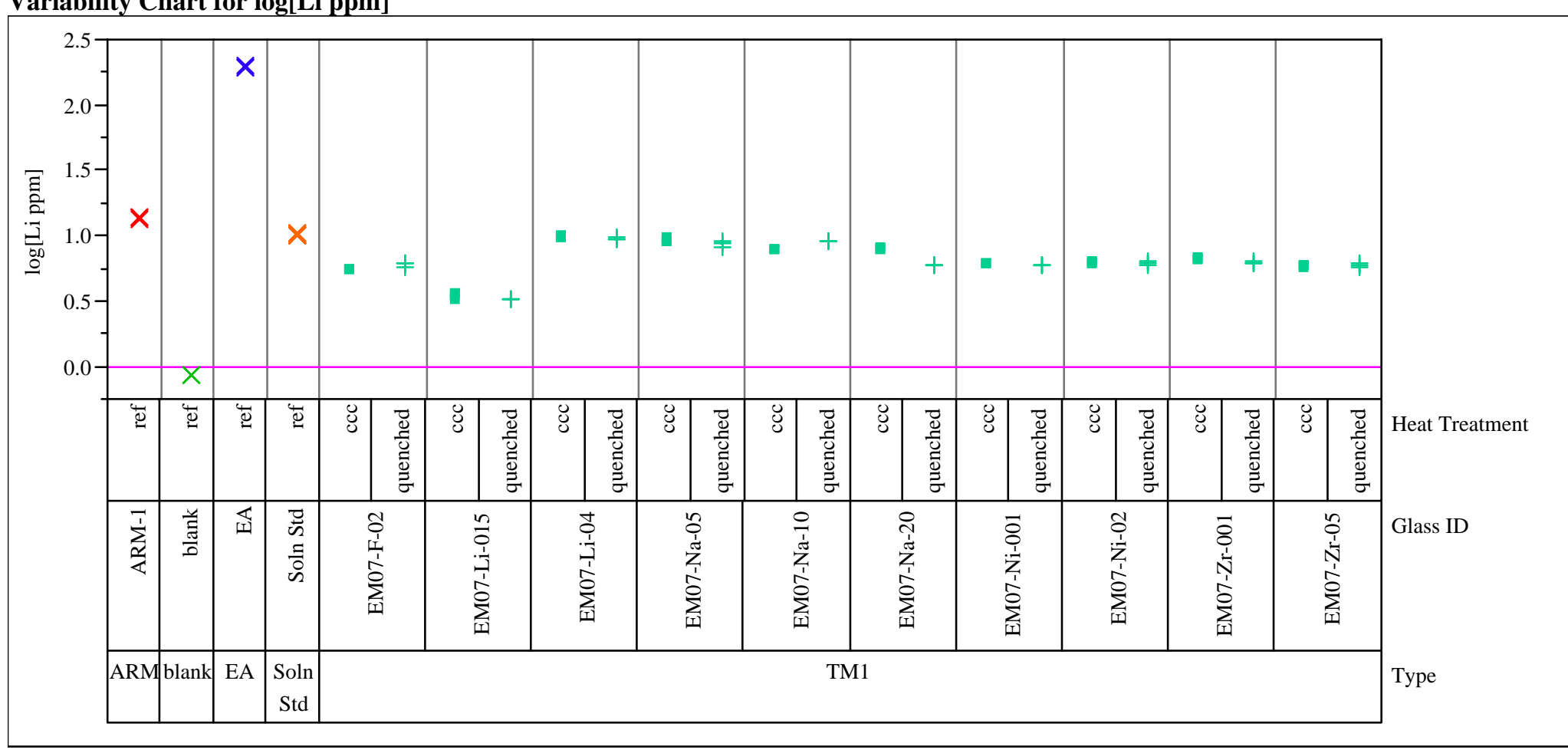


Figure B4. Laboratory PCT Measurements by Glass Number for Study Glasses and Standards (ppm and $\log (\mathrm{ppm})$ )

Variability Gage Set $=1$

Variability Chart for log[Na ppm]

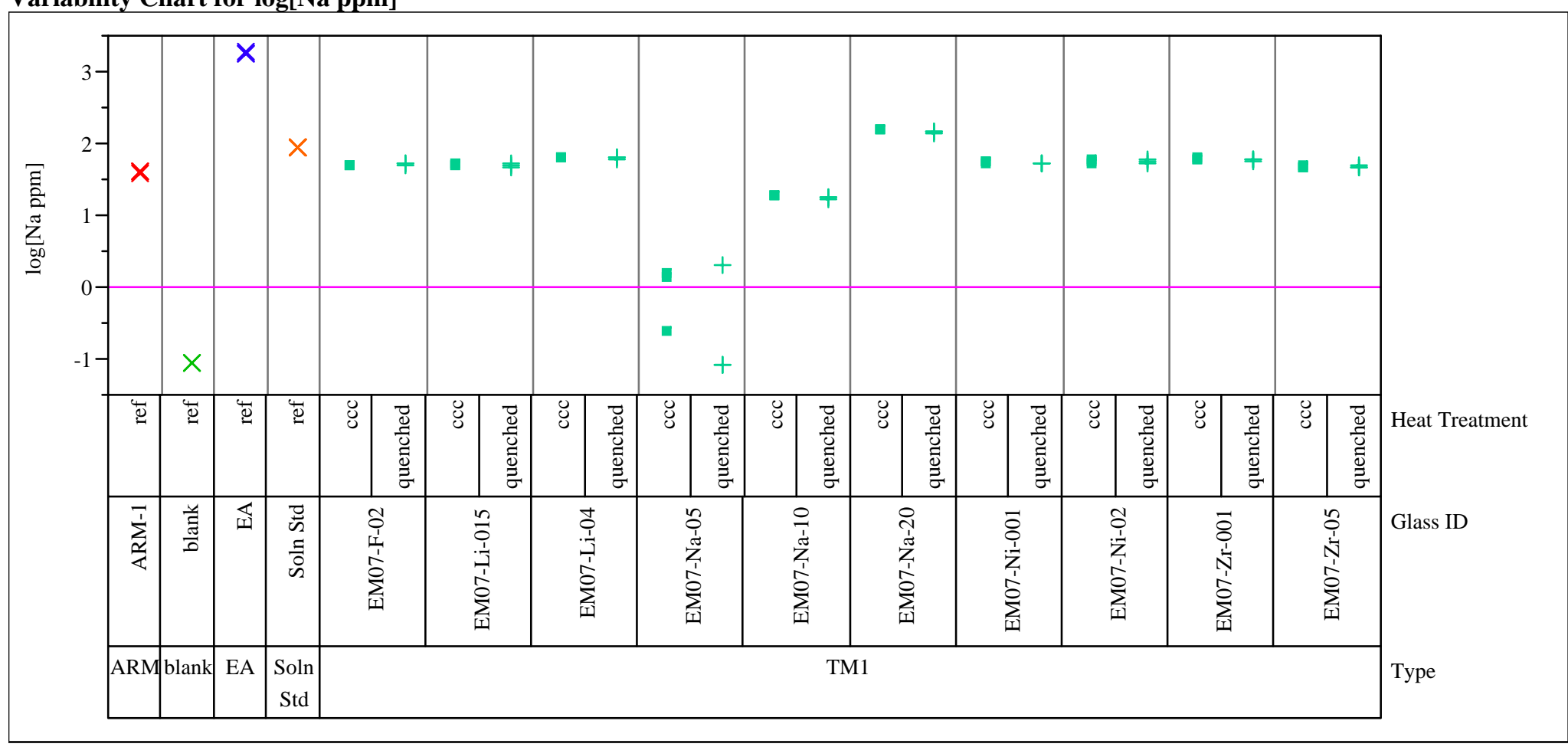


Figure B4. Laboratory PCT Measurements by Glass Number for Study Glasses and Standards (ppm and $\log (\mathrm{ppm})$ )

Variability Gage Set $=1$

Variability Chart for log[Si ppm]

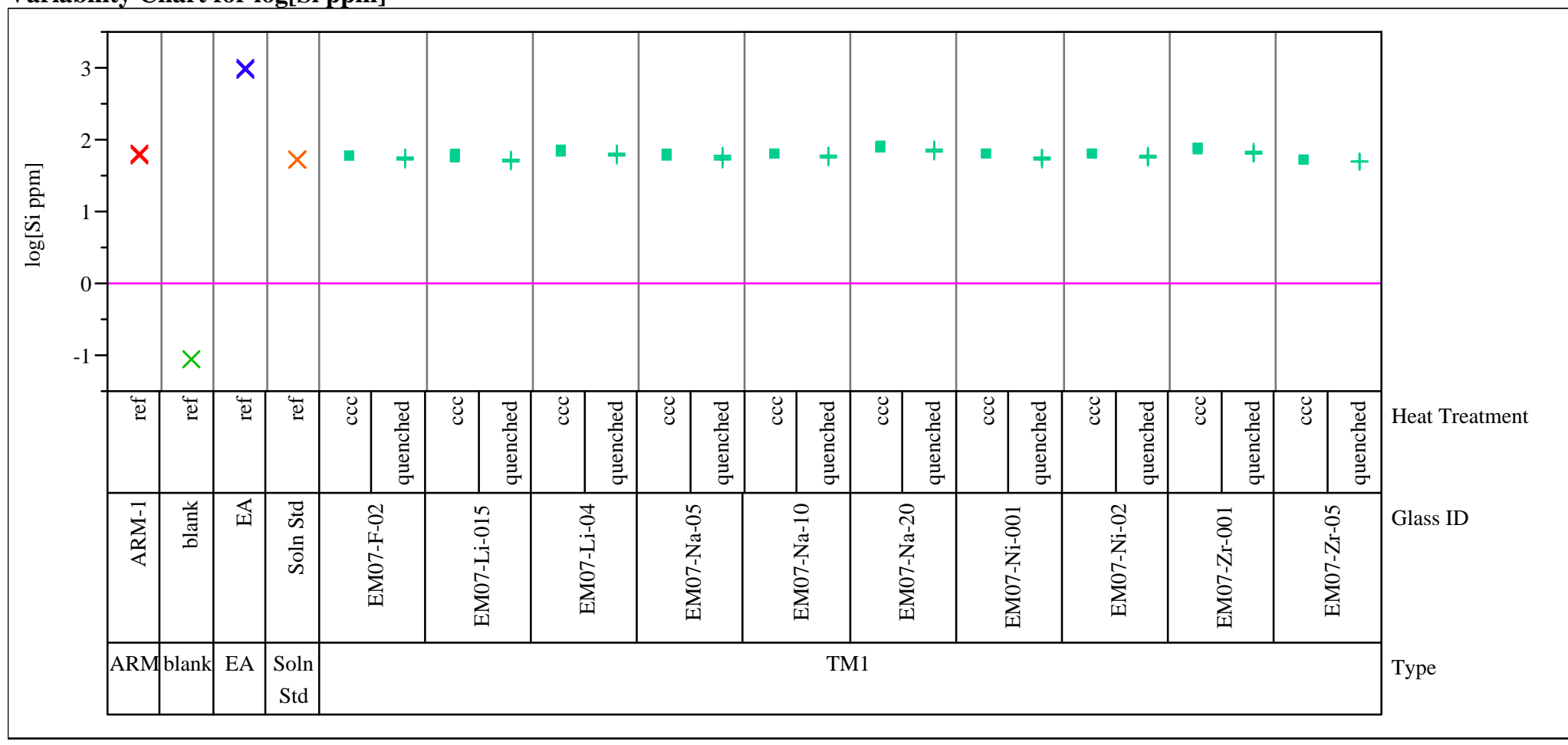


Figure B4. Laboratory PCT Measurements by Glass Number for Study Glasses and Standards (ppm and $\log (\mathrm{ppm})$ )

Variability Gage Set $=2$

Variability Chart for B (ppm)

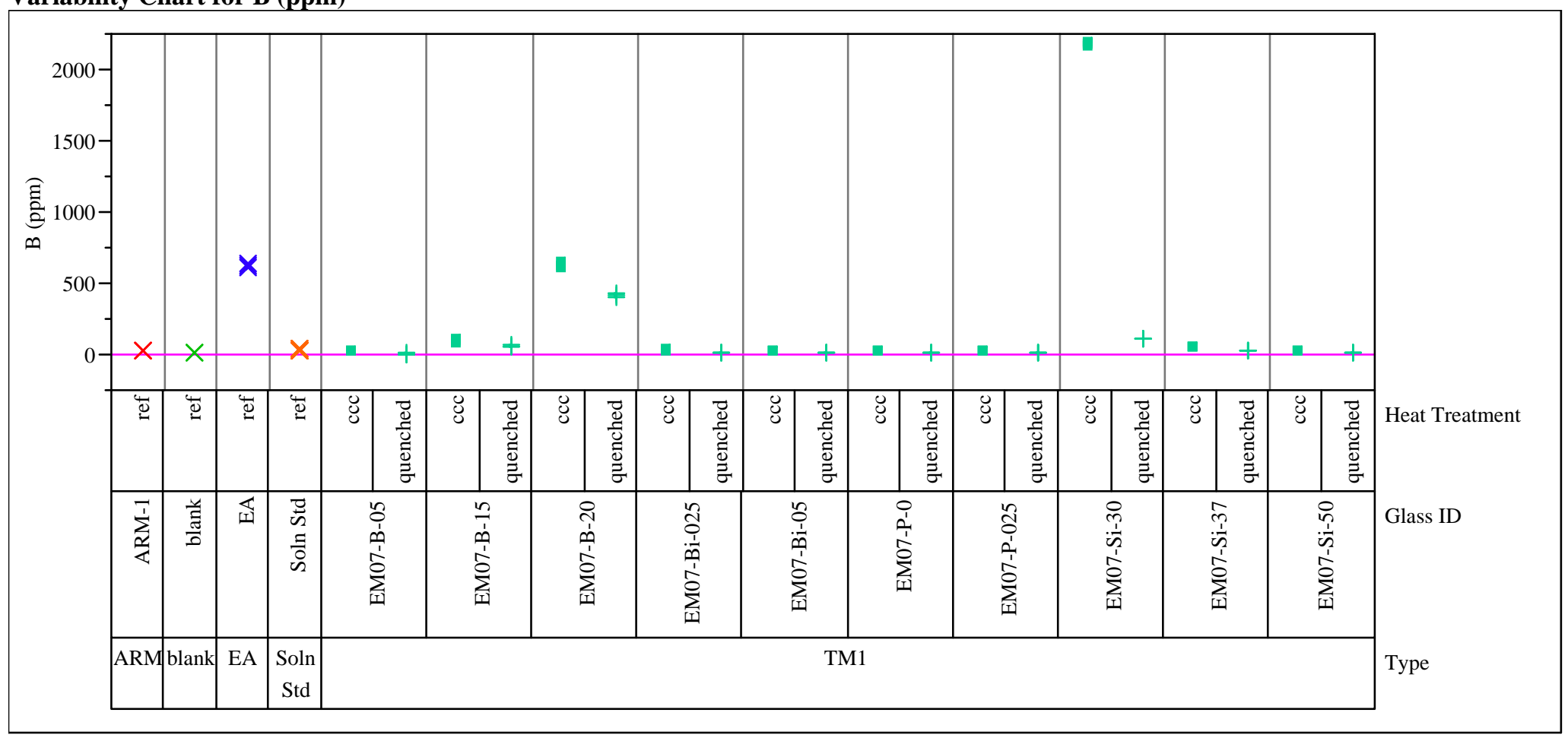


Figure B4. Laboratory PCT Measurements by Glass Number for Study Glasses and Standards (ppm and $\log (\mathrm{ppm})$ )

Variability Gage Set $=2$

Variability Chart for Li (ppm)

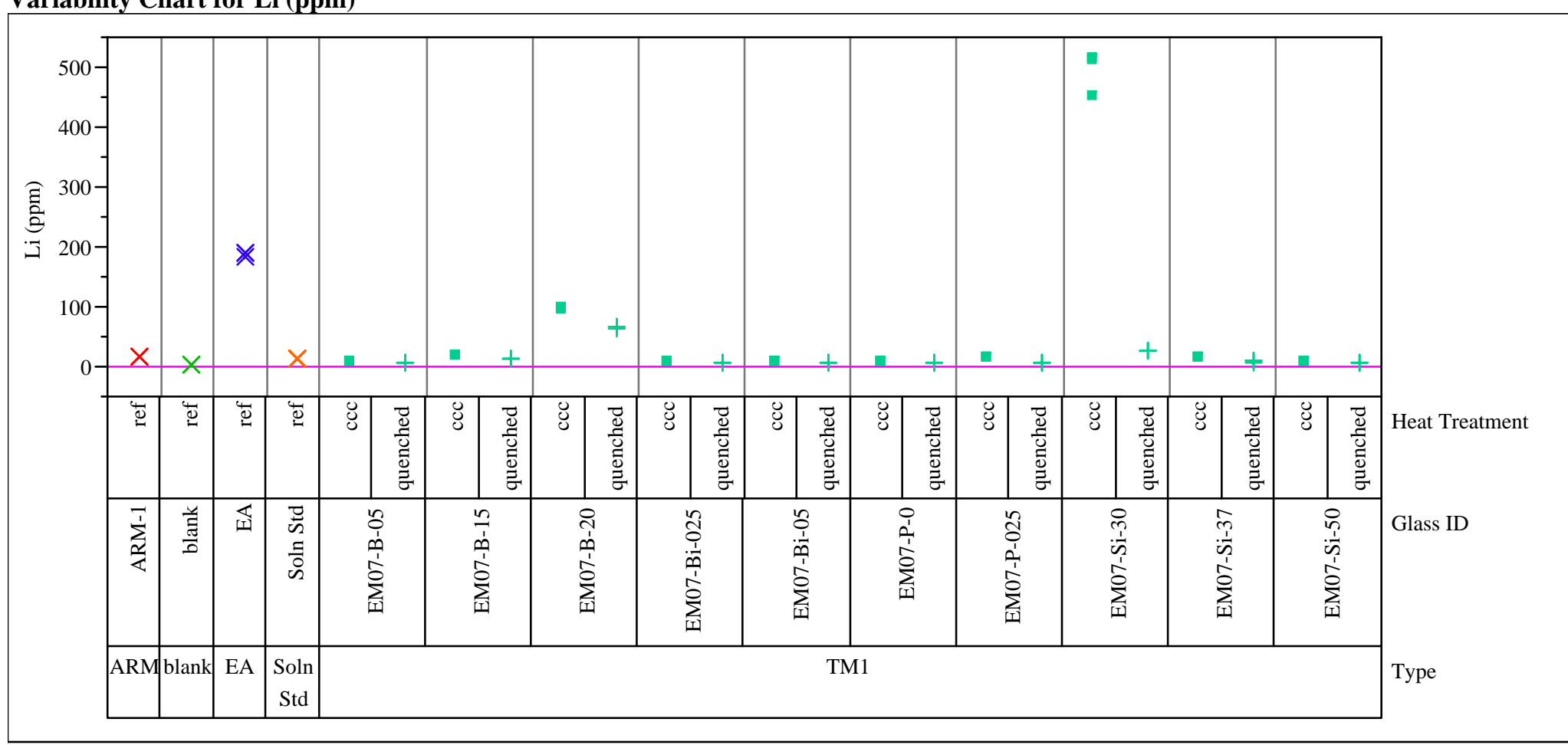


Figure B4. Laboratory PCT Measurements by Glass Number for Study Glasses and Standards (ppm and $\log (\mathrm{ppm})$ )

Variability Gage Set $=2$

Variability Chart for Na (ppm)

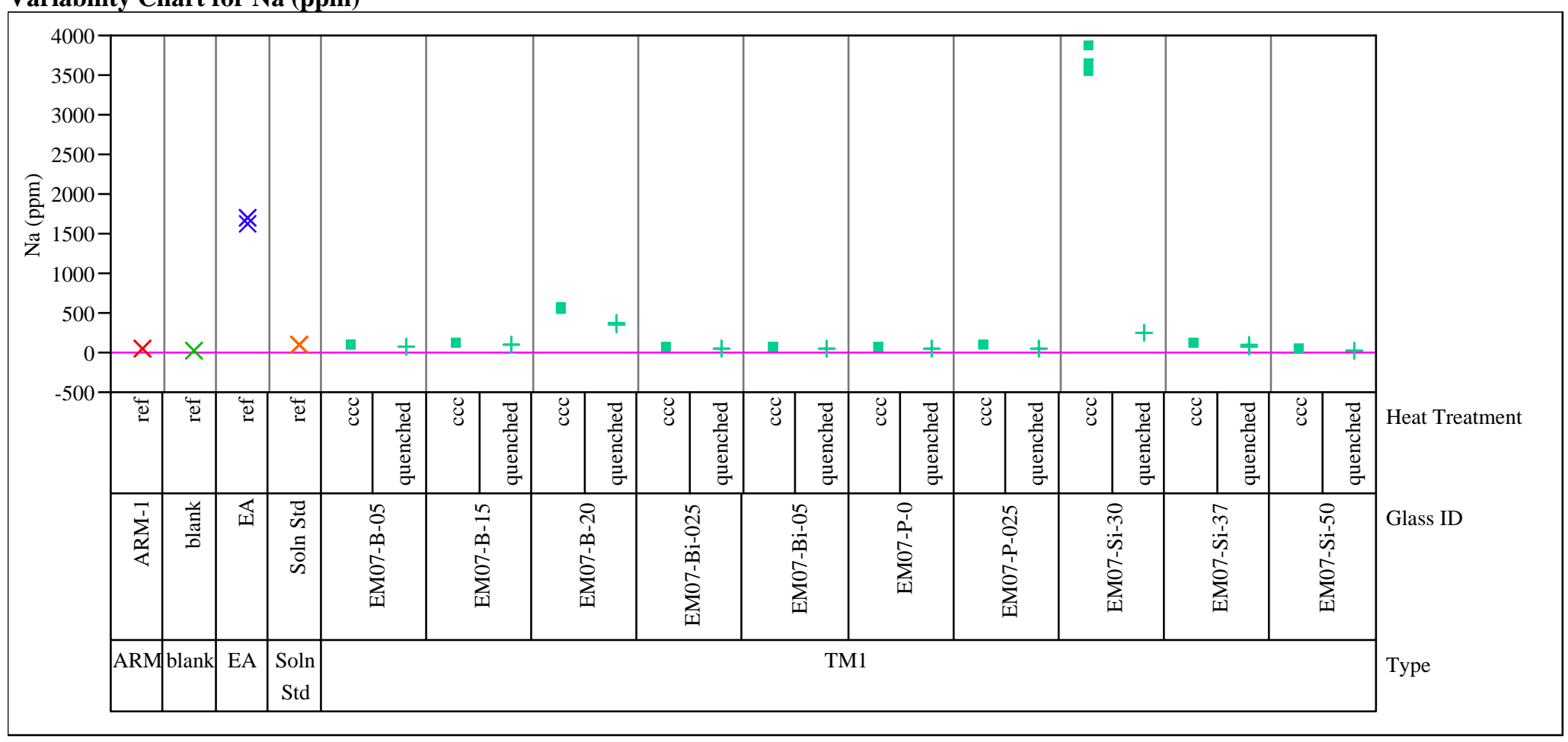


Figure B4. Laboratory PCT Measurements by Glass Number for Study Glasses and Standards (ppm and $\log (\mathrm{ppm})$ )

Variability Gage Set $=2$

Variability Chart for Si (ppm)

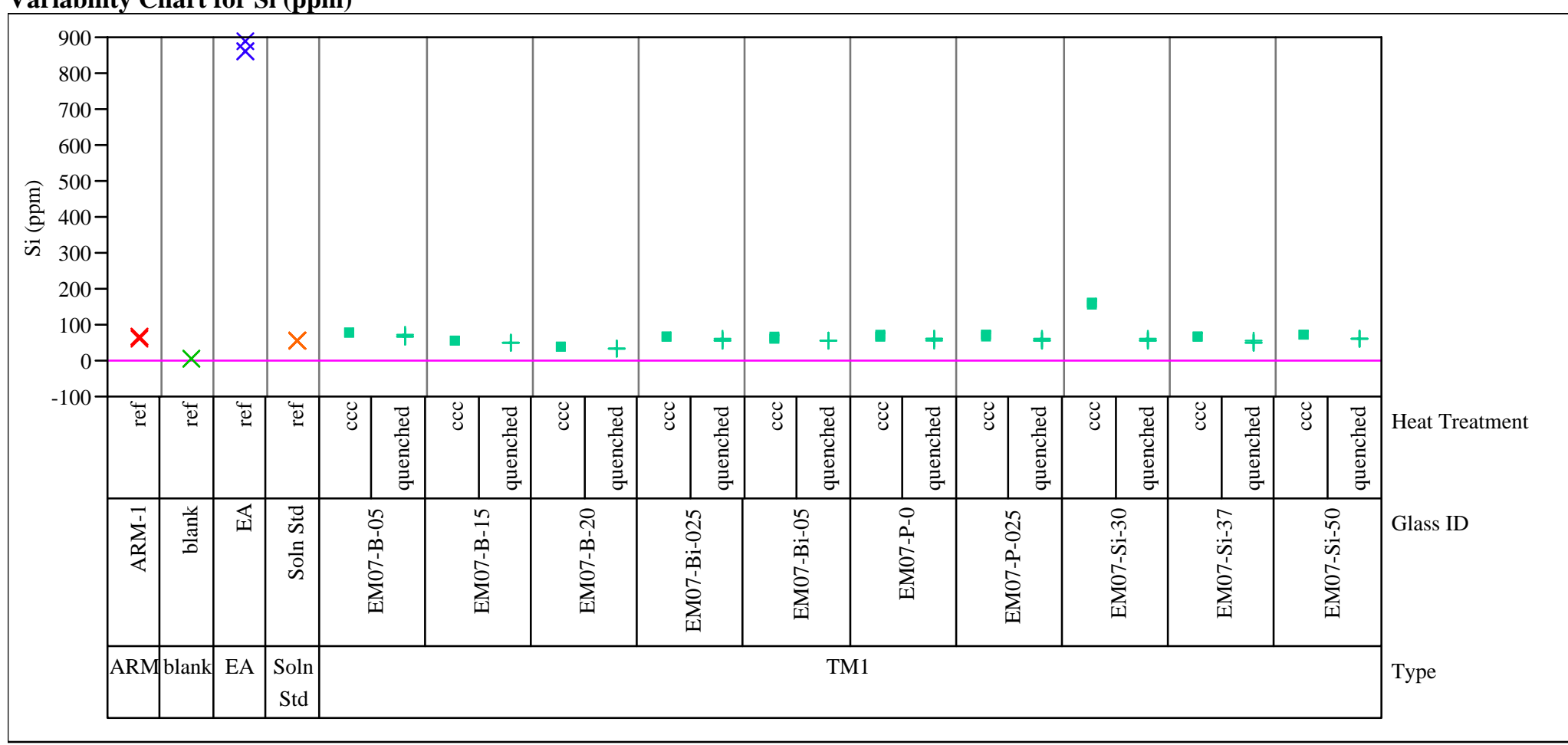


Figure B4. Laboratory PCT Measurements by Glass Number for Study Glasses and Standards (ppm and $\log (\mathrm{ppm})$ )

Variability Gage Set $=2$

Variability Chart for log[B ppm]

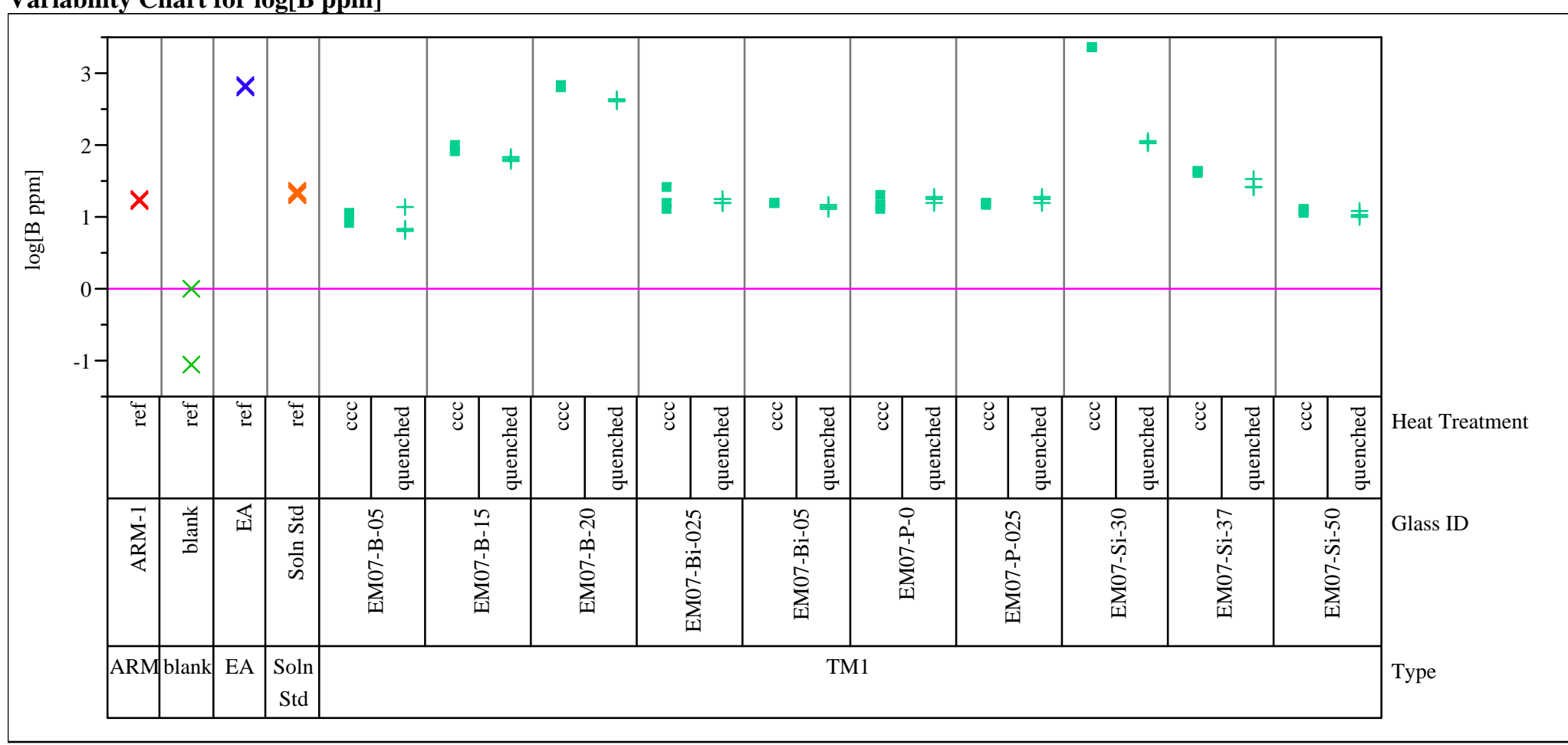


Figure B4. Laboratory PCT Measurements by Glass Number for Study Glasses and Standards (ppm and $\log (\mathrm{ppm})$ )

Variability Gage Set $=2$

Variability Chart for log[Li ppm]

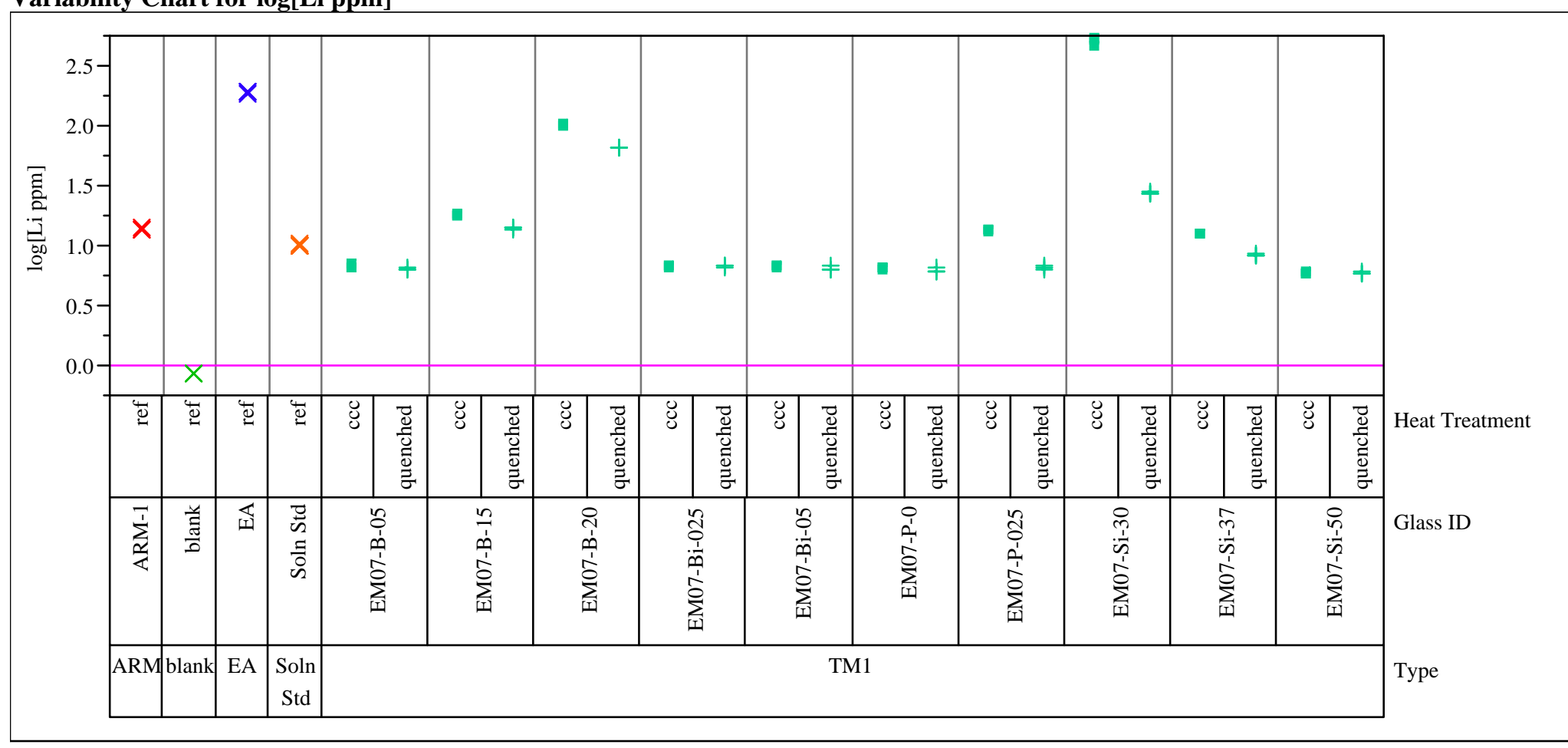


Figure B4. Laboratory PCT Measurements by Glass Number for Study Glasses and Standards (ppm and $\log (\mathrm{ppm})$ )

Variability Gage Set $=2$

Variability Chart for log[Na ppm]

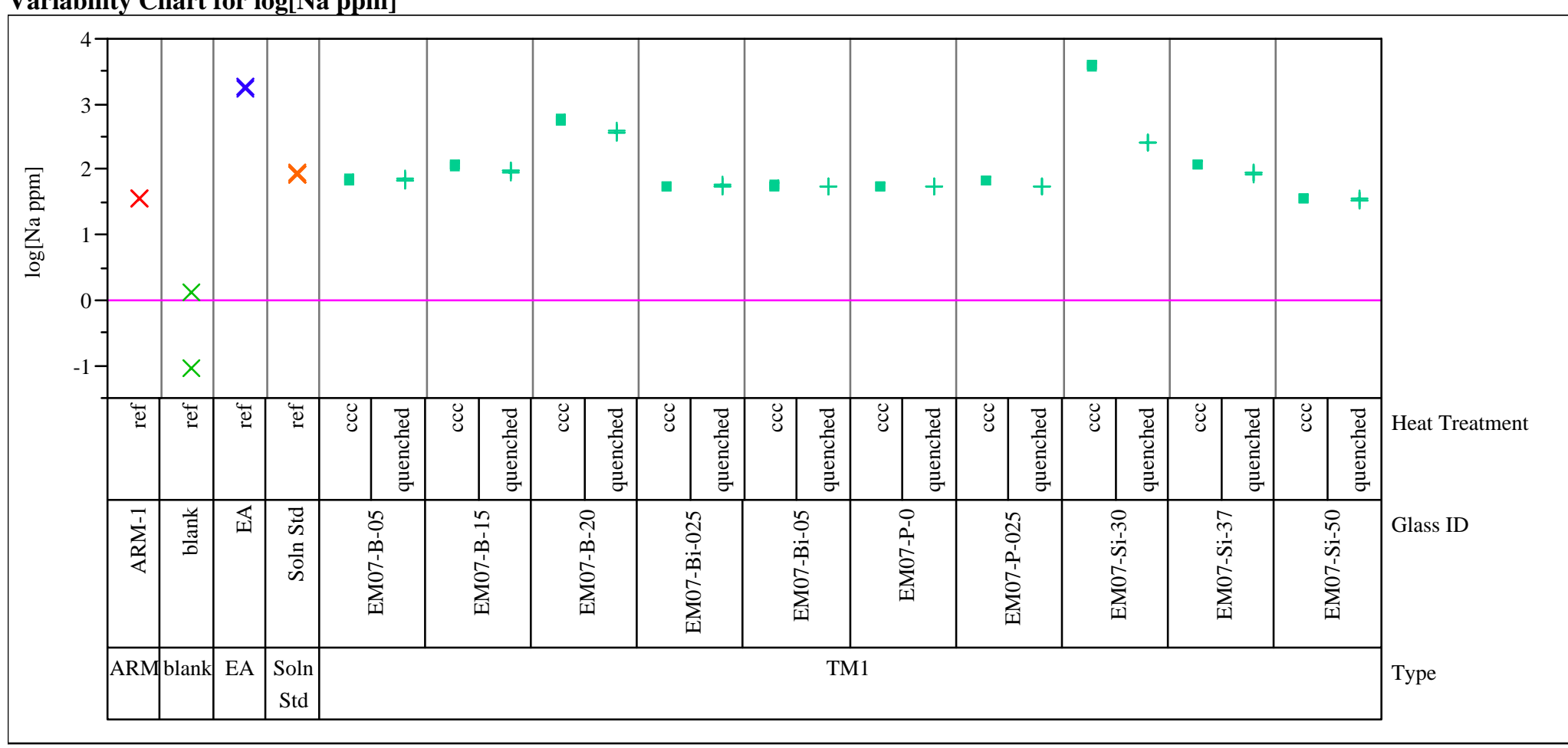


Figure B4. Laboratory PCT Measurements by Glass Number for Study Glasses and Standards (ppm and $\log (\mathrm{ppm})$ )

Variability Gage Set $=2$

Variability Chart for log[Si ppm]

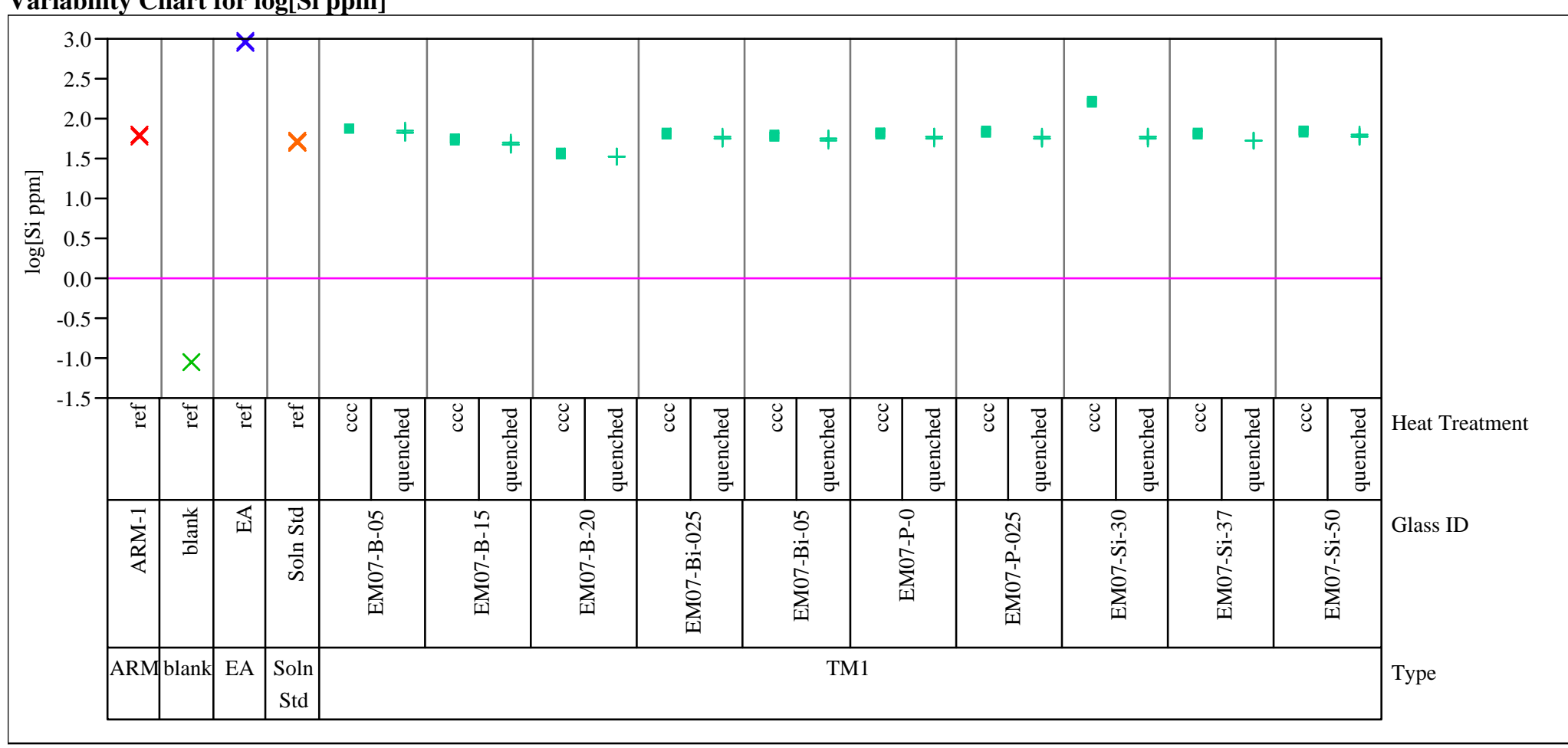


Figure B4. Laboratory PCT Measurements by Glass Number for Study Glasses and Standards (ppm and $\log (\mathrm{ppm})$ )

Variability Gage Set $=3$

Variability Chart for B (ppm)

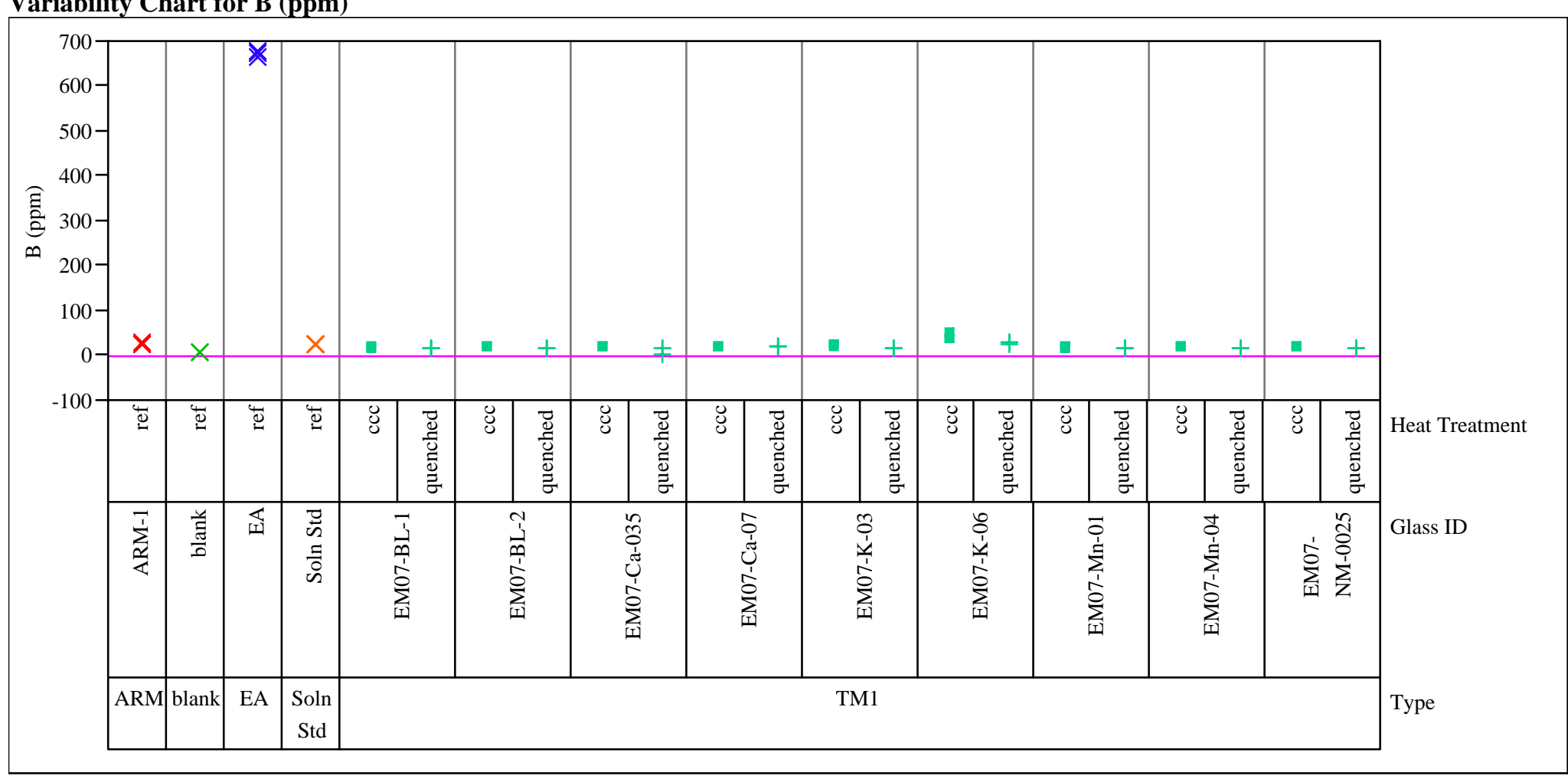


Figure B4. Laboratory PCT Measurements by Glass Number for Study Glasses and Standards (ppm and $\log (\mathrm{ppm})$ )

Variability Gage Set $=3$

Variability Chart for Li (ppm)

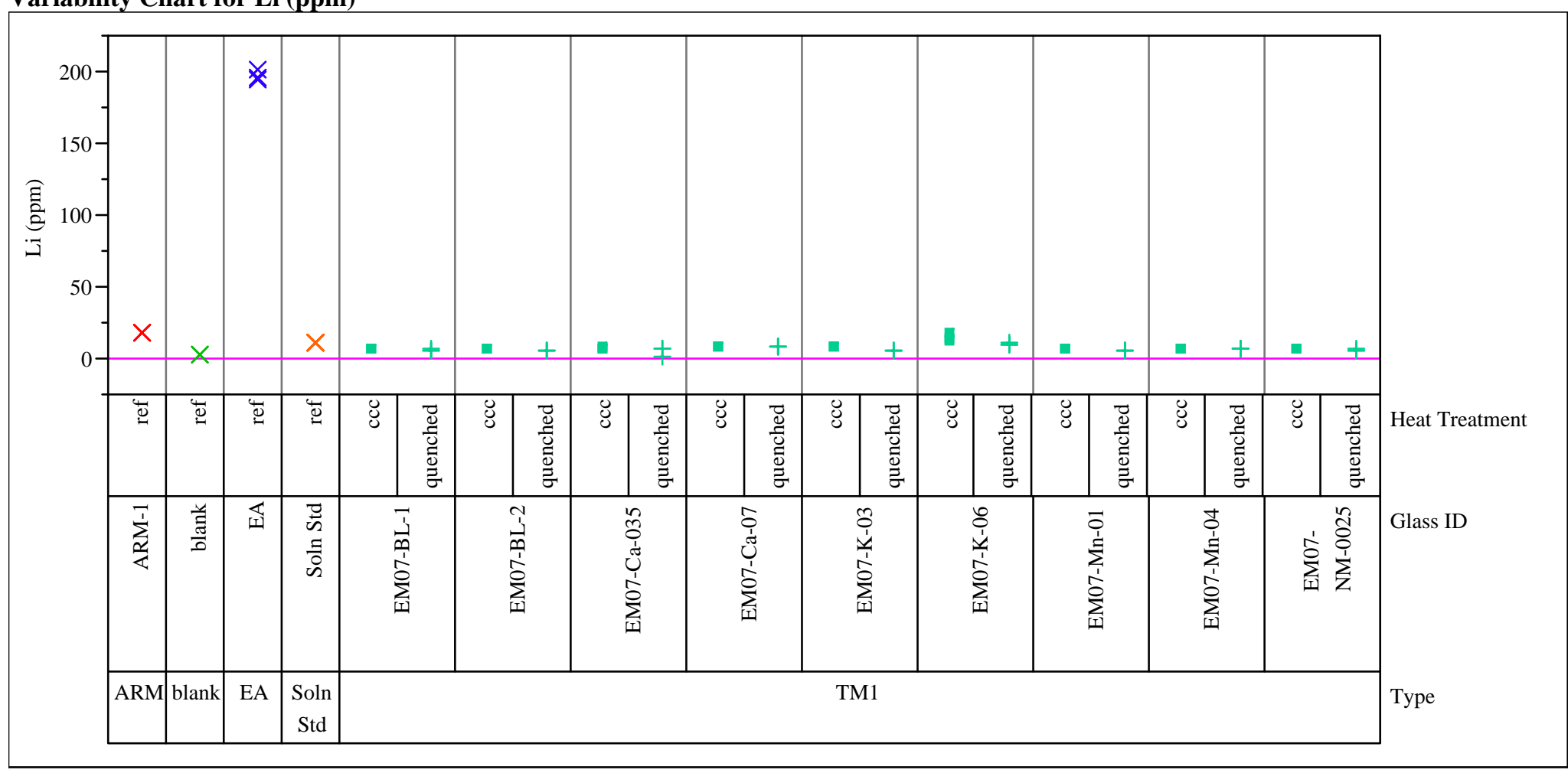


Figure B4. Laboratory PCT Measurements by Glass Number for Study Glasses and Standards (ppm and $\log (\mathrm{ppm})$ )

Variability Gage Set $=3$

Variability Chart for Na (ppm)

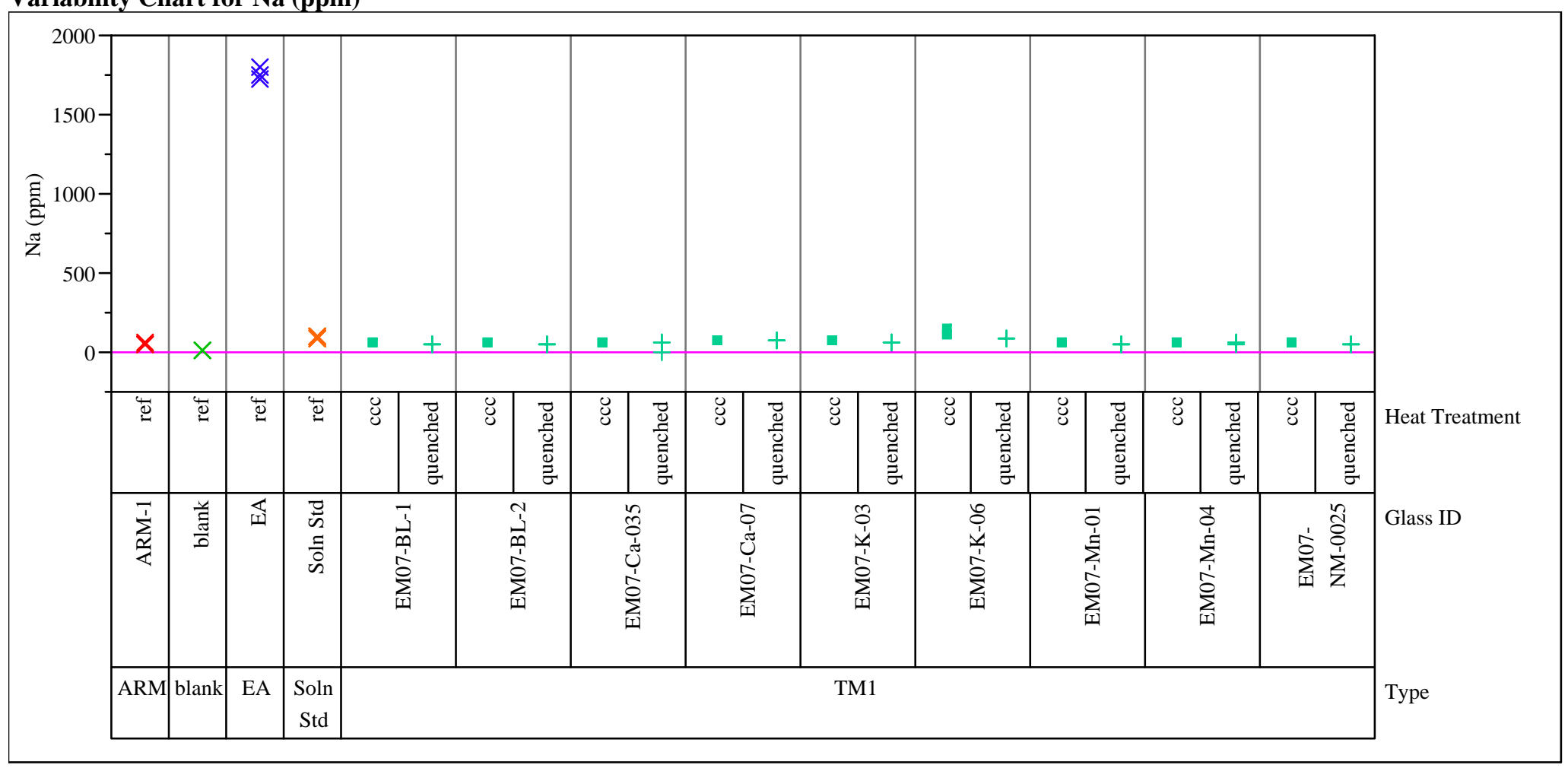


Figure B4. Laboratory PCT Measurements by Glass Number for Study Glasses and Standards (ppm and $\log (\mathrm{ppm})$ )

Variability Gage Set $=3$

Variability Chart for Si (ppm)

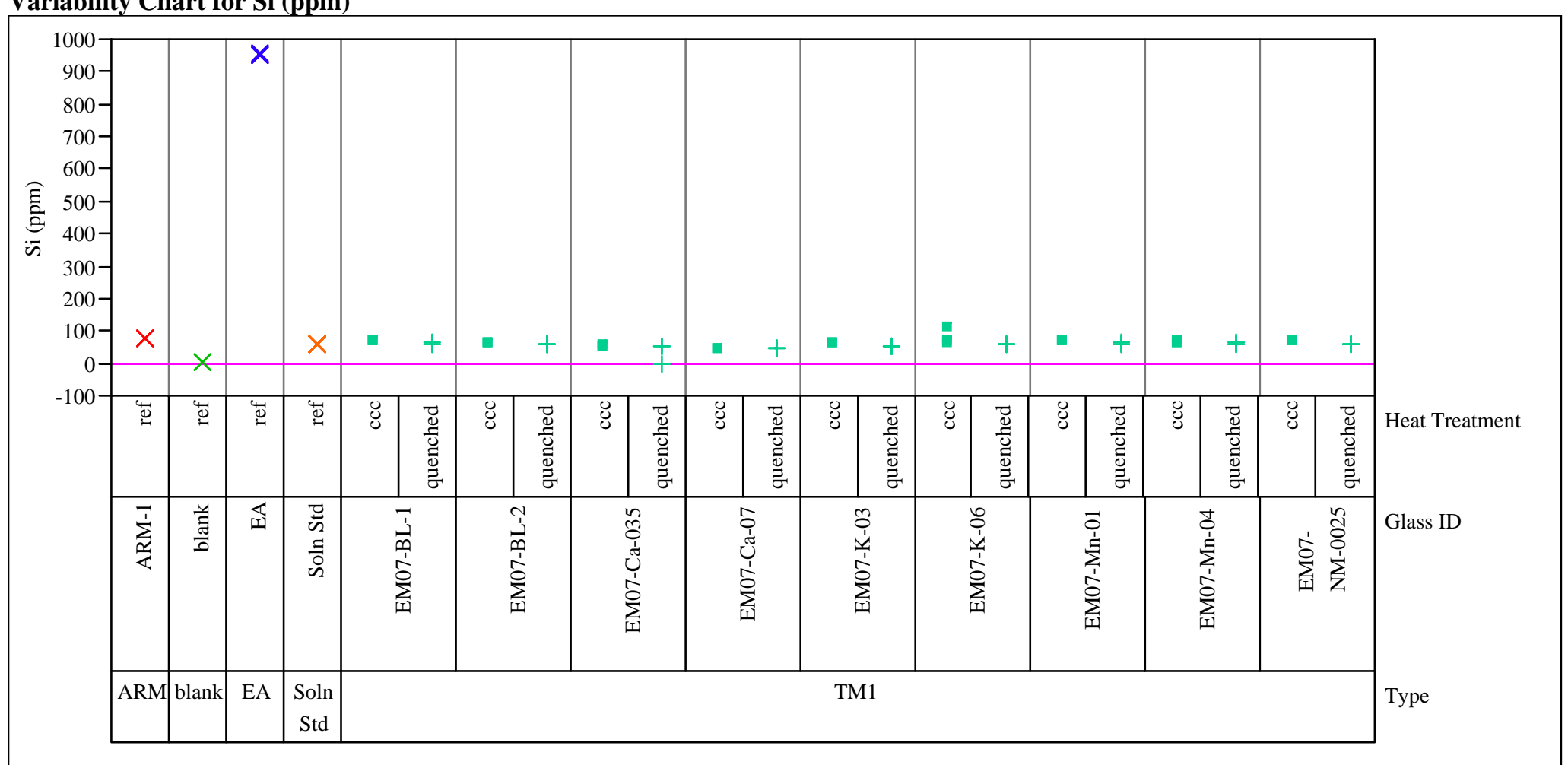


Figure B4. Laboratory PCT Measurements by Glass Number for Study Glasses and Standards (ppm and $\log (\mathrm{ppm})$ )

Variability Gage Set $=3$

Variability Chart for log[B ppm]

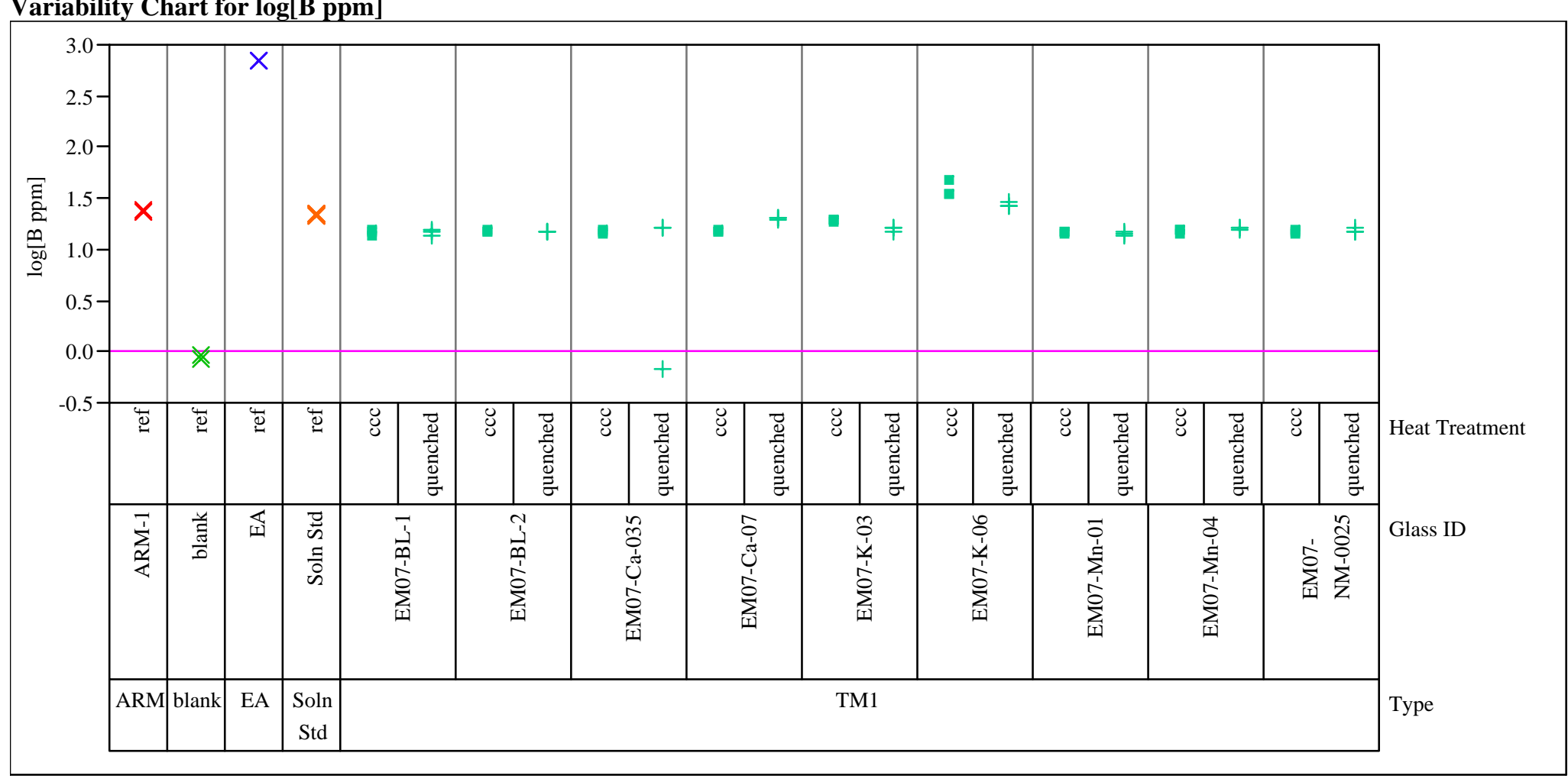


Figure B4. Laboratory PCT Measurements by Glass Number for Study Glasses and Standards (ppm and $\log (\mathrm{ppm})$ )

Variability Gage Set $=3$

Variability Chart for $\log [\mathrm{Li}$ ppm]

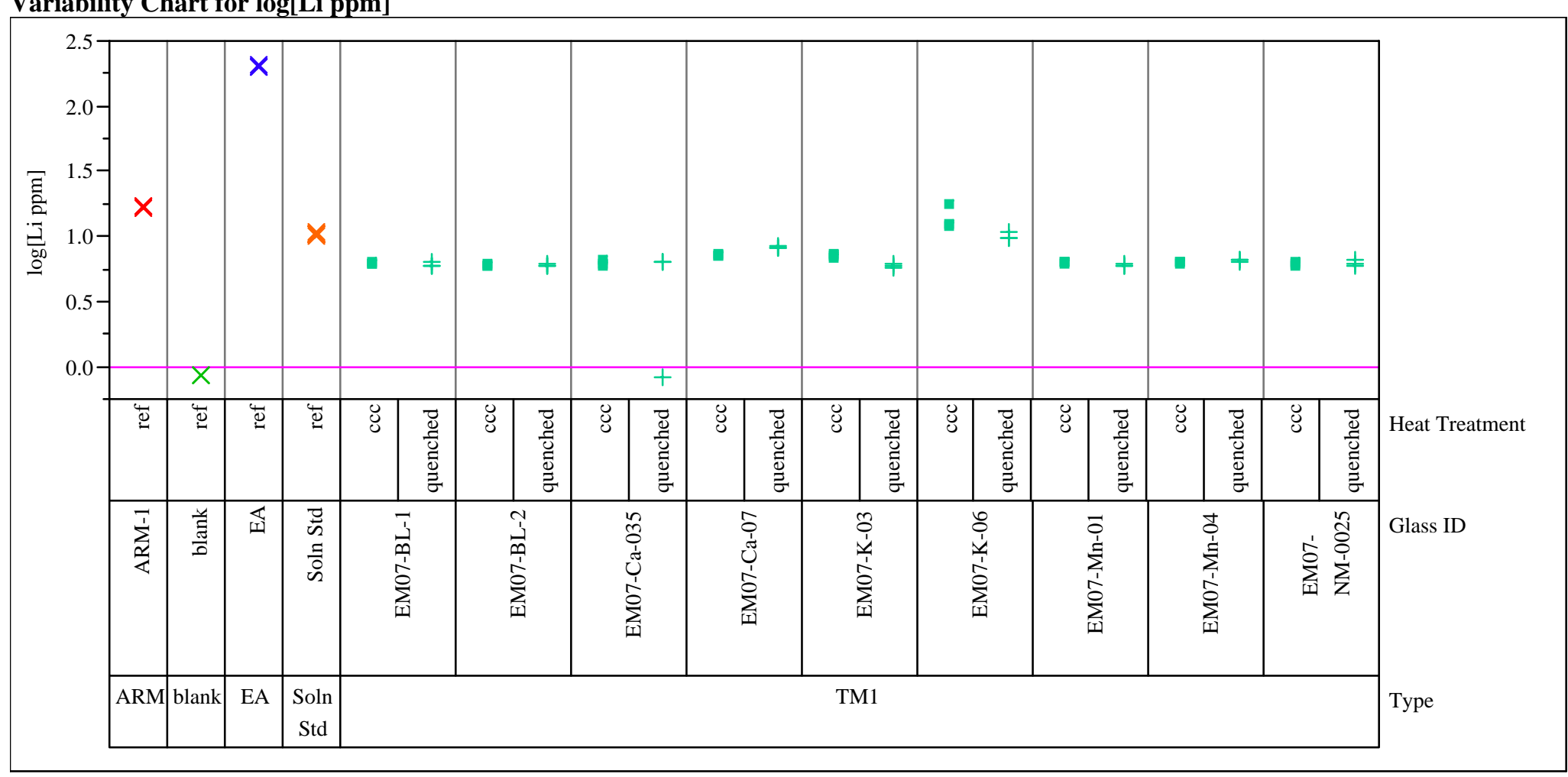


Figure B4. Laboratory PCT Measurements by Glass Number for Study Glasses and Standards (ppm and $\log (\mathrm{ppm})$ )

Variability Gage Set $=3$

Variability Chart for log[Na ppm]

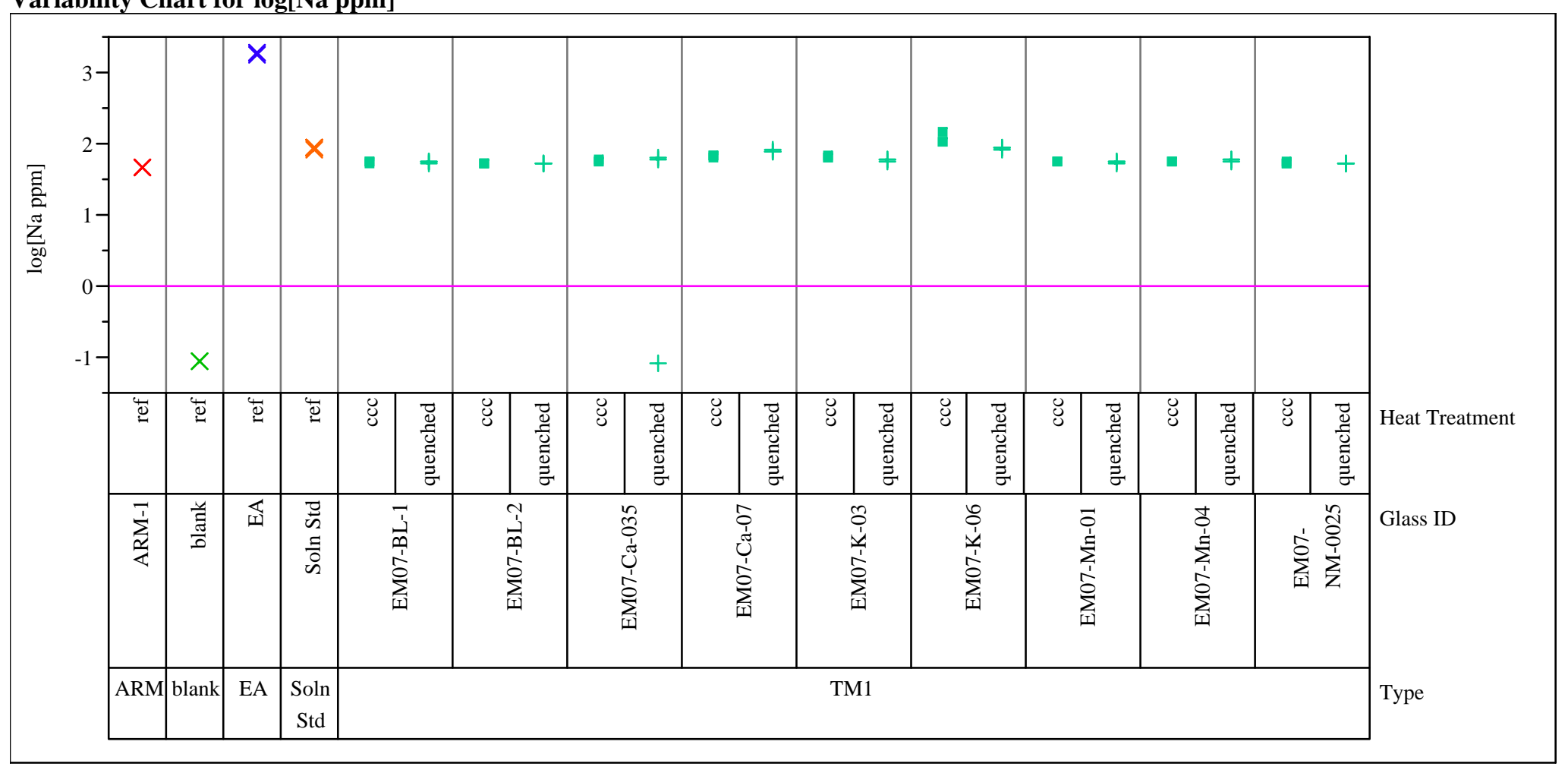


Figure B4. Laboratory PCT Measurements by Glass Number for Study Glasses and Standards (ppm and $\log (\mathrm{ppm})$ )

Variability Gage Set $=3$

Variability Chart for log[Si ppm]

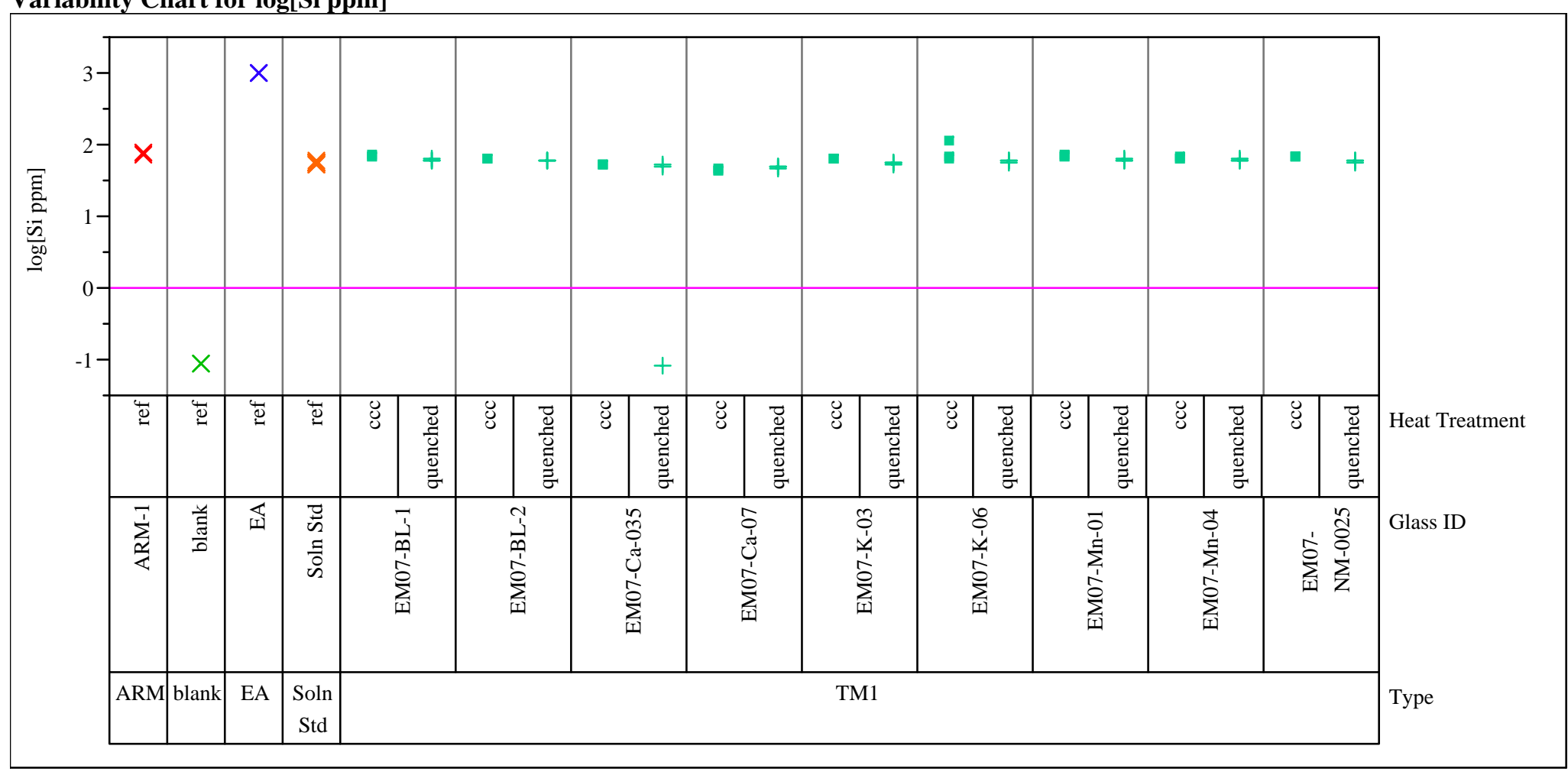


Figure B4. Laboratory PCT Measurements by Glass Number for Study Glasses and Standards (ppm and $\log (\mathrm{ppm})$ )

Variability Gage Set $=4$

Variability Chart for B (ppm)

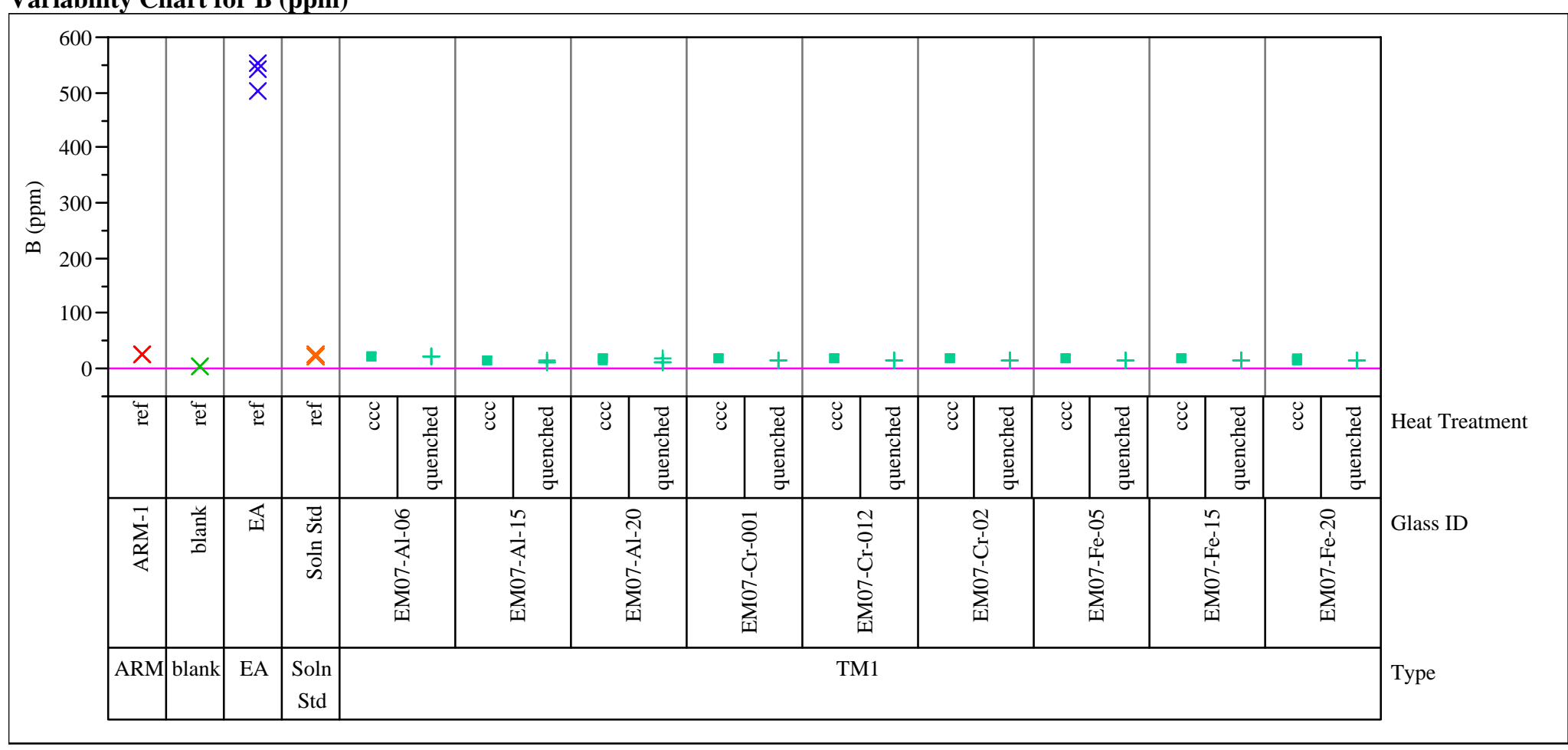


Figure B4. Laboratory PCT Measurements by Glass Number for Study Glasses and Standards (ppm and $\log (\mathrm{ppm})$ )

Variability Gage Set $=4$

Variability Chart for Li (ppm)

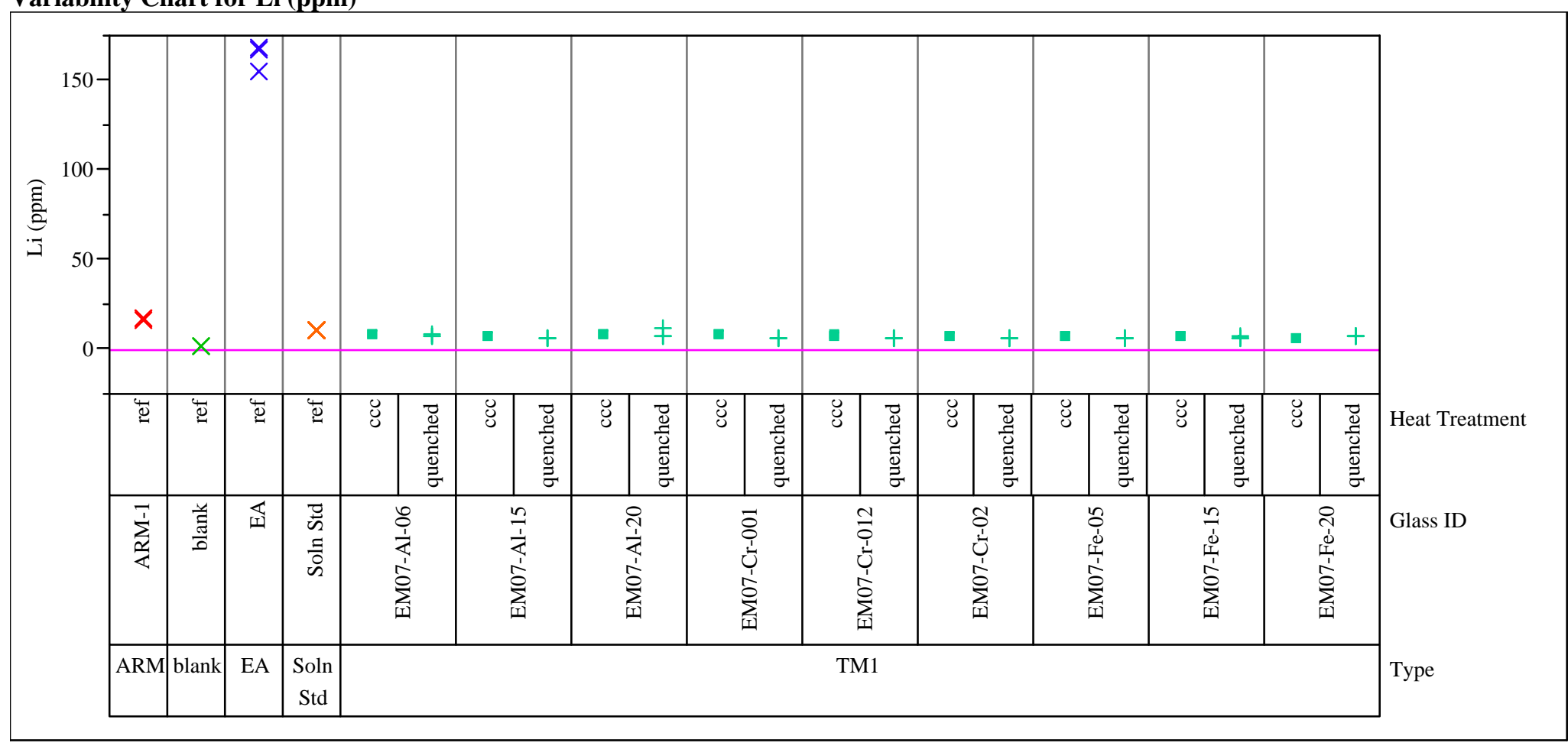


Figure B4. Laboratory PCT Measurements by Glass Number for Study Glasses and Standards (ppm and $\log (\mathrm{ppm})$ )

Variability Gage Set $=4$

Variability Chart for Na (ppm)

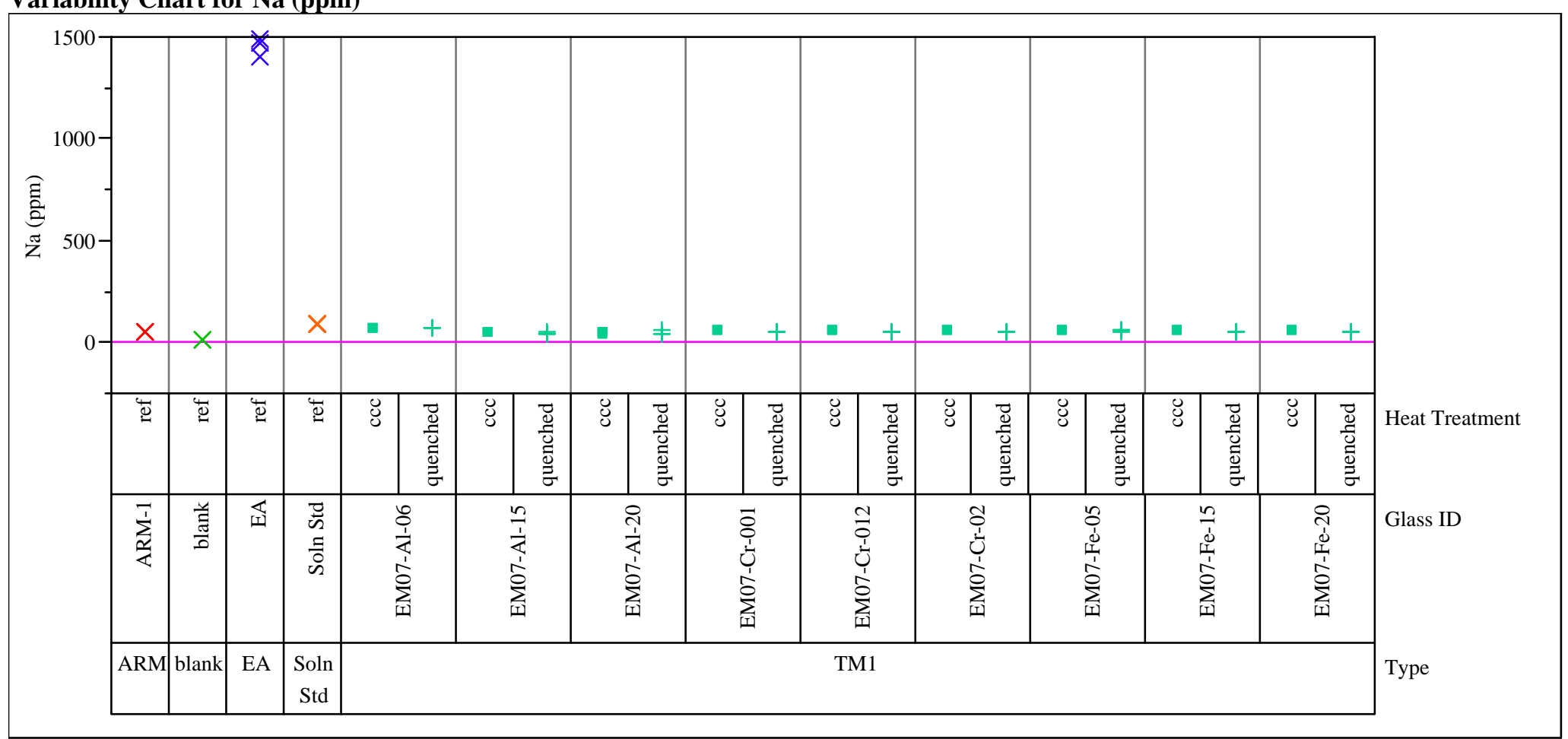


Figure B4. Laboratory PCT Measurements by Glass Number for Study Glasses and Standards (ppm and $\log (\mathrm{ppm})$ )

Variability Gage Set $=4$

Variability Chart for Si (ppm)

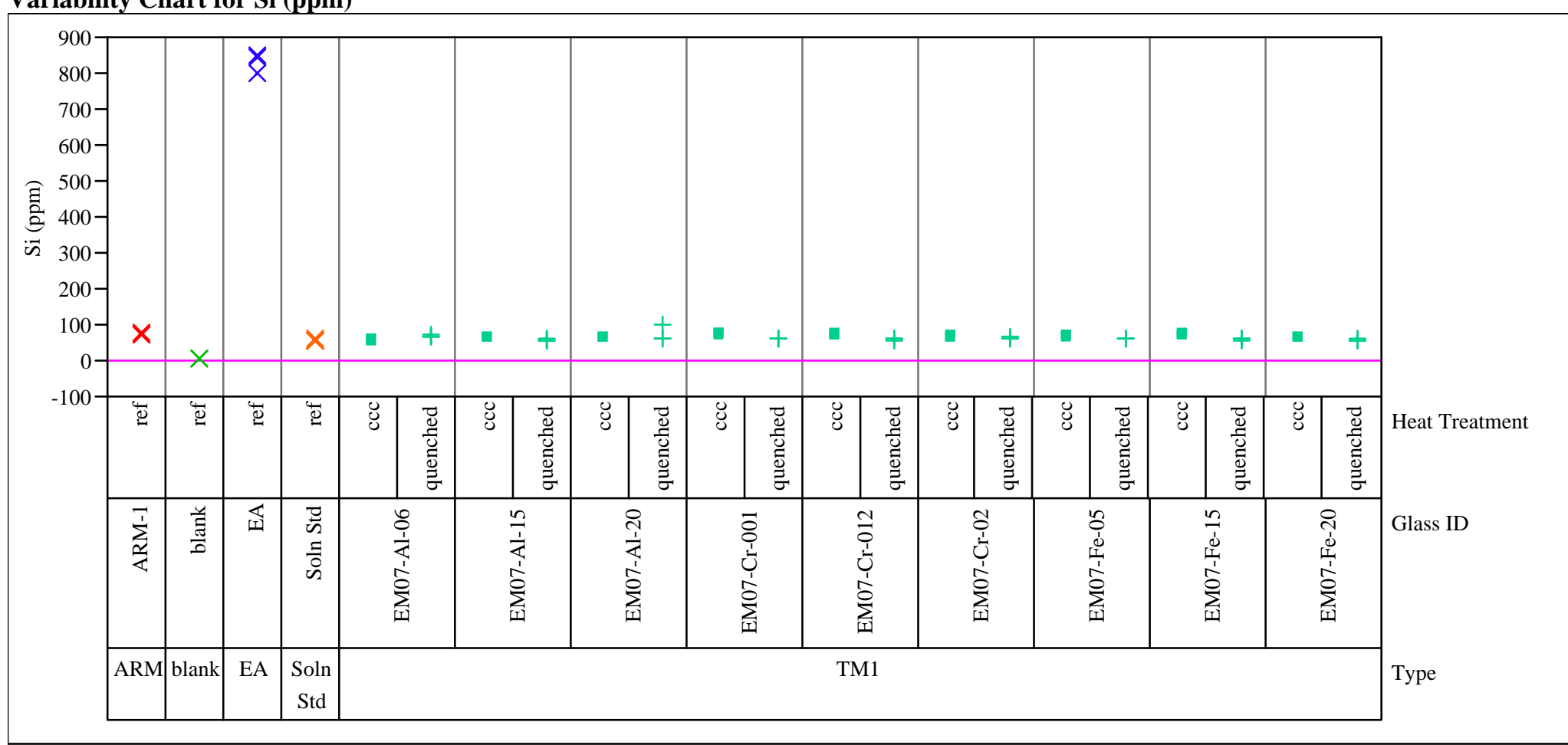


Figure B4. Laboratory PCT Measurements by Glass Number for Study Glasses and Standards (ppm and $\log (\mathrm{ppm})$ )

Variability Gage Set $=4$

Variability Chart for log[B ppm]

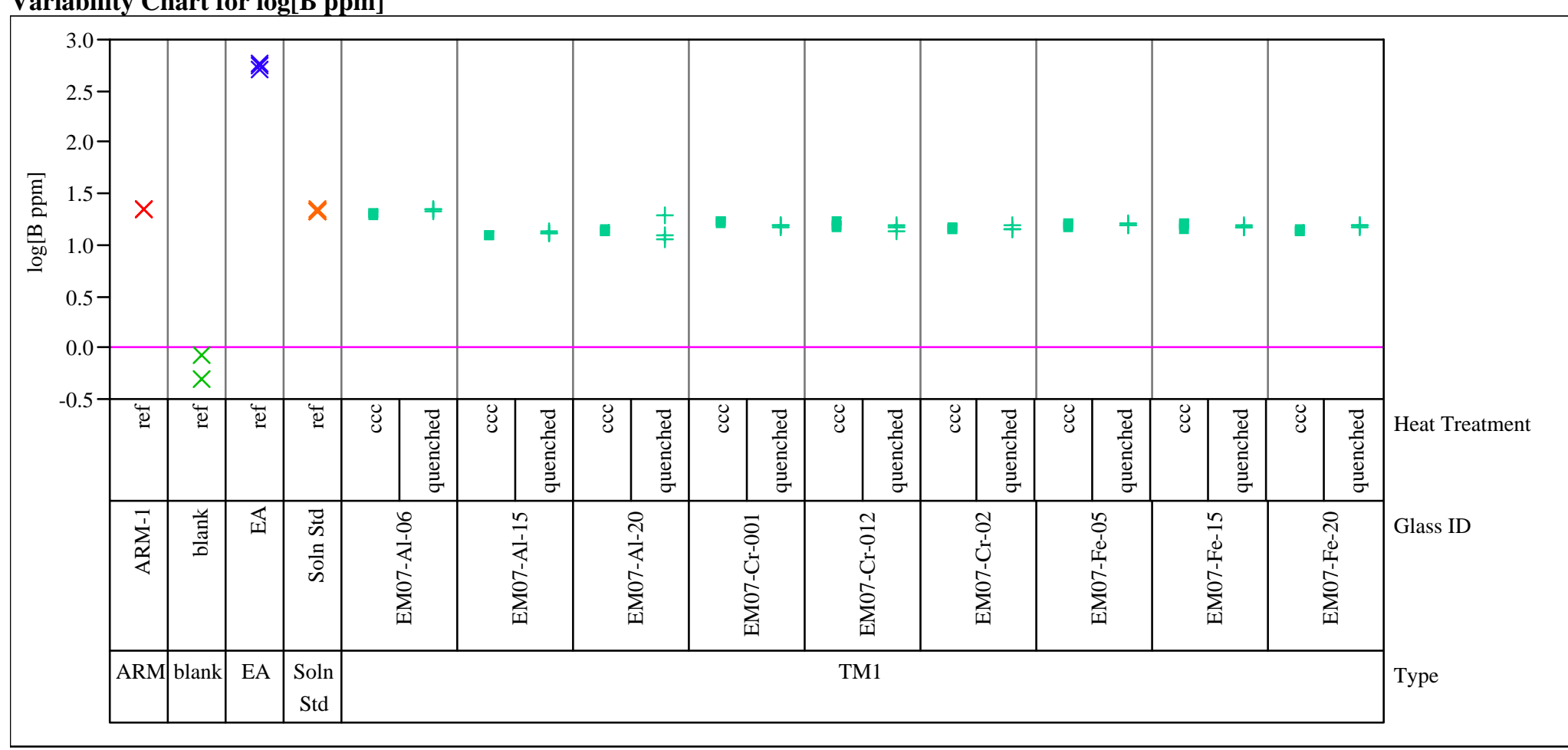


Figure B4. Laboratory PCT Measurements by Glass Number for Study Glasses and Standards (ppm and $\log (\mathrm{ppm})$ )

Variability Gage Set $=4$

Variability Chart for $\log [\mathrm{Li}$ ppm]

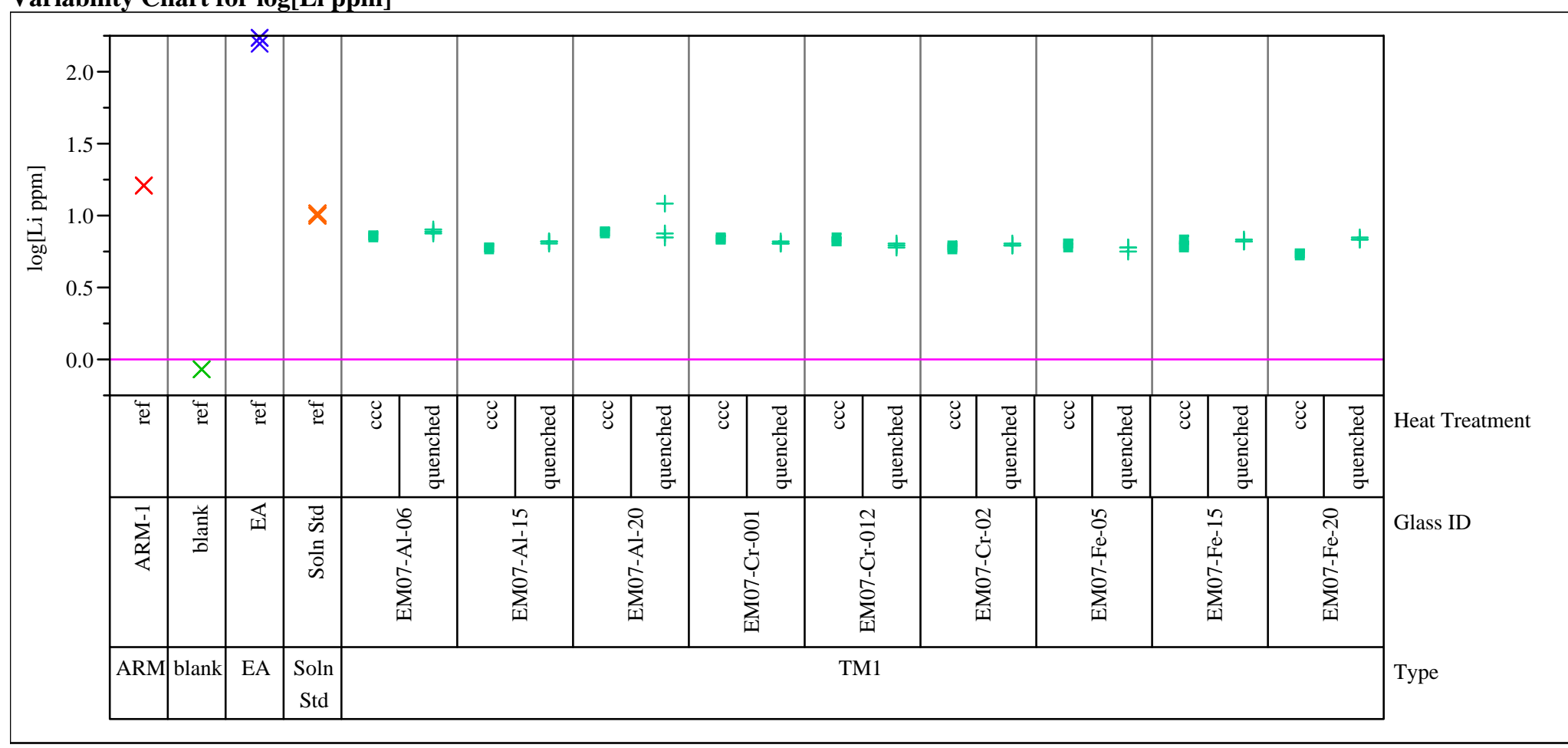


Figure B4. Laboratory PCT Measurements by Glass Number for Study Glasses and Standards (ppm and $\log (\mathrm{ppm})$ )

Variability Gage Set $=4$

Variability Chart for log[Na ppm]

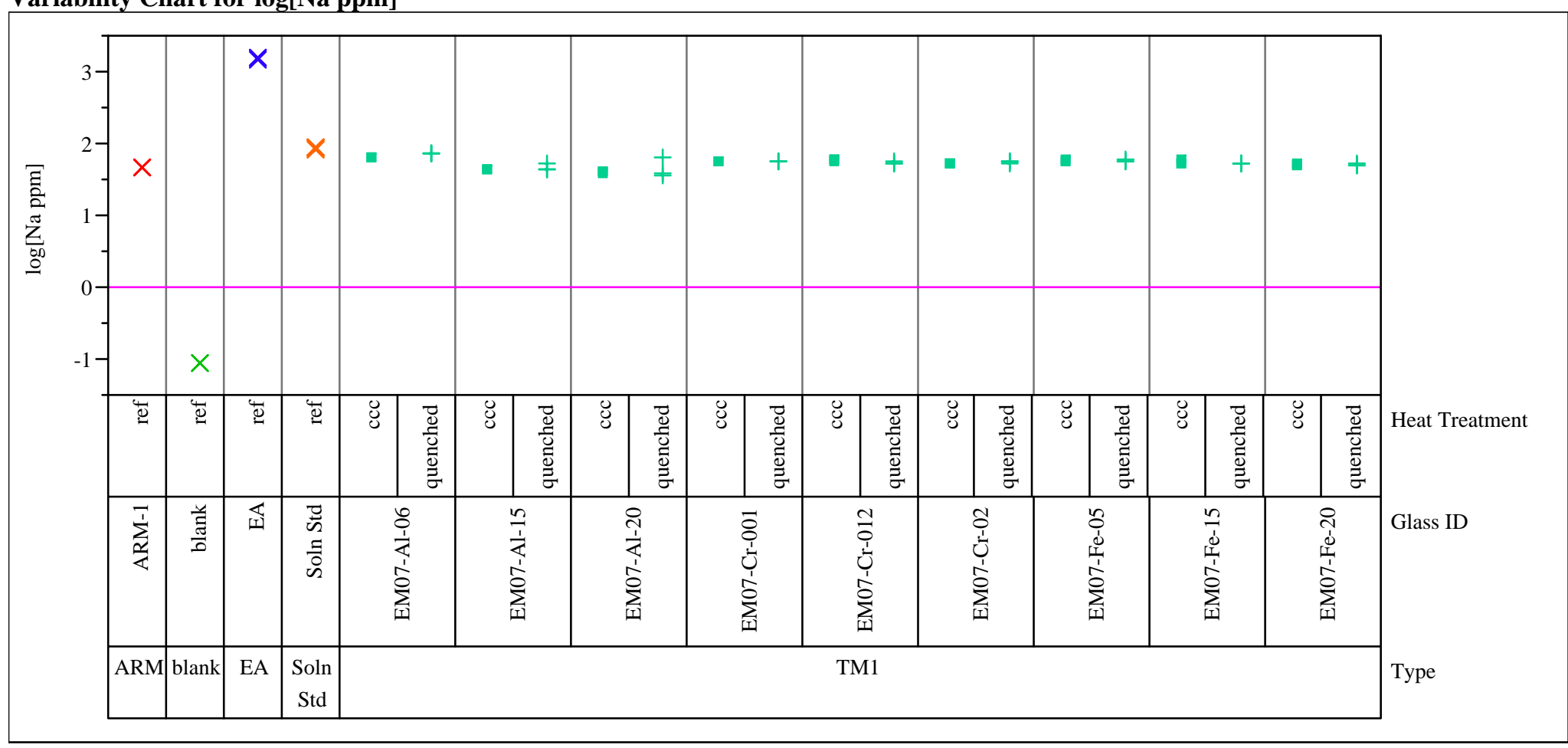


Figure B4. Laboratory PCT Measurements by Glass Number for Study Glasses and Standards (ppm and $\log (\mathrm{ppm})$ )

Variability Gage Set $=4$

Variability Chart for log[Si ppm]

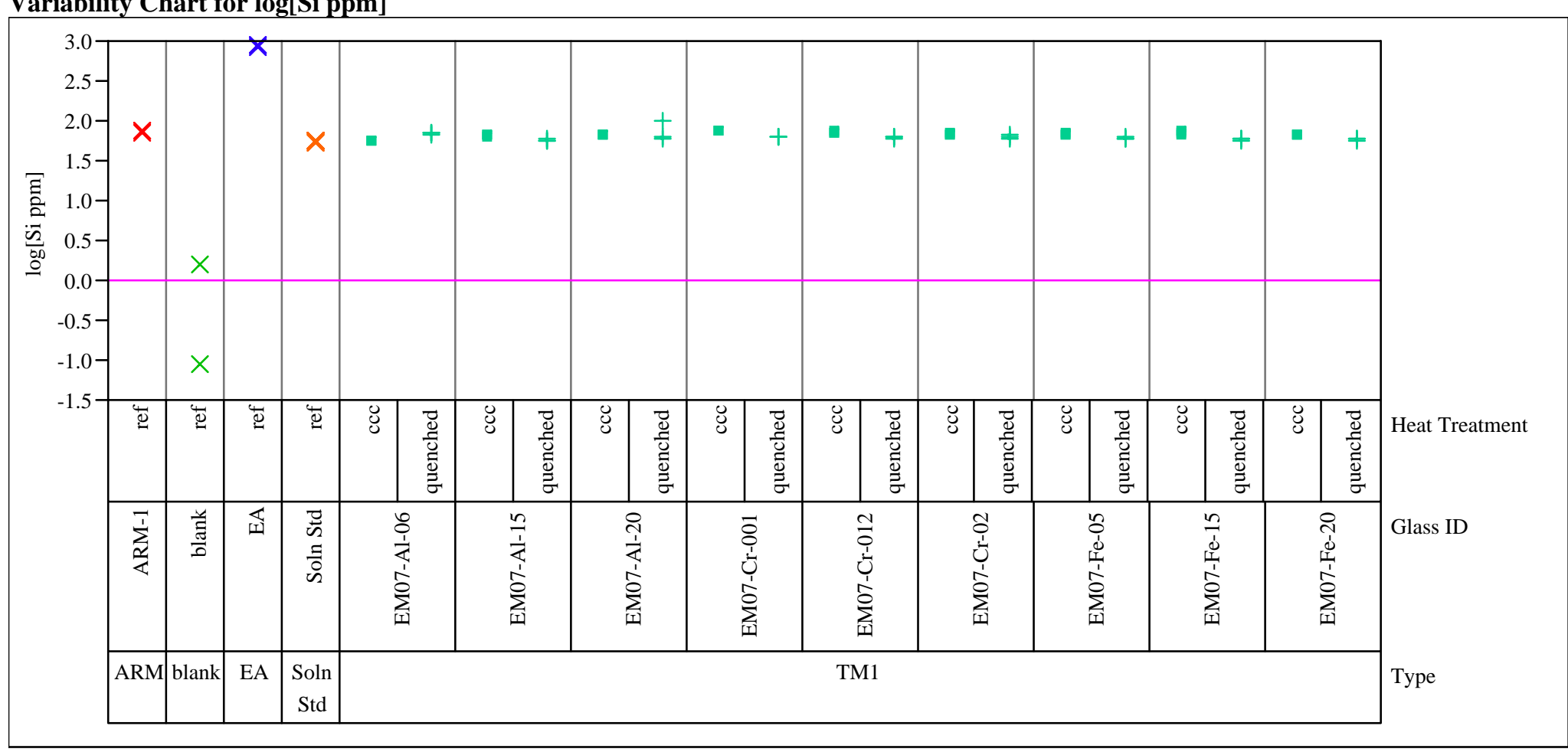


Figure B5. Correlations and Scatter Plots of Normalized PCTs Over All Compositional Views and Heat Treatments

$\begin{array}{lrrrr}\text { Correlations } & & & & \\ & \log \mathrm{NL}[\mathrm{B}(\mathrm{g} / \mathrm{L})] & \log \mathrm{NL}[\mathrm{Li}(\mathrm{g} / \mathrm{L})] & \log \mathrm{NL}[\mathrm{Na}(\mathrm{g} / \mathrm{L})] & \log \mathrm{NL}[\mathrm{Si}(\mathrm{g} / \mathrm{L})] \\ \log \mathrm{NL}[\mathrm{B}(\mathrm{g} / \mathrm{L})] & 1.0000 & 0.9647 & 0.8320 & 0.5757 \\ \log \mathrm{NL}[\mathrm{Li}(\mathrm{g} / \mathrm{L})] & 0.9647 & 1.0000 & 0.7308 & 0.5271 \\ \log \mathrm{NL}[\mathrm{Na}(\mathrm{g} / \mathrm{L})] & 0.8320 & 0.7308 & 1.0000 & 0.5650 \\ \log \mathrm{NL}[\mathrm{Si}(\mathrm{g} / \mathrm{L})] & 0.5757 & 0.5271 & 0.5650 & 1.0000\end{array}$

Scatterplot Matrix

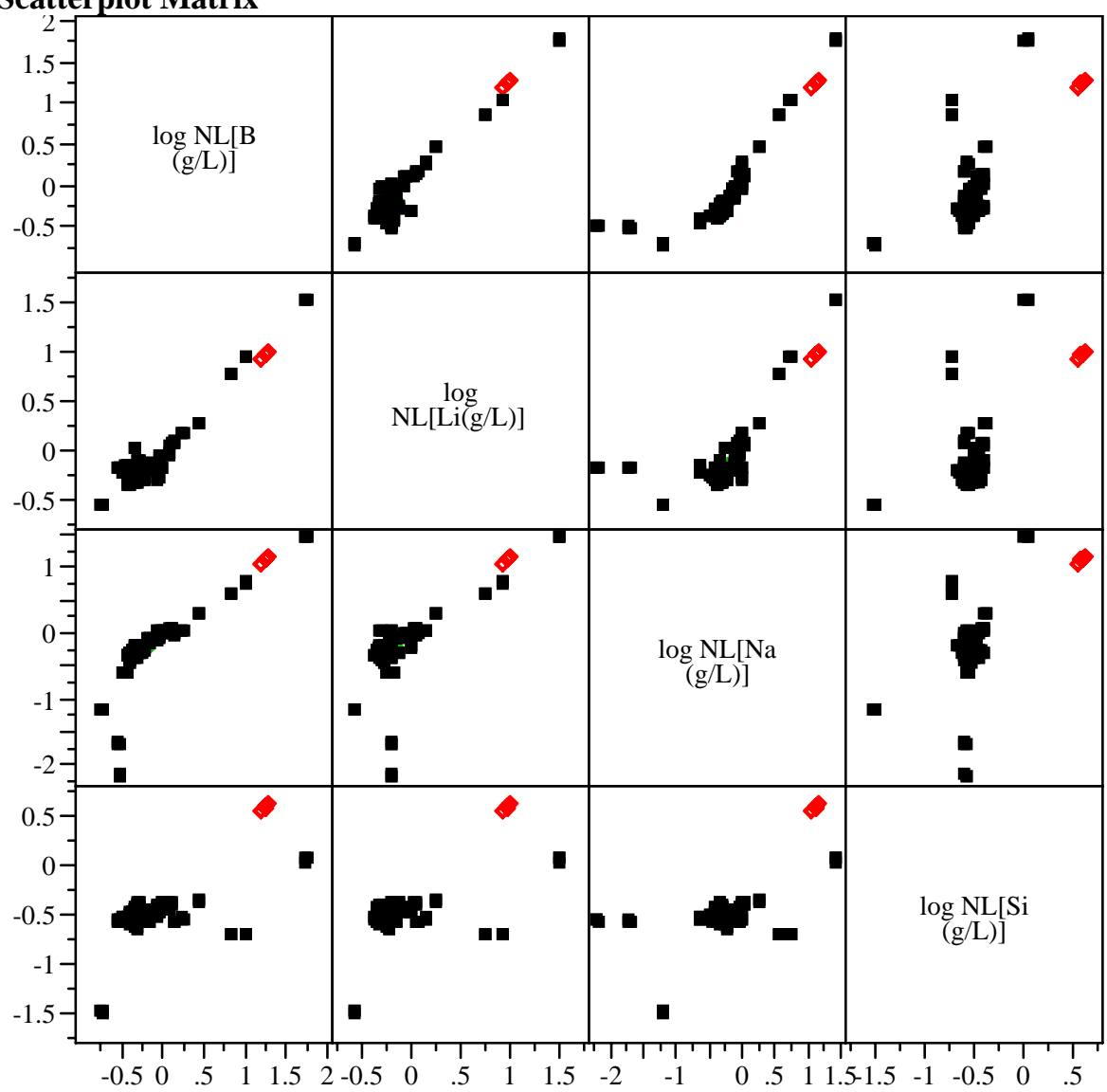


Figure B6. Effects of Heat Treatment (HT) on PCT $\log (\mathrm{ppm})$-Response of Study Glasses

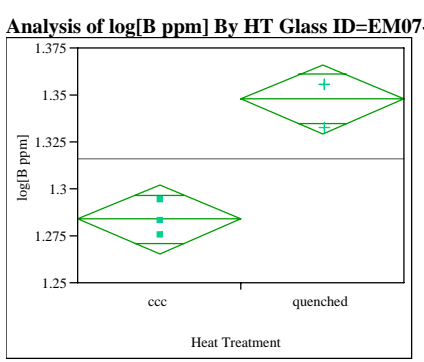

Anova

Summary of Fit

Rsquare

Root Mean Square Error $\quad 0.011511$

Observations (or Sum Wgts) $\begin{array}{r}1.315745 \\ \hline\end{array}$

t Test

Assuming equal variances

Difference $\quad 0.064003$ t Ratio $\quad 6.809767$

Std Err Dif 0.009399 DF $>1 t \quad 0.0024$

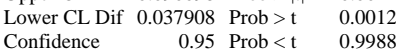

$\begin{array}{lrrrrr}\text { Source } & \text { DF } & \begin{array}{r}\text { Sum of } \\ \text { Squares }\end{array} & \begin{array}{r}\text { Mean } \\ \text { Square }\end{array} & \text { F Ratio } & \text { Prob > } \\ \text { HT } & 1 & 0.00614459 & 0.006145 & 46.3729 & 0.0024 \\ \text { Error } & 4 & 0.00053002 & 0.000133 & & \\ \text { C. } & 5 & 0.00667461 & & & \end{array}$

Means for Anova

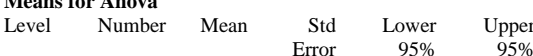

$\begin{array}{lrrrrr} & & & \text { Error } & 95 \% & 95 \% \\ \text { ccc } & 3 & 1.28374 & 0.00665 & 1.2653 & 1.3022\end{array}$

quenched 13 (1.347

Std Error uses a pooled estimate of error variance
Analysis of $\log [\mathrm{Li}$ ppm] By HT Glass ID=EM07-Al-06

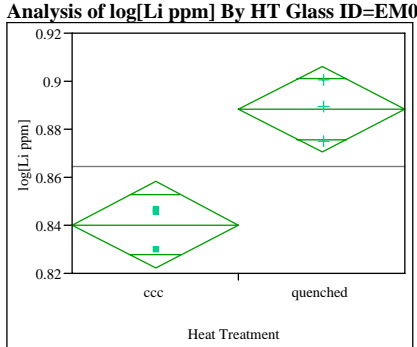

Anova

Summary of Fit

Rsquare

$\begin{array}{ll}\text { Adj Rsquare } & 0.87344 \\ \text { Root Mean Square Error } & 0.84245 \\ \text { D } & 0.011172\end{array}$

$\begin{array}{lr}\text { Mean of Response } & 0.864219 \\ \text { Observations (or Sum Wgts) } & 6\end{array}$

t Test

Assuming equal variances

Difference $\quad 0.048066$ t Ratio $\quad 5.266581$ $\begin{array}{lll}\text { Std Err Dif } 0.009127 & \mathrm{DF} & 4\end{array}$ Upper CL DIf 0.073405 Prob $>|l| 0.0062$ Confidence $\quad 0.95$ Prob $<t \quad 0.9969$

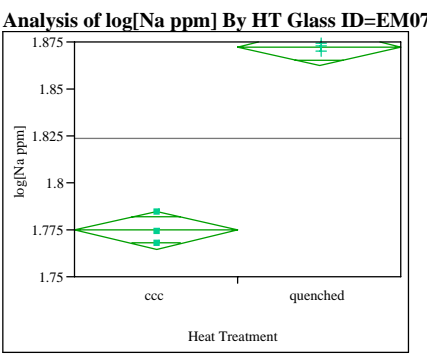

Anova

Summary of Fit

Rsquare

Root Mean Square Error $\quad 0.006247$

$\begin{array}{lr}\text { Mean of Response } & 1.82368 \\ \text { Observations (or Sum Wgts) } & 6\end{array}$

t Test

enched-ccc

Assuming equal variances

Difference $\quad 0.097611$ t Ratio $\quad 19.13553$ Uner CL Dif 0.111774 Prob $>|t|<0001$ $\begin{array}{lrll}\text { Lower CL Dif } & 0.083449 & \text { Prob }>t & <.0001 \\ \text { Confidence } & 0.95 & \text { Prob }<\mathrm{t} & 1.0000\end{array}$

$\begin{array}{llll}\text { Source DF Sum of Mean F Ratio Prob }> & \end{array}$

$\begin{array}{lrrrrr} & & \text { Squares } & \text { Square } & \text { F } \\ \text { HT } & 1 & 0.00346548 & 0.003465 & 27.7369 & 0.0062 \\ \text { Error } & 4 & 0.00049977 & 0.000125 & \end{array}$

C.

Means for Anova

Level Number Mean $\begin{array}{rrr}\text { Std } & \text { Lower } & \text { Upper } \\ \text { Error } & 95 \% & 95 \%\end{array}$ $\begin{array}{lllllll}\text { ccc } & & 3 & 0.840186 & 0.00645 & 0.82227 & 0.85810 \\ \text { quenched } & & 3 & 0.888252 & 0.00645 & 0.87033 & 0.90617\end{array}$ Std Error uses a pooled estimate of error variance

Std Error uses a pooled estimate of error variance
Analysis of log[Si ppm] By HT Glass ID=EM07-Al-06

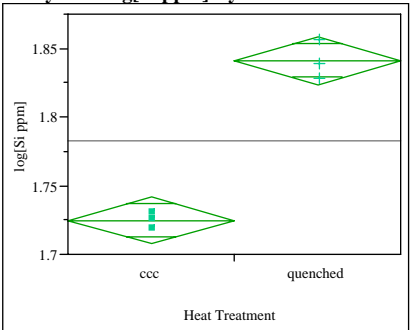

Anova
Summary of Fit

Rsquare
Adj Rsquare

Adj Rsquare $\quad 0.977712$ Mean of Response $\quad 0.01076$ Observations (or Sum Wgts)

t Test

Assuming equal variances

Difference $\quad 0.116428$ t Ratio 13.24635 Std Err Dif 0.008789 DF

$\begin{array}{lll} & 0.002 \\ \text { Upper } & & 0.000\end{array}$ Confidence $\quad 0.95$ Prob $<\mathrm{t} \quad 0.9999$

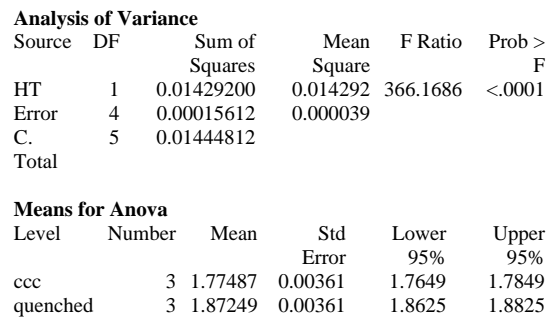

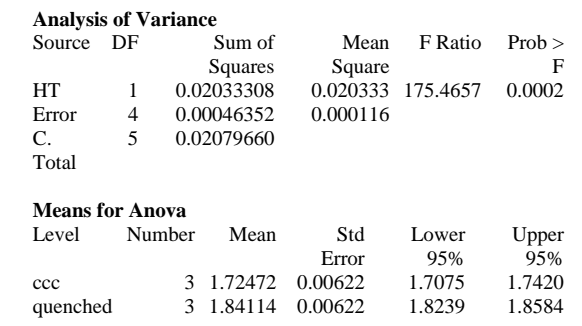

Std Error uses a pooled estimate of error variance 
Figure B6. Effects of Heat Treatment (HT) on PCT $\log (\mathrm{ppm})$-Response of Study Glasses

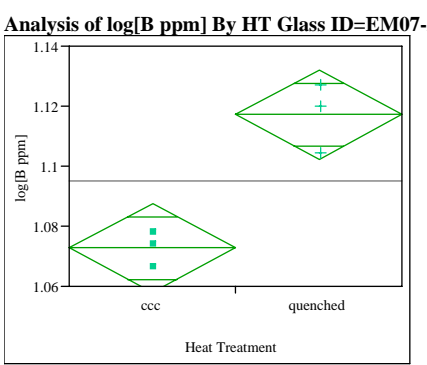

Anova
Summary of Fit

Rsquare

0.871565
Adj Rsquare

Mean of Response 1.094929

(1)

t Test
quenched-cce

Assuming equal variances

Difference $\quad 0.044495$ t Ratio $\quad 5.91017$ $\begin{array}{lll}\text { Std Err Dif } & 0.007529 & \mathrm{DF}\end{array}$ Upper CL Dif 0.065398 Prob $>|t| 0.0041$ $\begin{array}{lll} & 0.95 \mathrm{Prob}<\mathrm{t} & 0.9979\end{array}$

Analysis of $\log [\mathrm{Li}$ ppm] By HT Glass ID=EM07-Al-15

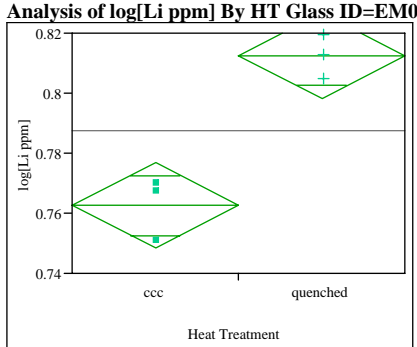

Anova
Summary of Fit

Rsquare

\begin{tabular}{lr} 
Adj Rsquare & 0.92237 \\
Root Mean Square Error & 0.902962 \\
\hline & 0.78858
\end{tabular}

$\begin{array}{ll}\text { Mean of Response } & 0.787517 \\ \text { Observations (or Sum Wgts) }\end{array}$

t Test

Assuming equal variances

Difference $\quad 0.049987$ t Ratio $\quad 6.893928$ Std Err Dif 0.007251 DF $\quad 4$ Upper CL DIf 0.072118 Prob $>|t|$ $\begin{array}{lll}\text { Confidence } & 0.95 \text { Prob }<\mathrm{t} \quad 0.9988\end{array}$

Analysis of Varianc $\begin{array}{llrrrr} & & \text { Squares } & \text { Square } & \text { F } \\ \text { HT } & 1 & 0.00296975 & 0.002970 & 34.9301 & 0.0041\end{array}$ $\begin{array}{lll} & 1 & 0.002965 \\ \text { C. } & 4 & 0.00034008 \\ \text { C. } & 5 & 0.00330982\end{array}$

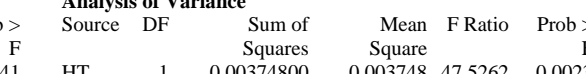
$\begin{array}{llrrrr}\text { HT } & 1 & 0.00374800 & 0.003748 & 47.5262 & 0.0023 \\ \text { Error } & 4 & 0.00031545 & 0.000079 & & \end{array}$ C. Total

$\begin{array}{lllll}\text { Means for Anova } & & & & \\ \text { Level Number } & \text { Mean } & \text { Std } & \text { Lower } & \text { Upper }\end{array}$ $\begin{array}{lrrrrr} & & & \text { Error } & \text { Lower } & 95 \% \\ \text { ccc } & 3 & 1.07268 & 0.00532 & 1.0579 & 1.0875 \\ & 3 & & & & \end{array}$ $\begin{array}{llllll}\text { quenched } & 3 & 1.11718 & 0.00532 & 1.1024 & 1.1320\end{array}$

Std Error uses a pooled estimate of error variance $\begin{array}{lllll}\text { Means for Anova } & & & \\ \text { Level Number Mean Std Lower Upper }\end{array}$ $\begin{array}{lrrrrr}\text { ccC } & 3 & 0.762524 & 0.00513 & 0.74829 & 0.77676 \\ & 3 & 0.8251 & 0.0513 & & \end{array}$ $\begin{array}{lllllll}\text { quenched } & 3 & 0.812510 & 0.00513 & 0.79828 & 0.82675\end{array}$ Std Error uses a pooled estimate of error variance
Analysis of $\log [\mathrm{Na}$ ppm] By HT Glass ID=EM07-Al-15

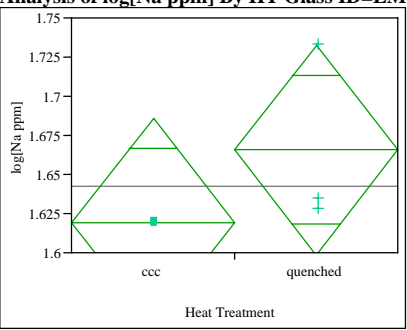

ummary of Fit

$\begin{array}{ll}\text { Rsquare } & 0.318448 \\ \text { Adj Rsquare } & 0.148061 \\ \text { Root Mean Square Error } & 0.041701\end{array}$

$\begin{array}{lr}\text { Mean of Response } & 1.642491 \\ \text { Observations (or Sum Wgts) } & 6\end{array}$

t Test

Assuming equal variances

$\begin{array}{lll}\text { Difference } & 0.04655 & \text { t Ratio } \\ 1.367\end{array}$ Upper CL Dif 0.14108 Prob > |t 0.2434 Lower CL Dif -0.04799 Prob $>$ t 0.1217 Confidence $\quad 0.95$ Prob $<\mathrm{t} 0.8783$

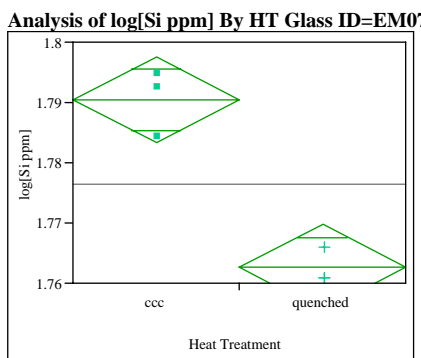

Rsquare
Adj Rsquare

Observations (or Sum Wgts) 1.776512

t Test

quenched-cce

Assuming equal variances

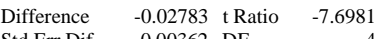
Std Err Dif 0.00362 DF $\quad-7.698$ Upper CL Dif -0.01779 Prob $>$ |L 0.0015 Confidence $\quad 0.95$ Prob $<t \quad 0.0008$

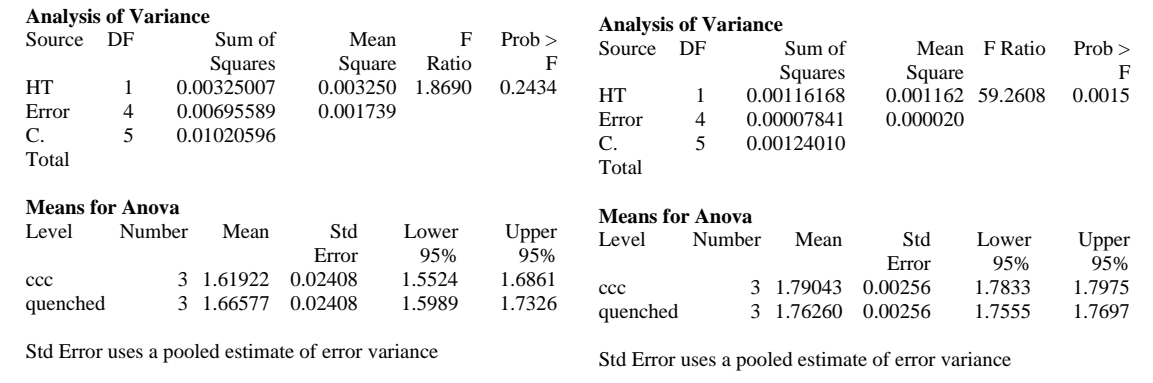


Figure B6. Effects of Heat Treatment (HT) on PCT log(ppm)-Response of Study Glasses

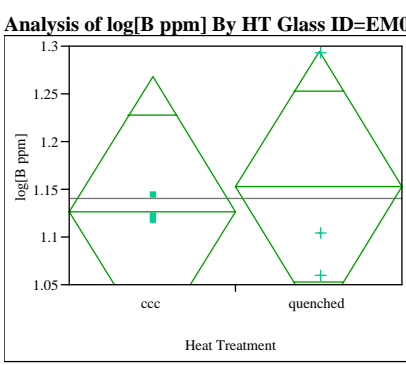

Anov

Summary of Fit

Rsquare
Adj Rsquare

Adj Rsquare 0.0304

Hare Error $\quad 0.088399$

Mean of Response
Observations (or Sum Wgts)
1.139752

t Test

Assuming equal variances

Difference $\quad 0.02558$ t Ratio $\quad 0.354436$ $\begin{array}{llll}\text { Std Err Dif } & 0.07218 \text { DF } & 4\end{array}$ $\begin{array}{llll}\text { Upper CL Dif } & 0.22598 & \text { Prob }>|t| & 0.7409 \\ \text { Lower CL Dif }-0.17482 & \text { Prob }>t & 0.3705\end{array}$ $\begin{array}{lll}\text { Confidence } & 0.95 \text { Prob }<\mathrm{t} & 0.6295\end{array}$

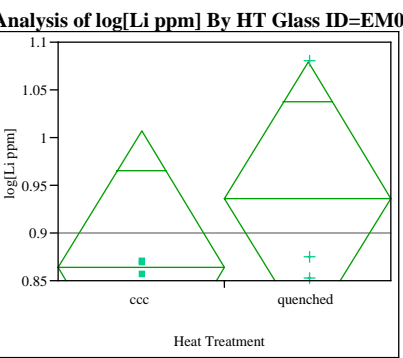

Anova

Summary of Fi

Rsquare

Root Mean Square Error $\quad 0.0890$

Observations (or Sum Wgts)

t Test
quenched-ccc

Assuming equal variances

Difference $\quad 0.07164 \mathrm{t}$ Ratio $\quad 0.985752$ Std Err Dif $\quad 0.07268$ DF

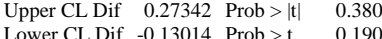
$\begin{array}{lr}\text { Confidence } & 0.95 \text { Prob }<\mathrm{t} \quad 0.8100\end{array}$

\section{Analysis of Variance}

Analysis of Varian

$\begin{array}{rrrrr}\text { Source DF } & \begin{array}{r}\text { Sum of } \\ \text { Squares }\end{array} & \begin{array}{r}\text { Mean } \\ \text { Square }\end{array} & \begin{array}{r}\text { F } \\ \text { Ratio }\end{array} & \text { Prob }> \\ & \text { F }\end{array}$ $\begin{array}{lllrrr}\text { HT } & 1 & 0.00098169 & 0.000982 & 0.1256 & 0.7409\end{array}$ Error $\quad 4 \quad 0.0312578$

$\begin{array}{lll}\text { C. } & 5 & 0.0322395\end{array}$ 0.00781

Means for Anova
Level Number Mean Std Lower Upper

$\begin{array}{lrrrrr} & & \text { Error } & \text { Lower } & \text { Upper } \\ \text { ccc } & 3 & 1.12696 & 0.05104 & 0.9853 & 1.2687 \\ & 3 & 1.525 & 0.5104 & 1.0108 & 1.294\end{array}$

$\begin{array}{lllllll} & & & & & & \\ \text { ccc } & & 1.12696 & 0.05104 & 0.9853 & 1.2687 \\ \text { quenched } & & 3 & 1.15254 & 0.05104 & 1.0108 & 1.2942\end{array}$

Std Error uses a pooled estimate of error variance

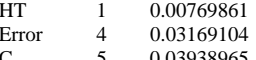

C.

Std Error uses a pooled estimate of error variance

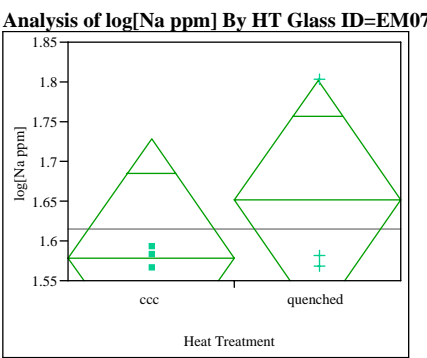

Anova

Summary of Fit

Rsquare
Adj Rsquare

$\begin{array}{ll}\text { Adj Rsquare } & -0.02374 \\ \text { Root Mean Square Error } \quad 0.093628\end{array}$ $\begin{array}{lr}\text { Mean of Response } & 1.614955 \\ \text { Observations (or Sum Wgts) } & 6\end{array}$

t Test

Assuming equal variances

$\begin{array}{lrr}\text { Difference } & 0.07188 \text { tRatio } & 0.940253 \\ \text { Std } & 0.07645 \text { D } & \end{array}$

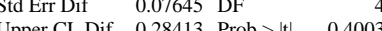
Lower CL Dif -0.14037 Prob $>t \quad 0.2002$ Confidence $\quad 0.95$ Prob $<\mathrm{t} 0.7998$

\begin{tabular}{lrrrr}
\multicolumn{2}{l}{ Analysis of Variance } & & & \\
Source DF & Sum of & Mean & F & Prob > \\
& Squares & Square & Ratio & F
\end{tabular} $\begin{array}{llrrrr}\text { HT } & 1 & 0.00775006 & 0.007750 & 0.8841 & 0.4003 \\ \text { Error } & 4 & 0.03506515 & 0.008766 & & \end{array}$

$\begin{array}{lll} & 1 & 0.077506 \\ \text { Enor } & 4 & 0.03506515\end{array}$

$\begin{array}{lllll}\text { Means for Anova } & & & \\ \text { Level Number Mean Std Lower Upper }\end{array}$

$\begin{array}{lrrrrr} & & \text { Error } & 95 \% & 95 \% \\ \text { ccc } & 3 & 0.864276 & 0.05139 & 0.72159 & 1.0070\end{array}$

$\begin{array}{lllllll}\text { quenched } & 3 & 0.935917 & 0.05139 & 0.79324 & 1.0786\end{array}$ $\begin{array}{lrrrrr} & & & \text { Error } & 95 \% & 95 \% \\ \text { ccc } & 3 & 1.57902 & 0.05406 & 1.4289 & 1.7291 \\ \text { quenched } & 3 & 1.65090 & 0.05406 & 1.5008 & 1.8010\end{array}$

Std Error uses a pooled estimate of error variance $\begin{array}{llll} & & & \\ \text { Level Number Mean Std Lower } & \text { Upper }\end{array}$

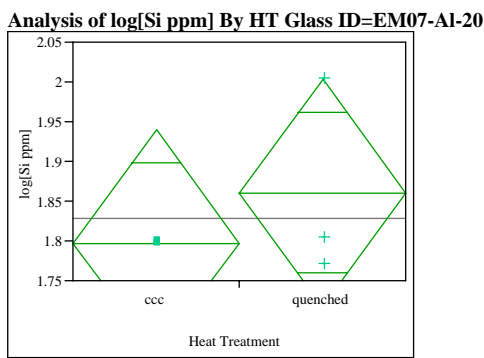

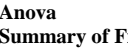

Rsquare
Adj Rsquare

\begin{tabular}{ll} 
Adj Rsquare & 0.158846 \\
Root Mean Square E & 0.05144 \\
\hline
\end{tabular} Mean of Response $\quad 0.08887$

Observations (or Sum Wgts)

t Test
quenched-ccc

Assuming equal variances

$\begin{array}{lll}\text { Difference } & 0.06307 \text { t Ratio } \quad 0.86912\end{array}$ Std Err Dif 0.07256 DF $>14 \quad 0.4338$ Uower CL Dif -0.13840 Prob $>t \quad 0.416$ Confidence $\quad 0.95$ Prob $<\mathrm{t} \quad 0.783$

\section{Analysis of Variance}

$\begin{array}{llll}\text { Sum of Mean } & F & \text { Prob }>\end{array}$ $\begin{array}{llrrrr}\text { HT } & 1 & 0.00596598 & 0.005966 & 0.7554 & 0.4338\end{array}$ \begin{tabular}{lllll} 
& Error & 4 & 0.03159233 & 0.007898 \\
\hline & 5 & 0.0375531 &
\end{tabular}

Total

Means for Anova

Level Number Mean Std Lower Upper

$\begin{array}{lrrrrr} & & & \text { Error } & 95 \% & 95 \% \\ \text { ccc } & 3 & 1.79742 & 0.05131 & 1.6550 & 1.9399 \\ \text { quenched } & 3 & 1.86049 & 0.05131 & 1.7180 & 2.0029\end{array}$

Std Error uses a pooled estimate of error variance 
Figure B6. Effects of Heat Treatment (HT) on PCT log(ppm)-Response of Study Glasses

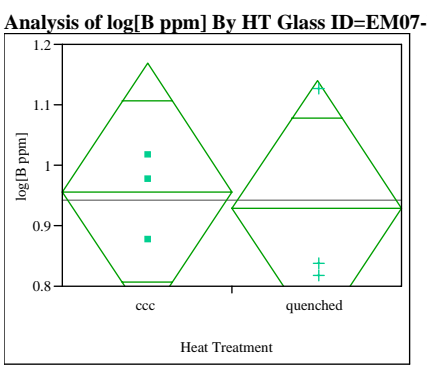

Anova
Summary of Fit

Rsquare

Adj Rsquare $\quad 0.016686$

Root Mean Square Error $\quad 0.132754$

Observations (or Sum Wgts)

Test

quenched-cce

Assuming equal variances

Difference $\quad-0.02824$ t Ratio $\quad-0.26053$ Std Err Dif 0.10839 DF

Upper CL Dif 0.27271 Prob $>|t| \quad 0.8073$

0.95 Prob $<1 \quad 0.4037$

Analysis of Variance

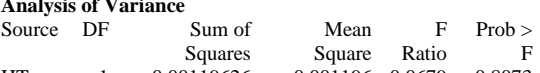
$\begin{array}{lrrrrr} & & \text { Squares } & \text { Square } & \text { Ratio } & \text { F } \\ \text { HT } & 1 & 0.00119626 & 0.001196 & 0.0679 & 0.8073\end{array}$

$\begin{array}{lll}\text { Error } & 4 & 0.07049469 \\ C & 5 & 0.07169095\end{array}$

Total

Means for Anova

Level Number Mean Std Lower Upper $\begin{array}{lrrrrr}\text { ccc } & 3 & 0.956195 & 0.07665 & 0.74339 & 1.1690 \\ \text { quenched } & 3 & 0.927955 & 0.07665 & 0.71515 & 1.1408\end{array}$

Std Error uses a pooled estimate of error variance

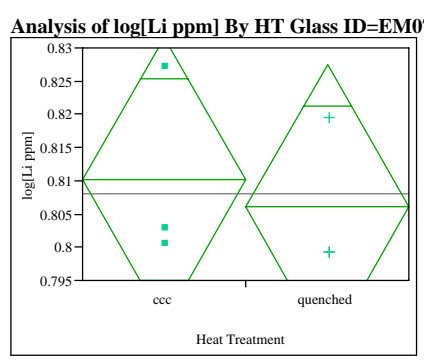

Anova

Summary of $F$

Rsquare

Root Mean Square Error $\quad 0.013314$

Mean of Response
Observations (or Sum Wgts)

t Test

t Test
quenched-ccc

Assuming equal variances

\begin{tabular}{lll} 
Difference & -0.00406 t Ratio & -0.37381 \\
\hline
\end{tabular}

$\begin{array}{llll}\text { Std Err Dif } & 0.01087 & \text { DF } & 4\end{array}$

$\begin{array}{lll}\text { Upper CL Dif } & 0.02612 \text { Prob }>|t| & 0.7275 \\ \text { Lower CL Dif }-0.03424 & \text { Pob }>t & 0.6362\end{array}$

$\begin{array}{lrl} & \\ \text { Confidence } & 0.95 \text { Prob }<t & 0.3638\end{array}$

$\begin{array}{rrrrr}\text { Source DF } & \begin{array}{r}\text { Sum of } \\ \text { Squares }\end{array} & \begin{array}{r}\text { Mean } \\ \text { Square }\end{array} & \begin{array}{r}\text { F } \\ \text { Ratio }\end{array} & \text { Prob > }\end{array}$

$\begin{array}{llrrrr}\text { HT } & 1 & 0.00002477 & 0.000025 & 0.1397 & 0.7275 \\ \text { Error } & 4 & 0.00070901 & 0.000177 & & \\ \text { C } & 5 & 0.007307 & & & \end{array}$

C.

Total

Means for Anova
Level Number Mean Std Lower Upper

$\begin{array}{lrrrrr} & & & \text { Error } & 95 \% & 95 \% \\ \text { ccc } & 3 & 0.810147 & 0.00769 & 0.78881 & 0.83149\end{array}$ $\begin{array}{llllll}\text { quenched } & 3 & 0.806084 & 0.00769 & 0.78474 & 0.82743\end{array}$

Std Error uses a pooled estimate of error variance

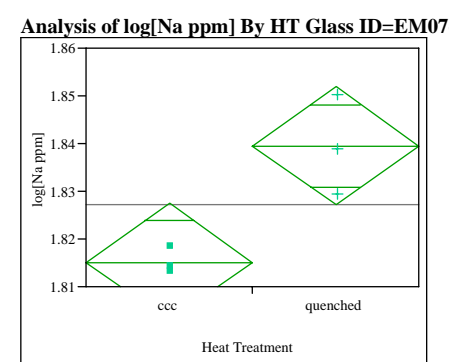

Anova

Anova
Summary of Fit

Rsquare
Adj Rsquare

\begin{tabular}{ll} 
Adj Rsquare & 0.789525 \\
Root Mean Squre Fror & 0.736907 \\
\hline & 0.007694
\end{tabular} Mean of Response $\quad 1.827305$ Observations (or Sum Wgts)

t Test
quenched-ccc

Assuming equal variances

$\begin{array}{llll}\text { Difference } & 0.024335 & \mathrm{t} \text { Ratio } & 3.873585 \\ \text { Std } & & \end{array}$ Std Err Dif 0.006282 DF $\quad 4$ Lower CL Dif 0.006893 Prob > t 0.0090 Confidence $\quad 0.95$ Prob $<\mathrm{t} \quad 0.9910$

Analysis of Variance

$\begin{array}{lllll} & & \\ \text { Source DF Sum of Mean } & \text { F Ratio Prob > }\end{array}$ $\begin{array}{lrrrrr} & & \text { Squares } & \text { Square } & \text { F } \\ \text { HT } & 1 & 0.00088831 & 0.000888 & 15.0047 & 0.0179 \\ \text { Error } & 4 & 0.00023681 & 0.000059 & & \end{array}$ C.

Means for Anova

$\begin{array}{llrr} & & & \\ \text { Level Number Mean Std } & \text { Lower } & \text { Upper } \\ & & & \end{array}$

$\begin{array}{lrrrrr} & & & \text { Error } & 95 \% & 95 \% \\ \text { ccc } & 3 & 1.81514 & 0.00444 & 1.8028 & 1.8275 \\ \text { quenched } & 3 & 1.83947 & 0.00444 & 1.8271 & 1.8518\end{array}$

Std Error uses a pooled estimate of error variance

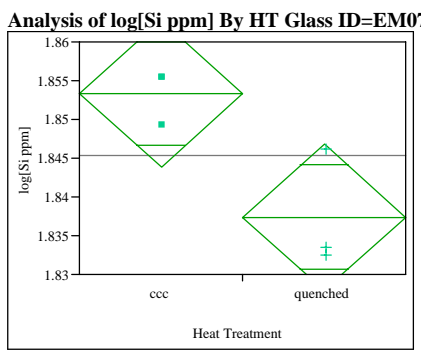

Anova

Summary of Fit

Rsquare

Root Mean Square Error $\quad 0.005908$ $\begin{array}{lr}\text { Mean of Response } & 1.845352 \\ \text { Observations (or Sum Wgts) } & 6\end{array}$

t Test
quenched-ccc

Assuming equal variances

Difference $\quad-0.01588$ t Ratio $\quad-3.2916$ Upper CL Dif -0.00249 Prob $>$ tt $\quad 0.0302$ Lower CL Dif -0.02927 Prob $>t \quad 0.9849$ Confidence $\quad 0.95$ Prob $<\mathrm{t} \quad 0.015$

Sum of Mean F Ratio Prob > $\begin{array}{llrrrr} & & \text { Squares } & \text { Square } & & \text { PT } \\ \text { Herror } & 1 & 0.00037822 & 0.000378 & 10.8347 & 0.0302 \\ \text { C } & 4 & 0.00013963 & 0.000035 & & \end{array}$ $\begin{array}{llll} & & & \\ \text { Error } & 4 & 0.00013963 & 0.000035\end{array}$

$\begin{array}{llrrr}\text { Means for Anova } & & & & \\ \text { Level Number } & \text { Mean Std } & \text { Lower } & \text { Upper }\end{array}$ $\begin{array}{lrrrrr} & & & \text { Error } & 95 \% & 95 \% \\ \text { ccc } & 3 & 1.85329 & 0.00341 & 1.8438 & 1.8628\end{array}$ $\begin{array}{lll}1.8279 & 1.8469\end{array}$

Std Error uses a pooled estimate of error variance 


\section{Figure B6. Effects of Heat Treatment (HT) on PCT log(ppm)-Response of Study Glasses}

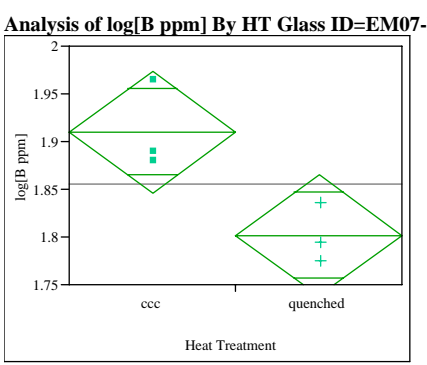

Anova

Summary of Fit

$\begin{array}{ll}\text { Rsquare } & 0.735208 \\ \text { Adj Rsquare } & 0.66902\end{array}$

0.66901
Root Mean Square Error $\quad 0.039822$ $\begin{array}{lr}\text { Mean of Response } & 1.856182 \\ \text { Observations (or Sum Wgts) } & 6\end{array}$

t Test
quenched-cc

Assuming equal variances

$\begin{array}{llr}\text { Difference } & -0.10836 \mathrm{t} \text { Ratio } & -3.33259 \\ \text { Std } & 0.03251 & -\end{array}$ $\begin{array}{llll}\text { Std Err Dif } & 0.03251 \text { DF } & 4 \\ \text { Upper CLDif } & -0.01808 \text { Prob }>|t| & 0.0290\end{array}$ $\begin{array}{llll}\text { Upper CL Dif } & -0.01808 & \text { Prob }>|t| & 0.0290 \\ \text { Lower CL Dif } & -0.19863 & \text { Prob }>t & 0.9855\end{array}$

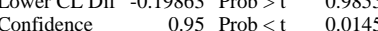

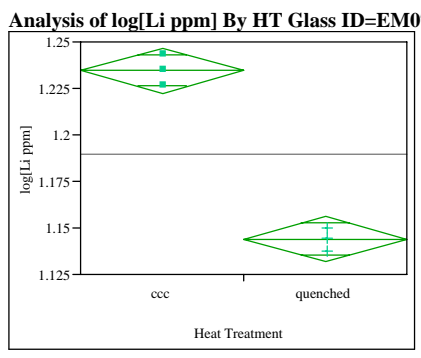

Anova

Summary of Fit

Rsquare

Root Mean Square Error $\quad 0.007536$

Mean of Response
Observations (or Sum Wgts)

t Test
quenched-cc

Assuming equal variances

$\begin{array}{llll}\text { Difference } & -0.09061 & t \text { Ratio } & -14.7254 \\ \text { Std Err Dif } & 0.00615 & \text { DF } & \end{array}$

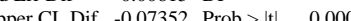

Lower CL Dif -0.10769 Prob $>t \quad 0.9999$

Confidence $\quad 0.95$ Prob $<\mathrm{t} \quad<.000$

$\begin{array}{lrrrr}\text { Source DF } & \begin{array}{r}\text { Sum of } \\ \text { Squares }\end{array} & \begin{array}{r}\text { Mean } \\ \text { Square }\end{array} & \text { F Ratio } & \text { Prob }> \\ & \text { F }\end{array}$

$\begin{array}{lrrrrr} & & \text { Squares } & \text { Square } & \text { F } \\ \text { HT } & 1 & 0.01231496 & 0.012315 & 216.8387 & 0.0001 \\ \text { Error } & 4 & 0.00022717 & 0.000057 & & \end{array}$

$\begin{array}{lrrrr}\begin{array}{l}\text { Means for Anova } \\ \text { Level }\end{array} \quad \text { Number } & \text { Mean } & \begin{array}{r}\text { Std } \\ \text { Error }\end{array} & \begin{array}{r}\text { Lower } \\ 95 \%\end{array} & \begin{array}{r}\text { Upper } \\ 95 \%\end{array}\end{array}$

$\begin{array}{lrrrrr} & & \text { Error } & 95 \% & 95 \% \\ \text { ccc } & 3 & 1.23464 & 0.00435 & 1.2226 & 1.2467\end{array}$

Std Error uses a pooled estimate of error variance

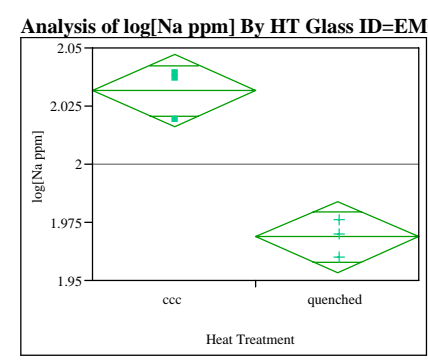

Anova

Aummary of Fit

$\begin{array}{ll}\text { Rsquare } & 0.941201 \\ \text { Adj Rsquare } & 0.926502\end{array}$

Root Mean Square Error $\quad 0.00962$

$\begin{array}{lr}\text { Mean of Response } & 2.000125 \\ \text { Observations (or Sum Wgts) } & 6\end{array}$

t Test

quenched-ccc

Assuming equal variances

$\begin{array}{lll}\text { Difference } & -0.06285 \text { t Ratio } & -8.00179\end{array}$

Upper CL Dif -0.04104 Prob $>$ tt 0.0013

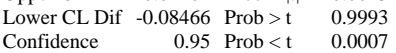

Analysis of Variance

$\begin{array}{rrrr}\text { Sum of } & \begin{array}{r}\text { Mean } \\ \text { Squares }\end{array} & \text { Patio } & \text { Prob > } \\ & \text { Square } & \text { F }\end{array}$

$\begin{array}{lrrrrr} & & \text { Squares } & \text { Square } & \text { F } \\ \text { HT } & 1 & 0.00592587 & 0.005926 & 64.0287 & 0.0013 \\ \text { Error } & 4 & 0.00037020 & 0.000093 & & \end{array}$

\begin{tabular}{lll} 
Error & 4 & 0.00037020 \\
\hline & 5 & 0.00620607
\end{tabular}

Means for Anova

Number

0.000093

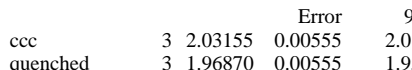

Std Error uses a pooled estimate of error variance

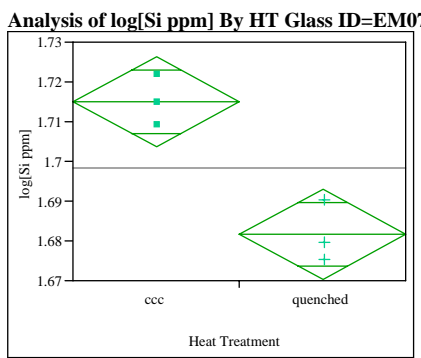

Anova

Summary of $F$

Rsquare
Adj Rsquare

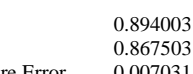

Root Mean Square Error $\quad 0.007031$ $\begin{array}{lr}\text { Mean of Response } & 1.69838 \\ \text { Observations (or Sum Wgts) } & 6\end{array}$

t Test
quenched-ccc

Assuming equal variances

$\begin{array}{lll}\text { Difference } & -0.03335 \mathrm{t} \text { Ratio } & -5.80834 \\ \text { Std } & & \end{array}$ Std Err Dif 0.00574 DF 10.40 $\begin{array}{lll}\text { Upper CL Dif } & -0.01741 & \text { Prob }>|t| \\ \text { Lower CL Dif }-0.04929 & \text { Prob }>t & 0.0094\end{array}$ Confidence $\quad 0.95$ Prob $<\mathrm{t} \quad 0.0022$

\section{Analysis of Variance}

$\begin{array}{llll} & \text { Sum of Mean F Ratio Prob > }\end{array}$ $\begin{array}{lrrrrr}\text { HT } & 1 & 0.00166794 & 0.001668 & 33.7368 & 0.0044 \\ \text { Error } & 4 & 0.00019776 & 0.000049 & \end{array}$ $\begin{array}{lll} & 1 & 0.0015694 \\ \text { C. } & 5 & 0.00019776 \\ & & 0.00186570\end{array}$ 0.000049

$\begin{array}{lllll}\text { Means for Anova } & & & \\ \text { Level Number } & \text { Mean Std } & \text { Lower } & \text { Upper }\end{array}$ \begin{tabular}{lrrrrr} 
& & Error & Lower & Upper \\
ccc & 3 & 1.71505 & 0.00406 & 1.7038 & 1.7263 \\
\hline
\end{tabular}

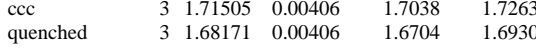
Std Error uses a pooled estimate of error variance 


\section{Figure B6. Effects of Heat Treatment (HT) on PCT $\log (\mathbf{p p m})$-Response of Study Glasses}

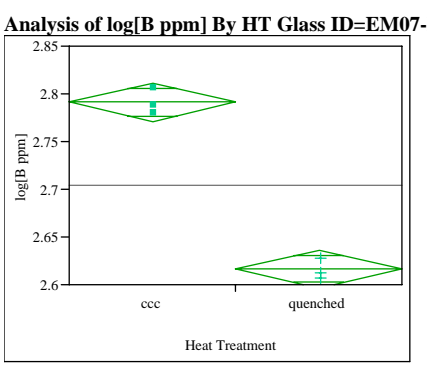

Anov

Summary of Fit

Rsquare

Error $\quad 0.012386$

$\begin{array}{lr}\text { Mean of Response } & 2.703652 \\ \text { Observations (or Sum Wgts) } & 6\end{array}$

t Test

Assuming equal variances

Difference $\quad-0.17487$ t Ratio $\quad-17.2916$

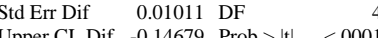
$\begin{array}{llll}\text { Upper CL Dif } & -0.14679 & \text { Prob }>|t| & <.0001 \\ \text { Lower CL Dif } & -0.20295 & \text { Prob }>t & 1.0000\end{array}$ $\begin{array}{lrl}\text { Confidence } & -0.95 \text { Prob }<\mathrm{t} & <.0001\end{array}$

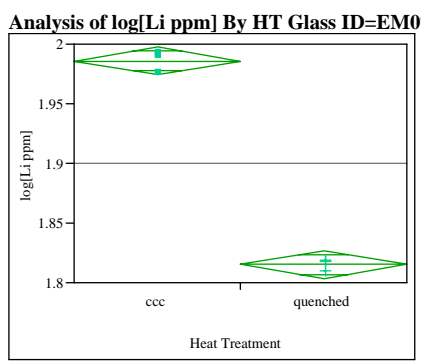

Anova

Summary of $\mathrm{Fi}$

Rsquare
Adj Rsquare

Square Error $\quad 0.00721$

Mean of Response
Observations (or Sum Wgts)

t Test
quenched-ccc

Assuming equal variances

Difference $\quad-0.17060$ t Ratio $\quad-28.9762$

Std Err Dif $\quad 0.00589$ DF $>14<0001$

Upper CL Dif -0.15425 Prob $>|t|<<0.000$

Confidence $\quad 0.95$ Prob $<t<0001$

Analysis of Variance

Analysis of Variance

Source DF Sum of Mean F Ratio Prob > $\begin{array}{llrrrrr}\text { HT } & & 1 & 0.04586835 & 0.045868 & 298.9994 & <.0001\end{array}$ $\begin{array}{lll}\text { Error } & 4 & 0.00061362\end{array}$

$\begin{array}{lll}\text { C. } & 5 & 0.04648197\end{array}$

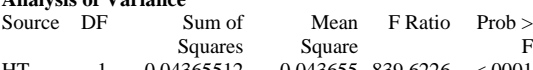
$\begin{array}{llllll}\text { HT } & 1 & 0.04365512 & 0.043655 & 839.6226 & <.0001 \\ \text { Error } & 4 & 0.00620797 & 0.000052 & & \end{array}$

C.

C.

Means for Anova

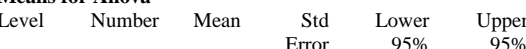

$\begin{array}{llrrrr} & 3 & 2.79109 & 0.00715 & 2.7712 & 2.85 \% \\ \text { ccc } & 3 & 2.61622 & 0.00715 & 2596 & 2.651\end{array}$

$\begin{array}{llllll}\text { quenched } & 3 & 2.61622 & 0.00715 & 2.5964 & 2.6361\end{array}$

Std Error uses a pooled estimate of error variance

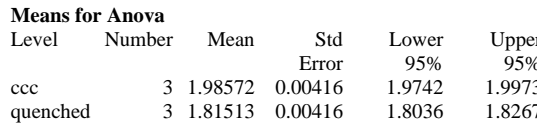

Std Error uses a pooled estimate of error variance

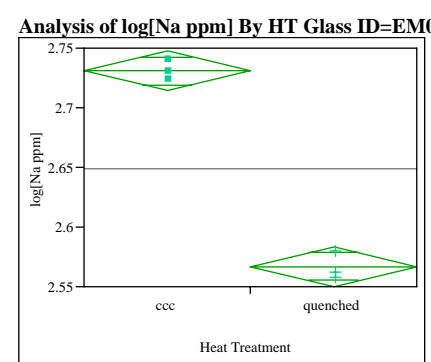

Anova

Summary of Fit

Rsquare

Root Mean Square Error $\quad 0.987278$

$\begin{array}{lr}\text { Mean of Response } & 2.648902 \\ \text { Observations (or Sum Wgts) } & 6\end{array}$

t Test

Assuming equal variances

$\begin{array}{llll}\text { Difference } & -0.16420 \text { t Ratio } & -19.7239\end{array}$

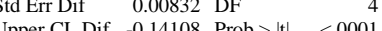
Lower CL Dif -0.18731 Prob $>t \quad 1.0000$

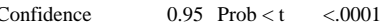

\begin{tabular}{lllll} 
Source DF Sum of Mean F Ratio Prob $>$ \\
\hline
\end{tabular} $\begin{array}{llrrrr} & & \text { Squares } & \text { Square } & \text { F } \\ \text { HT } & 1 & 0.04044088 & 0.040441 & 389.0306 & <.0001 \\ \text { Error } & 4 & 0.00041581 & 0.000104 & & \end{array}$

$\begin{array}{llll}\text { Means for Anova } & & & \\ \text { Level Number } & \text { Mean Std Lower Upper }\end{array}$

$\begin{array}{lrrrrr} & & \text { Error } & 95 \% & 95 \% \\ \text { ccc } & 3 & 2.73100 & 0.00589 & 2.7147 & 2.7473\end{array}$

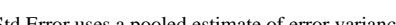

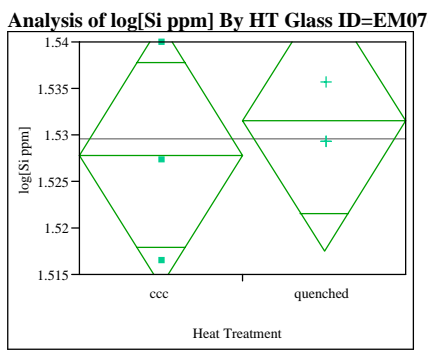

Anova

Summary of Fit

$\begin{array}{ll}\text { Rsquare } & 0.061596 \\ \text { Adj Rsquare } & -0.17301\end{array}$

Root Mean Square Error $\quad 0.008747$

Mean of Response 1.52964

Test

Assuming equal variances

$\begin{array}{lll}\text { Difference } & 0.00366 \text { t Ratio } \quad 0.512402\end{array}$ Upper CL Dif 0.02349 Prob > lt 0.6354 Lower CL Dif -0.01617 Prob $>t \quad 0.3177$ Confidence $\quad 0.95$ Prob $<\mathrm{t} \quad 0.6823$

Analysis of Variance

$\begin{array}{lllll}\text { Source DF Sum of Mean F } & \text { Prob > }\end{array}$ $\begin{array}{lrrrrr} & & \text { Squares } & \text { Square } & \text { Ratio } & \text { F } \\ \text { HT } & 1 & 0.00002009 & 0.000020 & 0.2626 & 0.6354 \\ \text { Error } & 4 & 0.00030603 & 0.000077 & & \end{array}$ \begin{tabular}{lll} 
Error & 4 & 0.00030603 \\
\hline & 5 & 0.00032612
\end{tabular}

$\begin{array}{lrrrr}\begin{array}{l}\text { Means for Anova } \\ \text { Level }\end{array} \text { Number } & \text { Mean } & \begin{array}{r}\text { Std } \\ \text { EIror }\end{array} & \begin{array}{r}\text { Lower } \\ 95 \%\end{array} & \begin{array}{r}\text { Upper } \\ 95 \%\end{array}\end{array}$ $\begin{array}{lrrrrr}\text { ccc } & 3 & 1.52782 & 0.00505 & 1.5138 & 1.5418 \\ \text { quenched } & 3 & 1.53148 & 0.00505 & 1.5175 & 1.5455\end{array}$

Std Error uses a pooled estimate of error variance 
Figure B6. Effects of Heat Treatment (HT) on PCT log(ppm)-Response of Study Glasses

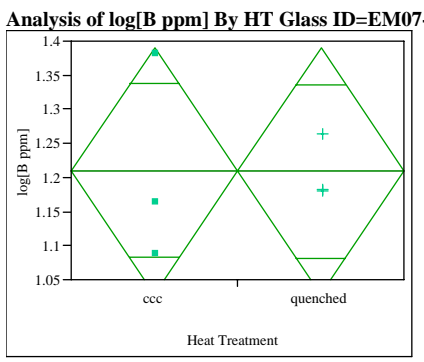

Anov

Anova

Rsquare

Adj Rsquare $5.124 \mathrm{e}-5$

$\quad 0.112809$

Mean of Response
Observations (or Sum Wgts)
1.209326

t Test

quenched-ccc

Assuming equal variances

Difference $\quad-0.00132$ t Ratio $\quad-0.01432$ $\begin{array}{llll}\text { Std Err Dif } & 0.09211 & \text { DF } & 4 \\ \text { Upper CL Dif } & 0.25441 & -0.04\end{array}$ $\begin{array}{llll}\text { Upper CL Dif } & 0.25441 & \text { Prob }>|t| & 0.9893 \\ \text { Lower CL Dif } & -0.25705 & \text { Prob }>t & 0.5054\end{array}$ $\begin{array}{lrlll} & \text { Confidence } & -0.25705 & \text { Prob }>t & 0.5054 \\ & 0.95 & \text { Prob }<t & 0.4946\end{array}$

Analysis of Variance

\begin{tabular}{lrrrrr}
\multicolumn{2}{l}{ Analysis of Variance } \\
Source & DF & Sum of & Mean & F & Prob > \\
HT & 1 & 0.00000261 & Square & Ratio & F \\
HTror & 4 & 0.05090345 & $0.0127-6$ & 0.0002 & 0.9893 \\
C. & 5 & 0.05090606 & & & \\
C. & & & & &
\end{tabular}

$\begin{array}{lrrrr}\text { Means for Anova } & & & & \\ \text { Level Number } & \text { Mean } \quad \begin{aligned} \text { Ltd } \\ \text { Lower }\end{aligned} & \text { Upper } \\ & & \text { Error } & 95 \% & 95 \%\end{array}$

$\begin{array}{lrrrrr} & & & \text { Error } & 95 \% & 95 \% \\ \text { ccc } & 3 & 1.20999 & 0.06513 & 1.0292 & 1.3908\end{array}$

$\begin{array}{llllll}\text { quenched } & 3 & 1.20867 & 0.06513 & 1.0278 & 1.3895\end{array}$

Std Error uses a pooled estimate of error variance

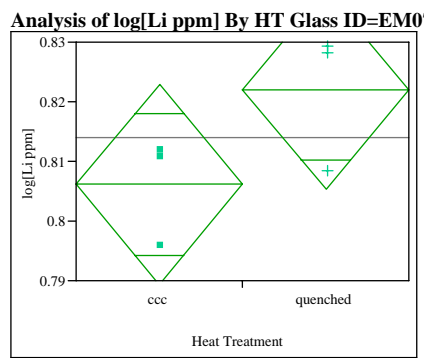

Anova

Summary of Fit

Rsquare
Adj Rsquare

Root Mean Square Error $\quad 0.010417$

Mean of Response ${ }_{\text {Observations (or Sum Wgts) }}^{0.814063}$

t Test
quenched-ccc

Assuming equal variances

Difference $\quad 0.01587$ t Ratio $\quad 1.865943$

Std Err Dif $\quad 0.00851$ DF

$\begin{array}{llll}\text { Upper CL Dif } & 0.03948 & \text { Prob }>|t| & 0.1355 \\ \text { Lower CL Dif }-0.00774 & \text { Prob }>t & 0.0677\end{array}$

$\begin{array}{lll}\text { Confidence } & 0.95 \text { Prob }<\mathrm{t} \quad 0.9323\end{array}$

Analysis of Varianc

$\begin{array}{lrrrr}\text { Source DF } & \text { Sum of } & \text { Mean } & \text { F } & \text { Prob > }\end{array}$ $\begin{array}{llllll}\text { HT } & 1 & 0.00037780 & 0.0000378 & 3.4817 & 0.1355 \\ \text { Error } & 4 & 0.00043404 & 0.000109 & & \end{array}$

Means for Anova

$\begin{array}{rrrrr} & \text { Error } & \text { Lower } & \text { Upp } \\ 95 \% & 95 \% \\ 3 & 0.806128 & 0.00601 & 0.78943 & 0.8228\end{array}$ $\begin{array}{lllllll} & & & & & & \\ \text { ccc } & 3 & 0.806128 & 0.00601 & 0.78943 & 0.82283 \\ \text { quenched } & & 3 & 0.821999 & 0.00601 & 0.80530 & 0.83870\end{array}$

Std Error uses a pooled estimate of error variance

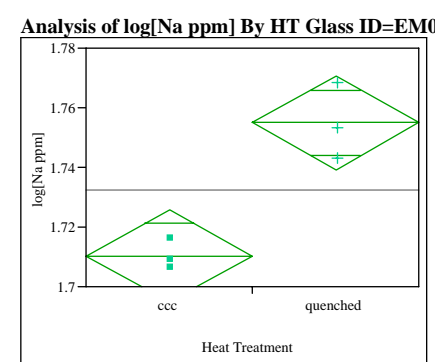

Anova

Summary of Fit

Rsquare

$\begin{array}{lr}\text { Adj Rsquare } & 0.887241 \\ \text { Root Mean Square Error } & 0.859051 \\ \text { Megn } & 0.00972\end{array}$ Mean of Response 1.73265

Observations (or Sum Wgts) $\quad 6$

t Test
quenched-ccc

Assuming equal variances

Difference $\quad 0.044522$ t Ratio 5.610164 Std Err Dif 0.007936 DF 15 Lower CL Dif 0.022488 Prob $>t \quad 0.0025$ Confidence $\quad 0.95$ Prob $<$ t $\quad 0.9975$

\section{Analysis of Variance}

$\begin{array}{rrrrr}\text { Squm of } & \text { Mean } & \text { F Ratio } & \text { Prob > } \\ \text { Square } & & \text { F }\end{array}$

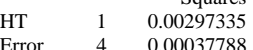

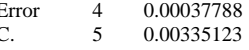

0.002973

Means for Anova

Level Number Mean Std Lower

\begin{tabular}{lrrrrr} 
& & Error & Lower & $95 \%$ & $95 \%$ \\
ccc & 3 & 1.71039 & 0.00561 & 1.6948 & 1.7260 \\
\hline
\end{tabular}

$\begin{array}{llllll}\text { quenched } & 3 & 1.75491 & 0.00561 & 1.7393 & 1.7705\end{array}$

Std Error uses a pooled estimate of error variance

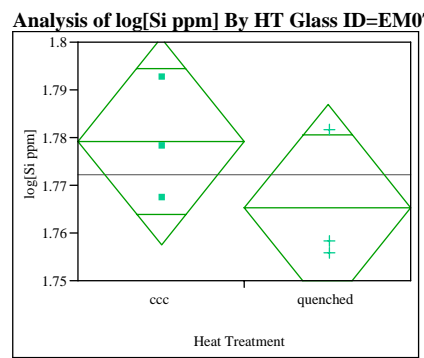

Anova

Summary of $F$

Rsquare
Adj Rsquare

$\begin{array}{ll} & 0.284562 \\ \text { Adj Rsquare } & 0.105703\end{array}$ Mean of Response $\quad 0.01348$

Observations (or Sum Wgts)

t Test
quenched-ccc

Assuming equal variances

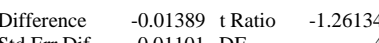

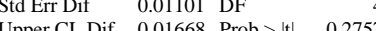
Lower CL Dif -0.04446 Prob $>t \quad 0.8621$ Confidence $\quad 0.95$ Prob $<\mathrm{t} \quad 0.1379$

\section{Analysis of Variance}

$\begin{array}{rlrr} & & & \\ & & \end{array}$ $\begin{array}{lrrrrr}\text { HT } & 1 & 0.00028928 & 0.000289 & 1.5910 & 0.2757\end{array}$ \begin{tabular}{lllll} 
Error & 4 & 0.00072730 & 0.000182 \\
\hline & 5 & 0.00101657 &
\end{tabular}

C.

Means for Anova

$\begin{array}{lllll} & & & \\ \text { Level Number Mean Std Lower Upper }\end{array}$

$\begin{array}{lrrrrr} & & & \text { Error } & 95 \% & 95 \% \\ \text { ccc } & 3 & 1.77924 & 0.00779 & 1.7576 & 1.8009 \\ \text { quenched } & 3 & 1.76535 & 0.00779 & 1.7437 & 1.7870\end{array}$

Std Error uses a pooled estimate of error variance 
Figure B6. Effects of Heat Treatment (HT) on PCT $\log (\mathrm{ppm})$-Response of Study Glasses

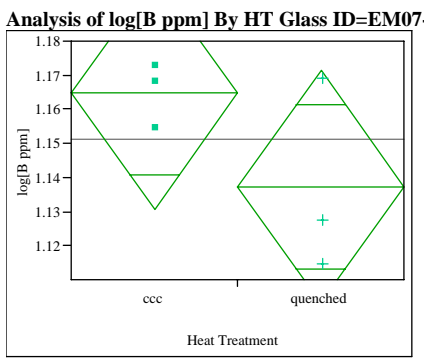

Anov

Summary of Fit

Rsquare
Adj Rsquare

Error $\quad 0.021314$

$\begin{array}{lr}\text { Mean of Response } & 1.151052 \\ \text { Observations (or Sum Wgts) } & 6\end{array}$

t Test

quenched-cce

Assuming equal variances

Difference $\quad-0.02780$ t Ratio $\quad-1.59758$ $\begin{array}{llll}\text { Std Err Dif } & 0.01740 \text { DF } & 4\end{array}$ $\begin{array}{llll}\text { Upper CL Dif } & 0.02052 & \text { Prob }>|t| & 0.1854 \\ \text { Lower CL Dif }-0.07612 & \text { Prob }>t & 0.9073\end{array}$ $\begin{array}{llll} & \text { Confidence } & -0.05 \text { Prob }<\mathrm{t} & 0.0927\end{array}$

Assuming equal variances

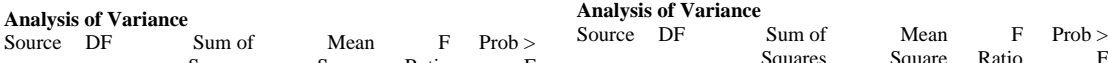

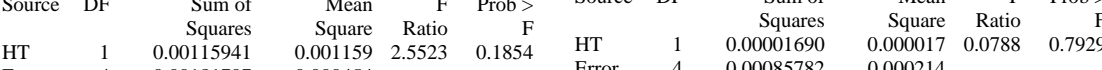
$\begin{array}{lll}\text { HT } & 1 & 0.0011594 \\ \text { Error } & 4 & 0.0018170 \\ \text { C. } & 5 & 0.009767\end{array}$

C. $\quad 5 \quad 0.0029764$ 0.00045

Means for Anova

Level Number Mean Std Lower $\begin{array}{rrr}\text { Upper } \\ 95 \%\end{array}$

$\begin{array}{lrrrrr} & & \text { Error } & 95 \% & 95 \% \\ \text { ccc } & 3 & 1.16495 & 0.01231 & 1.1308 & 1.1991\end{array}$

quenched $\begin{array}{lll}3 & 1.13715 & 0.01231\end{array}$

Std Error uses a pooled estimate of error variance
Analysis of $\log [\mathrm{Li}$ ppm] By HT Glass ID=EM07-Bi-05

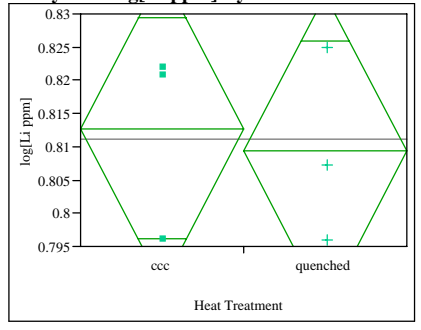

Anova

Rsquare

Root Mean Square Error $\quad 0.01464$

Mean of Response
Observations (or Sum Wgts)

t Test

quenched-ccc

Difference $\quad-0.00336$ t Ratio $\quad-0.28069$

Std Err Dif 0.01196 DF $\quad 4$

Upper CL Dif 0.02984 Prob $>|t| \quad 0.7929$

$\begin{array}{lrll}\text { Confidence } & -0.03655 & \text { Prob }>t & 0.6036 \\ & 0.95 & \text { Prob }<\mathrm{t} & 0.396\end{array}$ 0.00085782 0.000017

Total

$\begin{array}{lllll}\text { Means for Anova } & & & \\ \text { Number } & \text { Mean Std } & \text { Lower } & \text { Upper }\end{array}$ $\begin{array}{lrrrrr} & & \text { Error } & 95 \% & 95 \% \\ \text { ccc } & 3 & 0.812759 & 0.00845 & 0.78928 & 0.83623\end{array}$ \begin{tabular}{lllll}
3 & 0.812759 & 0.00845 & 0.7928 & 0.8363 \\
\hline & 0.78593 & 0.83288
\end{tabular} Std Error uses a pooled estimate of error variance

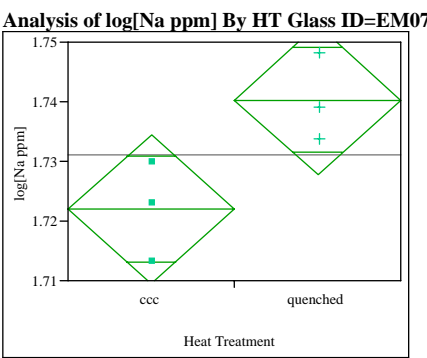

Anova
Summary of Fit

Rsquare

\begin{tabular}{lr} 
Adj Rsquare & 0.674672 \\
Root Mean Square Error & 0.59334 \\
\hline & 0.00786
\end{tabular} Mean of Response $\quad 1.73114$

Observations (or Sum Wgts)

t Test
quenched-ccc

Assuming equal variances

$\begin{array}{lll}\text { Difference } & 0.018381 \mathrm{t} \text { Ratio } \quad 2.880155 \\ & & \end{array}$ $\begin{array}{lll}\text { Std Err Dif } 0.006382 & \mathrm{DF} & 4 \\ \text { Unper } & & \end{array}$ Lower CL Dif 0.000662 Prob $>t \quad 0.0225$ Confidence $\quad 0.95$ Prob $<\mathrm{t} \quad 0.9775$

\section{Analysis of Variance}

\begin{tabular}{llrrrr} 
Source & DF & $\begin{array}{r}\text { Sum of } \\
\text { Squares }\end{array}$ & $\begin{array}{r}\text { Mean } \\
\text { Square }\end{array}$ & $\begin{array}{r}\text { F } \\
\text { Ratio }\end{array}$ & Prob $>$ \\
\hline & & F
\end{tabular} \begin{tabular}{llr} 
HT & 1 & 0.00050677 \\
\hline
\end{tabular} $\begin{array}{lll}\text { Error } & 4 & 0.00024436 \\ \text { C. } & 5 & 0.00075113\end{array}$

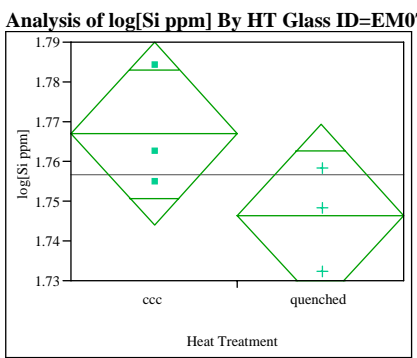

Anov

Summary of $F$

Rsquare
Adj Rsquare Mean of Response $\quad 0.014268$

Observations (or Sum Wgts)

t Test
quenched-ccc

Assuming equal variances

$\begin{array}{lll}\text { Difference } & -0.02064 \mathrm{t} \text { Ratio } & -1.77212\end{array}$ Std Err Dif 0.01165 DF $\quad-1.77212$ Lower CL Dif -0.05299 Prob $>t \quad 0.9245$ Confidence $\quad 0.95$ Prob $<\mathrm{t} \quad 0.0755$

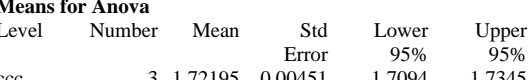

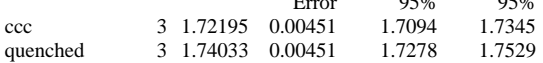

Std Error uses a pooled estimate of error variance
Analysis of Variance

$\begin{array}{rrrrr}\text { Source DF Sum of Mean } & F & \text { Prob > }\end{array}$ $\begin{array}{llllll}\text { HT } & 1 & 0.00063930 & 0.000639 & 3.1404 & 0.1511\end{array}$ $\begin{array}{llll}\text { Error } & 4 & 0.00081429 & 0.000204\end{array}$ C.

Means for Anova

$\begin{array}{lllll} & & & \\ \text { Level Number Mean Std Lower Upper }\end{array}$

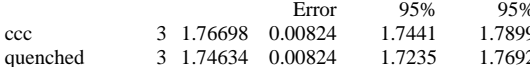

Std Error uses a pooled estimate of error variance 
Figure B6. Effects of Heat Treatment (HT) on PCT log(ppm)-Response of Study Glasses

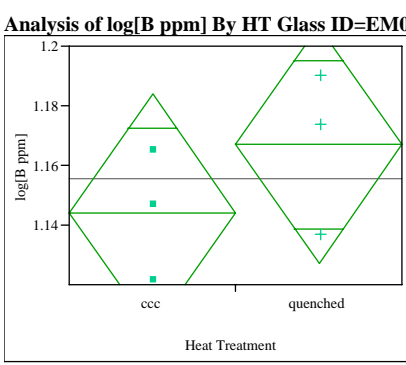

Anov

Summary of Fit

Rsquare
Adj Rsquare

$\begin{array}{lr}\text { Mean of Response } & 1.155565 \\ \text { Observations (or Sum Wgts) } & 6\end{array}$

t Test

Assuming equal variances

Difference $\quad 0.02270$ t Ratio $\quad 1.118808$ $\begin{array}{llll}\text { Std Err Dif } & 0.02029 & \text { DF } & 1.18 \\ \text { Upper CL Dif } & 0.07904 & \text { Prob }>\text { tt } & 0.3259\end{array}$ Upper CL Dif 0.07904 Prob $>|t| \quad 0.3259$ Confidence $\quad 0.95$ Prob $<\mathrm{t} \quad 0.8371$

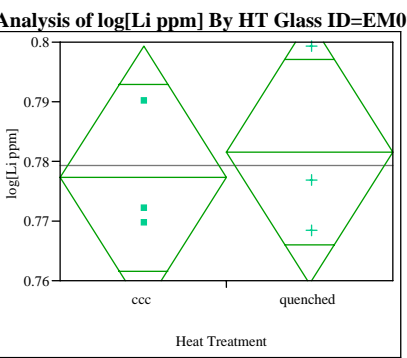

Anova

Summary of Fit

Rsquare

Root Mean Square Error $\quad 0.01378$

$\begin{array}{ll}\text { Mean of Response } & 0.779413 \\ \text { Observations (or Sum Wgts) }\end{array}$

t Test
quenched-ccc

Assuming equal variances

Difference $\quad 0.00431$ t Ratio 0.382868 $\begin{array}{lrrr}\text { Upper CL Dif } & 0.03555 & \text { Prob }>|t| & 0.7213 \\ \text { Lower CL Dif } & -0.02693 & \text { Prob }>t & 0.3606\end{array}$ Confidence $\quad 0.95$ Prob $<\mathrm{t} \quad 0.6394$ $\begin{array}{llll}\text { Std Err Dif } & 0.01125 & \mathrm{DF} & 4\end{array}$

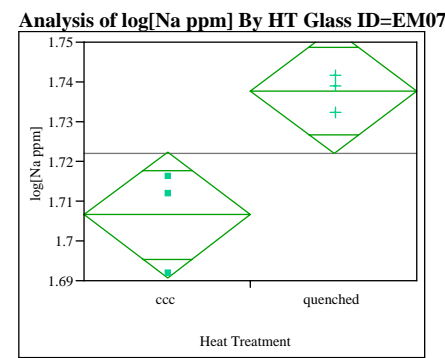

Anova

Anova
Summary of Fit

Rsquare

Adj Rsquare Mean of Response

t Test

Assuming equal variances $\begin{array}{lllr}\text { Std Err Dif } & 0.008002 & \mathrm{DF} & 4 \\ \text { Upper CL Dif } & 0.053428 & \text { Prob }>|t| & 0.0175\end{array}$ Lower CL Dif 0.008995 Prob $>t \quad 0.0088$ Confidence $\quad 0.95$ Prob $<\mathrm{t} \quad 0.9912$

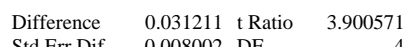

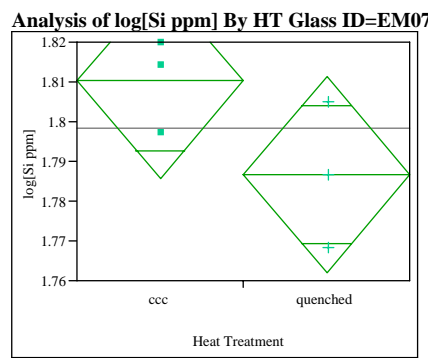

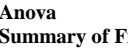

Rsquare
Adj Rsquare

$\begin{array}{ll}\text { Adj Rsquare } & 0.467644 \\ \text { Root Mean Square En } & 0.334555\end{array}$ Error $\quad 0.01538$ Observations (or Sum Wgts) $\quad 6$

t Test

Assuming equal variances

Difference $\quad-0.02355$ t Ratio $\quad-1.87451$ Std Err Dif $0.01256 \mathrm{DF}$

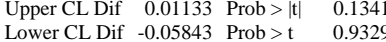
Confidence $\quad 0.95$ Prob $<\mathrm{t} \quad 0.067$

\begin{tabular}{|c|c|c|c|c|c|c|c|c|c|c|c|c|}
\hline \multicolumn{6}{|c|}{ Analysis of Variance } & \multicolumn{7}{|c|}{ Analysis of Variance } \\
\hline Source D & & $\begin{array}{l}\text { Sum of } \\
\text { Squares }\end{array}$ & $\begin{array}{l}\text { Mean } \\
\text { Square }\end{array}$ & $\begin{array}{r}\mathrm{F} \\
\text { Ratio }\end{array}$ & $\begin{array}{r}\text { Prob > } \\
F\end{array}$ & Source & DF & & $\begin{array}{l}\text { Sum of } \\
\text { Squares }\end{array}$ & $\begin{array}{l}\text { Mean } \\
\text { Square }\end{array}$ & $\begin{array}{r}\mathrm{F} \\
\mathrm{R}\end{array}$ & $\begin{array}{r}\text { Prob > } \\
F\end{array}$ \\
\hline HT & 1 & .00077314 & 0.000773 & 1.2517 & 0.3259 & HT & 1 & & 002784 & 0.000028 & 0.1466 & 0.7213 \\
\hline Error & 4 & .00247063 & 0.000618 & & & Error & 4 & & 0075965 & 0.000190 & & \\
\hline $\begin{array}{l}\text { C. } \\
\text { Total }\end{array}$ & & .00324377 & & & & $\begin{array}{l}\text { C. } \\
\text { Total }\end{array}$ & 5 & & 0078749 & & & \\
\hline Means & Anova & & & & & Means fo & or Ano & & & & & \\
\hline Level & Number & Mean & $\begin{array}{r}\text { Std } \\
\text { Frror }\end{array}$ & $\begin{array}{l}\text { Lower } \\
\text { Lo5\% }\end{array}$ & $\begin{array}{l}\text { Upper } \\
95 \%\end{array}$ & Level & Num & & Mean & $\begin{array}{l}\text { Std } \\
\text { Error }\end{array}$ & $\begin{array}{l}\text { Lower } \\
050 \%\end{array}$ & $\begin{array}{l}\text { Upper } \\
95 \%\end{array}$ \\
\hline $\mathrm{cc}$ & 3 & 1.14421 & 0.01435 & 1.1044 & 1.1841 & $\operatorname{ccc}$ & & 30 & .777259 & 0.00796 & 0.75517 & 0.79935 \\
\hline juenched & 3 & 1.16692 & 0.01435 & 1.1271 & 1.2068 & quenched & & 30 & . 781567 & 0.00796 & 0.75948 & 0.80366 \\
\hline
\end{tabular}

Std Error uses a pooled estimate of error variance

\section{Analysis of Variance \\ Analysis of Variance}

\begin{tabular}{llr} 
HT & 1 & 0.00146122 \\
\hline
\end{tabular}

Error $\quad 4 \quad 0.00038416$

$\begin{array}{lll}\text { C. } \quad 5 & 0.00184538\end{array}$

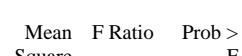
$\begin{array}{rrr}\text { Square } & \text { F } \\ 0.001461 & 15.2145 & 0.0175\end{array}$ 0.000096

Means for Anova

Level Number Mean Std Lower Upper

$\begin{array}{lrrrrr}\text { ccc } & 3 & 1.70650 & \text { Error } & 95 \% & 950566 \\ \text { quenched } & 3 & 1.75908 & 1.7222 \\ & & & & & \end{array}$

Std Error uses a pooled estimate of error variance
Analysis of Variance

$\begin{array}{llll}\text { DF Sum of Mean } F & \text { Prob > }\end{array}$ $\begin{array}{llrrrr}\text { HT } & 1 & 0.00083196 & 0.000832 & 3.5138 & 0.1341\end{array}$

$\begin{array}{llll}\text { Error } & 4 & 0.00094708 & 0.000237 \\ \text { C. } & 5 & 0.00177904 & \end{array}$

Means for Anova

Level Number Mean Std Lower Upper $\begin{array}{lrrrrr} & & & \text { Error } & 95 \% & 95 \% \\ \text { ccc } & 3 & 1.81021 & 0.00888 & 1.7855 & 1.8349 \\ \text { quenched } & 3 & 1.78666 & 0.00888 & 1.7620 & 1.8113\end{array}$

Std Error uses a pooled estimate of error variance 
Figure B6. Effects of Heat Treatment (HT) on PCT $\log (\mathrm{ppm})$-Response of Study Glasses

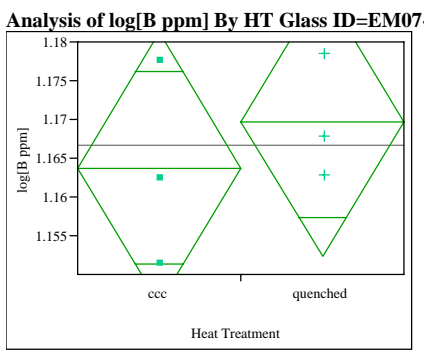

Anova

Summary of Fit

Rsquare
Adj Rsquare

0.101986
-0.12252

Root Mean Square Error $\quad 0.010903$

Mean of Response $\quad 1.166732$

Test

quenched-cc

Assuming equal variances

Difference $\quad 0.00600$ t Ratio $\quad 0.674$

Lower CL Dif -0.01872 Prob $>t \quad 0.268$

$\begin{array}{llll}\text { Confidence } & 0.95 \text { Prob }<\mathrm{t} \quad 0.7314\end{array}$

Analysis of Variance

$\begin{array}{lrrrrr}\text { Source } & \text { DF } & \begin{array}{r}\text { Sum of } \\ \text { Squares }\end{array} & \begin{array}{r}\text { Mean } \\ \text { Square }\end{array} & \begin{array}{r}\text { Ratio } \\ \text { Prob > }\end{array} \\ \text { HT } & 1 & 0.00005400 & 0.000054 & 0.4543 & 0.5373 \\ \text { Error } & 4 & 0.00047550 & 0.000119 & & \\ \text { C } & 5 & 0.00052950 & & & \end{array}$

C.

$\begin{array}{llrrr}\begin{array}{l}\text { Means for Anova } \\ \text { Level }\end{array} \text { Number } & \text { Mean } & \begin{array}{r}\text { Std } \\ \text { Error }\end{array} & \begin{array}{r}\text { Lower } \\ 95 \%\end{array} & \begin{array}{r}\text { Upper } \\ 95 \%\end{array}\end{array}$

$\begin{array}{lrrrrr} & & & \text { Error } & 95 \% & 95 \% \\ \text { ccc } & 3 & 1.16373 & 0.00629 & 1.1463 & 1.1812\end{array}$

$\begin{array}{lllll}\text { quenched } & 3 & 1.16973 & 0.00629 & 1.1523\end{array}$

Std Error uses a pooled estimate of error variance
Analysis of $\log [\mathrm{Li}$ ppm] By HT Glass ID=EM07-BL-2

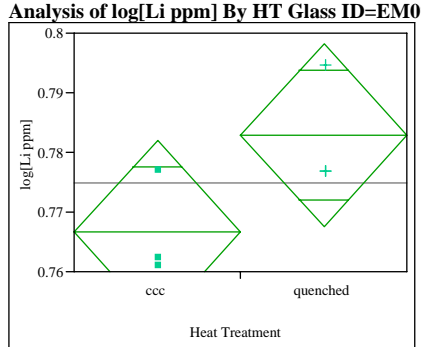

Anova

Summary of Fi

Rsquare
Adj Rsquare

Root Mean Square E

$\begin{array}{lr}\text { Mean of Response } & 0.774784 \\ \text { Observations (or Sum Wgts) } & 6\end{array}$

0.394358

t Test
quenched-ccc

Assuming equal variances

Difference $\quad 0.01619$ t Ratio $\quad 2.062935$

Std Err Dif 0.00785 DF

Upper CL Dif 0.03797 Prob $>|t| c \mid 0.108$

Confidence $\quad 0.95$ Prob $<\mathrm{t} \quad 0.9460$

$\begin{array}{llrrr}\text { Source DF } & \text { Sum of } & \text { Mean } & F & \text { Prob }>\end{array}$ $\begin{array}{llllll}\text { HT } & 1 & 0.00039302 & 0.000393 & 4.2557 & 0.1081 \\ \text { Error } & 4 & 0.00036941 & 0.000092 & & \end{array}$ C.

$\begin{array}{lllll}\text { Means for Anova } & & & \\ \text { Level Number Mean Std Lower Upper }\end{array}$

$\begin{array}{lrrrrr} & & \text { Error } & 95 \% & 95 \% \\ \text { ccc } & 3 & 0.766691 & 0.00555 & 0.75129 & 0.78210\end{array}$ $\begin{array}{lllllll}\text { quenched } & 3 & 0.782878 & 0.00555 & 0.76747 & 0.79828\end{array}$ Std Error uses a pooled estimate of error variance

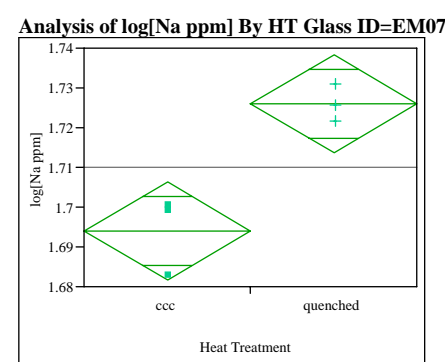

Anova

Anova
Summary of Fit

Rsquare
Adj Rsquare

$\begin{array}{ll}\text { Adj Rsquare } & 0.866046 \\ \text { Root Mean Square Error } & 0.832558 \\ & 0.007714\end{array}$ $\begin{array}{lr}\text { Mean of Response } & 1.710068 \\ \text { Observations (or Sum Wgts) } & 6\end{array}$

t Test

nched-ccc

Assuming equal variances

$\begin{array}{lll}\text { Difference } & 0.032032 & \mathrm{t} \text { Ratio } \\ 5.085371\end{array}$ Std Err Dif 0.006299 DF $>4$ Lower CL Dif 0.014543 Prob $>t \quad 0.0035$ Confidence $\quad 0.95$ Prob $<\mathrm{t} \quad 0.9965$

\section{Analysis of Variance}

$\begin{array}{lllll}\text { Source DF Sum of Mean F Ratio Prob > } & \end{array}$ $\begin{array}{llrrrr}\text { HT } & 1 & 0.00153903 & 0.001539 & 25.8610 & 0.0071\end{array}$ $\begin{array}{lll}\text { Error } & 4 & 0.00023805\end{array}$

$\begin{array}{lll}5 & 0.00177708\end{array}$ .000060

Level Number Mean Std Lower Upper

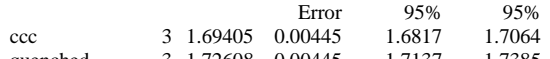

Std Error uses a pooled estimate of enor vange

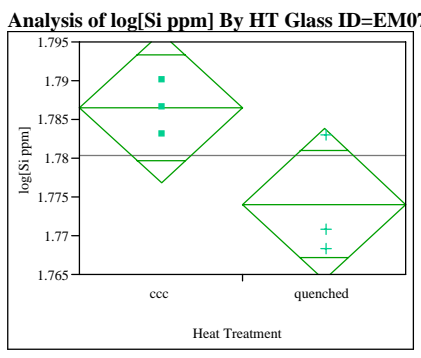

Anov

Summary of Fit

Rsquare

Root Mean Square Error $\quad 0.006055$ Mean of Response 1.78029

t Test
quenched-ccc

Assuming equal variances

Difference $\quad-0.01244$ t Ratio $\quad-2.51624$ Upper CL Dif 0.00129 Prob > |t $\quad 0.0656$ Lower CL Dif -0.02617 Prob $>$ t $\quad 0.9672$ Confidence $\quad 0.95$ Prob $<\mathrm{t} \quad 0.0328$

\section{Analysis of Variance}

Source DF Sum of Mean F Prob > $\begin{array}{lrrrrr} & & \text { Squares } & \text { Square } & \text { Ratio } & \text { F } \\ \text { HT } & 1 & 0.00023215 & 0.000232 & 6.3315 & 0.0656 \\ \text { Error } & 4 & 0.00014667 & 0.000037 & & \end{array}$

$\begin{array}{lllll}\text { Means for Anova } & & & \\ \text { Level Number Mean Std Lower Upper }\end{array}$ $\begin{array}{lrrrrr} & & & \text { Error } & 95 \% & 95 \% \\ \text { ccc } & 3 & 1.78651 & 0.00350 & 1.7768 & 1.7962\end{array}$ $0.00350 \quad 1.7$

Std Error uses a pooled estimate of error variance 
Figure B6. Effects of Heat Treatment (HT) on PCT log(ppm)-Response of Study Glasses

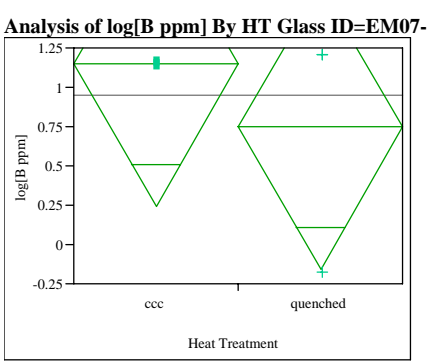

Anova

Summary of Fit

Rsquare

0.946318
Observations (or Sum Wgts)

t Test

quenched-cce

Assuming equal variances

Difference $\quad-0.4004$ t Ratio $\quad-0.86719$

$\begin{array}{lll} & 4 \\ \text { Un } & \end{array}$

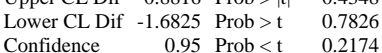

Analysis of $\log [\mathrm{Li}$ ppm] By HT Glass ID=EM07-Ca-035

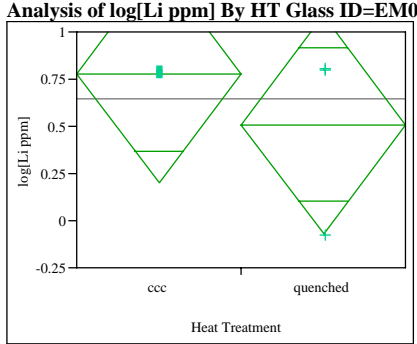

Anova

Summary of $F$

Rsquare

Root Mean Square Error $\quad 0.36118$

Mean of Response
Observations (or Sum Wgts)

t Test

quenched-ccc

Assuming equal variances

Difference $\quad-0.2692$ t Ratio $\quad-0.913$

Std Err Dif 0.2949 DF $>$ |t 0.4129

Lower CL Dif -1.0880 Prob $>t \quad 0.7936$

Confidence 0.95 Prob $<\mathrm{t} 0.2064$

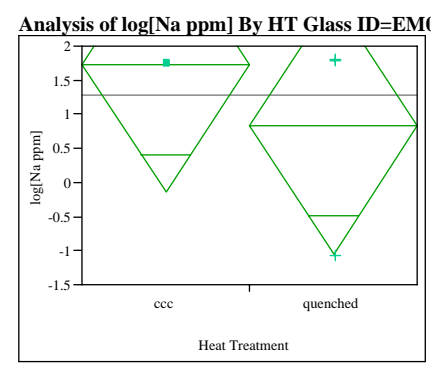

Anova

Summary of Fit

Rsquare

$\begin{array}{ll}\text { Adj Rsquare } & -0.180741 \\ \text { Root Mean Square Error } & -0.02407 \\ 1.172577\end{array}$

Observations (or Sum Wgts)

t Test
quenched-ccc

Assuming equal variances

$\begin{array}{llll}\text { Difference } & -0.8994 \text { t Ratio } & -0.93939\end{array}$ Std Err Dif 0.9574 DF 10.094 Lower CL Dif -3.5576 Prob >t 0.4996 Confidence $\quad 0.95$ Prob $<\mathrm{t} \quad 0.2004$ Mean of Response 1.285245

Analysis of $\log [\mathrm{Si}$ ppm] By HT Glass ID=EM07-Ca-035

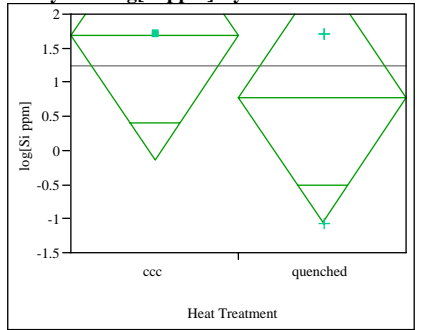

Anova

Summary of $F$

Rsquare
Adj Rsquare

$\begin{array}{ll}\text { Adj Rsquare } & 0.194688 \\ \text { Root Mean Square } & -0.06664\end{array}$ Mean of Response Error 1.137694 Observations (or Sum Wgts)

t Test
quenched-ccc

Assuming equal variances

$\begin{array}{lll}\text { Difference } & -0.9135 \text { t Ratio } \quad-0.98337\end{array}$ $\begin{array}{lll}\text { Std Err Dif } & 0.9289 \text { DF } & 4 \\ \text { Unper } & & \end{array}$

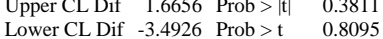

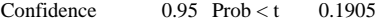

Analysis of Variance

Analysis of Variance

\begin{tabular}{lrrrrr}
\multicolumn{2}{l}{ Analysis of Variance } \\
Source & DF & Sum of & Mean & F & Prob > \\
& & Squares & Square & Ratio & F \\
HT & 1 & 0.2405280 & 0.240528 & 0.7520 & 0.4348 \\
Error & 4 & 1.2793866 & 0.319847 & & \\
C. & 5 & 1.5199145 & & & \\
Total & & & & &
\end{tabular}

Analysis of Vard
Source DF

Sum of

\begin{tabular}{llrrrr} 
HT & 1 & 0.10873976 & $\begin{array}{r}\text { Square } \\
\text { Ratio }\end{array}$ & Prob \\
\hline & 4 & 0.5308740 & 0.8336 & 0.4129
\end{tabular}

Error $\quad 4 \quad 0.5218064$

C. 0.130452

Analysis of Variance

$\begin{array}{lllll}\text { Source DF Sum of Mean F Prob > } & \end{array}$ \begin{tabular}{llr} 
HT & 1 & $\begin{array}{r}\text { Squares } \\
\text { H.2133301 }\end{array}$ \\
\hline & 4 & 5.79354
\end{tabular}

\begin{tabular}{lll} 
Error & 4 & 5.4997454 \\
C. & 5 & 6.7130754 \\
\hline
\end{tabular}

$\begin{array}{rrr}\text { Meaure } & \text { Ratio } & \text { F } \\ 1.21333 & 0.8825 & 0.4007 \\ 1.37494 & & \end{array}$

Means for Anova $\quad$ Std Lower Upper Means for Anova

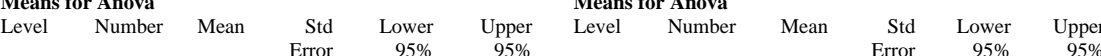

$\begin{array}{lrrrrrlrrrrr} & & & \text { Error } & 95 \% & 95 \% & & & & \text { Error } & 95 \% & 95 \% \\ \text { ccc } & 3 & 1.14654 & 0.32652 & 0.2400 & 2.0531 & \text { ccc } & 3 & 0.779550 & 0.20853 & 0.2006 & 1.3585 \\ \text { quenched } & 3 & 0.74610 & 0.32652 & -0.1605 & 1.6527 & \text { quenched } & 3 & 0.510304 & 0.20853 & -0.0687 & 1.0893\end{array}$

Std Error uses a pooled estimate of error variance
Std Error uses a pooled estimate of error variance

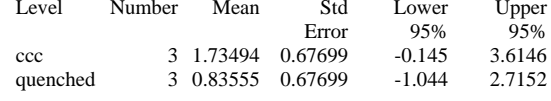

Std Error uses a pooled estimate of error variance
Analysis of Variance

Sum of Mean F Prob > $\begin{array}{lrrrrr}\text { HT } & 1 & 1.2516594 & 1.25166 & 0.9670 & 0.3811\end{array}$ $\begin{array}{llll} & & & \\ \text { Error } & 4 & 5.1773905 & 1.29435 \\ \text { C. } & 5 & 6.4290498 & \end{array}$

Means for Anova

Level Number Mean Std $\begin{array}{rrrr}\text { Lower } & \text { Upper } \\ & & & \end{array}$ $\begin{array}{lrrrrr} & & & \text { Error } & 95 \% & 95 \% \\ \text { ccc } & 3 & 1.69210 & 0.65685 & -0.132 & 3.5158 \\ \text { quenched } & 3 & 0.77862 & 0.65685 & -1.045 & 2.6023\end{array}$

Std Error uses a pooled estimate of error variance 


\section{Figure B6. Effects of Heat Treatment (HT) on PCT $\log (\mathbf{p p m})$-Response of Study Glasses}

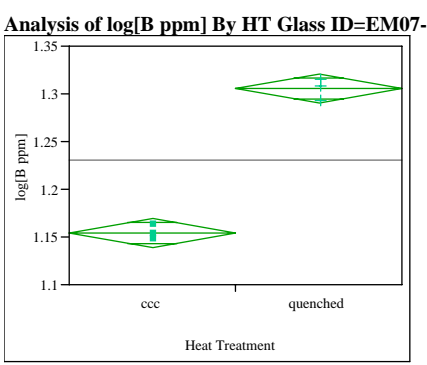

Anova

Summary of Fit

Rsquare
Adj Rsquare

Error $\quad 0.009518$

$\begin{array}{lr}\text { Mean of Response } & 1.230015 \\ \text { Observations (or Sum Wgts) } & 6\end{array}$

t Test

Assuming equal variances Std Err Dif 0.007772 DF 10.48 Confidence $\quad 0.95$ Prob $<\mathrm{t} \quad 1.0000$
Difference $\quad 0.151461$ t Ratio $\quad 19.4889$

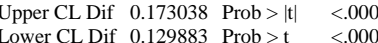

Assuming equal variances

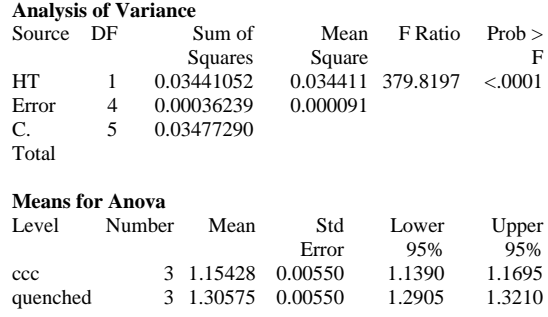

Std Error uses a pooled estimate of error variance
Analysis of $\log [\mathrm{Li}$ ppm] By HT Glass ID=EM07-Ca-07

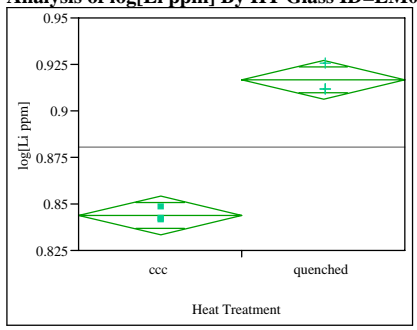

Anova

Summary of $F$

Rsquare

$\begin{array}{lr} & 0.9803 \\ \text { Adj Rsquare } & 0.975387\end{array}$

Root Mean Square Error $\quad 0.006335$

Mean of Response
Observations (or Sum Wgts)

t Test
quenched-ccc

Difference $\quad 0.072991$ t Ratio 14.11183 $\begin{array}{llll}\text { Std Err Dif } & 0.005172 & \text { DF } & 4\end{array}$ $\begin{array}{lll}\text { Upper CL Dif } & 0.087352 \text { Prob }>|t| & 0.0001\end{array}$

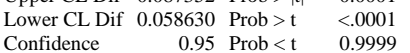

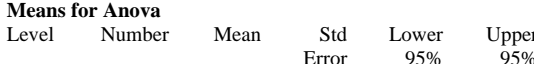

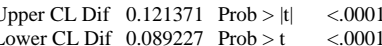
$\begin{array}{lrrrrr} & & & \text { Error } & 95 \% & 95 \% \\ \text { ccc } & 3 & 0.843714 & 0.00366 & 0.83356 & 0.85387\end{array}$ $\begin{array}{lllll}3 & 0.916705 & 0.00366 & 0.90655 & 0.92686\end{array}$ Std Error uses a pooled estimate of error variance

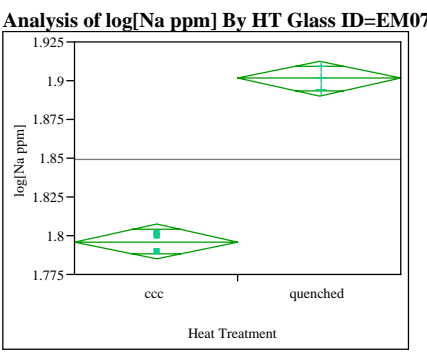

Anova

Summary of Fit

Rsquare

Adj Rsquare

Mean of Response

0.988056
0.98507

0.00709

Observations (or Sum Wgts) $\begin{array}{r}1.848893 \\ \quad 6\end{array}$

t Test

Assuming equal variances

Difference $\quad 0.105299$ t Ratio $\quad 18.19027$ S Err Dif 0.005789 DF $\quad 4$ $\begin{array}{lrl}\text { Confidence } & 0.95 \text { Prob }<\mathrm{t} & 1.0000\end{array}$

Analysis of Variance

$\begin{array}{lrrrrr}\text { Source } & \text { DF } & \begin{array}{r}\text { Sum of } \\ \text { Squares }\end{array} & \begin{array}{r}\text { Mean } \\ \text { Square }\end{array} & \text { F Ratio } & \text { Prob }> \\ & & & \text { F } \\ \text { HT } & 1 & 0.01663185 & 0.016632 & 330.8858 & <.0001\end{array}$ $\begin{array}{llllll}\text { HT } & 1 & 0.01663185 & 0.016632 & 330.8858 & <.0001 \\ \text { Error } & 4 & 0.00020106 & 0.000050 & & \end{array}$

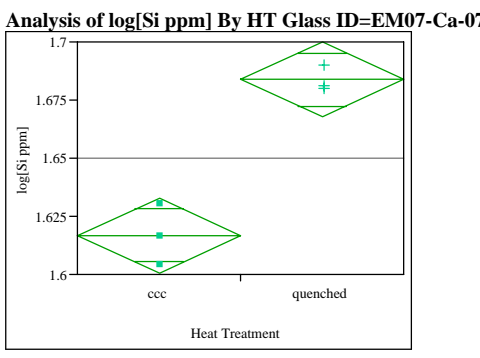

Anova

mary of Fit

Rsquare

\begin{tabular}{ll}
0.942806 \\
Root Mean Square & 0.928507 \\
\hline
\end{tabular} Observations (or Sum Wgts) $\quad 6$

quenched-ccc

Assuming equal variances

$\begin{array}{lll}\text { Difference } & 0.066971 & \mathrm{t} \text { Ratio } \quad 8.120184\end{array}$ Upper CL Dif 0.089870 Prob > |t 0.0013 Lower CL Dif 0.044072 Prob $>t \quad 0.0006$ Confidence $\quad 0.95$ Prob $<\mathrm{t} \quad 0.9994$ $\begin{array}{lrrrrr}\text { HT } & 1 & 0.00672767 & 0.006728 & 65.9374 & 0.0013 \\ \text { Error } & 4 & 0.00040813 & 0.000102 & & \end{array}$ \begin{tabular}{lll} 
Error & 4 & 0.00040813 \\
\hline & 5 & 0.0071550
\end{tabular}

Means for Anova

$\begin{array}{llrrr}\text { Level Number Mean } & \begin{array}{r}\text { Std } \\ \text { Error }\end{array} & \begin{array}{r}\text { Lower } \\ 95 \%\end{array} & \text { Upper } \\ & & 95 \%\end{array}$

$\begin{array}{lrrrrr} & & & \text { Error } & 95 \% & 95 \% \\ \text { ccc } & 3 & 1.79624 & 0.00409 & 1.7849 & 1.8076\end{array}$ $\begin{array}{llll}1.90154 & 0.00409 & 1.8902 & 1.9129\end{array}$

Std Error uses a pooled estimate of error variance

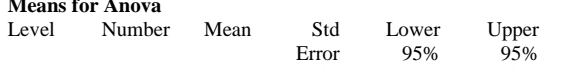
$\begin{array}{lrrrrr}\text { ccc } & 3 & 1.61676 & 0.00583 & 1.6006 & 1.6330\end{array}$

Std Error uses a pooled estimate of error variance 
Figure B6. Effects of Heat Treatment (HT) on PCT log(ppm)-Response of Study Glasses

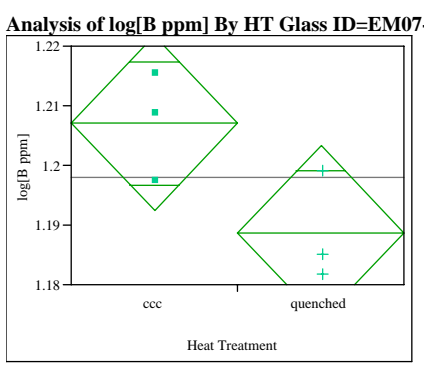

Anova

Anova

$\begin{array}{ll}\text { Adj Rsquare } & 0.502841 \\ \text { Root Mean Square Error } & 0.009134\end{array}$ $\begin{array}{lr}\text { Mean of Response } & 1.197893 \\ \text { Observations (or Sum Wgts) } & 6\end{array}$

t Test

quenched-ccc

Assuming equal variances

Difference $\quad-0.01835$ t Ratio $\quad-2.46113$ $\begin{array}{lll}\text { Std Err Dif } & 0.00746 \text { DF } & 4 \\ \text { Upper CL Dif } & 0.00235 & \end{array}$

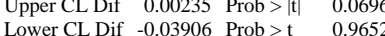
Confidence $\quad 0.95$ Prob $<\mathrm{t} \quad 0.0348$
Rsquare $\quad 0.602273$

\begin{tabular}{|c|c|c|c|c|c|c|c|c|c|c|c|}
\hline \multicolumn{5}{|c|}{ Analysis of Variance } & \multicolumn{7}{|c|}{ Analysis of Variance } \\
\hline Source DF & $\begin{array}{c}\text { Sum of } \\
\text { Squares }\end{array}$ & $\begin{array}{l}\text { Mean } \\
\text { Sayare }\end{array}$ & $F$ & Prob $>$ & Source & DF & & $\begin{array}{l}\text { Sum of } \\
\text { Squares }\end{array}$ & $\begin{array}{l}\text { Mean } \\
\text { Square }\end{array}$ & F Ratio & Prob > \\
\hline & 0.00050533 & 0.000505 & 6.0571 & 0.0696 & HT & 1 & & 0062760 & 0.000628 & 12.1272 & 0.0253 \\
\hline 4 & 0.00033371 & 0.000083 & & & Error & 4 & & 0020701 & 0.000052 & & \\
\hline 5 & 0.00083903 & & & & $\begin{array}{l}\text { C. } \\
\text { Total }\end{array}$ & 5 & & 083460 & & & \\
\hline or Ano & & & & & Means fo & or Ans & & & & & \\
\hline Num & Mean & $\begin{array}{r}\text { Std } \\
\text { Ftron }\end{array}$ & $\begin{array}{r}\text { Lower } \\
95 \%\end{array}$ & $\begin{array}{r}\text { Upper } \\
95 \%\end{array}$ & Level & Nun & & Mean & $\begin{array}{r}\text { Std } \\
\text { Frror }\end{array}$ & $\begin{array}{l}\text { Lower } \\
\text { groo }\end{array}$ & $\begin{array}{r}\text { Upper } \\
95 \%\end{array}$ \\
\hline & 31.20707 & 0.00527 & $\begin{array}{r}1.1924 \\
\end{array}$ & 1.2217 & $\mathrm{ccc}$ & & 3 & 0.829620 & 0.00415 & 0.81809 & 0.84115 \\
\hline hed & 31.18872 & 0.00527 & 1.1741 & 1.2034 & quenched & & & 0.809165 & 0.00415 & 0.79763 & 0.82070 \\
\hline
\end{tabular}

Std Error uses a pooled estimate of error variance

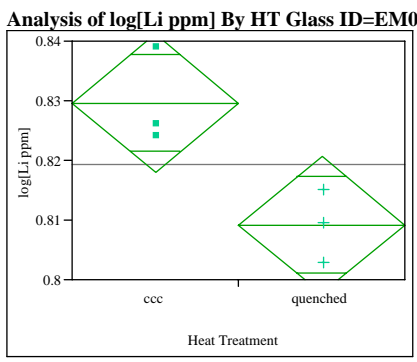

Anova

Summary of F

Rsquare

0.75197

Square Error $\quad 0.007194$

Mean of Response ${ }_{\text {Obervations (or Sum Wgts) }}^{0.819392}$

t Test
quenched-ccc

Assuming equal variances

$\begin{array}{lll}\text { Difference } & -0.02045 \text { t Ratio } & -3.48241\end{array}$

Std Err Dif 0.00587 DF -30.4024

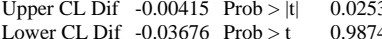

Confidence $\quad 0.95$ Prob $<\mathrm{t} \quad 0.0126$

Analysis of Variance

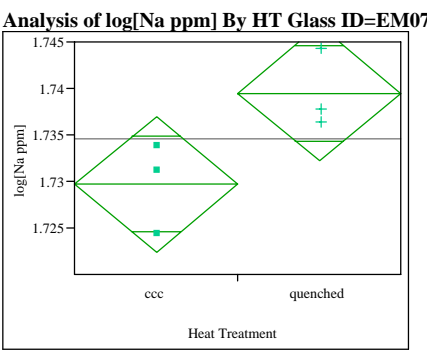

Anova

Summary of Fit

$\begin{array}{ll}\text { Rsquare } & 0.632971 \\ \text { Adj Rsquare } & 0.541214\end{array}$

Root Mean Square Error $\quad 0.004562$

$\begin{array}{lr}\text { Mean of Response } & 1.734587 \\ \text { Observations (or Sum Wgts) } & 6\end{array}$

t Test

quenched-ccc

Assuming equal variances

Difference $\quad 0.00978$ t Ratio 2.626466 Upper CL Dif 0.02013 Prob > tt $\quad 0.0584$ $\begin{array}{lll}\text { Lower CL Dif }-0.00056 \text { Prob }>t \quad 0.0292 & 0.95\end{array}$ Confidence $\quad 0.95$ Prob $<\mathrm{t} \quad 0.9708$

\begin{tabular}{|c|c|c|c|c|c|c|}
\hline \multicolumn{7}{|c|}{ Analysis of Variance } \\
\hline Source & DF & & $\begin{array}{l}\text { Sum of } \\
\text { Squares }\end{array}$ & $\begin{array}{l}\text { Mear } \\
\text { Square }\end{array}$ & $\begin{array}{r}F \\
\text { Ratio }\end{array}$ & \\
\hline HT & 1 & & 0014360 & 0.000144 & 6.8983 & 0.05 \\
\hline Error & 4 & & 0008327 & 0.00002 & & \\
\hline $\begin{array}{l}\text { C. } \\
\text { Total }\end{array}$ & 5 & & 0022686 & & & \\
\hline & & & & & & \\
\hline $\begin{array}{l}\text { Mevans } \\
\text { Level }\end{array}$ & $\begin{array}{l}r \text { Ano } \\
\text { Num }\end{array}$ & & Mean & & & \\
\hline & & & iveant & Error & $95 \%$ & \\
\hline ccc & & & 1.72 & 0.00263 & 1.7224 & \\
\hline quenched & & & 1.73948 & 0.00263 & 1.7322 & 78 \\
\hline
\end{tabular}

Std Error uses a pooled estimate of error variance

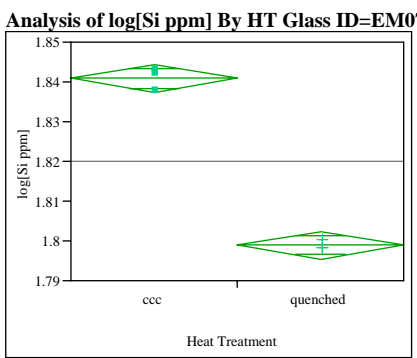

Anov

Summary of Fi

Rsquare
Adj Rsquare

$\begin{array}{ll}\text { Adj Rsquare } & 0.992929 \\ \text { Root Mean Square E } & 0.991161\end{array}$ Error $\quad 0.002169$ Observations (or Sum Wgts) 1.819955

t Test
quenched-ccc

Assuming equal variances

$\begin{array}{lll}\text { Difference } & -0.04198 \text { t Ratio } & -23.6995 \\ \text { Std } & & \end{array}$ Std Err Dif $0.00177 \mathrm{DF}$ $\begin{array}{llll}\text { Upper CL Dif } & 0.03706 & \text { Prob }>|t| & <.0001 \\ \text { Lower CL Dif }-0.04690 & \text { Prob }>t & 1.0000\end{array}$ Confidence $\quad 0.95$ Prob $<\mathrm{t} \quad<.0001$

\section{Analysis of Variance}

Source DF Sum of Mean F Ratio Prob > $\begin{array}{llrrrr}\text { HT } & 1 & 0.00264350 & 0.002644 & 561.6652 & <.0001\end{array}$

$\begin{array}{llll}\text { Error } & 4 & 0.00001883 & 4.707 \mathrm{e}-6 \\ \mathrm{C} & 5 & 0.00266233 & \end{array}$

Means for Anova

Level Number Mean Std Lower Upper $\begin{array}{lrrrrr} & & & \text { Error } & 95 \% & 95 \% \\ \text { ccc } & 3 & 1.84094 & 0.00125 & 1.8375 & 1.844\end{array}$ $\begin{array}{llllll}\text { quenched } & 3 & 1.749896 & 0.00125 & 1.8375 & 1.8444 \\ & & & & & \end{array}$ Std Error uses a pooled estimate of error variance 
Figure B6. Effects of Heat Treatment (HT) on PCT $\log (\mathrm{ppm})$-Response of Study Glasses

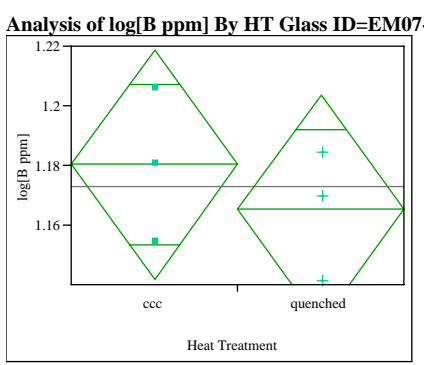

Anova

Summary of Fit

Rsquare
Adj Rsquare

Error $\quad 0.023865$

$\begin{array}{lr}\text { Mean of Response } & 1.172692 \\ \text { Observations (or Sum Wgts) } & 6\end{array}$

t Test

quenched-cce

Assuming equal variances

Difference $\quad-0.01507$ t Ratio $\quad-0.77357$

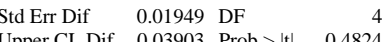
$\begin{array}{lrrr}\text { Upper CL Dif } & 0.03903 & \text { Prob }>|t| & 0.4824 \\ \text { Lower CL Dif } & -0.06917 & \text { Prob }>t & 0.7588\end{array}$ Confidence $\quad 0.95$ Prob $<\mathrm{t} \quad 0.2412$

Analysis of Variance

\begin{tabular}{lrrrrr}
\multicolumn{2}{l}{$\begin{array}{l}\text { Analysis of Variance } \\
\text { Source }\end{array}$} & DF & $\begin{array}{r}\text { Sum of } \\
\text { Squares }\end{array}$ & $\begin{array}{r}\text { Mean } \\
\text { Square }\end{array}$ & $\begin{array}{r}\text { Ratio } \\
\text { Prob > }\end{array}$ \\
HT & 1 & 0.00034082 & 0.000341 & 0.5984 & 0.4824 \\
Error & 4 & 0.00227816 & 0.000570 & &
\end{tabular}

$\begin{array}{ll}1 & 0.00034082 \\ & 0.00261898\end{array}$

$\begin{array}{lrrrr}\text { Means for Anova } & & & & \\ \text { Level Number Mean Std } & \text { Lower } & \text { Upper } \\ & & \text { Error } & 95 \% & 95 \%\end{array}$

$\begin{array}{lrrrrr} & & & \text { Error } & 95 \% & 95 \% \\ \text { ccc } & 3 & 1.18023 & 0.01378 & 1.1420 & 1.2185\end{array}$

$\begin{array}{llllll}\text { quenched } & 3 & 1.16516 & 0.01378 & 1.1269 & 1.2034\end{array}$

Std Error uses a pooled estimate of error variance

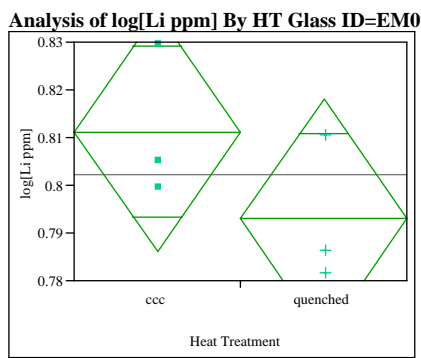

Anova

Summary of Fit

Rsquare

$\begin{array}{ll}\text { Adj Rsquare } & 0.335917 \\ \text { Root Mean Square Error } & 0.169896 \\ 0 & 0.015711\end{array}$

$\begin{array}{lr}\text { Mean of Response } & 0.802116 \\ \text { Observations (or Sum Wgts) } & 6\end{array}$

t Test

Assuming equal variances

$\begin{array}{lll}\text { Difference } & -0.01825 \text { t Ratio } & -1.42244 \\ \text { Std } & \end{array}$

Std Err Dif 0.01283 DF $\quad-1.4224$

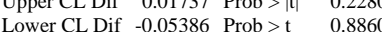

Confidence $\quad 0.95$ Prob $<t \quad 0.1140$

Analysis of Variance

$\begin{array}{llrlrl}\text { Source DF Sum of } & \text { Mean } & \text { F } & \text { Prob }>\end{array}$ $\begin{array}{llllll}\text { HT } & 1 & 0.00049943 & 0.000499 & 2.0233 & 0.2280 \\ \text { Error } & 4 & 0.00098734 & 0.000247 & & \end{array}$

$\begin{array}{lll}\text { Error } & 4 & 0.00098734 \\ C . & 5 & 0.001867\end{array}$

Total

Means for Anova

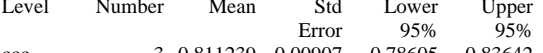

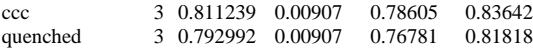

Std Error uses a pooled estimate of error variance

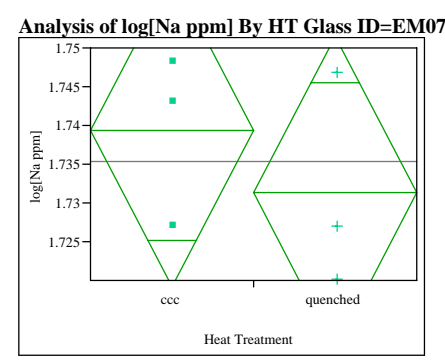

Anova

Summary of Fit

$\begin{array}{ll}\text { Rsquare } & 0.133463 \\ \text { Adj Rsquare } & -0.08317\end{array}$

Root Mean Square Error $\quad 0.012546$

$\begin{array}{lr}\text { Mean of Response } & 1.73538 \\ \text { Observations (or Sum Wgts) } & 6\end{array}$

t Test

quenched-ccc

Assuming equal variances

Difference $\quad-0.00804$ t Ratio $\quad-0.7849$ $\begin{array}{llll} & 0.01024 \text { DF } & \text { DFr Dif }\end{array}$

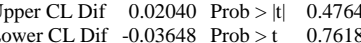
$\begin{array}{lrll}\text { Confidence } & -0.03648 & \text { Prob }>\text { t } & 0.7618 \\ & 0.95 & \text { Prob }<t & 0.2382\end{array}$

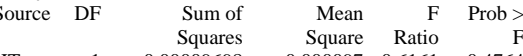

$\begin{array}{llrrrr}\text { HT } & 1 & 0.00009698 & 0.000097 & 0.6161 & 0.4764\end{array}$

$\begin{array}{lll}\text { Error } & 4 & 0.00062965 \\ \text { C } & 5 & 0.007265\end{array}$

Means for Anova

Level Number Mean Std Lower Upper

$\begin{array}{lrrrrr} & & & \text { Error } & 95 \% & 95 \% \\ \text { ccc } & 3 & 1.73940 & 0.00724 & 1.7193 & 1.7595 \\ & 3 & 1.7316 & & & \end{array}$

Std Error uses a pooled estimate of error variance

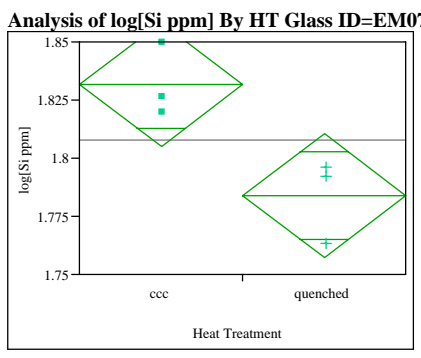

Anov

ummary of Fit

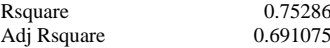

Root Mean Square Error $\quad 0.01674$

Mean of Response 1.807764

t Test
quenched-cce

Assuming equal variances

$\begin{array}{lrrr}\text { Difference } & -0.04771 & \mathrm{t} \text { Ratio } & -3.49073 \\ \text { Std Err Dif } & 0.01367 & \mathrm{DF} & \end{array}$ Upper CL Dif -0.00976 Prob $>|t| \quad 0.025$ Lower CL Dif -0.08566 Prob $>t \quad 0.9874$ Confidence $\quad 0.95$ Prob $<\mathrm{t} \quad 0.0126$

Analysis of Variance

Source DF Sum of Mean F Ratio Prob > $\begin{array}{llrrrr} & & \text { Squares } & \text { Square } & \text { HT } \\ \text { Herror } & 1 & 0.00341454 & 0.003415 & 12.1852 & 0.0251 \\ \text { C } & 4 & 0.00112088 & 0.000280 & & \end{array}$ $\begin{array}{lll}\text { Error } & 4 & 0.00112088 \\ \text { C } & 5 & 0.00453542\end{array}$

$\begin{array}{llrrr}\text { Means for Anova } & & & & \\ \text { Level Number } & \text { Mean Std } & \text { Lower } & \text { Upper }\end{array}$ $\begin{array}{lrrrrr} & & & \text { Error } & 95 \% & 95 \% \\ \text { ccc } & 3 & 1.83162 & 0.00966 & 1.8048 & 1.8585\end{array}$ quenched

Std Error uses a pooled estimate of error variance 
Figure B6. Effects of Heat Treatment (HT) on PCT log(ppm)-Response of Study Glasses

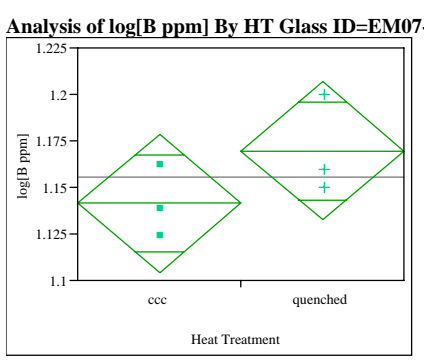

Anova

Summary of Fit

Rsquare
Adj Rsqu

0.358327
0.197909

Root Mean Square Error $\quad 0.023247$

Mean of Response 1.155534

t Test

t Test
quenched-cce

Assuming equal variances

Difference $\quad 0.02837$ t Ratio 1.494559 $\begin{array}{lll} & 0.2093\end{array}$

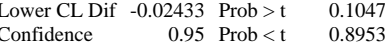

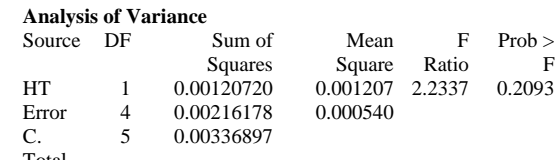

Means for Anova

$\begin{array}{rrrr}\text { Level Number Mean } & \begin{array}{r}\text { Std } \\ \text { Error }\end{array} & \begin{array}{r}\text { Lower } \\ 95 \%\end{array} & \text { Upper } \\ & & 95 \%\end{array}$

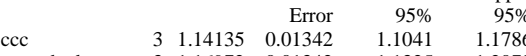

$1.1325 \quad 1.2070$

Std Error uses a pooled estimate of error variance
Analysis of $\log [\mathrm{Li}$ ppm] By HT Glass ID=EM07-Cr-02

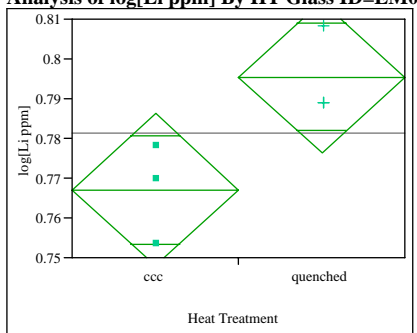

Anova

Summary of Fit

Rsquare

$\begin{array}{ll}\text { Adj Rsquare } & 0.677913 \\ \text { Root Mean Square Error } & 0.597391 \\ & 0.07892\end{array}$ Mean of Response $\quad 0.78122$

Observations (or Sum Wgts)

t Test
quenched-ccc

Assuming equal variances

Difference $\quad 0.028361$ t Ratio $\quad 2.90155$ $\begin{array}{lll}\text { Std Err Dif } 0.009775 \text { DF } & 4\end{array}$ Lower CL Dif 0.001223 Prob $>t \quad 0.0220$ $\begin{array}{lll}\text { Confidence } & 0.95 \text { Prob }<\mathrm{t} \quad 0.9780\end{array}$

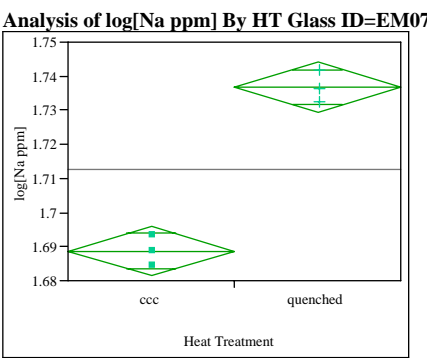

Anova

Summary of Fit

Rsquare

$\begin{array}{ll}\text { Adj Rsquare } & 0.976687 \\ \text { Root Mean Square Eror } & 0.970859\end{array}$ Mean of Response 1.71277

Observations (or Sum Wgts) $\quad 6$

t Test
quenched-ccc

Assuming equal variances

$\begin{array}{lll}\text { Difference } & 0.048121 & \text { t Ratio } \\ \text { Std } & 12.94531\end{array}$ Std Err Dif 0.003717 DF $>4$ Lower CL Dif 0.037800 Prob $>1 \quad 0.0001$ Confidence $\quad 0.95$ Prob $<$ t 0.9999

\section{Analysis of Variance \\ Analysis of Varian}

$\begin{array}{rrrr}\text { Sum of } & \text { Mean } & \text { F } & \text { Prob > } \\ \text { Squares } & \text { Square } & \text { Ratio } & \end{array}$ $\begin{array}{llllll}\text { HT } & 1 & 0.00120655 & 0.001207 & 8.4190 & 0.0440 \\ \text { Error } & 4 & 0.00057325 & 0.000143 & & \end{array}$

Means for Anova $\begin{array}{lrrrrr} & & \text { Error } & 95 \% & 95 \% \\ \text { ccc } & 3 & 0.767043 & 0.00691 & 0.74785 & 0.7862 \\ & 3 & 0.795404 & & & \end{array}$

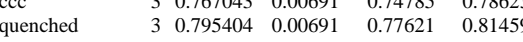
Std Error uses a pooled estimate of error variance $\begin{array}{lrrrr}\text { Source DF } & \text { Sum of } & \text { Mean } & \text { F Ratio Prob }>\end{array}$ $\begin{array}{lrrrrr} & & \text { Squares } & \text { Square } & \text { F } \\ \text { HT } & 1 & 0.00347347 & 0.003473 & 167.5811 & 0.0002 \\ \text { Error } & 4 & 0.00008291 & 0.000021 & & \end{array}$

Means for Anova

$\begin{array}{lllll} & & & \\ \end{array}$

$\begin{array}{lrrrrr}\text { ccc } & 3 & 1.68871 & 0.00263 & 1.6814 & 1.6960 \\ \text { quenched } & 3 & 1.73683 & 0.00263 & 1.7295 & \end{array}$

Std Error uses a pooled estimate of error variance

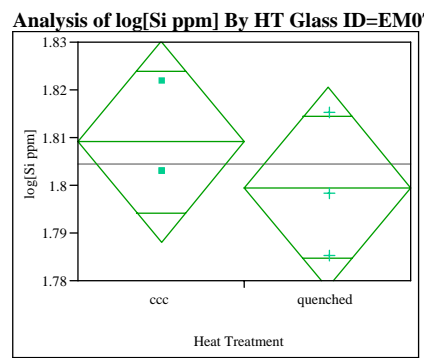

Anova

Summary of Fit

Rsquare
Adj Rsquare Mean of Response $\quad 0.01310$ Observations (or Sum Wgts)

t Test
quenched-ccc

Assuming equal variances

Difference $\quad-0.00954$ t Ratio $\quad-0.89198$ Std Err Dif $0.01070 \mathrm{DF}$

$\begin{array}{lll} & \\ \text { Uppeer CL Dif } & 0.02016 \text { Prob }>|t| & 0.4228\end{array}$ Confidence $\quad 0.95$ Prob $<\mathrm{t} \quad 0.2114$

Analysis of Variance

$\begin{array}{llrl}\text { Source DF Sum of Mean } & F & \text { Prob > }\end{array}$ $\begin{array}{lrrrrr}\text { HT } & 1 & 0.00013656 & 0.000137 & 0.7956 & 0.4228\end{array}$

$\begin{array}{llll}\text { Error } & 4 & 0.00068656 & 0.000172 \\ \text { C. } & 5 & 0.00082312 & \end{array}$

Means for Anova

$\begin{array}{lllll} & & & \\ \text { Level Number Mean Std } & \text { Lower } & \text { Upper } \\ 95 \% & 95 \%\end{array}$ $\begin{array}{lrrrrr}\text { ccc } & 3 & 1.80910 & 0.00756 & 1.7881 & 1.830 \\ \text { quenched } & 3 & 1.79956 & 0.00756 & 1.7786 & \end{array}$ $\begin{array}{llllll}\text { quenched } & 3 & 1.79956 & 0.00756 & 1.7786 & 1.8206\end{array}$ Std Error uses a pooled estimate of error variance 
Figure B6. Effects of Heat Treatment (HT) on PCT $\log (\mathrm{ppm})$-Response of Study Glasses

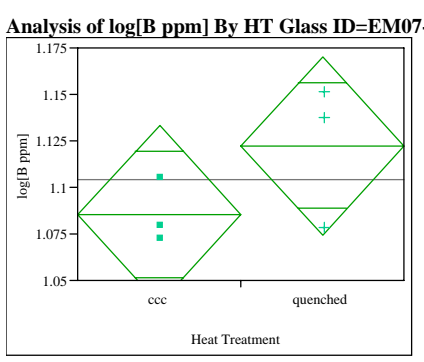

Anova

Summary of Fit

Rsquare
Adj Rsqu

0.363538

Root Mean Square Error $\quad 0.029838$

Mean of Response 1.103961

Test

quenched-cc

Assuming equal variances

Difference $\quad 0.03683$ t Ratio $\quad 1.511535$

$\begin{array}{llll}\text { Lower CL Dif }-0.03082 & \text { Prob }>t & 0.1026\end{array}$

Confidence $\quad 0.95$ Prob $<\mathrm{t} \quad 0.8974$

\begin{tabular}{lrrrrr}
\multicolumn{2}{l}{$\begin{array}{l}\text { Analysis of Variance } \\
\text { Source }\end{array}$} & DF & $\begin{array}{r}\text { Sum of } \\
\text { Squares }\end{array}$ & $\begin{array}{r}\text { Mean } \\
\text { Square }\end{array}$ & $\begin{array}{r}\text { Ratio } \\
\text { Prob }>\end{array}$ \\
HT & 1 & 0.00203414 & 0.002034 & 2.2847 & 0.2052 \\
Error & 4 & 0.000356127 & 0.000890 & & \\
C. & 5 & 0.00559541 & & & \\
Total & & & & &
\end{tabular}

Means for Anova

$\begin{array}{rrrr}\text { Std } & \text { Lower } & \text { Upper } \\ \text { Error } & 95 \% & 95 \%\end{array}$

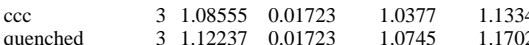

Std Error uses a pooled estimate of error variance
Analysis of $\log [\mathrm{Li}$ ppm] By HT Glass ID=EM07-F-02

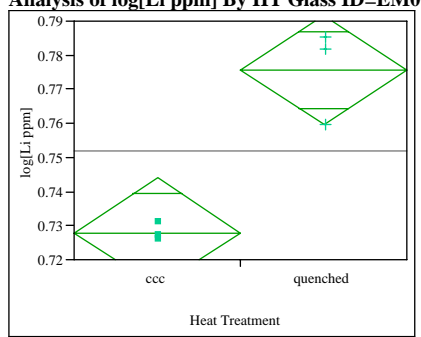

Anova

Summary of Fit

Rsquare

0.894519
Root Mean Square Error $\quad 0.868149$ Mean of Response $\quad 0.75174$

Observations (or Sum Wgts)

t Test
quenched-ccc

Assuming equal variances

Difference $\quad 0.047688$ t Ratio $\quad 5.824233$ Std Err Dif 0.008188 DF $\quad 4$ Lower CL Dif 0.024955 Prob $>t \quad 0.0022$

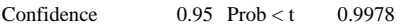

$\begin{array}{lllll}\text { Source DF Sum of Mean F Ratio Prob > } & \end{array}$ $\begin{array}{llllll}\text { HT } & 1 & 0.00341120 & 0.003411 & 33.9217 & 0.0043\end{array}$

0.00040224 000101

Means for Anova

Level Number Mean $\begin{array}{rrrr}\text { Std } & \text { Lower } & \text { Upper } \\ \text { Error } & 95 \% & 95 \%\end{array}$ $\begin{array}{lllllll}\text { ccc } & 3 & 0.727905 & 0.00579 & 0.71183 & 0.74398 \\ \text { quenched } & 3 & 0.775593 & 0.00579 & 0.75952 & 0.79167\end{array}$ Std Error uses a pooled estimate of error variance

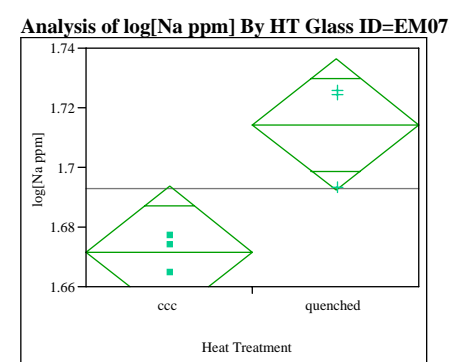

Anova

Summary of Fit

Rsquare

$\begin{array}{ll}\text { Adj Rsquare } & 0.729509 \\ \text { Root Mean Square Error } & 0.7213773\end{array}$ $\begin{array}{lr}\text { Mean of Response } & 1.692961 \\ \text { Observations (or Sum Wgts) } & 6\end{array}$

t Test

Assuming equal variances

Difference $\quad 0.042799$ t Ratio $\quad 3.805907$ Std Err Dif 0.01246 DF $\quad 4$ Lower CL Dif 0.011577 Prob $>t \quad 0.0095$ Confidence $\quad 0.95$ Prob $<$ t $\quad 0.9905$

\section{Analysis of Variance} $\begin{array}{lrrrrr}\text { Source } & \text { DF } & \begin{array}{r}\text { Sum of } \\ \text { Squares }\end{array} & \begin{array}{r}\text { Mean } \\ \text { Square }\end{array} & \text { F Ratio } & \text { Prob }> \\ \text { HT } & 1 & 0.00274769 & 0.002748 & 14.4849 & 0.0190\end{array}$

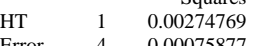

$\begin{array}{lll}\text { Error } & 4 & 0.00075877 \\ \text { C. } & 5 & 0.00350647\end{array}$ 0.000190

Means for Anova

Level Number Mean Std Lower Upper

$\begin{array}{lrrrrr}\text { ccc } & 3 & & \text { Error } & 95 \% & 95 \% \\ & 3 & 1.67156 & 0.00795 & 1.6495 & 1.6936\end{array}$

Std Error uses a pooled estinge of erior varice

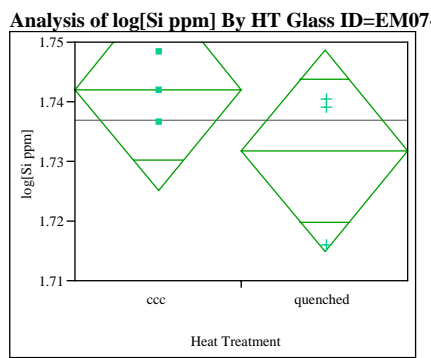

Summary of Fit

Rsquare Mean of Response Observations (or Sum Wgts)

t Test

Assuming equal variances

Difference $\quad-0.01028$ t Ratio -1.19394

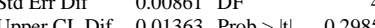
Lower CL Dif -0.03420 Prob $>t \quad 0.8508$ Confidence $\quad 0.95$ Prob $<\mathrm{t} \quad 0.1492$

\section{Analysis of Variance}

$\begin{array}{rrrrr}\text { Source DF } & \text { Sum of } & \text { Mean } & \text { F } & \text { Prob > }\end{array}$ $\begin{array}{llllrr}\text { HT } & 1 & 0.00015863 & 0.000159 & 1.4255 & 0.2985\end{array}$

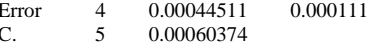

Means for Anova
Level Number $\quad$ Mean Std Lower $\quad$ Std \begin{tabular}{ccccc} 
& & & & \\
& Error & Lower & Upper \\
\hline & $95 \%$ & $95 \%$
\end{tabular}

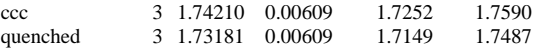
Std Error uses a pooled estimate of error variance 
Figure B6. Effects of Heat Treatment (HT) on PCT log(ppm)-Response of Study Glasses

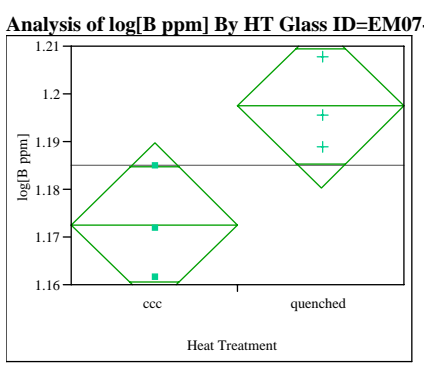

Anov

Summary of Fit

Rsquare

$\begin{array}{lr}\text { Mean of Response } & 1.184988 \\ \text { Observations (or Sum Wgts) } & 6\end{array}$

t Test

Assuming equal variances

Difference $\quad 0.024768$ t Ratio $\quad 2.843242$ $\begin{array}{llll}\text { Std Err Dif } & 0.008711 & \text { DF }\end{array}$

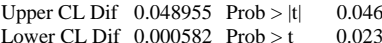
$\begin{array}{llll}\text { Confidence } & 0.95 \text { Prob }<\mathrm{t} & 0.9766\end{array}$

Analysis of Variance

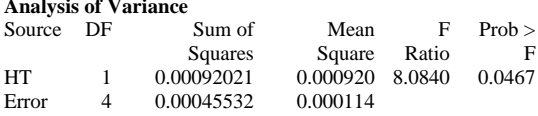

C. $\quad 5 \quad 0.0013755$

$\begin{array}{llll}\text { Means for Anova } & & & \\ \text { Level Number Mean Std Lower Upper }\end{array}$

$\begin{array}{lrrrrr} & & & \text { Std } & \text { Lower } & \text { Upper } \\ \text { ccc } & 3 & 1.17260 & 0.00616 & 1.15 \% 5 & 1.18 \% \\ & & & & & \end{array}$

$\begin{array}{llllll}\text { quenched } & 3 & 1.19737 & 0.00016 & 1.1555 & 1.1897 \\ & & & & & \end{array}$

Std Error uses a pooled estimate of error variance

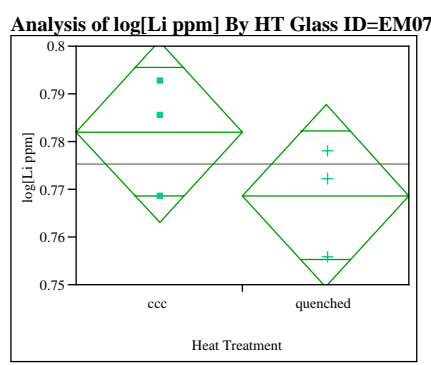

Anova

Summary of Fi

Rsquare

Root Mean Square Error $\quad 0.011932$

$\begin{array}{ll}\text { Mean of Response } & 0.775378 \\ \text { Observations (or Sum Wgts) }\end{array}$

t Test

Assuming equal variances

\begin{tabular}{lll} 
Difference & -0.01334 t Ratio & -1.36893 \\
\hline
\end{tabular}

Lower CL Dif -0.04039 Prob $>t \quad 0.878$

Confidence $\quad 0.95$ Prob $<\mathrm{t} \quad 0.1214$
Std Err Dif 0.00974 DF -1.3683

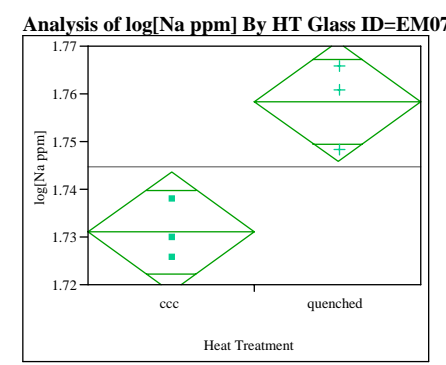

Anova

Anova
Summary of Fit

Rsquare

$\begin{array}{ll} & 0.821818 \\ \text { Adj Rsquare } & 0.777273 \\ & 0.00779\end{array}$

$\begin{array}{lr}\text { Mean of Response } & 1.744691 \\ \text { Observations (or Sum Wgts) } & 6\end{array}$

t Test

Assuming equal variances

$\begin{array}{lll}\text { Difference } & 0.027321 \mathrm{t} \text { Ratio } \quad 4.295228\end{array}$ Std Err Dif 0.006361 DF 14 Lower CL Dif 0.009661 Prob > t 0.0063 Confidence $\quad 0.95$ Prob $<\mathrm{t} \quad 0.9937$

\section{Analysis of Variance}

Analysis of Variance

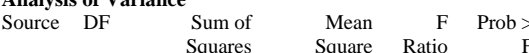

$\begin{array}{llllll}\text { HT } & 1 & 0.00026680 & 0.000267 & 1.8740 & 0.2429 \\ \text { Error } & 4 & 0.00056948 & 0.000142 & & \end{array}$

$\begin{array}{lllll}\text { Means for Anova } & & & & \\ \text { Level Number } & \text { Mean Std } & \text { Lower } & \text { Upper }\end{array}$

$\begin{array}{rrrrrr} & & \text { Error } & 95 \% & 95 \% \\ \text { ccc } & 3 & 0.782046 & 0.00689 & 0.76292 & 0.8011\end{array}$

$\begin{array}{lllllll}\text { quenched } & 3 & 0.768710 & 0.00689 & 0.74958 & 0.78784\end{array}$

Std Error uses a pooled estimate of error variance

Analysis of Variance

$\begin{array}{lll}\text { Error } & 4 & 0.00024276 \\ \text { C } & 5 & 0.00136241\end{array}$

Means for Anova

Std Error uses a pooled estimate of error variance

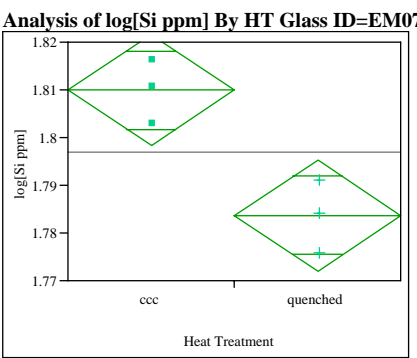

Anov

Summary of Fi

Rsquare
Adj Rsquare

$\begin{array}{ll}\text { Adj Rsquare } & 0.829337 \\ \text { Root Mean Square E } & 0.786671\end{array}$ Mean of Response $\quad 0.007278$ Observations (or Sum Wgts)

t Test
quenched-ccc

Assuming equal variances

Difference $\quad-0.02620$ t Ratio $\quad-4.40885$ Std Err Dif 0.00594 DF $\quad-4.408$ Lower CL Dif -0.04270 Prob $>t$ Confidence $\quad 0.95$ Prob $<\mathrm{t} \quad 0.0058$

\begin{tabular}{llr} 
HT & 1 & 0.00111966 \\
\hline & 4 & 0.002476
\end{tabular}

$\begin{array}{cc}\text { Mean } & \text { F Ratio Prob > } \\ \text { Square } & \end{array}$

$\begin{array}{llll} & & \\ \end{array}$

$\begin{array}{lrrrrr}\text { ccc } & 3 & 1.73103 & 0.00450 & 1.7185 & 1.7435 \\ \text { quenched } & 3 & 1.75835 & 0.00450 & 1.7459 & 1.7708\end{array}$

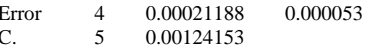

\section{Analysis of Variance}

DF Sum of Mean F Ratio Prob > $\begin{array}{llrrrr}\text { HT } & 1 & 0.00102965 & 0.001030 & 19.4380 & 0.0116\end{array}$

Means for Anova

$\begin{array}{lllll} & & & \\ \text { Level Number Mean Std } & \text { Lower } & \text { Upper } \\ 95 \% & 95 \%\end{array}$ $\begin{array}{lrrrrr} & & & \text { Error } & 95 \% & 95 \% \\ \text { ccc } & 3 & 1.80991 & 0.00420 & 1.7982 & 1.8216 \\ \text { quenched } & 3 & 1.78371 & 0.00420 & 1.7720 & 1.7954\end{array}$

Std Error uses a pooled estimate of error variance 
Figure B6. Effects of Heat Treatment (HT) on PCT log(ppm)-Response of Study Glasses

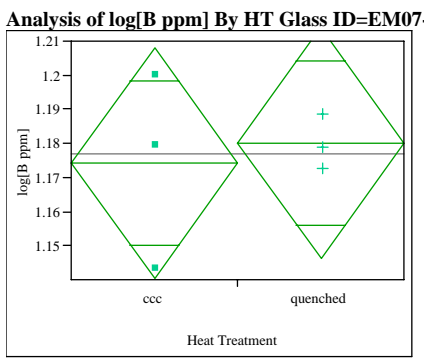

Anov

Anova

Rsquare

-0.21488
Root Mean Square Error $\quad 0.021173$

$\begin{array}{lr}\text { Mean of Response } & 1.177115 \\ \text { Observations (or Sum Wgts) } & 6\end{array}$

t Test
quenched-ccc

Assuming equal variances

Difference $\quad 0.00588$ t Ratio $\quad 0.340065$ $\begin{array}{lrrr}\text { Std Err Dif } & 0.01729 & \text { DF } & 4 \\ \text { Upper CIf } & 0.05388 \text { Prob > } & 0.7509\end{array}$ $\begin{array}{llll}\text { Upper CL Dif } & 0.05388 & \text { Prob }>|t| & 0.7509 \\ \text { Lower CL Dif } & -0.04212 & \text { Prob }>t & 0.3755\end{array}$ $\begin{array}{llll} & \text { Confidence } & -0.95 \text { Prob }<\mathrm{t} & 0.6245\end{array}$

Analysis of Variance

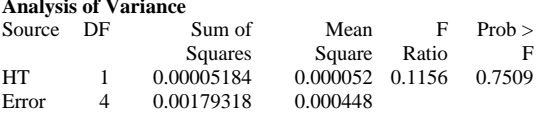

$\begin{array}{lll}\text { C. } & 5 & 0.00184503\end{array}$

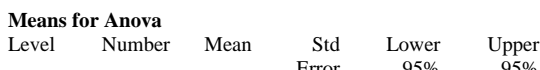

$\begin{array}{rrrrrr} & & \text { Etror } & \text { Lower } & \text { Upper } \\ \text { ccc } & 3 & 1.17418 & 050 & 95 \% \\ & 3 & 1.1805 & 0.01222 & 1.1402 & 1.2081\end{array}$

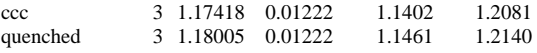

Std Error uses a pooled estimate of error variance

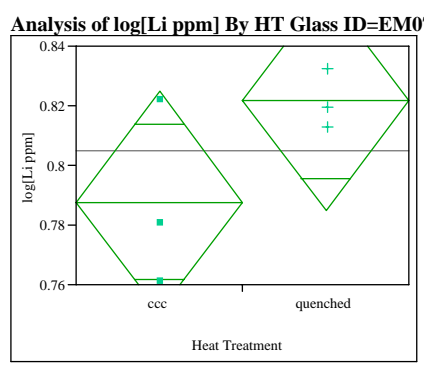

Anova

Summary of $F$

Rsquare
Adj Rsquare

\begin{tabular}{ll} 
Adj Rsquare & 0.448163 \\
Root Mean Square Error & 0.310204 \\
\hline & 0.023048
\end{tabular} Mean of Response 0.80470

Observations (or Sum Wgts)

t Test
quenched-ccc

Assuming equal variances

Difference $\quad 0.03392$ t Ratio 1.802364 Std Err Dif $\quad 0.01882$ DF $\quad 1.014$

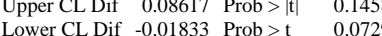
Confidence $\quad 0.95$ Prob $<\mathrm{t} \quad 0.927$

Analysis of Variance

$\begin{array}{llrrr}\text { Source DF Sum of Mean } & \text { F } & \text { Prob > }\end{array}$ $\begin{array}{llllll}\text { HT } & 1 & 0.00172559 & 0.001726 & 3.2485 & 0.1458 \\ \text { Error } & 4 & 0.0212477 & 0.00531 & & \end{array}$

$\begin{array}{lllll}\text { Means for Anova } & & & \\ \text { Level Number Mean Std Lower Upper }\end{array}$

$\begin{array}{rrrrrr} & & & \text { Error } & 95 \% & 95 \% \\ \text { ccc } & 3 & 0.787747 & 0.01331 & 0.75080 & 0.8246 \\ & 3 & 0.8264 & & & \end{array}$

$\begin{array}{lllllll}\text { quenched } & & 3 & 0.821664 & 0.01331 & 0.78472 & 0.85861\end{array}$

Std Error uses a pooled estimate of error variance

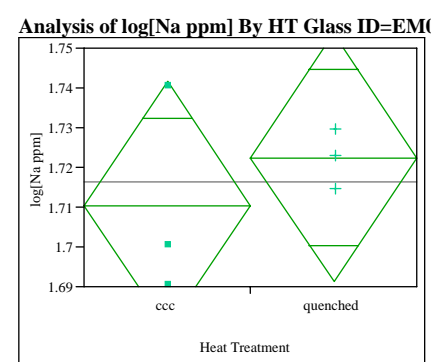

Anova

Summary of Fit

Rsquare

$\begin{array}{ll}\text { Adj Rsquare } & -0.09259 \\ \text { Root Mean Square Error } \quad 0.019493 & 0.7635\end{array}$ $\begin{array}{lr}\text { Mean of Response } & 1.716375 \\ \text { Observations (or Sum Wgts) } & 6\end{array}$

t Test

nched-ccc

Assuming equal variances

$\begin{array}{llll}\text { Difference } & 0.01208 \text { tRatio } \quad 0.759138\end{array}$ Std Err Dif 0.01592 DF $\quad 4 \quad 4$ Lower CL Dif -0.03211 Prob $>1 \quad 0.2450$ Confidence $\quad 0.95$ Prob $<\mathrm{t} \quad 0.7550$

\section{Analysis of Variance}

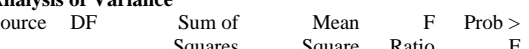
$\begin{array}{lllrrr}\text { HT } & 1 & 0.00021898 & 0.000219 & 0.5763 & 0.4900\end{array}$

$\begin{array}{lll} & & \\ \text { Error } & 4 & 0.00151995\end{array}$

$\begin{array}{lll}\text { c. } & 5 & 0.00173894\end{array}$

$\begin{array}{llll}\text { Means for Anova } & & & \\ \text { Level Number } & \text { Mean Std } \quad \text { Lower Upper }\end{array}$ 0.000380 $\begin{array}{lrrrrr} & & & \text { Error } & 95 \% & 95 \% \\ \text { ccc } & 3 & 1.71033 & 0.01125 & 1.6791 & 1.7416 \\ \text { quenched } & 3 & 1.72242 & 0.01125 & 1.6912 & 1.7537\end{array}$ Std Error uses a pooled estimate of error variance

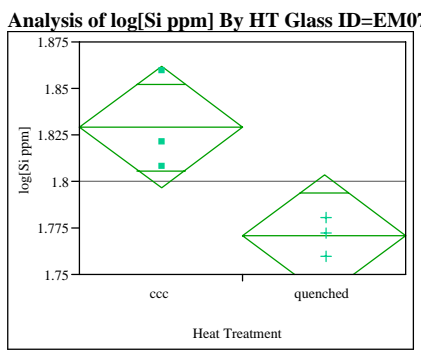

Anova

Summary of Fit

Rsquare

Root Mean Square Error $\quad 0.020503$ Mean of Response 1.7999

t Test
quenched-ccc

Assuming equal variances

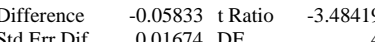
Upper CL Dif -0.01185 Prob $>|t| \quad 0.0253$ Lower CL Dif -0.10481 Prob $>t \quad 0.9874$ Confidence $\quad 0.95$ Prob $<\mathrm{t} \quad 0.012$

\section{Analysis of Variance}

Sum of Mean F Ratio Prob > $\begin{array}{llrrrr} & & \text { Squares } & \text { Square } & \text { F } \\ \text { HT } & 1 & 0.00510329 & 0.005103 & 12.1396 & 0.0253 \\ \text { Error } & 4 & 0.00168153 & 0.000420 & & \end{array}$ \begin{tabular}{lll} 
Error & 4 & 0.00168153 \\
\hline & 5 & 0.00678482
\end{tabular}

Means for Anova

Level Number Mean Std Lower Upper $\begin{array}{rrrrrr} & & & \text { Error } & 95 \% & 95 \% \\ \text { ccc } & 3 & 1.82910 & 0.01184 & 1.7962 & 1.8620\end{array}$ \begin{tabular}{l}
$-1.7379-1.8036$ \\
\hline
\end{tabular}

Std Error uses a pooled estimate of error varianc 
Figure B6. Effects of Heat Treatment (HT) on PCT $\log (\mathrm{ppm})$-Response of Study Glasses

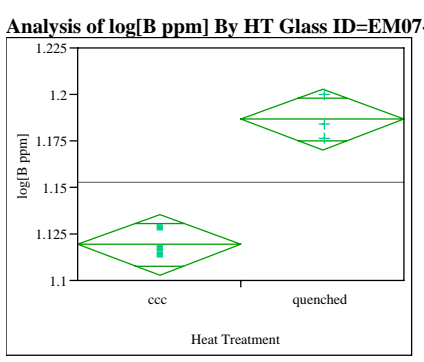

Anova

Summary of Fit

Rsquare
Adj Rsqu

0.928969
0.010127

Mean of Response 1.152944

Test

quenched-cce

Assuming equal variances

Difference $\quad 0.067372$ t Ratio $\quad 8.148139$ $\begin{array}{ll}0.0012 & 0\end{array}$ Lower CL Dif 0.044415 Prob $>t \quad 0.0006$ Confidence $\quad 0.95$ Prob $<\mathrm{t} \quad 0.9994$

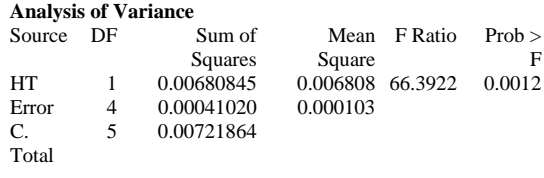

Total

Means for Anova

$\begin{array}{rrrr}\text { Level Number Mean } \begin{array}{rrr}\text { Std } \\ \text { Error }\end{array} & \begin{array}{r}\text { Lower } \\ 95 \%\end{array} & \text { Upper } \\ & 95 \%\end{array}$

$\begin{array}{lrrrrr} & & \text { Error } & 95 \% & 95 \% \\ \text { ccc } & 3 & 1.11926 & 0.00585 & 1.1030 & 1.1355\end{array}$

$1.1704 \quad 1.2029$

Std Error uses a pooled estimate of error variance

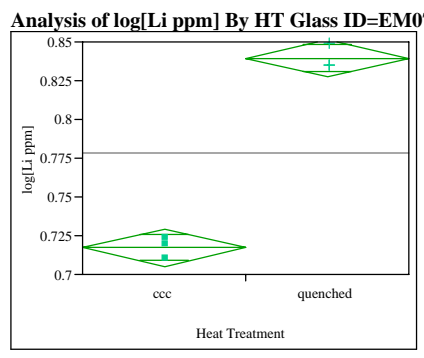

Anova

$\begin{array}{ll}\text { Rsquare } & 0.990132 \\ \text { Adj Rsquare } & 0.987664\end{array}$

Root Mean Square Error $\quad 0.007467$

Mean of Response
Observations (or Sum Wgts)

t Test

quenched-ccc

Assuming equal variances

Difference $\quad 0.122131$ t Ratio 20.03329 $\begin{array}{lllll}\text { Std Err Dif } & 0.006096 \text { DF } & 4\end{array}$ Upper CL Dif 0.139058 Prob $>|t| \quad<.0001$ $\begin{array}{lrr}\text { Lower CL Dif } & 0.105205 \text { Prob }>\mathrm{t} & <.0001 \\ \text { Confidence } & 0.95 \text { Prob }<\mathrm{t} & 1.0000\end{array}$

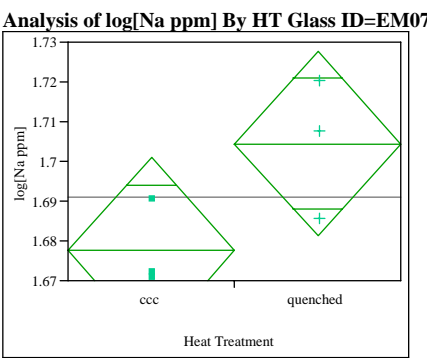

Anova
Summary of Fit

Rsquare

Adj Rsquare
Root Mean Squ Mean of Respons

\subsection{452216
0.014535}

t Test
quenched-ccc

Assuming equal variances

$\begin{array}{lll}\text { Difference } & 0.02687 & \text { t Ratio } \quad 2.264441\end{array}$ Std Err Dif 0.01187 DF $>4$ Lower CL Dif -0.00608 Prob $>t \quad 0.0431$ Confidence $\quad 0.95$ Prob $<\mathrm{t} 0.9569$

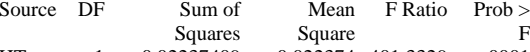
$\begin{array}{llrrrr} & & \text { Squares } & \text { Square } & & \\ \text { HT } & 1 & 0.02237409 & 0.022374 & 401.3329 & <.0001 \\ \text { Error } & 4 & 0.00022300 & 0.000056 & & \end{array}$

Means for Anova

Level Number Mean Std Lower $\begin{array}{llll}\text { Upper } \\ \end{array}$

$\begin{array}{lrrrrr} & & \text { Error } & 95 \% & 95 \% \\ \text { ccc } & 3 & 0.717371 & 0.00431 & 0.70540 & 0.72934\end{array}$

Std Error uses a pooled estimate of error variance
Analysis of Variance

$\begin{array}{lrrrrr}\text { Dource } & \text { DF } & \begin{array}{r}\text { Sum of } \\ \text { Squares }\end{array} & \begin{array}{r}\text { Mean } \\ \text { Square }\end{array} & \begin{array}{r}\text { Ratio } \\ \text { Rat }\end{array} & \begin{array}{r}\text { Prob > } \\ \text { F }\end{array} \\ & 1 & 0.00108332 & 0.001083 & 5.1277 & 00863\end{array}$

$\begin{array}{lll}\text { C. } & 4 & 0.00084508 \\ & 5 & 0.00192840\end{array}$ 0.000211

Means for Anova

$\begin{array}{rrrr}\text { Mtd } & \text { Lower } & \text { Upper } \\ \text { Error } & 95 \% & 95 \%\end{array}$

$\begin{array}{lrrrrr}\text { ccc } & 3 & 1.67763 & 0.00839 & 1.6543 & 1.75 \% \\ \text { quenched } & 3 & 1.70450 & 0.00839 & 1.6812 & 1.7278\end{array}$

Std Error uses a pooled estimate of error variance

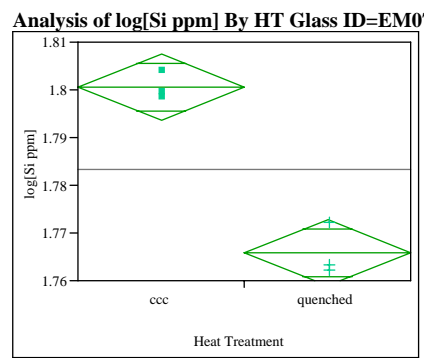

Anov

Summary of Fi

Rsquare
Adj Rsquare Mean of Response Error $\quad 0.004372$ Observations (or Sum Wgts)

t Test

Assuming equal variances

$\begin{array}{lll}\text { Difference } & -0.03459 \text { t Ratio } \quad-9.68993 \\ \text { Std } & 0.0037 \text { D D }\end{array}$ Std Err Dif 0.00357 DF -1009 $\begin{array}{rlll}\text { Lower CL Dif }-0.04450 & \text { Prob }>t & 0.0006 \\ \text { Cog } & 0.9997\end{array}$ Confidence $\quad 0.95$ Prob $<t \quad 0.0003$

\section{Analysis of Variance}

Source DF Sum of Mean F Ratio Prob > $\begin{array}{lrrrr} & & \text { Squares } & \text { Square } & \text { FT } \\ \text { HT } & 1 & 0.00179434 & 0.00179493 .8948 & 0.0006\end{array}$

$\begin{array}{llll}\text { Error } & 4 & 0.00007644 & 0.000019 \\ \text { C. } & 5 & 0.00187078 & \end{array}$

Means for Anova

Level Number Mean Std Lower Upper $\begin{array}{lrrrrr} & & & \text { Error } & 95 \% & 95 \% \\ \text { ccc } & 3 & 1.80049 & 0.00252 & 1.7935 & 1.8075\end{array}$ $\begin{array}{llllll}\text { quenched } & 3 & 1.76590 & 0.00252 & 1.7589 & 1.7729\end{array}$

Std Error uses a pooled estimate of error variance 
Figure B6. Effects of Heat Treatment (HT) on PCT log(ppm)-Response of Study Glasses

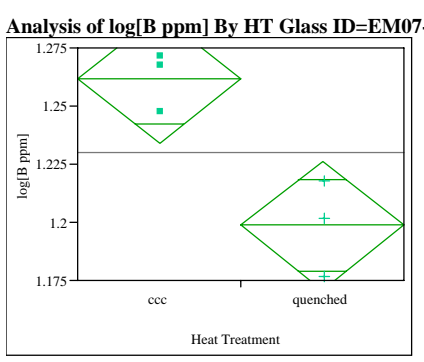

Anova

Summary of Fit

Rsquare
Adj Rsquare

Root Mean Square Error $\quad 0.017288$

$\begin{array}{lr}\text { Mean of Response } & 1.230223 \\ \text { Observations (or Sum Wgts) } & 6\end{array}$

t Test
quenched-ccc

Assuming equal variances

$\begin{array}{lll}\text { Difference } & -0.06317 \text { t Ratio } \quad-4.47485\end{array}$ Lower CL Dif -0.10236 Prob $>t \quad 0.9945$ $\begin{array}{lll}\text { Confidence } & 0.95 \mathrm{Prob}<\mathrm{t} \quad 0.0055\end{array}$

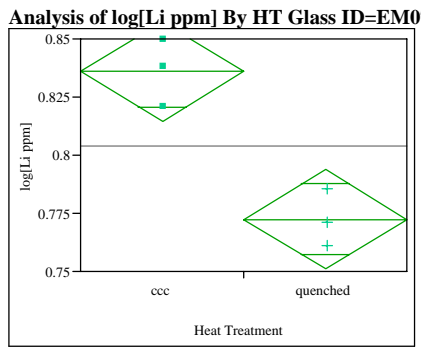

Anova

Summary of Fit

Rsquare

Root Mean Square Error $\quad 0.01337$

Mean of Response
Observations (or Sum Wgts)

t Test

quenched-ccc

Assuming equal variances

$\begin{array}{lll}\text { Difference } & -0.06352 \text { t Ratio } & -5.81826\end{array}$

Upper CL Dif -0.03321 Prob > It - 0.004

Lower CL Dif -0.09382 Prob $>t \quad 0.9978$

Confidence $\quad 0.95$ Prob $<\mathrm{t} \quad 0.0022$

Analysis of Variance
Source DF Sum of Mean F Ratio Prob >

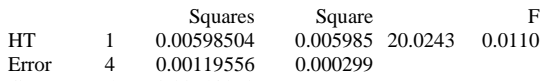

$5 \quad 0.00718060$

Total

Means for Anova

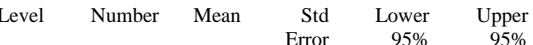

$\begin{array}{lrrrrr}\text { ccc } & 3 & 1.26181 & 0.00998 & 1.2341 & 1.2895 \\ & 3 & 1.19864 & 0.00998 & 1.1709 & \end{array}$

$\begin{array}{llllll}\text { quenched } & 3 & 1.19864 & 0.00998 & 1.1\end{array}$

Std Error uses a pooled estimate of error variance $\begin{array}{lll}\text { HT } & 1 & 0.00605129\end{array}$ $\begin{array}{llll}\text { Error } & 4 & 0.00071503 & 0.000179\end{array}$

c. Std Error uses a pooled estimate of error variance

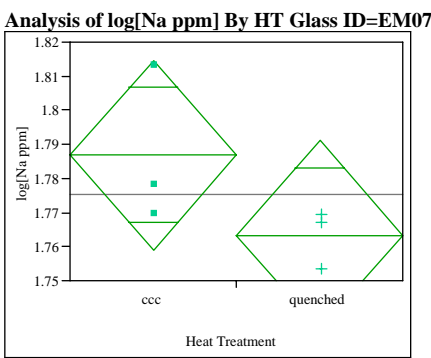

Anova

Summary of Fit

Rsquare
Adj Rsquare

\begin{tabular}{lr} 
Adj Rsquare & 0.407744 \\
Root Mean Square Error & 0.25968 \\
\hline
\end{tabular} $\begin{array}{lr}\text { Mean of Response } & 1.775141 \\ \text { Observations (or Sum Wgts) }\end{array}$

t Test
quenched-ccc

Assuming equal variances

Difference $\quad-0.02353$ t Ratio $\quad-1.65947$ \begin{tabular}{lll} 
Std Err Dif & 0.01418 DF & 4 \\
\hline
\end{tabular}

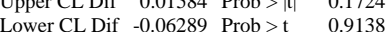

Analysis of Varianc

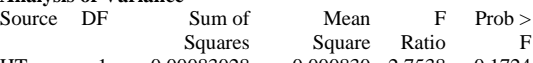
\begin{tabular}{llr} 
HT & 1 & 0.00083028 \\
\hline & 4 & 0.00203628
\end{tabular} \begin{tabular}{lll} 
Error & 4 & 0.000120200 \\
C & 5 & 0.00203628 \\
\hline & &
\end{tabular} $\begin{array}{rrr}\text { Square } & \text { Ratio } & \text { F } \\ 0.000830 & 2.7538 & 0.1724 \\ 0.000302 & & \end{array}$ 0.000302 $\begin{array}{rrr}\text { Square } & & \\ 0.006051 & 33.8521 & 0.00\end{array}$ To

\section{$\underset{\text { Level Number }}{\text { Means for Anova }}$ Mean Std Lower Upper Means for Anova}

Level Number Mean Std Lower Upper $\begin{array}{lllllll}\text { ccc } & 3 & 0.835893 & 0.00772 & 0.81446 & 0.85732 \\ \text { quenched } & & 3 & 0.772378 & 0.00772 & 0.75095 & 0.7938\end{array}$ $\begin{array}{llrrrr}\text { ccc } & 3 & 1.78690 & 0.01002 & 1.7591 & 1.8147 \\ \text { quenched } & 3 & 1.76338 & 0.01002 & 1.7355 & \end{array}$ Std Error uses a pooled estimate of error variance Confidence $\quad 0.95$ Prob $<\mathrm{t} \quad 0.0862$

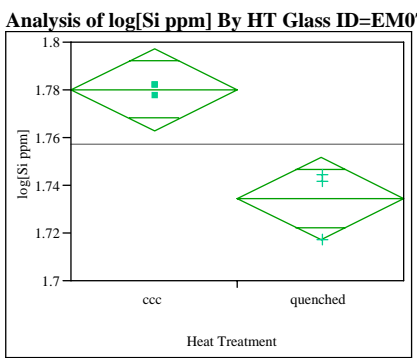

Anova

Summary of Fi

Rsquare
Adj Rsquare Mean of Response $\quad 0.01067$ Observations (or Sum Wgts)

t Test
quenched-ccc

Assuming equal variances

Difference $\quad-0.04570$ t Ratio $\quad-5.24477$ Std Err Dif $0.00871 \mathrm{DF} \quad-50.2477$ Lower CL Dif -0.06989 Prob $>t \quad 0.9968$ Confidence $\quad 0.95$ Prob $<\mathrm{t} \quad 0.0032$

\section{Analysis of Variance}

Source DF Sum of Mean F Ratio Prob > $\begin{array}{llrrrr}\text { HT } & 1 & 0.00313233 & 0.003132 & 27.5076 & 0.0063\end{array}$

$\begin{array}{llll}\text { Error } & 4 & 0.00045549 & 0.000114 \\ \text { C. } & 5 & 0.00358782 & \end{array}$

Means for Anova $\begin{array}{rrrrr}\text { Level Number } & \text { Mean } & \begin{array}{r}\text { Std } \\ \text { Error }\end{array} & \begin{array}{r}\text { Lower } \\ 95 \%\end{array} & \begin{array}{l}\text { Upper } \\ 95 \%\end{array}\end{array}$ $\begin{array}{llllll}\text { ccc } & 3 & 1.78016 & 0.00616 & 1.7631 & 1.7973 \\ \text { quenched } & 3 & 1.73446 & 0.00616 & 1.7174 & 1.7516\end{array}$

Std Error uses a pooled estimate of error variance 
Figure B6. Effects of Heat Treatment (HT) on PCT $\log (\mathrm{ppm})$-Response of Study Glasses

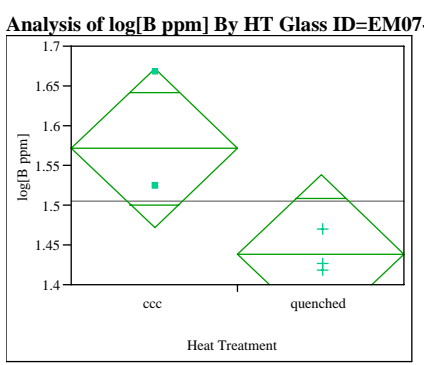

Anov

Summary of Fit

Rsquare
Adj Rsquare

0.062253
0.54

Observations (or Sum Wgts) $\begin{array}{r}1.504467 \\ 6\end{array}$

t Test

Assuming equal variances

\begin{tabular}{llll} 
Difference & -0.13322 t Ratio $\quad-2.62099$ \\
\hline
\end{tabular} $\begin{array}{llr}\text { Std Err Dif } & 0.05083 \text { DF } & 4 \\ \text { Upper CLD } & 0.00790 \text { Prob }>\text { th } & 0.0587\end{array}$ $\begin{array}{llll}\text { Upper CL Dif } & 0.00790 & \text { Prob }>|t| & 0.0587 \\ \text { Lower CL Dif } & -0.27435 & \text { Prob }>t & 0.9706\end{array}$ $\begin{array}{lrl} & \text { Confidence } & 0.95 \text { Prob }<\mathrm{t} \quad 0.0294\end{array}$

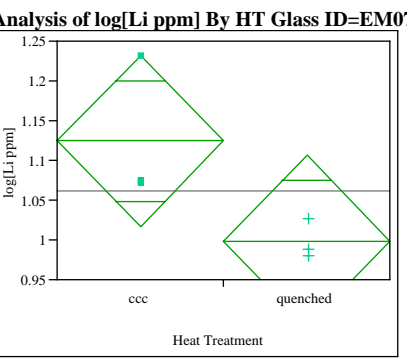

Anova

Summary of Fit

Rsquare Mean of Response 1.06156

Observations (or Sum Wgts)

t Test
quenched-ccc

Assuming equal variances

Difference $\quad-0.12595$ t Ratio -2.29956 Std Err Dif $0.05477 \mathrm{DF}$ Lower CL Dif - 0.027801 Prob $>t \quad 0.9585$ Confidence $\quad 0.95$ Prob $<\mathrm{t} \quad 0.0415$

\section{Analysis of Variance}

Analysis of Varian

\begin{tabular}{lrrrrr} 
Source & DF & $\begin{array}{r}\text { Sum of } \\
\text { Squares }\end{array}$ & $\begin{array}{r}\text { Mean } \\
\text { Square }\end{array}$ & $\begin{array}{r}\text { Ratio } \\
\text { Rato }\end{array}$ & F \\
\hline & 1 & 0.02662286 & 0.026623 & 6.8696 & 0.0587
\end{tabular}

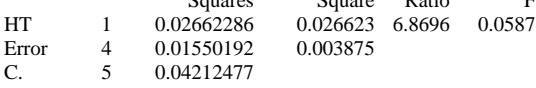

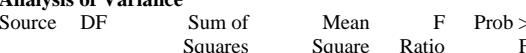

$\begin{array}{llrrrr}\text { HT } & 1 & 0.02379324 & 0.023793 & 5.2880 & 0.0830 \\ \text { Error } & 4 & 0.01799802 & 0.004500 & & \end{array}$

$\begin{array}{lllll}\text { Means for Anova } & & & \\ \text { Level Number Mean Std Lower Upper }\end{array}$

$\begin{array}{lrrrrr} & \text { Number } & \text { Mean } & \begin{array}{r}\text { Std } \\ \text { Error }\end{array} & \begin{array}{r}\text { Lower } \\ 95 \%\end{array} & \text { Upper } \\ \text { ccc } & 3 & 1.57108 & 0.03594 & 1.4713 & 1.670 \\ & 3 & 1.4378 & 0.03594 & 1.3381 & 1.536\end{array}$

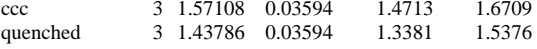

Std Error uses a pooled estimate of error variance

$\begin{array}{lllll}\text { Means for Anova } & & & \\ \text { Level Number Mean Std Lower Upper }\end{array}$

quenched

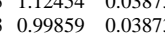

Std Error uses a pooled estimate of error variance $\begin{array}{ll}\text { Adj Rsquare } & 0.5691356 \\ \text { Root Mean Square Error } & 0.067078\end{array}$

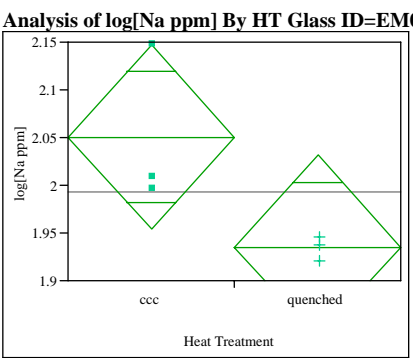
$\begin{array}{llrrrr}\text { HT } & 1 & 0.02008161 & 0.020082 & 5.5238 & 0.0785 \\ \text { Error } & 4 & 0.01454191 & 0.003635 & & \end{array}$

Error $4 \quad 0.01454191$

$\begin{array}{lrrrrr} & & \text { Error } & 95 \% & 95 \% \\ \text { ccc } & 3 & 1.12454 & 0.03873 & 1.0170 & 1.2321 \\ & 3 & 0.958 & 0.0383 & 0.891 & 1.06\end{array}$
Anova

Anova
Summary of Fit

Rsquare

0.474999
Root Mean Square Error $\quad 0.060295$ $\begin{array}{lr}\text { Mean of Response } & 1.992793 \\ \text { Observations (or Sum Wgts) } & 6\end{array}$

t Test

Assuming equal variances

$\begin{array}{llll}\text { Difference } & -0.11571 \text { t Ratio } & -2.35027\end{array}$ $\begin{array}{lrlr}\text { Std Err Dif } & 0.04923 \text { DF } & 4 \\ \text { Uper CL Dif } & 0.02098 \text { Prob > tt } & 0.0785\end{array}$ Lower CL Dif -0.25239 Prob $>t \quad 0.9608$ Confidence $\quad 0.95$ Prob $<\mathrm{t} \quad 0.0392$

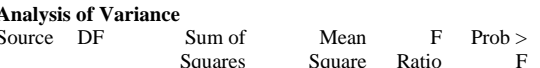

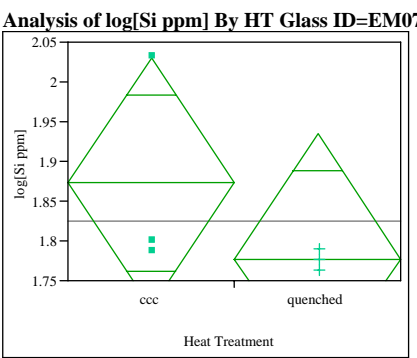

Anova

Summary of $F$

Rsquare
Adj Rsquare Mean of Response $\quad 0.09819$ Observations (or Sum Wgts)

t Test

Assuming equal variances

$\begin{array}{lll}\text { Difference } & -0.09584 \mathrm{t} \text { Ratio } & -1.19539\end{array}$ Std Err Dif 0.08017 DF 1.1929 Lower CL Dif -0.31843 Prob $>t \quad 0.8510$ Confidence $\quad 0.95$ Prob $<\mathrm{t} \quad 0.1490$

\section{Analysis of Variance}

$\begin{array}{lllll}\text { Source DF Sum of Mean F Prob > } & \end{array}$ $\begin{array}{llrrrr} & & \text { Squares } & \text { Square } & \text { Ratio } & \\ \text { HT } & 1 & 0.01377748 & 0.013777 & 1.4290 & 0.2980\end{array}$ $\begin{array}{llll}\text { Error } & 4 & 0.03856628 & 0.009642 \\ \text { C. } & 5 & 0.05234376 & \end{array}$

C.

Level Number Mean Std Lower Upper

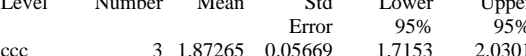

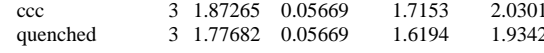

Std Error uses a pooled estimate of error variance 
Figure B6. Effects of Heat Treatment (HT) on PCT $\log (\mathrm{ppm})$-Response of Study Glasses

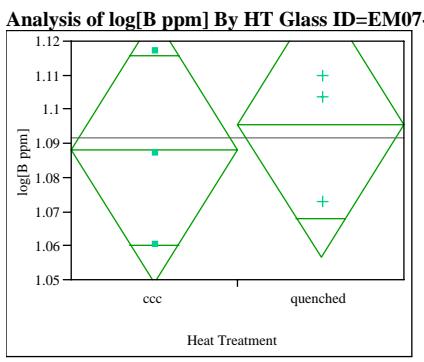

Anov

Anova
Summary of Fit

Rsquare
Adj Rsquare

Error $\quad 0.024431$

$\begin{array}{lr} & 1.091788 \\ \text { Observations (or Sum Wgts) } & 6\end{array}$

t Test

Assuming equal variances

Difference $\quad 0.00773$ t Ratio $\quad 0.387684$

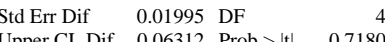
$\begin{array}{llll}\text { Upper CL Dif } & 0.06312 & \text { Prob }>|t| & 0.7180 \\ \text { Lower CL Dif } & -0.04765 & \text { Prob }>t & 0.3590\end{array}$ $\begin{array}{lrl}\text { Confidence } & -0.04 \text { Prob }<t \quad 0.6410\end{array}$

Analysis of Variance

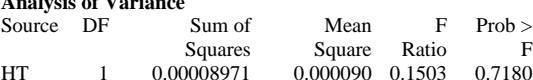

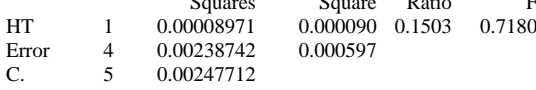

$\begin{array}{lrrrr}\text { Means for Anova } & & & & \\ \text { Level Number } & \text { Mean } \quad \text { Std } & \text { Lower } & \text { Upper } \\ & & \text { Error } & 95 \% & 95 \%\end{array}$

$\begin{array}{rrrrrr} & & & \text { Error } & 95 \% & 95 \% \\ \text { ccc } & 3 & 1.08792 & 0.01411 & 1.0488 & 1.127\end{array}$

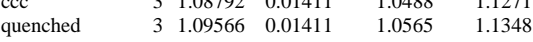

Std Error uses a pooled estimate of error variance

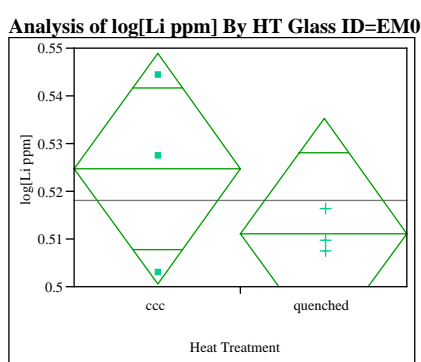

Anova

Summary of Fit

Rsquare
Adj Rsquare

$\begin{array}{lr} & 0.235376 \\ \text { Adj Rsquare } & 0.04422 \\ \text { Root Mean Square Error } & 0.015003\end{array}$ Mean of Response $\quad 0.51792$

Observations (or Sum Wgts)

t Test
quenched-ccc

Assuming equal variances

Difference $\quad-0.01359$ t Ratio $\quad-1.10965$ Std Err Dif 0.01225 DF $>14 \quad 0.3294$ Lower CL Dif -0.04760 Prob $>t \quad 0.8353$ $\begin{array}{lll}\text { Confidence } & 0.95 \text { Prob }<\mathrm{t} \quad 0.1647\end{array}$

Analysis of Variance

Source DF Sum of Mean F Prob > $\begin{array}{llllll}\text { HT } & 1 & 0.00027715 & 0.000277 & 1.2313 & 0.3294 \\ \text { Error } & 4 & 0.00090033 & 0.000225 & & \end{array}$

C.

Total

Means for Anova

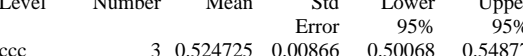

$\begin{array}{lllllll}\text { ccc } & 3 & 0.524725 & 0.00866 & 0.50068 & 0.54877 \\ \text { quenched } & & 3 & 0.511133 & 0.00866 & 0.48708 & 0.53518\end{array}$

Std Error uses a pooled estimate of error variance

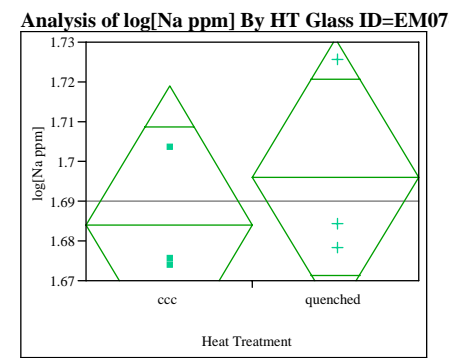

Anova

Summary of Fit

Rsquare

$\begin{array}{ll} & 0.102575 \\ \text { Adj Rsquare } & -0.12178 \\ \text { Root Mean Square Eror } & 0.02174\end{array}$ $\begin{array}{lr}\text { Mean of Response } & 1.690041 \\ \text { Observations (or Sum Wgts) } & 6\end{array}$

t Test

Assuming equal variances

Difference $\quad 0.01200$ tRatio $\quad 0.676162$

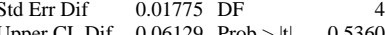
Lower CL Dif -0.03728 Prob $>t \quad 0.5360$ Confidence $\quad 0.95$ Prob $<\mathrm{t} \quad 0.7320$

\section{nalysis of Variance}

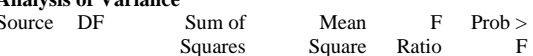
$\begin{array}{lllrrr}\text { HT } & 1 & 0.00021609 & 0.000216 & 0.4572 & 0.5360\end{array}$

$\begin{array}{lll}\text { E. } & 4 & 0.00189055 \\ \text { C. } & 5 & 0.00210664\end{array}$ 0.000473

\section{Means for Anova}

Level Number Mean Std Lower Upper

$\begin{array}{lrrrrr}\text { ccc } & 3 & 1.68404 & \text { Error } & 95 \% & 95 \% \\ & 3 & & 9.01255 & 1.6492 & 1.7189\end{array}$

Std Error uses a pooled estingte of enor vand

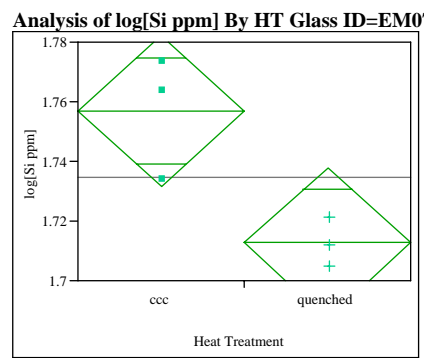

Anova

Summary of Fit

Rsquare
Adj Rsquare Mean of Response $\quad 0.015738$

Observations (or Sum Wgts)

t Test
quenched-ccc

Assuming equal variances

Difference $\quad-0.04413$ t Ratio $\quad-3.43426$ Std Err Dif 0.01285 DF $\quad-3.434$ Lower CL Dif -0.07981 Prob $>t \quad 0.9868$ Confidence $\quad 0.95$ Prob $<\mathrm{t} \quad 0.0132$

\section{Analysis of Variance}

DF Sum of Mean F Ratio Prob > $\begin{array}{llrrrr} & & \text { Squares } & \text { Square } & & \text { F } \\ \text { HT } & 1 & 0.00292134 & 0.002921 & 11.7941 & 0.0264 \\ \text { Error } & 4 & 0.00099078 & 0.000248 & & \end{array}$ $\begin{array}{llll}\text { Error } & 4 & 0.00099078 & 0.000248 \\ \text { C. } & 5 & 0.00391211 & \end{array}$

Means for Anova

$\begin{array}{lllll} & & & \\ \text { Level Number Mean Std Lower Upper }\end{array}$ $\begin{array}{lrrrrr} & & & \text { Error } & 95 \% & 95 \% \\ \text { ccc } & 3 & 1.75683 & 0.00909 & 1.7316 & 1.7821 \\ \text { quenched } & & 1.71270 & 0.00909 & 1.6875 & 1.7379\end{array}$ Std Error uses a pooled estimate of error variance 
Figure B6. Effects of Heat Treatment (HT) on PCT $\log (\mathrm{ppm})$-Response of Study Glasses

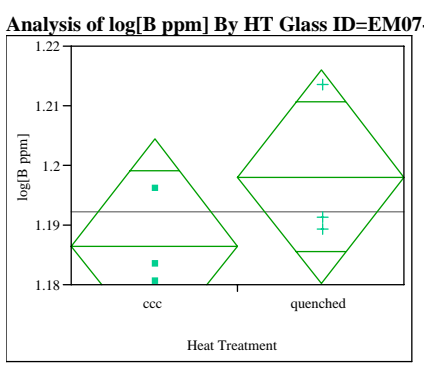

Anova

Anova

Rsquare
Adj Rsquare

Error $\quad 0.011136$

$\begin{array}{lr}\text { Mean of Response } & 1.192302 \\ \text { Observations (or Sum Wgts) } & 6\end{array}$

t Test

Assuming equal variances

$\begin{array}{lll}\text { Difference } & 0.01153 \text { t Ratio } \quad 1.268372\end{array}$ $\begin{array}{lllr}\text { Std Err Dif } & 0.00909 & \text { DF } & 4 \\ \text { Upper CL Dif } & 0.03678 \text { Prob }>\text { tt } & 0.2735\end{array}$ $\begin{array}{llll}\text { Upper CL Dif } & 0.03678 & \text { Prob }>|t| & 0.2735 \\ \text { Lower CL Dif }-0.01371 & \text { Prob }>t & 0.1367\end{array}$

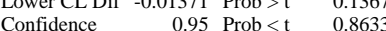

Root Mean Square Error $\quad 0.004462$

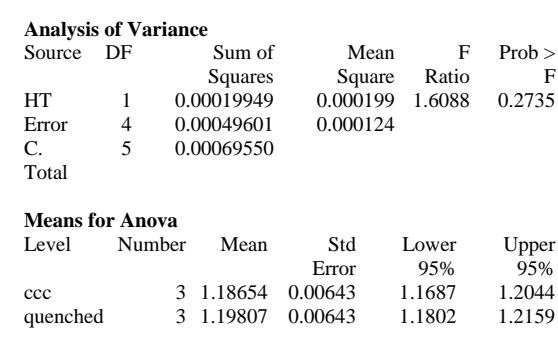

Std Error uses a pooled estimate of error variance

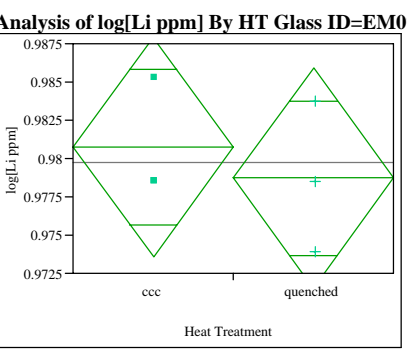

Anova

Summary of Fit

Rsquare

Adj Rsquare $\quad \begin{array}{ll}0.07198 \\ -0.16002\end{array}$

Observations (or Sum Wots)

t Test

quenched-ccc

Assuming equal variances

Difference $\quad-0.00203$ t Ratio $\quad-0.557$ $\begin{array}{llr}\text { Std Err Dif } & 0.00364 \text { DF } & 4 \\ \text { L } & \end{array}$

Upper CL Dif 0.00809 Prob $>|t| 0.6072$

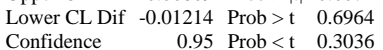

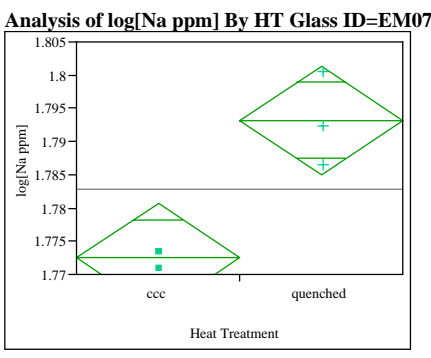

Anova

Summary of Fit

$\begin{array}{ll}\text { Rsquare } & 0.861939 \\ \text { Adj Rsquare } & 0.827424\end{array}$

Root Mean Square Error $\quad 0.005061$

$\begin{array}{lr}\text { Mean of Response } & 1.782816 \\ \text { Observations (or Sum Wgts) } & 6\end{array}$

t Test
quenched-ccc

Assuming equal variances

Difference $\quad 0.020648$ tRatio $\quad 4.997275$ 4
$\mathrm{DF}$ Lower CL Dif 0.009176 Prob $>t \quad 0.0038$ Confidence $\quad 0.95$ Prob $<\mathrm{t} \quad 0.9962$

Analysis of Variance

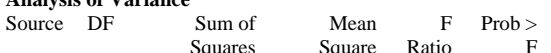
$\begin{array}{rrrrrr} & & \text { Squares } & \text { Square } & \text { Ratio } & \text { F } \\ \text { HT } & 1 & 0.00000618 & 6.177 \mathrm{e}-6 & 0.3103 & 0.6072\end{array}$ $5 \quad 0.00008581$

$\begin{array}{lllll}\text { Means for Anova } & & & & \\ \text { Level Number Mean Std Lower Upper }\end{array}$ $\begin{array}{lrrrrr} & & & \text { Error } & 95 \% & 95 \% \\ \text { ccc } & 3 & 0.980758 & 0.00258 & 0.97361 & 0.98791\end{array}$

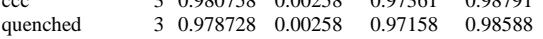

Std Error uses a pooled estimate of error variance

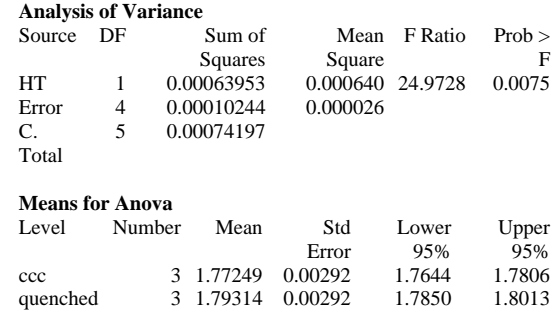

Std Error uses a pooled estimate of error variance

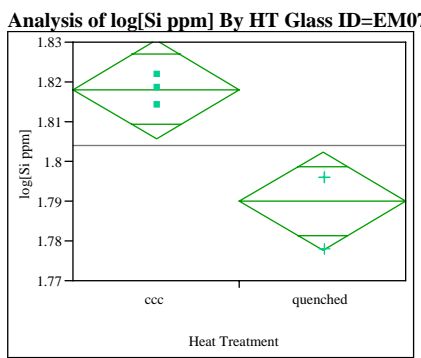

Anova

Summary of $F$

Rsquare
Adj Rsquare

\begin{tabular}{ll} 
Adj Rsquare & 0.831805 \\
Root Mean Square & 0.789757 \\
\hline
\end{tabular} Mean of Response $\quad 1.004737$

Observations (or Sum Wgts)

t Test
quenched-ccc

Assuming equal variances

Difference $\quad-0.02810$ t Ratio $\quad-4.44769$ Std Err Dif 0.00632 DF $>$ |t $\quad 0.411$ Lower CL Dif -0.04564 Prob $>t \quad 0.0944$ Confidence $\quad 0.95$ Prob $<\mathrm{t} \quad 0.0056$

\section{Analysis of Variance}

Sum of Mean F Ratio Prob > $\begin{array}{llrrrr} & & \text { Squares } & \text { Square } & \text { F } \\ \text { HT } & 1 & 0.00118419 & 0.001184 & 19.7820 & 0.0113\end{array}$

Error $\quad 4 \quad 0.00023945$

$\begin{array}{lll}\text { C. } \quad 5 & 0.0014236\end{array}$ 0.000060

Means for Anova

Level Number Mean Std Lower Upper $\begin{array}{lrrrrr} & & & \text { Error } & 95 \% & 95 \% \\ \text { ccc } & 3 & 1.81808 & 0.00447 & 1.8057 & 1.8305\end{array}$ $\begin{array}{llllll}\text { quenched } & 3 & 1.78998 & 0.00447 & 1.7776 & 1.802\end{array}$ Std Error uses a pooled estimate of error variance 
Figure B6. Effects of Heat Treatment (HT) on PCT log(ppm)-Response of Study Glasses

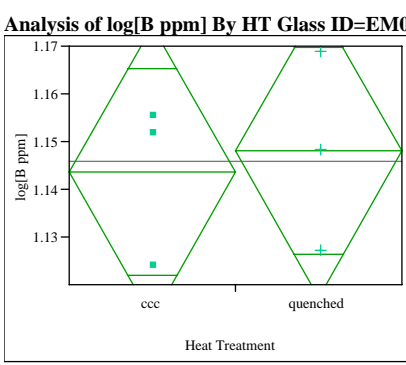

Anov

Summary of Fit

Rsquare
Adj Rsquare

$\begin{array}{lr}\text { Mean of Response } & 1.145851 \\ \text { Observations (or Sum Wgts) } & 6\end{array}$

t Test

Assuming equal variances

Difference $\quad 0.00437$ t Ratio $\quad 0.280059$ $\begin{array}{llll}\text { Std Err Dif } & 0.01562 \text { DF } & 4 \\ \text { Upper CIf } & 0.04744 \text { Prob }>\text { (t) } & 0.7933\end{array}$ Upper CL Dif 0.04774 Prob $>|t| \quad 0.7933$ Confidence $\quad 0.95$ Prob $<\mathrm{t} \quad 0.6033$

\begin{tabular}{|c|c|c|c|c|c|}
\hline \\
\hline \multicolumn{3}{|c|}{$\begin{array}{l}\text { Analysis of Variance } \\
\text { Source DF of }\end{array}$} & $\begin{array}{r}\text { Mean } \\
\text { Square }\end{array}$ & Ratio & $\begin{array}{r}\text { Prob }> \\
F\end{array}$ \\
\hline HT & 1 & 0.00002870 & 0.000029 & 0.0784 & 0.7933 \\
\hline Error & 4 & 0.00146356 & 0.000366 & & \\
\hline C. & 5 & .00149226 & & & \\
\hline \multirow{2}{*}{\multicolumn{6}{|c|}{ Means for Anova }} \\
\hline & & & & & \\
\hline & Number & IViean & $\begin{array}{l}\text { Std } \\
\text { Error }\end{array}$ & $\begin{array}{l}\text { Lower } \\
95 \%\end{array}$ & $\begin{array}{r}\text { Upper } \\
95 \%\end{array}$ \\
\hline ccc & & $\begin{array}{lll}3 & 1.14366\end{array}$ & 0.01104 & 1.1130 & 1.1743 \\
\hline quenched & & $\begin{array}{lll}3 & 1.14804\end{array}$ & 0.01104 & 1.1174 & 1.1787 \\
\hline
\end{tabular}

Std Error uses a pooled estimate of error variance

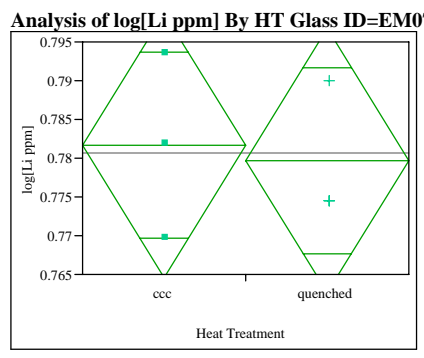

Anova

Rsquare

Root Mean Square Error $\quad 0.010575$

Mean of Response $\quad 0.780679$

t Test

quenched-ccc

Assuming equal variances

\begin{tabular}{lll} 
Difference & -0.00195 t Ratio & -0.22592 \\
\hline
\end{tabular}

$\begin{array}{llll}\text { Std Err Dif } 0.00863 \text { DF } & 4\end{array}$

$\begin{array}{llll}\text { Upper CL Dif } & 0.02202 & \text { Prob }>|t| & 0.8323 \\ \text { Cower CL Dif } & -0.0252 & \text { Prob }>t & 0.5838\end{array}$

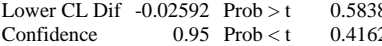

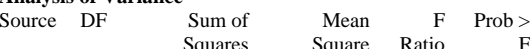

$\begin{array}{llrrrr} & & \text { Squares } & \text { Square } & \text { Ratio } & \\ \text { HT } & 1 & 0.00000571 & 5.708 \mathrm{e}-6 & 0.0510 & 0.8323\end{array}$

$\begin{array}{llll}\text { Error } & 4 & 0.00044730 & 0.000112\end{array}$

Means for Anova
Level Number $\quad$ Mean $\begin{array}{rrr}\text { Std } & \text { Lower } & \text { Upper } \\ & & \end{array}$

$\begin{array}{lrrrrr} & & \text { Error } & 95 \% & 95 \% \\ \text { ccc } & 3 & 0.781654 & 0.00611 & 0.76470 & 0.7986\end{array}$

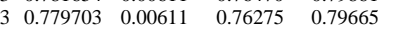

Std Error uses a pooled estimate of error variance

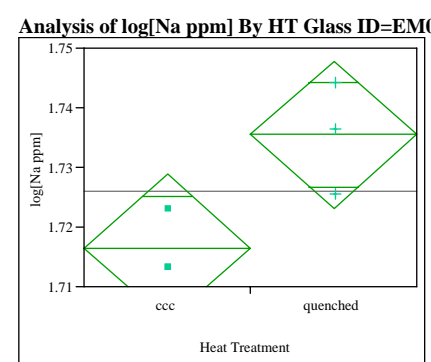

Anova

Summary of Fit

$\begin{array}{ll}\text { Rsquare } & 0.694644 \\ \text { Adj Rsquare } & 0.618305 \\ \text { Root Mean Square Error } & 0.007714\end{array}$ Mean of Response 1.725952

Observations (or Sum Wgts) 6

t Test
quenched-ccc

Assuming equal variances

$\begin{array}{lll}\text { Difference } & 0.019000 & \text { t Ratio } \\ 3.016529\end{array}$ Std Err Dif 0.006299 DF 1 Lower CL Dif 0.001512 Prob > t 0.0196 Confidence $\quad 0.95$ Prob $<$ t 0.9804

Analysis of Variance

$\begin{array}{lllll}\text { Source DF Sum of Mean } & F & \text { Prob }>\end{array}$ \begin{tabular}{llr} 
HT & 1 & 0.00054150 \\
\hline
\end{tabular}

$\begin{array}{lll}\text { Error } & 4 & 0.00023804 \\ \text { C. } & 5 & 0.00077954\end{array}$ $\begin{array}{rrr}\text { Square } & \text { Ratio } & \text { P } \\ 0.000542 & 9.0995 & 0.0393\end{array}$ 0.000060

Total

$\begin{array}{lllll} & & & \\ \text { Level Number Mean Std } & \text { Lower } & \text { Upper } \\ 95 \% & 95 \%\end{array}$

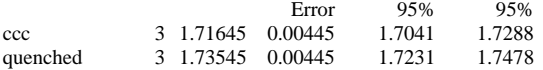

Std Error uses a pooled estimate of error variance

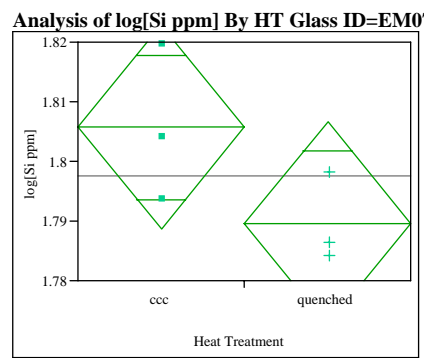

Anov

Summary of $F$

Rsquare
Adj Rsquare

\begin{tabular}{ll} 
Adj Rsquare & 0.459202 \\
Root Mean Square Enor & 0.324002 \\
\hline & 0.09067
\end{tabular} Mean of Response $\quad 0.0106$

Observations (or Sum Wgts)

t Test

Assuming equal variances

$\begin{array}{lll}\text { Difference } & -0.01606 \mathrm{t} \text { Ratio } & -1.84295 \\ \text { Std } & 0.0071 \text { DF }\end{array}$

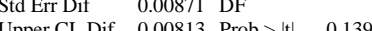
Lower CL Dif -0.04024 Prob $>t \quad 0.9304$ Confidence $\quad 0.95$ Prob $<\mathrm{t} \quad 0.0696$

\section{Analysis of Variance}

$\begin{array}{rrrr}\text { Sum of Mean } & F & \text { Prob > }\end{array}$ $\begin{array}{llllll}\text { HT } & 1 & 0.00038668 & 0.000387 & 3.3965 & 0.1391 \\ \text { Error } & 4 & 0.0004553 & 0.0114 & & \end{array}$ $\begin{array}{llll}\text { C. } & 5 & 0.00045539 & 0.000114 \\ \text { C. } & & 0.00084207 & \\ \end{array}$

Means for Anova

$\begin{array}{llrl}\text { Level Number for Anova } & & & \\ \text { Mean } \quad \text { Std } & \text { Lower } & \text { Upper }\end{array}$ $\begin{array}{lrrrrr} & & \text { Error } & 95 \% & 95 \% \\ \text { cсc } & 3 & 1.80568 & 0.00616 & 1.7886 & 1.8228\end{array}$ $\begin{array}{llllll} & & & & \\ \text { quenched } & 3 & 1.785662 & 0.00616 & 1.7886 & 1.8228 \\ & & & & & \end{array}$

Std Error uses a pooled estimate of error variance 
Figure B6. Effects of Heat Treatment (HT) on PCT log(ppm)-Response of Study Glasses

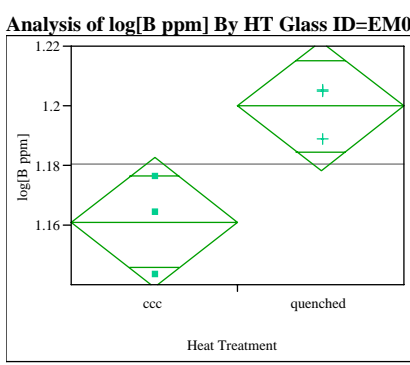

Anov

Summary of Fit

Rsquare
Adj Rsquare

$\begin{array}{lr}\text { Mean of Response } & 1.180407 \\ \text { Observations (or Sum Wgts) } & 6\end{array}$

t Test

Assuming equal variances Std Err Dif 0.011083 DF Upper CL Dif 0.069594 Prob $>|t| \quad 0.024$ Confidence $\quad 0.95$ Prob $<\mathrm{t} \quad 0.9876$
Difference $\quad 0.038821$ t Ratio $\quad 3.502652$
Analysis of $\log [\mathrm{Li}$ ppm] By HT Glass ID=EM07-Mn-04

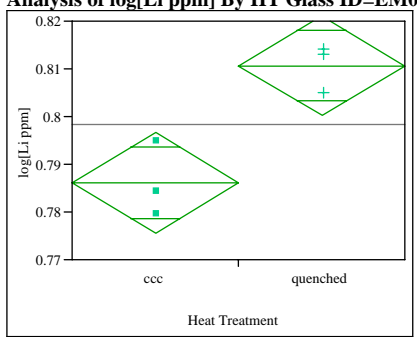

Anova

Summary of Fi

Rsquare

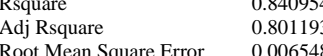
Mean of Response 0.798376

Observations (or Sum Wgts)

t Test
quenched-ccc

Assuming equal variances

Difference $\quad 0.024589$ t Ratio $\quad 4.598914$ Std Err Dif 0.005347 DF $\quad 4$ Lower CL Dif 0.009744 Prob $>t \quad 0.0050$ Confidence $\quad 0.95$ Prob $<\mathrm{t} \quad 0.9950$

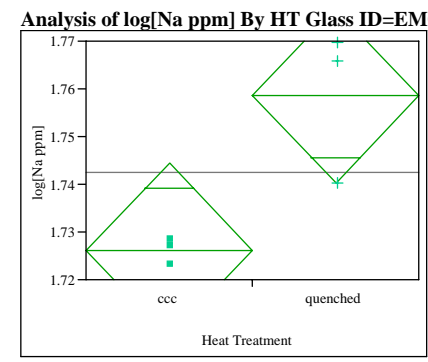

Anova

Summary of Fit

$\begin{array}{ll}\text { Rsquare } & 0.752111 \\ \text { Adj Rsquare } & 0.690138 \\ \text { Root Mean Square Eror } & 0.011443\end{array}$ Mean of Response 1.742369 Observations (or Sum Wgts) $\quad 6$

t Test
quenched-ccc

Assuming equal variances

$\begin{array}{lll}\text { Difference } & 0.032547 \text { t Ratio } \quad 3.483709\end{array}$ Std Err Dif 0.009343 DF $>4$ Lower CL Dif 0.006608 Prob $>1 \quad 0.0126$ Confidence $\quad 0.95$ Prob $<\mathrm{t} \quad 0.9874$

Analysis of Variance

Analysis of Variance

\begin{tabular}{lrrrrr}
\multicolumn{2}{l}{ Analysis of Variance } & & & \\
Source & DF & $\begin{array}{r}\text { Sum of } \\
\text { Squares }\end{array}$ & $\begin{array}{r}\text { Mean } \\
\text { Square }\end{array}$ & F Ratio & Prob > \\
HT & 1 & 0.00226066 & 0.002261 & 12.2686 & 0.0248 \\
Error & 4 & 0.00073706 & 0.000184 & &
\end{tabular}

$\begin{array}{lll}\text { C. } & 5 & 0.00299772\end{array}$

\section{Source DF Sum of}

$\begin{array}{llllll}\text { HT } & 1 & 0.00090696 & 0.000907 & 21.1500 & 0.0100\end{array}$

Error $\quad 4 \quad 0.0001715$

0.00107849

0.0000

Means for Anova

$\begin{array}{lllll} & & & & \\ \text { Level Number Mean } & \text { Std } & \text { Lower } & \text { Upper }\end{array}$

$\begin{array}{lrrrrr} & & & \text { Error } & 95 \% & 95 \% \\ \text { ccc } & 3 & 1.16100 & 0.00784 & 1.1392 & 1.1828\end{array}$

$\begin{array}{llllll}\text { quenched } & 3 & 1.19982 & 0.00784 & 1.1781 & 1.2216\end{array}$

Std Error uses a pooled estimate of error variance $\begin{array}{llll}\text { Means for Anova } & & & \\ \text { Level Number Mean Std Lower Upper }\end{array}$

$\begin{array}{lrrrrr} & & \text { Error } & 95 \% & 95 \% \\ \text { ccc } & 3 & 0.786081 & 0.00378 & 0.77558 & 0.79658\end{array}$

$\begin{array}{lllllll}\text { quenched } & 3 & 0.810671 & 0.00378 & 0.80017 & 0.82117\end{array}$

Std Error uses a pooled estimate of error variance

\begin{tabular}{|c|c|c|c|c|c|c|}
\hline Source & DF & & $\begin{array}{l}\text { Sum of } \\
\text { Squares }\end{array}$ & $\begin{array}{l}\text { Mean } \\
\text { Square }\end{array}$ & F Ratio & Prob > \\
\hline HT & 1 & & 0158901 & 0.001589 & 12.1362 & 0.0253 \\
\hline Error & 4 & & 0052372 & 0.000131 & & \\
\hline $\begin{array}{l}\text { C. } \\
\text { Total }\end{array}$ & 5 & & 0211273 & & & \\
\hline \multicolumn{7}{|c|}{ Means for Anova } \\
\hline Level & Numb & & Mean & Std & $\begin{array}{l}\text { Lower } \\
\text { 950\% }\end{array}$ & \\
\hline $\mathrm{ccc}$ & & 3 & & Error & $\begin{array}{r}95 \% \\
9078\end{array}$ & \\
\hline quenched & & 3 & 1.75864 & 0.00661 & 1.7403 & \\
\hline
\end{tabular}

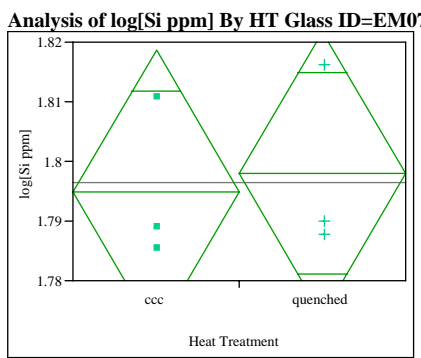

Anova

Summary of Fi

Rsquare
Adj Rsquare Mean of Response $\quad{ }_{1.014824}$ Observations (or Sum Wgts)

t Test
quenched-ccc

Assuming equal variances

$\begin{array}{lll}\text { Difference } & 0.00303 \text { t Ratio } & 0.250692\end{array}$ Std Err Dif 0.01210 DF $>0.014$ Lower CL Dif -0.03057 Prob $>t \quad 0.4072$ Confidence $\quad 0.95$ Prob $<\mathrm{t} \quad 0.5928$

\section{Analysis of Variance}

$\begin{array}{rrrrr}\text { Source DF } & \begin{array}{r}\text { Sum of } \\ \text { Squares }\end{array} & \begin{array}{r}\text { Mean } \\ \text { Square }\end{array} & \begin{array}{r}\text { F } \\ \text { Ratio }\end{array} & \text { Prob }> \\ & 1 & 0.00 \text { F }\end{array}$ $\begin{array}{llrrrr}\text { HT } & 1 & 0.00001381 & 0.000014 & 0.0628 & 0.8144\end{array}$ $\begin{array}{llll}\text { Error } & 4 & 0.00087900 & 0.000220\end{array}$

Means for Anova

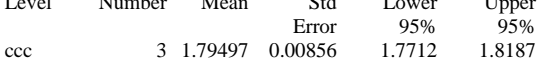

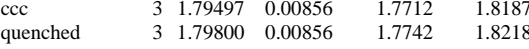

Std Error uses a pooled estimate of error variance 
Figure B6. Effects of Heat Treatment (HT) on PCT log(ppm)-Response of Study Glasses

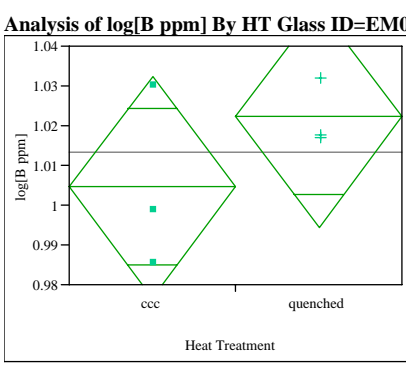

Anova

Summary of Fit

Rsquare
Adj Rsquare

$\begin{array}{lr}\text { Mean of Response } & 1.013464 \\ \text { Observations (or Sum Wgts) } & 6\end{array}$

t Test

quenched-ccc

Assuming equal variances

Difference $\quad 0.01765$ tRatio $\quad 1.246914$

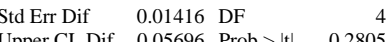

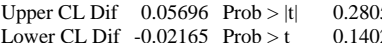

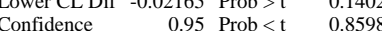

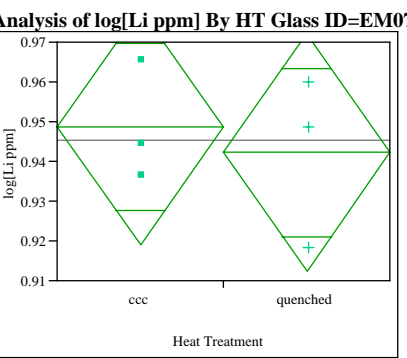

Anova

Summary of Fit

Rsquare

Root Mean Square Error $\quad 0.01857$

$\begin{array}{lr}\text { Mean of Response } & 0.945448 \\ \text { Observations (or Sum Wgts) } & 6\end{array}$

t Test
quenched-ccc

Assuming equal variances

$\begin{array}{lll}\text { Std Err Dif } & 0.01516 \text { DF } & \\ & \end{array}$

Upper CL Dif 0.03563 Prob $>|c| 0.6917$

$\begin{array}{lll}\text { Confidence } & 0.95 \text { Prob }<\mathrm{t} \quad 0.3458\end{array}$ $\begin{array}{lll}\text { Difference } & -0.00647 \quad \text { t Ratio } & -0.42653\end{array}$

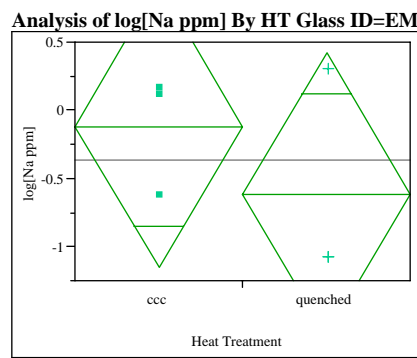

Anova

Summary of Fit

Rsquare
Adj Rsquar

$\begin{array}{lr}\text { Adj Rsquare } & -0.0234 \\ \text { Root Mean Square Error } & 0.646742\end{array}$ $\begin{array}{lr}\text { Mean of Response } & -0.36823 \\ \text { Observations (or Sum Wgts) } & 6\end{array}$

t Test

Assuming equal variances

Difference $\quad-0.4970$ t Ratio $\quad-0.9411$ Std Err Dif 0.5281 DF Lower CL Dif -1.9631 Prob $>t \quad 0.3000$ Confidence $\quad 0.95$ Prob $<\mathrm{t} \quad 0.2000$

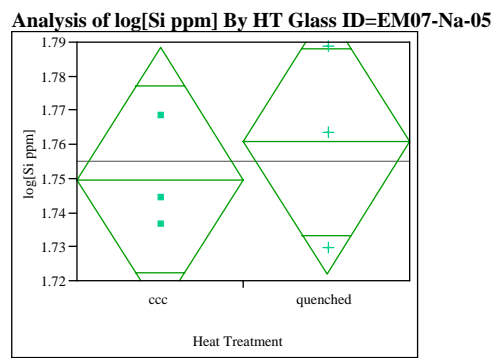

Anova

Anova
Summary of Fit

Rsquare

Root Mean Square Error $\quad 0.024071$ $\begin{array}{lr}\text { Mean of Response } & 1.75519 \\ \text { Observations (or Sum Wgts) } & 6\end{array}$

t Test

quenched-ccc

Assuming equal variances

Difference $\quad 0.01098$ t Ratio 0.558454 Std Err Dif 0.01965 DF Upper CL Dif 0.06554 Prob $>|t| 0.6063$ Confidence $\quad 0.95$ Prob $<\mathrm{t} \quad 0.6968$

\begin{tabular}{|c|c|c|c|c|c|c|c|c|c|c|c|}
\hline \multicolumn{6}{|c|}{ Analysis of Variance } & \multicolumn{6}{|c|}{ Analysis of Variance } \\
\hline Source I & DF & $\begin{array}{l}\text { Sum of } \\
\text { Squares }\end{array}$ & $\begin{array}{l}\text { Mean } \\
\text { Square }\end{array}$ & $\begin{array}{lr}\text { n } & \text { F } \\
\text { e } & \text { Ratio }\end{array}$ & $\begin{array}{r}\text { Prob }> \\
F\end{array}$ & Source I & DF & $\begin{array}{l}\text { Sum of } \\
\text { Squares }\end{array}$ & $\begin{array}{l}\text { Mean } \\
\text { Square }\end{array}$ & $\begin{array}{lr}\text { n } & \text { F } \\
\text { e } & \text { Ratio }\end{array}$ & $\begin{array}{r}\text { Prob > } \\
F\end{array}$ \\
\hline HT & 1 & 0.00046741 & 0.000467 & $\begin{array}{ll}7 & 1.5548\end{array}$ & 0.2805 & HT & 1 & 0.00006274 & 0.000063 & $3 \quad 0.1819$ & 0.6917 \\
\hline Error & 4 & 0.00120249 & 0.000301 & & & Error & 4 & 0.00137951 & 0.000345 & & \\
\hline C. & & 0.00166990 & & & & C. & & 0.00144226 & & & \\
\hline & & & & & & Tot & & & & & \\
\hline Means for & r An & & & & & secta & $r A$ & & & & \\
\hline Level & Numbe & Mean & $\begin{array}{r}\text { Std } \\
\text { Error }\end{array}$ & $\begin{array}{r}\text { Lower } \\
95 \%\end{array}$ & $\begin{array}{r}\text { Upper } \\
95 \%\end{array}$ & Level & Number & Mean & $\begin{array}{r}\text { Std } \\
\text { Etror }\end{array}$ & $\begin{array}{l}\text { Lower } \\
95 \%\end{array}$ & Upper \\
\hline & & $\begin{array}{ll}31.00464 \\
\end{array}$ & 0.01001 & 0.97684 & $\begin{array}{r}1.0324 \\
\end{array}$ & ccc & & 30.948682 & 0.01072 & 0.91891 & 0.97845 \\
\hline guenched & & $\begin{array}{ll}3 & 1.02229\end{array}$ & 0.01001 & 0.99450 & 1.0501 & quenched & & $\begin{array}{ll}3 & 0.942214\end{array}$ & 0.01072 & 0.91245 & 0.97198 \\
\hline
\end{tabular}

Analysis of Variance $\begin{array}{lrrrrr}\text { Source } & \text { DF } & \begin{array}{r}\text { Sum of } \\ \text { Squares }\end{array} & \begin{array}{r}\text { Mean } \\ \text { Square }\end{array} & \begin{array}{r}\text { Ratio } \\ \text { Prob }>\end{array} \\ \text { HT } & 1 & 0.3704648 & 0.370465 & 0.8857 & 0.3999 \\ \text { Error } & 4 & 1.6731014 & 0.418275 & & \end{array}$

\begin{tabular}{lll} 
& 1 & 0.370648 \\
C. & 4 & 1.6731014 \\
& 5 & 2.0435662 \\
\hline
\end{tabular}

Means for Anova

Level Number Mean Std Lower Upper

$\begin{array}{rrrrr} & & \text { Error } & 95 \% & 95 \% \\ \text { ccc } & 3 & -0.37340 & -1.156 & 0.91697\end{array}$

quenched $\quad 3 \begin{array}{rrrrr}0.11974 & -0.37340 & -1.156 & 0.91697 \\ & & 0.37340 & -1.653 & 0.42001\end{array}$

Std Error uses a pooled estimate of error variance
Analysis of Variance

$\begin{array}{lllll}\text { Source DF Sum of Mean } & F & \text { Prob > }\end{array}$ $\begin{array}{llrrrr} & & \text { Squares } & \text { Square } & \text { Ratio } & \\ \text { HT } & 1 & 0.00018070 & 0.000181 & 0.3119 & 0.6063 \\ \text { Error } & 4 & 0.00231758 & 0.000579 & & \end{array}$ $\begin{array}{llll}\text { Error } & 4 & 0.00231758 & 0.000579 \\ \text { C. } & 5 & 0.00249827 & \end{array}$

$\begin{array}{llrrr}\text { Means for Anova } & & & & \\ \text { Level Number } & \text { Mean } \quad \text { Std } & \text { Lower } & \text { Upper }\end{array}$ $\begin{array}{lrrrrr} & & \text { Error } & 95 \% & 95 \% \\ \text { ccc } & 3 & 1.74970 & 0.01390 & 1.7111 & 1.7883\end{array}$

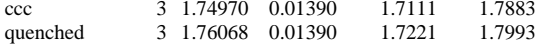
Std Error uses a pooled estimate of error variance 
Figure B6. Effects of Heat Treatment (HT) on PCT $\log (\mathrm{ppm})$-Response of Study Glasses

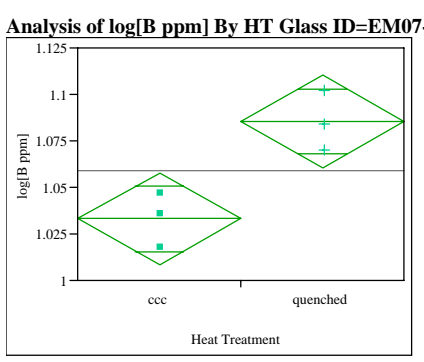

Anova

Summary of Fit

Rsquare
Adj Rsquare

Root Mean Square Error $\quad 0.015517$

Mean of Response 1.059208

t Test

quenched-cc

Assuming equal variances

Difference $\quad 0.052340$ tRatio $\quad 4.131259$

Drob > t| $\quad 0.0145$

Lower CL Dif 0.017165 Prob $>t \quad 0.0072$

$\begin{array}{lll}\text { Confidence } & 0.95 \mathrm{Prob}<\mathrm{t} \quad 0.9928\end{array}$

Analysis of Variance
$\begin{aligned} & \text { Source DF Sum of Mean F Ratio Prob > }\end{aligned}$

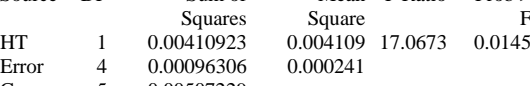

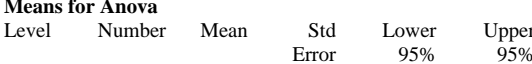

$\begin{array}{lrrrrr} & & \text { Error } & 95 \% & 95 \% \\ \text { ccc } & 3 & 1.03304 & 0.00896 & 1.0082 & 1.0579\end{array}$

quenched $-31.08530-0.00896 \quad 1.0605$

Std Error uses a pooled estimate of error variance
Analysis of $\log [\mathrm{Li}$ ppm] By HT Glass ID=EM07-Na-10

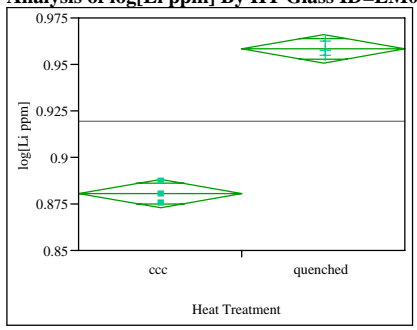

Anova

$\begin{array}{ll}\text { Rsquare } & 0.989908 \\ \text { Adj Rsquare } & 0.987385\end{array}$

Root Mean Square Error $\quad 0.004808$

Mean of Response
Observations (or Sum Wgts)

t Test

quenched-ccc

Assuming equal variances

Difference $\quad 0.077764$ t Ratio 19.80792 $\begin{array}{llll}\text { Std Err Dif } & 0.003926 \text { DF } & 4\end{array}$ Upper CL Dif 0.088664 Prob $>|t| \quad<.0001$

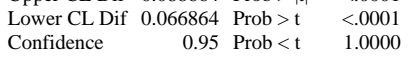

Analysis of Variance
Source DF Sum of Mean $\quad$ F Ratio Prob >

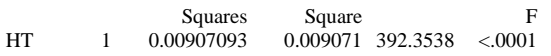
Error $\quad 4 \quad 0.00009248$

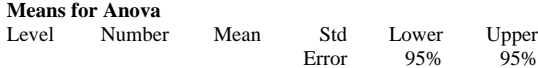

$\begin{array}{lrrrrr} & & \text { Error } & 95 \% & 95 \% \\ \text { ccc } & 3 & 0.880479 & 0.00278 & 0.87277 & 0.88819\end{array}$

Std Error uses a pooled estimate of error variance

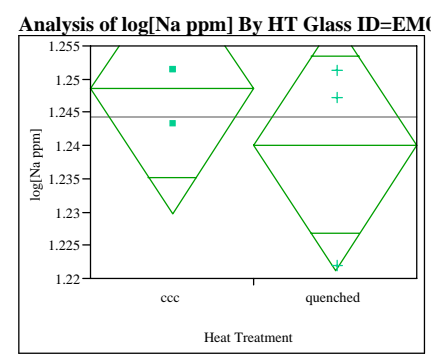

Anova

Aummary of Fit

Rsquare

Root Mean Square Error $\quad 0.011743$

$\begin{array}{lr}\text { Mean of Response } & 1.244299 \\ \text { Observations (or Sum Wgts) } & 6\end{array}$

t Test

quenched-ccc

Assuming equal variances

Difference $\quad-0.00842$ t Ratio $\quad-0.87844$ -0.8784
4

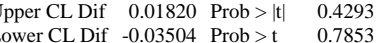
$\begin{array}{lrl}\text { Confidence } & 0.95 \text { Prob }<\mathrm{t} \quad 0.2147\end{array}$

$\begin{array}{lrrrrr} & \text { DF } & \begin{array}{r}\text { Sum of } \\ \text { Squares }\end{array} & \begin{array}{r}\text { Mean } \\ \text { Square }\end{array} & \begin{array}{r}\text { F } \\ \text { Ratio }\end{array} & \begin{array}{r}\text { Prob }> \\ \text { F }\end{array} \\ \text { HT } & 1 & 0.00010641 & 0.000106 & 0.7716 & 0.4293\end{array}$

$\begin{array}{lll}\text { C. } & 5 & 0.00065798\end{array}$ 0.000138

Means for Anova

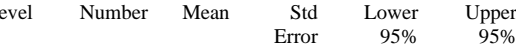

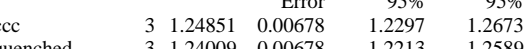

Std Error uses a pooled estimate of error variance

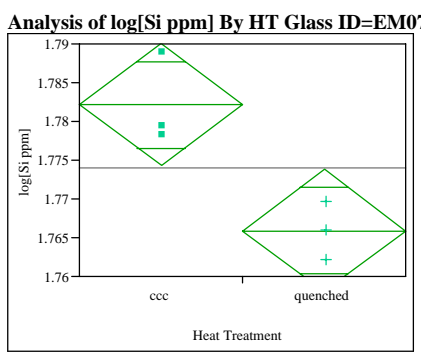

ummary of Fit

Rsquare

Root Mean Square Error 0.004918

Mean of Response 1.774025

t Test
quenched-ccc

Assuming equal variances

Difference $\quad-0.01622$ t Ratio $\quad-4.03982$

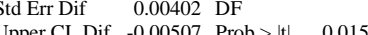
Lower CL Dif -0.02737 Prob $>t \quad 0.992$ Confidence $\quad 0.95$ Prob $<\mathrm{t} \quad 0.007$

Mean F Ratio Prob > $\begin{array}{llrrrr}\text { HT } & 1 & 0.00039469 & 0.000395 & 16.3201 & 0.0156 \\ \text { Error } & 4 & 0.00009674 & 0.000024 & & \end{array}$ \begin{tabular}{lll} 
Error & 4 & 0.00009674 \\
\hline & 5 & 0.00049143
\end{tabular}

Means for Anova

Level Number Mean Std Lower Upper $\begin{array}{lrrrrr} & & & \text { Error } & 95 \% & 95 \% \\ \text { ccc } & 3 & 1.78214 & 0.00284 & 1.7743 & 1.7900\end{array}$ $0.00284 \quad 1.7580$

Std Error uses a pooled estimate of error variance 


\section{Figure B6. Effects of Heat Treatment (HT) on PCT log(ppm)-Response of Study Glasses}

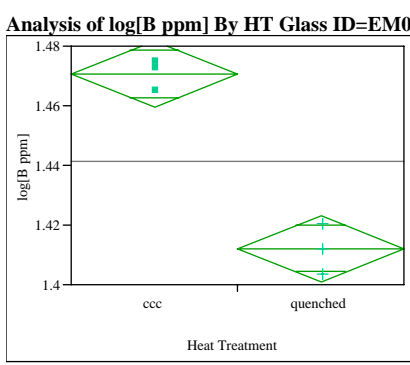

Anova

Anova
Summary of Fit

Rsquare
Adj Rsquare

$\begin{array}{lr}\text { Mean of Response } & 1.441381 \\ \text { Observations (or Sum Wgts) } & 6\end{array}$

t Test

Assuming equal variances

$\begin{array}{llll}\text { Difference } & -0.05849 \text { t Ratio } & -10.2974\end{array}$ $\begin{array}{llll}\text { Std Err Dif } & 0.00568 \text { DF } & 4 \\ \text { Upper CLDif } & -0.04272 \text { Prob }>\text { |t } & 0.0005\end{array}$ $\begin{array}{llll}\text { Upper CL Dif } & -0.04272 & \text { Prob }>|t| & 0.0005 \\ \text { Lower CL Dif } & -0.07426 & \text { Prob }>t & 0.9997\end{array}$ Confidence $\quad 0.95$ Prob $<\mathrm{t} \quad 0.0003$

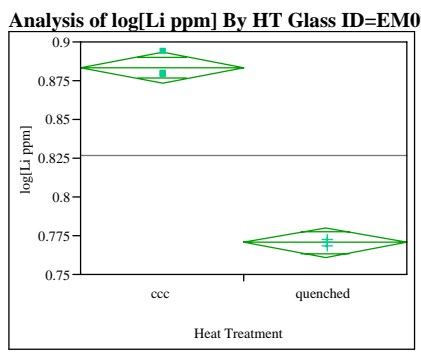

Anova

Summary of Fit

$\begin{array}{ll}\text { Rsquare } & 0.992233 \\ \text { Adj Rsquare } & 0.990292 \\ & 0.029674\end{array}$

Root Mean Square Error $\quad 0.006114$

Mean of Response
Observations (or Sum Wgts)

t Test
quenched-ccc

Assuming equal variances

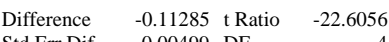

Upper CL Dif -0.09899 Prob > $>0004$

Lower CL Dif -0.12671 Prob $>t \quad 1.0000$

$\begin{array}{lll}\text { Confidence } & 0.95 \text { Prob }<\mathrm{t} \quad<.0001\end{array}$

Source DF Sum of $\begin{array}{r}\text { Mean } \\ \text { Square }\end{array}$ F Ratio Prob $>$

$\begin{array}{llrrrrr} & & \text { Squares } & \text { Square } & & \text { F } \\ \text { HT } & 1 & 0.01910298 & 0.019103 & 511.0128 & <.0001\end{array}$

$\begin{array}{lll}\text { Error } & 4 & 0.00014953 \\ C & 5 & 0.0195251\end{array}$

Means for Anova

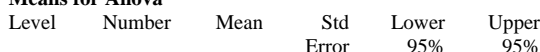

$\begin{array}{lrrrrr} & & \text { Error } & 95 \% & 95 \% \\ \text { ccc } & 3 & 0.883300 & 0.00353 & 0.87350 & 0.89310\end{array}$

$\begin{array}{lllllll}\text { quenched } & 3 & 0.770449 & 0.00353 & 0.76065 & 0.78025\end{array}$

Std Error uses a pooled estimate of error variance

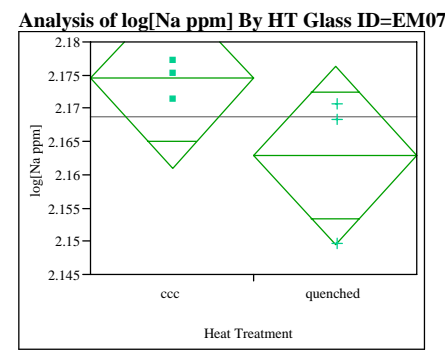

Anova

Summary of Fit

$\begin{array}{ll}\text { Rsquare } & 0.415079 \\ \text { Adj Rsquare } & 0.268849\end{array}$

Root Mean Square Error $\quad 0.008393$

$\begin{array}{lr}\text { Mean of Response } & 2.168709 \\ \text { Observations (or Sum Wgts) } & 6\end{array}$

t Test
quenched-ccc

Assuming equal variances

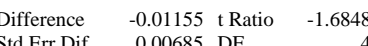

Upper CL Dif 0.00748 Prob $>$ |t 0.1673

Lower CL Dif -0.03057 Prob $>t \quad 0.9163$

Confidence $\quad 0.95$ Prob $<\mathrm{t} \quad 0.083$

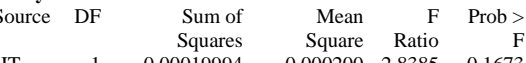

$\begin{array}{lrrrrr}\text { HT } & 1 & 0.00019994 & 0.000200 & 2.8385 & 0.1673 \\ \text { Error } & 4 & 0.00028174 & 0.000070 & & \end{array}$

$5 \quad 0.00048168$

$\begin{array}{rrrr} & & & \\ & & & \end{array}$

$\begin{array}{lrrrrr} & 3 & 2.17448 & 0.00485 & 2.1610 & 2.1879 \\ \text { ccc } & 3 & 2.1629 & 0.0045 & 2.1495 & \end{array}$

Std Error uses a pooled estimate of error variance

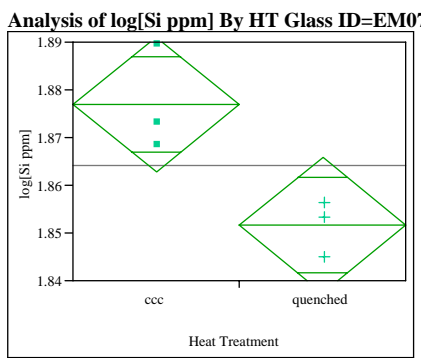

Anova

Summary of $F$

Rsquare
Adj Rsquare

Error $\quad 0.008808$

Observations (or Sum Wgts) $\begin{array}{r}1.864242 \\ 6\end{array}$

t Test
quenched-ccc

Assuming equal variances

$\begin{array}{lll}\text { Difference } & -0.02532 \text { t Ratio } & -3.5211 \\ \text { Std } & \end{array}$ Std Err Dif 0.00719 D

$\begin{array}{lll}\text { Upper CL Dif }-0.00536 & \text { Prob }>|t| & 0.0244\end{array}$

Confidence $\quad 0.95$ Prob $<\mathrm{t} \quad 0.0122$

Analysis of Variance

DF Sum of Mean F Ratio Prob > $\begin{array}{llrrrr} & & \text { Squares } & \text { Square } & \text { F } \\ \text { HT } & 1 & 0.00096176 & 0.000962 & 12.3982 & 0.0244 \\ \text { Error } & 4 & 0.00031029 & 0.000078 & & \end{array}$ $\begin{array}{lll} & & \\ \text { Error } & 4 & 0.00031029 \\ \text { C. } & 5 & 0.00127206\end{array}$ 0.000078

Means for Anova

$\begin{array}{lllll} & & & \\ \text { Level Number Mean Std } & \text { Lower } & \text { Upper } \\ & & & \end{array}$ $\begin{array}{lrrrrr} & & & \text { Error } & 95 \% & 95 \% \\ \text { ccc } & 3 & 1.87690 & 0.00509 & 1.8628 & 1.8910\end{array}$ $\begin{array}{llllll}\text { quenched } & 3 & 1.85158 & 0.00509 & 1.8375 & 1.8657\end{array}$

Std Error uses a pooled estimate of error variance 
Figure B6. Effects of Heat Treatment (HT) on PCT log(ppm)-Response of Study Glasses

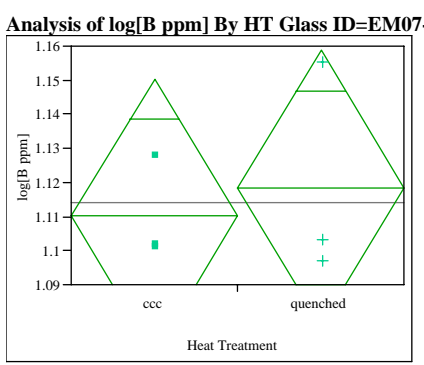

Anova

Anova
Summary of Fit

Rsquare
Adj Rsquare

$\begin{array}{ll}\text { Adj Rsquare } & -0.19898 \\ \text { Root Mean Square Error } & 0.025107\end{array}$ $\begin{array}{lr}\text { Mean of Response } & 1.114273 \\ \text { Observations (or Sum Wgts) } & 6\end{array}$

t Test
quenched-ccc

Assuming equal variances

Difference $\quad 0.00846$ t Ratio $\quad 0.412568$ $\begin{array}{llll}\text { Std Err Dif } & 0.02050 & \text { DF } & 4 \\ \text { Utp } & & \end{array}$ $\begin{array}{llll}\text { Upper CL Dif } & 0.06537 & \text { Prob }>|t| & 0.701 \\ \text { Lower CL Dif } & -0.04846 & \text { Prob }>t & 0.3505\end{array}$ $\begin{array}{llll} & \text { Confidence } & -0.95 \text { Prob }<\mathrm{t} & 0.6495\end{array}$

Analysis of $\log [\mathrm{Li}$ ppm] By HT Glass ID=EM07-Ni-001

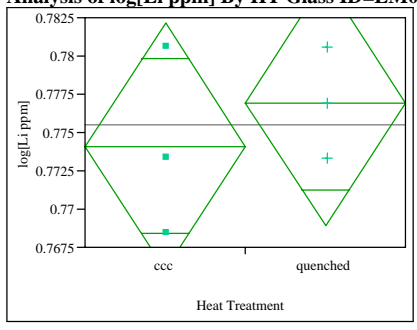

Anova

Summary of Fit

Rsquare

Adj Rsquare $\quad \begin{array}{rr}0.10743 \\ -0.11571\end{array}$

Root Mean Square Error $\quad 0.005032$

Observations (or Sum West)

t Test
quenched-c

Assuming equal variances

Difference $\quad 0.00285$ tRatio $\quad 0.69386$ $\begin{array}{lll}\text { Std Err Dif } \quad 0.00411 \mathrm{DF} & 4\end{array}$ Upper CL Dif 0.01426 Prob $>|t| 0.5260$ $\begin{array}{lrr}\text { Lower CL Dif } & -0.00856 \text { Prob }>t & 0.2630 \\ \text { Confidence } & 0.95 \text { Prob }<\mathrm{t} & 0.7370\end{array}$

\begin{tabular}{|c|c|c|c|c|c|c|c|c|c|c|c|}
\hline \multicolumn{12}{|c|}{ Analysis of Varianc } \\
\hline \multicolumn{2}{|c|}{ Analysis of Variance } & Sum of & Mean & & Prob $>$ & Source & & $\begin{array}{l}\text { Sum of } \\
\text { Saurase }\end{array}$ & $\begin{array}{l}\text { Mean } \\
\text { Sayre }\end{array}$ & $\begin{array}{r}F \\
\text { Ratio }\end{array}$ & Prob $>$ \\
\hline & & Squares & Square & Ratio & & & 1 & $\begin{array}{r}\text { Squares } \\
0.00001219\end{array}$ & $\begin{array}{r}\text { Square } \\
0.000012\end{array}$ & $\begin{array}{l}\text { e } \\
2 \\
2\end{array}$ & \\
\hline HT & 1 & 0.00010729 & 0.000107 & 0.1702 & 0.7011 & Erro & 4 & $\begin{array}{l}0.00010127 \\
0.000127\end{array}$ & & & \\
\hline Error & 4 & 0.00252136 & 0.000630 & & & & & & & & \\
\hline C. & 5 & 0.00262866 & & & & Total & & & & & \\
\hline \multirow{2}{*}{\multicolumn{6}{|c|}{ Means for Anova }} & \multicolumn{6}{|c|}{ Means for Anova } \\
\hline & & & & & & Level & Number & Mean & & & \\
\hline Level & Number & Mean & $\begin{array}{l}\text { Std } \\
\text { Error }\end{array}$ & Lower & Upper & & & & & & $95 \%$ \\
\hline & & 1.11004 & 0.01450 & & & & & & 0.00291 & 0.76603 & .78216 \\
\hline uenched & & $\begin{array}{lll}3 & 1.11850\end{array}$ & 0.01450 & 1.0783 & 1.1587 & quenched & & 30.776942 & 0.00291 & 0.76888 & 0.78501 \\
\hline
\end{tabular}

Std Error uses a pooled estimate of error variance

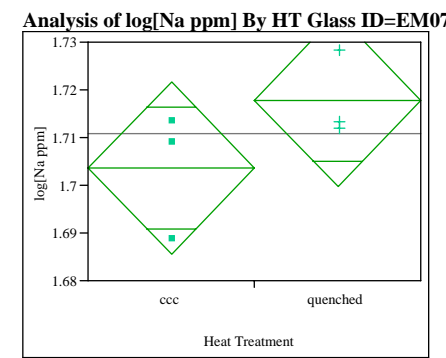

Anova

Summary of Fit

Rsquare

$\begin{array}{ll}\text { Adj Rsquare } & 0.370137 \\ \text { Root Mean Square Error } & 0.212671 \\ 0 & 0.011306\end{array}$ $\begin{array}{lr}\text { Mean of Response } & 1.710723 \\ \text { Observations (or Sum Wgts) } & 6\end{array}$

t Test

quenched-ccc

Assuming equal variances

Difference $\quad 0.01415$ t Ratio $\quad 1.533163$ $\begin{array}{llll}\text { Std Err Dif } & 0.00923 & \mathrm{DF} & 4 \\ \text { Uner } & 4\end{array}$ Lower CL Dif -0.01148 Prob $>t \quad 0.1000$ Confidence $\quad 0.95$ Prob $<\mathrm{t} 0.9000$

$\begin{array}{llrrr}\text { Source DF Sum of Mean } & F & \text { Prob }>\end{array}$ $\begin{array}{lrrrrr} & & \text { Squares } & \text { Square } & \text { Ratio } & \text { F } \\ \text { HT } & 1 & 0.00030044 & 0.000300 & 2.3506 & 0.2000 \\ \text { Error } & 4 & 0.00051126 & 0.000128 & & \end{array}$ $\begin{array}{lll}1 & & 0.003004 \\ \text { C. } \quad & 5 & 0.00081170\end{array}$ 0.000128

$\begin{array}{lllll} & & & & \\ \text { Level Number Mean Std } & \text { Lower } & \text { Upper }\end{array}$

$\begin{array}{lrrrrr} & & \text { Error } & 95 \% & 95 \% \\ \text { ccc } & 3 & 1.70365 & 0.00653 & 1.6855 & 1.7218\end{array}$

$\begin{array}{lllll} & & & & \text { Enenched } \\ \text { quench } & 3 & 1.71780 & 0.00653 & 1.65 \% \\ & & & \end{array}$

Std Error uses a pooled estimate of error variance

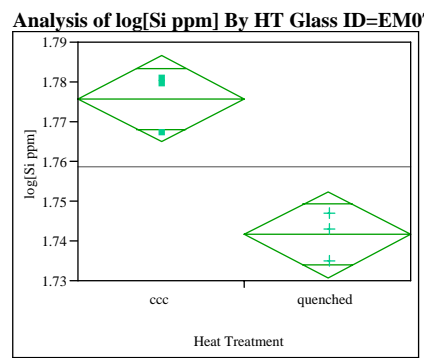

Anova
Summary of Fi

Rsquare
Adj Rsquare

$\begin{array}{ll}\text { Adj Rsquare } & 0.904939 \\ \text { Root Mean Square Eror } & 0.881174\end{array}$ Mean of Response $\quad 1.758678$ Observations (or Sum Wgts)

t Test
quenched-ccc

Assuming equal variances

Difference $\quad-0.03404$ t Ratio $\quad-6.17077$ Std Err Dif 0.00552 DF -0.17077 Lower CL Dif -0.04936 Prob >t 0 Confidence $\quad 0.95$ Prob $<\mathrm{t} \quad 0.0018$

\section{Analysis of Variance}

$\begin{array}{llll}\text { DF Sum of Mean F Ratio Prob > } & \end{array}$ $\begin{array}{llrrrr} & & \text { Squares } & \text { Square } & \text { F } \\ \text { HT } & 1 & 0.00173816 & 0.001738 & 38.0784 & 0.0035\end{array}$ \begin{tabular}{llll} 
Error & 4 & 0.00018259 & 0.000046 \\
\hline & 5 & 0.00192075 &
\end{tabular}

C.

Level Number Mean Std Lower $\begin{array}{lll}\text { Upper } \\ 95 \% & 950 \%\end{array}$ $\begin{array}{lrrrrr}\text { ccc } & 3 & 1.77570 & 0.00390 & 1.7649 & 1.7865 \\ \text { quenched } & 3 & 1.746 & 0.00390 & 1.730 & 1.7525\end{array}$ $\begin{array}{llllll}\text { quenched } & 3 & 1.74166 & 0.00390 & 1.7308 & 1.7525\end{array}$ Std Error uses a pooled estimate of error variance 
Figure B6. Effects of Heat Treatment (HT) on PCT log(ppm)-Response of Study Glasses

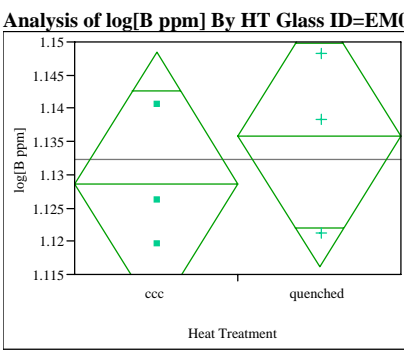

Anova
Summary of Fit

Rsquare

0.115048
-0.10619

Root Mean Square Error $\quad 0.012293$

$\begin{array}{lr}\text { Mean of Response } & 1.132262 \\ \text { Observations (or Sum Wgts) } & 6\end{array}$

t Test
quenched-ccc

Assuming equal variances

Difference $\quad 0.00724$ t Ratio $\quad 0.721123$ $\begin{array}{lll} & \end{array}$ Lower CL Dif -0.02063 Prob $>t \quad 0.2554$ $\begin{array}{lll}\text { Confidence } & 0.95 \mathrm{Prob}<\mathrm{t} \quad 0.7446\end{array}$

\section{Analysis of Variance $\begin{array}{lrrrrr}\text { Source } & \text { DF } & \begin{array}{r}\text { Sum of } \\ \text { Squares }\end{array} & \begin{array}{r}\text { Mean } \\ \text { Square }\end{array} & \begin{array}{r}\text { Ratio } \\ \text { Prob > }\end{array} \\ \text { HT } & 1 & 0.00007858 & 0.000079 & 0.5200 & 0.5107 \\ \text { Error } & 4 & 0.00060444 & 0.000151 & & \\ \text { C } & 5 & 0.00068302 & & & \end{array}$ \\ $5 \quad 0.00068302$}

Means for Anova

$\begin{array}{llrrr}\text { Level Number } & \text { Mean } & \begin{array}{r}\text { Std } \\ \text { Error }\end{array} & \begin{array}{rlr}\text { Lower } & \text { Upper } \\ 95 \% & 95 \%\end{array}\end{array}$

$\begin{array}{lrrrrr}\text { ccc } & 3 & 1.12864 & 0.00710 & 1.1089 & 1.1483 \\ & 3 & 1.13588 & 0.00710 & 1.1162 & 1.1556\end{array}$

Std Error uses a pooled estimate of error variance

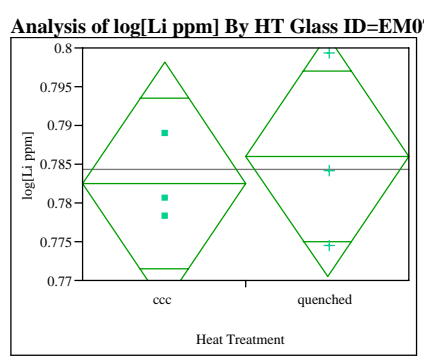

Anova

$\begin{array}{ll}\text { Rsquare } & 0.045809 \\ \text { Adj Rsquare } & -0.1927 \\ \text { Red } & 0.0784272\end{array}$

Root Mean Square Error $\quad 0.009703$

Mean of Response
Observations (or Sum Wgts)

t Test

quenched-ccc

Assuming equal variances

Difference $\quad 0.00347 \mathrm{t}$ Ratio $\quad 0.438214$

$\begin{array}{llll}\text { Difference } & 0.00347 & \text { tRatio } & 0.438214 \\ \text { Std Err Dif } & 0.00792 & \text { DF }\end{array}$

$\begin{array}{llll}\text { Upper CL Dif } & 0.02547 & \text { Prob }>|t| & 0.6839 \\ \text { Lener } & & 0.03419\end{array}$

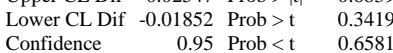

$\begin{array}{rrrrr}\text { Source DF } & \text { Sum of } & \text { Mean } & \text { F } & \text { Prob > }\end{array}$

$\begin{array}{lrrrrr} & & \text { Squares } & \text { Square } & \text { Ratio } & \text { F } \\ \text { HT } & 1 & 0.00001808 & 0.000018 & 0.1920 & 0.6839\end{array}$

$\begin{array}{llll}\text { Error } & 4 & 0.00037661 & 0.000094\end{array}$

$\begin{array}{llrrr}\text { Means for Anova } & \text { Level Number } & \text { Mean } \quad \begin{array}{r}\text { Std } \\ \text { Error }\end{array} & \begin{array}{r}\text { Lower } \\ 95 \%\end{array} & \begin{array}{l}\text { Upper } \\ 95 \%\end{array}\end{array}$

$\begin{array}{lrrrrr} & & \text { Error } & 95 \% & 95 \% \\ \text { ccc } & 3 & 0.782537 & 0.00560 & 0.76698 & 0.79809\end{array}$

Std Error uses a pooled estimate of error variance

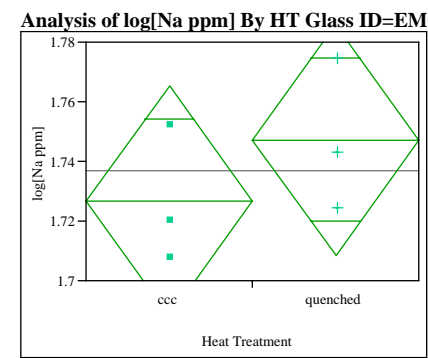

Anova

Summary of Fit

Rsquare

$\begin{array}{ll}\text { Adj Rsquare } & 0.214899 \\ \text { Root Mean Square Eror } & 0.018624 \\ 0.02419\end{array}$ Mean of Response 1.736935

Observations (or Sum Wgts) $\quad 6$

t Test
quenched-ccc

Assuming equal variances

$\begin{array}{lll}\text { Difference } & 0.02067 \text { t Ratio } \quad 1.046367\end{array}$ $\begin{array}{llll}\text { Std Err Dif } & 0.01975 \text { DF } & 4 \\ \text { Upper CL Dif } & 0.07551 \text { Prob > } & 0.3545\end{array}$

$\begin{array}{lrll}\text { Upper CL Dif } & 0.07551 & \text { Prob }>|t| & 0.3545 \\ \text { Lower CL Dif } & -0.03417 & \text { Prob }>t & 0.1772 \\ \text { Confidence } & 0.95 & \text { Prob }<t & 0.8228\end{array}$

Analysis of Variance

$\begin{array}{lrrrr}\text { Source DF } & \begin{array}{r}\text { Sum of } \\ \text { Squares }\end{array} & \begin{array}{r}\text { Mean } \\ \text { Square }\end{array} & \text { Ratio } & \text { Prob }> \\ & \text { Rat } & \text { F }\end{array}$

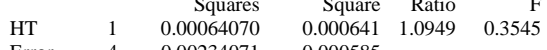
$\begin{array}{lll}\text { Error } & 4 & 0.00234071\end{array}$ $\begin{array}{ll}\text { C. } & 0.00298142\end{array}$ 0.000585

\section{Means for Anova}

$\begin{array}{llll} & & & \\ \text { Level Number Mean Std } & \text { Lower } & \text { Upper }\end{array}$

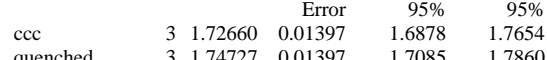

Std Error uses a pooled estimate of error variance

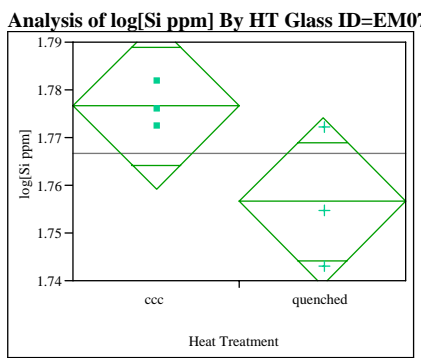

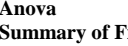

Rsquare
Adj Rsquare

$\begin{array}{ll}\text { Adj Rsquare } & 0.556458 \\ \text { Root Mean Square Enor } & 0.445573\end{array}$ Mean of Response $\quad{ }_{1.010916}$ Observations (or Sum Wgts)

t Test

enched-ccc

Assuming equal variances

$\begin{array}{lll}\text { Difference } & -0.01997 \text { t Ratio } & -2.24016\end{array}$

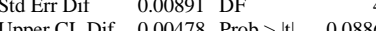

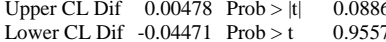
Confidence $\quad 0.95$ Prob $<\mathrm{t} \quad 0.0443$

\section{Analysis of Variance}

$\begin{array}{llrlrl}\text { Source DF Sum of Mean } & F & \text { Prob }>\end{array}$ $\begin{array}{llllrr}\text { HT } & 1 & 0.00059795 & 0.000598 & 5.0183 & 0.0886\end{array}$

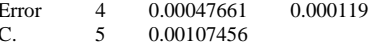

Means for Anova

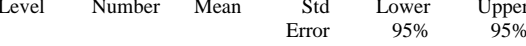

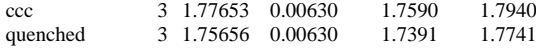
Std Error uses a pooled estimate of error variance 
Figure B6. Effects of Heat Treatment (HT) on PCT log(ppm)-Response of Study Glasses

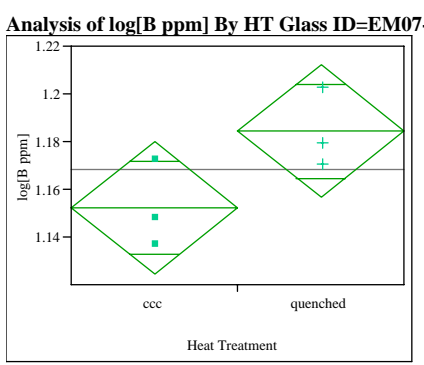

Anova

Summary of Fit

Rsquare
Adj Rsquare

Error $\quad 0.017382$

Mean of Response
Observations (or Sum Wgts)
1.168272

t Test

Assuming equal variances $\begin{array}{llll}\text { Std Err Dif } & 0.01419 & \mathrm{DF} & \\ \text { Upper CL Dif } & 0.07152 & \text { Prob }>|t| & 0.086\end{array}$

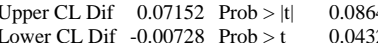
$\begin{array}{llll} & \text { Confidence } & 0.95 \text { Prob }<\mathrm{t} & 0.9568\end{array}$ $\begin{array}{llll}\text { Difference } & 0.03212 \text { t Ratio } \quad 2.263178\end{array}$

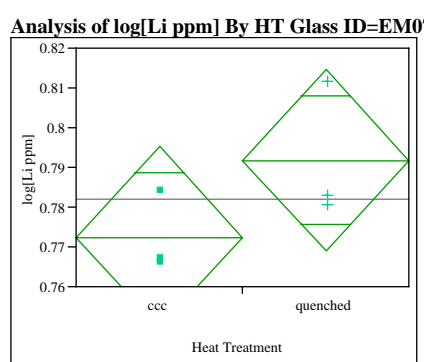

Anova

Summary of Fit

Rsquare

$\begin{array}{ll}\text { Adj Rsquare } & 0.409199 \\ \text { Root Mean Square Eror } & 0.261499\end{array}$ 0.014248

Observations (or Sum Wgts)

t Test
quenched-ccc

Assuming equal variances

Difference $\quad 0.01936$ t Ratio $\quad 1.664472$

Std Err Dif 0.01163 DF $\quad 1.66472$

Lower CL Dif -0.01294 Prob $>t \quad 0.0 .0857$

Confidence $\quad 0.95$ Prob $<\mathrm{t} \quad 0.9143$

Analysis of Variance

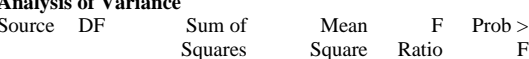

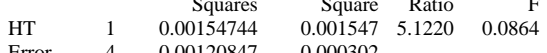

$\begin{array}{lll}\text { Error } & 4 & 0.00120847 \\ \text { C } & 5 & 0.00275591\end{array}$

$\begin{array}{ll}\text { C. } & 5 \\ -0.0027559\end{array}$ 0.000302

HT $\quad 1 \quad 0.00056244$

Means for Anova

$\begin{array}{llrr} & & & \\ \text { Level Number Mean Std } & \text { Lower } & \text { Uppe } \\ & & & \end{array}$

$\begin{array}{lllrrr} & & & \text { Error } & 95 \% & 95 \% \\ \text { ccc } & 3 & 1.15221 & 0.01004 & 1.1243 & 1.1801\end{array}$

Std Error uses a pooled estimate of error variance

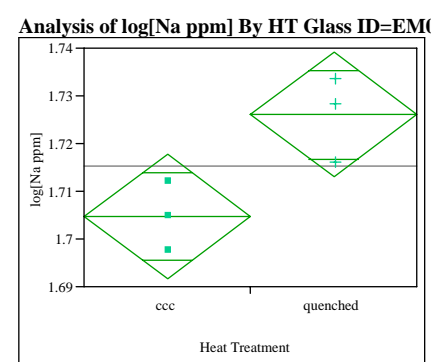

Anova

Anova
Summary of Fit

Rsquare
Adj Rsquare

$\begin{array}{lr}\text { Adj Rsquare } & 0.64857 \\ \text { Root Mean Square Error } & 0.008175\end{array}$ $\begin{array}{lr}\text { Mean of Response } & 1.715365 \\ \text { Observations (or Sum Wgts) } & 6\end{array}$

t Test

Assuming equal variances

Difference $\quad 0.021347$ t Ratio $\quad 3.19806$ Std Err Dif 0.006675 DF 4 Lower CL Dif 0.002814 Prob >t 0.0165 Confidence $\quad 0.95$ Prob $<\mathrm{t} \quad 0.9835$

\section{Analysis of Varianc}

\section{Source DF Sum of}

\begin{tabular}{llr} 
HT & 1 & 0.00068351 \\
\hline
\end{tabular}

\begin{tabular}{lll} 
Error & 4 & 0.00026732 \\
C. & 5 & 0.00095084 \\
\hline
\end{tabular}

Mean F Ratio Prob >

Means for Anova

Level Number Mean Std Lower Upper

$\begin{array}{lrrrrr} & & \text { Error } & 95 \% & 95 \% \\ \text { ccc } & 3 & 1.70469 & 0.00472 & 1.6916 & 1.7178\end{array}$

Std Error uses a pooled estimate of error variance

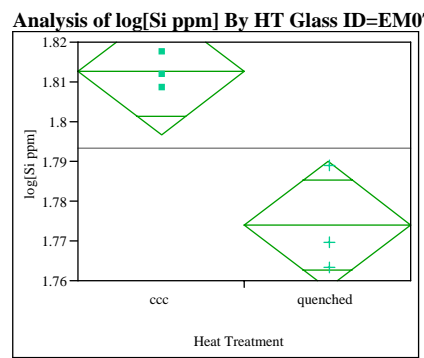

Anov

Summary of Fi

Rsquare
Adj Rsquare

\begin{tabular}{ll} 
Adj Rsquare & 0.850237 \\
Root Mean Square & 0.812796 \\
\hline & 0.00906
\end{tabular} Mean of Response $\quad 0.00990$

Observations (or Sum Wgts)

t Test
quenched-ccc

Assuming equal variances

Difference $\quad-0.03855$ t Ratio $\quad-4.76538$ Std Err Dif $0.00809 \mathrm{DF}$ Lower CL Dif -0.06101 Prob $>t \quad 0.9956$ Confidence $\quad 0.95$ Prob $<\mathrm{t} \quad 0.0044$

\section{Analysis of Variance}

$\begin{array}{lll}\text { DF Sum of Mean F Ratio Prob > } & \\ 1 \text { - }\end{array}$ $\begin{array}{llrrrr}\text { HT } & 1 & 0.00222926 & 0.002229 & 22.7089 & 0.0089\end{array}$

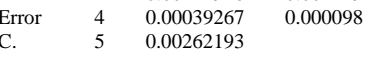

Means for Anova

\begin{tabular}{rrrr} 
& Error & $95 \%$ & Opper \\
\hline
\end{tabular}

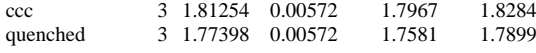

Std Error uses a pooled estimate of error variance 
Figure B6. Effects of Heat Treatment (HT) on PCT log(ppm)-Response of Study Glasses

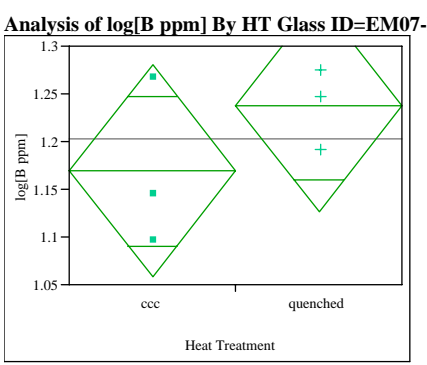

Anova

Summary of Fit

Rsquare
Adj Rsquare

o.069447

Mean of Response
Observations (or Sum Wgts)
1.203472

t Test

quenched-cce

Assuming equal variances

Difference $\quad 0.06864$ t Ratio $\quad 1.210467$ $\begin{array}{llll}\text { Std Err Dif } & 0.05670 & \text { DF } & 4 \\ \text { Upper CL Dif } & 0.22607 & \text { Prob }>\text { tt } & 0.2927\end{array}$ $\begin{array}{lrrr}\text { Upper CL Dif } & 0.22607 & \text { Prob }>|t| & 0.2927 \\ \text { Lower CL Dif } & -0.08880 & \text { Prob }>t & 0.1464\end{array}$

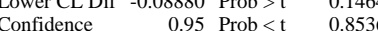

Analysis of $\log [$ Li ppm] By HT Glass ID=EM07-P-0

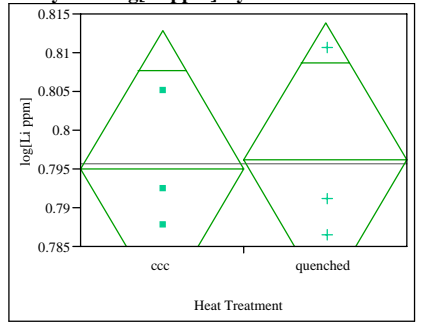

Anova

Rsquare

Root Mean Square Error $\quad 0.011061$

Mean of Response
Observations (or Sum Wgts)

t Test
quenched-cc

Assuming equal variances

Difference $\quad 0.00109 \mathrm{t}$ Ratio $\quad 0.121146$

Std Err Dif 0.00903 DF

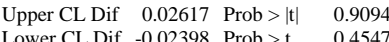

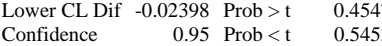

\begin{tabular}{|c|c|c|c|c|c|c|c|c|c|c|c|c|}
\hline \multicolumn{6}{|c|}{ Analysis of Variance } & \multicolumn{7}{|c|}{ Analysis of Variance } \\
\hline Source & DF & & & & & & DF & & Sum of & & & \\
\hline & & Squares & Square & Ratio & & & & & Squares & Square & & \\
\hline HT & 1 & 0.00706667 & 0.007067 & 1.4652 & 0.2927 & HT & 1 & & 000180 & $1.795 \mathrm{e}-6$ & 0.0147 & 0.9094 \\
\hline Error & 4 & 0.01929162 & 0.004823 & & & Error & 4 & & 048934 & 0.000122 & & \\
\hline C. & & 0.02635829 & & & & $\begin{array}{l}\text { C. } \\
\text { Total }\end{array}$ & 5 & & 049114 & & & \\
\hline & & & & & & & & & & & & \\
\hline & r A & & & & & Means fo & $\mathbf{r} \mathbf{A}$ & & & & & \\
\hline Level & Number & Mean & Std & Lower & Upper & Level & Nun & & Mean & Std & Lower & \\
\hline & & & Error & $95 \%$ & $95 \%$ & & & & & $\begin{array}{l}\text { Error } \\
000639\end{array}$ & $\begin{array}{r}95 \% \\
07732\end{array}$ & $\begin{array}{c}95 \% \\
95278\end{array}$ \\
\hline ccc & & $\begin{array}{ll}3 & 1.16915\end{array}$ & 0.04010 & 1.0578 & 1.2805 & cec & & & . & 0.00659 & 0.077842 & 0.81278 \\
\hline quenched & & 31.23779 & 0.04010 & 1.1265 & 1.3491 & & & & & & & \\
\hline
\end{tabular}

Std Error uses a pooled estimate of error variance
Analysis of $\log [\mathrm{Na}$ ppm] By HT Glass ID=EM07-P-0

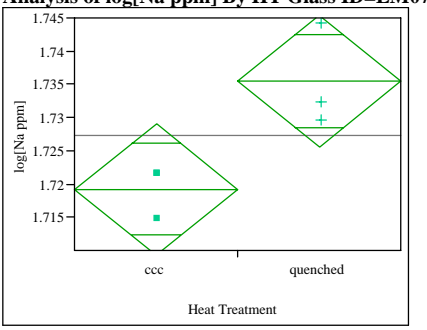

Anova

ummary of Fi

$\begin{array}{ll}\text { Rsquare } & 0.721647 \\ \text { Adj Rsquare } & 0.652059\end{array}$

Root Mean Square Error $\quad 0.006175$

$\begin{array}{lr}\text { Mean of Response } & 1.727354 \\ \text { Observations (or Sum Wgts) } & 6\end{array}$

t Test

Assuming equal variances

$\begin{array}{lll}\text { Difference } & 0.016237 & \text { t Ratio } \quad 3.220286\end{array}$

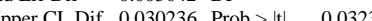
ower CL Dif 0.002238 Prob $>$ t 0.016 Confidence $\quad 0.95$ Prob $<\mathrm{t} \quad 0.9839$

Analysis of Variance

$\begin{array}{rrrrr}\text { Source DF } & \begin{array}{r}\text { Sum of } \\ \text { Squares }\end{array} & \begin{array}{r}\text { Mean } \\ \text { Square }\end{array} & \text { F Ratio } & \text { Prob > } \\ & & \text { S }\end{array}$ $\begin{array}{llrrrr}\text { HT } & 1 & 0.00039545 & 0.000395 & 10.3702 & 0.0323 \\ \text { Error } & 4 & 0.00015253 & 0.000038 & & \end{array}$ $\begin{array}{lll}\text { Error } & 4 & 0.00015253 \\ \text { C } & 5 & 0.00054799\end{array}$

Means for Anova

Level Number Mean Std Lower Upper $\begin{array}{lrrrrr} & & & \text { Error } & 95 \% & 95 \% \\ \text { ccc } & 3 & 1.71924 & 0.00357 & 1.7093 & 1.7291\end{array}$ $\begin{array}{lllll} & 3 & 1.73547 & 0.00357 & 1.7256\end{array}$

Std Error uses a pooled estimate of error variance

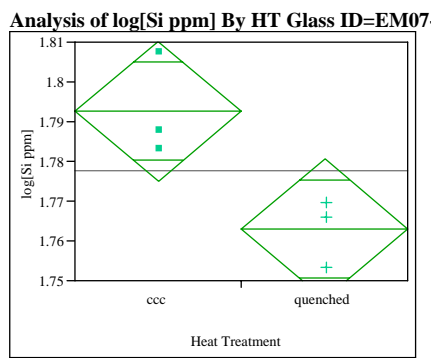

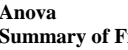

Rsquare
Adj Rsquare

$\begin{array}{ll}\text { Adj Rsquare } & 0.733969 \\ \text { Root Mean Square Eno } & 0.667462\end{array}$ Mean of Response $\quad 0.01094$

Observations (or Sum Wgts)

t Test
quenched-ccc

Assuming equal variances

$\begin{array}{lll}\text { Difference } & -0.02970 \quad t \text { Ratio } & -3.32203 \\ \text { Std } & & \end{array}$ Std Err Dif 0.00894 DF 1 $\begin{array}{lll}\text { Lower CL Dif }-0.05452 & \text { Prob }>t & 0.0293 \\ \text { Con } & 0.9853 & \text { Pot }\end{array}$ Confidence $\quad 0.95$ Prob $<\mathrm{t} \quad 0.0147$

\section{Analysis of Variance}

Sum of Mean F Ratio Prob >

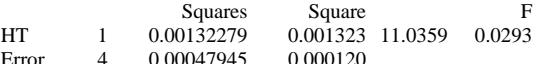

$\begin{array}{llll}\text { Error } & 4 & 0.00047945 & 0.000120 \\ \text { C. } & 5 & 0.00180224 & \end{array}$

Means for Anova

$\begin{array}{lllll} & & & \\ \text { Level Number Mean Std } & \text { Lower } & \text { Upper } \\ 95 \% & 95 \%\end{array}$ $\begin{array}{lrrrrr}\text { ccc } & 3 & 1.79266 & 0.00632 & 1.7751 & 1.8102 \\ \text { quenched } & 3 & 1.762 & 0.00632 & 1.7454 & 1.7005\end{array}$ $\begin{array}{llllll}\text { quenched } & 3 & 1.76296 & 0.00632 & 1.7454 & 1.7805\end{array}$ Std Error uses a pooled estimate of error variance 


\section{Figure B6. Effects of Heat Treatment (HT) on PCT $\log (\mathbf{p p m})$-Response of Study Glasses}

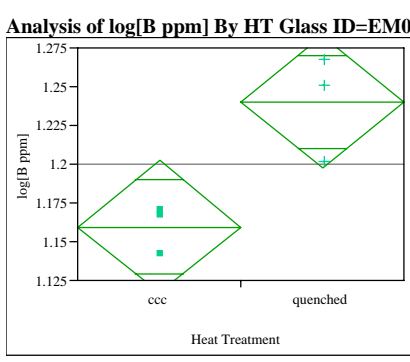

Anova

\section{Summary of Fit}

Rsquare

Root Mean Square Error $\quad 0.026557$

Mean of Response 1.199737

t Test
quenched-cc

Assuming equal variances

Difference $\quad 0.080411$ t Ratio 3.708388

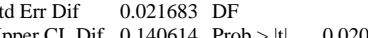

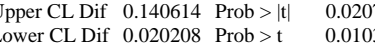
$\begin{array}{lrl}\text { Confidence } & 0.95 \text { Prob }<\mathrm{t} \quad 0.9897\end{array}$

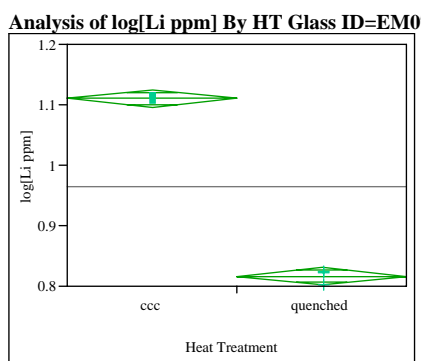

Anova
Summary of Fi

Rsquare

Adj Rsquare $\quad 0.996702$

Mean of Response 0.96344

Observations (or Sum Wgts)

tTest

quenched-ccc

Assuming equal variances

Difference $\quad-0.29388$ t Ratio $\quad-38.886$

Std Err Dif 0.00756 DF -38.04

Upper CL Dif -0.27290 Prob $>|t|<.000$

Confidence 0.95 Prob $<\mathrm{t} \quad<.0001$

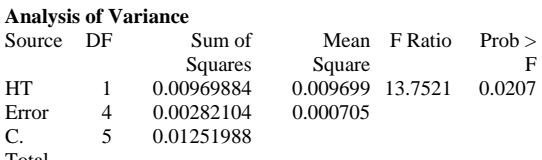

Total

$\begin{array}{lrrrr}\text { Level Number Mean } & \begin{array}{r}\text { Std } \\ \text { Error }\end{array} & \begin{array}{r}\text { Lower } \\ 95 \%\end{array} & \begin{array}{r}\text { Upper } \\ 95 \%\end{array}\end{array}$

$\begin{array}{lrrrrr} & & & \text { Error } & 95 \% & 95 \% \\ \text { ccc } & 3 & 1.15953 & 0.01533 & 1.1170 & 1.2021\end{array}$

$\begin{array}{lllll}\text { quenched } & 3 & 1.23994 & 0.01533 & 1.1974\end{array}$

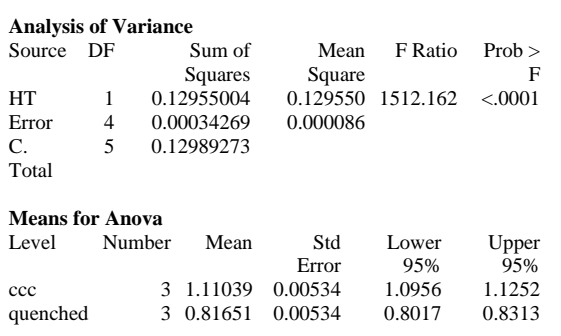

Std Error uses a pooled estimate of error variance

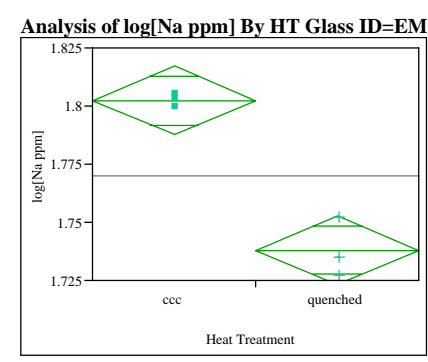

Anova

Summary of Fit

Rsquare

Adj Rsquare $\quad 0.947596$

Root Mean Square Error $\quad 0.009267$

$\begin{array}{lr}\text { Mean of Response } & 1.770221 \\ \text { Observations (or Sum Wgts) } & 6\end{array}$

t Test
quenched-cce

Assuming equal variances

$\begin{array}{llll}\text { Difference } & -0.06435 \text { t Ratio } & -8.50469\end{array}$

Upper CL Dif -0.04334 Prob > |t| 0.0010

$\begin{array}{lrr}\text { Lower CL Dif } & -0.08536 \text { Prob }>\mathrm{t} & 0.9995 \\ \text { Confidence } & 0.95 \text { Prob }<\mathrm{t} & 0.0005\end{array}$

Analysis of Variance

$\begin{array}{lllll}\text { Source DF Sum of Mean F Ratio Prob > } & \text { > }\end{array}$ $\begin{array}{lrrrrr} & & \text { Squares } & \text { Square } & \text { F } \\ \text { HT } & 1 & 0.00621127 & 0.006211 & 72.3297 & 0.0010\end{array}$

$\begin{array}{lll}\text { Error } & 4 & 0.00034350\end{array}$

Means for Anova

$\begin{array}{lllll}\text { Level Number } & \text { Mean Std Lower Upper }\end{array}$

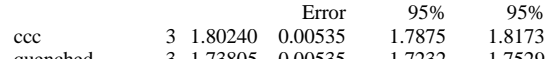

Std Error uses a pooled estimate of error variance

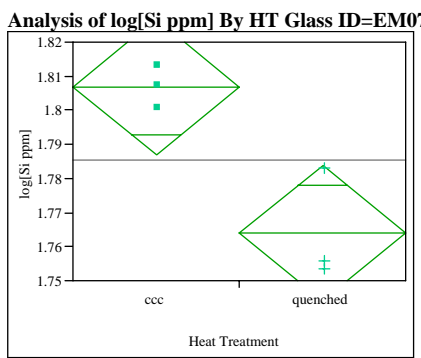

Anova

Summary of $F$

Rsquare
Adj Rsquare Mean of Response $\quad{ }_{1.012414}$

Observations (or Sum Wgts)

t Test
quenched-ccc

Assuming equal variances

$\begin{array}{lll}\text { Difference } & -0.04285 \text { t Ratio } & -4.22768\end{array}$

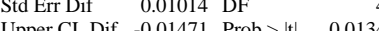
Lower CL Dif -0.07100 Prob $>t$ Confidence $\quad 0.95$ Prob $<\mathrm{t} \quad 0.0067$

\section{Analysis of Variance}

Sum of Mean F Ratio Prob > $\begin{array}{llrrrr} & & \text { Squares } & \text { Square } & \text { F } \\ \text { HT } & 1 & 0.00275453 & 0.002755 & 17.8733 & 0.0134 \\ \text { Error } & 4 & 0.00061646 & 0.000154 & & \end{array}$ $\begin{array}{llll} & & & \\ \text { Error } & 4 & 0.00061646 & 0.000154 \\ \text { C. } & 5 & 0.00337099 & \end{array}$

Means for Anova $\begin{array}{lrrrrr} & & \text { Error } & \text { rower } & \text { Opper } \\ \text { ccc } & 3 & 1.80691 & 0.00717 & 1.7870 & 1.8268 \\ & 3 & 1.7696 & & & \end{array}$ $\begin{array}{llllll}\text { quenched } & 3 & 1.76406 & 0.00717 & 1.7442 & 1.7840\end{array}$ Std Error uses a pooled estimate of error variance 


\section{Figure B6. Effects of Heat Treatment (HT) on PCT log(ppm)-Response of Study Glasses}

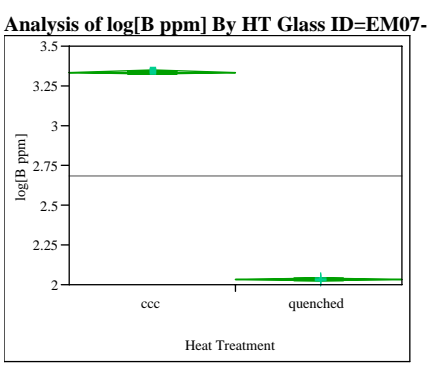

Anov

Summary of Fit

Rsquare
Adj Rsquare

e Error 0.007297

$\begin{array}{lr}\text { Mean of Response } & 2.68424 \\ \text { Observations (or Sum Wgts) } & 6\end{array}$

t Test

Assuming equal variances

$\begin{array}{llll}\text { Difference } & -1.3031 \text { t Ratio } & -218.722 \\ \text { Std Err Dif } & 0.0060 & \end{array}$ $\begin{array}{llll}\text { Std Err Dif } & 0.0060 & \mathrm{DF} & 4 \\ \text { Upper CL Dif } & -1.2866 & \text { Prob }>|t| & <.0001\end{array}$

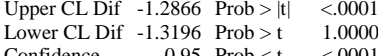

Analysis of Variance

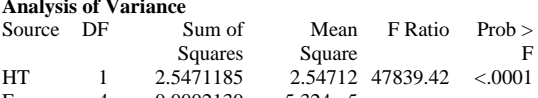

$\begin{array}{lll}\text { HT } & 1 & 2.5471185 \\ \text { Error } & 4 & 0.0002130\end{array}$

C. $5 \quad 2.5473315$

Level Number Mean Std Lower Upper

$\begin{array}{lrrrrr} & 3 & & \text { Error } & 95 \% & 95 \% \\ \text { ccc } & 3 & 33579 & 0.00421 & 3.3241 & 3.3475\end{array}$

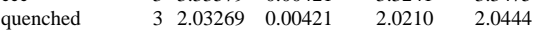

Std Error uses a pooled estimate of error variance
Analysis of $\log [\mathrm{Li}$ ppm] By HT Glass ID=EM07-Si-30

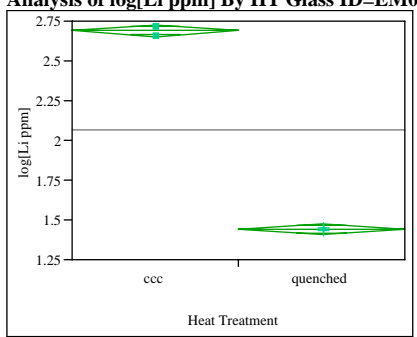

Anova

Summary of Fi

Rsquare
Adj Rsquare

0.99911
Root Mean Square Error $\quad 0.9988$
0.02850 Mean of Response 2.06550

Observations (or Sum Wgts)

t Test
quenched-ccc

Assuming equal variances

Difference $\quad-1.2489$ t Ratio $\quad-67.0462$ Std Err Dif 0.0186 DF 1 Lower CL Dif -1.3006 Prob $>t<1<0.0000$ Confidence $\quad 0.95$ Prob $<\mathrm{t} \quad<.0001$

\section{Analysis of Variance}

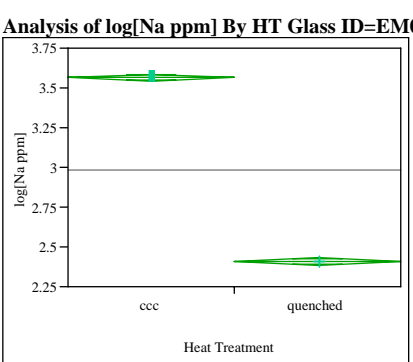

Anova

Summary of Fit

Rsquare

0.999483
Root Mean Square Error $\quad 0.014376$ $\begin{array}{lr}\text { Mean of Response } & 2.98634 \\ \text { Observations (or Sum Wgts) } & 6\end{array}$

t Test

Assuming equal variances

Difference $\quad-1.1539$ t Ratio $\quad-98.3076$ Sid Err Dif 0.0117 DF $>000$ Lower CL Dif -1.1865 Prob $>t<1.0000$ Confidence $\quad 0.95$ Prob $<\mathrm{t} \quad<.0001$

\section{Analysis of Variance}

$\begin{array}{lrrrrr} & \text { DF } & \begin{array}{r}\text { Sum of } \\ \text { Squares }\end{array} & \begin{array}{r}\text { Mean } \\ \text { Square }\end{array} & \text { F Ratio } & \text { Prob }> \\ \text { HT } & 1 & 2.3394851 & 2.33949 & 4495.190 & <0001\end{array}$

\begin{tabular}{lll} 
Error & 4 & 2.3394851 \\
\hline & 4 & 0.002081
\end{tabular}

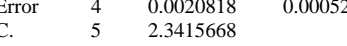

$\begin{array}{lllll}\text { Means for Anova } & & & \\ \text { Level Number Mean Std Lower Upper }\end{array}$

$\begin{array}{lrrrrr}\text { Level } & \text { Number } & \text { Mean } & \text { Std } & \text { Lower } & \text { Upper } \\ \text { ccc } & 3 & 2.68993 & 0.01317 & 95 \% & 95 \% \\ \text { quenched } & 3 & 1.44107 & 0.01317 & 1.4045 & 2.7265 \\ & & & & & \end{array}$

Std Error uses a pooled estimate of error variance

A

$\begin{array}{lll}\text { Error } & 4 & 1.00008267\end{array}$

$\begin{array}{ll}4 & 1.9981818\end{array}$

0.00021 Std Error uses a pooled estimate of error variance

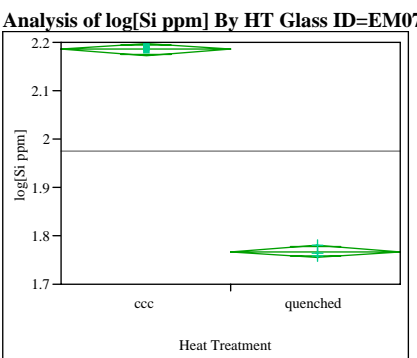

Anova
Summary of Fit

Rsquare

Observations (or Sum Wgts) 1.976246

t Tes

quenched-cce

Assuming equal variances

Difference $\quad-0.41751$ t Ratio -63.3257

Upper CL Dif -0.39920 Prob $>|t|<.0001$

0.95 Prob $<t \quad<0001$

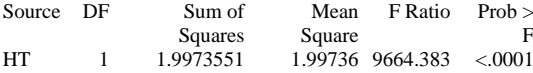

Analysis of Variance

Sum of Mean F Ratio Prob >

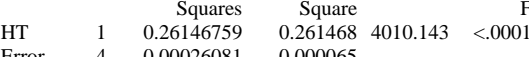
Error $\quad 4 \quad 0.00026081 \quad 0.000065$

Tot

\section{Means for Anova}

$\begin{array}{llll}\text { Leans for Anova } & \text { Number Mean Std Lower Upper }\end{array}$

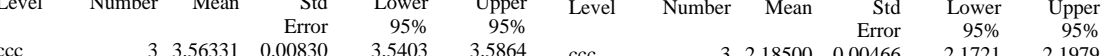
$\begin{array}{llllll}\text { ccC } & 3 & 3.56331 & 0.00830 & 3.5403 & 3.5864 \\ \text { quenched } & 3 & 2.40937 & 0.00830 & 2.3863 & 2.4324\end{array}$

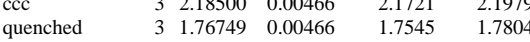

Std Error uses a pooled estimate of error variance 
Figure B6. Effects of Heat Treatment (HT) on PCT log(ppm)-Response of Study Glasses

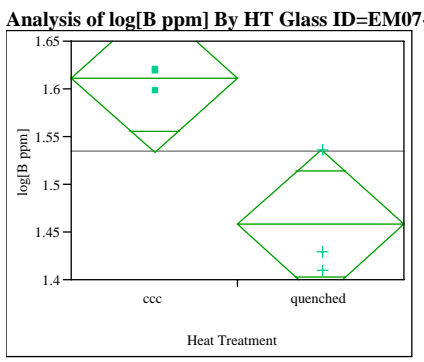

Anov

Anova

Rsquare

Error $\quad 0.048986$

$\begin{array}{lr}\text { Mean of Response } & 1.534708 \\ \text { Observations (or Sum Wgts) } & 6\end{array}$

t Test

quenched-ccc

Assuming equal variances

Difference $\quad-0.15356$ t Ratio $\quad-3.83928$ $\begin{array}{lrlr}\text { Std Err Dif } & 0.04000 & \mathrm{DF} & 4 \\ \text { Unper } & & \end{array}$

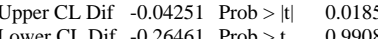
$\begin{array}{llll} & \text { Confidence } & -0.95 \text { Prob }<\mathrm{t} & 0.0092\end{array}$

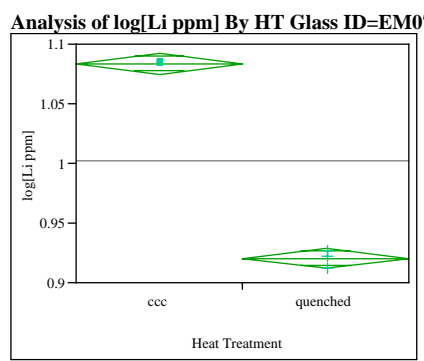

Anova

Summary of F

Rsquare

Root Mean Square Frror $\quad 0.005393$

Mean of Response 1.002042

t Test
quenched-ccc

Assuming equal variances

Difference $\quad-0.16310$ t Ratio $\quad-37.0378$

Std Err Dif 0.00440 DF

Upper CL Dif -0.15087 Prob $>|t|<\quad<.0001$

Confidence $\quad 0.95$ Prob $<t<0001$

Analysis of Variance

Source DF Sum of Mean $\quad$ F Ratio Prob > $\begin{array}{llrrrr} & & \text { Squares } & \text { Square } & & \\ \text { HT } & 1 & 0.03990118 & 0.039901 & 1371.798 & <.0001 \\ \text { Error } & 4 & 0.00011635 & 0.000029 & & \end{array}$ C.

$\begin{array}{ll}\text { Means for Anova } \\ \text { Level } & \text { Number } \quad \text { Mean Std Lower }\end{array}$

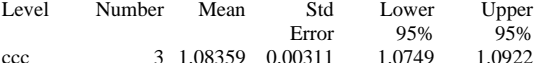

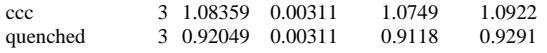

Std Error uses a pooled estimate of error variance

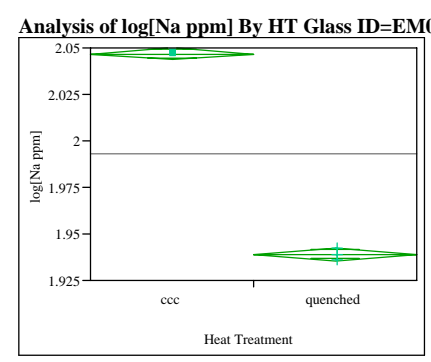

Anova

Summary of Fit

$\begin{array}{ll}\text { Rsquare } & 0.999005 \\ \text { Adj Rsquare } & 0.998757\end{array}$

Root Mean Square Error $\quad 0.002085$

$\begin{array}{lr}\text { Mean of Response } & 1.992908 \\ \text { Observations (or Sum Wgts) } & 6\end{array}$

t Test

quenched-ccc

Assuming equal variances

Difference $\quad-0.10788$ t Ratio $\quad-63.3818$ Upper CL Dif -0.10316 Prob $>|t|$ Lower CL Dif -0.11261 Prob $>t \quad 1.0000$ $\begin{array}{lll}\text { Confidence } \quad 0.95 \text { Prob }<t \quad<.0001 & 0\end{array}$

\section{Analysis of Variance}

$\begin{array}{lllll} & & \\ \text { Source DF } & \text { Sum of Mean } & \text { F Ratio Prob }>\end{array}$ $\begin{array}{llrrrrr} & & \text { Squares } & \text { Square } & \text { F } \\ \text { HT } & 1 & 0.01745846 & 0.017458 & 4017.253 & <.0001\end{array}$ $\begin{array}{lll}\text { Error } \quad 4 & 0.00001738\end{array}$

$\begin{array}{llll}\text { Means for Anova } & & \\ \text { Level Number Mean Std Lower Upper }\end{array}$ $\begin{array}{lrrrrr} & & & \text { Error } & 95 \% & 95 \% \\ \text { ccc } & 3 & 2.04685 & 0.00120 & 2.0435 & 2.0502\end{array}$ Std Error uses a pooled estimate of error variance

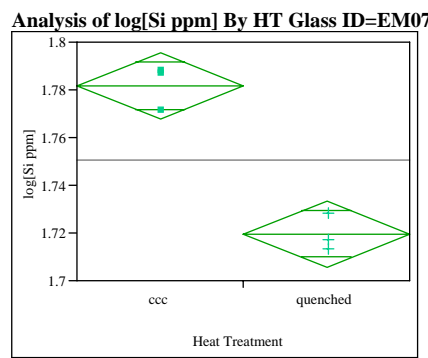

Anova

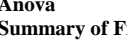

Rsquare
Adj Rsquare

$\begin{array}{ll}\text { Adj Rsquare } & 0.950733 \\ \text { Root Mean Square Eno } & 0.938416\end{array}$ Mean of Response $\quad \begin{array}{ll}0.008648 \\ 1750679\end{array}$ Observations (or Sum Wgts)

t Test

(1)

Assuming equal variances

$\begin{array}{lll}\text { Difference } & -0.06204 \text { t Ratio } & -8.78577 \\ \text { Std } & -0.00726 & \end{array}$ Std Err Dif 0.00706 DF 10.8 Lower CL Dif - -0.08164 Prob $>t \quad 0.00995$ Confidence $\quad 0.95$ Prob $<\mathrm{t} \quad 0.0005$

\section{Analysis of Variance}

$\begin{array}{llll}\text { DF Sum of Mean F Ratio Prob > } & \end{array}$ $\begin{array}{llrrrr} & & \text { Squares } & \text { Square } & \text { F } \\ \text { HT } & 1 & 0.00577258 & 0.005773 & 77.1898 & 0.0009\end{array}$ $\begin{array}{lllll}\text { Error } & 4 & 0.00029914 & 0.000075 \\ \text { C. } & 5 & 0.00607172 & \end{array}$

Means for Anova
Level Number Mean Std Lower Upper $\begin{array}{lrrrrr} & & & \text { Error } & \text { Lower } & \text { Upper } \\ \text { ccc } & 3 & 1.78170 & 0.00499 & 1.7678 & 1.7956 \\ & 3 & 1.7196 & 0.00499 & 1.7058 & 1.7335\end{array}$

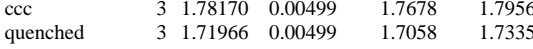
Std Error uses a pooled estimate of error variance 
Figure B6. Effects of Heat Treatment (HT) on PCT log(ppm)-Response of Study Glasses

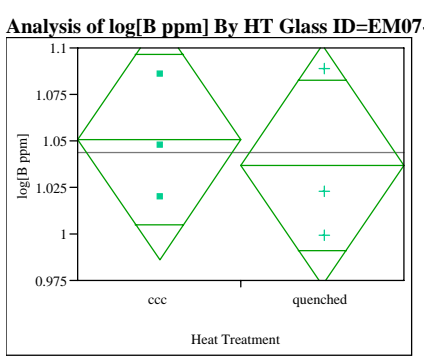

Anova

Summary of Fit

Rsquare
Adj Rsquare

Root Mean Square Error $\quad 0.040325$

Mean of Response 1.043697

6

t Test

quenched-ccc

Assuming equal variances

Difference $\quad-0.01366$ t Ratio $\quad-0.41503$

Lower CL Dif -0.10508 Prob $>t \quad 0.6503$

$\begin{array}{lll}\text { Confidence } & 0.95 \text { Prob }<\mathrm{t} \quad 0.3497\end{array}$

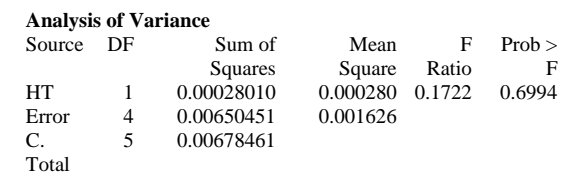

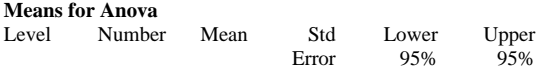

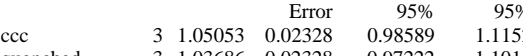

$\begin{array}{lllll}\text { quenched } & 3 & 1.03686 & 0.02328 & 0.9722\end{array}$

Std Error uses a pooled estimate of error variance

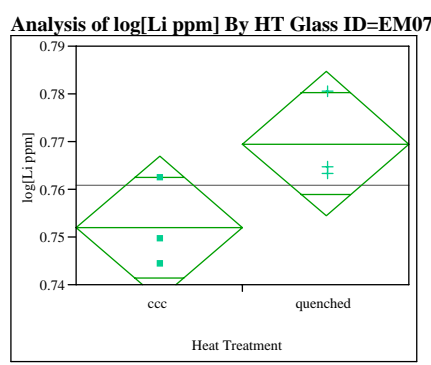

Anova

Summary of $F$

Rsquare

$\begin{array}{ll}\text { Adj Rsquare } & 0.568289 \\ \text { Root Mean Square Error } & 0.460362\end{array}$

Mean of Response 0.76077

Observations (or Sum Wgts)

t Test
quenched-ccc

Assuming equal variances

$\begin{array}{lll}\text { Difference } & 0.01757 \text { t Ratio } \quad 2.29466\end{array}$ Std Err Dif $0.00766 \mathrm{DF}$ $\begin{array}{lll}\text { Lower CL Dif }-0.00369 & \text { Prob }>t & 0.0417\end{array}$ Confidence $\quad 0.95$ Prob $<\mathrm{t} \quad 0.9583$

$\begin{array}{llrrr}\text { Source DF Sum of Mean } & \text { F } & \text { Prob }>\end{array}$ $\begin{array}{llllll}\text { HT } & 1 & 0.00046305 & 0.000463 & 5.2655 & 0.0834 \\ \text { Error } & 4 & 0.00035176 & 0.000088 & & \end{array}$ C.

Means for Anova
Level Number Mean Std Lower Upper

$\begin{array}{lrrrrr} & & \text { Error } & 95 \% & 95 \% \\ \text { ccc } & 3 & 0.751992 & 0.00541 & 0.73696 & 0.7670 \\ & 3 & 0.76562 & & & \end{array}$

$\begin{array}{lllllll}\text { quenched } & 3 & 0.769562 & 0.00541 & 0.75453 & 0.78459\end{array}$

Std Error uses a pooled estimate of error variance

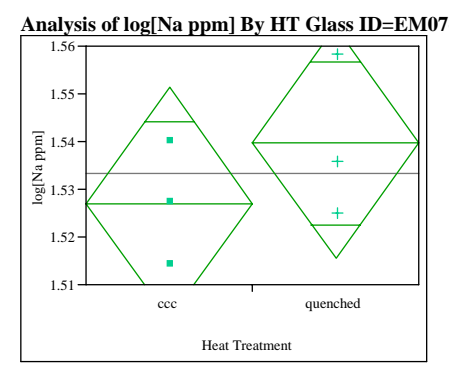

Anova

Anova
Summary of Fit

Rsquare

$\begin{array}{ll}\text { Adj Rsquare } & 0.00984 \\ \text { Root Mean Square Error } & 0.015083\end{array}$ $\begin{array}{lr}\text { Mean of Response } & 1.53339 \\ \text { Observations (or Sum Wgts) } & 6\end{array}$

t Test

nched-ccc

Assuming equal variances

$\begin{array}{llll}\text { Difference } & 0.01262 & \text { tRatio } & 1.024557\end{array}$ Std Err Dif 0.01231 DF $\quad 4$ $\begin{array}{llll} & \\ \text { Lower CL Dif }-0.02157 & \text { Prob }>t & 0.3635 \\ \text { L } & 0.9517\end{array}$ Confidence $\quad 0.95$ Prob $<\mathrm{t} 0.8183$

\section{Analysis of Varianc}

$\begin{array}{llrrr}\text { Source DF Sum of Mean } & \text { F } & \text { Prob > }\end{array}$ \begin{tabular}{llr} 
HT & 1 & 0.00023880 \\
\hline
\end{tabular}

\begin{tabular}{lll} 
Error & 4 & 0.00090995 \\
C. & 5 & 0.00114875 \\
\hline
\end{tabular} $\begin{array}{rrr}\text { Square } & \text { Ratio } & \mathrm{F} \\ 0.000239 & 1.0497 & 0.3635\end{array}$ 0.000227

Tota

Level Number Mean Std Lower Upper

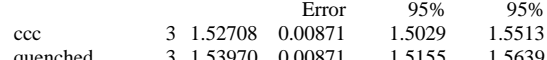

Std Error uses a pooled estimate of error variance

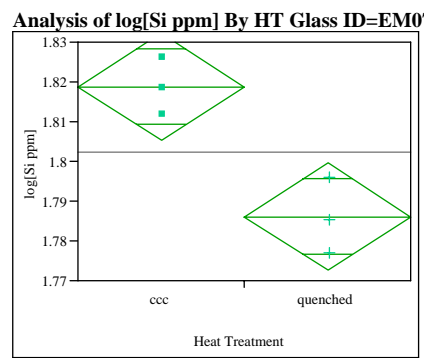

Anova

Summary of $F$

Rsquare
Adj Rsquare

$\begin{array}{ll}\text { Adj Rsquare } & 0.850575 \\ \text { Root Mean Square Enor } & 0.813219\end{array}$ Mean of Response $\quad 1.00242$

Observations (or Sum Wgts)

t Test
quenched-ccc

Assuming equal variances

$\begin{array}{lll}\text { Difference } & -0.03272 \text { t Ratio } & -4.77172\end{array}$ Std Err Dif 0.00686 DF 10.47 Lower CL Dif -0.05176 Prob > $t$ Confidence $\quad 0.95$ Prob $<\mathrm{t} \quad 0.0044$

\section{Analysis of Variance}

\begin{tabular}{ll} 
Sum of Mean F Ratio Prob > \\
\hline D
\end{tabular} $\begin{array}{llrrrr} & & \text { Squares } & \text { Square } & & \\ \text { HT } & 1 & 0.00160609 & 0.001606 & 22.7693 & 0.0088\end{array}$ $\begin{array}{lll}\text { Error } & 4 & 0.00028215\end{array}$

$\begin{array}{llll}\text { C. } & 5 & 0.00188824 & 0.000071\end{array}$

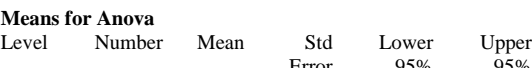

$\begin{array}{lrrrrr} & & \text { Error } & 95 \% & 95 \% \\ \text { ccc } & 3 & 1.81878 & 0.00485 & 1.8053 & 1.8322\end{array}$

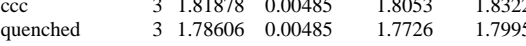

Std Error uses a pooled estimate of error variance 
Figure B6. Effects of Heat Treatment (HT) on PCT $\log (\mathrm{ppm})$-Response of Study Glasses

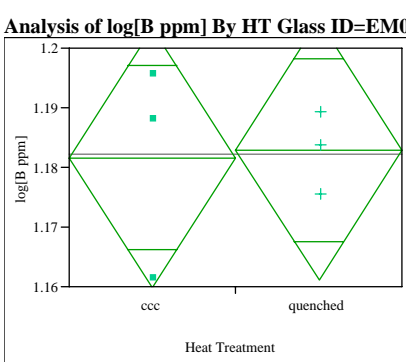

Anova

Summary of Fit

Rsquare

-0.2456
Root Mean Square Error $\quad 0.013581$ $\begin{array}{lr}\text { Mean of Response } & 1.182266 \\ \text { Observations (or Sum Wgts) } & 6\end{array}$

t Test

(Tenched-cce

Assuming equal variances

Difference $\quad 0.00132$ t Ratio $\quad 0.118815$ $\begin{array}{llll}\text { Std Err Dif } & 0.01109 \text { DF } & 4\end{array}$ Upper CL Dif 0.03210 Prob $>|t| \quad 0.911$

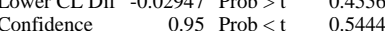

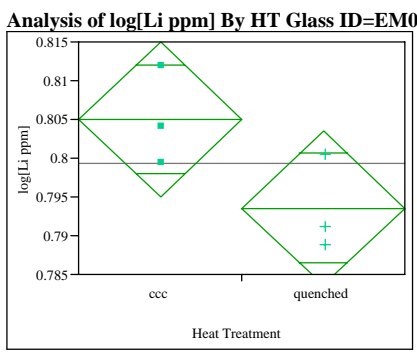

Anova

Summary of $\mathrm{Fit}$

Rsquare

Root Mean Square Error $\quad 0.006222$

Mean of Response
Observations (or Sum Wgts)

t Test

quenched-ccc

Assuming equal variances

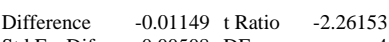

$0.00508 \mathrm{DF} \quad-2.263$

$\begin{array}{llll}\text { Upper CL Dif } & 0.00262 & \text { Prob }>|t| & 0.0865 \\ \text { Lower CL Dif }-0.02559 & \text { Pob }>t & 0.9567\end{array}$

$\begin{array}{lrl} & \\ \text { Confidence } & 0.95 \text { Prob }<t & 0.043\end{array}$

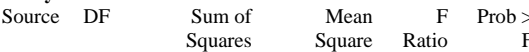

$\begin{array}{llrrrr}\text { HT } & 1 & 0.00019798 & 0.000198 & 5.1145 & 0.086\end{array}$

$\begin{array}{llll}\text { Error } & 4 & 0.00015483 & 0.000039\end{array}$

$\begin{array}{lrrrr}\text { Leans for Anova } & \text { Number } & \text { Mean } \quad \begin{array}{r}\text { Std } \\ \text { Lever }\end{array} & \begin{array}{r}\text { Lower } \\ 95 \%\end{array} & 95 \%\end{array}$

$\begin{array}{lrrrrr} & & & \text { Error } & 95 \% & 95 \% \\ \text { ccc } & 3 & 0.805026 & 0.00359 & 0.79505 & 0.81500\end{array}$

$\begin{array}{lrlll}\text { quenched } & 3 & 0.793537 & 0.00359 & 0.78356\end{array}$

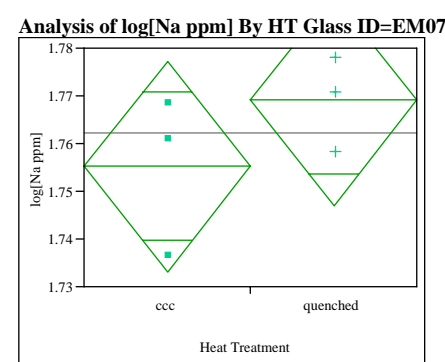

Anova

Anova
Summary of Fit

Rsquare

$\begin{array}{ll}\text { Adj Rsquare } & 0.276125 \\ \text { Root Mean Square Error } & 0.095156 \\ 0.013781\end{array}$ Mean of Response $\quad 1.762196$ Observations (or Sum Wgts) $\quad 6$

t Test
quenched-ccc

Assuming equal variances

Difference $\quad 0.01390$ t Ratio $\quad 1.235238$ $\begin{array}{llll}\text { Std Err Dif } & 0.01125 \text { DF } & 4 \\ \text { Uper CL Dif } & 0.04514 \text { Prob }> & 0.283\end{array}$

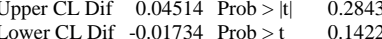
Confidence $\quad 0.95$ Prob $<\mathrm{t} \quad 0.8578$

\section{Analysis of Variance}

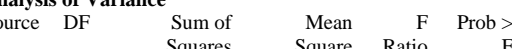
\begin{tabular}{llr} 
HT & 1 & 0.00028978 \\
\hline
\end{tabular} $\begin{array}{lll}\text { Error } & 4 & 0.00075968 \\ \mathrm{C} & 5 & 0.00104946\end{array}$

Means for Anova

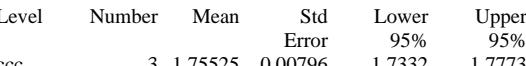

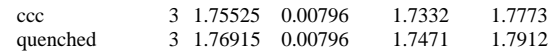
Std Error uses a pooled estimate of error variance

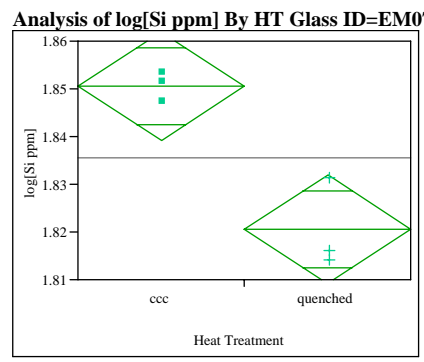

Anova

Anova
Summary of Fit

Rsquare
Adj Rsquare

$\begin{array}{ll}\text { Adj Rsquare } & 0.871367 \\ \text { Root Mean Square } & 0839209\end{array}$ Mean of Response $\quad 0.007059$

Observations (or Sum Wgts)

t Test
quenched-ccc

Assuming equal variances

Difference $\quad-0.03000 \quad t$ Ratio $\quad-5.2054$ $\begin{array}{lll}\text { Std Err Dif } & 0.00576 \text { DF } & 4 \\ \text { Unper CL Dif } & -0.01400 \text { Prob }>\text { tt } & 0.0065\end{array}$ Lower CL Dif -0.04600 Prob $>t \quad 0.9968$ Confidence $\quad 0.95$ Prob $<\mathrm{t} \quad 0.0032$

\section{Analysis of Variance}

$\begin{array}{llll}\text { Source DF Sum of Mean F Ratio Prob > } & \end{array}$ $\begin{array}{llrrrr} & & \text { Squares } & \text { Square } & \text { F } \\ \text { HT } & 1 & 0.00135006 & 0.001350 & 27.0962 & 0.0065\end{array}$ $\begin{array}{lllll} & \text { Error } & 4 & 0.00019930 & 0.000050 \\ \text { C. } & 5 & 0.00154936 & \end{array}$

Means for Anova

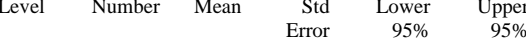
$\begin{array}{llllrr}\text { ccc } & 3 & 1.85058 & 0.00408 & 1.8393 & 1.8619 \\ \text { quenched } & 3 & 1.82058 & 0.00408 & & \end{array}$ $\begin{array}{llllll}\text { quenched } & 3 & 1.82058 & 0.00408 & 1.8093 & 1.8319\end{array}$

Std Error uses a pooled estimate of error variance 
Figure B6. Effects of Heat Treatment (HT) on PCT $\log (\mathrm{ppm})$-Response of Study Glasses

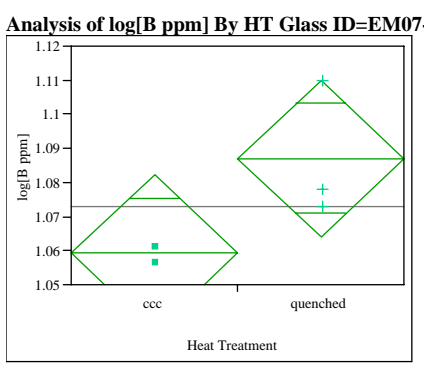

Anov

Summary of Fit

Rsquare

$\begin{array}{lr}\text { Mean of Response } & 1.073138 \\ \text { Observations (or Sum Wgts) } & 6\end{array}$

t Test

quenched-cce

Assuming equal variances

Difference $\quad 0.02781$ t Ratio $\quad 2.382349$ $\begin{array}{llll}\text { Std Err Dif } & 0.01168 & \mathrm{DF} & 4 \\ \text { Unper CL Dif } & 0.06023 & \text { Prob }>\text { tt } & 0.0758\end{array}$

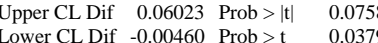
$\begin{array}{llll}\text { Confidence } & -0.00465 \text { Prob }<t \quad 0.9621\end{array}$

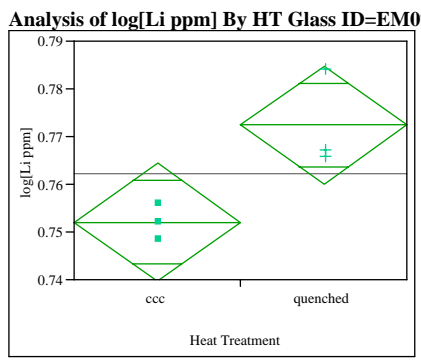

Anova

Summary of $\mathrm{Fi}$

Rsquare

$\begin{array}{ll}\text { Adj Rsquare } & 0.724231 \\ \text { Root Mean Square Error } & 0.65288\end{array}$ Mean of Response $\quad 0.7622$

Observations (or Sum Wgts)

t Test
quenched-ccc

Assuming equal variances

Difference $\quad 0.020368$ tRatio $\quad 3.24112$ $\begin{array}{llll}\text { Std Err Dif } 0.006284 & \mathrm{DF} & 4\end{array}$ Lower CL Dif 0.002920 Prob $>t \quad 0.015$ Confidence $\quad 0.95 \mathrm{Prob}<\mathrm{t} \quad 0.9842$
Analysis of Variance

\begin{tabular}{|c|c|c|c|c|c|c|c|c|c|c|c|}
\hline \multicolumn{6}{|c|}{ Analysis of Variance } & \multicolumn{6}{|c|}{ Analysis of Variance } \\
\hline Source & DF & $\begin{array}{l}\text { Sum of } \\
\text { Squares }\end{array}$ & $\begin{array}{l}\text { Mean } \\
\text { Square }\end{array}$ & $\begin{array}{r}F \\
\text { Ratio }\end{array}$ & $\begin{array}{r}\text { Prob > } \\
\text { F }\end{array}$ & Source D & DF & $\begin{array}{l}\text { Sum of } \\
\text { Squares }\end{array}$ & $\begin{array}{l}\text { Mean } \\
\text { Square }\end{array}$ & F Ratio & $\begin{array}{r}\text { Prob > } \\
\text { F }\end{array}$ \\
\hline HT & 1 & .00116047 & 0.001160 & 5.6756 & 0.0758 & HT & 1 & 0.00062226 & 0.000622 & 10.5049 & 0.0316 \\
\hline Error & 4 & .00081787 & 0.000204 & & & Error & 4 & 0.00023694 & 0.000059 & & \\
\hline C. & 5 & .00197833 & & & & C. & & 0.00085920 & & & \\
\hline & & & & & & & & & & & \\
\hline Means fo & Anova & & & & & Means for & $\mathrm{r}$ An & & & & \\
\hline Level & Number & Mean & $\begin{array}{r}\text { Std } \\
\text { Error }\end{array}$ & $\begin{array}{r}\text { Lower } \\
95 \%\end{array}$ & $\begin{array}{r}\text { Upper } \\
95 \%\end{array}$ & Level & Number & Mean & $\begin{array}{r}\text { Std } \\
\text { Error }\end{array}$ & $\begin{array}{r}\text { Lower } \\
95 \%\end{array}$ & $\begin{array}{l}\text { Upper } \\
95 \%\end{array}$ \\
\hline $\mathrm{ccc}$ & & 1.05923 & 0.00826 & 1.0363 & 1.0822 & $\mathrm{ccc}$ & & 30.752046 & 0.00444 & 0.73971 & 0.76438 \\
\hline uenched & & 1.08704 & 0.00826 & 1.0641 & 1.1100 & quenched & & 30.772413 & 0.00444 & 0.76008 & 0.78475 \\
\hline
\end{tabular}

Std Error uses a pooled estimate of error variance
Std Error uses a pooled estimate of error variance

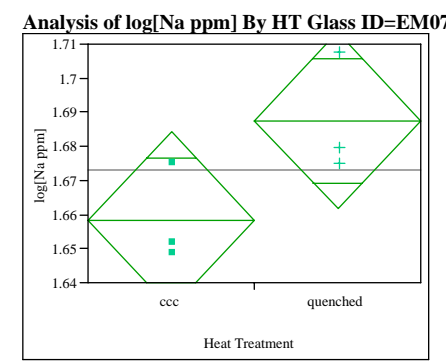

Anova

Summary of Fit

Rsquare
Adj Rsquare

$\begin{array}{ll}\text { Adj Rsquare } & 0.436543 \\ \text { Root Mean Square Error } & 0.016151\end{array}$ $\begin{array}{lr}\text { Mean of Response } & 1.672941 \\ \text { Observations (or Sum Wgts) } & 6\end{array}$

t Test

Assuming equal variances

$\begin{array}{llll}\text { Difference } & 0.02911 \text { t Ratio } \quad 2.207667\end{array}$ $\begin{array}{lll}\text { Std Err Dif } & 0.01319 \text { DF } & 4 \\ & & \end{array}$ Lower CL Dif -0.00750 Prob $>t \quad 0.0459$ Confidence $\quad 0.95$ Prob $<\mathrm{t} \quad 0.9541$

Analysis of Variance

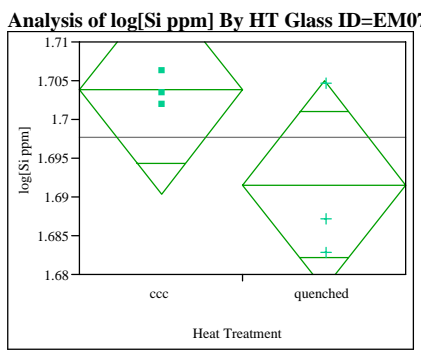

Anova

Summary of Fit

$\begin{array}{lr}\text { Rsquare } & 0.44426 \\ \text { Adj Rsquare } & 0.305325\end{array}$

Root Mean Square Error $\quad 0.008355$

Mean of Response 1.697675

t Test
quenched-ccc

Assuming equal variances

Difference $\quad-0.01220$ t Ratio $\quad-1.78819$ Upper CL Dif 0.00674 Prob > It 0.148 Lower CL Dif -0.03114 Prob $>t \quad 0.9259$ Confidence $\quad 0.95$ Prob $<\mathrm{t} \quad 0.074$

\begin{tabular}{|c|c|c|c|c|c|c|}
\hline \multicolumn{7}{|c|}{ malysis or variance } \\
\hline Source & DF & & $\begin{array}{l}\text { Sum of } \\
\text { Squares }\end{array}$ & $\begin{array}{r}\text { Mear } \\
\text { Square }\end{array}$ & $\begin{array}{rr}n & \text { F } \\
\text { e } & \text { Ratio }\end{array}$ & \\
\hline HT & 1 & & .00127137 & 0.00127 & $\begin{array}{ll}1 & 4.8738\end{array}$ & \\
\hline Error & 4 & & .00104344 & 0.000261 & & \\
\hline $\begin{array}{l}\text { C. } \\
\text { Total }\end{array}$ & 5 & & .00231481 & & & \\
\hline ans fo & $\operatorname{man}_{2}$ & & & & & \\
\hline Level & $\mathrm{Nu}$ & & Mean & $\begin{array}{r}\text { Std } \\
\text { Frror }\end{array}$ & $\begin{array}{l}\text { Lower } \\
95 \%\end{array}$ & \\
\hline ccc & & & $1.65 \varepsilon$ & $\begin{array}{l}\text { Error } \\
0.00932\end{array}$ & $\begin{array}{r}95 \% \\
1.6325\end{array}$ & \\
\hline quenched & & & 1.68750 & 0.00932 & 1.6616 & \\
\hline
\end{tabular}

Source DF Sum of Mean F Prob $>$ $\begin{array}{rrrrrr} & & \text { Squares } & \text { Square } & \text { Ratio } & \text { F } \\ \text { HT } & 1 & 0.00022320 & 0.000223 & 3.1976 & 0.1483\end{array}$ Error $\quad 4 \quad 0.0002792$

$\begin{array}{lllll}\text { Means for Anova } & & & \\ \text { Level Number Mean Std Lower Upper }\end{array}$

Std Error uses a pooled estimate of error variance $\begin{array}{lrrrrr} & & & \text { Error } & 95 \% & 95 \% \\ \text { ccc } & 3 & 1.70377 & 0.00482 & 1.6904 & 1.7172\end{array}$
Std Error uses a pooled estimate of error variance 


\section{Figure B7. Effects of Heat Treatment on Normalized PCT Results}

\section{Comp View=measured}

Variability Chart for $\log \mathrm{NL}[\mathrm{B}(\mathrm{g} / \mathrm{L})]$

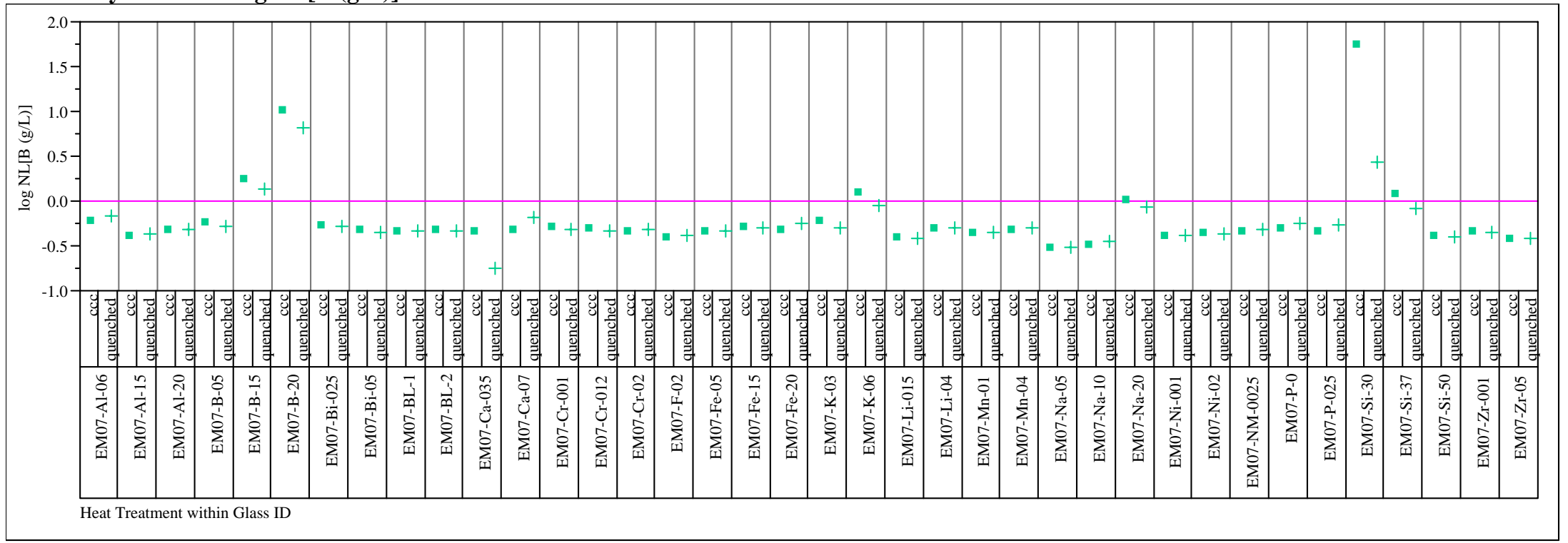




\section{Figure B7. Effects of Heat Treatment on Normalized PCT Results}

\section{Comp View=measured}

Variability Chart for $\log \mathrm{NL}[\mathrm{Li}(\mathrm{g} / \mathrm{L})]$

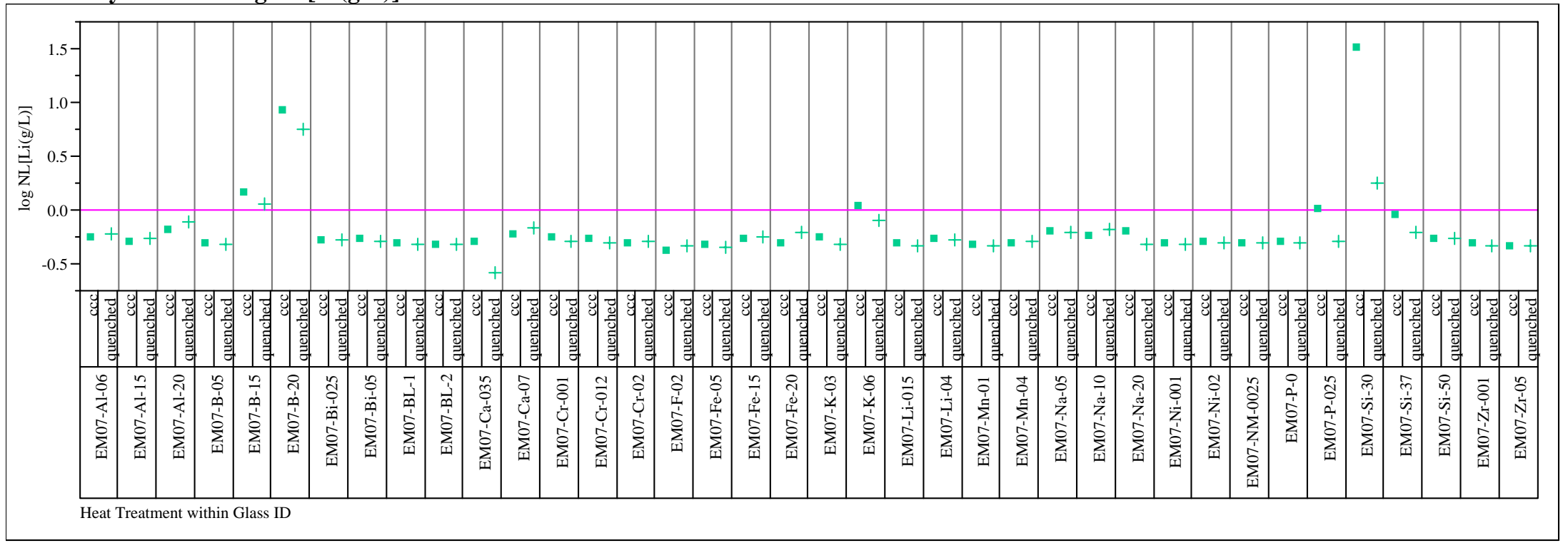




\section{Figure B7. Effects of Heat Treatment on Normalized PCT Results}

\section{Comp View=measured}

Variability Chart for $\log \mathrm{NL}[\mathrm{Na}(\mathrm{g} / \mathrm{L})]$

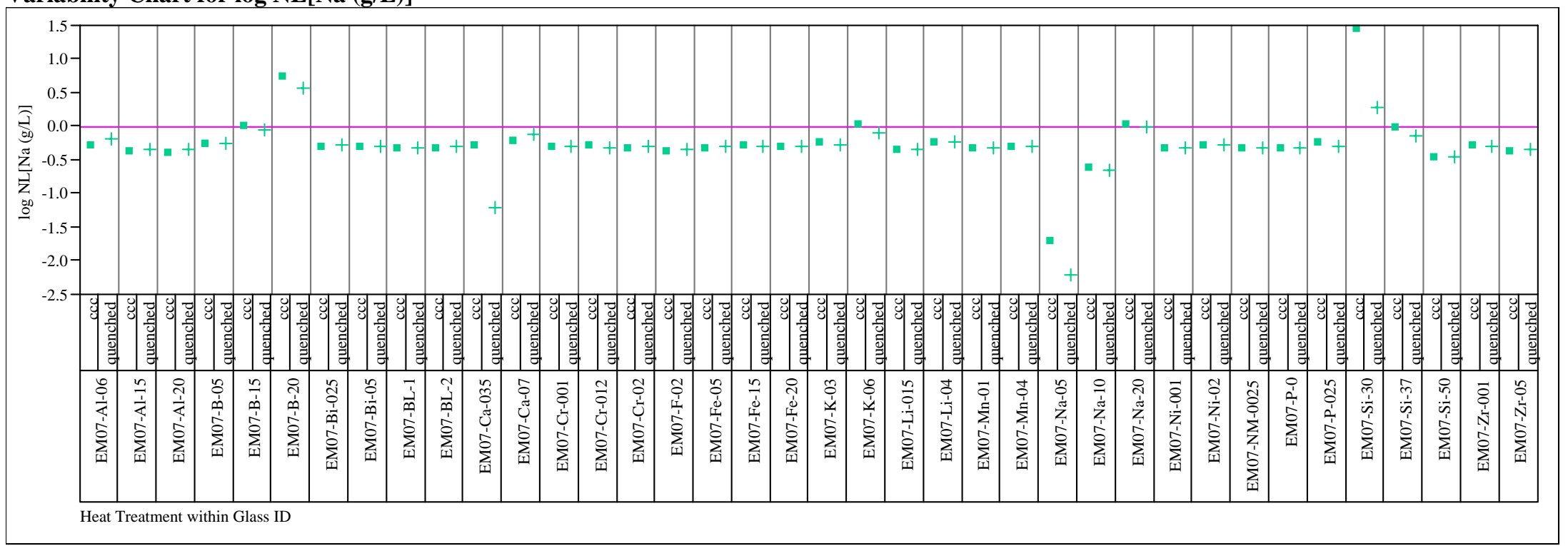




\section{Figure B7. Effects of Heat Treatment on Normalized PCT Results}

\section{Comp View=measured}

Variability Chart for $\log \mathrm{NL}[\mathrm{Si}(\mathrm{g} / \mathrm{L})]$

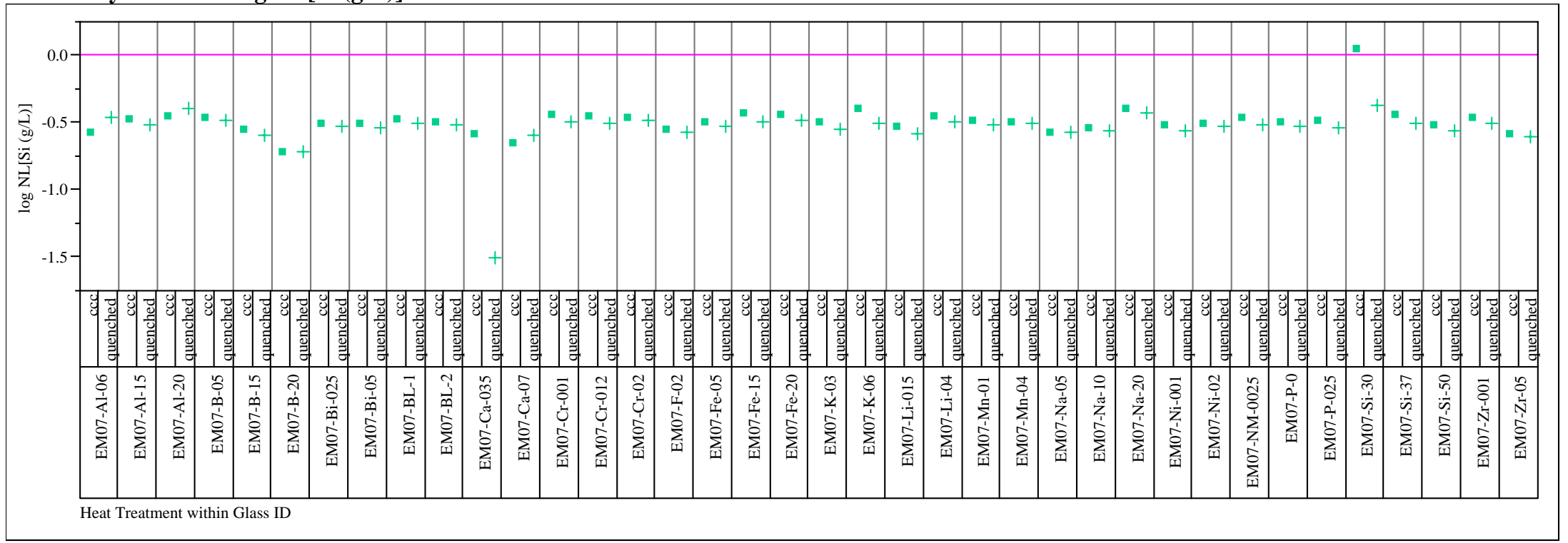


Figure B7. Effects of Heat Treatment on Normalized PCT Results

Comp View=measured bc

Variability Chart for $\log \mathrm{NL}[\mathrm{B}(\mathrm{g} / \mathrm{L})]$

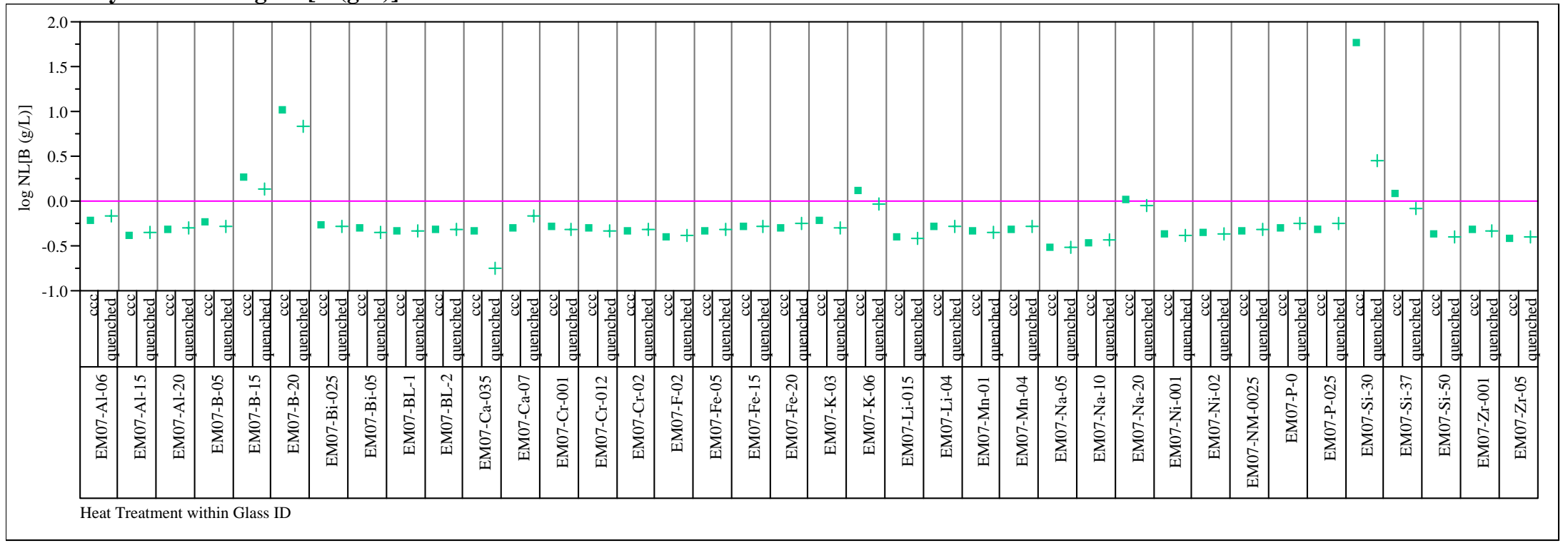




\section{Figure B7. Effects of Heat Treatment on Normalized PCT Results}

\section{Comp View=measured bc}

Variability Chart for $\log \mathrm{NL}[\mathrm{Li}(\mathrm{g} / \mathrm{L})]$

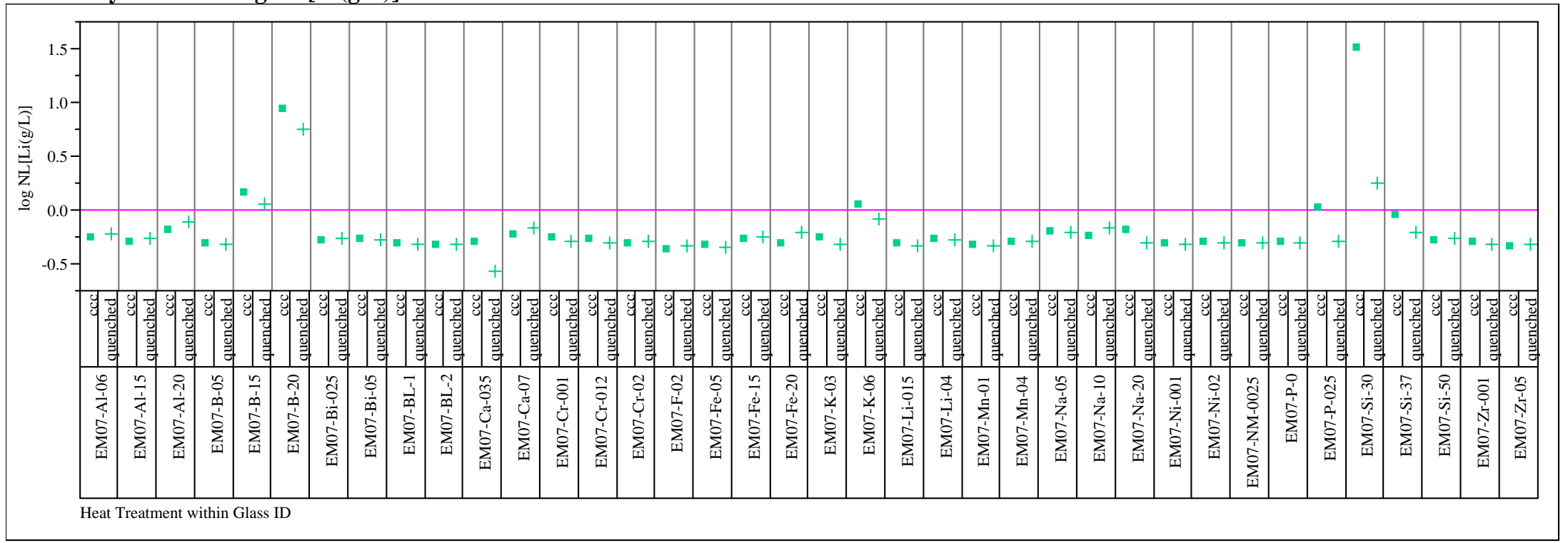




\section{Figure B7. Effects of Heat Treatment on Normalized PCT Results}

\section{Comp View=measured bc}

Variability Chart for log NL[Na (g/L)]

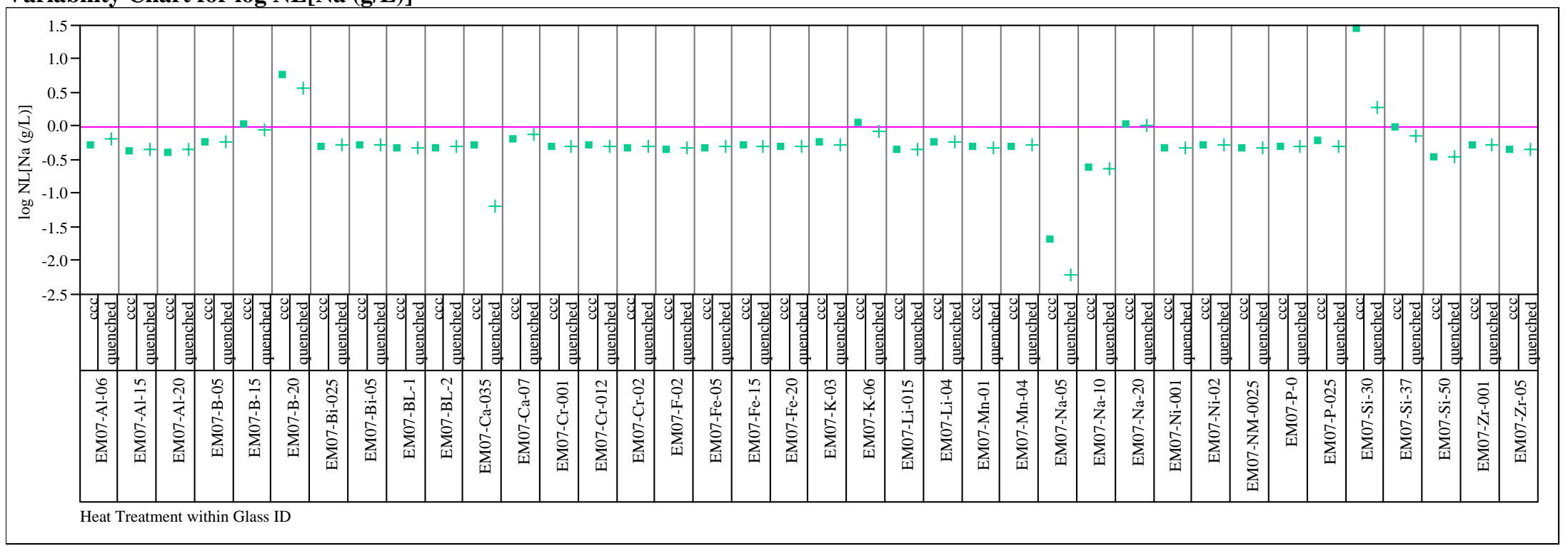




\section{Figure B7. Effects of Heat Treatment on Normalized PCT Results}

\section{Comp View=measured bc}

Variability Chart for $\log \mathrm{NL}[\mathrm{Si}(\mathrm{g} / \mathrm{L})]$

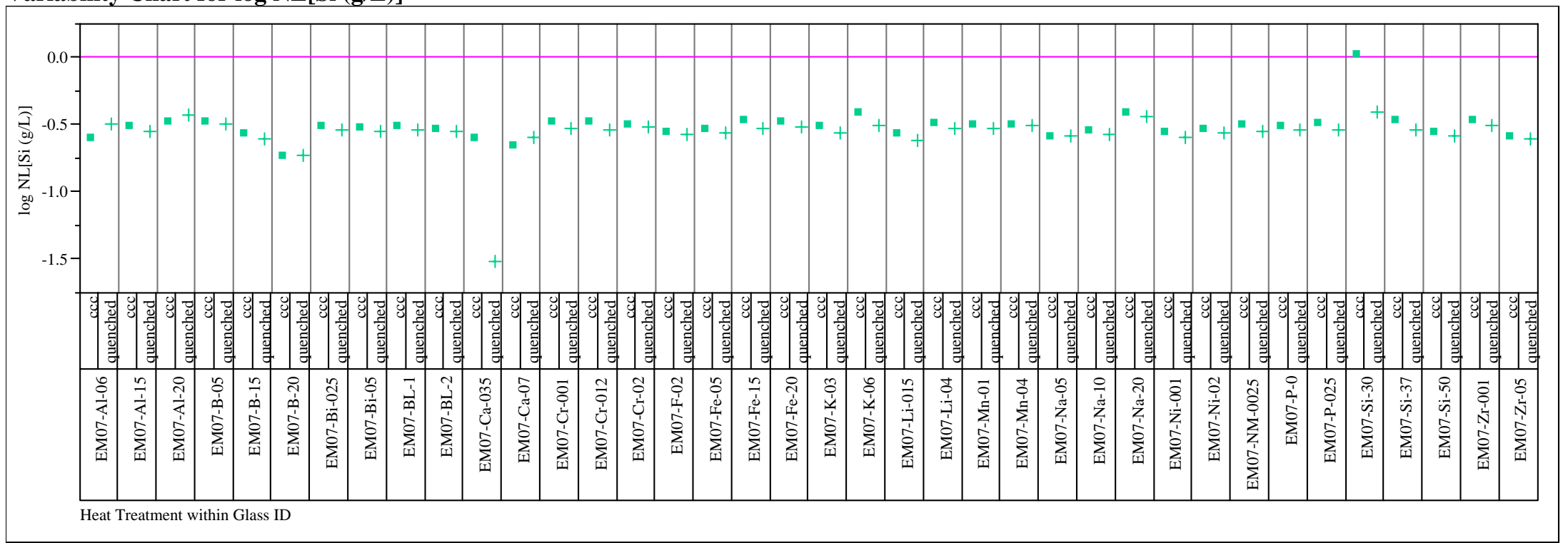




\section{Figure B7. Effects of Heat Treatment on Normalized PCT Results}

\section{Comp View=targeted}

Variability Chart for $\log \mathrm{NL}[\mathrm{B}(\mathrm{g} / \mathrm{L})]$

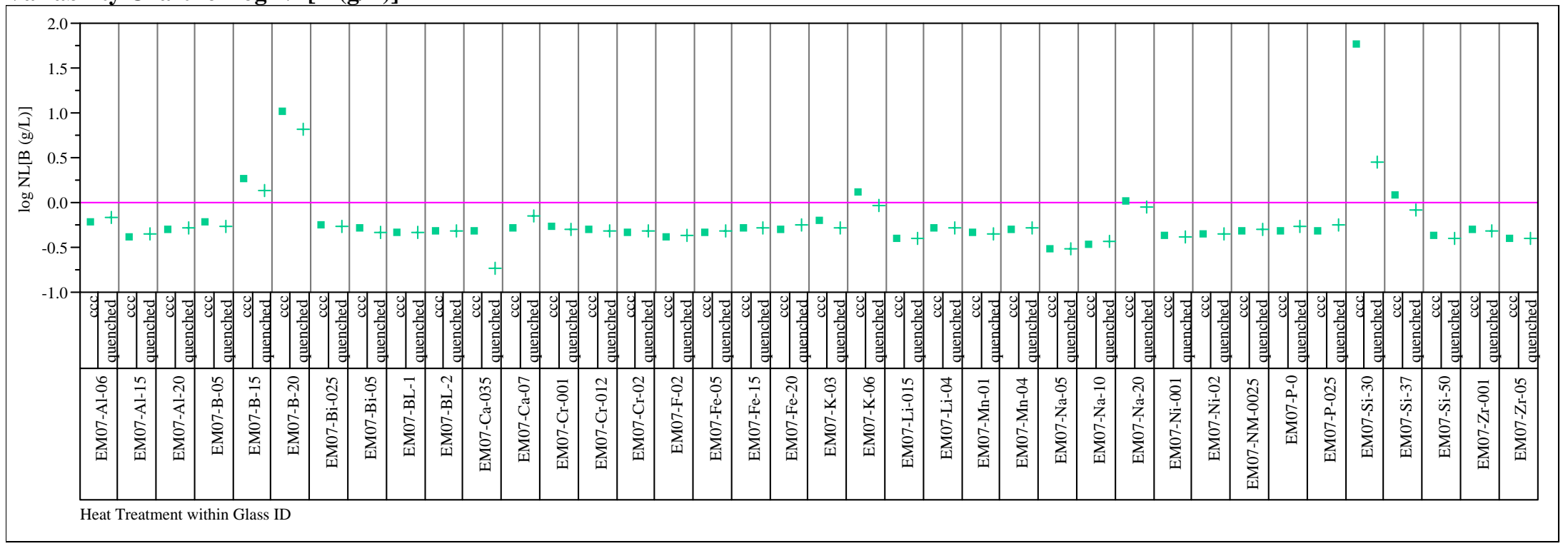




\section{Figure B7. Effects of Heat Treatment on Normalized PCT Results}

\section{Comp View=targeted}

Variability Chart for $\log \mathrm{NL}[\mathrm{Li}(\mathrm{g} / \mathrm{L})]$

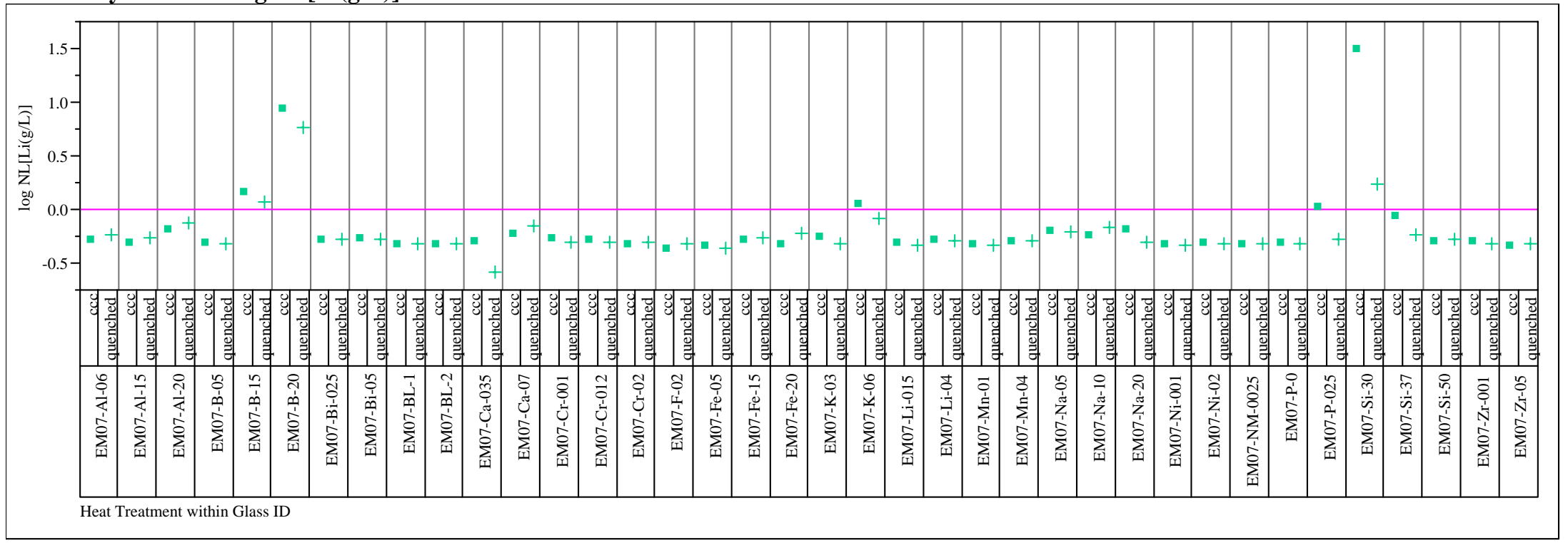




\section{Figure B7. Effects of Heat Treatment on Normalized PCT Results}

\section{Comp View=targeted}

Variability Chart for log NL[Na (g/L)]

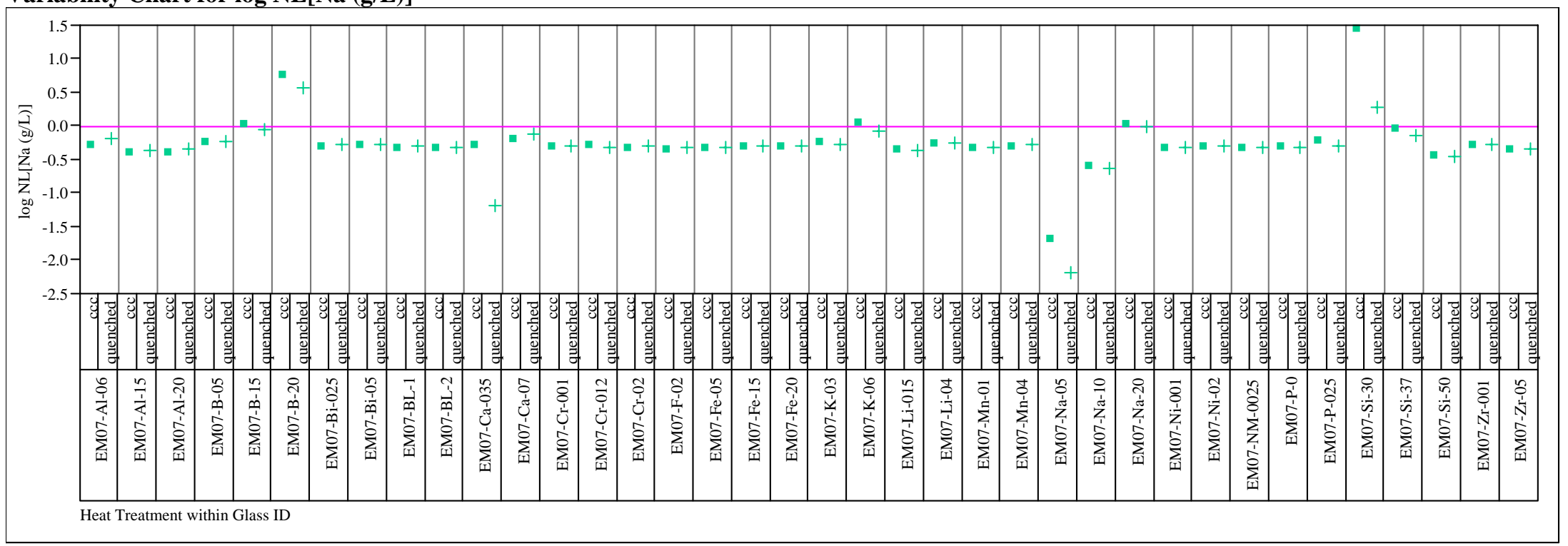




\section{Figure B7. Effects of Heat Treatment on Normalized PCT Results}

\section{Comp View=targeted}

Variability Chart for $\log \mathrm{NL}[\mathrm{Si}(\mathrm{g} / \mathrm{L})]$

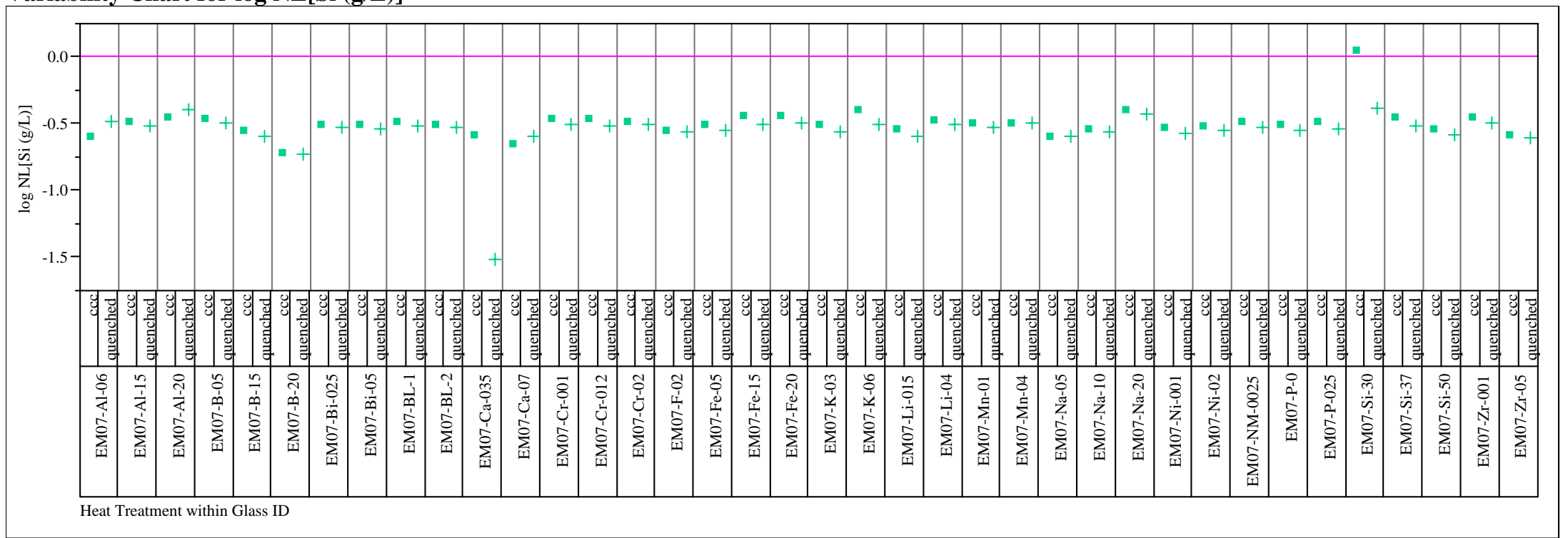




\section{Distribution}
A.B. Barnes, 999-W
D.R. Best, 999-W
T.B. Edwards, 999-W
K.M. Fox, 999-W
J.C. Griffin, 773-A
C.C. Herman, 999-W
J.E. Marra, 773-A
T.A. Nance, 773-42A
D.K. Peeler, 999-W
F.C. Raszewski, 999-W
P.C. Suggs, 766-H
J.P. Vaughan, 773-41A
PNNL
J. Crum
P. Hrma
D-S. Kim
B. Riley
C. Rodriguez
M. Schweiger
J. Vienna

$\underline{\text { DOE-HQ }}$

T. Chee

K. Gerdes 